\title{
Lipid Concentrations in Standard Reference Material (SRM) 1950: Results from an Interlaboratory Comparison Exercise for Lipidomics
}

John A. Bowden

Alan Heckert

Candice Z. Ulmer

Christina M. Jones 
NISTIR 8185

\section{Lipid Concentrations in Standard Reference Material (SRM) 1950: Results from an Interlaboratory Comparison Exercise for Lipidomics}

John A. Bowden Candice Z. Ulmer Christina M. Jones

Chemical Sciences Division Material Measurement Laboratory

Alan Heckert Statistical Engineering Division Information Technology Laboratory

This publication is available free of charge from:

https://doi.org/10.6028/NIST.IR.8185

August 2017

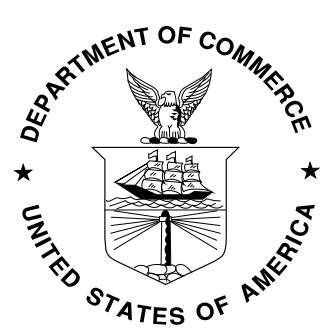

U.S. Department of Commerce Wilbur L. Ross, Jr., Secretary 


\section{OVERVIEW OF EXERCISE}

The continued growth of the lipidomics research community, combined with a concomitant increase in the number of lipidomic applications, has culminated in an emerging need for the harmonization and standardization of lipidomics measurement. Harmonization and standardization of lipidomic measurement is a considerable undertaking, owing to the vast structural diversity and complexity of lipids, which also subsequently coincides with the use of a broad range of qualitative and quantitative measurement strategies employed by the lipidomics community. The lipidomics community needs to address the variability present in current lipidomics measurement before harmonization and standardization can begin to occur. Accordingly, this work encompasses the first community-supported harmonization effort via an interlaboratory comparison exercise, focused on ascertaining sources of lipidomic measurement variability and/or agreement, while also highlighting measurement challenges in regards to lipid quantitation.

The main objectives of the interlaboratory comparison exercise were to 1) generate consensus estimates in $\mathrm{nmol} / \mathrm{mL}$ for those lipids routinely measured by participants, 2) determine the extent of agreement present within the community using current quantitation lipidomics workflows, 3) and identify those lipids or lipid classes that require more attention. The basic framework of the National Institute of Standards and Technology (NIST) interlaboratory comparison for lipidomics was to distribute one vial of Standard Reference Material (SRM) 1950 - Metabolites in Frozen Human Plasma to each participating laboratory, and to encourage each participant to employ the analytical methodologies that they typically use to quantify lipids in their laboratory. SRM 1950 was chosen as the vehicle for the comparison exercise as it has been previously recognized and promoted as an appropriate reference material for metabolomics (1-5). In addition, SRM 1950 was constructed to approximate "normal" blood plasma indicative of the United States population (see http://srm1950.nist.gov/). Invitations were sent to a cohort of laboratories that were representative of the diverse cross-section of lipid measurement methodologies present within the lipidomics community. Consensus estimates (at sum composition level), with corresponding uncertainties, were generated for those lipids measured by at least five laboratories. Additional analyses were performed to further assess the collective submitted data, including coefficient of dispersion (COD) for each consensus estimate and zetascores ( $\zeta$-scores). COD values were used to evaluate the quality or "usefulness" of the consensus estimates. $\zeta$-scores were used to determine the relative measurement agreement amongst the consensus estimates by lipid species and lipid class.

The final consensus estimates and associated uncertainties generated from this exercise hold considerable potential for the lipidomics community, both to serve as inter- and intralaboratory benchmarks but also to initiate follow-up efforts to continue measurement harmonization within the lipidomics community. 


\section{MATERIALS AND METHODS}

\section{Guidelines for Laboratory Participation}

Upon receipt of SRM 1950, each laboratory was instructed to identify and quantify those lipids that they routinely measure in their laboratories. We required that the lipids be quantitatively measured in triplicate and the final lipid concentration reported for each replicate in $\mathrm{nmol} / \mathrm{mL}$ plasma. To aid in the data submission process, an Excel template was provided (Appendix A) that included comment boxes to record method information (e.g., laboratory profile, sample preparation/extraction, sample introduction and chromatography, mass spectrometric analysis, and data handling/processing) and tabs for each potential target lipid class present in SRM 1950. Possible lipid classes included ceramides (CER), cholesteryl esters (CE), diacylglycerols (DAG), free fatty acids (FFA), lysophosphatidylcholines (LPC), lysophosphatidylethanolamines (LPE), phosphatidic acids (PA), phosphatidylcholines (PC), phosphatidylethanolamines (PE), phosphatidylglycerols (PG), phosphatidylinositols (PI), phosphatidylserines (PS), sphingomyelins (SM), and triacylglycerols (TAG). The target list for each lipid class consisted of potential individual lipid species. In addition, an "other" tab was included, which allowed for the submission of bile acids (BA) and eicosanoids. We also encouraged laboratories to provide values for lipids not included in the target lists. In total, 320 lipid species (designated by the sum composition annotation, lipid class and total carbons (C) and degrees of unsaturation in the fatty acyl chains, DB) were listed as potential targets in the submission template (i.e., lipid species with a high probability to be present in SRM 1950 based on the previous LIPID MAPS consortium report (6)). The 320 target lipid species are listed in Appendix A.

\section{Handling of Submitted Lipidomic Data}

Lipid identifications and quantitative measurements of lipid species in triplicate for each participating laboratory were submitted to NIST via the provided data template. Each laboratory was given a random laboratory identification number. Therefore, the laboratory identification number is not correlated with the order of the participants listed in this document. For each laboratory submission, mean and standard deviation calculations were performed for each lipid species submitted with three replicate concentrations above zero. Only the lipid species with mean and standard deviation calculated were included in the final consensus estimation (unless otherwise indicated). Data was validated, with respect to proper annotation, $\mathrm{m} / \mathrm{z}$ value, and adduct measured using LipidPioneer (7). Any corrections to the original laboratory submission were sent to each laboratory for approval. We received data from a diverse collection of participating laboratories, encompassing a wide range of lipidomic methodologies (see Table 1), thus we obtained lipid data annotated at the sum compositional level and/or the fatty acyl level. To allow for comparison amongst all laboratory submissions, all lipid data were converted to sum composition level. Thus, the final lipid concentration for each lipid was calculated by summing all the isomers for each replicate, then calculating the average and standard deviation of the summed triplicate concentrations. A lipid isomer was included in the summation if it was reported by at least two laboratories. 


\section{Calculation of Final Consensus Locations and Uncertainties}

Lipid consensus estimates and associated uncertainties were determined using three methods, the Vangel-Rukhin (VR), the DerSimonian-Laird (DSL), and the median of means (MEDM). The VR method $(8,9)$ is a weighted mean based on maximum likelihood. The derivation and mathematical details of the VR mean and its associated standard uncertainty are given in a previous publication $(8,9)$. This method incorporates both inter-laboratory and intra-laboratory variances in determining the weights. Maximum likelihood approaches have excellent statistical properties; however, the VR method is not robust against extreme outliers. In these cases, the VR weighting method gives the outlying laboratory value greater influence and results in unrepresentative estimates (i.e., the resulting value reflects neither the non-outlying values or the outlying value). Thus, to obtain a reasonable mean using this approach, the extreme outlying lipid values would need to be omitted from the estimation. The VR means were calculated with no outliers omitted.

The DSL method (10) is a weighted mean based on the method of moments. The associated standard uncertainties for the DSL mean were determined using several approaches, including the original method (10), a parametric bootstrap (11), and the Horn-Horn Duncan (HHD) (12). As with VR, DSL incorporates both inter- and intra-laboratory variances in determining the weights. However, the DSL weighting scheme is more dependent on the intra-laboratory variances than the VR method. Thus, the DSL method is more robust against extreme outliers in the laboratory means if the associated variance of the outlying laboratory is large relative to the variances of the nonoutlying laboratories. When the variance of the extreme outlier is not significantly greater than the variances of the non-outlying laboratories, this robustness breaks down. In these cases, as with VR, these extreme outlier laboratories would need to be omitted to obtain a reasonable mean estimate. Another consequence of the greater emphasis on intra-laboratory variances is that when the smallest or largest laboratory means, excluding the extreme outliers, had significantly smaller variances than the other laboratories, the resulting DSL mean may be over-weighted to these most extreme (but non-outlier) laboratory means. The DSL means were calculated with no outliers omitted.

The median of the means (MEDM) method (13) uses the median of the laboratory means as the estimate of the location. The associated uncertainty, $u$, is $\sqrt{ }(\pi / 2 m) \times 1.483 \times$ MAD, where $m$ and MAD denote the number of laboratories and the median absolute deviation of the laboratory means, respectively. The MEDM method is robust to outliers in the laboratory means regardless of the nature of the intra-laboratory variances. This method will give a "reasonable" location (i.e., half the laboratory values fall above the location and half fall below the location) without omitting any laboratories. In addition, the MEDM location estimate is less likely than VR or DSL to be distorted. The trade-off is that the MEDM method makes no use of the intra-laboratory variance (i.e., all laboratories have equal weight).

Since we aimed to include all laboratory submissions in the lipid concentration estimation, the MEDM method was chosen as it consistently generates a reasonable location value. The final locations (in $\mathrm{nmol} / \mathrm{mL}$ ) were reported using the MEDM method for those lipids measured by at least five laboratories. The consensus estimates determined using all three estimation approaches, with number of laboratories reporting and the standard uncertainty, are shown in Tables 2 to 6 . The five lipid categories reported in this study are organized as the following: fatty acyl lipids (FA), glycerolipids (GL), glycerophospholipids (GP), sphingolipids (SL), and sterol lipids (ST), 
respectively. Sample coefficient of dispersion (COD) values (14), expressed as a percentage (listed in Tables 2 to 6 ), were calculated for each lipid with $n \geq 5$ laboratories reporting using the equation: $100 * u / M E D M$. The COD values were used to assess the quality or "usefulness" of each final MEDM location. We designated lipids with a COD $<40 \%$ as acceptable for use with quality control activities.

\section{Final MEDM Location Plots}

MEDM location plots were generated for all lipids $(n=339)$ that were reported by at least five laboratories. On each plot, the calculated mean and standard deviation of the mean $(\mathrm{nmol} / \mathrm{mL})$, from the triplicate measurement made by each laboratory, is shown, as well as the calculated DSL, VR, and MEDM consensus estimates with standard uncertainties. For enhanced visualization, the location plots were often truncated on the $\mathrm{y}$-axis to remove outlying lipid concentrations from laboratories. The final selected MEDM location and associated uncertainty for each lipid is also provided on the plot.

\section{Calculation of Zeta-Scores}

Zeta( $\zeta$ )-scores were calculated for each lipid ( $n \geq 5$ laboratories reporting) using the final calculated MEDM location, standard uncertainty, and the calculated means from the submitted lipid concentration replicates reported by each laboratory. Zeta-scores were obtained using the following equation (15):

$$
Z e t a(i)=\frac{X(i)-X_{p t}}{\sqrt{u_{x(i)}^{2}+u_{p t}^{2}}}
$$

where $X_{p t}$ is the MEDM location, $X(i)$ is the laboratory's submitted average concentration value, $u_{x}$ is the laboratory's standard uncertainty, and $u_{p t}$ is the standard uncertainty of the MEDM location. $\zeta$-scores were used to evaluate the following: 1 ) collective laboratory values relative to the MEDM location on a per lipid basis and 2) individual laboratory values relative to the MEDM location for lipids of a single lipid class.

\section{RESULTS AND DISCUSSION}

\section{Summary of Laboratories and Lipids Reported in SRM 1950}

To initiate the interlaboratory comparison exercise, 100 invitations were sent to a comprehensive and diverse collection of laboratories, with the mindset that this methodological diversity would lead to the creation of robust consensus mean estimates. Upon the data submission closing date, 31 laboratories submitted data for the exercise (one laboratory submitted lipidomic data using two different MS platforms, for a total number of 32 laboratory submissions). Examination of the participating laboratories revealed that $45 \%$ were international (outside of the United States), 52 \% were from laboratories that employ global lipidomic methodologies (reported lipids from three or more lipid categories), and $78 \%$ of the laboratories self-identified as academic. These self-reported classifications, along with some additional information (extraction type and instrument platforms used) are found in Table 1. The collective data from all participating laboratories resulted in the identification of 1527 unique lipid species at the sum compositional level ( $n \geq 1$ laboratories reporting). Further dissection of the 1527 lipids revealed that five lipid 
categories were represented (Appendix B) as follows: FA $(n=177)$, GL $(n=317)$, GP $(n=679)$, SP $(n=236)$, and ST $(n=118)$. In Appendix B, all unique lipid species are provided, including a summary table that breaks down the lipid categories into lipid classes and sub-classes.

Consensus estimates were calculated for lipid species reported by at least five laboratories, using the VR, DSL, and MEDM methods. In total, final consensus locations and associated uncertainties were determined using the MEDM method for 339 lipids at the sum composition level ( $n \geq 5$ laboratories reporting). The 339 lipids were represented in lipid categories as follows: FA $(n=14)$, GL $(n=83)$, GP $(n=150)$, SP $(n=58)$, and ST $(n=34)$. Upon further examination of the MEDM locations $(n=339)$, several location estimates had large uncertainties, thus limiting their application as a useful quality control benchmark. Thus, to classify the usefulness of the MEDM locations, we calculated COD values for each MEDM location. To be considered acceptable for use with quality control activities, among other validation purposes, we set a requirement that the corresponding COD value must be $\leq 40 \%$, with smaller COD values indicating that the lipid was measured with increased robustness and was minimally impacted by the methodology employed. In total, there were 254 lipids that fit this criterion and the lipid breakdown was BA $(n=14)$, CE $(n=15)$, CER $(n=8)$, free cholesterol, DAG $(n=5)$, eicosanoids $(n=3)$, FFA $(n=5)$, HexCer $(n=4)$, LPC $(n=25)$, LPE $(n=6)$, PC $(n=53)$, PE $(n=29)$, PI $(n$ $=13)$, PG $(n=1)$, SM $(n=30)$, and TAG $(n=42)$, representing all the major lipid classes, and are shown in Tables 2 to 6.

For those lipids ( $n=85)$ with COD values $>40 \%$ (i.e. the lipids not measured consistently within the exercise), the lipid class breakdown (number of lipid species) was as follows: CE ( $n=$ 4), CER $(n=7)$, DAG $(n=19)$, FFA $(n=6)$, HexCer $(n=1)$, LPE $(n=2)$, PC $(n=10)$, PE $(n=$ $6)$, PG $(n=2)$, PI $(n=2)$, PS $(n=1)$, SM $(n=8)$, and TAG $(n=17)$. Furthermore, four lipid classes had $\geq 25 \%$ of the lipid species present with COD $>40 \%$, including (number of species with COD $>40 \%$ / total number of species with $n \geq 5$ laboratories reporting): TAG (17/59=29\%), CER $(8 / 15=53 \%)$, FFA $(6 / 11=55 \%)$, and DAG $(19 / 24=79 \%)$. These finding indicate that measurement of these lipid classes were the most frequently inconsistent. We strongly suggest that the lipids with COD > $40 \%$ not be used for validation purposes, rather we note that the measurement of these lipids is problematic and future improvements should be made.

MEDM location plots for each lipid ( $n \geq 5$ laboratories reporting) are shown in Appendix

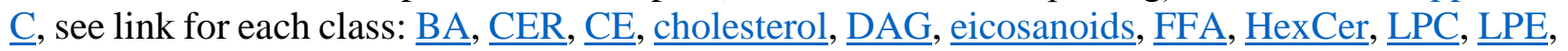
$\underline{\text { PC, }} \underline{\mathrm{PE}}, \underline{\mathrm{PG}}, \underline{\mathrm{PI}}, \underline{\mathrm{PS}}, \underline{\mathrm{SM}}$, and TAG. On each plot, submitted lipid data from each laboratory (calculated mean and standard deviation of the mean shown in $\mathrm{nmol} / \mathrm{mL}$ from the triplicate measurement) are shown to the right of the plot (right of the dashed line). On the left of the plot, consensus estimates calculated using the DSL, VR, and MEDM methods are shown with standard uncertainties. Extreme values were truncated from the plot but were not removed from the estimation. The final MEDM location is provided at the bottom of each figure.

Evaluation of all MEDM location COD values, in relation to number of laboratories reporting, showed a generally decreasing COD as the number of laboratories reporting a lipid increased as expected (Figure 1). In addition, it is expected that the impact of outlier laboratories decreases as the total number of laboratories reporting for a lipid increases. To further dissect trends in the final MEDM locations ( $n=339$ ), we examined the top-50 and bottom-50 lipids by 
concentration (nmol/mL) (Appendix D). The top-50 comprised CE $(n=11)$, FFA $(n=4)$, LPC $(n$ $=4)$, PC $(n=11), \mathrm{SM}(n=7)$, and TAG $(n=13)$. The average number of laboratories reporting for the top-50 consensus lipids was (15 \pm 4$)$ laboratories with an average COD of (26 \pm 11$) \%$. The bottom-50 lipids comprised BA $(n=10)$, CER $(n=9)$, DAG $(n=2)$, eicosanoids $(n=3)$, HexCer $(n=1)$, LPC $(n=9)$, LPE $(n=1)$, PC $(n=8)$, PE $(n=2)$, SM $(n=4)$, and TAG $(n=1)$. The average number of laboratories reporting for the bottom-50 consensus lipids was $(7 \pm 2)$ laboratories with an average COD of (35 \pm 19$) \%$. This comparison suggests that laboratories measure more concentrated lipid species more precisely and with better harmonization within the community. This is further supported by the top-50 lipids having only three lipid species with COD $>40 \%$ (three fatty acids), while the bottom-50 had 18 lipid species with COD > $40 \%$.

To increase the coverage of the lipidome examined in this exercise, we extended the analysis to lipids measured by only three or four laboratories. We investigated the utility of these lipids (i.e., lipids could potentially be useful if measured uniformly within the community despite having < 5 laboratories reporting). The MEDM method was not used to determine these estimates, as the associated uncertainty calculations for the MEDM procedure may understate the uncertainty when there are fewer than five laboratories (13). In addition, it is recommended that there be at least six laboratories for the maximum likelihood asymptotics of the VR method to be valid (16). Thus, for the estimation of these lipids ( $n=192$, Table 7 and Table 8), with only three to four laboratories reporting, the DSL estimation method was employed as its uncertainty estimation remains valid for a small number of laboratories (10). To examine the usefulness of these additional DSL means, we set the criteria to be COD $\leq 40 \%$ and a percent difference between the DSL mean and the MEDM location $\leq 20 \%$, which ensures both a generally reliable consensus estimate and avoids lipids with extreme outliers. For the 192 lipid species, there were 62 lipids which fit the criteria for acceptance as "tentative" consensus means for validation purposes. For the 62 lipid species, the following lipid classes were represented: BA $(n=2)$, CE $(n=3)$, CER ( $n$ $=4)$, DAG $(n=1)$, dihydroceramides (DHC, $n=4)$, dhSph-1P $(n=1)$, eicosanoids $(n=20)$, FFA $(n=3)$, HexCer $(n=1)$, LPC $(n=4)$, PC $(n=4)$, PE $(n=2)$, PG $(n=2)$, PS $(n=2)$, SM $(n=2)$, Sph-1P, TAG $(n=7)$, and total cholesterol.

\section{$\zeta$-Score Analysis}

To determine the relative agreement of each participating laboratory, in relation to the community-derived MEDM location, $\zeta$-scores were calculated for each submitted lipid concentration. While z-scores are often employed, which subtract the consensus estimate from the laboratory submitted value and divide by the standard uncertainty of the consensus estimate, we employed the zeta( $\zeta)$-score, a variant of the z-score, which takes into account laboratory variances (15).

Upon closer inspection of the collective calculated $\zeta$-scores, several community-wide trends were observed based on lipid species measured (for lipids with $\mathrm{n} \geq 5$ laboratories reporting). $\zeta$-scores were implemented to normalize the submitted data from all laboratories and to compare values relative to the MEDM location by lipid species (Appendix E) or by laboratory (Appendix F). Untruncated $\zeta$-score averages are included in all plots to help identify those lipids with a higher frequency of atypical measurement in relation to the collective data submitted. For each $\zeta$-score plot, each dot represents the number of combined standard uncertainties a single laboratory- 
reported lipid value is from the MEDM location. With normally distributed data, approximately $95 \%$ of the data should fall within \pm 2 standard deviations (two solid lines on plots). This normality guideline assumes that the participating laboratories are approximately equal in ability, and thus the differences observed reflect random measurement error. For those lipids by which a majority of the dots reside outside the guidelines $( \pm 2)$, the $\zeta$-scores indicate that the capability of measuring those lipids is poor for the majority of the laboratories reporting and is not just random measurement error. According to the $\zeta$-scores on a per lipid species basis (all the laboratories scores on the same plot for a lipid species, Appendix E), the ten most variably measured lipids in relation to the MEDM location by untruncated $\zeta$-scores (in parentheses) were SM d34:0 (6.47), CE 22:4 (6.51), CE 22:5 (7.37), SM d41:1 (7.38), PC O-40:6/P-40:5/39:6 (8.71), PC 38:7 (8.76), PE O-40:5/P-40:4/39:5 (8.8), SM d38:0 (9.31), CER d38:1 (9.61), and DAG 38:0 (10.48). The untruncated $\zeta$-score averages indicate that, on average, the lipids were measured more than six times (and greater) the standard uncertainty away from the MEDM location.

The second manner in which $\zeta$-scores were organized was by laboratory on a per lipid class basis. In these plots (Appendix F), the $\zeta$-scores were used to identify which laboratories consistently measured lipid species, organized by lipid class, at different concentrations relative to the MEDM location. The untruncated average $\zeta$-scores reveal how well each laboratory performed relative to the MEDM location. Further examination of the $\zeta$-scores by laboratory, on a per lipid class basis, revealed that five lipid classes had $\geq 3$ laboratories with untruncated $\zeta$-score averages above five (i.e., a $\zeta$-score value of 5 for a lipid class indicates, that on average, the laboratory $\zeta$ scores were four times the combined standard uncertainties away from the MEDM location). The five lipid classes were (with number of laboratories with $\zeta$-score averages above five): DAG (3), LPE (3), PE (3), SM (4), and LPC (5).

\section{SUMMARY}

To date, there is no community-wide accepted workflow for performing and analyzing lipidomics experiments. Furthermore, there are no lipid benchmark concentrations in complex mixtures that can be used to assess current lipidomic workflows and methods. This interlaboratory study is the first step towards providing a means to increase harmonization and initiating a conversation regarding potential efforts required to improve standardization in lipid measurement within the lipidomics community.

\section{DISCLAIMER}

Certain commercial equipment, instruments, or materials are identified in this paper to specify adequately the experimental procedures. Such identification does not imply recommendation or endorsement by the National Institute of Standards and Technology; nor does it imply that the materials or equipment identified are necessarily the best for the purpose.

\section{ACKNOWLEDGEMENTS}

In addition to acknowledging the contributions (and funding sources) for each participating laboratory (Appendix G), the authors of this report would also like to acknowledge the contributions of Rebecca Pugh, Debra Ellisor, Amanda Moors, David Duewer, Katrice Lippa, John 
Kucklick, Stephen Somerville, Theresa Cantu, Jackie Bangma, and Jeremy Koelmel, which helped make this interlaboratory exercise successful.

\section{REFERENCES}

1. Beger, R. D., W. Dunn, M. A. Schmidt, S. S. Gross, J. A. Kirwan, M. Cascante, L. Brennan, D. S. Wishart, M. Oresic, and T. Hankemeier. 2016. Metabolomics enables precision medicine: “a white paper, community perspective”. Metabolomics 12: 149.

2. Phinney, K. W., G. Ballihaut, M. Bedner, B. S. Benford, J. E. Camara, S. J. Christopher, W. C. Davis, N. G. Dodder, G. Eppe, and B. E. Lang. 2013. Development of a Standard Reference Material for metabolomics research. Anal. Chem. 85: 11732-11738.

3. Simon-Manso, Y., M. S. Lowenthal, L. E. Kilpatrick, M. L. Sampson, K. H. Telu, P. A. Rudnick, W. G. Mallard, D. W. Bearden, T. B. Schock, and D. V. Tchekhovskoi. 2013. Metabolite profiling of a NIST Standard Reference Material for human plasma (SRM 1950): GC-MS, LC-MS, NMR, and clinical laboratory analyses, libraries, and web-based resources. Anal. Chem. 85: 11725-11731.

4. Telu, K. H., X. Yan, W. E. Wallace, S. E. Stein, and Y. Simón-Manso. 2016. Analysis of human plasma metabolites across different liquid chromatography/mass spectrometry platforms: Cross-platform transferable chemical signatures. Rapid Comm. Mass Spectrom. 30: 581-593.

5. Koal, T., and H.-P. Deigner. 2010. Challenges in mass spectrometry based targeted metabolomics. Curr. Mol. Med. 10: 216-226.

6. Quehenberger, O., A. M. Armando, A. H. Brown, S. B. Milne, D. S. Myers, A. H. Merrill, S. Bandyopadhyay, K. N. Jones, S. Kelly, and R. L. Shaner. 2010. Lipidomics reveals a remarkable diversity of lipids in human plasma. J. Lipid Res. 51: 3299-3305.

7. Ulmer, C. Z., J. P. Koelmel, J. M. Ragland, T. J. Garrett, and J. A. Bowden. 2017. LipidPioneer: A Comprehensive User-Generated Exact Mass Template for Lipidomics. J. Am. Soc. Mass Spectrom. 28: 562-565.

8. Rukhin, A. L., and M. G. Vangel. 1998. Estimation of a common mean and weighted means statistics. J. Am. Stat. Assoc. 93: 303-308.

9. Vangel, M. G., and A. L. Rukhin. 1999. Maximum Likelihood Analysis for Heteroscedastic One-W ay Random Effects ANOVA in Interlaboratory Studies. Biometrics 55: 129-136.

10. DerSimonian, R., and N. Laird. 1986. Meta-analysis in clinical trials. Control. Clin. Trials 7: 177-188. 
11. Efron, B., and R. J. Tibshirani. 1993. An introduction to the bootstrap. Number 57 in Monographs on statistics and applied probability. Chapman \& Hall, New York.

12. Horn, S. D., R. A. Horn, and D. B. Duncan. 1975. Estimating heteroscedastic variances in linear models. Journal of the American Statistical Association 70: 380-385.

13. CCQM. 2013. CCQM Guidance note: Estimation of a consensus KCRV and associated degrees of equivalence: Version 10 http://www.bipm.org/cc/CCQM/Allowed/19/CCQM13-22_Consensus_KCRV_v10.pdf.

14. Bonett, D. G., Seier, E. 2006. Confidence Interval for a Coefficient of Dispersion in Nonnormal Distributions. Biometrics 48: 144-148.

15. ISO 13528. 2015. Statistical methods for use in proficiency testing by interlaboratory comparison. $2^{\text {nd }}$ Edition. 1-89.

16. Vangel, M. G. personal communication. 
Table 1. Participating laboratory and methodological information

\begin{tabular}{|c|c|c|c|c|c|c|c|c|c|c|c|}
\hline \multirow[b]{2}{*}{ Lab \# } & \multirow{2}{*}{$\begin{array}{c}\text { International/ } \\
\text { National* }\end{array}$} & \multirow[b]{2}{*}{ Lab Type* } & \multirow{2}{*}{$\begin{array}{l}\text { Target/ } \\
\text { Global }\end{array}$} & \multirow{2}{*}{\multicolumn{2}{|c|}{ Extraction Type }} & \multicolumn{2}{|c|}{ Instrument \#1 } & \multicolumn{2}{|c|}{ Instrument \#2 } & \multicolumn{2}{|c|}{ Instrument \#3 } \\
\hline & & & & & & $\begin{array}{c}\text { Sample } \\
\text { Introduction }\end{array}$ & MS Used & $\begin{array}{c}\text { Sample } \\
\text { Introduction }\end{array}$ & $\begin{array}{c}\text { MS } \\
\text { Used }\end{array}$ & $\begin{array}{c}\text { Sample } \\
\text { Introduction }\end{array}$ & $\begin{array}{l}\text { MS } \\
\text { Used }\end{array}$ \\
\hline 1 & International & Academia & Global & Bligh-Dyer & & HPLC & HRMS/MS & & & & \\
\hline 2 & International & Industry & Global & Matyash & & DI & HRMS/MS & & & & \\
\hline 3 & International & Academia & Global & Matyash & & UHPLC & HRMS/MS & & & & \\
\hline $4 \dagger$ & & & & & & UHPLC & MS/MS & GC & MS & & \\
\hline $5 \dagger$ & International & Industry & Global & Folch & & UHPLC & MS/MS & & & & \\
\hline 6 & National & Academia & Global & Bligh-Dyer & & DI & MS/MS & GC & MS & & \\
\hline 7 & International & Academia & Global & Modified Bligh-Dyer & & DI & MS/MS & & & & \\
\hline 8 & National & Academia & Targeted & SPE & Modified Bligh-Dyer & HPLC & MS/MS & GC & MS & & \\
\hline 9 & National & Academia & Global & Matyash & & UHPLC & HRMS/MS & & & & \\
\hline 10 & International & Academia & Global & Butanol/Methanol & Methanol Precipitation & UHPLC & MS/MS & & & & \\
\hline 11 & International & Industry & Global & SPE & Methanol Precipitation & DI & MS/MS & GC & MS/MS & UHPLC & MS/MS \\
\hline 12 & National & Academia & Global & Matyash & & UHPLC & HRMS & & & & \\
\hline 13 & National & Academia & Global & SPE & & DI & MS/MS & HPLC & MS/MS & & \\
\hline 14 & National & Academia & Targeted & Isopropanol/Ethyl acetate & Chloroform/water/ $1 \mathrm{~N} \mathrm{NaCl}$ & HPLC & MS/MS & & & & \\
\hline 15 & National & Academia & Global & Matyash & & HPLC & HRMS/MS & & & & \\
\hline 16 & National & Government & Global & Modified Bligh-Dyer & & DI & MS/MS & & & & \\
\hline 17 & National & Academia & Targeted & Folch & & HPLC & MS/MS & & & & \\
\hline 18 & International & Industry & Global & Modified Folch & & UHPLC & HRMS & & & & \\
\hline 19 & National & Industry & Global & Folch & & UHPLC & HRMS/MS & & & & \\
\hline 20 & International & Academia & Targeted & SPE & Modified Bligh-Dyer & UHPLC & MS/MS & & & & \\
\hline 21 & National & Academia & Targeted & SPE & & UHPLC & MS/MS & & & & \\
\hline 22 & International & Academia & Targeted & SPE & & UHPLC & MS/MS & & & & \\
\hline 23 & International & Academia & Global & Matyash & & DI & HRMS & & & & \\
\hline 24 & National & Academia & Targeted & Acetonitrile Precipitation & & UHPLC & MS & & & & \\
\hline 25 & National & Academia & Targeted & Acetonitrile Precipitation & & UHPLC & MS/MS & & & & \\
\hline 26 & National & Academia & Targeted & Methanol Precipitation & & DI & MS/MS & & & & \\
\hline 27 & International & Academia & Targeted & Bligh-Dyer & & DI & MS/MS & GC & MS & HPLC & MS/MS \\
\hline 28 & International & Academia & Targeted & Isopropanol & & HPLC & HRMS/MS & & & & \\
\hline 30 & National & Academia & Targeted & SPE & & UHPLC & MS/MS & & & & \\
\hline 31 & National & Academia & Targeted & Methanol Precipitation & & UHPLC & MS/MS & & & & \\
\hline 32 & International & Industry & Targeted & Ethyl Acetate & & HPLC & MS/MS & & & & \\
\hline 34 & National & Academia & Targeted & Acetonitrile Precipitation & & UPLC & MS/MS & & & & \\
\hline
\end{tabular}

* Self-reported by participating laboratory. † Submissions for laboratory ID 4 and 5 are from the same laboratory, the two submissions were performed on two different mass spectrometric platforms. N/A indicates that the information was not provided. Global $=$ reported $\geq 3$ lipid categories, Targeted $=$ reported $<3$ lipid categories. HRMS - high resolution-mass spectrometry, MS - low resolution-mass spectrometry, MS/MS - tandem mass spectrometry 
Table 2. Consensus estimates and associated uncertainties for fatty acyl lipids (FA) measured by at least five laboratories in SRM 1950

\begin{tabular}{|c|c|c|c|c|c|c|c|c|c|}
\hline Lipid & $\begin{array}{l}\text { \# of } \\
\text { Labs } \\
\end{array}$ & Units & $\begin{array}{l}\text { DSL } \\
\text { Mean } \\
\end{array}$ & $\begin{array}{c}\text { DSL } \\
\text { Standard } \\
\text { Uncertainty } \\
\end{array}$ & $\begin{array}{c}\text { VR } \\
\text { Mean } \\
\end{array}$ & $\begin{array}{c}\text { VR } \\
\text { Standard } \\
\text { Uncertainty } \\
\end{array}$ & $\begin{array}{c}\text { MEDM } \\
\text { Location } \\
\end{array}$ & $\begin{array}{c}\text { MEDM } \\
\text { Standard } \\
\text { Uncertainty } \\
\end{array}$ & COD (\%) \\
\hline FFA 16:0 & 5 & $\mathrm{nmol} / \mathrm{mL}$ & 19 & 20 & 75 & 39 & 43 & 13 & 31 \\
\hline FFA $16: 1$ & 6 & $\mathrm{nmol} / \mathrm{mL}$ & 8.4 & 3.0 & 8.5 & 2.7 & 6.1 & 2.9 & 48 \\
\hline FFA $18: 0$ & 5 & $\mathrm{nmol} / \mathrm{mL}$ & 34 & 15 & 34 & 13 & 15 & 9.0 & 62 \\
\hline FFA $18: 1$ & 6 & $\mathrm{nmol} / \mathrm{mL}$ & 37 & 12 & 100 & 30 & 110 & 53 & 48 \\
\hline FFA 18:2 & 6 & $\mathrm{nmol} / \mathrm{mL}$ & 64 & 25 & 64 & 23 & 44 & 22 & 49 \\
\hline FFA 18:3 & 6 & $\mathrm{nmol} / \mathrm{mL}$ & 3.6 & 0.64 & 3.6 & 0.57 & 2.9 & 0.62 & 21 \\
\hline FFA 20:3 & 5 & $\mathrm{nmol} / \mathrm{mL}$ & 2.7 & 1.9 & 3.0 & 1.8 & 1.3 & 0.62 & 47 \\
\hline FFA $20: 4$ & 7 & $\mathrm{nmol} / \mathrm{mL}$ & 8.7 & 4.8 & 16 & 11 & 4.7 & 1.5 & 31 \\
\hline FFA $20: 5$ & 7 & $\mathrm{nmol} / \mathrm{mL}$ & 0.42 & 0.059 & 0.82 & 0.44 & 0.42 & 0.056 & 13 \\
\hline FFA 22:5 & 5 & $\mathrm{nmol} / \mathrm{mL}$ & 1.4 & 0.67 & 1.4 & 0.61 & 1.1 & 0.56 & 52 \\
\hline FFA 22:6 & 8 & $\mathrm{nmol} / \mathrm{mL}$ & 2.8 & 1.2 & 2.9 & 1.3 & 1.5 & 0.17 & 11 \\
\hline 12-HETE & 5 & $\mathrm{pmol} / \mathrm{mL}$ & 11 & 2.9 & 11 & 2.6 & 6.8 & 1.5 & 23 \\
\hline 15-HETE & 5 & $\mathrm{pmol} / \mathrm{mL}$ & 1.8 & 0.19 & 2.0 & 0.21 & 2.4 & 0.64 & 27 \\
\hline 5-HETE & 5 & $\mathrm{pmol} / \mathrm{mL}$ & 10 & 1.3 & 11 & 1.6 & 10 & 1.3 & 13 \\
\hline
\end{tabular}

The DSL uncertainty is the Horn-Horn Duncan. The COD (\%) was calculated using the MEDM location and standard uncertainty. Final MEDM locations are in bold. The abbreviations identify free fatty acids (FFA) and hydroxyeicosatetraenoic acids (HETE). 
Table 3. Consensus estimates and associated uncertainties for glycerolipids measured by at least five laboratories in SRM 1950

\begin{tabular}{|c|c|c|c|c|c|c|c|c|c|}
\hline Lipid & $\begin{array}{l}\text { \# of } \\
\text { Labs } \\
\end{array}$ & Units & $\begin{array}{l}\text { DSL } \\
\text { Mean } \\
\end{array}$ & $\begin{array}{c}\text { DSL } \\
\text { Standard } \\
\text { Uncertainty }\end{array}$ & $\begin{array}{c}\text { VR } \\
\text { Mean } \\
\end{array}$ & $\begin{array}{c}\text { VR } \\
\text { Standard } \\
\text { Uncertainty } \\
\end{array}$ & $\begin{array}{c}\text { MEDM } \\
\text { Location } \\
\end{array}$ & $\begin{array}{c}\text { MEDM } \\
\text { Standard } \\
\text { Uncertainty } \\
\end{array}$ & COD (\%) \\
\hline DAG 30:0 & 7 & $\mathrm{nmol} / \mathrm{mL}$ & 0.55 & 0.15 & 0.65 & 0.13 & 0.83 & 0.17 & 20 \\
\hline DAG 32:0 & 11 & $\mathrm{nmol} / \mathrm{mL}$ & 0.56 & 0.35 & 5.3 & 2.0 & 2.6 & 1.2 & 44 \\
\hline DAG 32:1 & 10 & $\mathrm{nmol} / \mathrm{mL}$ & 0.71 & 0.24 & 1.00 & 0.29 & 1.2 & 0.62 & 51 \\
\hline DAG 32:2 & 11 & $\mathrm{nmol} / \mathrm{mL}$ & 0.29 & 0.088 & 0.62 & 0.20 & 0.62 & 0.29 & 48 \\
\hline DAG 34:0 & 10 & $\mathrm{nmol} / \mathrm{mL}$ & 5.9 & 3.7 & 11 & 3.6 & 6.5 & 3.6 & 56 \\
\hline DAG 34:1 & 16 & $\mathrm{nmol} / \mathrm{mL}$ & 4.1 & 1.2 & 22 & 9.6 & 6.1 & 2.4 & 40 \\
\hline DAG 34:2 & 14 & $\mathrm{nmol} / \mathrm{mL}$ & 2.7 & 0.71 & 5.2 & 1.4 & 4.4 & 1.9 & 43 \\
\hline DAG 34:3 & 7 & $\mathrm{nmol} / \mathrm{mL}$ & 0.16 & 0.070 & 8.7 & 4.5 & 0.31 & 0.20 & 63 \\
\hline DAG 36:0 & 9 & $\mathrm{nmol} / \mathrm{mL}$ & 3.7 & 1.9 & 13 & 7.3 & 1.6 & 0.98 & 60 \\
\hline DAG 36:1 & 12 & $\mathrm{nmol} / \mathrm{mL}$ & 5.2 & 2.2 & 6.1 & 2.8 & 2.6 & 1.1 & 43 \\
\hline DAG 36:2 & 16 & $\mathrm{nmol} / \mathrm{mL}$ & 2.4 & 0.89 & 25 & 12 & 6.2 & 2.2 & 36 \\
\hline DAG 36:3 & 15 & $\mathrm{nmol} / \mathrm{mL}$ & 4.2 & 1.6 & 6.8 & 1.6 & 8.4 & 3.3 & 39 \\
\hline DAG 36:4 & 12 & $\mathrm{nmol} / \mathrm{mL}$ & 0.68 & 0.29 & 2.6 & 0.60 & 2.8 & 1.0 & 38 \\
\hline DAG 36:5 & 6 & $\mathrm{nmol} / \mathrm{mL}$ & 0.61 & 0.28 & 2.9 & 2.0 & 0.89 & 0.54 & 61 \\
\hline DAG 38:0 & 7 & $\mathrm{nmol} / \mathrm{mL}$ & 1.8 & 1.2 & 1.9 & 1.1 & 0.24 & 0.13 & 55 \\
\hline DAG 38:1 & 5 & $\mathrm{nmol} / \mathrm{mL}$ & 0.39 & 0.35 & 0.65 & 0.28 & 0.51 & 0.39 & 77 \\
\hline DAG 38:2 & 5 & $\mathrm{nmol} / \mathrm{mL}$ & 0.69 & 0.54 & 2.2 & 0.98 & 1.5 & 1.2 & 81 \\
\hline DAG 38:3 & 5 & $\mathrm{nmol} / \mathrm{mL}$ & 0.45 & 0.32 & 2.8 & 1.4 & 1.3 & 1.0 & 80 \\
\hline DAG 38:4 & 11 & $\mathrm{nmol} / \mathrm{mL}$ & 0.57 & 0.19 & 2.0 & 0.99 & 0.95 & 0.38 & 40 \\
\hline DAG 38:5 & 11 & $\mathrm{nmol} / \mathrm{mL}$ & 1.2 & 0.48 & 1.6 & 0.42 & 1.8 & 0.82 & 47 \\
\hline DAG 38:6 & 9 & $\mathrm{nmol} / \mathrm{mL}$ & 1.6 & 0.79 & 2.0 & 1.1 & 0.77 & 0.37 & 47 \\
\hline DAG 40:5 & 5 & $\mathrm{nmol} / \mathrm{mL}$ & 0.046 & 0.021 & 0.043 & 0.017 & 0.084 & 0.053 & 63 \\
\hline DAG 40:6 & 6 & $\mathrm{nmol} / \mathrm{mL}$ & 0.18 & 0.072 & 0.18 & 0.064 & 0.28 & 0.17 & 60 \\
\hline DAG 40:7 & 5 & $\mathrm{nmol} / \mathrm{mL}$ & 0.060 & 0.039 & 0.49 & 0.22 & 0.89 & 0.68 & 77 \\
\hline TAG 42:0 & 5 & $\mathrm{nmol} / \mathrm{mL}$ & 0.48 & 0.20 & 0.73 & 0.30 & 0.38 & 0.19 & 50 \\
\hline TAG 42:1 & 5 & $\mathrm{nmol} / \mathrm{mL}$ & 0.29 & 0.057 & 0.29 & 0.048 & 0.37 & 0.17 & 45 \\
\hline TAG 42:2 & 6 & $\mathrm{nmol} / \mathrm{mL}$ & 0.12 & 0.031 & 0.12 & 0.026 & 0.16 & 0.064 & 41 \\
\hline TAG 44:0 & 5 & $\mathrm{nmol} / \mathrm{mL}$ & 1.8 & 0.82 & 2.1 & 0.78 & 1.2 & 0.73 & 62 \\
\hline TAG 44:1 & 7 & $\mathrm{nmol} / \mathrm{mL}$ & 1.1 & 0.32 & 9.3 & 6.9 & 1.7 & 0.84 & 50 \\
\hline TAG 44:2 & 6 & $\mathrm{nmol} / \mathrm{mL}$ & 1.1 & 0.41 & 3.9 & 2.7 & 0.90 & 0.40 & 45 \\
\hline
\end{tabular}

The DSL uncertainty is the Horn-Horn Duncan. The COD (\%) was calculated using the MEDM location and standard uncertainty. Final MEDM locations are in bold. The abbreviations identify diacylglycerols (DAG) and triacylglycerols (TAG). 
Table 3. (cont...)

\begin{tabular}{|c|c|c|c|c|c|c|c|c|c|}
\hline Lipid & $\begin{array}{l}\text { \# of } \\
\text { Labs }\end{array}$ & Units & $\begin{array}{c}\text { DSL } \\
\text { Mean }\end{array}$ & $\begin{array}{c}\text { DSL } \\
\text { Standard } \\
\text { Uncertainty }\end{array}$ & $\begin{array}{c}\text { VR } \\
\text { Mean }\end{array}$ & $\begin{array}{c}\text { VR } \\
\text { Standard } \\
\text { Uncertainty }\end{array}$ & $\begin{array}{c}\text { MEDM } \\
\text { Location }\end{array}$ & $\begin{array}{c}\text { MEDM } \\
\text { Standard } \\
\text { Uncertainty }\end{array}$ & COD (\%) \\
\hline TAG 46:0 & 6 & $\mathrm{nmol} / \mathrm{mL}$ & 2.0 & 0.66 & 3.5 & 1.2 & 2.8 & 1.6 & 56 \\
\hline TAG 46:1 & 8 & $\mathrm{nmol} / \mathrm{mL}$ & 6.0 & 2.1 & 17 & 11 & 5.7 & 2.6 & 46 \\
\hline TAG 46:2 & 8 & $\mathrm{nmol} / \mathrm{mL}$ & 2.4 & 0.63 & 2.8 & 0.63 & 3.6 & 1.3 & 37 \\
\hline TAG 46:3 & 5 & $\mathrm{nmol} / \mathrm{mL}$ & 0.71 & 0.21 & 0.70 & 0.18 & 0.76 & 0.34 & 45 \\
\hline TAG 48:0 & 10 & $\mathrm{nmol} / \mathrm{mL}$ & 5.3 & 1.3 & 5.3 & 1.2 & 4.5 & 1.2 & 26 \\
\hline TAG 48:1 & 16 & $\mathrm{nmol} / \mathrm{mL}$ & 24 & 11 & 28 & 14 & 13 & 3.2 & 24 \\
\hline TAG 48:2 & 15 & $\mathrm{nmol} / \mathrm{mL}$ & 21 & 6.9 & 14 & 2.0 & 16 & 2.8 & 18 \\
\hline TAG 48:3 & 11 & $\mathrm{nmol} / \mathrm{mL}$ & 5.1 & 1.5 & 7.3 & 3.0 & 3.3 & 1.3 & 41 \\
\hline TAG 48:4 & 5 & $\mathrm{nmol} / \mathrm{mL}$ & 1.2 & 0.13 & 1.8 & 0.47 & 1.3 & 0.23 & 18 \\
\hline TAG 49:1 & 9 & $\mathrm{nmol} / \mathrm{mL}$ & 1.9 & 0.32 & 2.4 & 0.48 & 2.0 & 0.42 & 21 \\
\hline TAG 49:2 & 6 & $\mathrm{nmol} / \mathrm{mL}$ & 1.5 & 0.41 & 1.6 & 0.37 & 1.8 & 0.56 & 31 \\
\hline TAG 50:0 & 11 & $\mathrm{nmol} / \mathrm{mL}$ & 4.6 & 1.2 & 4.9 & 0.97 & 3.8 & 0.83 & 22 \\
\hline TAG 50:1 & 14 & $\mathrm{nmol} / \mathrm{mL}$ & 17 & 6.9 & 33 & 5.6 & 38 & 10.0 & 26 \\
\hline TAG 50:2 & 15 & $\mathrm{nmol} / \mathrm{mL}$ & 18 & 5.4 & 48 & 7.4 & 47 & 12 & 26 \\
\hline TAG 50:3 & 16 & $\mathrm{nmol} / \mathrm{mL}$ & 27 & 4.3 & 27 & 4.1 & 23 & 6.6 & 29 \\
\hline TAG 50:4 & 15 & $\mathrm{nmol} / \mathrm{mL}$ & 8.5 & 1.7 & 8.3 & 1.6 & 8.7 & 2.9 & 34 \\
\hline TAG 50:5 & 7 & $\mathrm{nmol} / \mathrm{mL}$ & 1.7 & 0.37 & 1.8 & 0.35 & 1.6 & 0.64 & 40 \\
\hline TAG 51:1 & 7 & $\mathrm{nmol} / \mathrm{mL}$ & 1.2 & 0.46 & 1.5 & 0.33 & 1.8 & 0.48 & 27 \\
\hline TAG 51:2 & 8 & $\mathrm{nmol} / \mathrm{mL}$ & 4.0 & 0.48 & 4.9 & 0.62 & 4.8 & 1.1 & 22 \\
\hline TAG 51:3 & 5 & $\mathrm{nmol} / \mathrm{mL}$ & 3.6 & 0.79 & 3.6 & 0.68 & 4.8 & 1.9 & 39 \\
\hline TAG 51:4 & 6 & $\mathrm{nmol} / \mathrm{mL}$ & 0.96 & 0.31 & 1.2 & 0.32 & 1.4 & 0.62 & 43 \\
\hline TAG 52:0 & 8 & $\mathrm{nmol} / \mathrm{mL}$ & 2.3 & 0.76 & 5.8 & 2.1 & 3.4 & 1.8 & 54 \\
\hline TAG 52:1 & 11 & $\mathrm{nmol} / \mathrm{mL}$ & 20 & 4.5 & 17 & 3.5 & 14 & 2.9 & 20 \\
\hline TAG 52:2 & 16 & $\mathrm{nmol} / \mathrm{mL}$ & 49 & 23 & 71 & 14 & 44 & 14 & 33 \\
\hline TAG 52:3 & 16 & $\mathrm{nmol} / \mathrm{mL}$ & 22 & 5.4 & 170 & 66 & 100 & 29 & 28 \\
\hline TAG 52:4 & 15 & $\mathrm{nmol} / \mathrm{mL}$ & 41 & 26 & 60 & 11 & 48 & 17 & 35 \\
\hline TAG 52:5 & 13 & $\mathrm{nmol} / \mathrm{mL}$ & 17 & 3.5 & 17 & 3.3 & 15 & 5.7 & 39 \\
\hline TAG 52:6 & 8 & $\mathrm{nmol} / \mathrm{mL}$ & 3.0 & 0.78 & 3.1 & 0.72 & 4.0 & 1.4 & 35 \\
\hline ТАC 52.7 & 5 & $\mathrm{nmol} / \mathrm{mI}$ & 037 & 0064 & 037 & 0055 & 030 & 012 & כ2 \\
\hline
\end{tabular}

The DSL uncertainty is the Horn-Horn Duncan. The COD (\%) was calculated using the MEDM location and standard uncertainty. Final MEDM locations are in bold. The abbreviations identify diacylglycerols (DAG) and triacylglycerols (TAG). 
Table 3. (cont...)

\begin{tabular}{|c|c|c|c|c|c|c|c|c|c|}
\hline Lipid & $\begin{array}{l}\text { \# of } \\
\text { Labs }\end{array}$ & Units & $\begin{array}{l}\text { DSL } \\
\text { Mean }\end{array}$ & $\begin{array}{c}\text { DSL } \\
\text { Standard } \\
\text { Uncertainty }\end{array}$ & $\begin{array}{c}\text { VR } \\
\text { Mean }\end{array}$ & $\begin{array}{c}\text { VR } \\
\text { Standard } \\
\text { Uncertainty }\end{array}$ & $\begin{array}{c}\text { MEDM } \\
\text { Location }\end{array}$ & $\begin{array}{c}\text { MEDM } \\
\text { Standard } \\
\text { Uncertainty }\end{array}$ & COD (\%) \\
\hline TAG 53:2 & 9 & $\mathrm{nmol} / \mathrm{mL}$ & 1.9 & 0.20 & 2.0 & 0.20 & 1.9 & 0.41 & 21 \\
\hline TAG 53:3 & 6 & $\mathrm{nmol} / \mathrm{mL}$ & 3.1 & 0.72 & 15 & 11 & 3.7 & 1.1 & 29 \\
\hline TAG 53:4 & 6 & $\mathrm{nmol} / \mathrm{mL}$ & 1.6 & 0.40 & 4.4 & 2.3 & 2.4 & 0.76 & 32 \\
\hline TAG 53:5 & 6 & $\mathrm{nmol} / \mathrm{mL}$ & 0.67 & 0.21 & 0.91 & 0.28 & 0.84 & 0.37 & 44 \\
\hline TAG 54:0 & 9 & $\mathrm{nmol} / \mathrm{mL}$ & 1.3 & 0.59 & 4.2 & 1.5 & 2.4 & 1.3 & 51 \\
\hline TAG 54:1 & 10 & $\mathrm{nmol} / \mathrm{mL}$ & 3.0 & 0.61 & 20 & 17 & 3.2 & 0.91 & 29 \\
\hline TAG 54:2 & 13 & $\mathrm{nmol} / \mathrm{mL}$ & 23 & 12 & 47 & 34 & 8.2 & 2.6 & 31 \\
\hline TAG 54:3 & 15 & $\mathrm{nmol} / \mathrm{mL}$ & 33 & 6.2 & 35 & 7.3 & 26 & 9.8 & 37 \\
\hline TAG 54:4 & 15 & $\mathrm{nmol} / \mathrm{mL}$ & 12 & 5.9 & 37 & 6.5 & 36 & 13 & 35 \\
\hline TAG 54:5 & 15 & $\mathrm{nmol} / \mathrm{mL}$ & 5.7 & 2.4 & 30 & 5.6 & 27 & 11 & 38 \\
\hline TAG 54:6 & 16 & $\mathrm{nmol} / \mathrm{mL}$ & 2.3 & 2.3 & 15 & 2.9 & 14 & 5.1 & 37 \\
\hline TAG 54:7 & 7 & $\mathrm{nmol} / \mathrm{mL}$ & 4.5 & 1.3 & 4.5 & 1.2 & 5.6 & 1.5 & 26 \\
\hline TAG 56:2 & 5 & $\mathrm{nmol} / \mathrm{mL}$ & 0.67 & 0.14 & 0.67 & 0.13 & 0.69 & 0.23 & 33 \\
\hline TAG 56:3 & 6 & $\mathrm{nmol} / \mathrm{mL}$ & 1.1 & 0.27 & 1.1 & 0.23 & 1.4 & 0.14 & 10 \\
\hline TAG 56:4 & 10 & $\mathrm{nmol} / \mathrm{mL}$ & 2.4 & 0.81 & 5.8 & 3.7 & 2.0 & 0.56 & 28 \\
\hline TAG 56:5 & 12 & $\mathrm{nmol} / \mathrm{mL}$ & 3.9 & 0.86 & 4.9 & 1.0 & 4.1 & 1.4 & 33 \\
\hline TAG 56:6 & 15 & $\mathrm{nmol} / \mathrm{mL}$ & 8.0 & 1.7 & 8.1 & 1.7 & 6.4 & 2.7 & 42 \\
\hline TAG 56:7 & 8 & $\mathrm{nmol} / \mathrm{mL}$ & 22 & 13 & 23 & 13 & 13 & 2.7 & 20 \\
\hline TAG 56:8 & 11 & $\mathrm{nmol} / \mathrm{mL}$ & 3.1 & 0.78 & 3.1 & 0.74 & 3.3 & 1.3 & 40 \\
\hline TAG 56:9 & 5 & $\mathrm{nmol} / \mathrm{mL}$ & 0.75 & 0.23 & 0.74 & 0.20 & 0.71 & 0.27 & 38 \\
\hline TAG 58:6 & 5 & $\mathrm{nmol} / \mathrm{mL}$ & 1.5 & 0.39 & 1.5 & 0.36 & 1.6 & 0.68 & 42 \\
\hline TAG 58:7 & 5 & $\mathrm{nmol} / \mathrm{mL}$ & 3.2 & 1.7 & 11 & 8.2 & 2.0 & 0.64 & 32 \\
\hline TAG 58:8 & 9 & $\mathrm{nmol} / \mathrm{mL}$ & 0.97 & 0.28 & 0.96 & 0.25 & 0.68 & 0.21 & 31 \\
\hline TAG 58:9 & 6 & $\mathrm{nmol} / \mathrm{mL}$ & 1.5 & 0.72 & 1.9 & 0.90 & 1.2 & 0.27 & 22 \\
\hline
\end{tabular}

The DSL uncertainty is the Horn-Horn Duncan. The COD (\%) was calculated using the MEDM location and standard uncertainty. Final MEDM locations are in bold. The abbreviations identify diacylglycerols (DAG) and triacylglycerols (TAG). 
Table 4. Consensus estimates and associated uncertainties for glycerophospholipids measured by at least five laboratories in SRM 1950

\begin{tabular}{|c|c|c|c|c|c|c|c|c|c|}
\hline Lipid & $\begin{array}{l}\# \text { of } \\
\text { Labs }\end{array}$ & Units & $\begin{array}{c}\text { DSL } \\
\text { Mean }\end{array}$ & $\begin{array}{c}\text { DSL } \\
\text { Standard } \\
\text { Uncertainty }\end{array}$ & $\begin{array}{c}\text { VR } \\
\text { Mean }\end{array}$ & $\begin{array}{c}\text { VR } \\
\text { Standard } \\
\text { Uncertainty }\end{array}$ & $\begin{array}{c}\text { MEDM } \\
\text { Location }\end{array}$ & $\begin{array}{c}\text { MEDM } \\
\text { Standard } \\
\text { Uncertainty }\end{array}$ & COD (\%) \\
\hline LPC 14:0 & 16 & $\mathrm{nmol} / \mathrm{mL}$ & 0.99 & 0.19 & 1.1 & 0.19 & 1.0 & 0.20 & 19 \\
\hline LPC $15: 0$ & 9 & $\mathrm{nmol} / \mathrm{mL}$ & 0.85 & 0.27 & 0.86 & 0.26 & 0.52 & 0.11 & 22 \\
\hline LPC 16:0 & 20 & $\mathrm{nmol} / \mathrm{mL}$ & 85 & 44 & 79 & 12 & 73 & 11 & 15 \\
\hline LPC O-16:0 & 10 & $\mathrm{nmol} / \mathrm{mL}$ & 0.49 & 0.11 & 0.50 & 0.10 & 0.55 & 0.16 & 29 \\
\hline LPC P-16:0 & 8 & $\mathrm{nmol} / \mathrm{mL}$ & 0.35 & 0.088 & 310 & 290 & 0.46 & 0.13 & 27 \\
\hline LPC 16:1 & 19 & $\mathrm{nmol} / \mathrm{mL}$ & 1.00 & 0.21 & 2.7 & 0.51 & 2.4 & 0.35 & 15 \\
\hline LPC 17:0 & 6 & $\mathrm{nmol} / \mathrm{mL}$ & 1.3 & 0.045 & 680 & 620 & 1.4 & 0.24 & 18 \\
\hline LPC $17: 1$ & 6 & $\mathrm{nmol} / \mathrm{mL}$ & 0.31 & 0.12 & 54 & 50 & 0.25 & 0.071 & 29 \\
\hline LPC 18:0 & 20 & $\mathrm{nmol} / \mathrm{mL}$ & 16 & 5.0 & 30 & 4.9 & 27 & 3.3 & 12 \\
\hline LPC O-18:0 & 6 & $\mathrm{nmol} / \mathrm{mL}$ & 0.49 & 0.39 & 0.61 & 0.44 & 0.16 & 0.058 & 36 \\
\hline LPC 18:1 & 19 & $\mathrm{nmol} / \mathrm{mL}$ & 22 & 5.2 & 20 & 2.9 & 18 & 2.3 & 13 \\
\hline LPC 18:2 & 19 & $\mathrm{nmol} / \mathrm{mL}$ & 25 & 11 & 25 & 3.8 & 22 & 2.9 & 13 \\
\hline LPC 18:3 & 18 & $\mathrm{nmol} / \mathrm{mL}$ & 1.4 & 0.80 & 1.8 & 1.2 & 0.44 & 0.13 & 30 \\
\hline LPC 20:0 & 7 & $\mathrm{nmol} / \mathrm{mL}$ & 0.18 & 0.057 & 65 & 60 & 0.10 & 0.034 & 34 \\
\hline LPC 20:1 & 13 & $\mathrm{nmol} / \mathrm{mL}$ & 0.19 & 0.030 & 0.29 & 0.084 & 0.19 & 0.024 & 12 \\
\hline LPC 20:2 & 9 & $\mathrm{nmol} / \mathrm{mL}$ & 0.43 & 0.19 & 0.44 & 0.18 & 0.23 & 0.044 & 19 \\
\hline LPC 20:3 & 18 & $\mathrm{nmol} / \mathrm{mL}$ & 1.4 & 0.34 & 2.4 & 0.50 & 1.8 & 0.26 & 15 \\
\hline LPC 20:4 & 20 & $\mathrm{nmol} / \mathrm{mL}$ & 2.8 & 0.62 & 6.4 & 1.0 & 6.0 & 0.60 & 10 \\
\hline LPC 20:5 & 15 & $\mathrm{nmol} / \mathrm{mL}$ & 0.47 & 0.11 & 0.48 & 0.11 & 0.33 & 0.092 & 28 \\
\hline LPC 22:0 & 5 & $\mathrm{nmol} / \mathrm{mL}$ & 0.023 & 0.0032 & 15 & 13 & 0.025 & 0.0017 & 7 \\
\hline LPC 22:1 & 5 & $\mathrm{nmol} / \mathrm{mL}$ & 0.015 & 0.0053 & 12 & 11 & 0.013 & 0.0046 & 36 \\
\hline LPC 22:4 & 8 & $\mathrm{nmol} / \mathrm{mL}$ & 0.17 & 0.077 & 0.18 & 0.071 & 0.12 & 0.041 & 33 \\
\hline LPC 22:5 & 12 & $\mathrm{nmol} / \mathrm{mL}$ & 0.46 & 0.12 & 0.56 & 0.13 & 0.43 & 0.13 & 30 \\
\hline LPC 22:6 & 17 & $\mathrm{nmol} / \mathrm{mL}$ & 1.3 & 0.45 & 1.3 & 0.41 & 0.77 & 0.14 & 18 \\
\hline LPC 24:0 & 5 & $\mathrm{nmol} / \mathrm{mL}$ & 0.055 & 0.017 & 27 & 24 & 0.046 & 0.015 & 33 \\
\hline LPE 16:0 & 14 & $\mathrm{nmol} / \mathrm{mL}$ & 0.69 & 0.17 & 0.80 & 0.14 & 0.91 & 0.27 & 29 \\
\hline LPE 18:0 & 15 & $\mathrm{nmol} / \mathrm{mL}$ & 2.8 & 1.1 & 3.6 & 1.5 & 1.6 & 0.55 & 34 \\
\hline LPE 18:1 & 14 & $\mathrm{nmol} / \mathrm{mL}$ & 2.0 & 0.61 & 1.8 & 0.53 & 1.4 & 0.47 & 35 \\
\hline
\end{tabular}

The DSL uncertainty is the Horn-Horn Duncan. The COD (\%) was calculated using the MEDM location and standard uncertainty. Final MEDM locations are in bold. For PC and PE lipid classes, the isobaric species (ether-linked) were summed and the possibilities observed by the participants are separated by a "’”. The abbreviations identify lysophosphatidylcholines (LPC) and lysophosphatidylethanolamines (LPE). 
Table 4. (cont....)

\begin{tabular}{|c|c|c|c|c|c|c|c|c|c|}
\hline Lipid & $\begin{array}{l}\text { \# of } \\
\text { Labs }\end{array}$ & Units & $\begin{array}{l}\text { DSL } \\
\text { Mean }\end{array}$ & $\begin{array}{c}\text { DSL } \\
\text { Standard } \\
\text { Uncertainty }\end{array}$ & $\begin{array}{c}\text { VR } \\
\text { Mean }\end{array}$ & $\begin{array}{c}\text { VR } \\
\text { Standard } \\
\text { Uncertainty }\end{array}$ & $\begin{array}{l}\text { MEDM } \\
\text { Location }\end{array}$ & $\begin{array}{c}\text { MEDM } \\
\text { Standard } \\
\text { Uncertainty }\end{array}$ & COD (\%) \\
\hline LPE 18:2 & 16 & $\mathrm{nmol} / \mathrm{mL}$ & 2.9 & 0.89 & 3.5 & 1.2 & 1.9 & 0.56 & 30 \\
\hline LPE 20:3 & 5 & $\mathrm{nmol} / \mathrm{mL}$ & 0.47 & 0.25 & 0.71 & 0.37 & 0.52 & 0.38 & 72 \\
\hline LPE 20:4 & 14 & $\mathrm{nmol} / \mathrm{mL}$ & 1.5 & 0.60 & 2.3 & 0.98 & 1.1 & 0.41 & 37 \\
\hline LPE 22:1 & 5 & $\mathrm{nmol} / \mathrm{mL}$ & 0.017 & 0.019 & 0.090 & 0.062 & 0.036 & 0.030 & 82 \\
\hline LPE 22:6 & 12 & $\mathrm{nmol} / \mathrm{mL}$ & 0.57 & 0.11 & 0.62 & 0.12 & 0.52 & 0.18 & 34 \\
\hline PC 30:0 & 11 & $\mathrm{nmol} / \mathrm{mL}$ & 1.8 & 0.32 & 2.0 & 0.34 & 1.6 & 0.32 & 20 \\
\hline PC 30:1 & 5 & $\mathrm{nmol} / \mathrm{mL}$ & 0.76 & 0.54 & 0.84 & 0.41 & 0.76 & 0.43 & 57 \\
\hline PC O-30:0/29:0 & 7 & $\mathrm{nmol} / \mathrm{mL}$ & 0.083 & 0.028 & 0.41 & 0.30 & 0.072 & 0.026 & 36 \\
\hline PC O-30:1/P-30:0 & 7 & $\mathrm{nmol} / \mathrm{mL}$ & 0.071 & 0.022 & 0.13 & 0.068 & 0.047 & 0.0096 & 20 \\
\hline PC 32:0 & 18 & $\mathrm{nmol} / \mathrm{mL}$ & 7.0 & 0.53 & 8.4 & 0.77 & 7.2 & 1.0 & 14 \\
\hline PC O-32:0/31:0 & 11 & $\mathrm{nmol} / \mathrm{mL}$ & 1.4 & 0.25 & 1.4 & 0.24 & 1.5 & 0.41 & 28 \\
\hline PC 32:1 & 18 & $\mathrm{nmol} / \mathrm{mL}$ & 9.2 & 1.0 & 13 & 1.3 & 13 & 1.9 & 15 \\
\hline PC O-32:1/P-32:0/31:1 & 11 & $\mathrm{nmol} / \mathrm{mL}$ & 1.7 & 0.22 & 1.7 & 0.20 & 1.6 & 0.24 & 14 \\
\hline PC O-32:2/P-32:1/31:2 & 8 & $\mathrm{nmol} / \mathrm{mL}$ & 0.29 & 0.062 & 18 & 16 & 0.34 & 0.093 & 28 \\
\hline PC $32: 3$ & 8 & $\mathrm{nmol} / \mathrm{mL}$ & 0.27 & 0.089 & 6.7 & 6.0 & 0.42 & 0.14 & 34 \\
\hline PC P-33:1/32:2 & 16 & $\mathrm{nmol} / \mathrm{mL}$ & 2.1 & 0.18 & 3.2 & 0.45 & 2.6 & 0.37 & 14 \\
\hline PC 34:0 & 12 & $\mathrm{nmol} / \mathrm{mL}$ & 3.3 & 1.4 & 3.6 & 1.5 & 2.1 & 0.37 & 18 \\
\hline PC O-34:0/33:0 & 10 & $\mathrm{nmol} / \mathrm{mL}$ & 0.70 & 0.10 & 0.70 & 0.099 & 0.76 & 0.17 & 22 \\
\hline PC $34: 1$ & 19 & $\mathrm{nmol} / \mathrm{mL}$ & 33 & 33 & 1,500 & 1,300 & 120 & 21 & 17 \\
\hline PC O-34:1/P-34:0/33:1 & 17 & $\mathrm{nmol} / \mathrm{mL}$ & 3.1 & 0.77 & 5.0 & 0.69 & 4.9 & 0.86 & 17 \\
\hline PC O-34:2/P-34:1/33:2 & 17 & $\mathrm{nmol} / \mathrm{mL}$ & 4.7 & 0.52 & 6.5 & 0.86 & 5.2 & 1.3 & 25 \\
\hline PC O-34:3/P-34:2/33:3 & 12 & $\mathrm{nmol} / \mathrm{mL}$ & 4.5 & 0.67 & 250 & 230 & 4.7 & 0.88 & 19 \\
\hline PC O-34:4/P-34:3 & 6 & $\mathrm{nmol} / \mathrm{mL}$ & 0.084 & 0.037 & 0.11 & 0.044 & 0.12 & 0.079 & 66 \\
\hline PC P-35:1/34:2 & 18 & $\mathrm{nmol} / \mathrm{mL}$ & 140 & 11 & 2,400 & 2,100 & 240 & 47 & 19 \\
\hline PC P-35:2/34:3 & 18 & $\mathrm{nmol} / \mathrm{mL}$ & 9.1 & 0.90 & 11 & 1.1 & 12 & 1.7 & 14 \\
\hline PC O-35:4/34:4 & 9 & $\mathrm{nmol} / \mathrm{mL}$ & 0.97 & 0.15 & 0.98 & 0.14 & 1.0 & 0.25 & 24 \\
\hline PC $34: 5$ & 5 & $\mathrm{nmol} / \mathrm{mL}$ & 0.047 & 0.016 & 0.048 & 0.014 & 0.034 & 0.0045 & 13 \\
\hline PC 36:1 & 17 & $\mathrm{nmol} / \mathrm{mL}$ & 26 & 1.6 & 29 & 3.6 & 26 & 4.6 & 17 \\
\hline PC O-36:0/35:0 & 5 & $\mathrm{nmol} / \mathrm{mL}$ & 0.72 & 0.36 & 0.69 & 0.31 & 0.72 & 0.53 & 74 \\
\hline
\end{tabular}

The DSL uncertainty is the Horn-Horn Duncan. The COD (\%) was calculated using the MEDM location and standard uncertainty. Final MEDM locations are in bold. For PC and PE lipid classes, the isobaric species (ether-linked) were summed and the possibilities observed by the participants are separated by a "»". Abbreviations identify lysophosphatidylethanolamines (LPE) and phosphatidylcholines (PC). 
Table 4. (cont...)

\begin{tabular}{|c|c|c|c|c|c|c|c|c|c|}
\hline Lipid & $\begin{array}{l}\text { \# of } \\
\text { Labs }\end{array}$ & Units & $\begin{array}{l}\text { DSL } \\
\text { Mean }\end{array}$ & $\begin{array}{c}\text { DSL } \\
\text { Standard } \\
\text { Uncertainty }\end{array}$ & $\begin{array}{c}\text { VR } \\
\text { Mean }\end{array}$ & $\begin{array}{c}\text { VR } \\
\text { Standard } \\
\text { Uncertainty }\end{array}$ & $\begin{array}{l}\text { MEDM } \\
\text { Location }\end{array}$ & $\begin{array}{c}\text { MEDM } \\
\text { Standard } \\
\text { Uncertainty }\end{array}$ & COD (\%) \\
\hline PC O-36:1/P-36:0/35:1 & 16 & $\mathrm{nmol} / \mathrm{mL}$ & 2.7 & 0.55 & 3.3 & 0.64 & 3.5 & 0.99 & 28 \\
\hline PC 36:2 & 18 & $\mathrm{nmol} / \mathrm{mL}$ & 100 & 4.9 & 160 & 18 & 140 & 25 & 17 \\
\hline PC O-36:2/P-36:1/35:2 & 17 & $\mathrm{nmol} / \mathrm{mL}$ & 3.2 & 1.7 & 7.7 & 1.2 & 7.4 & 1.7 & 22 \\
\hline PC $36: 3$ & 17 & $\mathrm{nmol} / \mathrm{mL}$ & 82 & 7.1 & 1,600 & 1,400 & 100 & 14 & 14 \\
\hline PC O-36:3/P-36:2/35:3 & 12 & $\mathrm{nmol} / \mathrm{mL}$ & 4.0 & 0.73 & 4.4 & 0.77 & 3.7 & 0.82 & 22 \\
\hline PC 36:4 & 19 & $\mathrm{nmol} / \mathrm{mL}$ & 48 & 27 & 130 & 18 & 150 & 28 & 19 \\
\hline PC O-36:4/P-36:3/35:4 & 17 & $\mathrm{nmol} / \mathrm{mL}$ & 12 & 1.2 & 440 & 410 & 12 & 1.4 & 12 \\
\hline PC $36: 5$ & 16 & $\mathrm{nmol} / \mathrm{mL}$ & 9.2 & 0.55 & 12 & 1.5 & 11 & 1.8 & 17 \\
\hline PC O-36:5/P-36:4/35:5 & 11 & $\mathrm{nmol} / \mathrm{mL}$ & 7.9 & 1.6 & 290 & 270 & 6.9 & 1.6 & 23 \\
\hline PC P-36:5/35:6 & 5 & $\mathrm{nmol} / \mathrm{mL}$ & 0.25 & 0.056 & 0.25 & 0.049 & 0.30 & 0.094 & 31 \\
\hline PC 36:6 & 8 & $\mathrm{nmol} / \mathrm{mL}$ & 0.32 & 0.065 & 70 & 66 & 0.28 & 0.088 & 32 \\
\hline PC 38:0 & 6 & $\mathrm{nmol} / \mathrm{mL}$ & 1.9 & 0.50 & 2.0 & 0.47 & 2.0 & 0.85 & 42 \\
\hline PC 38:1 & 6 & $\mathrm{nmol} / \mathrm{mL}$ & 0.34 & 0.089 & 0.36 & 0.086 & 0.37 & 0.17 & 47 \\
\hline PC 38:2 & 15 & $\mathrm{nmol} / \mathrm{mL}$ & 2.9 & 0.49 & 3.3 & 0.71 & 2.3 & 0.20 & 9 \\
\hline PC O-38:2/37:2 & 6 & $\mathrm{nmol} / \mathrm{mL}$ & 0.91 & 0.21 & 0.90 & 0.18 & 0.98 & 0.32 & 32 \\
\hline PC $38: 3$ & 14 & $\mathrm{nmol} / \mathrm{mL}$ & 26 & 0.83 & 26 & 3.3 & 26 & 5.2 & 20 \\
\hline PC O-38:3/P-38:2/37:3 & 14 & $\mathrm{nmol} / \mathrm{mL}$ & 1.5 & 0.38 & 1.8 & 0.39 & 1.5 & 0.51 & 34 \\
\hline PC 38:4 & 18 & $\mathrm{nmol} / \mathrm{mL}$ & 59 & 4.5 & 80 & 8.4 & 84 & 14 & 17 \\
\hline PC O-38:4/P-38:3/37:4 & 12 & $\mathrm{nmol} / \mathrm{mL}$ & 5.6 & 1.6 & 8.5 & 1.3 & 7.4 & 2.0 & 27 \\
\hline PC 38:5 & 18 & $\mathrm{nmol} / \mathrm{mL}$ & 32 & 4.2 & 410 & 360 & 42 & 7.9 & 19 \\
\hline PC O-38:5/P-38:4/37:5 & 16 & $\mathrm{nmol} / \mathrm{mL}$ & 8.2 & 0.79 & 12 & 1.5 & 11 & 1.6 & 14 \\
\hline PC 38:6 & 18 & $\mathrm{nmol} / \mathrm{mL}$ & 39 & 2.2 & 40 & 4.4 & 41 & 4.4 & 11 \\
\hline PC O-38:6/P-38:5/37:6 & 12 & $\mathrm{nmol} / \mathrm{mL}$ & 3.6 & 0.60 & 120 & 120 & 3.6 & 1.0 & 29 \\
\hline PC P-38:6/36:0 & 10 & $\mathrm{nmol} / \mathrm{mL}$ & 1.1 & 0.28 & 1.1 & 0.26 & 1.2 & 0.39 & 33 \\
\hline PC 38:7 & 8 & $\mathrm{nmol} / \mathrm{mL}$ & 2.5 & 1.8 & 73 & 66 & 0.79 & 0.35 & 44 \\
\hline PC 40:2 & 8 & $\mathrm{nmol} / \mathrm{mL}$ & 0.19 & 0.054 & 0.21 & 0.064 & 0.23 & 0.10 & 44 \\
\hline PC $40: 3$ & 7 & $\mathrm{nmol} / \mathrm{mL}$ & 0.21 & 0.084 & 0.26 & 0.081 & 0.27 & 0.14 & 51 \\
\hline PC $40: 4$ & 18 & $\mathrm{nmol} / \mathrm{mL}$ & 2.8 & 0.16 & 3.2 & 0.39 & 2.9 & 0.37 & 13 \\
\hline PC O-40:2/P-40:1 & 5 & $\mathrm{nmol} / \mathrm{mL}$ & 0.15 & 0.10 & 14 & 12 & 0.069 & 0.021 & 30 \\
\hline
\end{tabular}

The DSL uncertainty is the Horn-Horn Duncan. The COD (\%) was calculated using the MEDM location and standard uncertainty. Final MEDM locations are in bold. For PC and PE lipid classes, the isobaric species (ether-linked) were summed and the possibilities observed by the participants are separated by a "’”. Abbreviations identify phosphatidylcholines (PC). 
Table 4. (cont...)

\begin{tabular}{|c|c|c|c|c|c|c|c|c|c|}
\hline Lipid & $\begin{array}{l}\text { \# of } \\
\text { Labs }\end{array}$ & Units & $\begin{array}{l}\text { DSL } \\
\text { Mean }\end{array}$ & $\begin{array}{c}\text { DSL } \\
\text { Standard } \\
\text { Uncertainty }\end{array}$ & $\begin{array}{c}\text { VR } \\
\text { Mean }\end{array}$ & $\begin{array}{c}\text { VR } \\
\text { Standard } \\
\text { Uncertainty }\end{array}$ & $\begin{array}{c}\text { MEDM } \\
\text { Location }\end{array}$ & $\begin{array}{c}\text { MEDM } \\
\text { Standard } \\
\text { Uncertainty } \\
\end{array}$ & COD (\%) \\
\hline PC O-40:4/P-40:3/39:4 & 8 & $\mathrm{nmol} / \mathrm{mL}$ & 1.1 & 0.31 & 1.1 & 0.28 & 0.95 & 0.38 & 40 \\
\hline PC 40:5 & 18 & $\mathrm{nmol} / \mathrm{mL}$ & 4.3 & 0.50 & 6.9 & 0.82 & 6.7 & 1.1 & 16 \\
\hline PC O-40:5/P-40:4/39:5 & 12 & $\mathrm{nmol} / \mathrm{mL}$ & 2.0 & 0.43 & 2.0 & 0.41 & 1.7 & 0.45 & 27 \\
\hline PC 40:6 & 17 & $\mathrm{nmol} / \mathrm{mL}$ & 13 & 1.6 & 15 & 1.8 & 14 & 2.6 & 19 \\
\hline PC O-40:6/P-40:5/39:6 & 11 & $\mathrm{nmol} / \mathrm{mL}$ & 4.0 & 2.1 & 81 & 75 & 1.8 & 0.74 & 42 \\
\hline PC 40:7 & 16 & $\mathrm{nmol} / \mathrm{mL}$ & 2.8 & 0.59 & 3.8 & 0.55 & 3.5 & 0.76 & 21 \\
\hline PC O-40:7/P-40:6/39:7 & 9 & $\mathrm{nmol} / \mathrm{mL}$ & 1.5 & 0.34 & 41 & 37 & 1.1 & 0.23 & 20 \\
\hline PC 40:8 & 14 & $\mathrm{nmol} / \mathrm{mL}$ & 1.1 & 0.33 & 1.1 & 0.31 & 0.73 & 0.20 & 28 \\
\hline PC O-42:5/P-42:4 & 7 & $\mathrm{nmol} / \mathrm{mL}$ & 1.1 & 0.23 & 1.1 & 0.21 & 0.79 & 0.12 & 15 \\
\hline PC 42:6 & 5 & $\mathrm{nmol} / \mathrm{mL}$ & 0.090 & 0.041 & 0.12 & 0.061 & 0.079 & 0.041 & 52 \\
\hline PE 32:1 & 6 & $\mathrm{nmol} / \mathrm{mL}$ & 0.33 & 0.092 & 0.33 & 0.085 & 0.34 & 0.12 & 36 \\
\hline PE 34:0 & 5 & $\mathrm{nmol} / \mathrm{mL}$ & 1.4 & 0.53 & 1.4 & 0.47 & 1.6 & 1.1 & 65 \\
\hline PE 34:1 & 14 & $\mathrm{nmol} / \mathrm{mL}$ & 1.2 & 0.17 & 1.1 & 0.13 & 1.2 & 0.17 & 14 \\
\hline PE O-34:1/P-34:0 & 6 & $\mathrm{nmol} / \mathrm{mL}$ & 0.62 & 0.36 & 1.0 & 0.55 & 0.46 & 0.22 & 48 \\
\hline PE 34:2 & 16 & $\mathrm{nmol} / \mathrm{mL}$ & 2.9 & 0.58 & 3.1 & 0.60 & 2.2 & 0.26 & 12 \\
\hline PE O-34:2/P-34:1 & 11 & $\mathrm{nmol} / \mathrm{mL}$ & 0.72 & 0.15 & 0.72 & 0.13 & 0.78 & 0.17 & 22 \\
\hline PE O-34:3/P-34:2 & 11 & $\mathrm{nmol} / \mathrm{mL}$ & 2.0 & 0.42 & 1.9 & 0.39 & 1.5 & 0.41 & 27 \\
\hline PE 36:0 & 11 & $\mathrm{nmol} / \mathrm{mL}$ & 0.63 & 0.29 & 9.3 & 8.1 & 0.28 & 0.10 & 36 \\
\hline PE 36:1 & 14 & $\mathrm{nmol} / \mathrm{mL}$ & 2.7 & 1.4 & 4.1 & 2.0 & 1.3 & 0.26 & 20 \\
\hline PE 36:2 & 16 & $\mathrm{nmol} / \mathrm{mL}$ & 6.9 & 1.0 & 6.7 & 0.92 & 6.7 & 0.79 & 12 \\
\hline PE O-36:2/P-36:1/35:2 & 12 & $\mathrm{nmol} / \mathrm{mL}$ & 1.1 & 0.25 & 16 & 14 & 0.93 & 0.22 & 23 \\
\hline PE 36:3 & 16 & $\mathrm{nmol} / \mathrm{mL}$ & 2.9 & 0.65 & 2.9 & 0.63 & 2.4 & 0.38 & 16 \\
\hline PE O-36:3/P-36:2/35:3 & 15 & $\mathrm{nmol} / \mathrm{mL}$ & 2.9 & 0.51 & 2.9 & 0.49 & 3.2 & 0.76 & 24 \\
\hline PE 36:4 & 16 & $\mathrm{nmol} / \mathrm{mL}$ & 3.0 & 0.43 & 2.9 & 0.38 & 3.1 & 0.39 & 13 \\
\hline PE O-36:4/P-36:3 & 14 & $\mathrm{nmol} / \mathrm{mL}$ & 2.1 & 0.47 & 2.1 & 0.45 & 1.6 & 0.29 & 18 \\
\hline PE 36:5 & 11 & $\mathrm{nmol} / \mathrm{mL}$ & 0.27 & 0.071 & 0.27 & 0.067 & 0.26 & 0.13 & 48 \\
\hline PE O-36:5/P-36:4 & 15 & $\mathrm{nmol} / \mathrm{mL}$ & 4.6 & 1.2 & 120 & 110 & 4.9 & 1.9 & 38 \\
\hline PE O-36:6/P-36:5 & 7 & $\mathrm{nmol} / \mathrm{mL}$ & 0.47 & 0.27 & 0.56 & 0.24 & 0.70 & 0.49 & 70 \\
\hline
\end{tabular}

The DSL uncertainty is the Horn-Horn Duncan. The COD (\%) was calculated using the MEDM location and standard uncertainty. Final MEDM locations are in bold. For PC and PE lipid classes, the isobaric species (ether-linked) were summed and the possibilities observed by the participants are separated by a "/”. The abbreviations identify phosphatidylcholines (PC) and phosphatidylethanolamines (PE). 
Table 4. (cont...)

\begin{tabular}{|c|c|c|c|c|c|c|c|c|c|}
\hline Lipid & $\begin{array}{l}\text { \# of } \\
\text { Labs }\end{array}$ & Units & $\begin{array}{c}\text { DSL } \\
\text { Mean }\end{array}$ & $\begin{array}{c}\text { DSL } \\
\text { Standard } \\
\text { Uncertainty }\end{array}$ & $\begin{array}{c}\text { VR } \\
\text { Mean }\end{array}$ & $\begin{array}{c}\text { VR } \\
\text { Standard } \\
\text { Uncertainty }\end{array}$ & $\begin{array}{c}\text { MEDM } \\
\text { Location }\end{array}$ & $\begin{array}{c}\text { MEDM } \\
\text { Standard } \\
\text { Uncertainty }\end{array}$ & COD (\%) \\
\hline PE 38:1 & 7 & $\mathrm{nmol} / \mathrm{mL}$ & 6.2 & 3.9 & 6.5 & 3.4 & 2.6 & 1.7 & 67 \\
\hline PE 38:2 & 7 & $\mathrm{nmol} / \mathrm{mL}$ & 3.1 & 1.5 & 5.7 & 3.2 & 1.9 & 1.2 & 64 \\
\hline PE 38:3 & 14 & $\mathrm{nmol} / \mathrm{mL}$ & 1.0 & 0.16 & 0.84 & 0.098 & 0.95 & 0.20 & 21 \\
\hline PE 38:4 & 16 & $\mathrm{nmol} / \mathrm{mL}$ & 6.9 & 2.8 & 7.8 & 0.87 & 8.1 & 1.2 & 15 \\
\hline PE O-38:4/P-38:3/37:4 & 9 & $\mathrm{nmol} / \mathrm{mL}$ & 1.1 & 0.20 & 1.1 & 0.18 & 0.94 & 0.18 & 19 \\
\hline PE 38:5 & 12 & $\mathrm{nmol} / \mathrm{mL}$ & 2.5 & 0.34 & 2.4 & 0.32 & 2.7 & 0.47 & 17 \\
\hline PE O-38:5/P-38:4 & 17 & $\mathrm{nmol} / \mathrm{mL}$ & 2.8 & 1.1 & 6.7 & 1.4 & 5.8 & 1.9 & 33 \\
\hline PE 38:6 & 15 & $\mathrm{nmol} / \mathrm{mL}$ & 2.9 & 0.53 & 11 & 6.8 & 3.2 & 0.59 & 19 \\
\hline PE O-38:6/P-38:5 & 16 & $\mathrm{nmol} / \mathrm{mL}$ & 2.2 & 0.52 & 5.3 & 1.1 & 4.9 & 1.2 & 25 \\
\hline PE O-38:7/P-38:6 & 8 & $\mathrm{nmol} / \mathrm{mL}$ & 3.6 & 0.62 & 49 & 43 & 3.5 & 0.98 & 28 \\
\hline PE 40:4 & 10 & $\mathrm{nmol} / \mathrm{mL}$ & 0.23 & 0.047 & 0.22 & 0.041 & 0.26 & 0.082 & 31 \\
\hline PE 40:5 & 12 & $\mathrm{nmol} / \mathrm{mL}$ & 0.67 & 0.13 & 0.67 & 0.12 & 0.73 & 0.23 & 31 \\
\hline PE O-40:5/P-40:4/39:5 & 12 & $\mathrm{nmol} / \mathrm{mL}$ & 2.4 & 1.2 & 2.4 & 1.1 & 0.73 & 0.13 & 17 \\
\hline PE 40:6 & 14 & $\mathrm{nmol} / \mathrm{mL}$ & 1.8 & 0.29 & 1.8 & 0.27 & 1.8 & 0.36 & 20 \\
\hline PE O-40:6/P-40:5/39:6 & 14 & $\mathrm{nmol} / \mathrm{mL}$ & 1.7 & 0.45 & 1.8 & 0.47 & 1.3 & 0.31 & 23 \\
\hline PE 40:7 & 11 & $\mathrm{nmol} / \mathrm{mL}$ & 0.91 & 0.21 & 1.1 & 0.22 & 0.77 & 0.26 & 33 \\
\hline PE O-40:7/P-40:6/39:7 & 14 & $\mathrm{nmol} / \mathrm{mL}$ & 2.3 & 0.41 & 2.3 & 0.40 & 2.5 & 0.72 & 29 \\
\hline PI 32:1 & 10 & $\mathrm{nmol} / \mathrm{mL}$ & 0.45 & 0.041 & 0.45 & 0.037 & 0.56 & 0.11 & 19 \\
\hline PI 34:1 & 14 & $\mathrm{nmol} / \mathrm{mL}$ & 2.7 & 0.39 & 2.9 & 0.44 & 2.4 & 0.42 & 17 \\
\hline PI 34:2 & 14 & $\mathrm{nmol} / \mathrm{mL}$ & 2.7 & 0.14 & 2.7 & 0.17 & 2.8 & 0.38 & 14 \\
\hline PI 36:1 & 13 & $\mathrm{nmol} / \mathrm{mL}$ & 2.0 & 0.30 & 2.0 & 0.29 & 2.1 & 0.59 & 28 \\
\hline PI 36:2 & 15 & $\mathrm{nmol} / \mathrm{mL}$ & 9.3 & 1.3 & 8.5 & 0.90 & 7.7 & 0.93 & 12 \\
\hline PI 36:3 & 14 & $\mathrm{nmol} / \mathrm{mL}$ & 2.0 & 0.21 & 1.9 & 0.19 & 2.2 & 0.29 & 14 \\
\hline PI 36:4 & 14 & $\mathrm{nmol} / \mathrm{mL}$ & 2.6 & 0.25 & 2.6 & 0.24 & 3.0 & 0.48 & 16 \\
\hline PI 38:2 & 8 & $\mathrm{nmol} / \mathrm{mL}$ & 0.22 & 0.073 & 0.32 & 0.089 & 0.34 & 0.16 & 47 \\
\hline PI 38:3 & 14 & $\mathrm{nmol} / \mathrm{mL}$ & 3.0 & 0.36 & 3.0 & 0.34 & 3.4 & 0.54 & 16 \\
\hline
\end{tabular}

The DSL uncertainty is the Horn-Horn Duncan. The COD (\%) was calculated using the MEDM location and standard uncertainty. Final MEDM locations are in bold. For PC and PE lipid classes, the isobaric species (ether-linked) were summed and the possibilities observed by the participants are separated by a "’”. The abbreviations identify phosphatidylethanolamines (PE) and phosphatidylinositols (PI). 
Table 4. (cont...)

\begin{tabular}{|c|c|c|c|c|c|c|c|c|c|}
\hline Lipid & \# of Labs & Units & $\begin{array}{l}\text { DSL } \\
\text { Mean }\end{array}$ & $\begin{array}{c}\text { DSL } \\
\text { Standard } \\
\text { Uncertainty }\end{array}$ & $\begin{array}{c}\text { VR } \\
\text { Mean }\end{array}$ & $\begin{array}{c}\text { VR } \\
\text { Standard } \\
\text { Uncertainty }\end{array}$ & $\begin{array}{c}\text { MEDM } \\
\text { Location }\end{array}$ & $\begin{array}{c}\text { MEDM } \\
\text { Standard } \\
\text { Uncertainty }\end{array}$ & $\begin{array}{c}\text { COD } \\
(\%)\end{array}$ \\
\hline PI 38:4 & 17 & $\mathrm{nmol} / \mathrm{mL}$ & 19 & 2.6 & 19 & 2.4 & 19 & 2.2 & 11 \\
\hline PI 38:5 & 15 & $\mathrm{nmol} / \mathrm{mL}$ & 2.5 & 0.27 & 2.3 & 0.22 & 2.5 & 0.44 & 18 \\
\hline PI 38:6 & 10 & $\mathrm{nmol} / \mathrm{mL}$ & 0.28 & 0.019 & 0.29 & 0.018 & 0.32 & 0.031 & 10 \\
\hline PI 40:4 & 7 & $\mathrm{nmol} / \mathrm{mL}$ & 0.34 & 0.10 & 0.34 & 0.091 & 0.30 & 0.042 & 14 \\
\hline PI 40:5 & 8 & $\mathrm{nmol} / \mathrm{mL}$ & 0.51 & 0.13 & 0.58 & 0.13 & 0.63 & 0.26 & 40 \\
\hline PI 40:6 & 12 & $\mathrm{nmol} / \mathrm{mL}$ & 0.80 & 0.094 & 0.80 & 0.089 & 0.84 & 0.16 & 19 \\
\hline PG 34:1 & 5 & $\mathrm{nmol} / \mathrm{mL}$ & 0.82 & 0.60 & 1.2 & 0.38 & 1.3 & 0.60 & 45 \\
\hline PG 36:1 & 5 & $\mathrm{nmol} / \mathrm{mL}$ & 0.45 & 0.31 & 0.50 & 0.24 & 0.83 & 0.61 & 73 \\
\hline PG 36:2 & 6 & $\mathrm{nmol} / \mathrm{mL}$ & 0.54 & 0.20 & 6.0 & 5.1 & 0.67 & 0.24 & 36 \\
\hline PS 38:4 & 6 & $\mathrm{nmol} / \mathrm{mL}$ & 0.26 & 0.21 & 2.3 & 0.96 & 2.2 & 1.6 & 74 \\
\hline
\end{tabular}

The DSL uncertainty is the Horn-Horn Duncan. The COD (\%) was calculated using the MEDM location and standard uncertainty. Final MEDM locations are in bold. For PC and PE lipid classes, the isobaric species (ether-linked) were summed and the possibilities observed by the participants are separated by a "’”. The abbreviations identify phosphatidylglycerols (PG), phosphatidylinositols (PI), and phosphatidylserines (PS). 
Table 5. Consensus estimates and associated uncertainties for sphingolipids measured by at least five laboratories in SRM 1950

\begin{tabular}{|c|c|c|c|c|c|c|c|c|c|}
\hline Lipid & $\begin{array}{l}\text { \# of } \\
\text { Labs }\end{array}$ & Units & $\begin{array}{l}\text { DSL } \\
\text { Mean }\end{array}$ & $\begin{array}{c}\text { DSL } \\
\text { Standard } \\
\text { Uncertainty }\end{array}$ & $\begin{array}{c}\text { VR } \\
\text { Mean }\end{array}$ & $\begin{array}{c}\text { VR } \\
\text { Standard } \\
\text { Uncertainty }\end{array}$ & $\begin{array}{c}\text { MEDM } \\
\text { Location }\end{array}$ & $\begin{array}{c}\text { MEDM } \\
\text { Standard } \\
\text { Uncertainty } \\
\end{array}$ & COD (\%) \\
\hline HexCer d34:1 & 6 & $\mathrm{nmol} / \mathrm{mL}$ & 0.69 & 0.18 & 0.81 & 0.12 & 0.86 & 0.21 & 25 \\
\hline HexCer d36:1 & 5 & $\mathrm{nmol} / \mathrm{mL}$ & 0.13 & 0.033 & 0.14 & 0.032 & 0.13 & 0.043 & 34 \\
\hline HexCer d40:1 & 5 & $\mathrm{nmol} / \mathrm{mL}$ & 2.1 & 0.48 & 2.1 & 0.43 & 2.4 & 0.68 & 28 \\
\hline HexCer d42:1 & 6 & $\mathrm{nmol} / \mathrm{mL}$ & 2.3 & 0.56 & 2.4 & 0.51 & 2.7 & 0.73 & 27 \\
\hline HexCer d42:2 & 6 & $\mathrm{nmol} / \mathrm{mL}$ & 1.4 & 0.43 & 1.4 & 0.39 & 1.1 & 0.59 & 51 \\
\hline CER d32:1 & 8 & $\mathrm{nmol} / \mathrm{mL}$ & 0.034 & 0.0092 & 0.034 & 0.0084 & 0.051 & 0.021 & 42 \\
\hline CER d34:0 & 5 & $\mathrm{nmol} / \mathrm{mL}$ & 0.025 & 0.013 & 0.022 & 0.0098 & 0.045 & 0.031 & 70 \\
\hline CER d34:1 & 17 & $\mathrm{nmol} / \mathrm{mL}$ & 0.38 & 0.11 & 0.40 & 0.11 & 0.28 & 0.044 & 16 \\
\hline CER d36:1 & 14 & $\mathrm{nmol} / \mathrm{mL}$ & 0.12 & 0.017 & 0.11 & 0.014 & 0.12 & 0.021 & 17 \\
\hline CER d36:2 & 7 & $\mathrm{nmol} / \mathrm{mL}$ & 0.012 & 0.0047 & 0.015 & 0.0047 & 0.026 & 0.014 & 56 \\
\hline CER d38:1 & 16 & $\mathrm{nmol} / \mathrm{mL}$ & 0.42 & 0.27 & 9.4 & 7.5 & 0.11 & 0.021 & 20 \\
\hline CER d40:1 & 18 & $\mathrm{nmol} / \mathrm{mL}$ & 0.80 & 0.13 & 0.81 & 0.12 & 0.65 & 0.12 & 18 \\
\hline CER d40:2 & 6 & $\mathrm{nmol} / \mathrm{mL}$ & 0.13 & 0.029 & 4.7 & 4.2 & 0.15 & 0.021 & 14 \\
\hline CER d41:1 & 7 & $\mathrm{nmol} / \mathrm{mL}$ & 0.90 & 0.27 & 1.0 & 0.28 & 0.67 & 0.27 & 40 \\
\hline CER d42:0 & 6 & $\mathrm{nmol} / \mathrm{mL}$ & 0.32 & 0.19 & 0.56 & 0.36 & 0.28 & 0.18 & 63 \\
\hline CER d42:1 & 19 & $\mathrm{nmol} / \mathrm{mL}$ & 3.0 & 0.57 & 2.9 & 0.54 & 1.9 & 0.47 & 24 \\
\hline CER d42:2 & 19 & $\mathrm{nmol} / \mathrm{mL}$ & 1.0 & 0.19 & 1.0 & 0.17 & 0.82 & 0.10 & 12 \\
\hline CER d42:3 & 5 & $\mathrm{nmol} / \mathrm{mL}$ & 0.21 & 0.094 & 4.5 & 3.9 & 0.23 & 0.14 & 62 \\
\hline CER d44:1 & 7 & $\mathrm{nmol} / \mathrm{mL}$ & 0.069 & 0.020 & 0.071 & 0.018 & 0.063 & 0.031 & 49 \\
\hline CER d44:2 & 7 & $\mathrm{nmol} / \mathrm{mL}$ & 0.048 & 0.018 & 0.033 & 0.0089 & 0.044 & 0.022 & 49 \\
\hline SM d31:1 & 5 & $\mathrm{nmol} / \mathrm{mL}$ & 0.14 & 0.036 & 50 & 45 & 0.19 & 0.049 & 25 \\
\hline SM d32:0 & 9 & $\mathrm{nmol} / \mathrm{mL}$ & 0.93 & 0.51 & 8.5 & 6.7 & 0.47 & 0.22 & 47 \\
\hline SM d32:1 & 14 & $\mathrm{nmol} / \mathrm{mL}$ & 4.8 & 1.2 & 12 & 3.1 & 8.4 & 1.4 & 17 \\
\hline SM d32:2 & 10 & $\mathrm{nmol} / \mathrm{mL}$ & 1.0 & 0.39 & 1.2 & 0.40 & 0.66 & 0.24 & 36 \\
\hline SM d33:1 & 14 & $\mathrm{nmol} / \mathrm{mL}$ & 6.2 & 1.5 & 6.6 & 1.6 & 4.7 & 0.64 & 14 \\
\hline SM d34:0 & 14 & $\mathrm{nmol} / \mathrm{mL}$ & 11 & 5.1 & 43 & 33 & 5.8 & 1.3 & 22 \\
\hline SM d34:1 & 21 & $\mathrm{nmol} / \mathrm{mL}$ & 81 & 9.0 & 110 & 13 & 100 & 15 & 15 \\
\hline SM d34:2 & 17 & $\mathrm{nmol} / \mathrm{mL}$ & 9.2 & 1.4 & 22 & 4.4 & 16 & 2.2 & 14 \\
\hline SM d35:1 & 9 & $\mathrm{nmol} / \mathrm{mL}$ & 2.2 & 0.42 & 2.2 & 0.35 & 2.5 & 0.58 & 23 \\
\hline
\end{tabular}

The DSL uncertainty is the Horn-Horn Duncan. The COD (\%) was calculated using the MEDM location and standard uncertainty. Final MEDM locations are in bold. The abbreviations identify hexosylceramides (HexCer), ceramides (CER), and sphingomyelins (SM). 
Table 5. (cont...)

\begin{tabular}{|c|c|c|c|c|c|c|c|c|c|}
\hline Lipid & $\begin{array}{l}\text { \# of } \\
\text { Labs }\end{array}$ & Units & $\begin{array}{l}\text { DSL } \\
\text { Mean }\end{array}$ & $\begin{array}{c}\text { DSL } \\
\text { Standard } \\
\text { Uncertainty }\end{array}$ & $\begin{array}{c}\text { VR } \\
\text { Mean }\end{array}$ & $\begin{array}{c}\text { VR } \\
\text { Standard } \\
\text { Uncertainty }\end{array}$ & $\begin{array}{l}\text { MEDM } \\
\text { Location }\end{array}$ & $\begin{array}{c}\text { MEDM } \\
\text { Standard } \\
\text { Uncertainty }\end{array}$ & COD (\%) \\
\hline SM d35:2 & 6 & $\mathrm{nmol} / \mathrm{mL}$ & 0.34 & 0.090 & 0.44 & 0.099 & 0.52 & 0.21 & 39 \\
\hline SM d36:0 & 11 & $\mathrm{nmol} / \mathrm{mL}$ & 1.9 & 0.62 & 2.0 & 0.58 & 2.0 & 0.49 & 24 \\
\hline SM d36:1 & 22 & $\mathrm{nmol} / \mathrm{mL}$ & 11 & 1.9 & 19 & 2.4 & 20 & 3.7 & 18 \\
\hline SM d36:2 & 22 & $\mathrm{nmol} / \mathrm{mL}$ & 9.3 & 1.2 & 8.8 & 1.0 & 9.6 & 1.5 & 16 \\
\hline SM d36:3 & 13 & $\mathrm{nmol} / \mathrm{mL}$ & 1.5 & 0.30 & 1.7 & 0.37 & 1.3 & 0.41 & 31 \\
\hline SM d37:1 & 11 & $\mathrm{nmol} / \mathrm{mL}$ & 0.99 & 0.20 & 24 & 22 & 1.0 & 0.23 & 23 \\
\hline SM d37:2 & 5 & $\mathrm{nmol} / \mathrm{mL}$ & 0.22 & 0.086 & 0.26 & 0.091 & 0.21 & 0.10 & 50 \\
\hline SM d38:0 & 8 & $\mathrm{nmol} / \mathrm{mL}$ & 2.3 & 1.3 & 28 & 17 & 0.92 & 0.51 & 55 \\
\hline SM d38:1 & 17 & $\mathrm{nmol} / \mathrm{mL}$ & 6.0 & 0.84 & 14 & 2.4 & 11 & 3.1 & 27 \\
\hline SM d38:2 & 17 & $\mathrm{nmol} / \mathrm{mL}$ & 2.0 & 1.4 & 4.2 & 0.63 & 5.2 & 1.3 & 25 \\
\hline SM d38:3 & 8 & $\mathrm{nmol} / \mathrm{mL}$ & 0.56 & 0.16 & 0.47 & 0.12 & 0.61 & 0.24 & 39 \\
\hline SM d39:1 & 14 & $\mathrm{nmol} / \mathrm{mL}$ & 5.2 & 1.2 & 110 & 100 & 3.6 & 1.0 & 29 \\
\hline SM d39:2 & 9 & $\mathrm{nmol} / \mathrm{mL}$ & 0.66 & 0.16 & 1.3 & 0.63 & 0.61 & 0.16 & 26 \\
\hline SM d40:0 & 10 & $\mathrm{nmol} / \mathrm{mL}$ & 1.4 & 0.59 & 33 & 26 & 1.5 & 0.65 & 43 \\
\hline SM d40:1 & 17 & $\mathrm{nmol} / \mathrm{mL}$ & 7.8 & 6.2 & 34 & 9.0 & 20 & 5.1 & 25 \\
\hline SM d40:2 & 15 & $\mathrm{nmol} / \mathrm{mL}$ & 5.0 & 2.5 & 16 & 3.2 & 12 & 2.8 & 24 \\
\hline SM d40:3 & 8 & $\mathrm{nmol} / \mathrm{mL}$ & 1.7 & 0.37 & 1.6 & 0.29 & 2.2 & 0.79 & 37 \\
\hline SM d41:1 & 14 & $\mathrm{nmol} / \mathrm{mL}$ & 15 & 4.3 & 300 & 270 & 7.7 & 2.1 & 27 \\
\hline SM d41:2 & 14 & $\mathrm{nmol} / \mathrm{mL}$ & 3.8 & 0.95 & 8.0 & 1.6 & 5.8 & 1.4 & 24 \\
\hline SM d $41: 3$ & 7 & $\mathrm{nmol} / \mathrm{mL}$ & 0.79 & 0.22 & 66 & 60 & 0.77 & 0.30 & 39 \\
\hline SM d42:1 & 21 & $\mathrm{nmol} / \mathrm{mL}$ & 7.3 & 0.94 & 27 & 5.4 & 20 & 5.4 & 28 \\
\hline SM d42:2 & 18 & $\mathrm{nmol} / \mathrm{mL}$ & 29 & 3.9 & 70 & 13 & 44 & 11 & 25 \\
\hline SM d42:3 & 12 & $\mathrm{nmol} / \mathrm{mL}$ & 11 & 4.0 & 22 & 4.2 & 17 & 4.7 & 27 \\
\hline SM d42:4 & 8 & $\mathrm{nmol} / \mathrm{mL}$ & 1.9 & 0.48 & 3.1 & 0.62 & 4.2 & 1.8 & 42 \\
\hline SM d43:1 & 9 & $\mathrm{nmol} / \mathrm{mL}$ & 0.66 & 0.22 & 0.68 & 0.23 & 0.62 & 0.28 & 45 \\
\hline SM d43:2 & 10 & $\mathrm{nmol} / \mathrm{mL}$ & 1.3 & 0.39 & 1.4 & 0.39 & 1.0 & 0.29 & 29 \\
\hline SM d44:1 & 9 & $\mathrm{nmol} / \mathrm{mL}$ & 0.15 & 0.064 & 0.42 & 0.20 & 0.25 & 0.12 & 49 \\
\hline SM d44:2 & 9 & $\mathrm{nmol} / \mathrm{mL}$ & 0.41 & 0.096 & 11 & 9.7 & 0.40 & 0.13 & 32 \\
\hline SM d44:3 & 5 & $\mathrm{nmol} / \mathrm{mL}$ & 0.62 & 0.40 & 4.3 & 3.5 & 0.27 & 0.19 & 71 \\
\hline
\end{tabular}

The DSL uncertainty is the Horn-Horn Duncan. The COD (\%) was calculated using the MEDM location and standard uncertainty. Final MEDM locations are in bold. The abbreviations identify hexosylceramides (HexCer), ceramides (CER), and sphingomyelins (SM). 
Table 6. Consensus estimates and associated uncertainties for sterol lipids measured by at least five laboratories in SRM 1950

\begin{tabular}{|c|c|c|c|c|c|c|c|c|c|}
\hline Lipid & $\begin{array}{l}\text { \# of } \\
\text { Labs } \\
\end{array}$ & Units & $\begin{array}{c}\text { DSL } \\
\text { Mean } \\
\end{array}$ & $\begin{array}{c}\text { DSL } \\
\text { Standard } \\
\text { Uncertainty }\end{array}$ & $\begin{array}{c}\text { VR } \\
\text { Mean } \\
\end{array}$ & $\begin{array}{c}\text { VR } \\
\text { Standard } \\
\text { Uncertainty }\end{array}$ & $\begin{array}{c}\text { MEDM } \\
\text { Location } \\
\end{array}$ & $\begin{array}{c}\text { MEDM } \\
\text { Standard } \\
\text { Uncertainty }\end{array}$ & COD (\%) \\
\hline CE 14:0 & 7 & $\mathrm{nmol} / \mathrm{mL}$ & 16 & 3.7 & 13 & 2.0 & 16 & 6.0 & 37 \\
\hline CE 15:0 & 6 & $\mathrm{nmol} / \mathrm{mL}$ & 5.5 & 1.2 & 5.1 & 0.95 & 5.3 & 1.8 & 34 \\
\hline CE 16:0 & 13 & $\mathrm{nmol} / \mathrm{mL}$ & 49 & 16 & 170 & 31 & 210 & 58 & 28 \\
\hline CE 16:1 & 11 & $\mathrm{nmol} / \mathrm{mL}$ & 20 & 8.6 & 92 & 19 & 100 & 27 & 27 \\
\hline CE 16:2 & 5 & $\mathrm{nmol} / \mathrm{mL}$ & 1.8 & 0.32 & 1.8 & 0.28 & 1.9 & 0.46 & 25 \\
\hline CE 17:0 & 6 & $\mathrm{nmol} / \mathrm{mL}$ & 2.7 & 0.57 & 8.9 & 4.2 & 6.0 & 2.5 & 42 \\
\hline CE $17: 1$ & 9 & $\mathrm{nmol} / \mathrm{mL}$ & 6.2 & 0.23 & 6.8 & 0.51 & 8.2 & 1.0 & 13 \\
\hline CE 18:0 & 7 & $\mathrm{nmol} / \mathrm{mL}$ & 15 & 1.9 & 15 & 2.0 & 15 & 3.7 & 25 \\
\hline CE 18:1 & 14 & $\mathrm{nmol} / \mathrm{mL}$ & 180 & 81 & 520 & 120 & 450 & 110 & 25 \\
\hline CE 18:2 & 14 & $\mathrm{nmol} / \mathrm{mL}$ & 110 & 63 & 2,900 & 1,100 & 1,700 & 430 & 26 \\
\hline CE 18:3 & 13 & $\mathrm{nmol} / \mathrm{mL}$ & 14 & 10 & 230 & 93 & 84 & 24 & 28 \\
\hline CE 20:1 & 6 & $\mathrm{nmol} / \mathrm{mL}$ & 0.51 & 0.11 & 0.77 & 0.20 & 1.3 & 0.66 & 51 \\
\hline CE 20:2 & 9 & $\mathrm{nmol} / \mathrm{mL}$ & 4.9 & 2.3 & 5.0 & 2.1 & 5.8 & 3.1 & 53 \\
\hline CE 20:3 & 13 & $\mathrm{nmol} / \mathrm{mL}$ & 12 & 2.0 & 100 & 47 & 35 & 12 & 35 \\
\hline CE 20:4 & 14 & $\mathrm{nmol} / \mathrm{mL}$ & 210 & 130 & 960 & 400 & 350 & 58 & 17 \\
\hline CE 20:5 & 12 & $\mathrm{nmol} / \mathrm{mL}$ & 23 & 1.4 & 99 & 35 & 38 & 8.6 & 23 \\
\hline CE 22:4 & 7 & $\mathrm{nmol} / \mathrm{mL}$ & 6.9 & 5.9 & 8.0 & 4.1 & 1.2 & 0.70 & 59 \\
\hline CE 22:5 & 6 & $\mathrm{nmol} / \mathrm{mL}$ & 25 & 19 & 26 & 17 & 4.1 & 1.6 & 39 \\
\hline CE 22:6 & 11 & $\mathrm{nmol} / \mathrm{mL}$ & 32 & 8.7 & 140 & 74 & 37 & 9.5 & 26 \\
\hline Free Cholesterol & 8 & $\mathrm{nmol} / \mathrm{mL}$ & 710 & 160 & 780 & 70 & 770 & 110 & 14 \\
\hline CDCA & 7 & $\mathrm{nmol} / \mathrm{mL}$ & 0.58 & 0.26 & 0.67 & 0.31 & 0.30 & 0.11 & 38 \\
\hline CA & 9 & $\mathrm{nmol} / \mathrm{mL}$ & 0.16 & 0.036 & 0.24 & 0.093 & 0.12 & 0.034 & 28 \\
\hline
\end{tabular}

The DSL uncertainty is the Horn-Horn Duncan. The COD (\%) was calculated using the MEDM location and standard uncertainty. Final MEDM locations are in bold. The abbreviations identify chenodeoxycholic acid (CDCA), cholic acid (CA), and cholesteryl ester (CE). 
Table 6. (cont...)

\begin{tabular}{|c|c|c|c|c|c|c|c|c|c|}
\hline Lipid & \# of Labs & Units & $\begin{array}{l}\text { DSL } \\
\text { Mean }\end{array}$ & $\begin{array}{c}\text { DSL } \\
\text { Standard } \\
\text { Uncertainty }\end{array}$ & $\begin{array}{c}\text { VR } \\
\text { Mean }\end{array}$ & $\begin{array}{c}\text { VR } \\
\text { Standard } \\
\text { Uncertainty }\end{array}$ & $\begin{array}{l}\text { MEDM } \\
\text { Location }\end{array}$ & $\begin{array}{c}\text { MEDM } \\
\text { Standard } \\
\text { Uncertainty }\end{array}$ & COD (\%) \\
\hline DCA & 9 & $\mathrm{nmol} / \mathrm{mL}$ & 0.51 & 0.11 & 3.3 & 2.7 & 0.35 & 0.083 & 24 \\
\hline GCDCA & 8 & $\mathrm{nmol} / \mathrm{mL}$ & 1.0 & 0.15 & 1.0 & 0.14 & 1.1 & 0.18 & 17 \\
\hline GDCA & 7 & $\mathrm{nmol} / \mathrm{mL}$ & 0.38 & 0.064 & 0.37 & 0.056 & 0.43 & 0.069 & 16 \\
\hline GLCA & 6 & $\mathrm{nmol} / \mathrm{mL}$ & 0.025 & 0.0053 & 0.025 & 0.0045 & 0.025 & 0.0018 & 7 \\
\hline GUDCA & 6 & $\mathrm{nmol} / \mathrm{mL}$ & 0.15 & 0.016 & 0.15 & 0.015 & 0.15 & 0.024 & 16 \\
\hline GCA & 6 & $\mathrm{nmol} / \mathrm{mL}$ & 0.28 & 0.064 & 0.28 & 0.060 & 0.24 & 0.069 & 29 \\
\hline LCA & 8 & $\mathrm{nmol} / \mathrm{mL}$ & 0.015 & 0.0029 & 0.69 & 0.64 & 0.014 & 0.0036 & 26 \\
\hline TCDCA & 9 & $\mathrm{nmol} / \mathrm{mL}$ & 0.085 & 0.0071 & 0.10 & 0.024 & 0.084 & 0.0050 & 6 \\
\hline TCA & 9 & $\mathrm{nmol} / \mathrm{mL}$ & 0.042 & 0.016 & 0.047 & 0.019 & 0.026 & 0.0056 & 22 \\
\hline TDCA & 8 & $\mathrm{nmol} / \mathrm{mL}$ & 0.048 & 0.0089 & 0.048 & 0.0090 & 0.040 & 0.0064 & 16 \\
\hline TLCA & 5 & $\mathrm{nmol} / \mathrm{mL}$ & 0.0023 & 0.00041 & 0.0026 & 0.00044 & 0.0027 & 0.00069 & 26 \\
\hline UDCA & 8 & $\mathrm{nmol} / \mathrm{mL}$ & 0.15 & 0.043 & 0.38 & 0.19 & 0.11 & 0.024 & 22 \\
\hline
\end{tabular}

The DSL uncertainty is the Horn-Horn Duncan. The COD (\%) was calculated using the MEDM location and standard uncertainty. Final MEDM locations are in bold. The abbreviations identify deoxycholic acid (DCA), glycochenodeoxycholic acid (GCDCA), glycodeoxycholic acid (GDCA), glycolithocholic acid (GLCA), glycoursodeoxycholic acid (GUDCA), glyocholic acid (GCA), lithocholic acid (LCA), taurochenodeoxycholic acid (TCDCA), taurocholic acid (TCA), taurodeoxycholic acid (TDCA), taurolithocholic acid (TLCA), ursodeoxycholic acid (UDCA). 
Table 7. Consensus estimates and corresponding uncertainties for those lipids measured by three to four laboratories in SRM 1950.

\begin{tabular}{|c|c|c|c|c|c|c|c|}
\hline Lipid & $\begin{array}{l}\# \text { of } \\
\text { Labs }\end{array}$ & Units & $\begin{array}{l}\text { DSL } \\
\text { Mean }\end{array}$ & $\begin{array}{c}\text { DSL } \\
\text { Standard } \\
\text { Uncertainty }\end{array}$ & $\begin{array}{c}\text { MEDM } \\
\text { Location }\end{array}$ & $\begin{array}{c}\text { DSL COD } \\
(\%) \\
\end{array}$ & $\begin{array}{c}\text { Percent } \\
\text { Difference } \\
(\%) \\
\end{array}$ \\
\hline Chenodeoxycholic acid-3-Sulfate & 3 & $\mathrm{nmol} / \mathrm{mL}$ & 0.013 & 0.051 & 0.14 & 389 & 165 \\
\hline Hyodeoxycholic acid & 4 & $\mathrm{nmol} / \mathrm{mL}$ & 0.023 & 0.019 & 0.035 & 84 & 42 \\
\hline Taurolithocholic acid sulfate & 3 & $\mathrm{nmol} / \mathrm{mL}$ & 0.088 & 0.018 & 0.10 & 21 & 15 \\
\hline$\alpha$-Muricholic acid & 4 & $\mathrm{nmol} / \mathrm{mL}$ & 0.0048 & 0.0048 & 0.012 & 101 & 85 \\
\hline$\beta$-Muricholic acid & 4 & $\mathrm{nmol} / \mathrm{mL}$ & 0.0018 & 0.00082 & 0.0033 & 46 & 60 \\
\hline$\omega$-Muricholic acid & 3 & $\mathrm{nmol} / \mathrm{mL}$ & 0.0056 & 0.00013 & 0.0057 & 2 & 1 \\
\hline CE 14:1 & 4 & $\mathrm{nmol} / \mathrm{mL}$ & 0.93 & 0.26 & 1.2 & 28 & 22 \\
\hline CE 20:0 & 4 & $\mathrm{nmol} / \mathrm{mL}$ & 1.4 & 0.21 & 3.1 & 15 & 79 \\
\hline CE 22:0 & 4 & $\mathrm{nmol} / \mathrm{mL}$ & 0.35 & 0.034 & 0.36 & 10 & 2 \\
\hline CE 22:1 & 4 & $\mathrm{nmol} / \mathrm{mL}$ & 0.41 & 0.21 & 0.52 & 52 & 23 \\
\hline CE 24:0 & 3 & $\mathrm{nmol} / \mathrm{mL}$ & 0.28 & 0.052 & 0.31 & 18 & 7 \\
\hline CE 24:1 & 3 & $\mathrm{nmol} / \mathrm{mL}$ & 0.15 & 0.014 & 0.15 & 9 & 1 \\
\hline Total Cholesterol & 3 & $\mathrm{nmol} / \mathrm{mL}$ & 4,000 & 24 & 4,000 & 1 & 0 \\
\hline LacCer d34:1 & 3 & $\mathrm{nmol} / \mathrm{mL}$ & 2.7 & 0.72 & 2.1 & 26 & 28 \\
\hline HexCer d38:1 & 3 & $\mathrm{nmol} / \mathrm{mL}$ & 0.20 & 0.00015 & 0.20 & 0 & 0 \\
\hline LacCer d42:1 & 3 & $\mathrm{nmol} / \mathrm{mL}$ & 0.30 & 0.079 & 0.28 & 26 & 9 \\
\hline CerG3(d18:1/16:0) & 3 & $\mathrm{nmol} / \mathrm{mL}$ & 0.27 & 0.33 & 0.98 & 119 & 113 \\
\hline CerG3(d18:1/24:0) & 3 & $\mathrm{nmol} / \mathrm{mL}$ & 0.065 & 0.13 & 0.37 & 195 & 141 \\
\hline CER d16:0 & 3 & $\mathrm{nmol} / \mathrm{mL}$ & 0.052 & 0.0075 & 0.056 & 14 & 9 \\
\hline CER d18:0 & 3 & $\mathrm{nmol} / \mathrm{mL}$ & 0.031 & 0.012 & 0.042 & 41 & 31 \\
\hline CER d20:0 & 3 & $\mathrm{nmol} / \mathrm{mL}$ & 0.028 & 0.0076 & 0.030 & 28 & 8 \\
\hline CER d22:0 & 3 & $\mathrm{nmol} / \mathrm{mL}$ & 0.084 & 0.029 & 0.11 & 35 & 30 \\
\hline CER d24:0 & 3 & $\mathrm{nmol} / \mathrm{mL}$ & 0.090 & 0.033 & 0.084 & 37 & 6 \\
\hline
\end{tabular}

The final mean was calculated using the DSL estimation method (grey). The uncertainty associated with MEDM was not provided as it does not hold up when less than five laboratories are reporting. COD (\%) is calculated using the DSL mean and standard uncertainty. The percent difference (\%) was calculated comparing the DSL mean and MEDM location values. Abbreviations: cholesteryl ester (CE), ceramide (CER), hexosylceramide (HexCer), and lactosylceramide (LacCer). 
Table 7. (cont...)

\begin{tabular}{|c|c|c|c|c|c|c|c|}
\hline Lipid & \# of Labs & Units & $\begin{array}{l}\text { DSL } \\
\text { Mean }\end{array}$ & $\begin{array}{c}\text { DSL } \\
\text { Standard } \\
\text { Uncertainty }\end{array}$ & $\begin{array}{l}\text { MEDM } \\
\text { Location }\end{array}$ & $\begin{array}{c}\text { DSL COD } \\
(\%)\end{array}$ & $\begin{array}{c}\text { Percent } \\
\text { Difference } \\
\text { (\%) }\end{array}$ \\
\hline CER d24:1 & 3 & $\mathrm{nmol} / \mathrm{mL}$ & 0.11 & 0.095 & 0.21 & 88 & 63 \\
\hline CER d34:2 & 3 & $\mathrm{nmol} / \mathrm{mL}$ & 0.12 & 0.13 & 0.033 & 107 & 116 \\
\hline CER d38:2 & 3 & $\mathrm{nmol} / \mathrm{mL}$ & 0.013 & 0.0052 & 0.019 & 39 & 33 \\
\hline CER d38:5 & 3 & $\mathrm{nmol} / \mathrm{mL}$ & 0.0013 & 0.027 & 0.37 & 2015 & 199 \\
\hline CER d39:1 & 4 & $\mathrm{nmol} / \mathrm{mL}$ & 0.093 & 0.016 & 0.11 & 18 & 15 \\
\hline CER d40:0 & 4 & $\mathrm{nmol} / \mathrm{mL}$ & 0.12 & 0.062 & 0.17 & 51 & 34 \\
\hline CER d41:2 & 3 & $\mathrm{nmol} / \mathrm{mL}$ & 0.099 & 0.042 & 0.14 & 43 & 35 \\
\hline CER d43:1 & 3 & $\mathrm{nmol} / \mathrm{mL}$ & 0.12 & 0.048 & 0.16 & 40 & 30 \\
\hline CER d44:3 & 3 & $\mathrm{nmol} / \mathrm{mL}$ & 0.32 & 0.27 & 0.22 & 84 & 38 \\
\hline DHC d16:0 & 3 & $\mathrm{nmol} / \mathrm{mL}$ & 5.3 & 0.67 & 5.5 & 13 & 4 \\
\hline DHC d22:0 & 3 & $\mathrm{nmol} / \mathrm{mL}$ & 0.26 & 0.046 & 0.29 & 18 & 12 \\
\hline DHC d24:0 & 3 & $\mathrm{nmol} / \mathrm{mL}$ & 0.40 & 0.050 & 0.33 & 12 & 20 \\
\hline DHC d24:1 & 3 & $\mathrm{nmol} / \mathrm{mL}$ & 0.97 & 0.14 & 1.1 & 15 & 13 \\
\hline DAG 30:1 & 3 & $\mathrm{nmol} / \mathrm{mL}$ & 0.41 & 0.26 & 0.51 & 64 & 22 \\
\hline DAG 32:3 & 4 & $\mathrm{nmol} / \mathrm{mL}$ & 0.24 & 0.38 & 0.73 & 154 & 101 \\
\hline DAG 34:4 & 4 & $\mathrm{nmol} / \mathrm{mL}$ & 0.44 & 0.38 & 0.71 & 86 & 46 \\
\hline DAG 40:0 & 3 & $\mathrm{nmol} / \mathrm{mL}$ & 0.53 & 0.32 & 0.56 & 60 & 5 \\
\hline DAG 40:1 & 3 & $\mathrm{nmol} / \mathrm{mL}$ & 0.017 & 0.0053 & 0.021 & 32 & 22 \\
\hline DAG 40:4 & 4 & $\mathrm{nmol} / \mathrm{mL}$ & 0.91 & 0.34 & 0.83 & 38 & 10 \\
\hline FFA 14:0 & 3 & $\mathrm{nmol} / \mathrm{mL}$ & 2.1 & 3.4 & 5.1 & 167 & 85 \\
\hline FFA $17: 0$ & 3 & $\mathrm{nmol} / \mathrm{mL}$ & 1.7 & 0.23 & 1.7 & 14 & 1 \\
\hline FFA $17: 1$ & 3 & $\mathrm{nmol} / \mathrm{mL}$ & 0.86 & 0.15 & 0.86 & 18 & 0 \\
\hline FFA 20:1 & 3 & $\mathrm{nmol} / \mathrm{mL}$ & 1.8 & 0.17 & 1.6 & 9 & 9 \\
\hline FFA 20:2 & 4 & $\mathrm{nmol} / \mathrm{mL}$ & 1.00 & 0.34 & 1.3 & 34 & 23 \\
\hline
\end{tabular}

The final mean was calculated using the DSL estimation method (grey). The uncertainty associated with MEDM was not provided as it does not hold up when less than five laboratories are reporting. COD (\%) is calculated using the DSL mean and standard uncertainty. The percent difference (\%) was calculated comparing the DSL mean and MEDM location values. Abbreviations: ceramide (CER), dihydroceramide (DHC), diacylglycerol (DAG), and free fatty acid (FFA). 
Table 7. (cont...)

\begin{tabular}{|c|c|c|c|c|c|c|c|}
\hline Lipid & \# of Labs & Units & $\begin{array}{l}\text { DSL } \\
\text { Mean }\end{array}$ & $\begin{array}{c}\text { DSL } \\
\text { Standard } \\
\text { Uncertainty }\end{array}$ & $\begin{array}{l}\text { MEDM } \\
\text { Location }\end{array}$ & $\begin{array}{c}\text { DSL COD } \\
(\%)\end{array}$ & $\begin{array}{c}\text { Percent } \\
\text { Difference } \\
\text { (\%) }\end{array}$ \\
\hline FFA 22:4 & 4 & $\mathrm{nmol} / \mathrm{mL}$ & 1.0 & 0.32 & 1.3 & 32 & 22 \\
\hline FFA 24:1 & 3 & $\mathrm{nmol} / \mathrm{mL}$ & 0.66 & 0.61 & 1.0 & 93 & 45 \\
\hline LPC 14:1 & 4 & $\mathrm{nmol} / \mathrm{mL}$ & 0.0013 & 0.0012 & 0.12 & 92 & 196 \\
\hline LPC P-18:0 & 4 & $\mathrm{nmol} / \mathrm{mL}$ & 0.37 & 0.14 & 0.68 & 39 & 60 \\
\hline LPC O-18:1 & 3 & $\mathrm{nmol} / \mathrm{mL}$ & 0.41 & 0.13 & 0.35 & 31 & 16 \\
\hline LPC 19:0 & 4 & $\mathrm{nmol} / \mathrm{mL}$ & 0.94 & 0.94 & 1.6 & 99 & 54 \\
\hline LPC 19:1 & 3 & $\mathrm{nmol} / \mathrm{mL}$ & 0.023 & 0.014 & 0.037 & 60 & 46 \\
\hline LPC O-20:0 & 4 & $\mathrm{nmol} / \mathrm{mL}$ & 0.023 & 0.0049 & 0.025 & 22 & 9 \\
\hline LPC O-20:1 & 3 & $\mathrm{nmol} / \mathrm{mL}$ & 0.072 & 0.075 & 0.024 & 104 & 100 \\
\hline LPC O-22:0 & 3 & $\mathrm{nmol} / \mathrm{mL}$ & 1.2 & 1.4 & 0.029 & 112 & 191 \\
\hline LPC 24:1 & 3 & $\mathrm{nmol} / \mathrm{mL}$ & 0.022 & 0.0071 & 0.023 & 33 & 4 \\
\hline LPC 26:0 & 3 & $\mathrm{nmol} / \mathrm{mL}$ & 0.015 & 0.0031 & 0.016 & 21 & 3 \\
\hline LPE 16:1 & 3 & $\mathrm{nmol} / \mathrm{mL}$ & 0.15 & 0.21 & 0.77 & 137 & 133 \\
\hline LPE P-18:1 & 3 & $\mathrm{nmol} / \mathrm{mL}$ & 0.23 & 0.17 & 0.40 & 73 & 54 \\
\hline LPE $18: 3$ & 3 & $\mathrm{nmol} / \mathrm{mL}$ & 0.00034 & 0.0011 & 0.45 & 307 & 200 \\
\hline LPE 20:1 & 4 & $\mathrm{nmol} / \mathrm{mL}$ & 0.00046 & 0.0089 & 1.4 & 1947 & 200 \\
\hline LPE 20:2 & 3 & $\mathrm{nmol} / \mathrm{mL}$ & 2.4 & 2.1 & 4.4 & 87 & 59 \\
\hline LPE 20:5 & 3 & $\mathrm{nmol} / \mathrm{mL}$ & 0.0084 & 0.080 & 0.54 & 942 & 194 \\
\hline LPE 22:0 & 3 & $\mathrm{nmol} / \mathrm{mL}$ & 0.51 & 0.76 & 1.0 & 147 & 66 \\
\hline LPE 22:4 & 3 & $\mathrm{nmol} / \mathrm{mL}$ & 2.5 & 1.8 & 1.0 & 73 & 84 \\
\hline LPE 22:5 & 3 & $\mathrm{nmol} / \mathrm{mL}$ & 0.45 & 0.71 & 1.7 & 158 & 115 \\
\hline PC 26:0 & 3 & $\mathrm{nmol} / \mathrm{mL}$ & 0.23 & 0.21 & 0.44 & 94 & 64 \\
\hline PC 28:0 & 4 & $\mathrm{nmol} / \mathrm{mL}$ & 0.16 & 0.025 & 0.15 & 15 & 5 \\
\hline PC 40:1 & 3 & $\mathrm{nmol} / \mathrm{mL}$ & 0.30 & 0.25 & 0.29 & 82 & 4 \\
\hline
\end{tabular}

The final mean was calculated using the DSL estimation method (grey). The uncertainty associated with MEDM was not provided as it does not hold up when less than five laboratories are reporting. COD (\%) is calculated using the DSL mean and standard uncertainty. The percent difference (\%) was calculated comparing the DSL mean and MEDM location values. Abbreviations: free fatty acid (FFA), lysophosphatidylcholine (LPC), lysophosphatidylethanolamine (LPE), and phosphatidylcholine (PC). 
Table 7. (cont...)

\begin{tabular}{|c|c|c|c|c|c|c|c|}
\hline Lipid & \# of Labs & Units & $\begin{array}{l}\text { DSL } \\
\text { Mean }\end{array}$ & $\begin{array}{c}\text { DSL } \\
\text { Standard } \\
\text { Uncertainty }\end{array}$ & $\begin{array}{l}\text { MEDM } \\
\text { Location }\end{array}$ & $\begin{array}{c}\text { DSL COD } \\
(\%)\end{array}$ & $\begin{array}{c}\text { Percent } \\
\text { Difference } \\
\text { (\%) }\end{array}$ \\
\hline PC O-40:8/P-40:7 & 4 & $\mathrm{nmol} / \mathrm{mL}$ & 0.083 & 0.033 & 0.11 & 40 & 24 \\
\hline PC 42:2 & 3 & $\mathrm{nmol} / \mathrm{mL}$ & 0.097 & 0.065 & 0.17 & 66 & 56 \\
\hline PC 42:4 & 3 & $\mathrm{nmol} / \mathrm{mL}$ & 0.067 & 0.053 & 0.14 & 79 & 68 \\
\hline PC O-42:4/P-42:3 & 4 & $\mathrm{nmol} / \mathrm{mL}$ & 0.47 & 0.14 & 0.58 & 31 & 22 \\
\hline PC 42:5 & 4 & $\mathrm{nmol} / \mathrm{mL}$ & 0.14 & 0.061 & 0.19 & 42 & 26 \\
\hline PC O-42:6/P-42:5 & 4 & $\mathrm{nmol} / \mathrm{mL}$ & 0.46 & 0.14 & 0.55 & 29 & 18 \\
\hline PC O-42:7 & 3 & $\mathrm{nmol} / \mathrm{mL}$ & 0.067 & 0.0011 & 0.078 & 2 & 15 \\
\hline PC O-44:5/P-44:4 & 3 & $\mathrm{nmol} / \mathrm{mL}$ & 1.3 & 0.30 & 1.4 & 24 & 6 \\
\hline PC O-44:6e/P-44:5 & 4 & $\mathrm{nmol} / \mathrm{mL}$ & 0.69 & 0.32 & 0.97 & 46 & 34 \\
\hline PE 34:3 & 4 & $\mathrm{nmol} / \mathrm{mL}$ & 0.14 & 0.020 & 0.15 & 14 & 8 \\
\hline PE 35:1 & 4 & $\mathrm{nmol} / \mathrm{mL}$ & 0.15 & 0.045 & 0.14 & 30 & 5 \\
\hline PE P-38:2/37:3 & 3 & $\mathrm{nmol} / \mathrm{mL}$ & 0.91 & 0.40 & 1.3 & 44 & 34 \\
\hline PE O-40:8/P-40:7 & 4 & $\mathrm{nmol} / \mathrm{mL}$ & 11 & 9.5 & 2.6 & 86 & 124 \\
\hline PE P-42:5 & 3 & $\mathrm{nmol} / \mathrm{mL}$ & 0.30 & 0.13 & 0.55 & 44 & 60 \\
\hline PE P-42:6 & 3 & $\mathrm{nmol} / \mathrm{mL}$ & 0.86 & 0.69 & 0.66 & 80 & 26 \\
\hline PG 34:2 & 4 & $\mathrm{nmol} / \mathrm{mL}$ & 0.55 & 0.22 & 0.45 & 40 & 19 \\
\hline PG 36:3 & 4 & $\mathrm{nmol} / \mathrm{mL}$ & 0.50 & 0.11 & 0.43 & 22 & 15 \\
\hline PG 38:4 & 3 & $\mathrm{nmol} / \mathrm{mL}$ & 0.040 & 0.020 & 0.065 & 51 & 47 \\
\hline PG 40:6 & 3 & $\mathrm{nmol} / \mathrm{mL}$ & 0.025 & 0.023 & 0.048 & 94 & 63 \\
\hline LPI 18:0 & 4 & $\mathrm{nmol} / \mathrm{mL}$ & 0.14 & 0.078 & 0.22 & 56 & 44 \\
\hline LPI 18:1 & 3 & $\mathrm{nmol} / \mathrm{mL}$ & 0.15 & 0.12 & 0.27 & 77 & 56 \\
\hline LPI 18:2 & 4 & $\mathrm{nmol} / \mathrm{mL}$ & 0.17 & 0.083 & 0.28 & 48 & 45 \\
\hline LPI 20:4 & 4 & $\mathrm{nmol} / \mathrm{mL}$ & 0.21 & 0.082 & 0.26 & 40 & 23 \\
\hline PI 32:0 & 3 & $\mathrm{nmol} / \mathrm{mL}$ & 0.24 & 0.072 & 0.30 & 30 & 21 \\
\hline
\end{tabular}

The final mean was calculated using the DSL estimation method (grey). The uncertainty associated with MEDM was not provided as it does not hold up when less than five laboratories are reporting. COD (\%) is calculated using the DSL mean and standard uncertainty. The percent difference (\%) was calculated comparing the DSL mean and MEDM location values. Abbreviations: phosphatidylcholine (PC), phosphatidylethanolamine (PE), phosphatidylglycerol (PG), lysophosphatidylinositol (LPI), and phosphatidylinositol (PI). 
Table 7. (cont...)

\begin{tabular}{|c|c|c|c|c|c|c|c|}
\hline Lipid & \# of Labs & Units & $\begin{array}{l}\text { DSL } \\
\text { Mean } \\
\end{array}$ & $\begin{array}{c}\text { DSL } \\
\text { Standard } \\
\text { Uncertainty }\end{array}$ & $\begin{array}{c}\text { MEDM } \\
\text { Location }\end{array}$ & $\begin{array}{c}\text { DSL COD } \\
(\%) \\
\end{array}$ & $\begin{array}{c}\text { Percent } \\
\text { Difference } \\
(\%)\end{array}$ \\
\hline PI 34:0 & 3 & $\mathrm{nmol} / \mathrm{mL}$ & 0.056 & 0.0078 & 0.086 & 14 & 43 \\
\hline PI 36:5 & 4 & $\mathrm{nmol} / \mathrm{mL}$ & 0.13 & 0.12 & 1.7 & 91 & 172 \\
\hline PS 36:1 & 4 & $\mathrm{nmol} / \mathrm{mL}$ & 0.16 & 0.066 & 0.18 & 43 & 11 \\
\hline PS $36: 2$ & 4 & $\mathrm{nmol} / \mathrm{mL}$ & 0.090 & 0.20 & 0.73 & 224 & 156 \\
\hline PS $36: 4$ & 3 & $\mathrm{nmol} / \mathrm{mL}$ & 0.19 & 0.19 & 0.36 & 104 & 63 \\
\hline PS 38:1 & 3 & $\mathrm{nmol} / \mathrm{mL}$ & 0.23 & 0.046 & 0.25 & 20 & 9 \\
\hline PS 38:2 & 3 & $\mathrm{nmol} / \mathrm{mL}$ & 0.24 & 0.040 & 0.27 & 16 & 11 \\
\hline PS $40: 4$ & 3 & $\mathrm{nmol} / \mathrm{mL}$ & 0.39 & 0.81 & 2.0 & 206 & 134 \\
\hline PS 40:5 & 3 & $\mathrm{nmol} / \mathrm{mL}$ & 0.37 & 0.93 & 7.4 & 253 & 181 \\
\hline SM d30:1 & 4 & $\mathrm{nmol} / \mathrm{mL}$ & 0.71 & 0.47 & 0.70 & 67 & 2 \\
\hline SM d33:0 & 4 & $\mathrm{nmol} / \mathrm{mL}$ & 13 & 14 & 0.026 & 108 & 199 \\
\hline SM d33:2 & 3 & $\mathrm{nmol} / \mathrm{mL}$ & 0.037 & 0.021 & 0.056 & 55 & 41 \\
\hline SM d34:3 & 3 & $\mathrm{nmol} / \mathrm{mL}$ & 0.14 & 0.041 & 0.18 & 30 & 27 \\
\hline SM d35:0 & 3 & $\mathrm{nmol} / \mathrm{mL}$ & 0.044 & 0.0072 & 0.050 & 16 & 13 \\
\hline SM d36:4 & 3 & $\mathrm{nmol} / \mathrm{mL}$ & 0.60 & 0.43 & 1.0 & 71 & 51 \\
\hline SM d38:4 & 3 & $\mathrm{nmol} / \mathrm{mL}$ & 0.16 & 0.11 & 0.25 & 68 & 44 \\
\hline SM d40:4 & 4 & $\mathrm{nmol} / \mathrm{mL}$ & 0.15 & 0.068 & 0.26 & 44 & 53 \\
\hline SM d42:5 & 3 & $\mathrm{nmol} / \mathrm{mL}$ & 0.36 & 0.021 & 0.37 & 6 & 4 \\
\hline SM d43:3 & 4 & $\mathrm{nmol} / \mathrm{mL}$ & 0.18 & 0.010 & 1.4 & 6 & 153 \\
\hline dhSph-1P & 3 & $\mathrm{nmol} / \mathrm{mL}$ & 0.10 & 0.035 & 0.10 & 35 & 4 \\
\hline Sph-1P & 3 & $\mathrm{nmol} / \mathrm{mL}$ & 0.42 & 0.0076 & 0.41 & 2 & 2 \\
\hline TAG 38:0 & 3 & $\mathrm{nmol} / \mathrm{mL}$ & 0.25 & 0.20 & 0.14 & 81 & 53 \\
\hline TAG 40:0 & 4 & $\mathrm{nmol} / \mathrm{mL}$ & 0.33 & 0.11 & 0.44 & 33 & 31 \\
\hline TAG 40:1 & 3 & $\mathrm{nmol} / \mathrm{mL}$ & 0.15 & 0.12 & 0.83 & 75 & 137 \\
\hline
\end{tabular}

The final mean was calculated using the DSL estimation method (grey). The uncertainty associated with MEDM was not provided as it does not hold up when less than five laboratories are reporting. COD (\%) is calculated using the DSL mean and standard uncertainty. The percent difference (\%) was calculated comparing the DSL mean and MEDM location values. Abbreviations: phosphatidylinositol (PI), phosphatidylserine (PS), sphingomyelin (SM), and triacylglycerol (TAG). 
Table 7. (cont...)

\begin{tabular}{|c|c|c|c|c|c|c|c|}
\hline Lipid & \# of Labs & Units & $\begin{array}{l}\text { DSL } \\
\text { Mean } \\
\end{array}$ & $\begin{array}{c}\text { DSL } \\
\text { Standard } \\
\text { Uncertainty }\end{array}$ & $\begin{array}{c}\text { MEDM } \\
\text { Location }\end{array}$ & $\begin{array}{c}\text { DSL COD } \\
(\%) \\
\end{array}$ & $\begin{array}{c}\text { Percent } \\
\text { Difference } \\
(\%)\end{array}$ \\
\hline TAG 40:2 & 3 & $\mathrm{nmol} / \mathrm{mL}$ & 0.058 & 0.025 & 0.085 & 43 & 39 \\
\hline TAG $44: 3$ & 4 & $\mathrm{nmol} / \mathrm{mL}$ & 0.18 & 0.0094 & 0.18 & 5 & 1 \\
\hline TAG 45:0 & 3 & $\mathrm{nmol} / \mathrm{mL}$ & 1.6 & 1.5 & 0.54 & 91 & 99 \\
\hline TAG 46:4 & 3 & $\mathrm{nmol} / \mathrm{mL}$ & 0.27 & 0.20 & 0.50 & 73 & 61 \\
\hline TAG 46:5 & 3 & $\mathrm{nmol} / \mathrm{mL}$ & 0.044 & 0.098 & 1.1 & 222 & 184 \\
\hline TAG 47:0 & 3 & $\mathrm{nmol} / \mathrm{mL}$ & 0.43 & 0.10 & 0.53 & 23 & 22 \\
\hline TAG 47:1 & 4 & $\mathrm{nmol} / \mathrm{mL}$ & 0.47 & 0.17 & 0.59 & 36 & 24 \\
\hline TAG 47:2 & 3 & $\mathrm{nmol} / \mathrm{mL}$ & 0.21 & 0.027 & 0.22 & 13 & 7 \\
\hline TAG 47:3 & 3 & $\mathrm{nmol} / \mathrm{mL}$ & 0.078 & 0.035 & 0.10 & 44 & 29 \\
\hline TAG 48:5 & 3 & $\mathrm{nmol} / \mathrm{mL}$ & 1.1 & 0.92 & 2.1 & 85 & 64 \\
\hline TAG 48:6 & 3 & $\mathrm{nmol} / \mathrm{mL}$ & 0.029 & 0.056 & 1.3 & 193 & 191 \\
\hline TAG 48:7 & 3 & $\mathrm{nmol} / \mathrm{mL}$ & 0.018 & 0.093 & 0.53 & 510 & 187 \\
\hline TAG 49:0 & 3 & $\mathrm{nmol} / \mathrm{mL}$ & 0.31 & 0.055 & 0.29 & 18 & 5 \\
\hline TAG 49:3 & 4 & $\mathrm{nmol} / \mathrm{mL}$ & 0.43 & 0.20 & 0.36 & 45 & 18 \\
\hline TAG 50:6 & 4 & $\mathrm{nmol} / \mathrm{mL}$ & 0.24 & 0.059 & 0.30 & 24 & 22 \\
\hline TAG 50:7 & 3 & $\mathrm{nmol} / \mathrm{mL}$ & 0.077 & 0.088 & 0.51 & 115 & 148 \\
\hline TAG 51:0 & 4 & $\mathrm{nmol} / \mathrm{mL}$ & 0.42 & 0.19 & 0.35 & 45 & 18 \\
\hline TAG 51:5 & 3 & $\mathrm{nmol} / \mathrm{mL}$ & 0.55 & 0.28 & 0.52 & 51 & 5 \\
\hline TAG 53:0 & 3 & $\mathrm{nmol} / \mathrm{mL}$ & 0.053 & 0.039 & 0.39 & 74 & 152 \\
\hline TAG 53:1 & 3 & $\mathrm{nmol} / \mathrm{mL}$ & 0.73 & 0.49 & 0.44 & 67 & 50 \\
\hline TAG 54:8 & 4 & $\mathrm{nmol} / \mathrm{mL}$ & 0.92 & 0.30 & 0.90 & 32 & 2 \\
\hline TAG 55:3 & 3 & $\mathrm{nmol} / \mathrm{mL}$ & 0.43 & 0.15 & 0.35 & 34 & 20 \\
\hline TAG 55:4 & 3 & $\mathrm{nmol} / \mathrm{mL}$ & 0.32 & 0.029 & 0.42 & 9 & 28 \\
\hline TAG 55:6 & 3 & $\mathrm{nmol} / \mathrm{mL}$ & 0.17 & 0.073 & 0.39 & 44 & 78 \\
\hline
\end{tabular}

The final mean was calculated using the DSL estimation method (grey). The uncertainty associated with MEDM was not provided as it does not hold up when less than five laboratories are reporting. COD (\%) is calculated using the DSL mean and standard uncertainty. The percent difference (\%) was calculated comparing the DSL mean and MEDM location values. Abbreviations: triacylglycerol (TAG). 
Table 7. (cont...)

\begin{tabular}{|c|c|c|c|c|c|c|c|}
\hline Lipid & \# of Labs & Units & $\begin{array}{l}\text { DSL } \\
\text { Mean }\end{array}$ & $\begin{array}{c}\text { DSL } \\
\text { Standard } \\
\text { Uncertainty }\end{array}$ & $\begin{array}{c}\text { MEDM } \\
\text { Location }\end{array}$ & $\begin{array}{c}\text { DSL COD } \\
(\%)\end{array}$ & $\begin{array}{c}\text { Percent } \\
\text { Difference } \\
(\%)\end{array}$ \\
\hline TAG 56:1 & 4 & $\mathrm{nmol} / \mathrm{mL}$ & 0.68 & 0.28 & 0.83 & 42 & 20 \\
\hline TAG 57:6 & 3 & $\mathrm{nmol} / \mathrm{mL}$ & 0.034 & 0.023 & 0.056 & 66 & 48 \\
\hline TAG 57:7 & 3 & $\mathrm{nmol} / \mathrm{mL}$ & 0.049 & 0.026 & 0.069 & 54 & 35 \\
\hline TAG 58:10 & 4 & $\mathrm{nmol} / \mathrm{mL}$ & 0.92 & 0.56 & 0.55 & 61 & 51 \\
\hline TAG 58:11 & 3 & $\mathrm{nmol} / \mathrm{mL}$ & 0.11 & 0.018 & 0.13 & 16 & 13 \\
\hline TAG 58:2 & 3 & $\mathrm{nmol} / \mathrm{mL}$ & 0.53 & 0.21 & 0.66 & 39 & 22 \\
\hline TAG 58:3 & 3 & $\mathrm{nmol} / \mathrm{mL}$ & 0.19 & 0.019 & 0.21 & 10 & 8 \\
\hline TAG 60:10 & 3 & $\mathrm{nmol} / \mathrm{mL}$ & 0.26 & 0.088 & 0.20 & 34 & 24 \\
\hline TAG 60:11 & 4 & $\mathrm{nmol} / \mathrm{mL}$ & 0.42 & 0.37 & 0.13 & 89 & 107 \\
\hline TAG 60:12 & 3 & $\mathrm{nmol} / \mathrm{mL}$ & 0.34 & 0.37 & 0.082 & 109 & 123 \\
\hline
\end{tabular}

The final mean was calculated using the DSL estimation method (grey). The uncertainty associated with MEDM was not provided as it does not hold up when less than five laboratories are reporting. COD (\%) is calculated using the DSL mean and standard uncertainty. The percent difference (\%) was calculated comparing the DSL mean and MEDM location values. Abbreviations: triacylglycerols (TAG). 
Table 8. Consensus estimates and associated uncertainties for eicosanoids measured by three to four laboratories in SRM 1950

\begin{tabular}{|c|c|c|c|c|c|c|c|}
\hline Lipid & $\begin{array}{l}\text { \# of } \\
\text { Labs } \\
\end{array}$ & Units & $\begin{array}{l}\text { DSL } \\
\text { Mean } \\
\end{array}$ & $\begin{array}{c}\text { DSL } \\
\text { Standard } \\
\text { Uncertainty }\end{array}$ & $\begin{array}{c}\text { MEDM } \\
\text { Location } \\
\end{array}$ & $\begin{array}{c}\text { DSL COD } \\
(\%) \\
\end{array}$ & $\begin{array}{c}\text { Percent } \\
\text { Difference } \\
(\%) \\
\end{array}$ \\
\hline 11,12-DiHETrE & 3 & $\mathrm{pmol} / \mathrm{mL}$ & 0.82 & 0.28 & 1.0 & 34 & 20 \\
\hline 11-HDoHE & 3 & $\mathrm{pmol} / \mathrm{mL}$ & 0.63 & 0.092 & 0.61 & 15 & 3 \\
\hline 11-HEPE & 3 & $\mathrm{pmol} / \mathrm{mL}$ & 0.22 & 0.14 & 0.38 & 63 & 54 \\
\hline 11-HETE & 4 & $\mathrm{pmol} / \mathrm{mL}$ & 2.1 & 1.0 & 1.5 & 49 & 32 \\
\hline 12,13-DiHOME & 3 & $\mathrm{pmol} / \mathrm{mL}$ & 5.1 & 0.38 & 5.0 & 7 & 3 \\
\hline 12,13-ЕрОМЕ & 3 & $\mathrm{pmol} / \mathrm{mL}$ & 6.9 & 2.0 & 7.8 & 28 & 12 \\
\hline 12-HEPE & 4 & $\mathrm{pmol} / \mathrm{mL}$ & 2.1 & 1.2 & 0.98 & 59 & 71 \\
\hline 12-HHTrE & 3 & $\mathrm{pmol} / \mathrm{mL}$ & 0.23 & 0.053 & 0.27 & 23 & 18 \\
\hline 13-HODE & 3 & $\mathrm{pmol} / \mathrm{mL}$ & 21 & 9.5 & 13 & 46 & 47 \\
\hline 13-HOTrE & 3 & $\mathrm{pmol} / \mathrm{mL}$ & 0.54 & 0.056 & 0.56 & 10 & 4 \\
\hline 14,15-DiHETrE & 3 & $\mathrm{pmol} / \mathrm{mL}$ & 1.6 & 0.75 & 1.1 & 47 & 37 \\
\hline 14-HDoHE & 4 & $\mathrm{pmol} / \mathrm{mL}$ & 1.3 & 0.11 & 1.3 & 8 & 2 \\
\hline 15-HEPE & 4 & $\mathrm{pmol} / \mathrm{mL}$ & 0.28 & 0.16 & 0.42 & 57 & 39 \\
\hline 17-HDoHE & 3 & $\mathrm{pmol} / \mathrm{mL}$ & 0.82 & 0.036 & 0.84 & 4 & 2 \\
\hline 18-HEPE & 3 & $\mathrm{pmol} / \mathrm{mL}$ & 0.28 & 0.069 & 0.25 & 25 & 10 \\
\hline 19,20-DiHDoPE (19,20-DiHDPA) & 3 & $\mathrm{pmol} / \mathrm{mL}$ & 0.96 & 0.15 & 1.6 & 16 & 50 \\
\hline 20-HETE & 3 & $\mathrm{pmol} / \mathrm{mL}$ & 2.1 & 0.53 & 2.0 & 25 & 7 \\
\hline 4-HDoHE & 4 & $\mathrm{pmol} / \mathrm{mL}$ & 2.5 & 0.40 & 3.9 & 16 & 43 \\
\hline 5,15-DiHETE & 4 & $\mathrm{pmol} / \mathrm{mL}$ & 0.16 & 0.045 & 0.25 & 28 & 43 \\
\hline 5,6-DiHETrE & 3 & $\mathrm{pmol} / \mathrm{mL}$ & 1.2 & 0.34 & 1.5 & 30 & 26 \\
\hline 5,6-EET & 3 & $\mathrm{pmol} / \mathrm{mL}$ & 0.82 & 0.28 & 1.0 & 34 & 20 \\
\hline 5-HEPE & 4 & $\mathrm{pmol} / \mathrm{mL}$ & 0.85 & 0.016 & 0.86 & 2 & 2 \\
\hline 5-HETrE & 3 & $\mathrm{pmol} / \mathrm{mL}$ & 0.99 & 0.27 & 1.2 & 27 & 21 \\
\hline
\end{tabular}

The final mean was calculated using the DSL estimation method (grey). The uncertainty associated with MEDM was not provided as it does not hold up when less than five laboratories are reporting. COD (\%) is calculated using the DSL mean and standard uncertainty. The percent difference (\%) was calculated comparing the DSL mean and MEDM location values. For nomenclature of eicosanoids, see the LIPID MAPS Structure Database Fatty Acyls [FA] (W) --> Eicosanoids [FA03] (http://www.lipidmaps.org/data/structure/LMSDSearch.php). 
Table 8. (cont...)

\begin{tabular}{|c|c|c|c|c|c|c|c|}
\hline Lipid & \# of Labs & Units & $\begin{array}{l}\text { DSL } \\
\text { Mean }\end{array}$ & $\begin{array}{c}\text { DSL } \\
\text { Standard } \\
\text { Uncertainty }\end{array}$ & $\begin{array}{c}\text { MEDM } \\
\text { Location }\end{array}$ & $\begin{array}{c}\text { DSL COD } \\
(\%)\end{array}$ & $\begin{array}{c}\text { Percent } \\
\text { Difference } \\
(\%)\end{array}$ \\
\hline 5-oxoETE (5-KETE) & 3 & $\mathrm{pmol} / \mathrm{mL}$ & 0.37 & 0.13 & 0.48 & 34 & 25 \\
\hline 8,9-DiHETrE & 3 & $\mathrm{pmol} / \mathrm{mL}$ & 0.51 & 0.22 & 0.65 & 43 & 25 \\
\hline 8-HDoHE & 3 & $\mathrm{pmol} / \mathrm{mL}$ & 4.3 & 3.7 & 1.0 & 87 & 124 \\
\hline 8-HEPE & 4 & $\mathrm{pmol} / \mathrm{mL}$ & 0.30 & 0.24 & 0.89 & 80 & 100 \\
\hline 8-HETE & 4 & $\mathrm{pmol} / \mathrm{mL}$ & 0.98 & 0.22 & 1.1 & 22 & 15 \\
\hline 8-HETrE & 3 & $\mathrm{pmol} / \mathrm{mL}$ & 0.38 & 0.093 & 0.46 & 24 & 19 \\
\hline 9,10-ЕрОМЕ & 3 & $\mathrm{pmol} / \mathrm{mL}$ & 7.5 & 3.3 & 4.2 & 45 & 56 \\
\hline 9,10-DiHOME & 3 & $\mathrm{pmol} / \mathrm{mL}$ & 6.7 & 0.44 & 7.0 & 7 & 5 \\
\hline 9-НЕРE & 4 & $\mathrm{pmol} / \mathrm{mL}$ & 0.43 & 0.087 & 0.50 & 20 & 15 \\
\hline 9-HETE & 4 & $\mathrm{pmol} / \mathrm{mL}$ & 0.85 & 0.082 & 0.85 & 10 & 0 \\
\hline 9-HODE & 3 & $\mathrm{pmol} / \mathrm{mL}$ & 10 & 2.6 & 9.7 & 25 & 5 \\
\hline 9-HOTrE & 3 & $\mathrm{pmol} / \mathrm{mL}$ & 0.62 & 0.18 & 0.80 & 28 & 25 \\
\hline 9-OxоODE (9-KODE) & 3 & $\mathrm{pmol} / \mathrm{mL}$ & 7.3 & 1.3 & 6.8 & 18 & 7 \\
\hline LTB4 & 3 & $\mathrm{pmol} / \mathrm{mL}$ & 0.019 & 0.0069 & 0.030 & 37 & 47 \\
\hline PGD2 & 4 & $\mathrm{pmol} / \mathrm{mL}$ & 0.082 & 0.048 & 0.17 & 58 & 71 \\
\hline PGE2 & 4 & $\mathrm{pmol} / \mathrm{mL}$ & 0.035 & 0.014 & 0.040 & 40 & 14 \\
\hline
\end{tabular}

The final mean was calculated using the DSL estimation method (grey). The uncertainty associated with MEDM was not provided as it does not hold up when less than five laboratories are reporting. COD (\%) is calculated using the DSL mean and standard uncertainty. The percent difference $\%)$ was calculated comparing the DSL mean and MEDM location values. For nomenclature of eicosanoids, see the LIPID MAPS Structure Database Fatty Acyls [FA] (W) --> Eicosanoids [FA03] (http://www.lipidmaps.org/data/structure/LMSDSearch.php). 


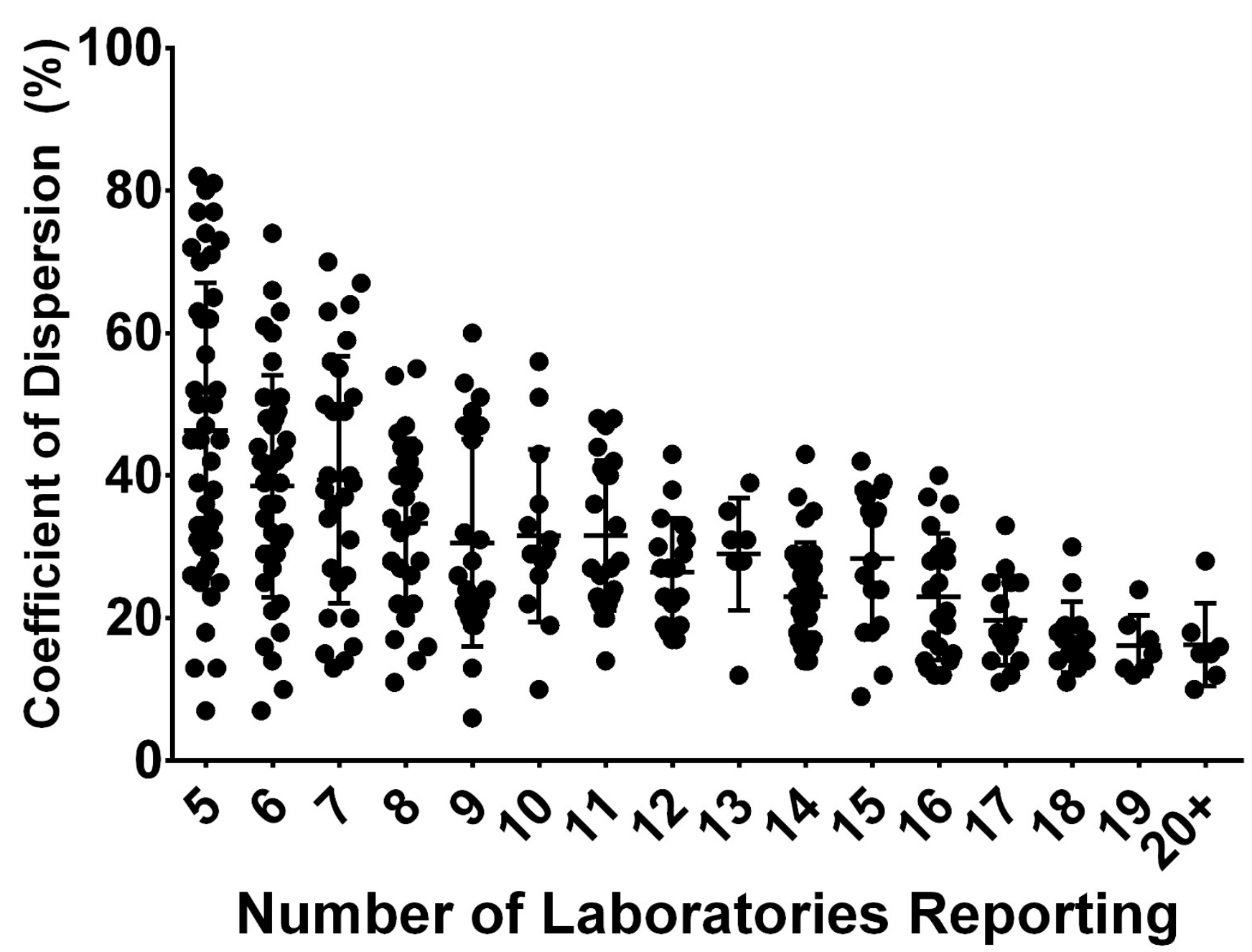

Figure 1. Coefficient of dispersion (COD, \%) values plotted against the number of laboratories reporting for each lipid ( $n=339, n \geq 5$ laboratories reporting) measured in SRM 1950. The COD for each lipid was calculated using the MEDM location and standard uncertainty. 


\section{Appendix A}

Upon agreement to participate in the interlaboratory comparison exercise, this Excel template was provided to each participating laboratory for data submission. The template included tabs for laboratories to provide details on the laboratory and methodological information including sample preparation, sample introduction and/or chromatography, mass spectrometric methods, and data processing software. Additional tabs were provided containing potential target lipid species (see Table S7 for all 320 target lipids). An example is shown for cholesteryl esters (CE, Table S6). On these target lipid tabs, we required the exact mass of lipid identified and the corresponding adduct used for scanning.

Table S1. Data submission template for basic laboratory information

\begin{tabular}{|l|l|}
\hline \multicolumn{2}{|l|}{ Please fill out what you can } \\
\hline Date(s) of Analyses & \\
\hline Main Principal Investigator (PI): & \\
\hline PI Affiliation: & \\
\hline Type of affiliation (academia, industry, core lab, etc.): & \\
\hline Interest in publication? (y/n) & $\begin{array}{l}\text { provide names as you } \\
\text { want them to appear }\end{array}$ \\
\hline If yes, who would be listed as a co-author? & $\begin{array}{l}\text { provide names as you } \\
\text { want them to appear }\end{array}$ \\
\hline If yes, who would be listed as an acknowledgement? & \\
\hline & \\
\hline $\begin{array}{l}\text { Do you use quality controls materials for your lipidomics } \\
\text { measurements? (y/n) }\end{array}$ & \\
\hline $\begin{array}{l}\text { Do you think Standard Reference Materials (SRMs) are needed for } \\
\text { lipidomics? (y/n) }\end{array}$ & \\
\hline If so, what matrices would you find most valuable? & \\
\hline & \\
\hline $\begin{array}{l}\text { Would you be interested in participating in a follow-up interlab } \\
\text { exercise? (y/n) }\end{array}$ & \\
\hline & \\
\hline
\end{tabular}


Table S2. Data submission template for sample preparation

\begin{tabular}{|c|c|}
\hline Please fill out what you can & \\
\hline \multicolumn{2}{|l|}{ How much material did you use for extraction? } \\
\hline \multirow{2}{*}{\multicolumn{2}{|c|}{$\begin{array}{l}\text { What type of extraction did you perform (BD, Folch, MTBE, etc.)? } \\
\text { Extraction protocol: }\end{array}$}} \\
\hline & \\
\hline \multicolumn{2}{|l|}{ What did you reconstitute extract in? } \\
\hline \multicolumn{2}{|l|}{ How long from sample thaw to the samples run on instrument? } \\
\hline How do you prepare internal standard solutions? (gravimetrically)? & Amt Spiked in? \\
\hline \multicolumn{2}{|l|}{ What internal standards did you employ (for each class)? } \\
\hline \multicolumn{2}{|l|}{ * Fatty acids (FFA) } \\
\hline \multicolumn{2}{|l|}{ * Triacylglycerols (TAG) } \\
\hline \multicolumn{2}{|l|}{ * Diacylglycerols (DAG) } \\
\hline \multicolumn{2}{|l|}{ * Cholesterol (CHOL) } \\
\hline \multicolumn{2}{|l|}{ * Cholesteryl esters (CE) } \\
\hline \multicolumn{2}{|l|}{ * Phosphatidylcholines (PC) } \\
\hline \multicolumn{2}{|l|}{ * Lysophosphatidylcholines (LPC) } \\
\hline \multicolumn{2}{|l|}{ * Phosphatidylethanolamines (PE) } \\
\hline \multicolumn{2}{|l|}{ * Lysophosphatidylethanolamines (LPE) } \\
\hline \multicolumn{2}{|l|}{ * Phosphatidylserines (PS) } \\
\hline \multicolumn{2}{|l|}{ * Phosphatidylinositols (PI) } \\
\hline \multicolumn{2}{|l|}{ * Phosphatidic acids (PA) } \\
\hline \multicolumn{2}{|l|}{ * Sphingomyelins (SM) } \\
\hline \multicolumn{2}{|l|}{$*$ Ceramides (CER) } \\
\hline * Other & \\
\hline
\end{tabular}


Table S3. Data submission template for sample introduction/chromatography

\begin{tabular}{|l|l|}
\hline \multicolumn{2}{|l|}{ Please fill out what you can } \\
\hline If shotgun & \\
\hline Type of instrument: & \\
\hline Manual, Flow-Injection, Nanomate, etc. & \\
\hline Shotgun settings: & \\
\hline Injection amount: & \\
\hline Which lipids monitored with approach: & \\
\hline & \\
\hline If LC & \\
\hline Type of LC system: & \\
\hline Column information: & \\
\hline & \\
\hline Solvent composition/gradient: & \\
\hline Other LC settings: & \\
\hline Injection amount: & \\
\hline Which lipids monitored with approach: & \\
\hline
\end{tabular}


Table S4. Data submission template for mass spectrometric parameters

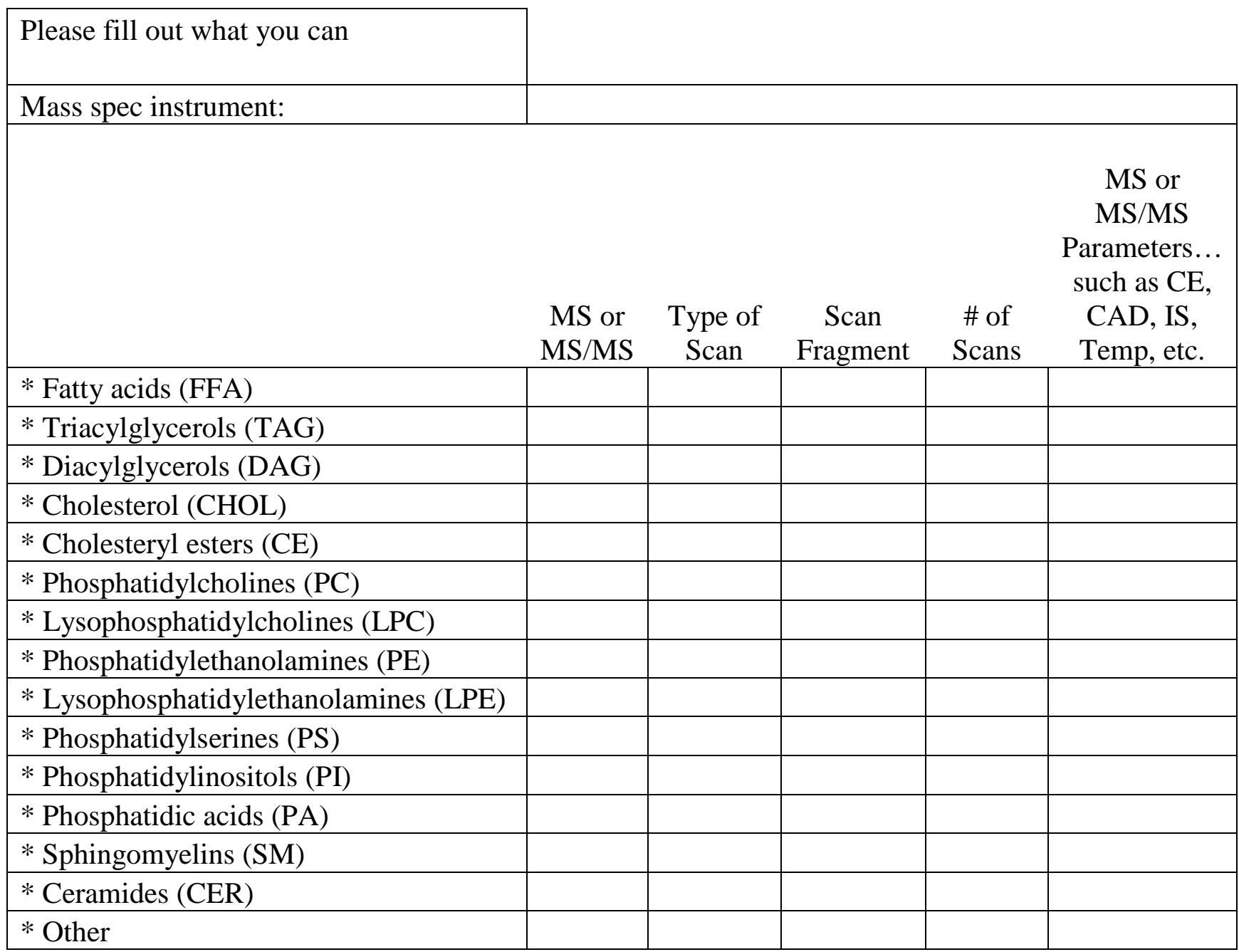




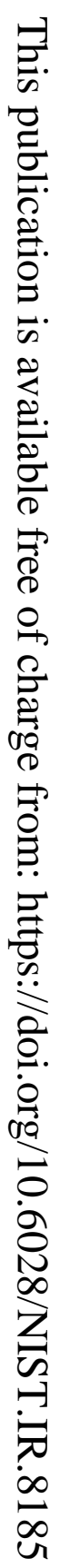

Table S5. Data submission template for data processing information Please fill out what you can

Software employed:

Correction factors employed:

Response factors employed:

Other processing details: 
Table S6. Example of a data submission tab for cholesteryl esters (CE), which includes potential targets species, $\mathrm{m} / \mathrm{z}$ value used for identification, lipid adduct employed, and triplicate concentrations reported in $\mathrm{nmol} / \mathrm{mL}$

\begin{tabular}{|c|c|c|c|c|}
\hline \multicolumn{5}{|c|}{ Type of adduct: $[\mathrm{M}+\mathrm{H}]^{+},[\mathrm{M}+\mathrm{Na}]^{+},\left[\mathrm{M}+\mathrm{NH}_{4}\right]^{+},[\mathrm{M}+\mathrm{Li}]^{+}$, [M-H]$]^{-}$, etc...: } \\
\hline \multirow[b]{3}{*}{ Lipid Species } & \multirow[b]{3}{*}{$\mathrm{m} / \mathrm{z}$ value } & \multicolumn{3}{|c|}{ in $\mathrm{nmol} / 1 \mathrm{~mL}$ plasma } \\
\hline & & Rep 1 & Rep 2 & Rep 3 \\
\hline & & SRM 1950 & SRM 1950 & SRM 1950 \\
\hline \multicolumn{5}{|l|}{$14: 1$} \\
\hline \multicolumn{5}{|l|}{ 14:0 } \\
\hline \multicolumn{5}{|l|}{$15: 1$} \\
\hline \multicolumn{5}{|l|}{$15: 0$} \\
\hline \multicolumn{5}{|l|}{ 16:1 } \\
\hline \multicolumn{5}{|l|}{ 16:0 } \\
\hline \multicolumn{5}{|l|}{$17: 1$} \\
\hline \multicolumn{5}{|l|}{$17: 0$} \\
\hline \multicolumn{5}{|l|}{$18: 3$} \\
\hline \multicolumn{5}{|l|}{$18: 2$} \\
\hline \multicolumn{5}{|l|}{ 18:1 } \\
\hline \multicolumn{5}{|l|}{ 18:0 } \\
\hline \multicolumn{5}{|l|}{$20: 5$} \\
\hline \multicolumn{5}{|l|}{$20: 4$} \\
\hline \multicolumn{5}{|l|}{$20: 3$} \\
\hline \multicolumn{5}{|l|}{$20: 2$} \\
\hline \multicolumn{5}{|l|}{$20: 1$} \\
\hline \multicolumn{5}{|l|}{ 20:0 } \\
\hline \multicolumn{5}{|l|}{$22: 6$} \\
\hline \multicolumn{5}{|l|}{$22: 4$} \\
\hline \multicolumn{5}{|l|}{$22: 2$} \\
\hline 22:1 & & & & \\
\hline $22: 0$ & & & & \\
\hline
\end{tabular}

Cholesteryl esters are listed in total carbons of fatty acyl chains by total number of double bonds in fatty acyl chains (C:DB). 
Table S7. Target lipid species (by class, $n=320$ ) provided in the data submission template

\begin{tabular}{|c|c|c|c|c|c|c|c|c|c|c|c|c|c|c|c|c|}
\hline$\underline{\mathrm{CE}}$ & $\underline{\text { FFA }}$ & $\underline{\text { TAG }}$ & $\underline{\mathrm{DAG}}$ & $\underline{\mathrm{PC}}$ & $\underline{\mathrm{PC}}$ & $\underline{\mathrm{LPC}}$ & $\underline{\mathrm{PE}}$ & $\underline{\mathrm{PE}}$ & $\underline{\mathrm{LPE}}$ & $\underline{\text { PS }}$ & $\underline{\mathrm{PA}}$ & $\underline{\mathrm{PG}}$ & $\underline{\mathrm{PI}}$ & $\underline{\text { CER }}$ & $\underline{\mathrm{SM}}$ & $\underline{\mathrm{SM}}$ \\
\hline 14:1 & $12: 0$ & $48: 2$ & $30: 2$ & $30: 1$ & 38:1 & $14: 1$ & $32: 1$ & O-40:6 & 16:0 & 32:1 & $32: 1$ & $34: 2$ & $32: 1$ & 14:0 & 31:0 & 41:3 \\
\hline 14:0 & $14: 1$ & $48: 1$ & $30: 1$ & $32: 2$ & 38:0 & $14: 0$ & P-34:2 & $40: 6$ & $18: 2$ & $34: 2$ & 32:0 & $34: 1$ & $34: 2$ & 16:0 & $32: 2$ & $41: 2$ \\
\hline $15: 1$ & $14: 0$ & $50: 4$ & $30: 0$ & $32: 1$ & $40: 8$ & $16: 1$ & $34: 2$ & O-40:5 & 18:1 & $34: 1$ & $34: 2$ & $36: 5$ & $34: 1$ & $17: 0$ & 32:1 & 41:1 \\
\hline 15:0 & 15:0 & $50: 3$ & $32: 3$ & $32: 0$ & $40: 7$ & P-16:0 & P-34:1 & $40: 5$ & 18:0 & 34:0 & $34: 1$ & $36: 4$ & 34:0 & 18:1 & 32:0 & $42: 4$ \\
\hline $16: 1$ & $16: 1$ & $50: 2$ & $32: 2$ & $34: 3$ & $40: 6$ & O-16:0 & $34: 1$ & $40: 4$ & $20: 4$ & $36: 4$ & $34: 0$ & $36: 3$ & $36: 5$ & 18:0 & 33:1 & $42: 3$ \\
\hline 16:0 & $16: 0$ & $50: 1$ & $32: 1$ & O-34:2 & $40: 5$ & $16: 0$ & 34:0 & $40: 1$ & $22: 1$ & $36: 3$ & $36: 4$ & $36: 2$ & $36: 4$ & 20:0 & 33:0 & $42: 2$ \\
\hline $17: 1$ & $17: 1$ & 50:0 & $32: 0$ & $34: 2$ & $40: 4$ & $18: 3$ & O-36:5/P-36:4 & $42: 7$ & $22: 6$ & $36: 2$ & $36: 3$ & $36: 1$ & $36: 3$ & 22:0 & $34: 2$ & $42: 1$ \\
\hline $17: 0$ & $17: 0$ & $52: 5$ & $34: 4$ & O-34:1 & $40: 3$ & $18: 2$ & $36: 5$ & P-42:6 & & $36: 1$ & $36: 2$ & 38:6 & $36: 2$ & $24: 1$ & $34: 1$ & 43:3 \\
\hline $18: 3$ & $18: 3$ & $52: 4$ & $34: 3$ & $34: 1$ & $40: 2$ & $18: 1$ & O-36:4/P-36:3 & $42: 6$ & & $36: 0$ & $36: 1$ & $38: 5$ & $36: 1$ & 24:0 & 34:0 & 43:2 \\
\hline 18:2 & $18: 2$ & $52: 3$ & $34: 2$ & $34: 0$ & & O-18:0 & $36: 4$ & P-42:5 & & $38: 6$ & $36: 0$ & $38: 4$ & $36: 0$ & $26: 2$ & $35: 1$ & $43: 1$ \\
\hline 18:1 & $18: 1$ & $52: 2$ & $34: 1$ & $36: 5$ & & 18:0 & O-36:3/P-36:2 & $42: 5$ & & $38: 5$ & 38:6 & $40: 9$ & 38:6 & $26: 1$ & $35: 0$ & $44: 3$ \\
\hline 18:0 & 18:0 & $52: 1$ & $34: 0$ & O-36:4/P-36:3 & & $20: 5$ & $36: 3$ & $42: 1$ & & $38: 4$ & $38: 5$ & $40: 8$ & $38: 5$ & $26: 0$ & $36: 3$ & $44: 2$ \\
\hline $20: 5$ & $20: 5$ & $52: 0$ & $36: 5$ & $36: 4$ & & $20: 4$ & O-36:2/P-36:1 & & & $38: 3$ & $38: 4$ & $40: 7$ & $38: 4$ & & $36: 2$ & $44: 1$ \\
\hline $20: 4$ & $20: 4$ & $54: 6$ & $36: 4$ & $36: 3$ & & $20: 3$ & $36: 2$ & & & $38: 2$ & $38: 3$ & $40: 6$ & $38: 3$ & & $36: 1$ & \\
\hline $20: 3$ & $20: 3$ & $54: 5$ & $36: 3$ & O-36:2/P-36:1 & & $20: 1$ & $36: 1$ & & & $38: 1$ & $38: 2$ & $40: 5$ & $38: 2$ & & $36: 0$ & \\
\hline $20: 2$ & $20: 2$ & $54: 4$ & $36: 2$ & $36: 2$ & & 22:6 & $36: 0$ & & & $40: 7$ & & $40: 4$ & 40:6 & & $37: 1$ & \\
\hline $20: 1$ & $20: 1$ & $54: 3$ & $36: 1$ & O-36:1/P-36:0 & & 22:5 & O-38:6/P-38:5 & & & $40: 6$ & & & $40: 5$ & & $38: 3$ & \\
\hline 20:0 & $20: 0$ & $54: 2$ & $36: 0$ & $36: 1$ & & $22: 4$ & $38: 6$ & & & $40: 5$ & & & $40: 4$ & & $38: 2$ & \\
\hline $22: 6$ & $22: 6$ & $54: 1$ & $38: 5$ & $36: 0$ & & & O-38:5/P-38:4 & & & $40: 4$ & & & $40: 2$ & & $38: 1$ & \\
\hline $22: 4$ & $22: 5$ & $54: 0$ & $38: 4$ & $38: 6$ & & & $38: 5$ & & & $40: 3$ & & & & & 38:0 & \\
\hline $22: 2$ & $22: 4$ & $56: 6$ & $38: 3$ & O-38:5/P-38:4 & & & $38: 4$ & & & & & & & & 39:2 & \\
\hline $22: 1$ & $22: 3$ & $56: 5$ & $38: 2$ & $38: 5$ & & & $38: 3$ & & & & & & & & 39:1 & \\
\hline \multirow[t]{2}{*}{$22: 0$} & $22: 1$ & $56: 4$ & $38: 1$ & $38: 4$ & & & $38: 2$ & & & & & & & & $40: 3$ & \\
\hline & $22: 0$ & & $38: 0$ & O-38:3/P-38:2 & & & $38: 1$ & & & & & & & & $40: 2$ & \\
\hline \multirow[t]{4}{*}{ CHOL } & $24: 1$ & & $40: 7$ & $38: 2$ & & & O-40:7 & & & & & & & & $40: 1$ & \\
\hline & $24: 0$ & & $40: 6$ & & & & $40: 7$ & & & & & & & & $40: 0$ & \\
\hline & $26: 0$ & & $40: 5$ & & & & & & & & & & & & & \\
\hline & & & $40: 4$ & & & & & & & & & & & & & \\
\hline
\end{tabular}

The list was partially derived from the lipids detected in the LIPID MAPS consortium analysis of SRM 1950 (6). 
The total number of lipids reported in SRM $1950(n=1527)$ measured by at least one laboratory at the sum composition level. The 1527 lipids were measured across five lipid categories, as shown in Table S8. Lipid class abbreviations are also provided. Individual lipids reported for each lipid category are shown in Tables S9 to S13, for lipid categories FA, GL, GP, SP, and ST, respectively. 
Table S8: Total number of lipids detected in SRM 1950 organized by lipid category and class

\begin{tabular}{|c|c|c|c|}
\hline Lipid Group & Lipid Class & Abbreviation & $\begin{array}{l}\text { \# of Lipid Species } \\
\text { (Sum Composition) }\end{array}$ \\
\hline \multirow[t]{3}{*}{ Fatty acyls (FA) } & free fatty acids & $\overline{\text { FFA }}$ & 27 \\
\hline & eicosanoids & & 141 \\
\hline & (o-acyl)-1-hydroxy fatty acid & OAHFA & 9 \\
\hline \multirow{4}{*}{ Glycerolipids (GL) } & monoacylglycerol & MAG & 27 \\
\hline & diacylglycerol & DAG & 75 \\
\hline & triacylglycerol & TAG & 214 \\
\hline & monogalactosyldiacylglycerol & MGDG & 1 \\
\hline \multirow[t]{20}{*}{ Glycerophospholipids (GP) } & bis(monoacylglycero)phosphate & BMP & 1 \\
\hline & cardiolipin & $\mathrm{CL}$ & 2 \\
\hline & lysophosphatidic acid & LPA & 6 \\
\hline & phosphatidic acid & $\mathrm{PA}$ & 17 \\
\hline & lysophosphatidylcholine & LPC & 84 \\
\hline & phosphatidylcholine & PC & 219 \\
\hline & lysophosphatidylethanolamine & LPE & 35 \\
\hline & phosphatidylethanolamine & $\mathrm{PE}$ & 99 \\
\hline & lysophosphatidylglycerol & LPG & 5 \\
\hline & phosphatidylglycerol & PG & 19 \\
\hline & lysophosphatidylinositol & LPI & 11 \\
\hline & phosphatidylinositol & PI & 43 \\
\hline & phosphatidylinositol monophosphate & PIP & 1 \\
\hline & lysophosphatidylserine & LPS & 2 \\
\hline & phosphatidylserine & PS & 22 \\
\hline & dimethylphosphatidylethanolamine & $\mathrm{dMePE}$ & 75 \\
\hline & lysodimethylphosphatidylethanolamine & LdMePE & 19 \\
\hline & cyclic phosphatidic acid & $\mathrm{cPA}$ & 3 \\
\hline & phosphatidylethanol & Pet & 14 \\
\hline & phosphatidylmethanol & PMe & 2 \\
\hline \multirow[t]{20}{*}{ Sphingolipids (SP) } & ceramide & Cer & 57 \\
\hline & ceramide-1-phosphate & CerP & 1 \\
\hline & dihydroceramide & $\mathrm{CerOH}$ & 10 \\
\hline & glucosylceramide & GlcCer & 1 \\
\hline & hexosylceramide & HexCer & 13 \\
\hline & dihexosylceramide & Hex2Cer & 6 \\
\hline & trihexosylceramide & Hex3Cer & 6 \\
\hline & lactosylceramide & LacCer & 13 \\
\hline & GM1 ganglioside & GM1Cer & 6 \\
\hline & GM2 ganglioside & GM2Cer & 4 \\
\hline & GM3 ganglioside & GM3Cer & 6 \\
\hline & acylceramide & 1-O-Cer/2-O-Cer & 8 \\
\hline & sphingomyelin & SM & 91 \\
\hline & sphingosine/sphinganine & SPH/dhSPH & 2 \\
\hline & lysosphingomyelin & LSM & 1 \\
\hline & sphingosine-1-phosphate & S1P & 3 \\
\hline & sphinganine-1-phosphate & dhS1P & 1 \\
\hline & phytosphingosine & phSM & 5 \\
\hline & psychosine & HexSph & 1 \\
\hline & lactosyl sphingosine & LacSph & 1 \\
\hline \multirow{4}{*}{ Sterol lipids (ST) } & cholesteryl ester & $\mathrm{CE}$ & 38 \\
\hline & free cholesterol/cholesterol derivatives & FC/CHOL & 3 \\
\hline & zymosteryl & ZyE & 4 \\
\hline & bile acids and derivatives & $\mathrm{BA}$ & 73 \\
\hline
\end{tabular}


Table S9. Individual fatty acyl lipid species measured by at least one laboratory in SRM 1950

\begin{tabular}{|c|c|c|}
\hline FA 12:0 & 15d-D12,14-PGJ3 & 9,10-DiHOME \\
\hline FA 14:0 & 15-HEDE & 9,12,13-TriHOME \\
\hline FA $14: 1$ & 15-HEPE & 9-HEPE \\
\hline FA 15:0 & 15-HETE & 9-HETE \\
\hline FA 16:0 & 15-HETrE & 9-HODE \\
\hline FA 16:1 & 15-keto PGE2 & 9-HOTrE \\
\hline FA $17: 0$ & 15-OxoEDE (15-KEDE) & 9-OxoOTrE (9-KOTrE) \\
\hline FA $17: 1$ & 15-OxoETE (15-KETE) & 9-OxoODE (9-KODE) \\
\hline FA 18:0 & 16,17-EpDPE & Bicyclo PGE2 \\
\hline FA 18:1 & 16-HDoHE & D12-PGJ2 \\
\hline FA $18: 2$ & 16-HETE & D17-PGE1 \\
\hline FA 18:3 & 17,18-DiHETE & Dihomo PGF2a \\
\hline FA $18: 4$ & 17,18-ЕрЕТЕ & EKODE \\
\hline FA 20:0 & 17-HDoHE & iPF2a-IV \\
\hline FA 20:1 & 17-HETE & LTB4 \\
\hline FA 20:2 & 18-HEPE & LTC4 \\
\hline FA 20:3 & 18-HETE & LTD4 \\
\hline FA 20:4 & 19,20-EpDPE & LTE4 \\
\hline FA 20:5 & 19(R)-hydroxy PGE1 & LXA4 \\
\hline FA 22:0 & 19(R)-OH PGF2a & LXA5 \\
\hline FA 22:1 & 19,20-DiHDoPE (19,20-DiHDPA) & LXB4 \\
\hline FA 22:2 & 19-HETE & PD1 \\
\hline FA 22:4 & 2,3 dinor 11b PGF2a & PGA2 \\
\hline FA 22:5 & 20-COOH AA & PGB2 \\
\hline FA 22:6 & 20-СOOH LTB4 & PGD2 \\
\hline FA 24:0 & 20-HDoHE & PGD3 \\
\hline FA $24: 1$ & 20-HETE & PGE1 \\
\hline 10-HDoHE & 20-OH LTB4 & PGE2 \\
\hline 10S,17S-DiHDoHE & 4,17-DiHDoHE & PGE3 \\
\hline 11-HEDE & 4-HDoHE & PGF1a \\
\hline 11,12-DiHET (DHET) & 4S,14S-diHDHA & PGF2a \\
\hline 11,12-DiHETrE & 5,15-DiHETE & PGJ2 \\
\hline 11,12-EET & 5,6 DiHET (DHET) & RvD1 \\
\hline 11,12-EpETrE & 5,6-DiHETE & RvD5 \\
\hline 11b PGE2 & 5,6-DiHETE(EPA) & RvD6 \\
\hline 11-HDoHE & 5,6-DiHETrE & RvE2 \\
\hline 11-HEPE & 5,6-EET & RvE3 \\
\hline 11-HETE & 5,6-EpETrE & tetranor 12-HETE \\
\hline 12,13-DiHOME & 5-HEPE & tetranor-PGEM \\
\hline 12,13-EpODE & 5-HETE & TXB1 \\
\hline 12,13-ЕрОМЕ & 5-HETrE & TXB2 \\
\hline 12epi LTB4 & 5-iso PGF2a VI & TXB3 \\
\hline 12-HEPE & 5-oxoETE (5-KETE) & OAHFA 34:0 \\
\hline 12-HETE & 5S,12S-diHETE & OAHFA 36:0 \\
\hline
\end{tabular}




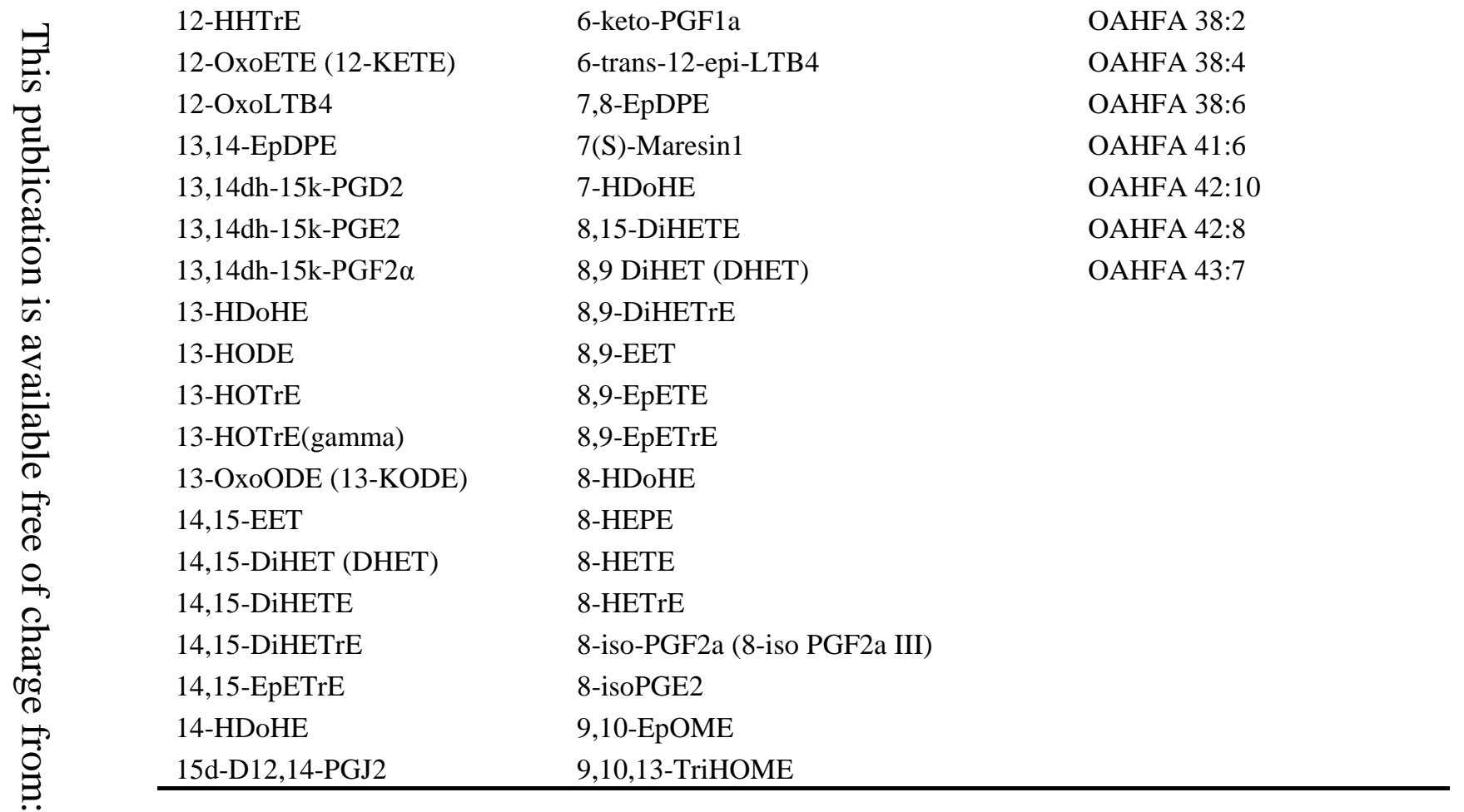


Table S10. Individual glycerolipid species measured by at least one laboratory in SRM 1950

\begin{tabular}{|c|c|c|}
\hline MAG 12:0 & TAG 33:2 & TAG 52:5 \\
\hline MAG 14:0 & TAG 33:3 & TAG 52:6 \\
\hline MAG 16:1 & TAG 34:0 & TAG 52:7 \\
\hline MAG 16:0 & TAG 34:1 & TAG 52:8 \\
\hline MAG $18: 4$ & TAG 35:0 & TAG 53:0 \\
\hline MAG 18:3 & TAG 35:3 & TAG 53:1 \\
\hline MAG 18:2 & TAG 35:4 & TAG 53:2 \\
\hline MAG 18:1 & TAG 36:0 & TAG 53:3 \\
\hline MAG 18:0 & TAG 36:2 & TAG 53:4 \\
\hline MAG 20:5 & TAG 36:3 & TAG 53:5 \\
\hline MAG 20:4 & TAG 37:0 & TAG 53:6 \\
\hline MAG 20:3 & TAG 37:1 & TAG 53:7 \\
\hline MAG 20:2 & TAG 38:0 & TAG 53:8 \\
\hline MAG 20:1 & TAG 38:1 & TAG 54:0 \\
\hline MAG 20:0 & TAG 38:2 & TAG 54:1 \\
\hline MAG 21:2 & TAG 38:3 & TAG 54:2 \\
\hline MAG 22:6 & TAG 39:0 & TAG 54:3 \\
\hline MAG 22:5 & TAG 39:1 & TAG 54:4 \\
\hline MAG 22:4 & TAG 39:2 & TAG 54:5 \\
\hline MAG 22:3 & TAG 40:0 & TAG 54:6 \\
\hline MAG 22:2 & TAG 40:1 & TAG 54:7 \\
\hline MAG 22:1 & TAG 40:2 & TAG 54:8 \\
\hline MAG 22:0 & TAG 40:3 & TAG 54:9 \\
\hline MAG 24:1 & TAG 40:4 & TAG 55:0 \\
\hline MAG 24:0 & TAG 41:0 & TAG 55:1 \\
\hline MAG 26:0 & TAG 41:1 & TAG 55:2 \\
\hline MAG 27:4 & TAG 41:2 & TAG 55:3 \\
\hline DAG 20:0 & TAG 41:3 & TAG 55:4 \\
\hline DAG 22:0 & TAG 42:0 & TAG 55:5 \\
\hline DAG 23:0 & TAG 42:1 & TAG 55:6 \\
\hline DAG 24:1 & TAG 42:2 & TAG 55:7 \\
\hline DAG 25:1 & TAG 42:3 & TAG 55:8 \\
\hline DAG 26:2 & TAG 42:4 & TAG 55:9 \\
\hline DAG 28:0 & TAG 42:5 & TAG 56:0 \\
\hline DAG 29:1 & TAG 42:6 & TAG 56:1 \\
\hline DAG 29:2 & TAG 43:0 & TAG 56:10 \\
\hline DAG 29:3 & TAG 43:1 & TAG 56:11 \\
\hline DAG 29:5 & TAG 43:2 & TAG 56:2 \\
\hline DAG 30:0 & TAG 43:3 & TAG 56:3 \\
\hline DAG 30:1 & TAG 43:4 & TAG 56:4 \\
\hline DAG 30:2 & TAG 44:0 & TAG 56:5 \\
\hline DAG 30:5 & TAG 44:1 & TAG 56:6 \\
\hline DAG 30:6 & TAG 44:2 & TAG 56:7 \\
\hline DAG 31:0 & TAG 44:3 & TAG 56:8 \\
\hline
\end{tabular}


DAG 31:1

DAG 31:2

DAG 32:0

DAG 32:1

DAG 32:2

DAG 32:3

DAG 33:0

DAG 33:1

DAG 33:2

DAG 33:3

DAG 33:4

DAG 33:5

DAG 34:0

DAG 34:1

DAG 34:2

DAG 34:3

DAG 34:4

DAG 35:0

DAG 35:1

DAG 35:2

DAG 35:3

DAG 36:0

DAG 36:1

DAG 36:2

DAG 36:3

DAG 36:4

DAG 36:5

DAG 37:0

DAG 37:2

DAG 38:0

DAG 38:1

DAG 38:2

DAG 38:3

DAG 38:4

DAG 38:5

DAG 38:6

DAG 38:7

DAG 39:0

DAG 40:0

DAG 40:1

DAG 40:2

DAG 40:3

DAG 40:4

DAG 40:5

DAG 40:6
TAG 44:4

TAG 44:5

TAG 44:6

TAG 45:0

TAG 45:1

TAG 45:2

TAG 45:3

TAG 45:4

TAG 45:5

TAG 45:6

TAG 46:0

TAG 46:1

TAG 46:2

TAG 46:3

TAG 46:4

TAG 46:5

TAG 46:6

TAG 47:0

TAG 47:1

TAG 47:2

TAG 47:3

TAG 47:4

TAG 47:5

TAG 47:6

TAG 48:0

TAG 48:1

TAG 48:2

TAG 48:3

TAG 48:4

TAG 48:5

TAG 48:6

TAG 48:7

TAG 49:0

TAG 49:1

TAG 49:2

TAG 49:3

TAG 49:4

TAG 49:5

TAG 49:6

TAG 49:7

TAG 50:0

TAG 50:1

TAG 50:2

TAG 50:3

TAG 50:4
TAG 56:9

TAG 57:1

TAG 57:2

TAG 57:3

TAG 57:4

TAG 57:5

TAG 57:6

TAG 57:7

TAG 57:8

TAG 57:9

TAG 58:0

TAG 58:1

TAG 58:10

TAG 58:11

TAG 58:12

TAG 58:13

TAG 58:2

TAG 58:3

TAG 58:4

TAG 58:5

TAG 58:6

TAG 58:7

TAG 58:8

TAG 58:9

TAG 59:1

TAG 59:2

TAG 59:5

TAG 59:9

TAG 60:0

TAG 60:1

TAG 60:10

TAG 60:11

TAG 60:12

TAG 60:13

TAG 60:14

TAG 60:15

TAG 60:2

TAG 60:3

TAG 60:4

TAG 60:5

TAG 60:7

TAG 60:8

TAG 60:9

TAG 62:1

TAG 62:12 


\begin{tabular}{|c|c|c|c|}
\hline & DAG 40:7 & TAG 50:5 & TAG 62:13 \\
\hline & DAG 40:8 & TAG 50:6 & TAG 62:14 \\
\hline & DAG 41:0 & TAG 50:7 & TAG 62:16 \\
\hline & DAG 42:0 & TAG 50:8 & TAG 62:2 \\
\hline & DAG 42:1 & TAG 51:0 & TAG 62:3 \\
\hline & DAG 42:2 & TAG 51:1 & TAG 62:5 \\
\hline & DAG 42:5 & TAG 51:2 & TAG 62:9 \\
\hline & DAG 42:6 & TAG 51:3 & TAG O-48:0 \\
\hline & DAG 42:7 & TAG 51:4 & TAG O-50:0 \\
\hline & DAG 48:6 & TAG 51:5 & TAG O-50:1 \\
\hline & DAG 54:6 & TAG 51:6 & TAG O-50:2 \\
\hline & DAG 56:6 & TAG 51:7 & TAG O-52:1 \\
\hline & DAG 56:7 & TAG 52:0 & TAG O-52:2 \\
\hline & TAG 24:0 & TAG 52:1 & TAG P-52:1 \\
\hline & TAG 28:0 & TAG 52:2 & TAG P-52:2 \\
\hline & TAG 32:2 & TAG 52:3 & MGDG 36:4 \\
\hline & TAG 33:1 & TAG 52:4 & \\
\hline
\end{tabular}


Table S11. Individual glycerophospholipid species measured by at least one laboratory in SRM 1950

\begin{tabular}{|c|c|c|}
\hline LPC 12:0 & PC O-42:0 & PG 36:5 \\
\hline LPC 13:0 & PC O-42:1 & PG 37:0 \\
\hline LPC 14:0 & PC O-16:0 & PG 37:2 \\
\hline LPC $14: 1$ & PC O-21:1 & PG 38:4 \\
\hline LPC 15:0 & PC O-23:0 & PG 38:5 \\
\hline LPC $15: 1$ & PC O-31:0 & PG 38:6 \\
\hline LPC 15:2 & PC O-32:4 & PG 40:4 \\
\hline LPC 16:0 & PC O-33:0 & PG 40:6 \\
\hline LPC 16:1 & PC O-34:5 & PG 40:7 \\
\hline LPC 16:2 & PC O-34:6 & PG 43:7 \\
\hline LPC 17:0 & PC O-36:6 & PG 46:0 \\
\hline LPC 17:1 & PC O-38:0 & PG 50:10 \\
\hline LPC 17:2 & PC O-38:1 & LPI 16:0 \\
\hline LPC $17: 3$ & PC 0-38:7 & LPI 16:1 \\
\hline LPC 17:4 & PC O-39:4 & LPI 18:0 \\
\hline LPC 18:0 & PC O-39:5 & LPI 18:1 \\
\hline LPC 18:1 & PC O-39:6 & LPI 18:2 \\
\hline LPC 18:2 & PC O-40:1 & LPI 20:3 \\
\hline LPC 18:3 & PC O-40:3 & LPI 20:4 \\
\hline LPC 18:4 & PC O-41:1 & LPI 20:5 \\
\hline LPC 19:0 & PC O-41:6 & LPI 22:4 \\
\hline LPC 19:1 & PC O-42:2 & LPI 22:5 \\
\hline LPC 19:2 & PC O-42:3 & LPI 22:6 \\
\hline LPC 19:3 & PC O-42:7 & PI 32:0 \\
\hline LPC 19:4 & PC O-43:4 & PI 32:1 \\
\hline LPC 19:5 & PC O-43:6 & PI 32:2 \\
\hline LPC 20:0 & PC O-44:4 & PI 33:1 \\
\hline LPC 20:1 & PC O-44:7 & PI 33:2 \\
\hline LPC 20:2 & PC 18:0 & PI 34:0 \\
\hline LPC 20:3 & PC 23:0 & PI 34:1 \\
\hline LPC 20:4 & PC $27: 1$ & PI 34:2 \\
\hline LPC 20:5 & PC 29:1 & PI 34:3 \\
\hline LPC 21:3 & PC O-30:1/P-30:0 & PI 35:1 \\
\hline LPC 21:5 & PC O-30:2/P-30:1 & PI 35:2 \\
\hline LPC 22:0 & PC P-30:3 & PI 36:0 \\
\hline LPC 22:1 & PC P-31:0 & PI 36:1 \\
\hline LPC 22:2 & PC P-31:3 & PI 36:2 \\
\hline LPC 22:3 & PC P-32:2 & PI 36:3 \\
\hline LPC 22:4 & PC P-32:4 & PI 36:4 \\
\hline LPC 22:5 & PC P-33:0 & PI 36:5 \\
\hline LPC 22:6 & PC P-33:2 & PI 37:1 \\
\hline LPC 23:0 & PC O-34:4/P-34:3 & PI 37:2 \\
\hline LPC 23:1 & PC P-34:4 & PI 37:4 \\
\hline LPC 24:0 & PC P-35:6 & PI 38:1 \\
\hline
\end{tabular}




\begin{tabular}{|c|c|c|c|}
\hline & LPC 24:1 & PC P-36:6 & PI 38:2 \\
\hline & LPC $24: 2$ & PC P-36:7 & PI 38:3 \\
\hline & LPC 24:3 & PC P-37:2 & PI 38:4 \\
\hline & LPC 24:4 & PC P-37:4 & PI 38:5 \\
\hline & LPC 24:5 & PC P-37:6 & PI 38:6 \\
\hline & LPC 24:6 & PC P-38:7 & PI 39:4 \\
\hline & LPC 26:0 & PC P-39:2 & PI 40:3 \\
\hline & LPC 26:1 & PC P-39:6 & PI 40:4 \\
\hline & LPC 26:2 & PC O-40:2/P-40:1 & PI 40:5 \\
\hline & LPC 26:4 & PC P-40:2 & PI 40:6 \\
\hline & LPC 28:0 & PC O-40:8/P-40:7 & PI 40:7 \\
\hline & LPC 33:5 & PC P-42:2 & PI 40:8 \\
\hline & LPC 35:4 & PC O-42:4/P-42:3 & PI 42:10 \\
\hline & LPC 35:5 & PC O-42:5/P-42:4 & PI 42:11 \\
\hline & LPC 36:2 & PC O-42:6/P-42:5 & PI 42:9 \\
\hline & LPC 37:4 & PC P-42:6 & PI O-36:2 \\
\hline & LPC 37:5 & PC P-42:7 & PI O-36:4 \\
\hline & LPC 37:6 & PC P-42:9 & PI O-38:4 \\
\hline & LPC 38:4 & PC P-44:2 & PI O-38:5 \\
\hline & LPC 38:5 & PC P-44:3 & PI O-38:6 \\
\hline & LPC O-14:0 & PC O-44:5/P-44:4 & PI P-38:4 \\
\hline & LPC O-16:0 & PC O-44:6/P-44:5 & PI P-40:6 \\
\hline & LPC O-16:1 & PC P-44:7 & LPS 18:0 \\
\hline & LPC O-18:0 & LPA 16:0 & LPS 18:1 \\
\hline & LPC O-18:1 & LPA 18:0 & PS 32:1 \\
\hline & LPC O-18:2 & LPA $18: 1$ & PS 34:0 \\
\hline & LPC O-20:0 & LPA 18:2 & PS 34:1 \\
\hline & LPC O-20:1 & LPA 20:4 & PS $34: 2$ \\
\hline & LPC O-22:0 & LPA 22:6 & PS 36:0 \\
\hline & LPC O-22:1 & PA 32:1 & PS 36:1 \\
\hline & LPC O-24:0 & PA 32:0 & PS 36:2 \\
\hline & LPC O-24:1 & PA 34:0 & PS $36: 3$ \\
\hline & LPC O-24:2 & PA 34:1 & PS $36: 4$ \\
\hline & LPC P-16:0 & PA 34:2 & PS 38:1 \\
\hline & LPC P-16:1 & PA 36:0 & PS 38:2 \\
\hline & LPC P-18:0 & PA 36:1 & PS 38:3 \\
\hline & LPC P-18:1 & PA 36:2 & PS $38: 4$ \\
\hline & LPC P-18:2 & PA 36:3 & PS 38:5 \\
\hline & LPC P-20:0 & PA 36:4 & PS 38:6 \\
\hline & LPC P-20:1 & PA 38:2 & PS 40:3 \\
\hline & PC 16:0 & PA $38: 3$ & PS 40:4 \\
\hline & PC $17: 0$ & PA 38:4 & PS 40:5 \\
\hline & PC 18:0 & PA 38:5 & PS 40:6 \\
\hline & PC 18:1 & PA 38:6 & PS 40:7 \\
\hline & PC 20:0 & PA P-40:4 & PS P-36:3 \\
\hline
\end{tabular}




\begin{tabular}{|c|}
\hline PC 20:2 \\
\hline PC 22:0 \\
\hline PC 22:2 \\
\hline PC 22:3 \\
\hline PC 23:0 \\
\hline PC 24:0 \\
\hline PC 26:0 \\
\hline PC 26:1 \\
\hline PC 26:3 \\
\hline PC 27:0 \\
\hline PC 28:0 \\
\hline PC 28:1 \\
\hline PC 28:2 \\
\hline PC 28:3 \\
\hline PC 29:1 \\
\hline PC 30:0 \\
\hline PC O-30:0/29:0 \\
\hline PC 30:1 \\
\hline PC $30: 2$ \\
\hline PC $30: 3$ \\
\hline PC $30: 4$ \\
\hline PC 32:0 \\
\hline PC O-32:0/31:0 \\
\hline PC 32:1 \\
\hline PC O-32:1/P-32:0/31:1 \\
\hline PC O-32:2/P-32:1/31:2 \\
\hline PC 32:3 \\
\hline PC 32:4 \\
\hline PC 32:5 \\
\hline PC P-33:1/32:2 \\
\hline PC 33:4 \\
\hline PC 34:0 \\
\hline PC O-34:0/33:0 \\
\hline PC 34:1 \\
\hline PC O-34:1/P-34:0/33:1 \\
\hline PC O-34:2/P-34:1/33:2 \\
\hline PC O-34:3/P-34:2/33:3 \\
\hline PC 34:5 \\
\hline PC 34:6 \\
\hline PC P-35:1/34:2 \\
\hline PC P-35:2/34:3 \\
\hline PC O-35:4/34:4 \\
\hline PC $35: 7$ \\
\hline PC O-36:0/35:0 \\
\hline PC 36:1 \\
\hline
\end{tabular}

PA 48:7
LPE 12:0
LPE 14:0
LPE 16:0
LPE 16:1
LPE 17:0
LPE 18:0
LPE 18:1
LPE 18:2
LPE 18:3
LPE 19:0
LPE 19:1
LPE 20:0
LPE 20:1
LPE 20:2
LPE 20:3
LPE 20:4
LPE 20:5
LPE 22:0
LPE 22:1
LPE 22:2
LPE 22:3
LPE 22:4
LPE 22:5
LPE 22:6
LPE 24:0
LPE 34:1
LPE O-16:0
LPE O-16:1
LPE O-20:1
PE 34:3
PP 35:1
PP P-16:0
LPE P-16:1
LPE P-18:0
LPE P-18:1
LPE P-20:0
LPE P-20:1
PE 19:2
PE
P2:0

PS P-38:4

BMP 18:1/18:1

PIP O-34:4

CL 66:3

CL 72:8

dMePE 24:0

dMePE 30:0

dMePE 32:1

dMePE 32:2

dMePE 33:2

dMePE 34:0

dMePE 34:1

dMePE 34:2

dMePE 34:3

dMePE 34:5

dMePE 35:1

dMePE 35:2

dMePE 35:3

dMePE 36:1

dMePE 36:2

dMePE 36:3

dMePE 36:4

dMePE 36:5

dMePE 36:6

dMePE 37:5

dMePE 37:6

dMePE 37:7

dMePE 38:3

dMePE 38:4

dMePE 38:5

dMePE 38:6

dMePE 38:7

dMePE 38:8

dMePE 40:4

dMePE 43:6

dMePE 44:11

dMePE 48:1

dMePE 48:2

dMePE 48:3

dMePE 50:2

dMePE 50:3

dMePE 50:4

dMePE 50:5

dMePE 50:6

dMePE 51:5 


\begin{tabular}{|c|c|c|c|}
\hline & PC O-36:1/P-36:0/35:1 & PE 36:0 & dMePE 52:2 \\
\hline & PC $36: 2$ & PE 36:1 & dMePE 52:3 \\
\hline & PC O-36:2/P-36:1/35:2 & PE 36:2 & dMePE 52:4 \\
\hline & PC 36:3 & PE O-36:2/P-36:1/35:2 & dMePE 52:5 \\
\hline & PC O-36:3/P-36:2/35:3 & PE 36:3 & dMePE 52:6 \\
\hline & PC 36:4 & PE O-36:3/P-36:2/35:3 & dMePE 52:7 \\
\hline & PC O-36:4/P-36:3/35:4 & PE 36:4 & dMePE 52:8 \\
\hline & PC $36: 5$ & PE 36:5 & dMePE 53:5 \\
\hline & PC O-36:5/P-36:4/35:5 & PE 37:1 & dMePE 53:6 \\
\hline & PC P-36:5/35:6 & PE 37:2 & dMePE 54:3 \\
\hline & PC 36:6 & PE 37:5 & dMePE 54:5 \\
\hline & PC 36:7 & PE 37:6 & dMePE 54:6 \\
\hline & PC $37: 1$ & PE 38:1 & dMePE 54:7 \\
\hline & PC $37: 7$ & PE 38:2 & dMePE 54:8 \\
\hline & PC $37: 8$ & PE P-38:2/37:3 & dMePE 54:9 \\
\hline & PC $37: 9$ & PE 38:3 & dMePE 56:10 \\
\hline & PC 38:0 & PE 38:4 & dMePE 56:11 \\
\hline & PC 38:1 & PE O-38:4/P-38:3/37:4 & dMePE 56:5 \\
\hline & PC 38:2 & PE 38:5 & dMePE 56:6 \\
\hline & PC O-38:2/37:2 & PE 38:6 & dMePE 56:7 \\
\hline & PC 38:3 & PE 38:7 & dMePE 56:8 \\
\hline & PC O-38:3/P-38:2/37:3 & PE 39:1 & dMePE 56:9 \\
\hline & PC 38:4 & PE 39:3 & dMePE 58:10 \\
\hline & PC O-38:4/P-38:3/37:4 & PE 39:4 & dMePE 58:6 \\
\hline & PC $38: 5$ & PE 40:1 & dMePE 58:9 \\
\hline & PC O-38:5/P-38:4/37:5 & PE 40:2 & dMePE O-32:0 \\
\hline & PC 38:6 & PE 40:3 & dMePE O-36:4 \\
\hline & PC O-38:6/P-38:5/37:6 & PE 40:4 & dMePE P-34:0 \\
\hline & PC P-38:6/36:0 & PE 40:5 & dMePE P-34:2 \\
\hline & PC 38:7 & PE O-40:5/P-40:4/39:5 & dMePE P-38:1 \\
\hline & PC $38: 8$ & PE 40:6 & dMePE P-38:2 \\
\hline & PC 38:9 & PE O-40:6/P-40:5/39:6 & dMePE P-38:4 \\
\hline & PC 39:10 & PE 40:7 & dMePE P-40:4 \\
\hline & PC 39:2 & PE O-40:7/P-40:6/39:7 & dMePE P-40:5 \\
\hline & PC 39:3 & PE 40:8 & dMePE P-40:8 \\
\hline & PC 40:0 & PE 41:4 & LdMePE 14:0 \\
\hline & PC 40:1 & PE 42:1 & LdMePE 15:0 \\
\hline & PC 40:10 & PE 42:5 & LdMePE 16:0 \\
\hline & PC 40:2 & PE 42:6 & LdMePE 16:1 \\
\hline & PC $40: 3$ & PE 42:7 & LdMePE 17:0 \\
\hline & PC $40: 4$ & PE 43:5 & LdMePE 17:1 \\
\hline & PC O-40:4/P-40:3/39:4 & PE 43:6 & LdMePE 18:0 \\
\hline & PC 40:5 & PE 44:5 & LdMePE 18:1 \\
\hline & PC O-40:5/P-40:4/39:5 & PE 45:8 & LdMePE 18:2 \\
\hline & PC 40:6 & PE 46:13 & LdMePE 18:3 \\
\hline
\end{tabular}




\begin{tabular}{|c|c|c|c|}
\hline$\mapsto$ & PC O-40:6/P-40:5/39:6 & PE 47:12 & LdMePE 20:0 \\
\hline ت. & PC 40:7 & PE 47:9 & LdMePE 20:1 \\
\hline 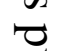 & PC O-40:7/P-40:6/39:7 & PE 54:2 & LdMePE 20:2 \\
\hline 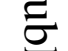 & PC 40:8 & PE O-16:0 & LdMePE 20:3 \\
\hline$\overline{\widehat{n}}$ & PC 40:9 & PE O-17:0 & LdMePE 20:4 \\
\hline 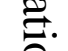 & PC 41:4 & PE O-18:0 & LdMePE 20:5 \\
\hline 电 & PC 41:5 & PE O-20:0 & LdMePE 22:4 \\
\hline$\omega$ & PC 41:6 & PE O-22:0 & LdMePE 22:5 \\
\hline 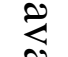 & PC 41:7 & PE O-34:0 & LdMePE 22:6 \\
\hline تص & PC 41:8 & PE O-36:1 & cPA 16:0 \\
\hline صِ & PC 41:9 & PE O-38:1 & cPA 18:0 \\
\hline$D$ & PC 42:0 & PE P-16:0 & cPA 18:1 \\
\hline $\overrightarrow{8}$ & PC 42:1 & PE P-18:0 & PEt 33:0 \\
\hline 0 & PC 42:10 & PE P-20:0 & PEt 37:0 \\
\hline$\hat{D}$ & PC 42:11 & PE P-32:0 & PEt 37:2 \\
\hline 㘴 & PC 42:12 & PE P-32:1 & PEt 41:6 \\
\hline OD & PC 42:2 & PE O-34:1/P-34:0 & PEt 43:5 \\
\hline 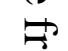 & PC 42:3 & PE O-34:2/P-34:1 & PEt 43:6 \\
\hline 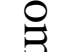 & PC 42:4 & PE O-34:3/P-34:2 & PEt 43:8 \\
\hline & PC 42:5 & PE P-34:3 & PEt $44: 4$ \\
\hline 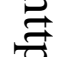 & PC 42:6 & PE P-34:4 & PEt 44:6 \\
\hline$\because$ & PC 42:7 & PE P-35:1 & PEt 45:7 \\
\hline$\overline{\widehat{\partial}}$ & PC 42:8 & PE P-35:2 & PEt 46:8 \\
\hline$\stackrel{0}{?}$ & PC 42:9 & PE P-36:0 & PEt 52:9 \\
\hline & PC 43:10 & PE O-36:4/P-36:3 & PEt O-34:6 \\
\hline$\risingdotseq$ & PC 43:4 & PE O-36:5/P-36:4 & PEt P-40:4 \\
\hline & PC 43:9 & PE O-36:6/P-36:5 & PMe 38:0 \\
\hline N & PC 44:1 & PE P-37:1 & PMe 47:7 \\
\hline a & PC 44:11 & PE P-37:2 & \\
\hline 2 & PC 44:12 & PE P-37:4 & \\
\hline $\mathcal{H}$ & PC 44:2 & PE P-38:1 & \\
\hline$\theta$ & PC 44:4 & PE O-38:5/P-38:4 & \\
\hline ? & PC 44:5 & PE O-38:6/P-38:5 & \\
\hline$\varpi_{\infty}$ & PC 44:6 & PE O-38:7/P-38:6 & \\
\hline & PC 45:11 & PE P-39:3 & \\
\hline & PC 45:13 & PE O-40:2/P-40:1 & \\
\hline & PC 46:4 & PE P-40:2 & \\
\hline & PC 46:5 & PE O-40:4/P-40:3 & \\
\hline & PC 46:6 & PE P-40:7 & \\
\hline & PC 46:7 & PE O-40:8 & \\
\hline & PC 49:3 & PE P-40:8 & \\
\hline & PC 49:4 & PE P-42:4 & \\
\hline & PC 51:3 & PE P-42:5 & \\
\hline & PC 52:8 & PE P-42:6 & \\
\hline & PC 53:13 & PE P-42:7 & \\
\hline
\end{tabular}




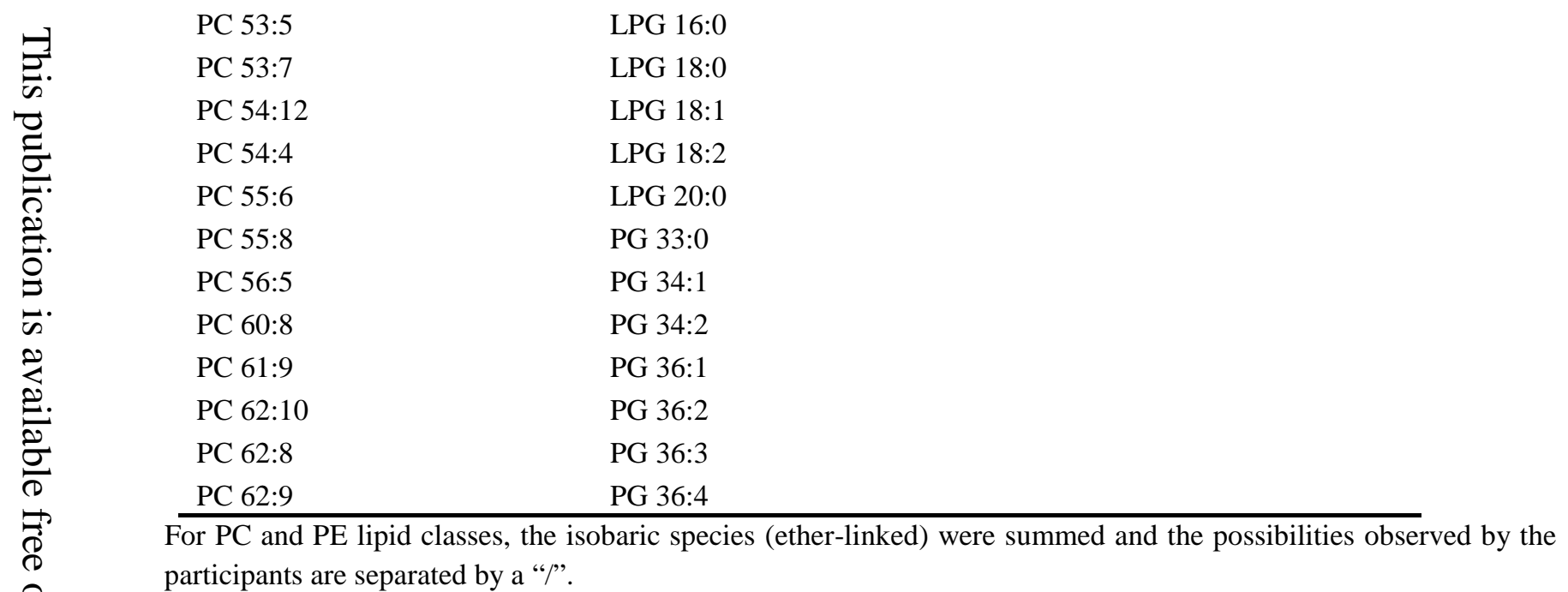

옹

官

ơ

官

!

宫

ิ․

웅

ó

○

용

N

必 
Table S12. Individual sphingolipid species measured by at least one laboratory in SRM 1950

\begin{tabular}{|c|c|c|}
\hline Cer d14:0 & HexCer d40:2 & SM d36:1 \\
\hline Cer d16:0 & HexCer d42:1 & SM d36:2 \\
\hline Cer d18:0 & HexCer d42:2 & SM d36:3 \\
\hline Cer d18:1 & HexCer d44:1 & SM d36:4 \\
\hline Cer d20:0 & HexCer d44:2 & SM d36:5 \\
\hline Cer d20:1 & Hex2Cer d34:1 & SM d36:6 \\
\hline Cer d22:0 & Hex2Cer d36:1 & SM d37:1 \\
\hline Cer d22:1 & Hex2Cer d38:1 & SM d37:2 \\
\hline Cer d24:0 & Hex2Cer d40:1 & SM d37:4 \\
\hline Cer d24:1 & Hex2Cer d42:1 & SM d37:5 \\
\hline Cer d26:0 & Hex2Cer d42:2 & SM d38:0 \\
\hline Cer d26:1 & LacCer d30:1 & SM d38:1 \\
\hline Cer d28:1 & LacCer d32:1 & SM d38:2 \\
\hline Cer d30:1 & LacCer d34:1 & SM d38:3 \\
\hline Cer d32:1 & LacCer d36:1 & SM d38:4 \\
\hline Cer d32:2 & LacCer d36:2 & SM d38:5 \\
\hline Cer d33:0 & LacCer d38:1 & SM d38:7 \\
\hline Cer d33:1 & LacCer d38:2 & SM d39:0 \\
\hline Cer d34:0 & LacCer d40:1 & SM d39:1 \\
\hline Cer d34:1 & LacCer d40:2 & SM d39:2 \\
\hline Cer d34:2 & LacCer d42:1 & SM d39:3 \\
\hline Cer d35:0 & LacCer d42:2 & SM d40:0 \\
\hline Cer d35:1 & LacCer d44:1 & SM d40:1 \\
\hline Cer d35:2 & LacCer d44:2 & SM d40:2 \\
\hline Cer d36:0 & Hex3Cer d34:1 & SM d $40: 3$ \\
\hline Cer d36:1 & Hex3Cer d36:1 & SM d $40: 4$ \\
\hline Cer d36:2 & Hex3Cer d38:1 & SM d40:5 \\
\hline Cer d36:3 & Hex3Cer d40:1 & SM d $40: 7$ \\
\hline Cer d36:7 & Hex3Cer d42:1 & SM d41:1 \\
\hline Cer d37:1 & Hex3Cer d42:2 & SM d $41: 2$ \\
\hline Cer d38:0 & GM1-Cer d41:1 & SM d $41: 3$ \\
\hline Cer d38:1 & GM1-Cer d34:1 & SM d $41: 4$ \\
\hline Cer d38:2 & GM1-Cer d40:1 & SM d42:0 \\
\hline Cer d38:4 & GM1-Cer d42:1 & SM d42:1 \\
\hline Cer d38:5 & GM1-Cer d42:2 & SM d $42: 2$ \\
\hline Cer d39:0 & GM1-Cer d42:2 & SM d $42: 3$ \\
\hline Cer d39:1 & GM2-Cer d34:1 & SM d42:4 \\
\hline Cer d40:0 & GM2-Cer d42:1 & SM d42:5 \\
\hline Cer d40:1 & GM2-Cer d42:2 & SM d42:6 \\
\hline Cer d40:2 & GM2-Cer d34:2 & SM d $42: 7$ \\
\hline Cer d40:4 & GM3-Cer d34:1 & SM d43:1 \\
\hline Cer d41:0 & GM3-Cerd 36:1 & SM d43:2 \\
\hline Cer d41:1 & GM3-Cer d38:1 & SM d43:3 \\
\hline Cer d41:2 & GM3-Cer d40:1 & SM d43:4 \\
\hline
\end{tabular}




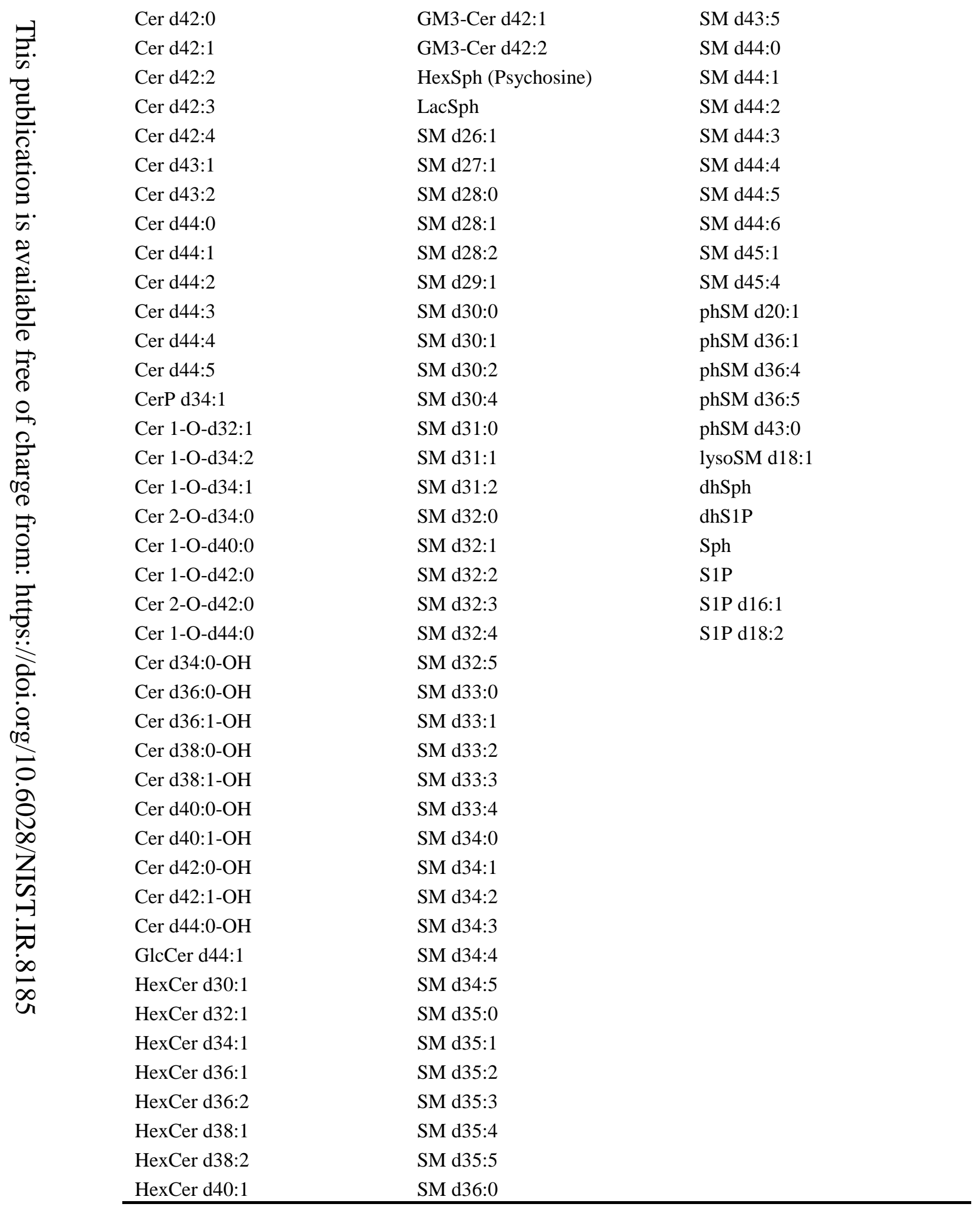


Table S13. Individual sterol lipid species measured by at least one laboratory in SRM 1950

\begin{tabular}{|c|c|c|}
\hline CE 14:0 & Thyroxine & Hyocholic acid \\
\hline CE $14: 1$ & 12-Ketochenodeoxycholic acid & Hyodeoxycholic acid \\
\hline CE 15:0 & 12-Ketolithocholic acid & isoDeoxycholic acid \\
\hline CE 15:1 & 3-Oxocholic acid & isoLithocholic acid \\
\hline CE 16:0 & $3 \alpha, 6 \alpha, 7 \alpha, 12 \alpha$-Tetetrahydroxyl bile acid & Lithocholic acid \\
\hline CE 16:1 & $3 \alpha, 6 \beta, 7 \alpha, 12 \alpha$-Tetrahydroxyl bile acid & Lithocholic acid-Sulfate (3) \\
\hline CE 16:2 & 6,7-Diketolithocholic acid & Murideoxycholic acid \\
\hline CE 17:0 & 7-Ketodeoxycholic acid & Murocholic acid \\
\hline CE $17: 1$ & 7-Ketolithocholic acid & Norcholic acid \\
\hline CE 17:2 & 7-oxo-Lithocholic acid & Nordeoxycholic acid \\
\hline CE $17: 3$ & Allocholic acid & Tauro-3 $\alpha, 6 \alpha, 7 \alpha, 12 \alpha$-tetrahydroxyl bile acid \\
\hline CE 18:0 & Alloisolithocholic acid & Tauroallocholic acid \\
\hline CE 18:1 & Chenodeoxycholic acid & Taurochenodeoxycholic acid \\
\hline CE 18:2 & Chenodeoxycholic acid-3-Sulfate & Taurochenodeoxycholic acid-Sulfate \\
\hline CE 18:3 & Cholic acid & Taurocholic acid \\
\hline CE 19:1 & Cholic acid-3-Sulfate & Taurodeoxycholic acid \\
\hline CE 19:2 & Dehydrocholic acid & Taurodeoxycholic acid-Sulfate \\
\hline CE 19:3 & Dehydrolithocholic acid & Taurohyodeoxyocholic acid \\
\hline CE 20:0 & Deoxycholic acid & Taurolithocholic acid \\
\hline CE 20:1 & Deoxycholic acid-3-Sulfate & Taurolithocholic acid sulfate \\
\hline CE 20:2 & Dioxolithocholic acid & Tauromuricholic acid (a+b) \\
\hline CE 20:3 & Glycoallocholic acid & Tauroursodeoxycholic acid \\
\hline CE 20:4 & Glycochenodeoxycholic acid-Sulfate & Tauroursodeoxycholic acid-3-sulfate \\
\hline CE 20:5 & Glycochenodeoxycholic acid & Tauro- $\alpha$-muricholic acid \\
\hline CE 21:5 & Glycocholic acid & Tauro- $\beta$-muricholic acid \\
\hline CE 22:0 & Glycocholic acid-3-sulfate & Tauro- $\omega$-muricholic acid \\
\hline CE 22:1 & Glycodeoxycholic acid & $\begin{array}{l}\text { Total of Tauroursodexycholic } \\
\text { acid/Taurohyocholic acid }\end{array}$ \\
\hline CE 22:2 & Glycodeoxycholic acid-Sulfate & Ursocholic acid \\
\hline CE 22:3 & Glycohyocholic acid & Ursodeoxycholic acid \\
\hline CE 22:4 & Glycohyodeoxycholic acid & Ursodeoxycholic acid-3-Sulfate \\
\hline CE 22:5 & Glycolithocholic acid & $\alpha$-Muricholic acid \\
\hline CE 22:6 & Glycolithocholic acid sulfate & $\beta$-Muricholic acid \\
\hline CE 24:0 & Glycomuricholic acid & $\lambda$-muricholic acid \\
\hline CE 24:1 & Glycoursocholic acid & $\omega$-Muricholic acid \\
\hline CE 24:4 & Glycoursodeoxycholic acid & ZyE 18:2 \\
\hline CE 24:5 & Glycoursodeoxycholic acid-Sulfate & ZyE 18:3 \\
\hline CE 24:6 & Glyco- $\alpha$-muriholic acid & ZyE 20:4 \\
\hline CE 26:0 & Glyco- $\beta$-muricholic acid & ZyE 23:0 \\
\hline cholesterol & Glyco- $\omega$-muricholic acid & \\
\hline total cholesterol & Glyocholic acid & \\
\hline
\end{tabular}


Plots were created for all lipids measured by at least five laboratories $(n=339)$. On each plot, every laboratory submission for each lipid species is displayed (calculated mean and standard deviation of the mean in $\mathrm{nmol} / \mathrm{mL}$ from a triplicate measurement). To enhance visual inspection, the plots were truncated at the $y$-axis in the presence of extreme outliers (outlying values and laboratory reported on bottom right of the plot). On the left of each plot, calculated consensus estimates using the DSL, VR, or MEDM methods are shown with standard uncertainties. For a few lipids, the VR mean was truncated to improve visualization. The uncertainty values for laboratory 8 are not standard deviation, rather standard error of the mean. If no point is present on the plot for a listed laboratory ID number, this indicates that the laboratory did not report a concentration for that lipid.

The final determined MEDM location is provided at the bottom left of each figure with standard uncertainty. A star $(\star)$ on a plot indicates that the uncertainty of this data point is not shown. For plasmanyl lipid species, lipids are identified in the plots using "e" (same as "O-"), while plasmenyl lipids are identified in the plots using a lowercase "p" (same as "P-"). 


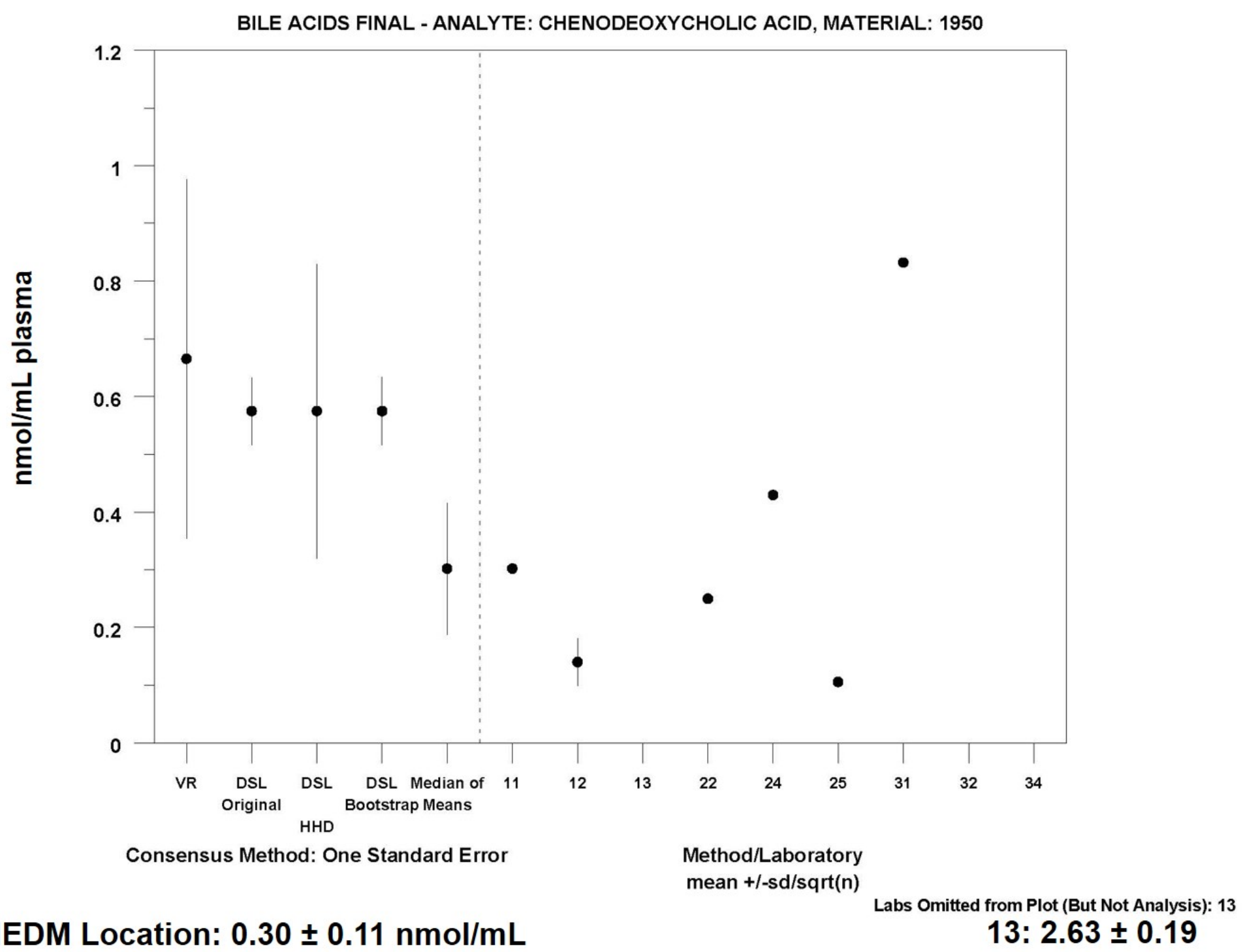




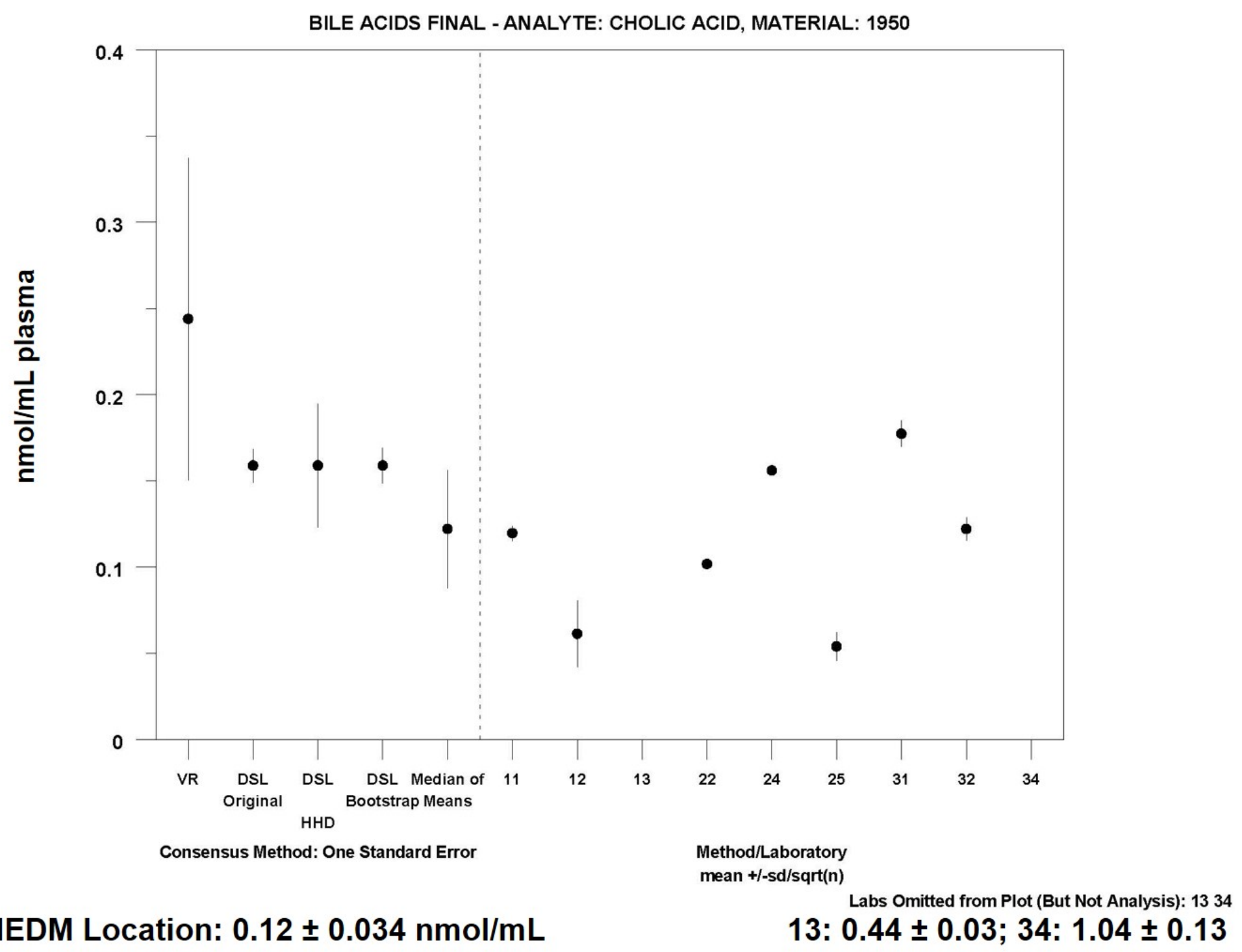




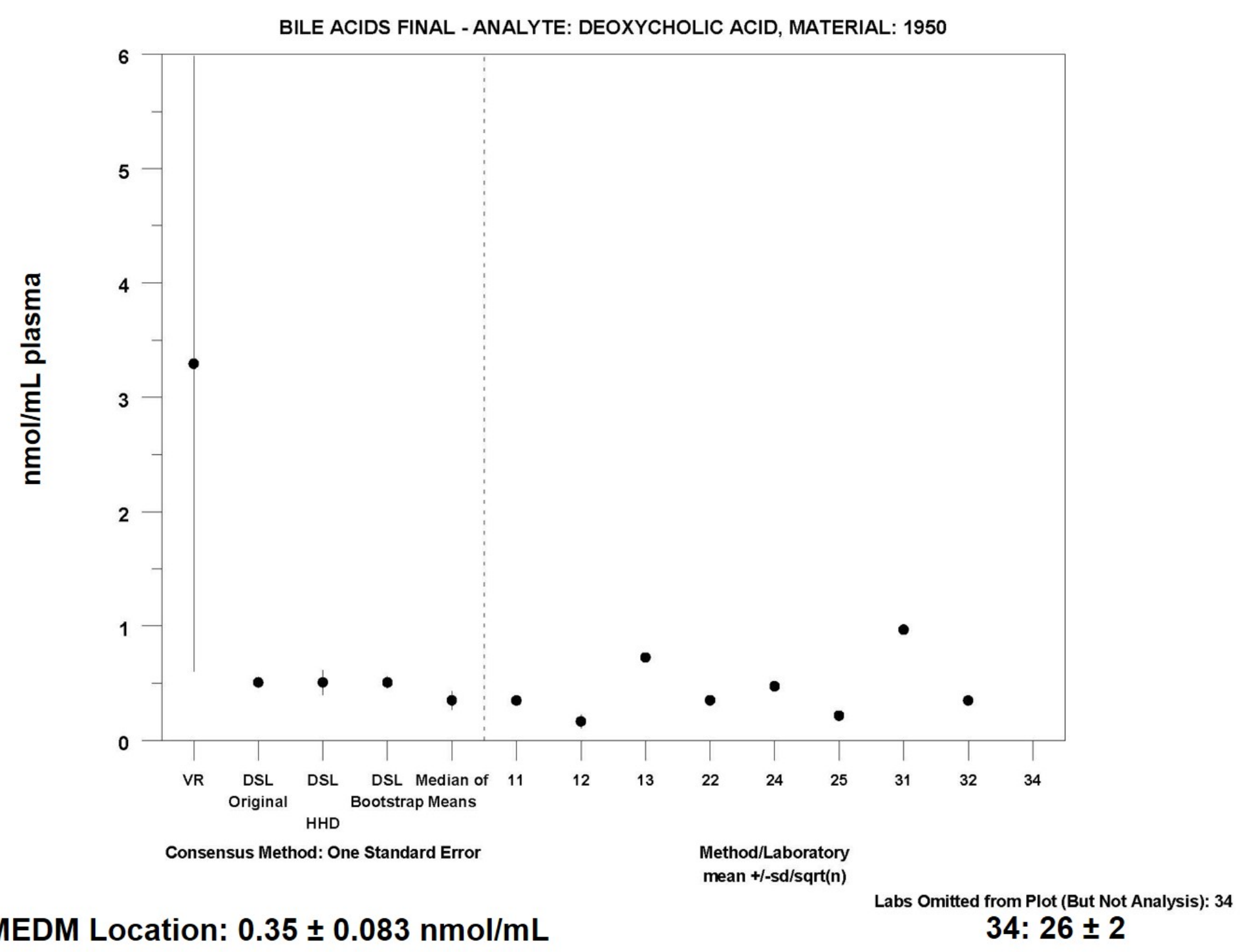




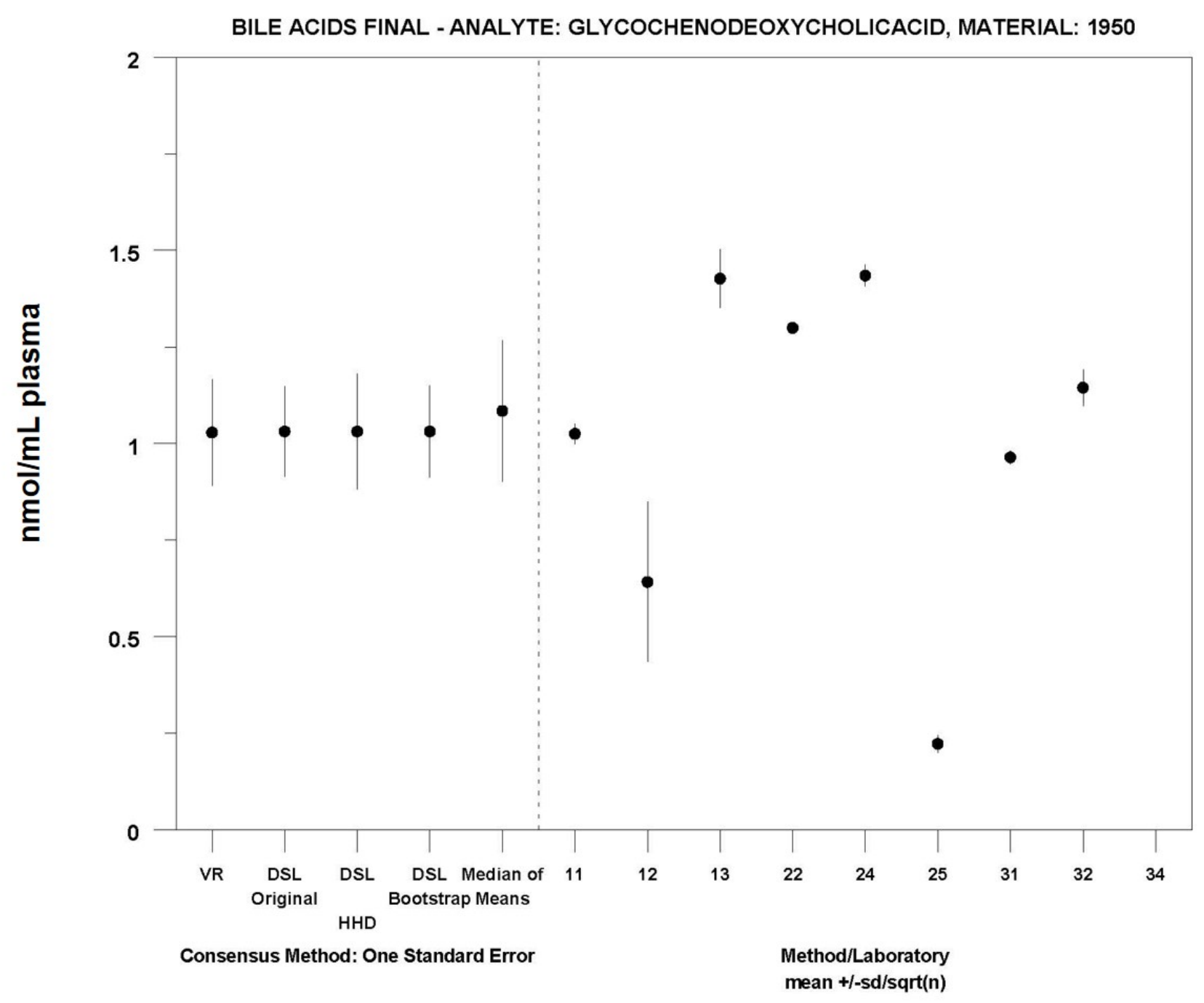

MEDM Location: $1.1 \pm 0.18 \mathrm{nmol} / \mathrm{mL}$ 


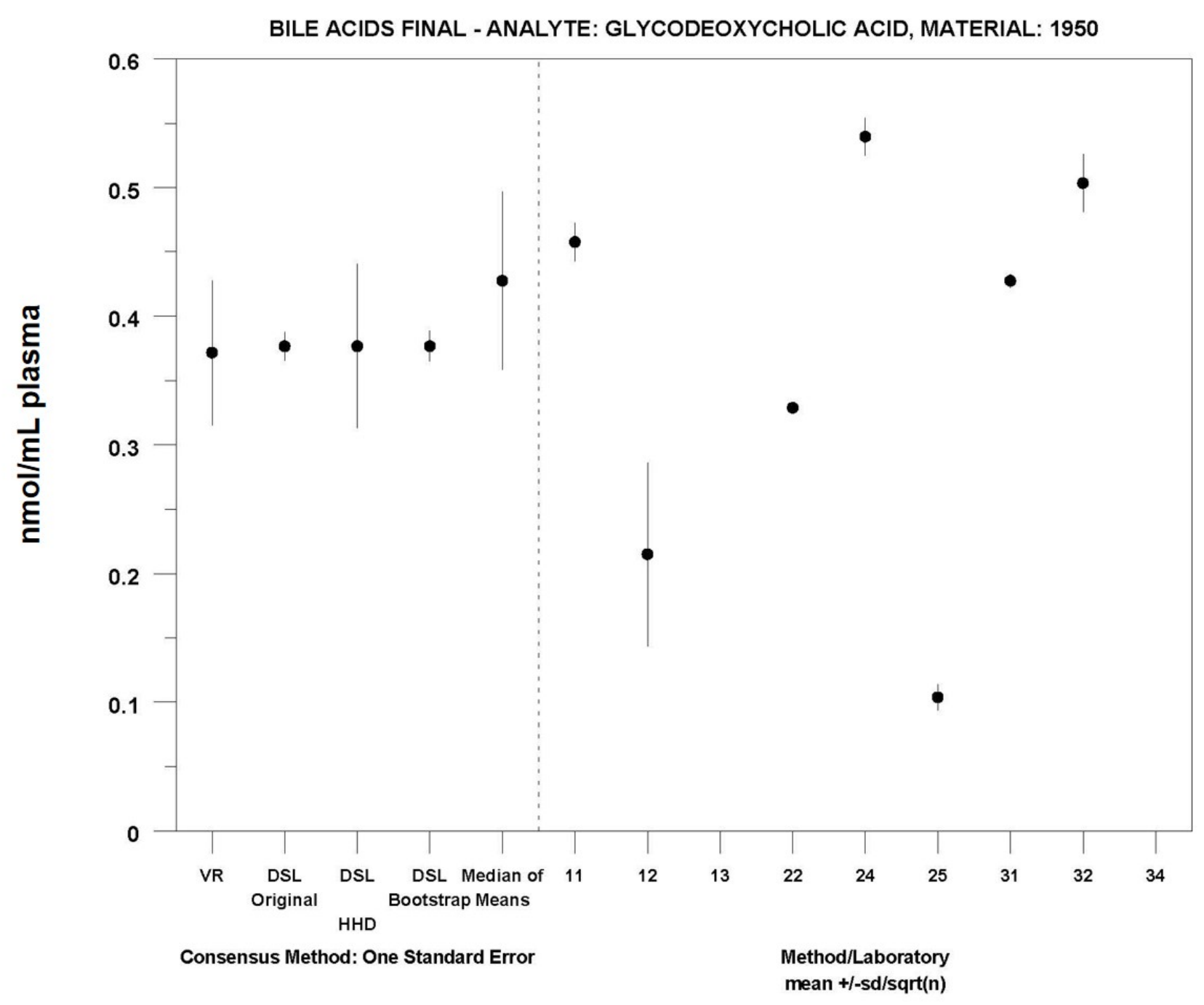

MEDM Location: $0.43 \pm 0.069 \mathrm{nmol} / \mathrm{mL}$ 


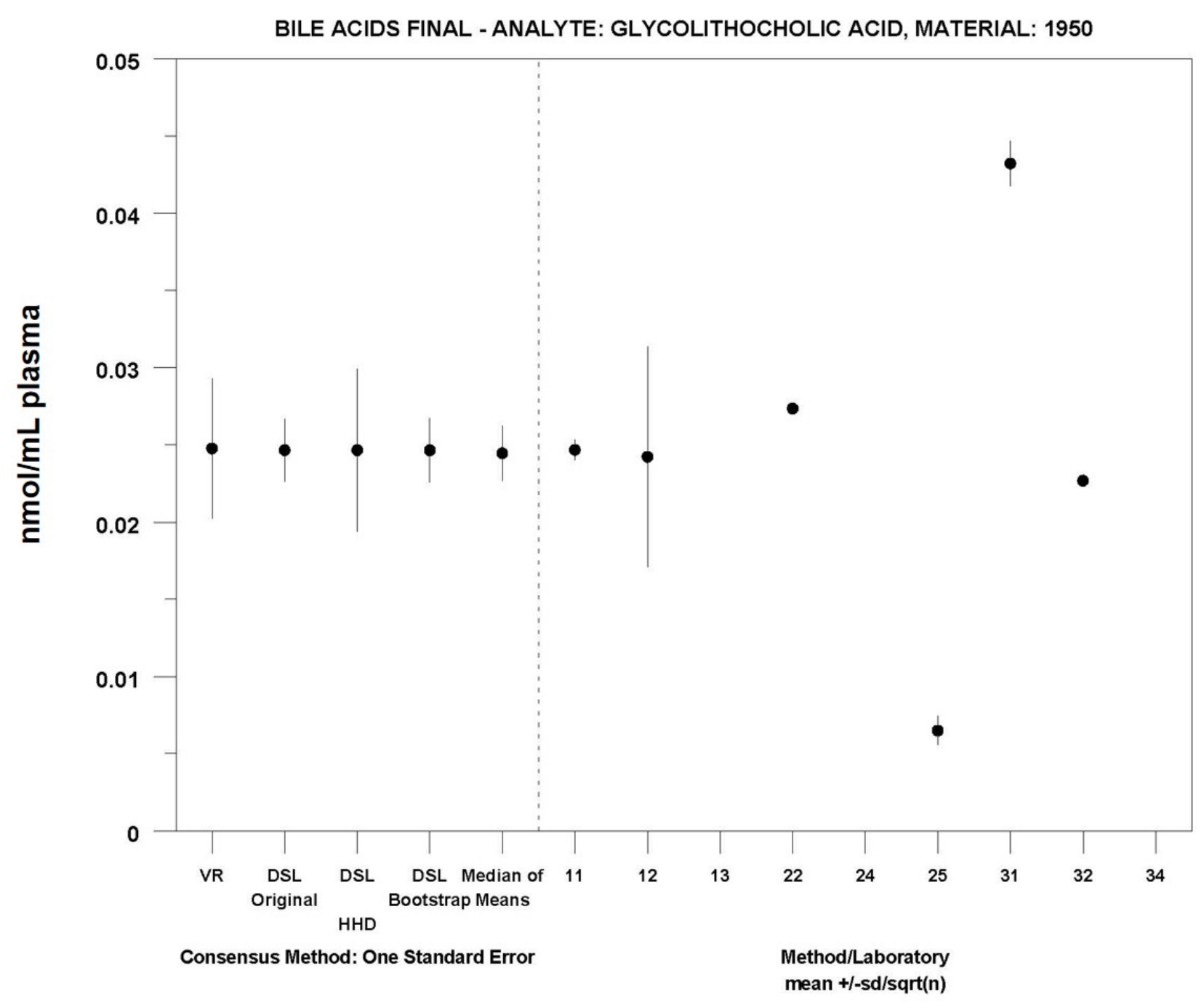

MEDM Location: $0.025 \pm 0.0018 \mathrm{nmol} / \mathrm{mL}$ 


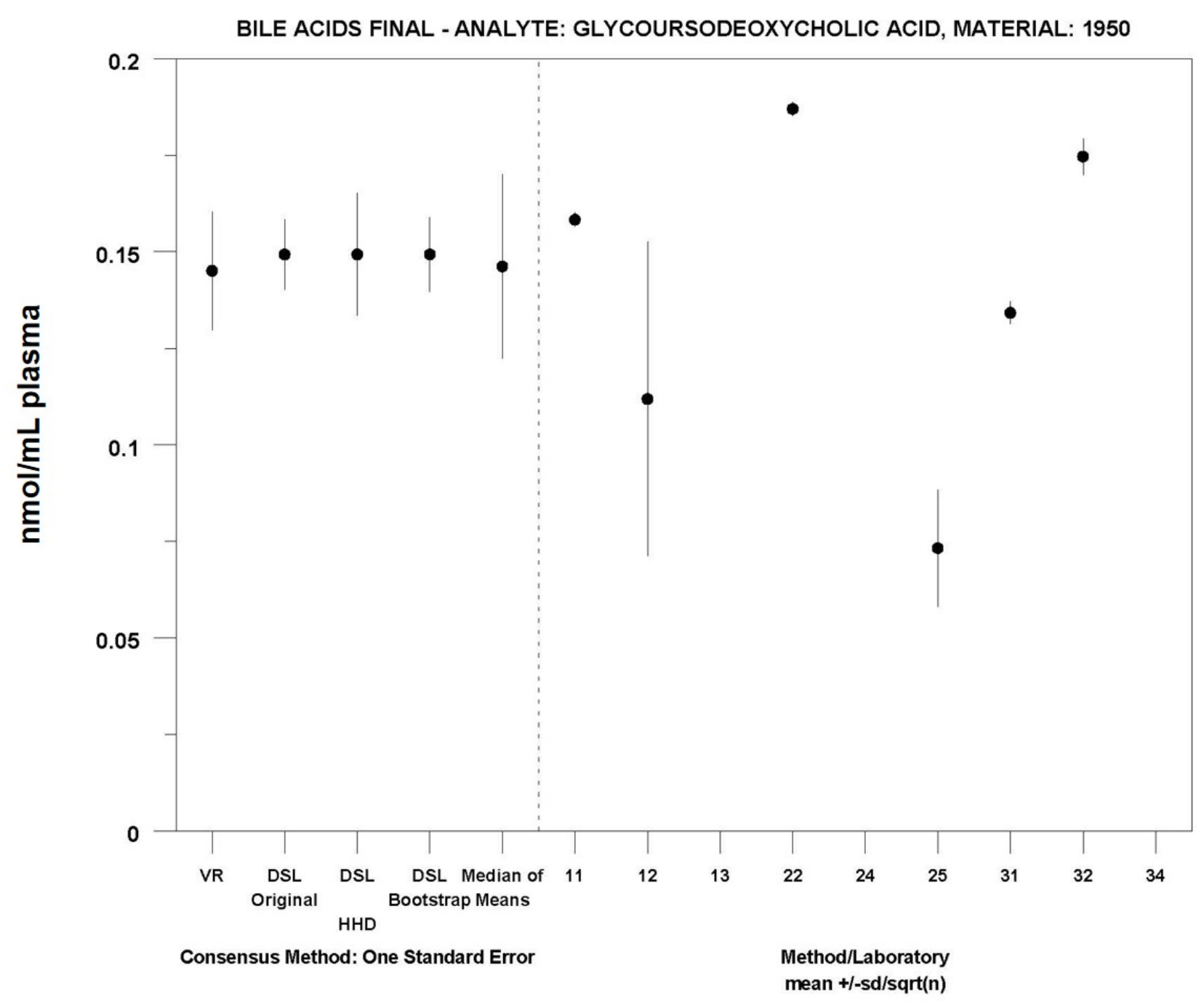

MEDM Location: $0.15 \pm 0.024 \mathrm{nmol} / \mathrm{mL}$ 


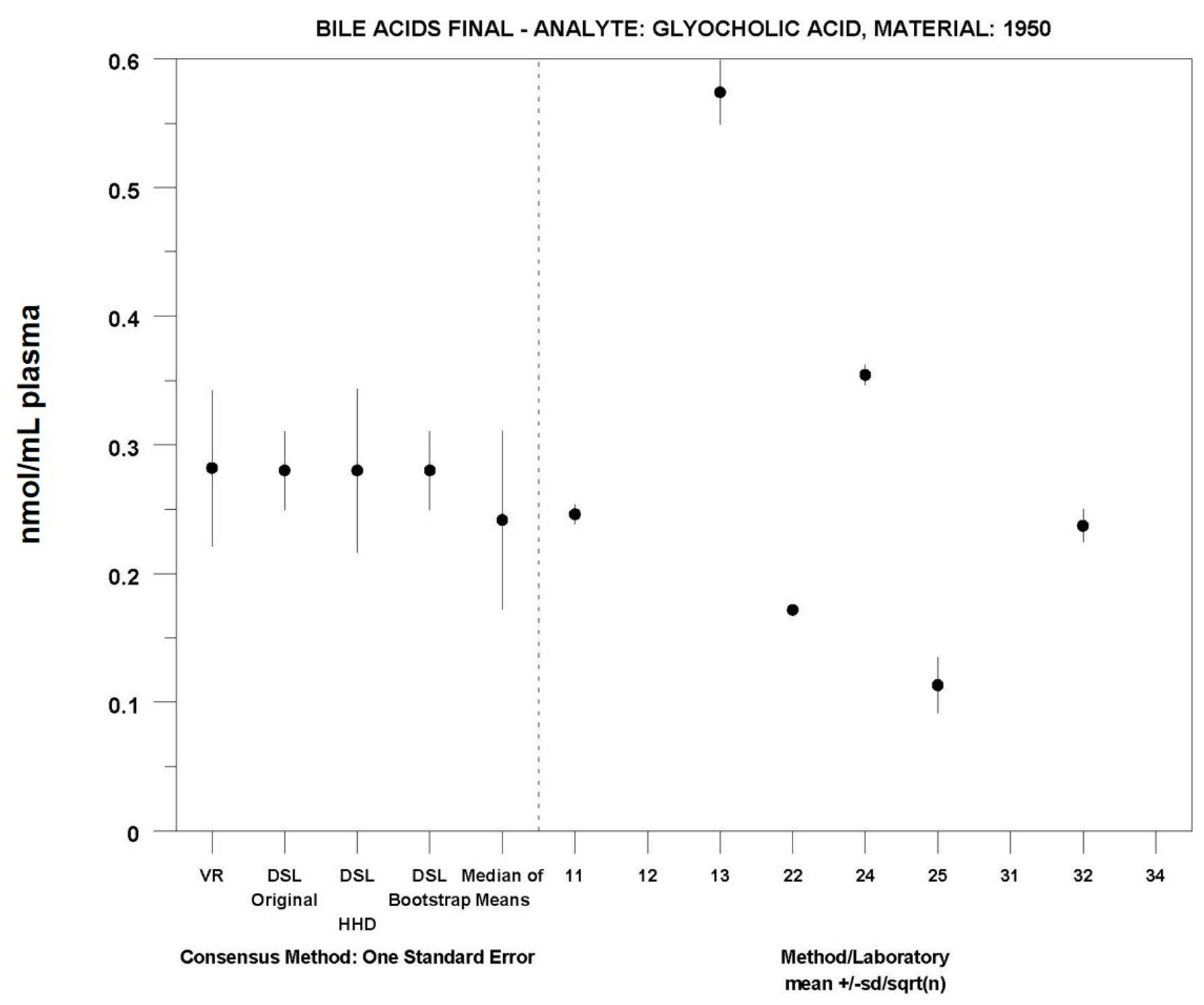

MEDM Location: $0.24 \pm 0.069 \mathrm{nmol} / \mathrm{mL}$ 


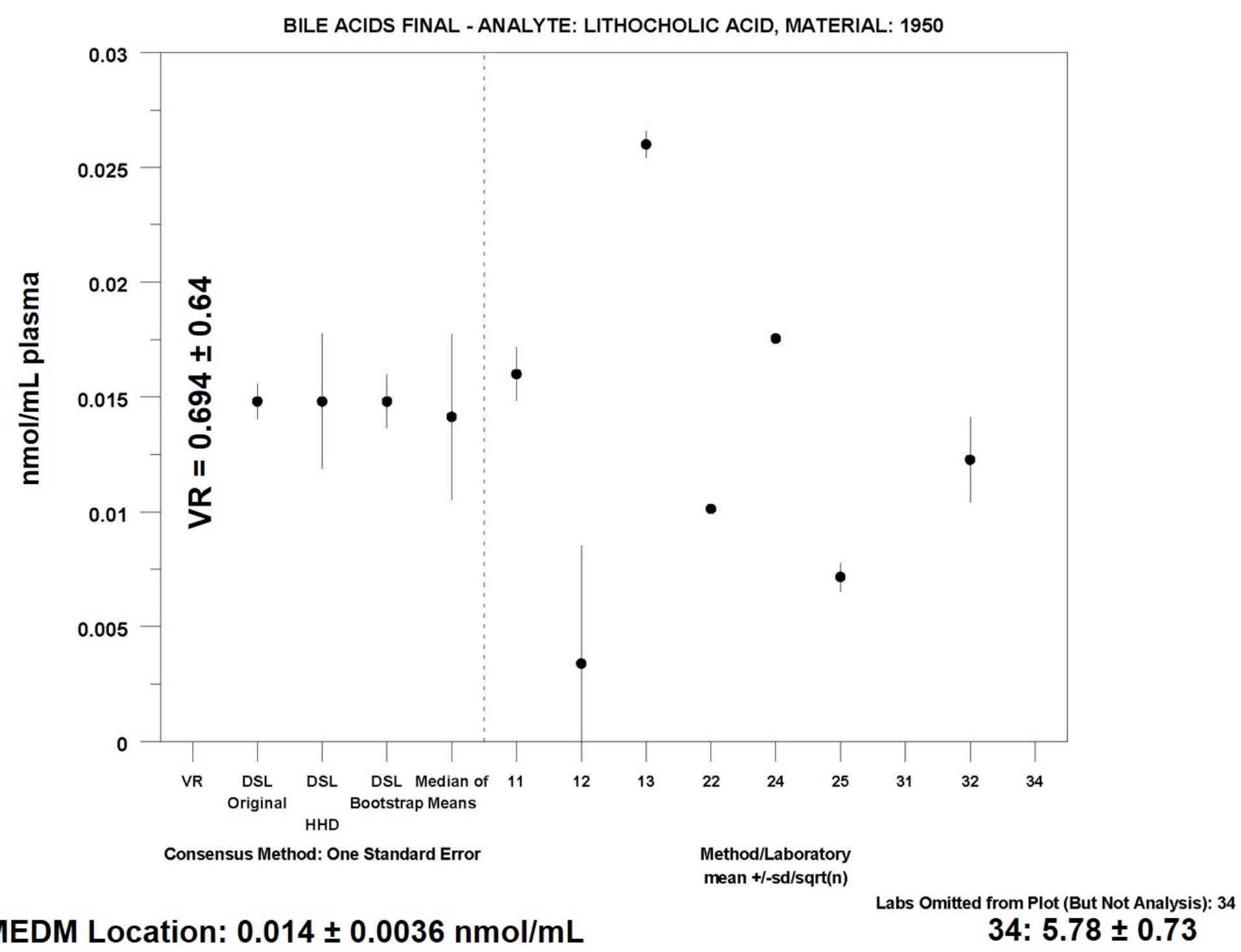




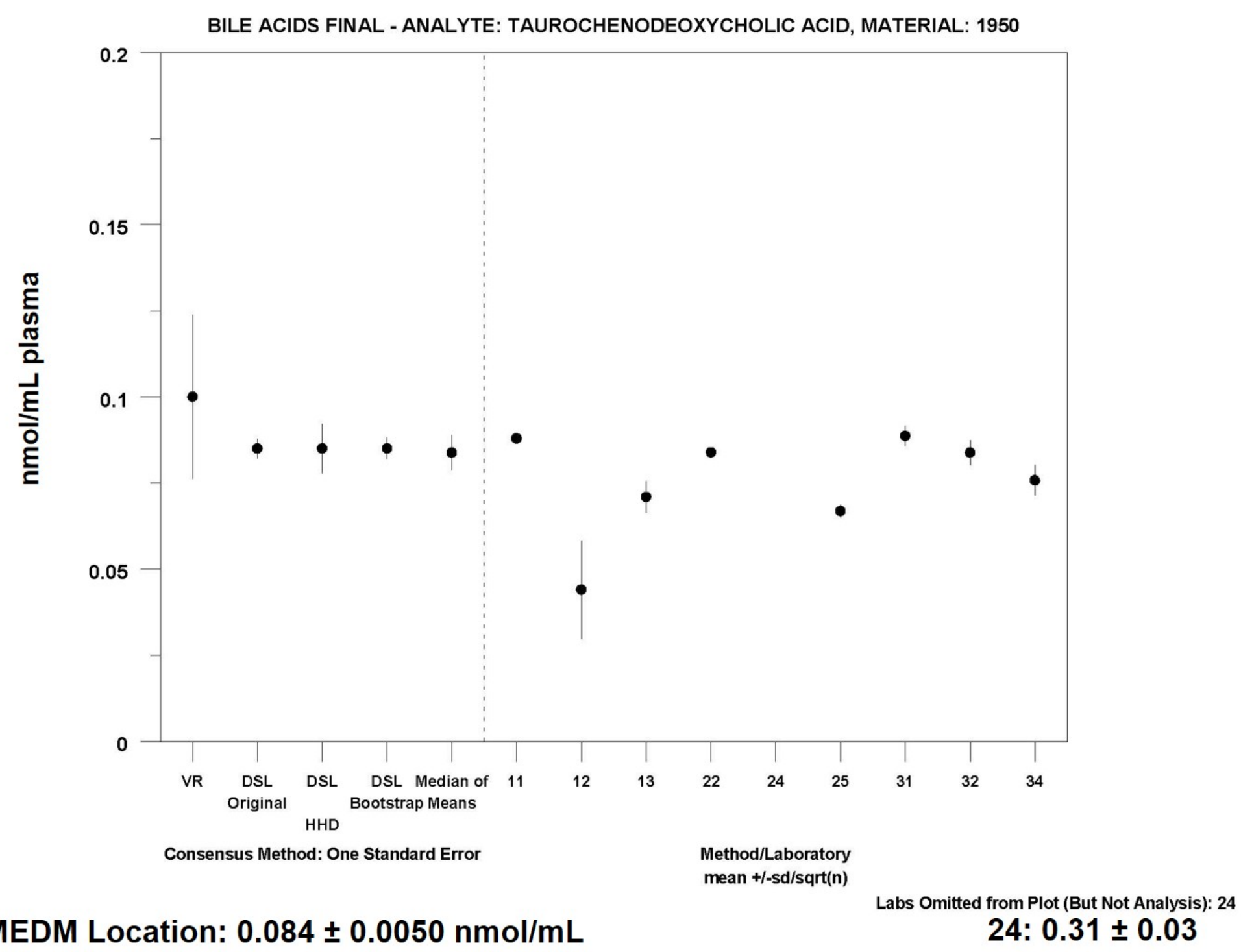




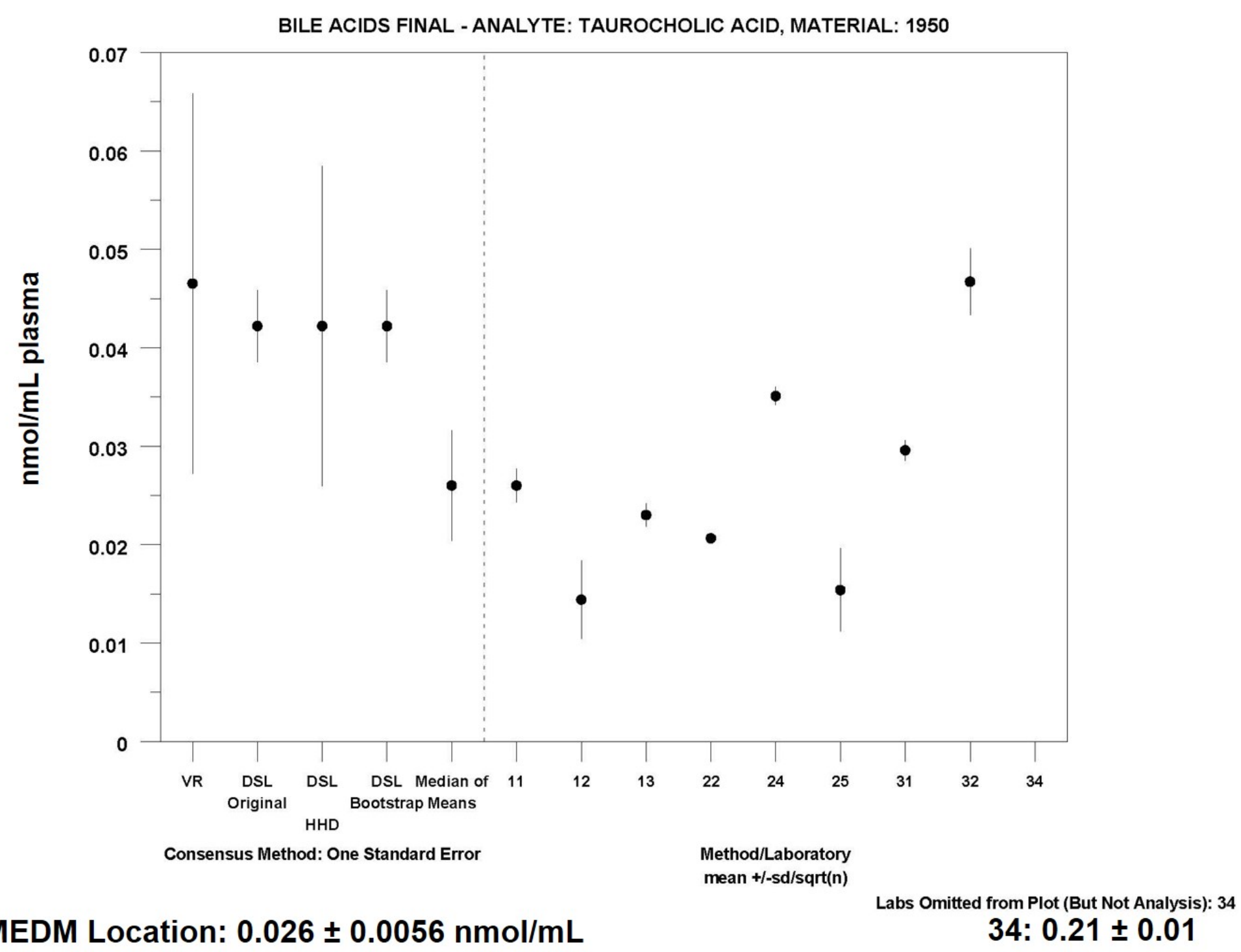




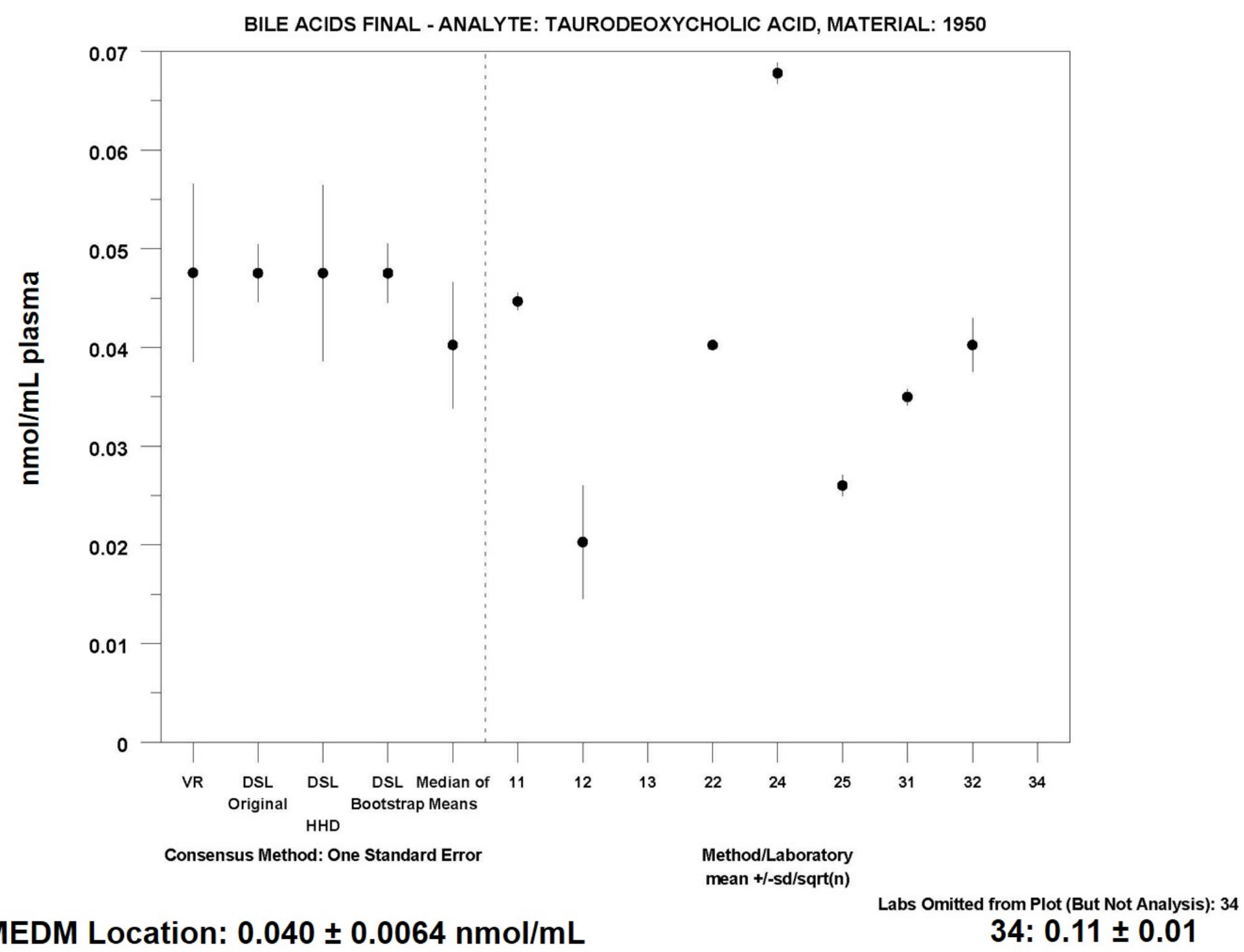




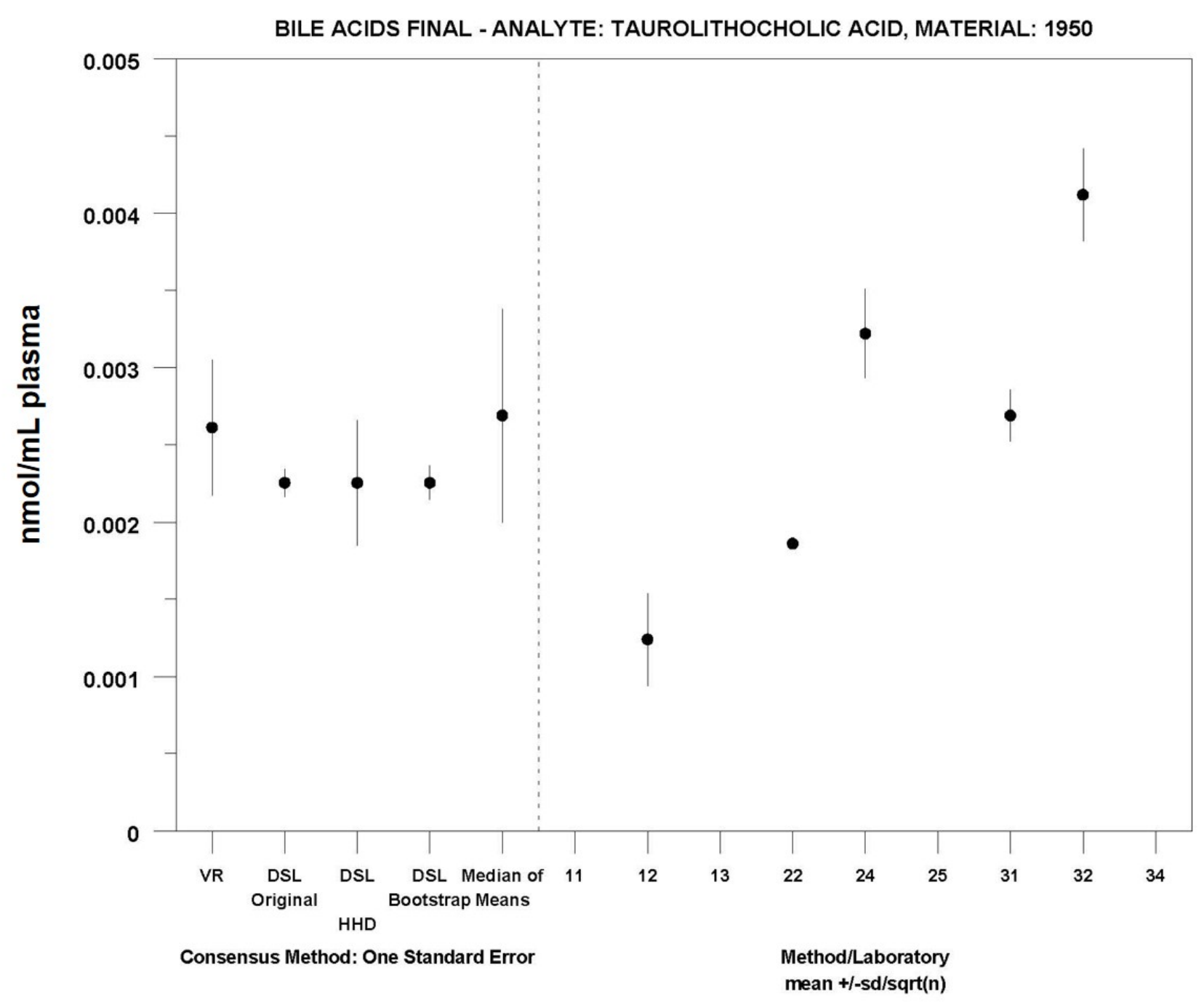

MEDM Location: $0.0027 \pm 0.00069 \mathrm{nmol} / \mathrm{mL}$ 


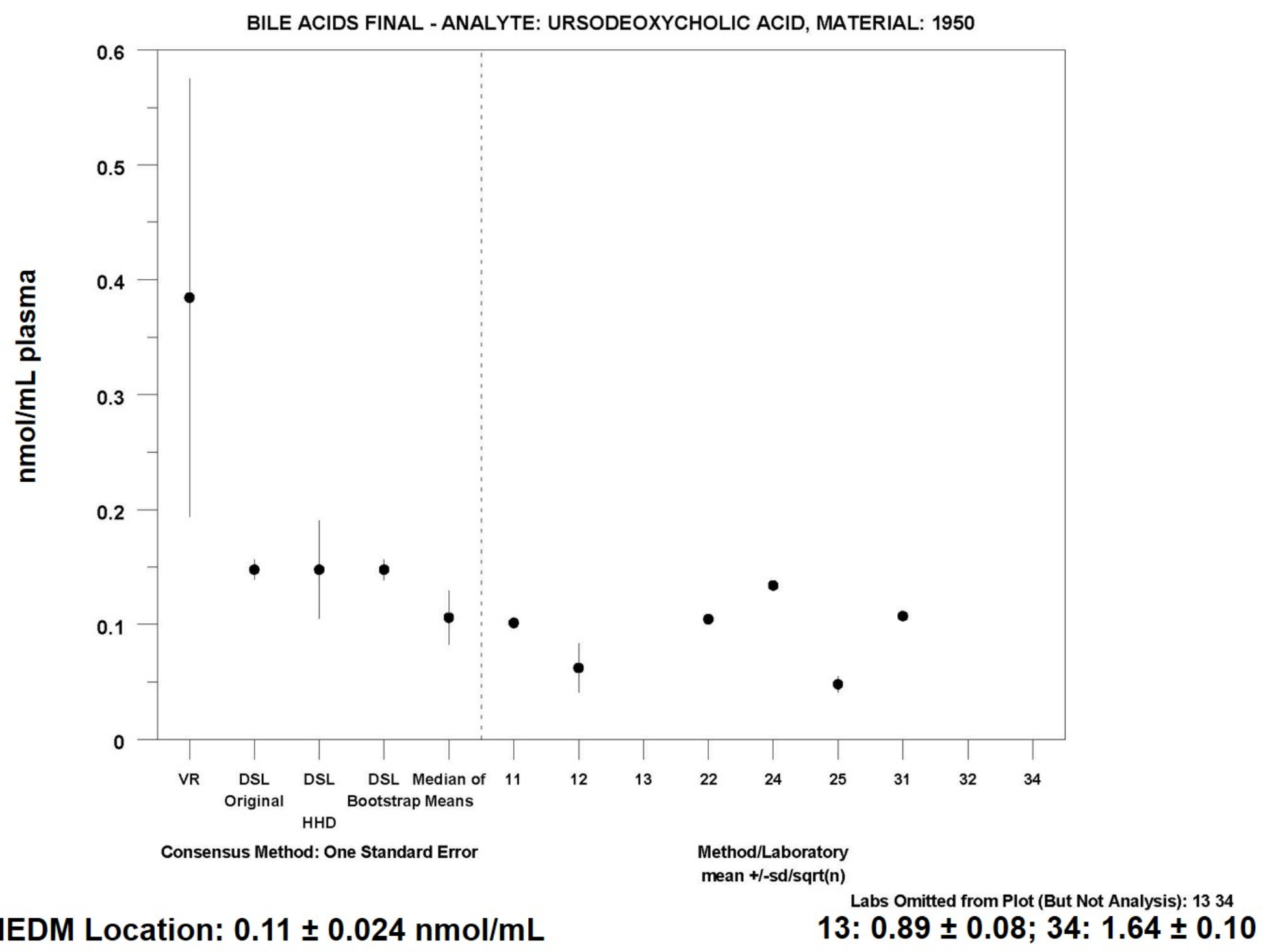




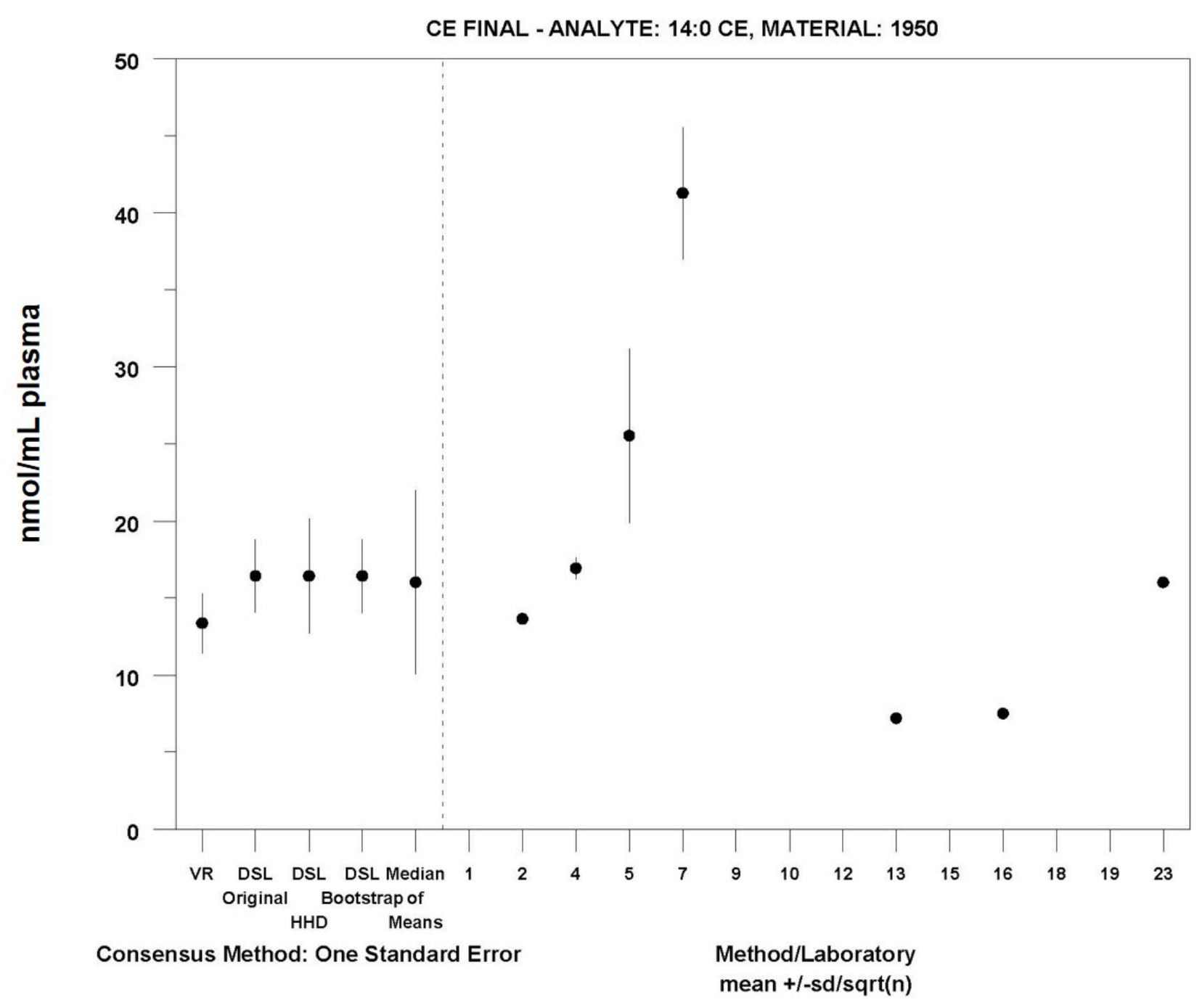

MEDM Location: $16.0 \pm 6.0 \mathrm{nmol} / \mathrm{mL}$ 


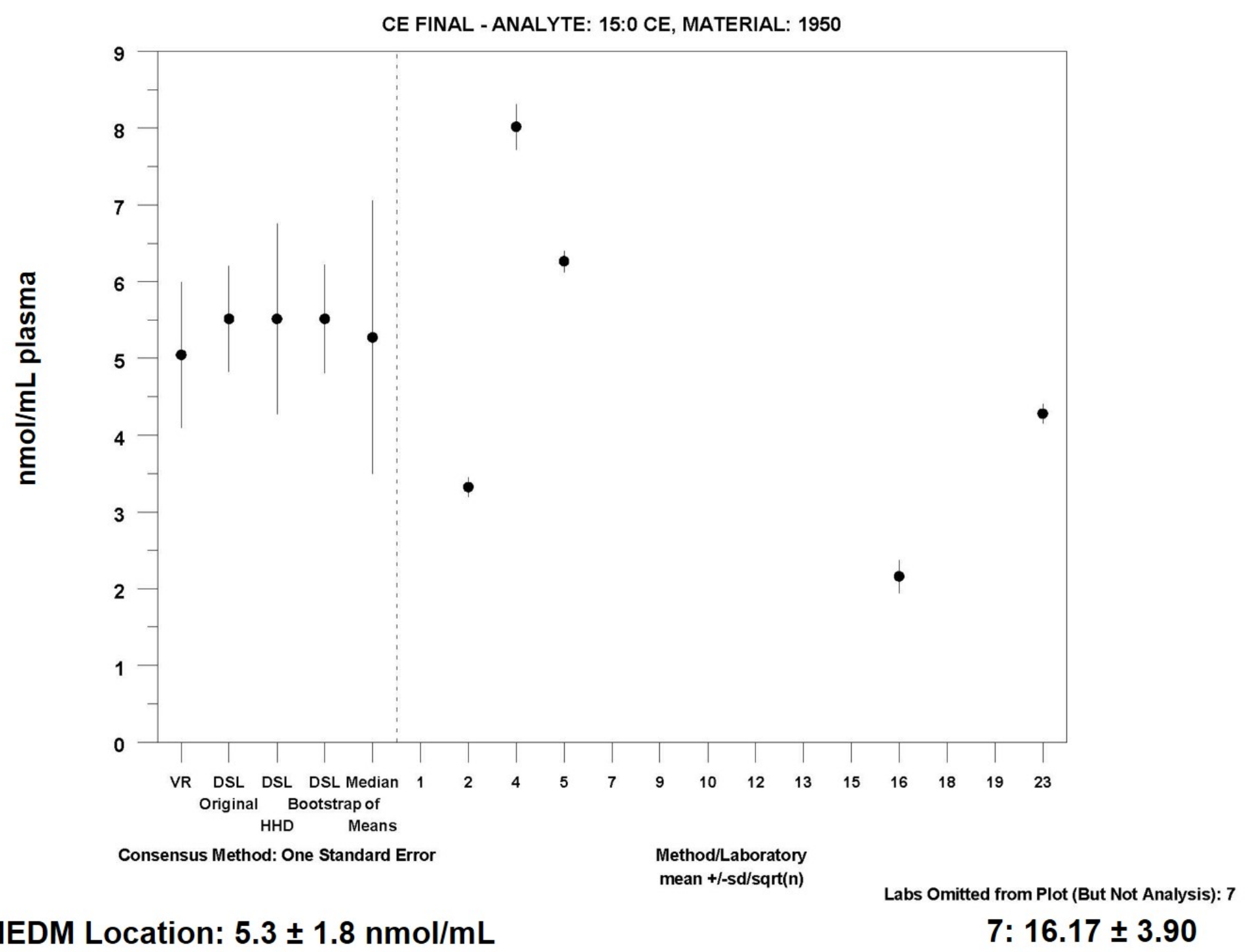




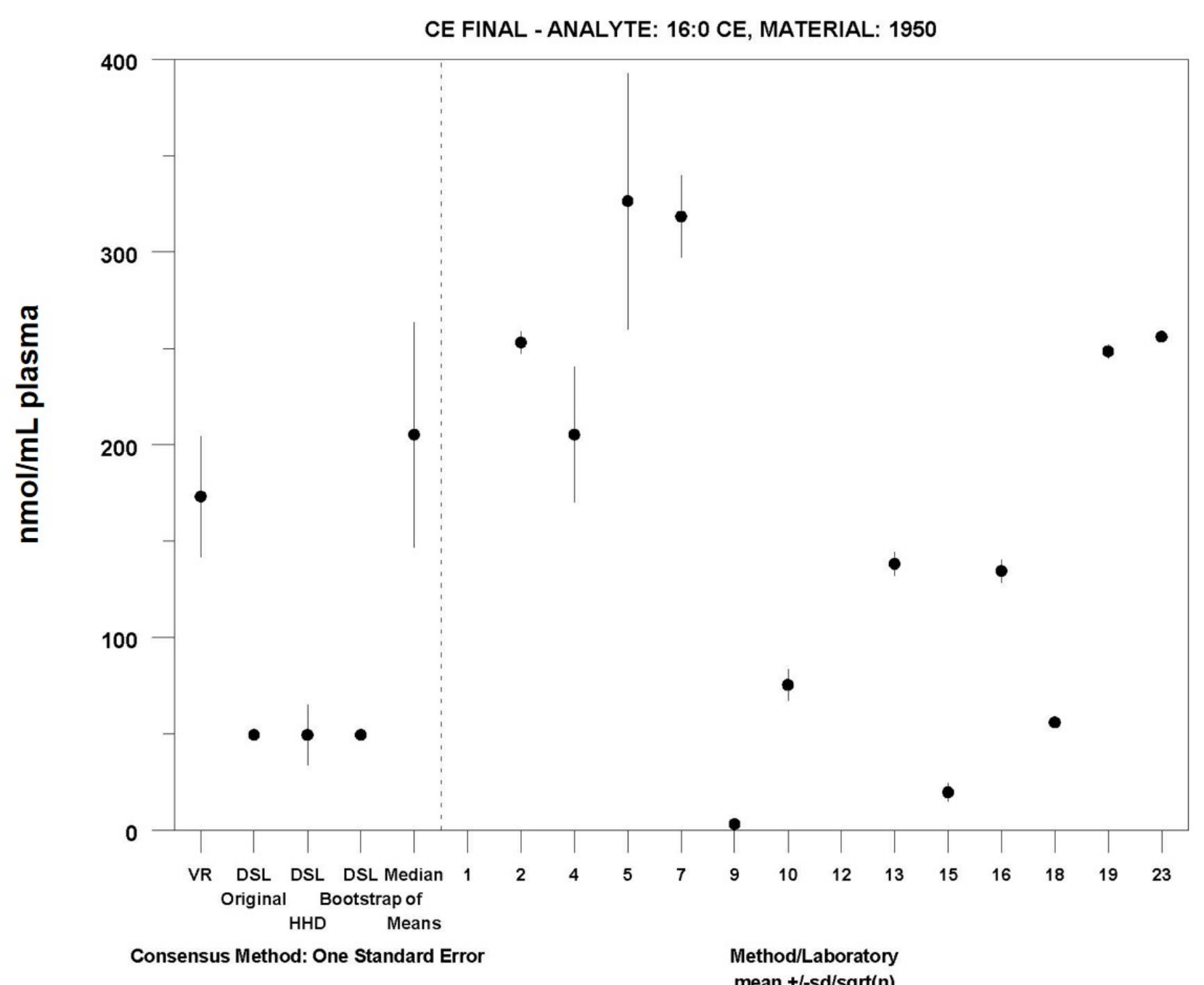

MEDM Location: $210 \pm 58 \mathrm{nmol} / \mathrm{mL}$

Labs Omitted from Plot (But Not Analysis): 1

1: $504.6 \pm 185.9$ 


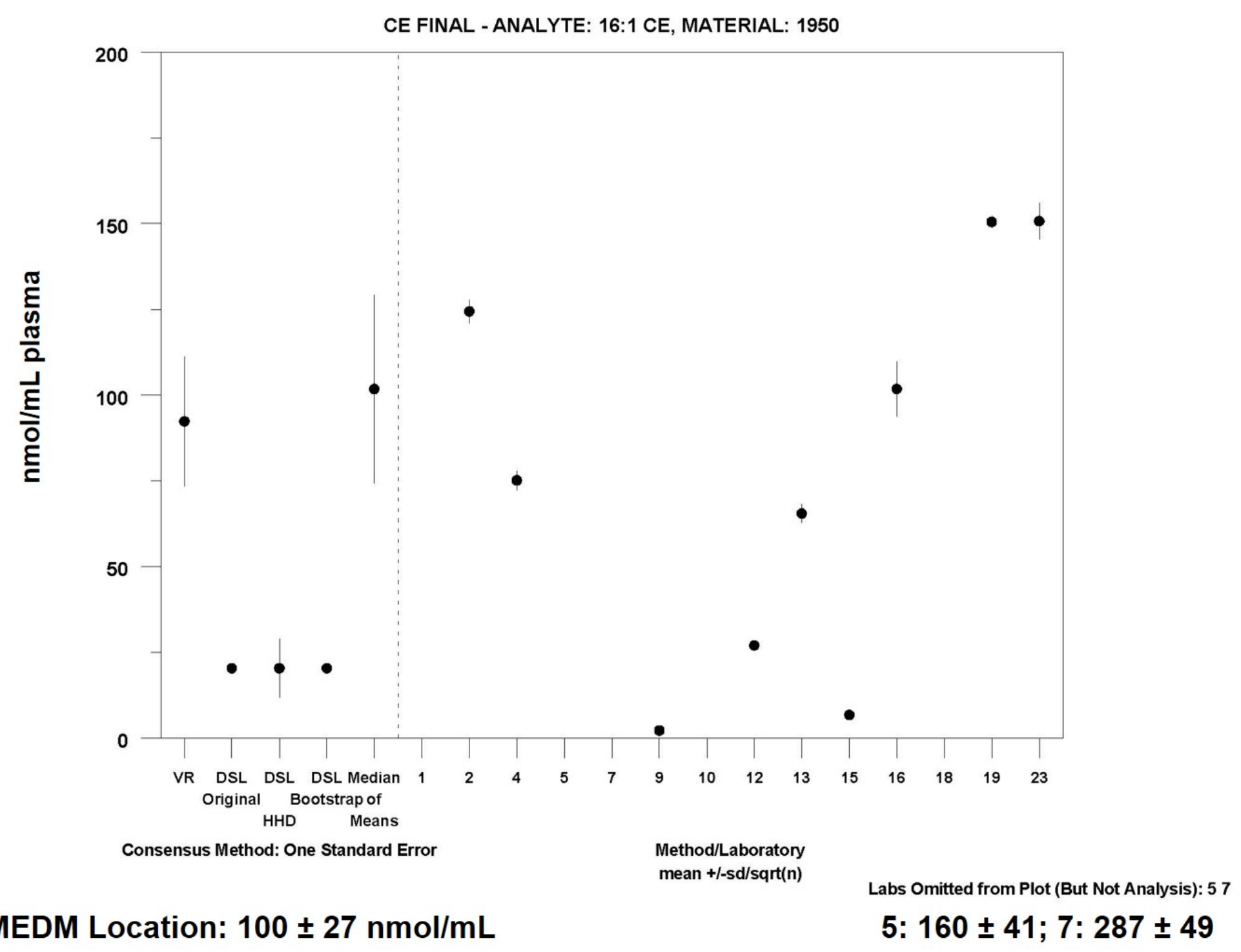




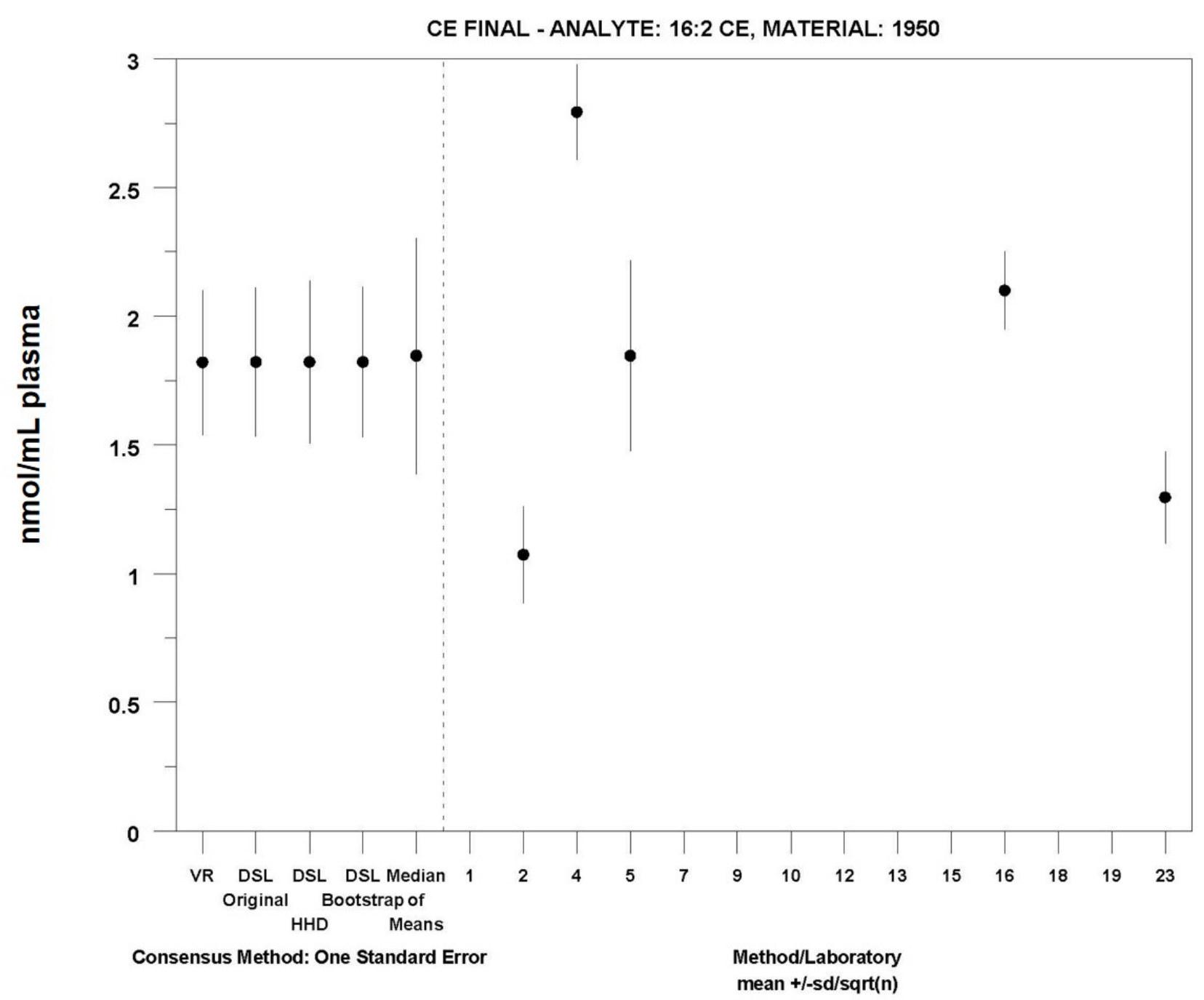

MEDM Location: $1.9 \pm 0.46 \mathrm{nmol} / \mathrm{mL}$ 


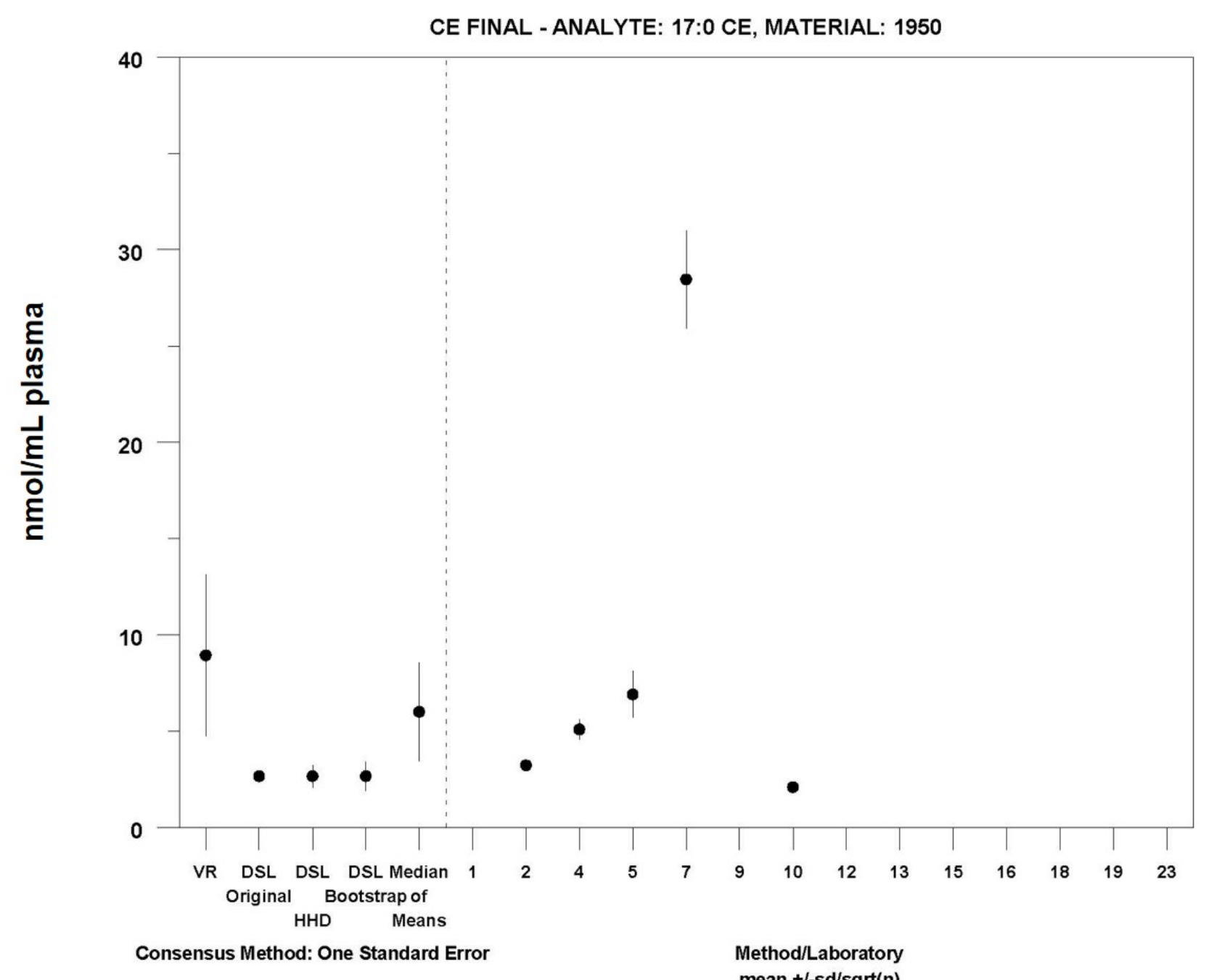

MEDM Location: $6.0 \pm 2.5 \mathrm{nmol} / \mathrm{mL}$

Labs Omitted from Plot (But Not Analysis): 1

1: $664 \pm 245$ 


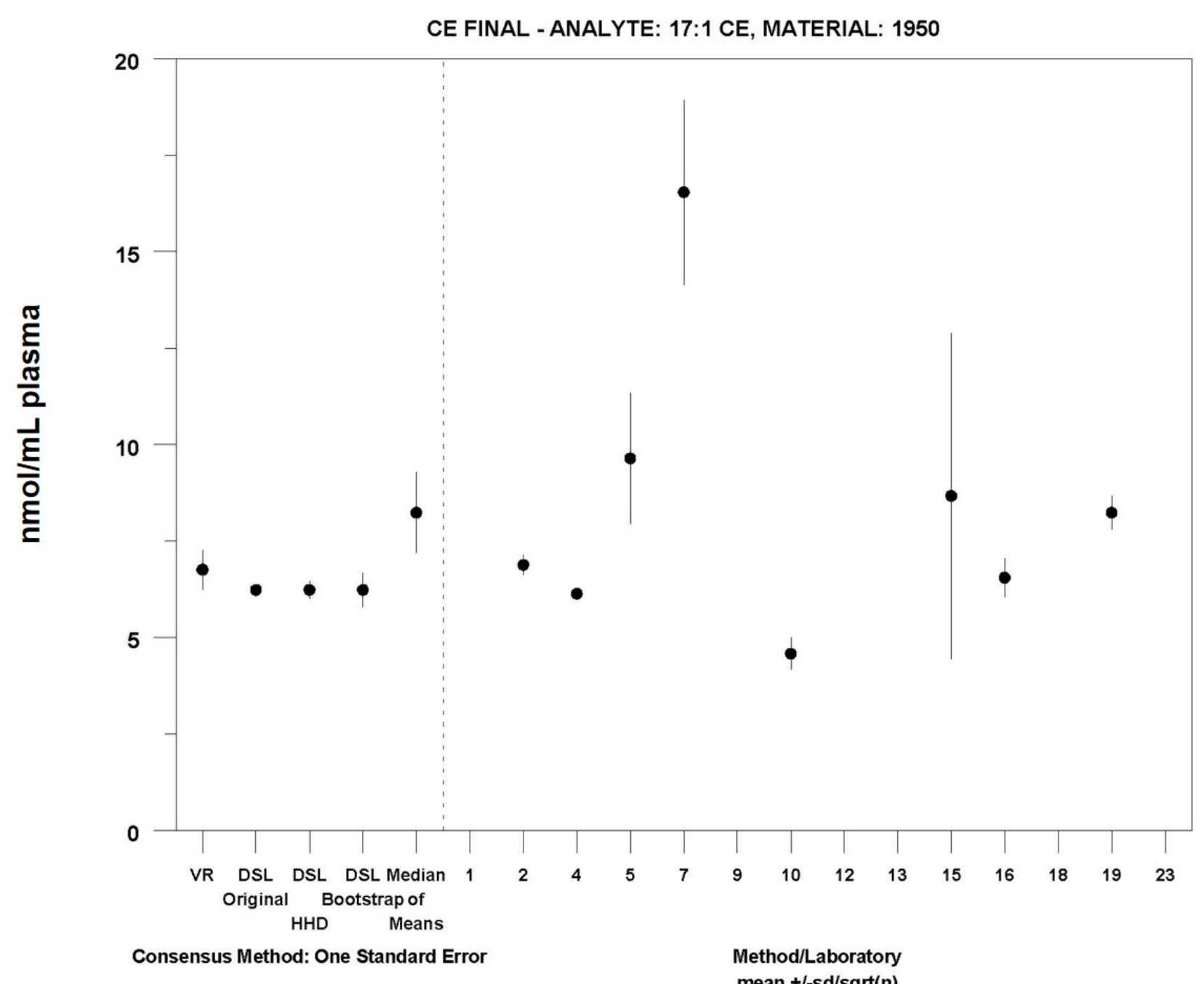

MEDM Location: $8.2 \pm 1.0 \mathrm{nmol} / \mathrm{mL}$

Labs Omitted from Plot (But Not Analysis): 1

1: $369 \pm 135$ 


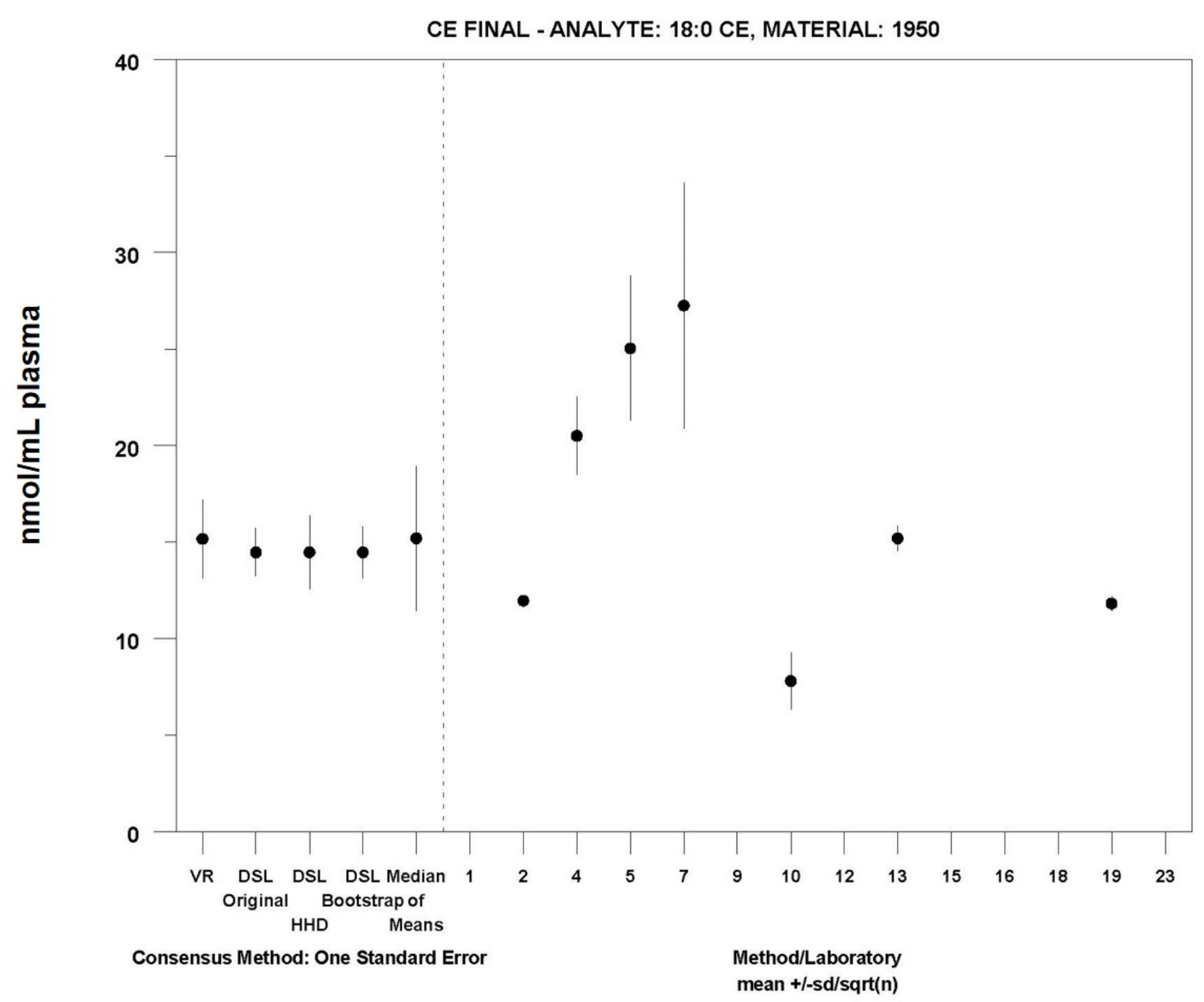

MEDM Location: $15 \pm 3.7 \mathrm{nmol} / \mathrm{mL}$ 


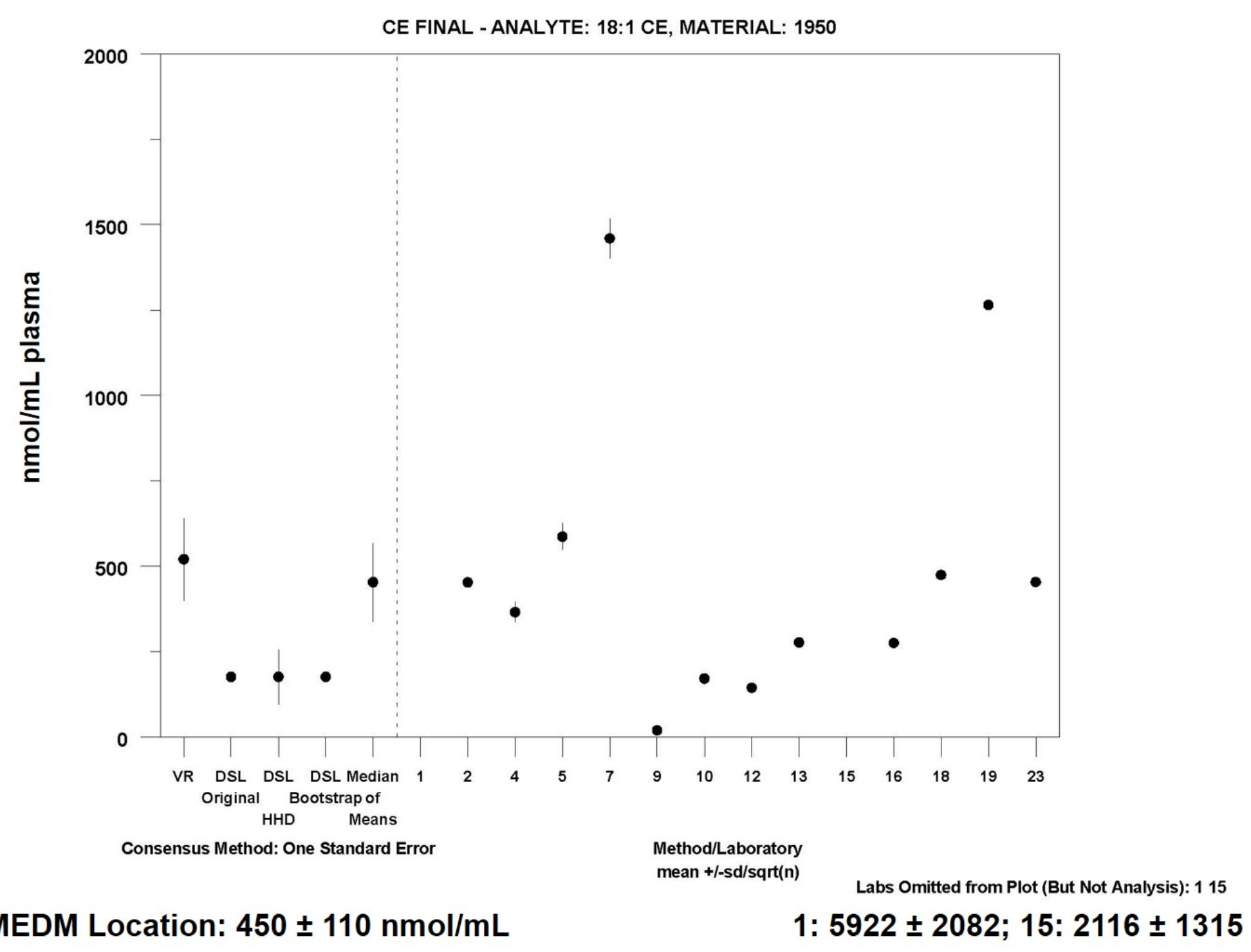




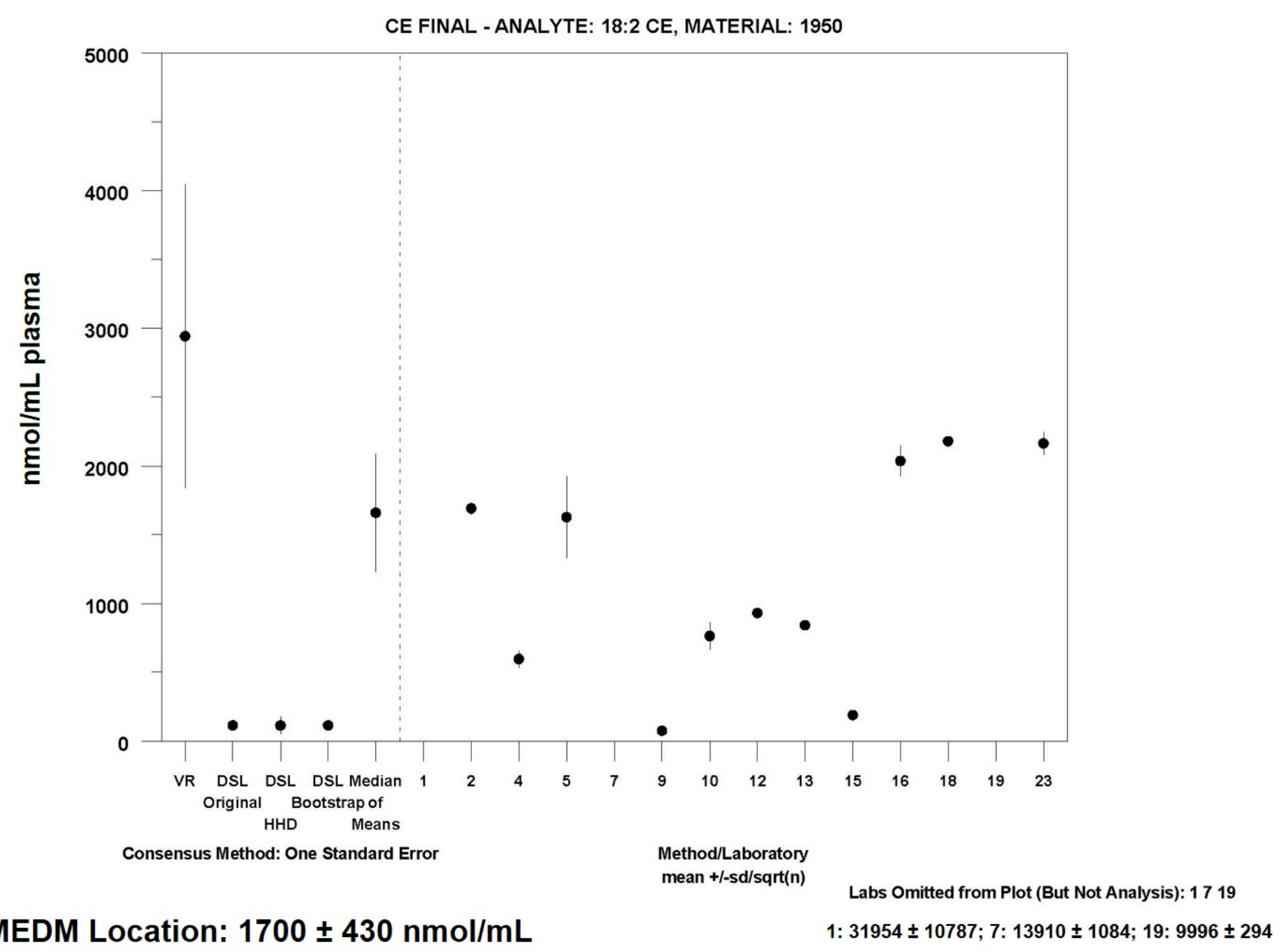




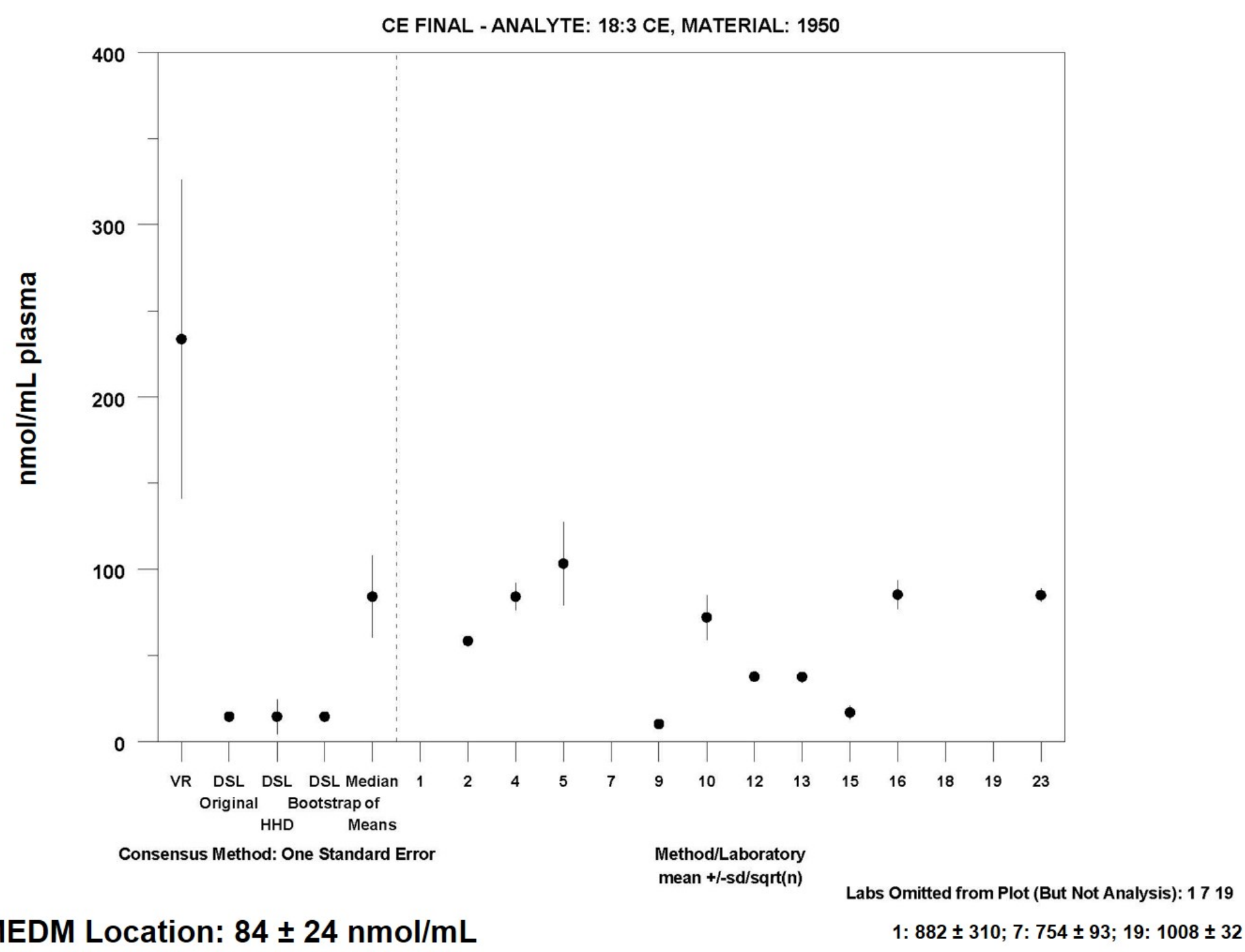




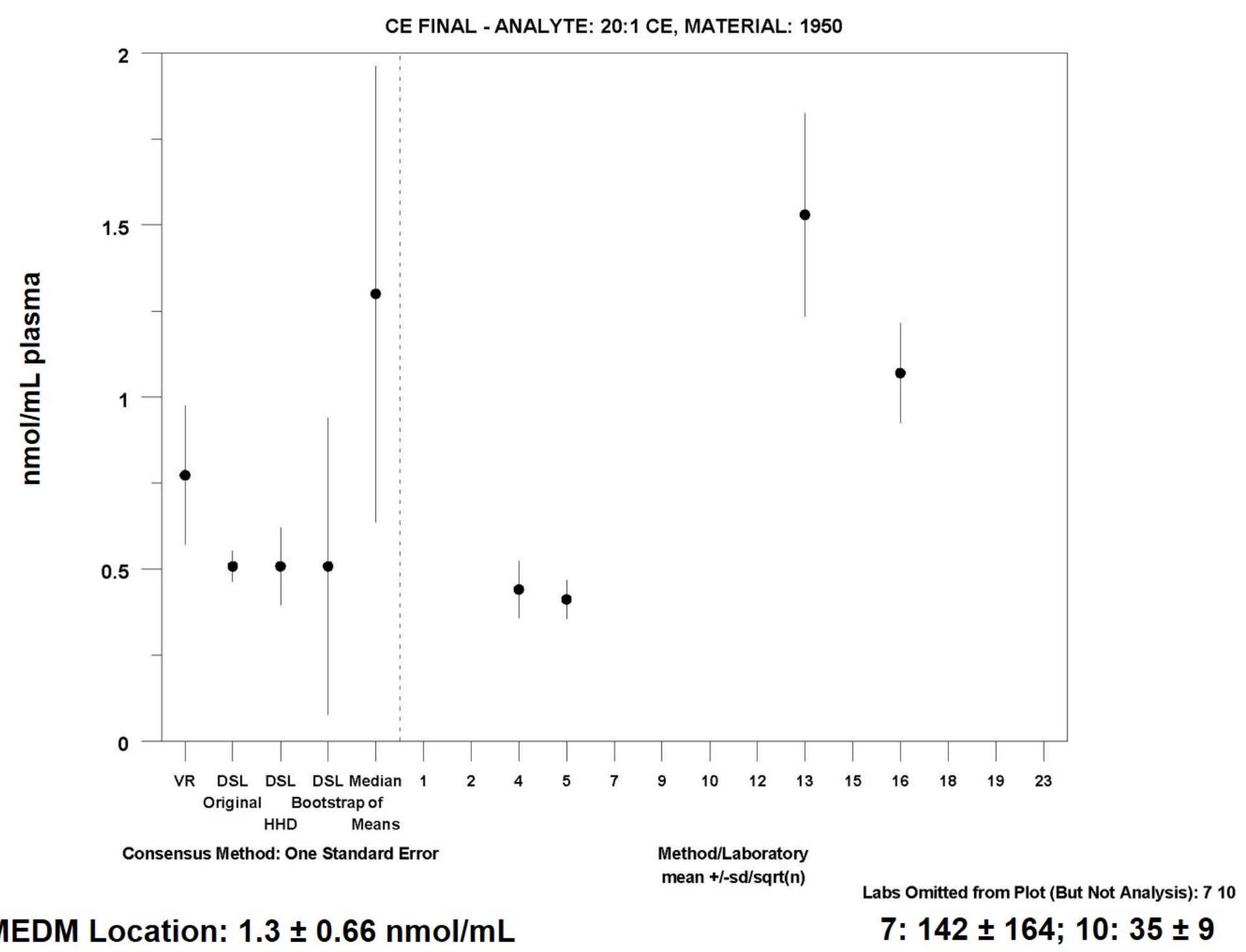




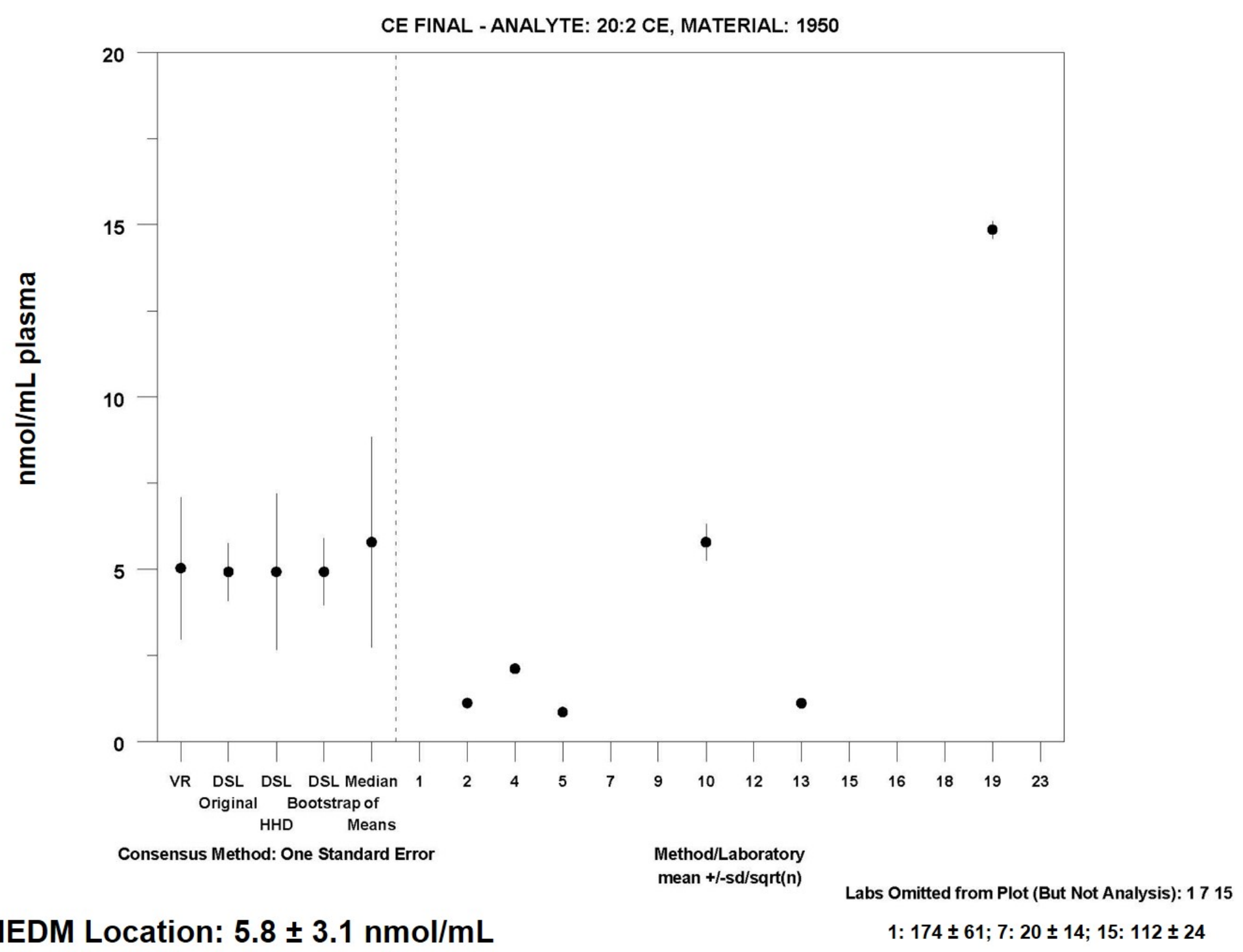




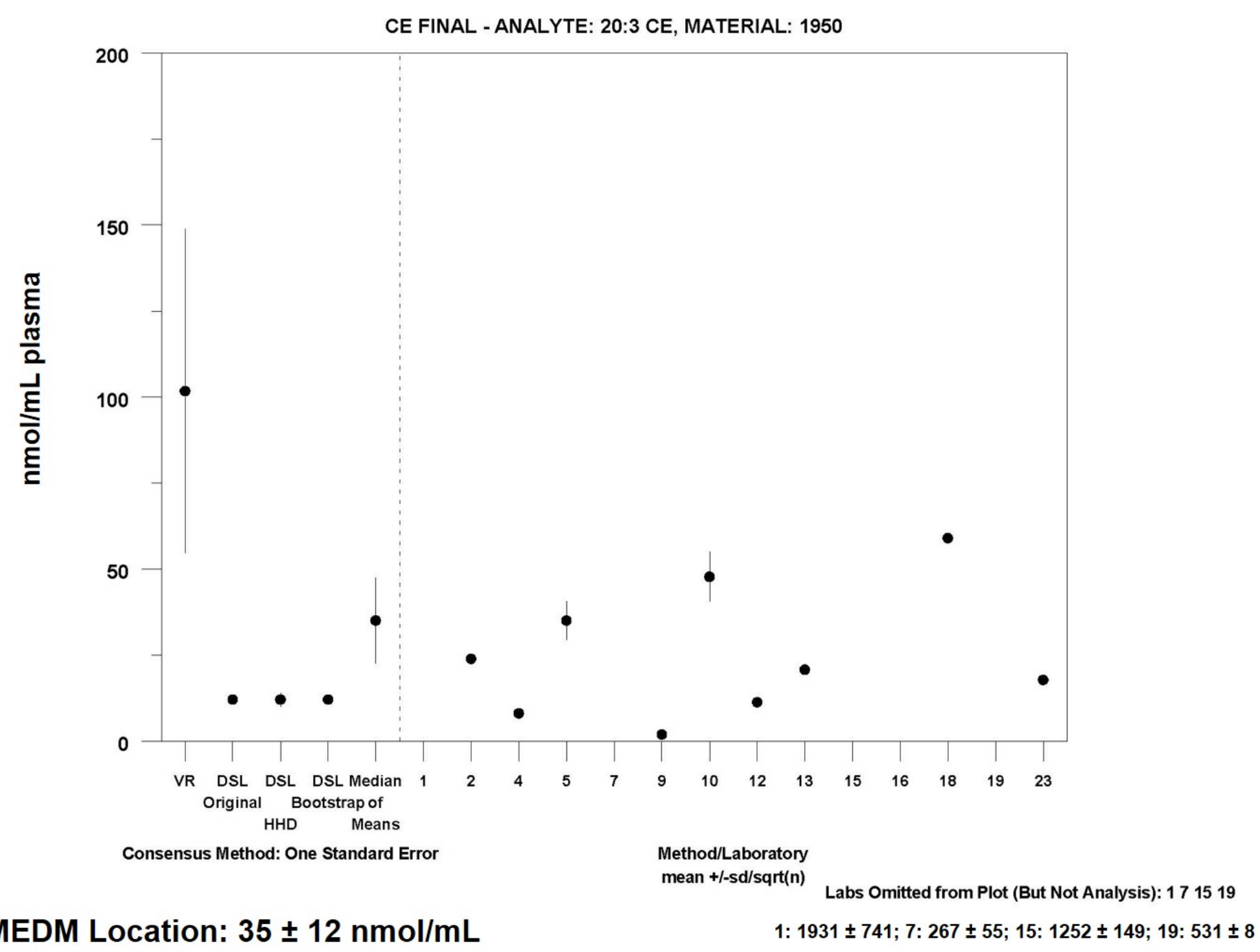




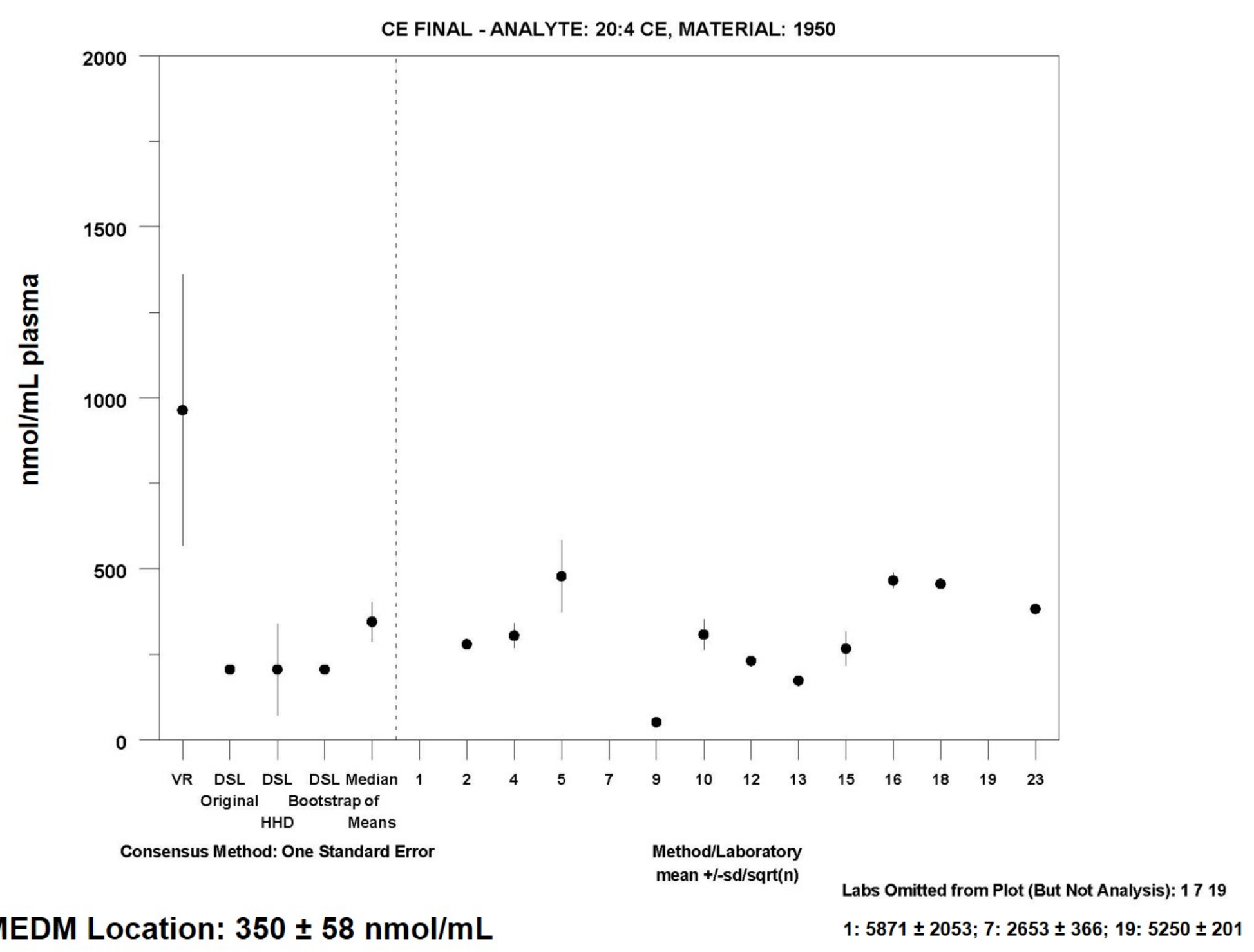




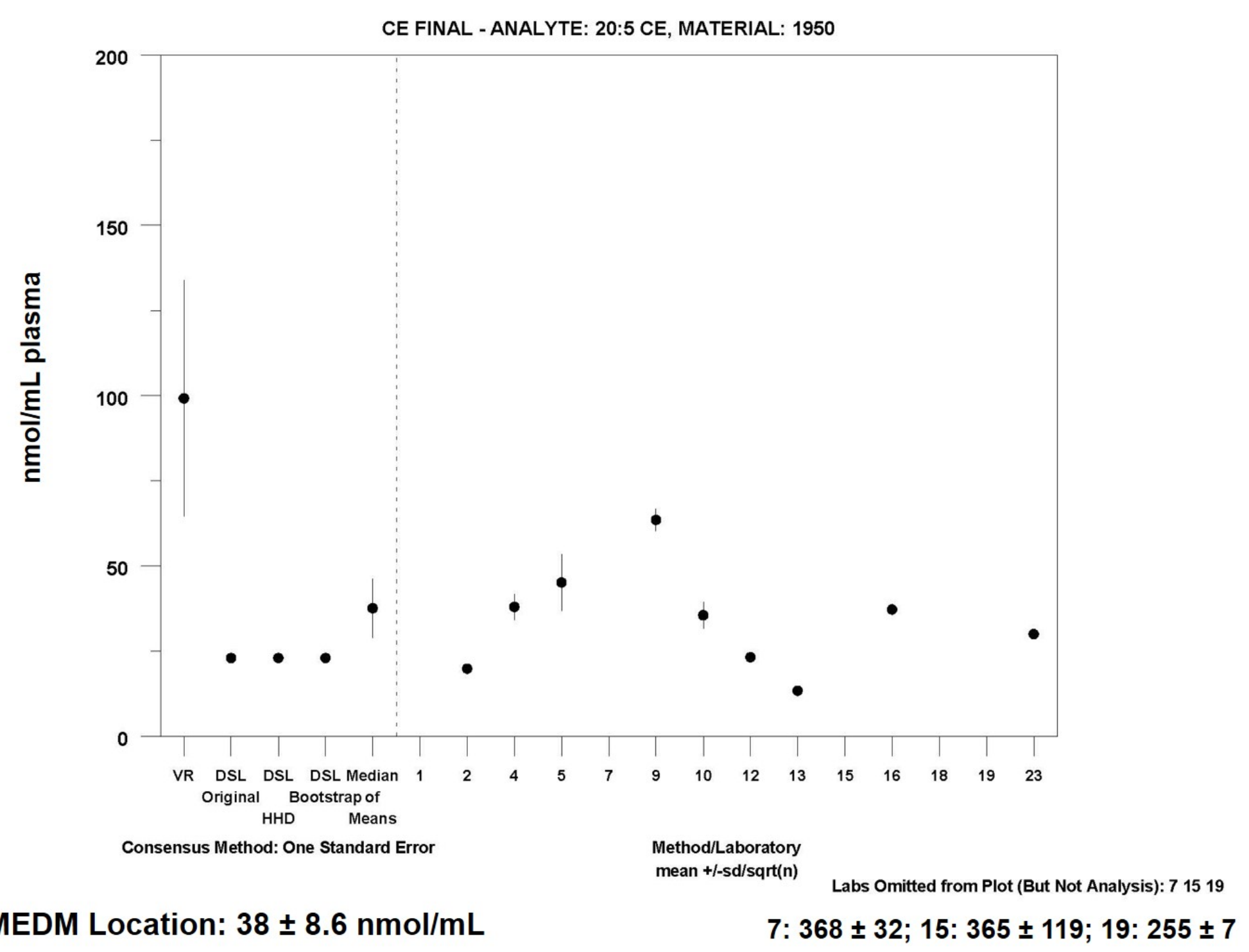




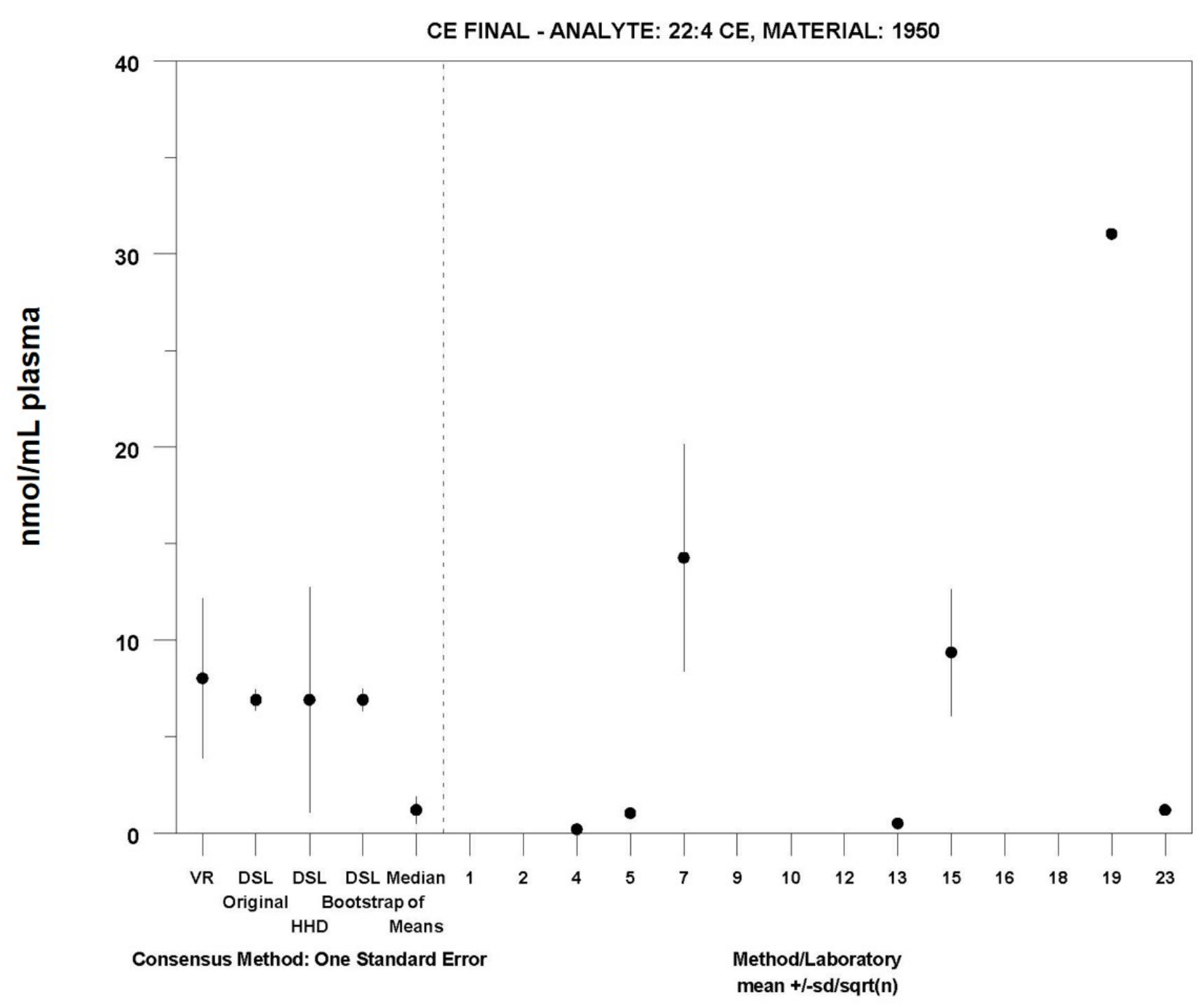

MEDM Location: $1.2 \pm 0.70 \mathrm{nmol} / \mathrm{mL}$ 


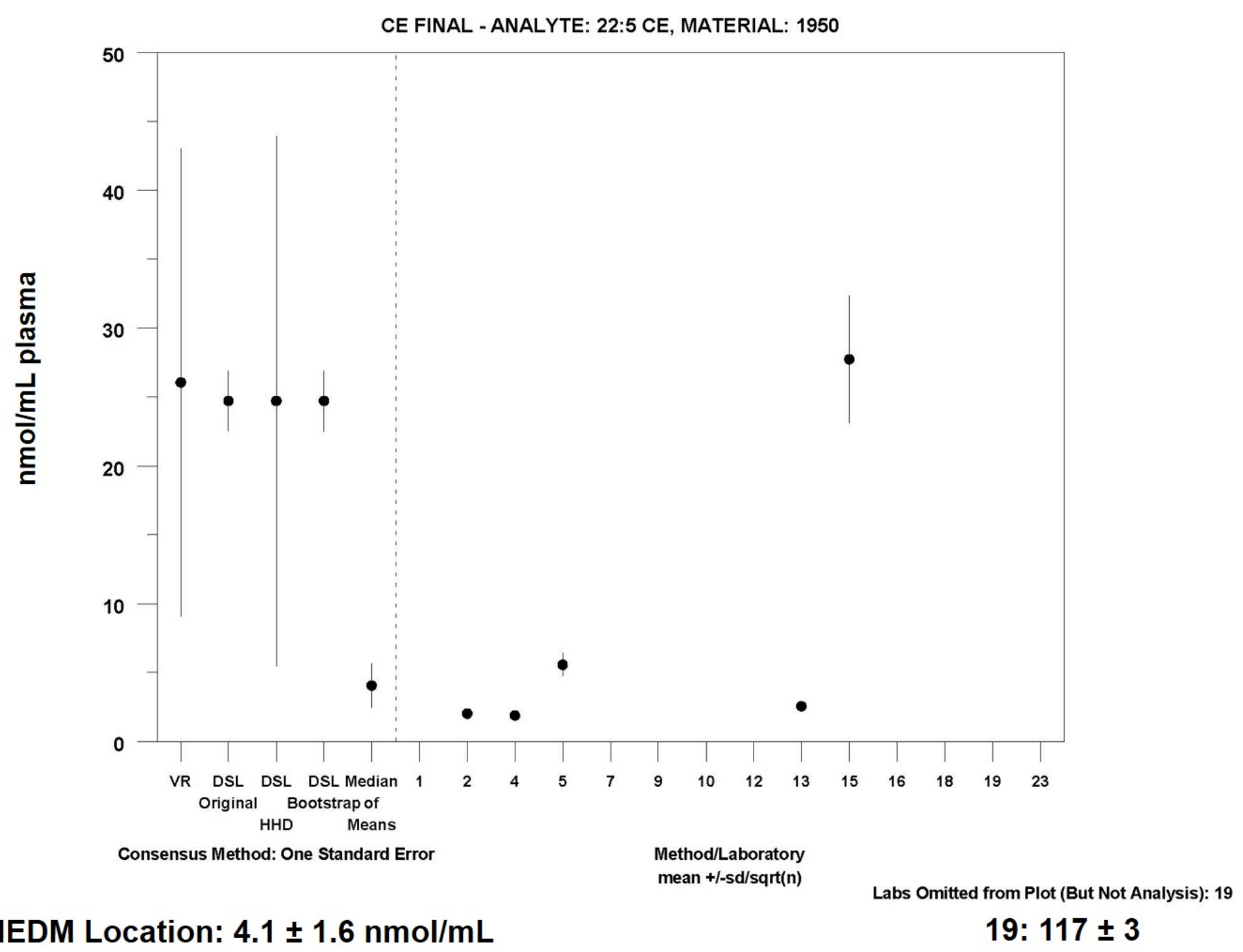




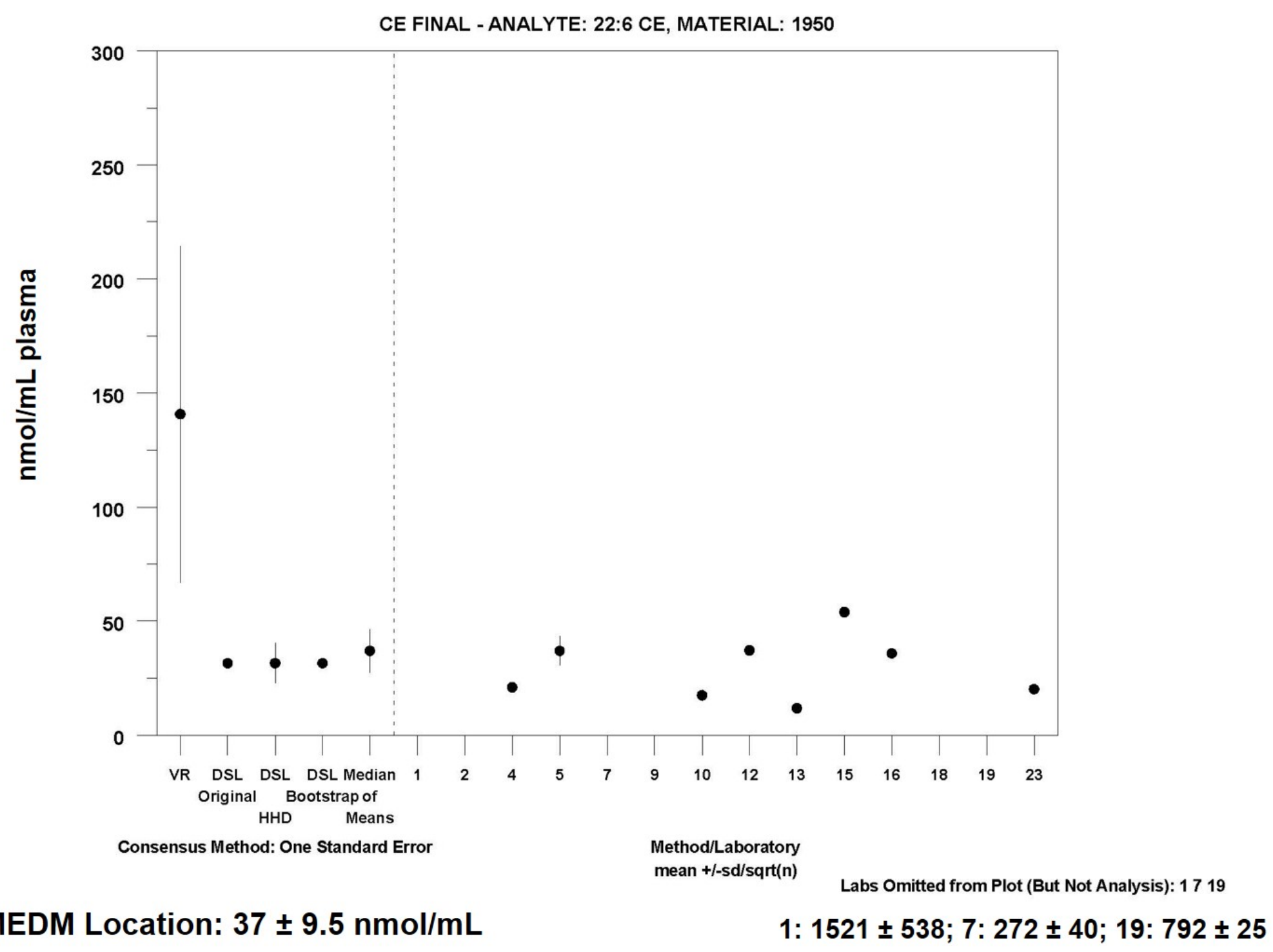




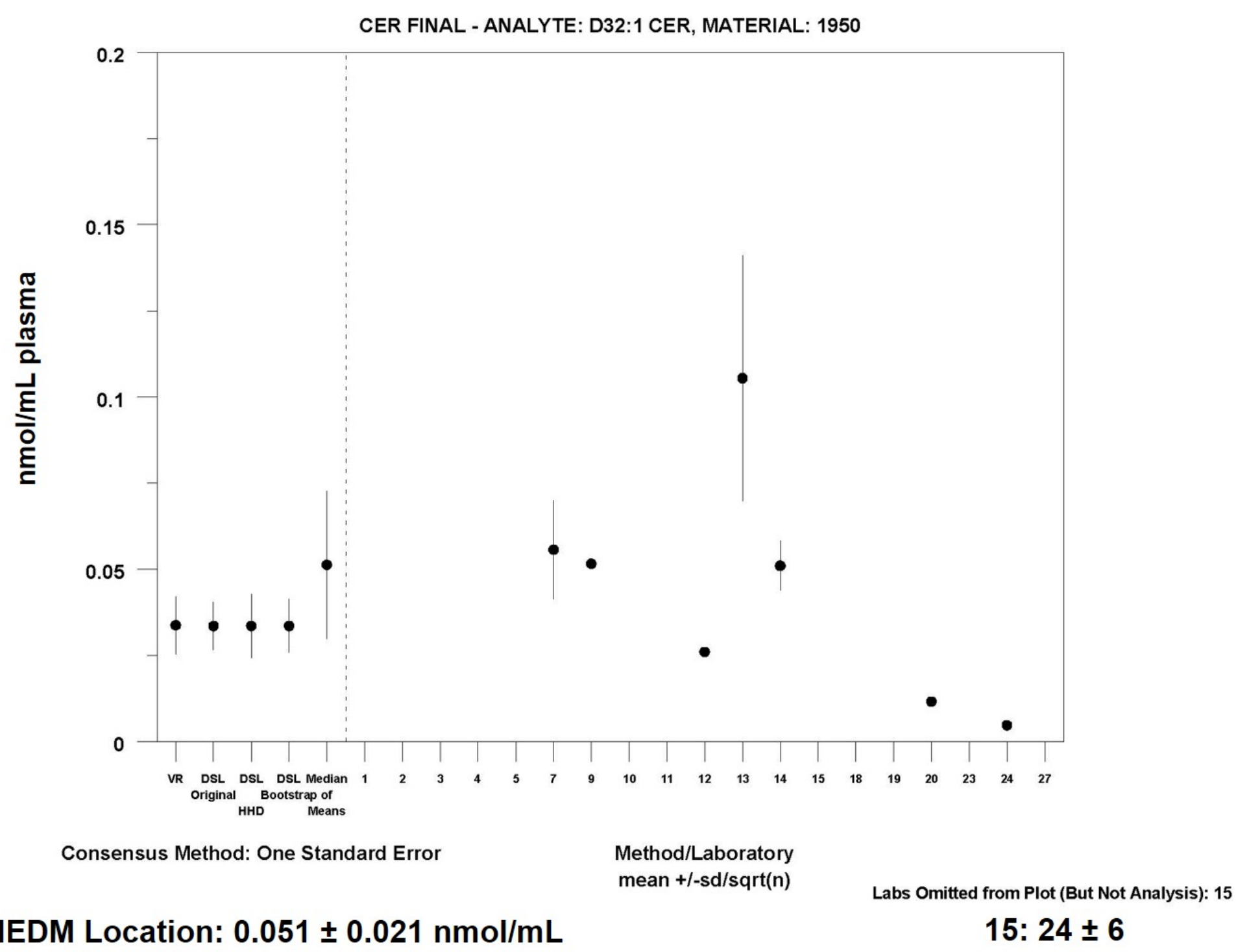




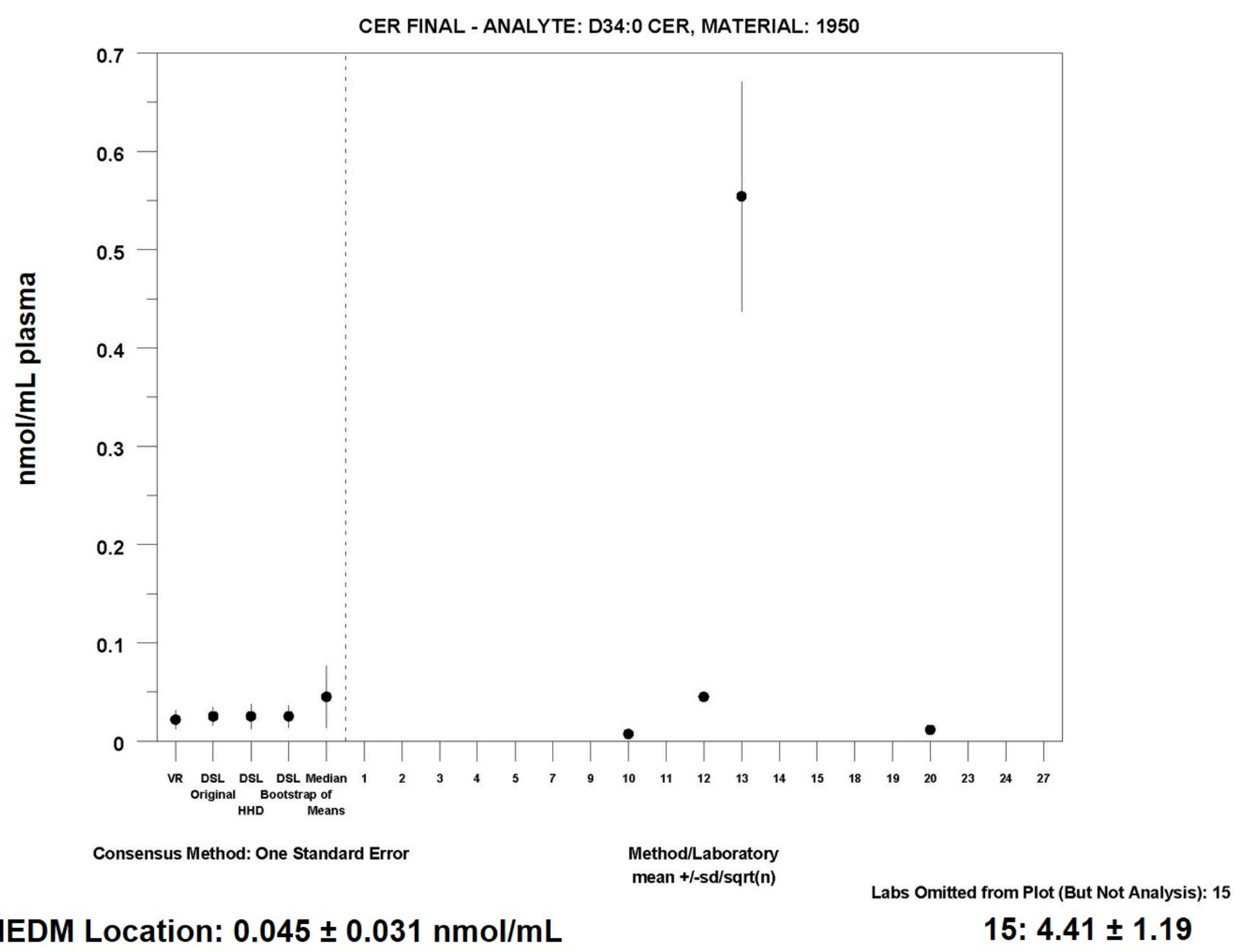




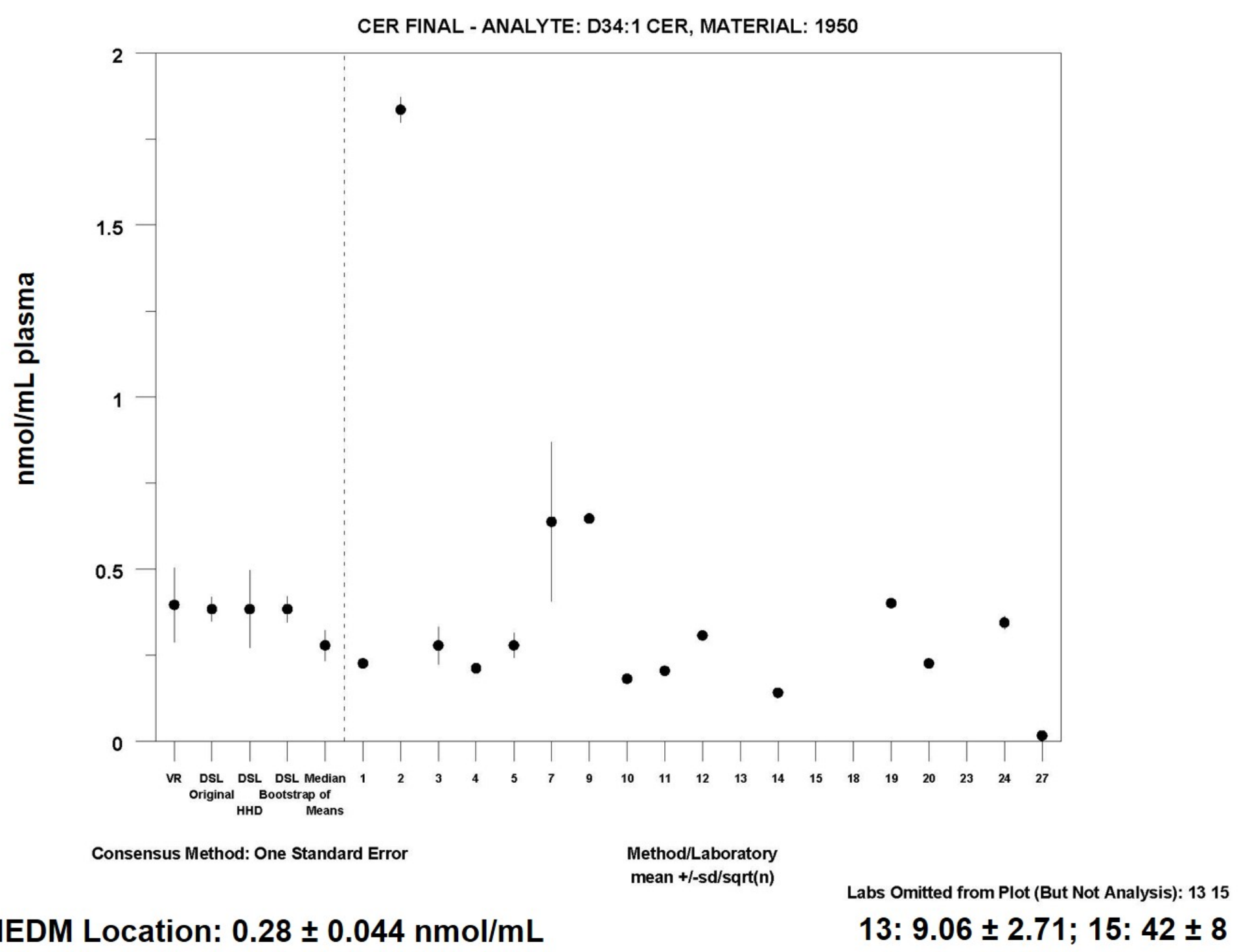




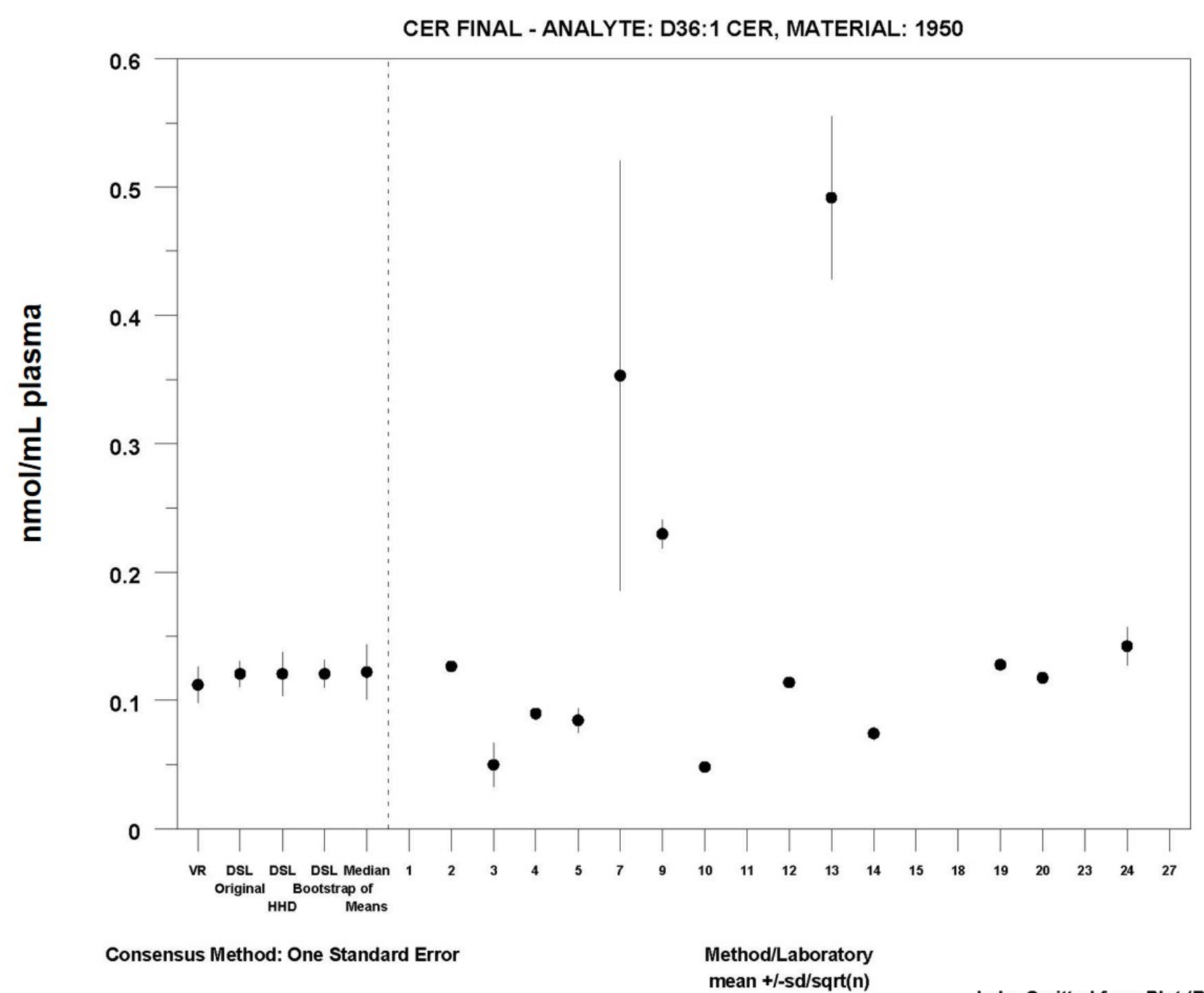

mean $+/$-sd/sqrt(n)

Labs Omitted from Plot (But Not Analysis): 15

MEDM Location: $0.12 \pm 0.021 \mathrm{nmol} / \mathrm{mL}$

15: $16 \pm 1.3$ 


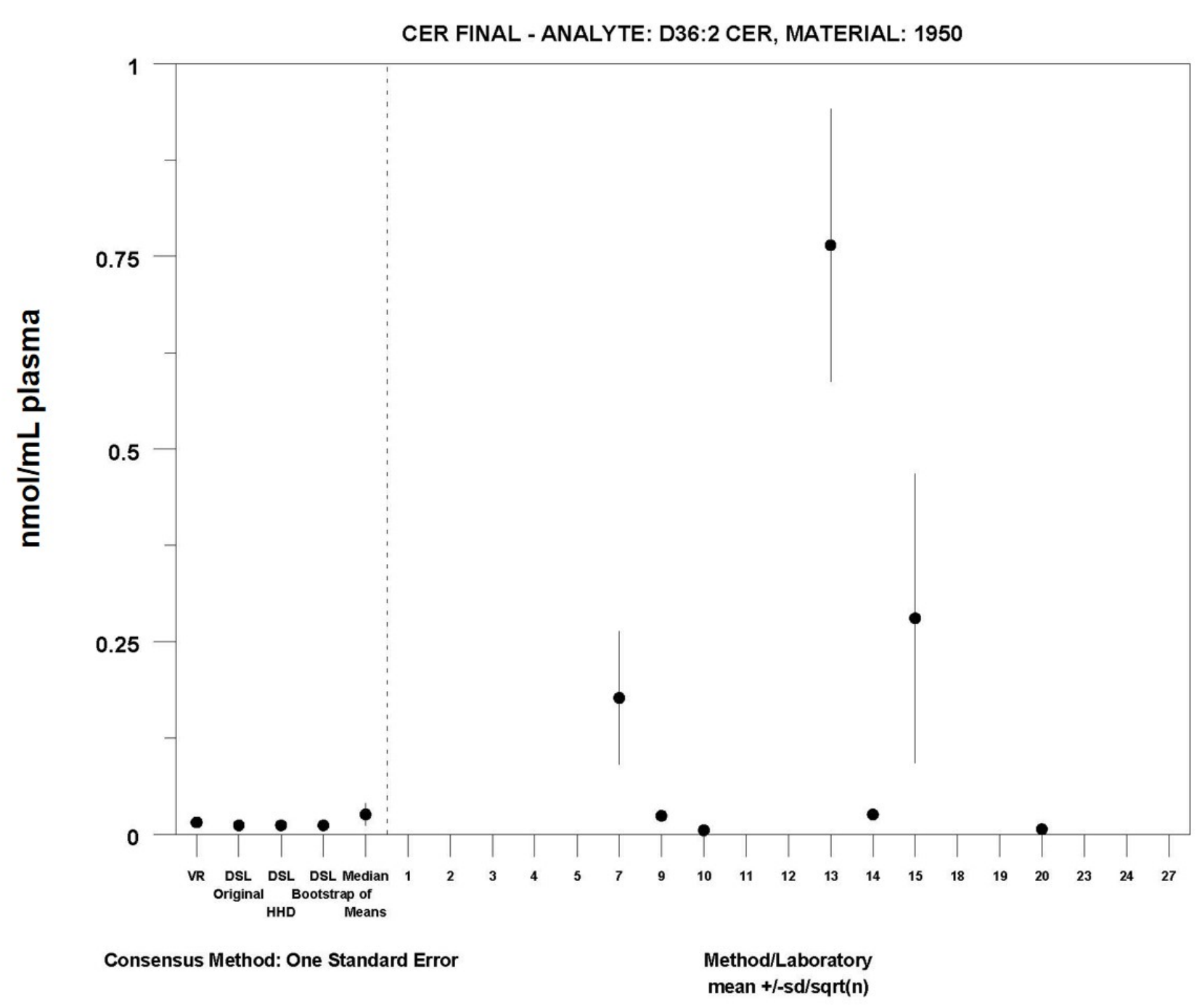

MEDM Location: $0.026 \pm 0.014 \mathrm{nmol} / \mathrm{mL}$ 


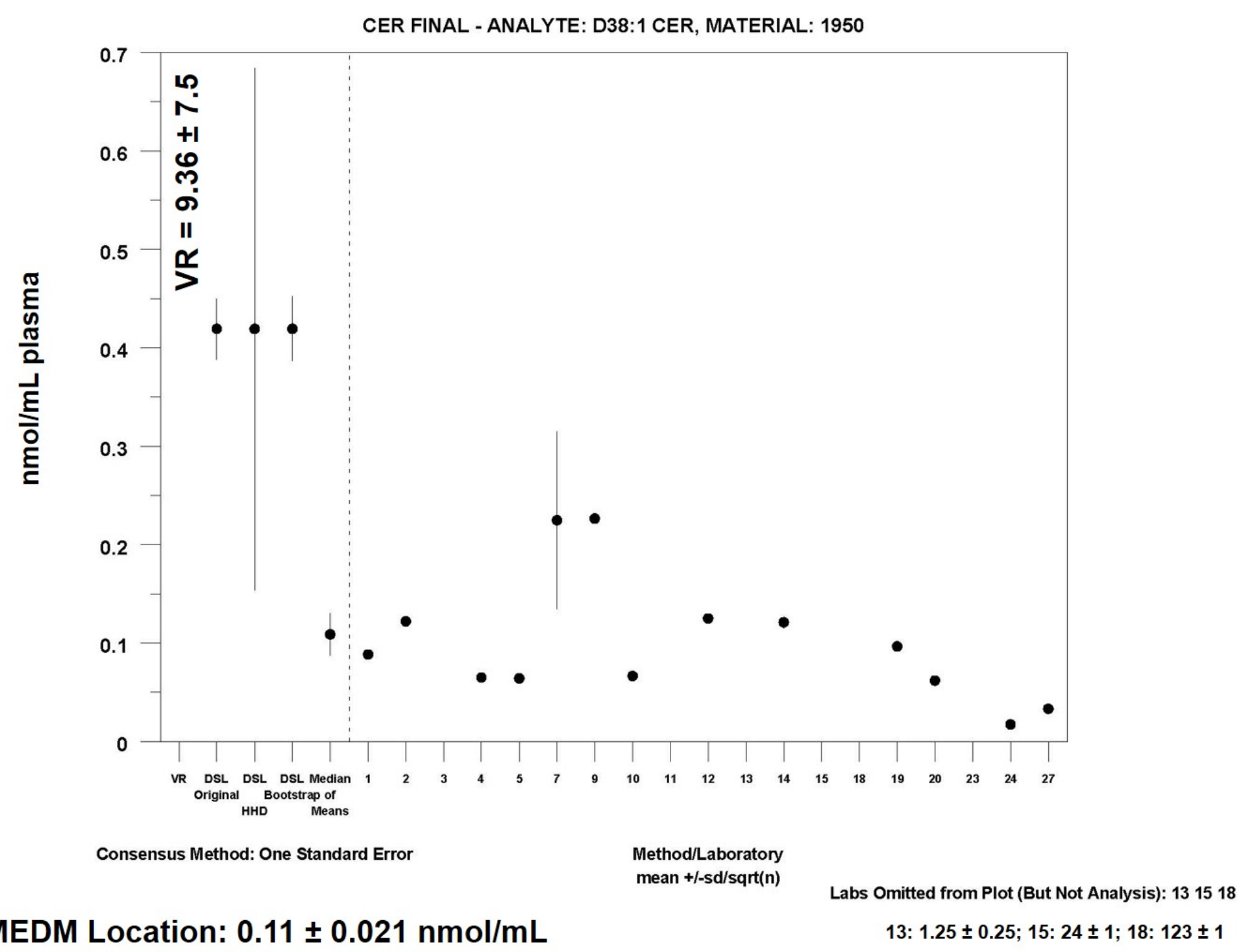




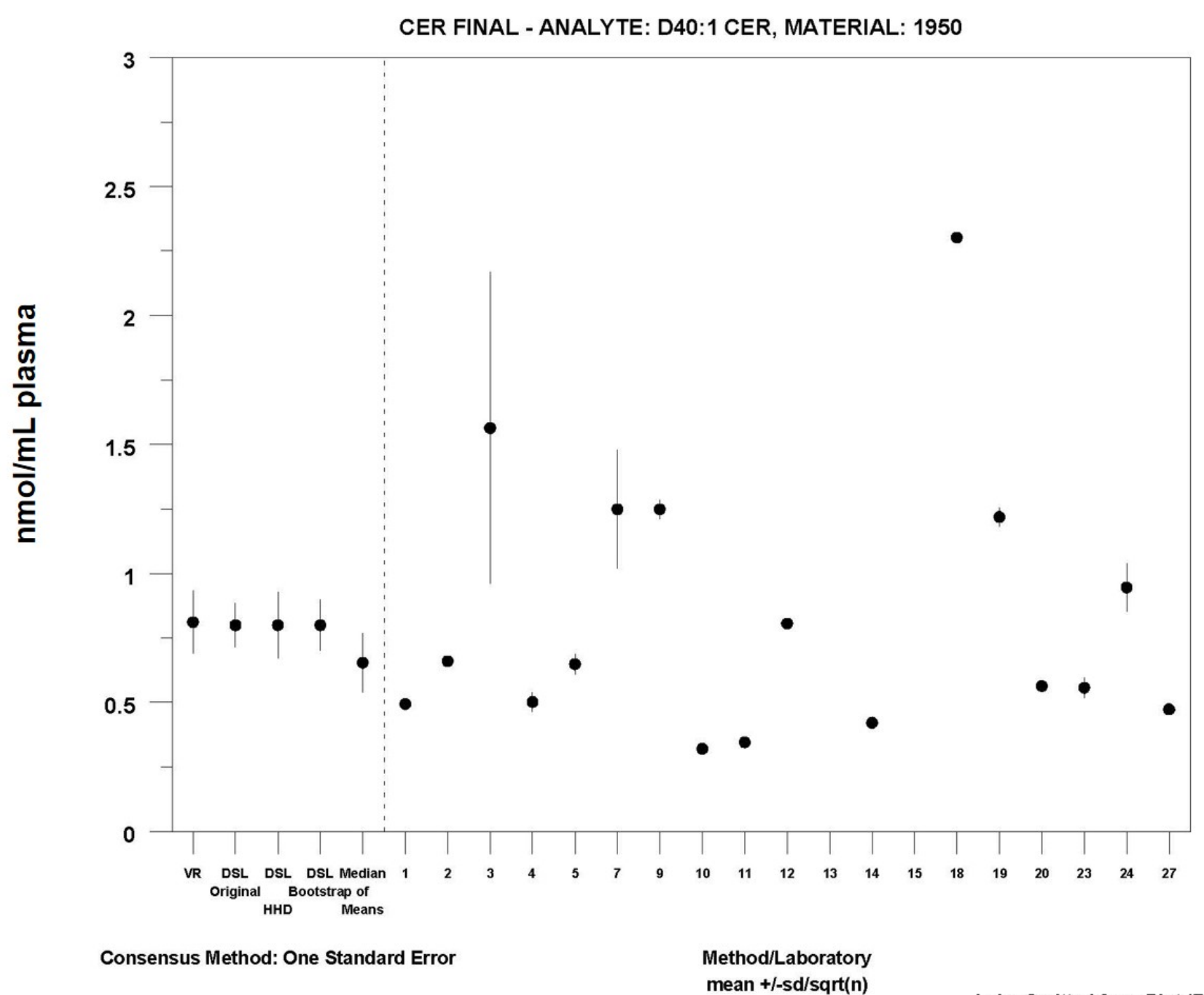

mean + -sd/sqrt(n)

Labs Omitted from Plot (But Not Analysis): 15

MEDM Location: $0.65 \pm 0.12 \mathrm{nmol} / \mathrm{mL}$

15: $188 \pm 100$ 


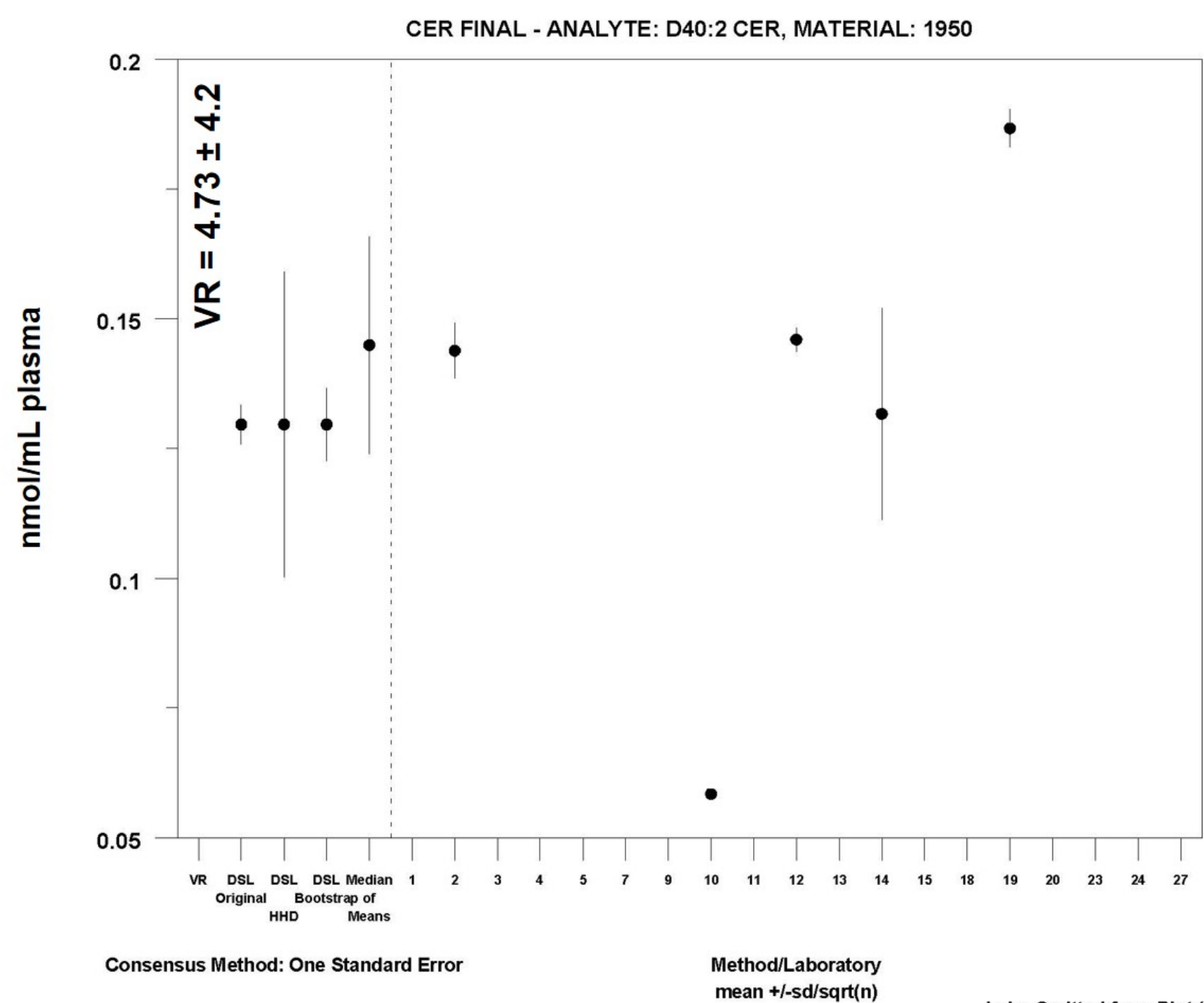

mean + -sd/sqrt(n)

Labs Omitted from Plot (But Not Analysis): 15

MEDM Location: $0.15 \pm 0.021 \mathrm{nmol} / \mathrm{mL}$

15: $28 \pm 1$ 


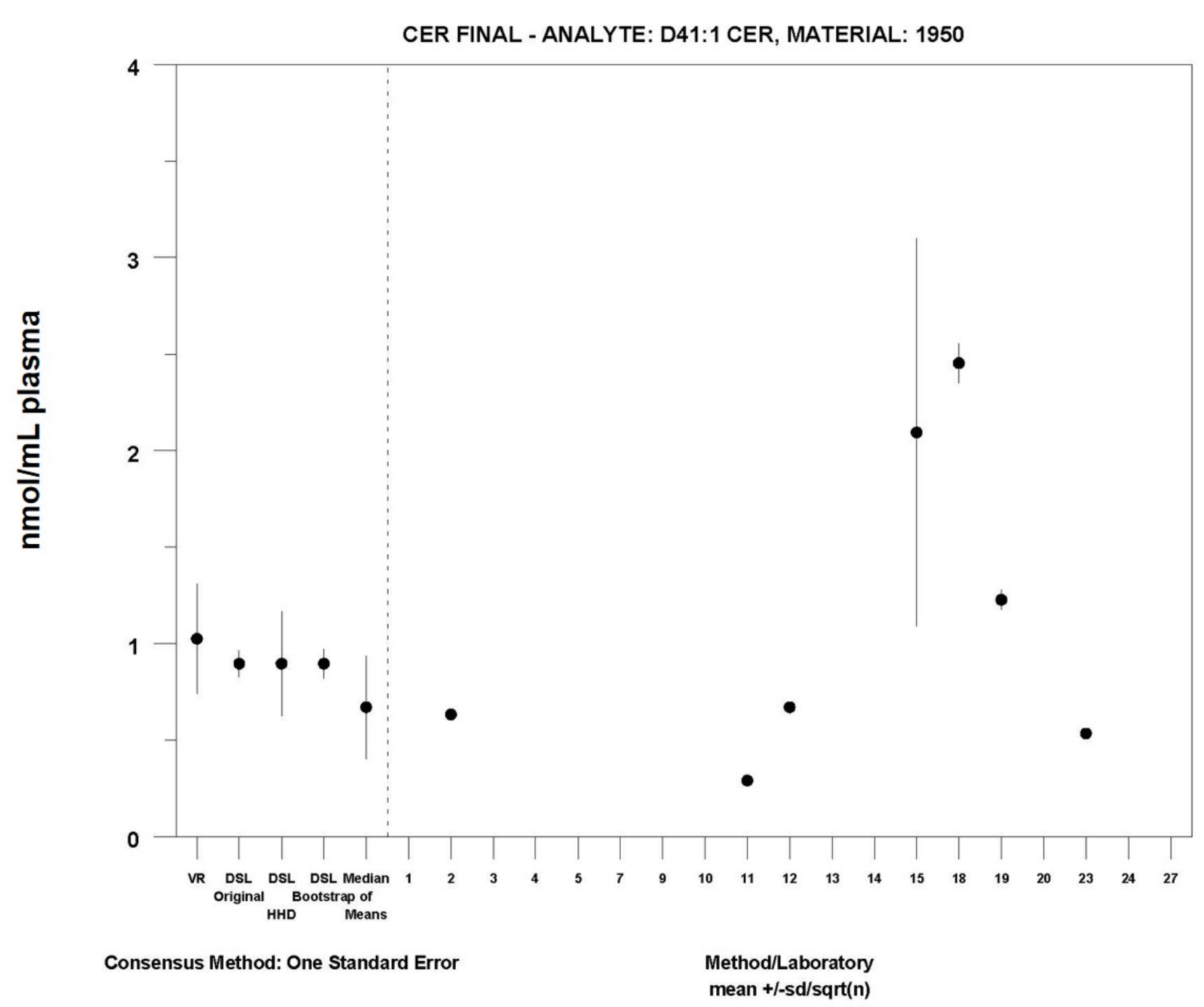

MEDM Location: $0.67 \pm 0.27 \mathrm{nmol} / \mathrm{mL}$ 


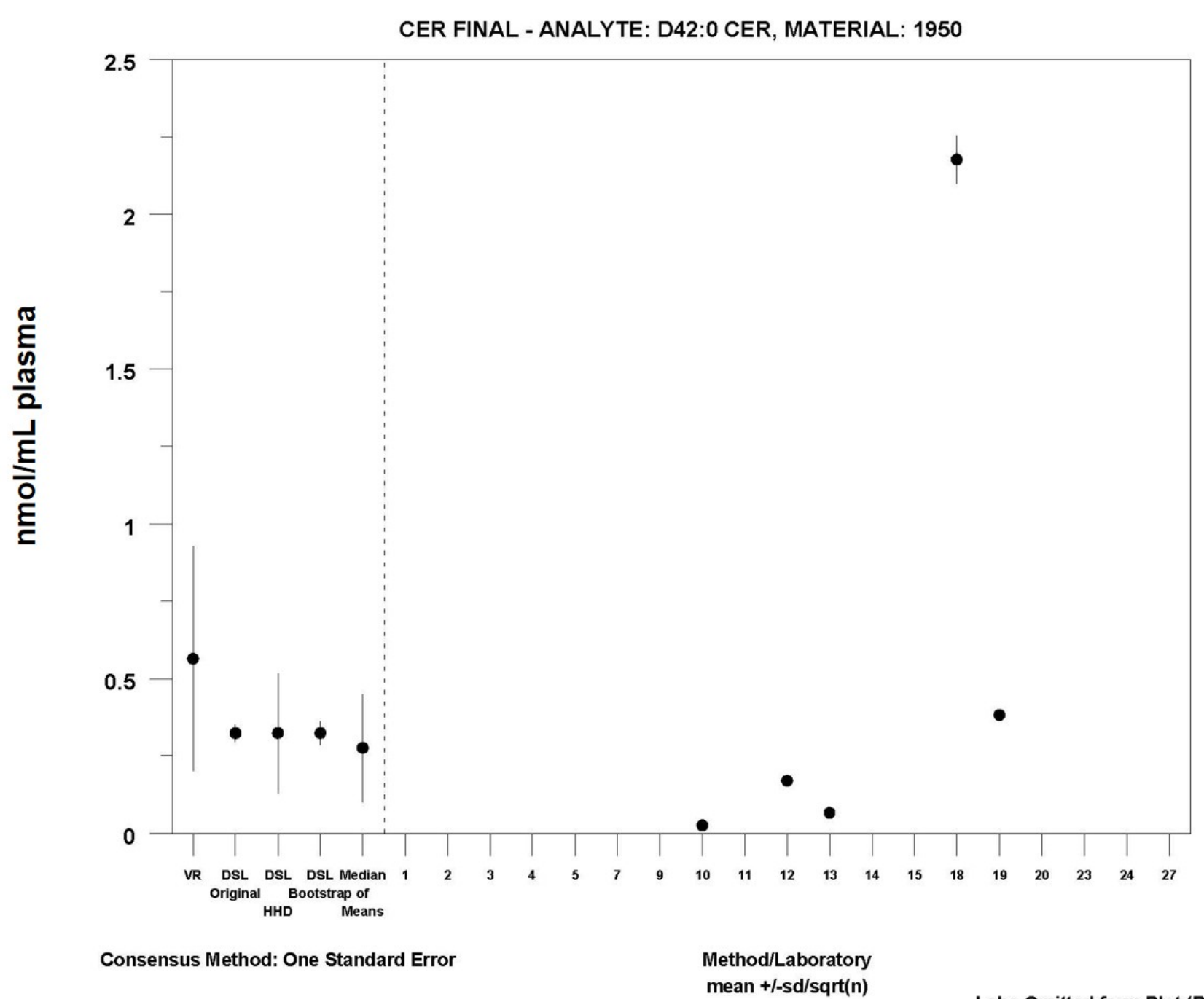

mean +l-sd/sqrt(n)

Labs Omitted from Plot (But Not Analysis): 15

MEDM Location: $0.28 \pm 0.18 \mathrm{nmol} / \mathrm{mL}$

15: $129 \pm 40$ 


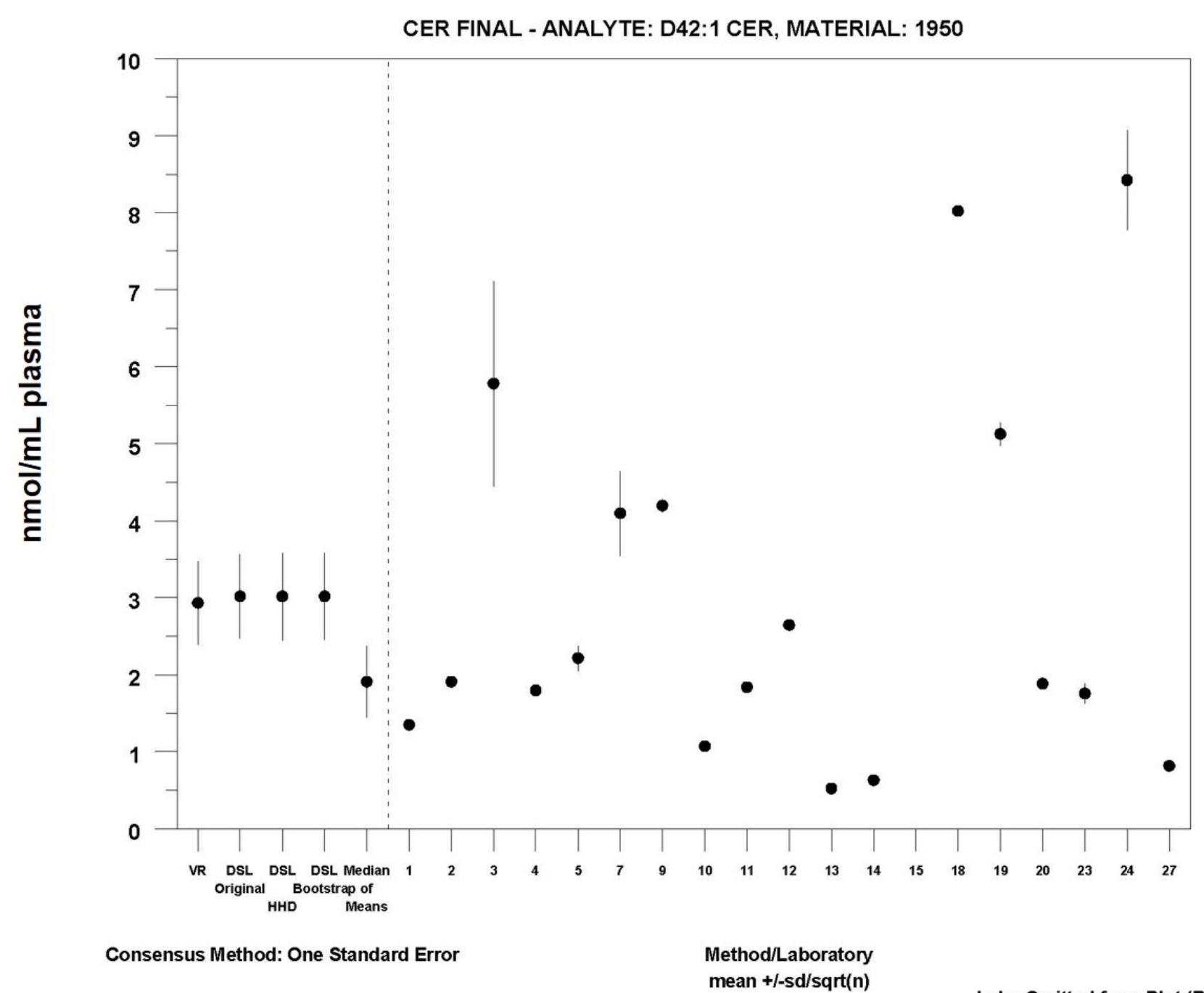

mean +l-sd/sqrt(n)

Labs Omitted from Plot (But Not Analysis): 15

MEDM Location: $1.9 \pm 0.47 \mathrm{nmol} / \mathrm{mL}$

15: $844 \pm 98$ 


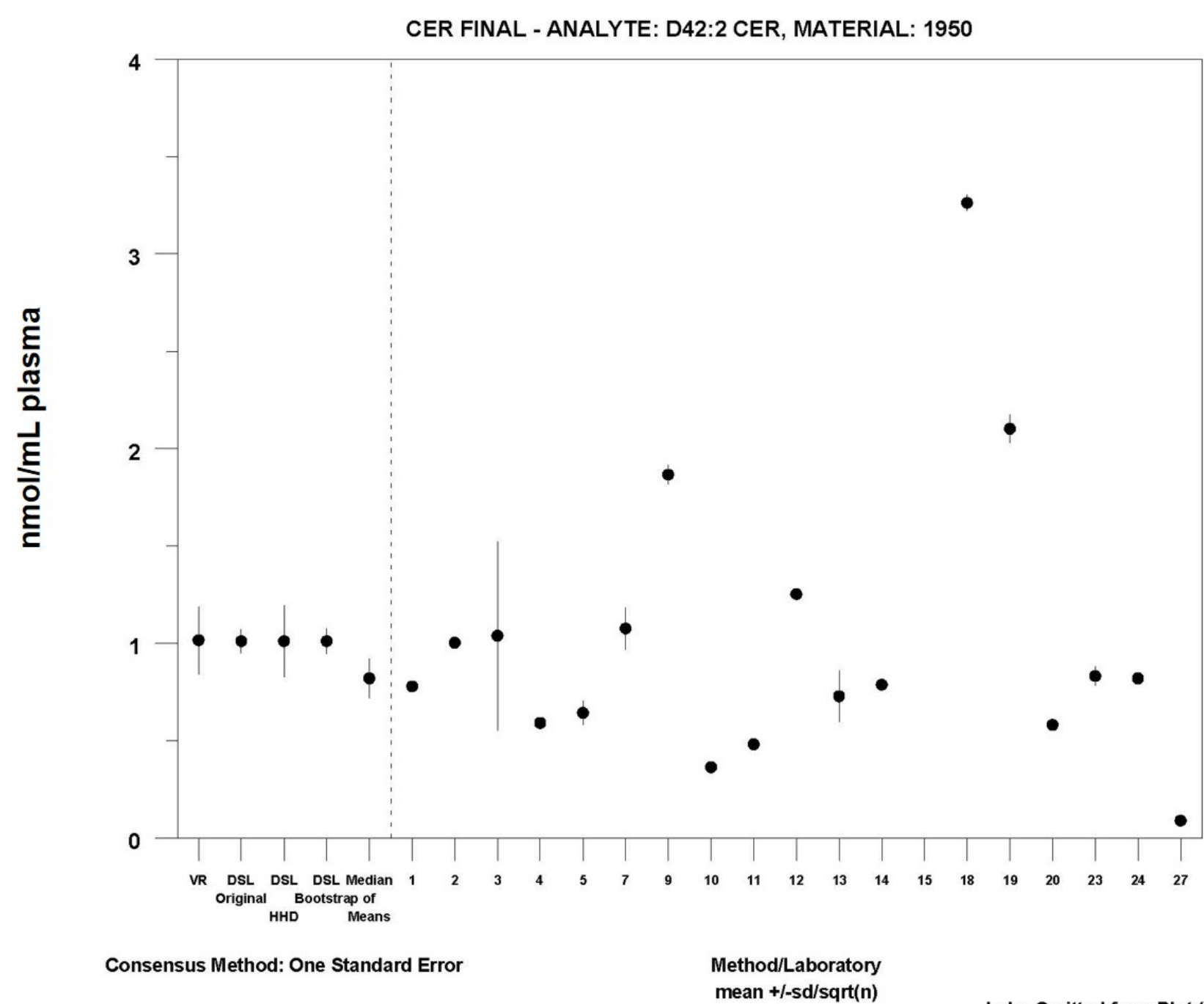

MEDM Location: $0.82 \pm 0.10 \mathrm{nmol} / \mathrm{mL}$

Labs Omitted from Plot (But Not Analysis): 15

15: $172 \pm 87$ 


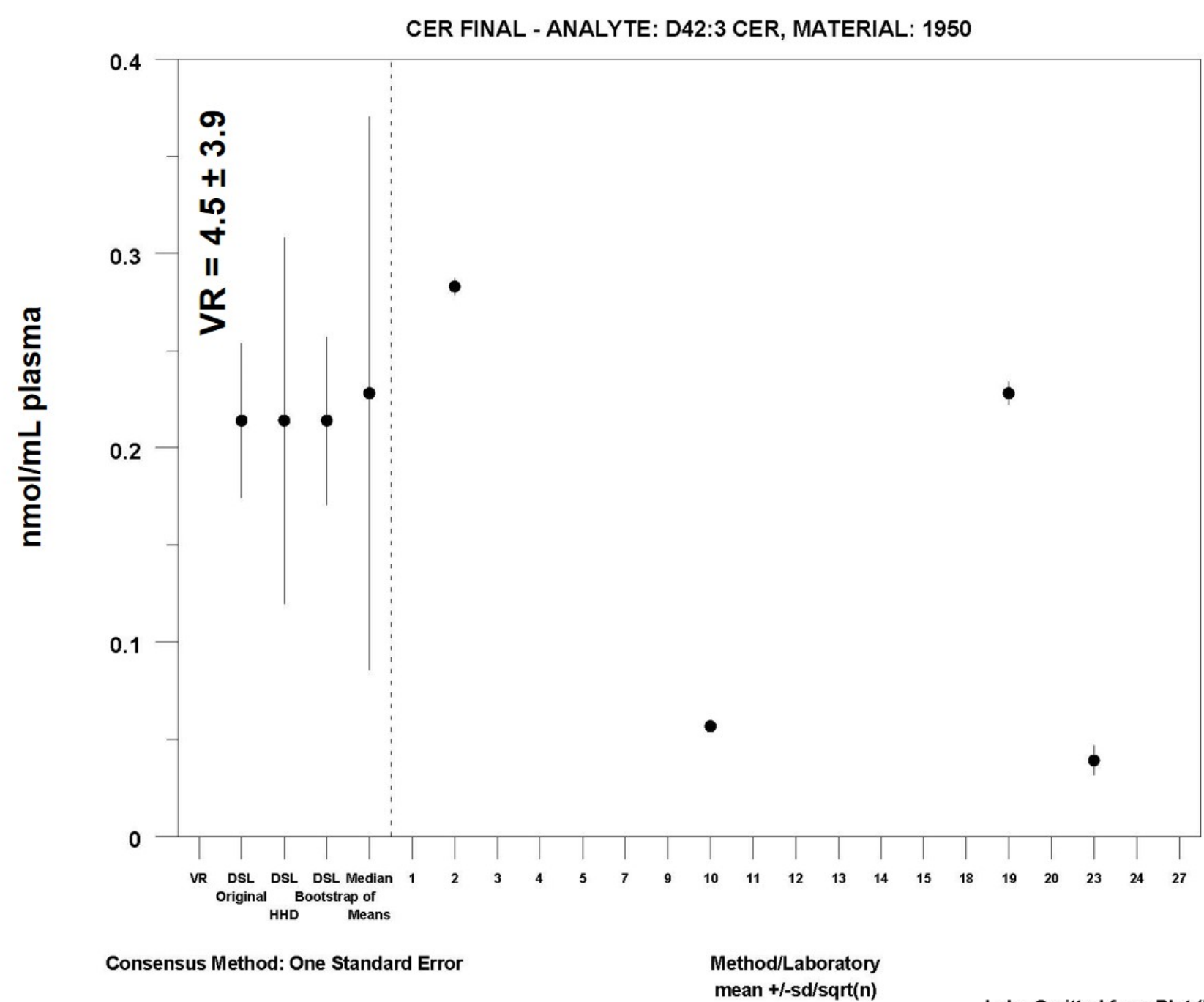

mean + -sd/sqrt(n)

Labs Omitted from Plot (But Not Analysis): 15

MEDM Location: $0.23 \pm 0.14 \mathrm{nmol} / \mathrm{mL}$

15: $22 \pm 1$ 


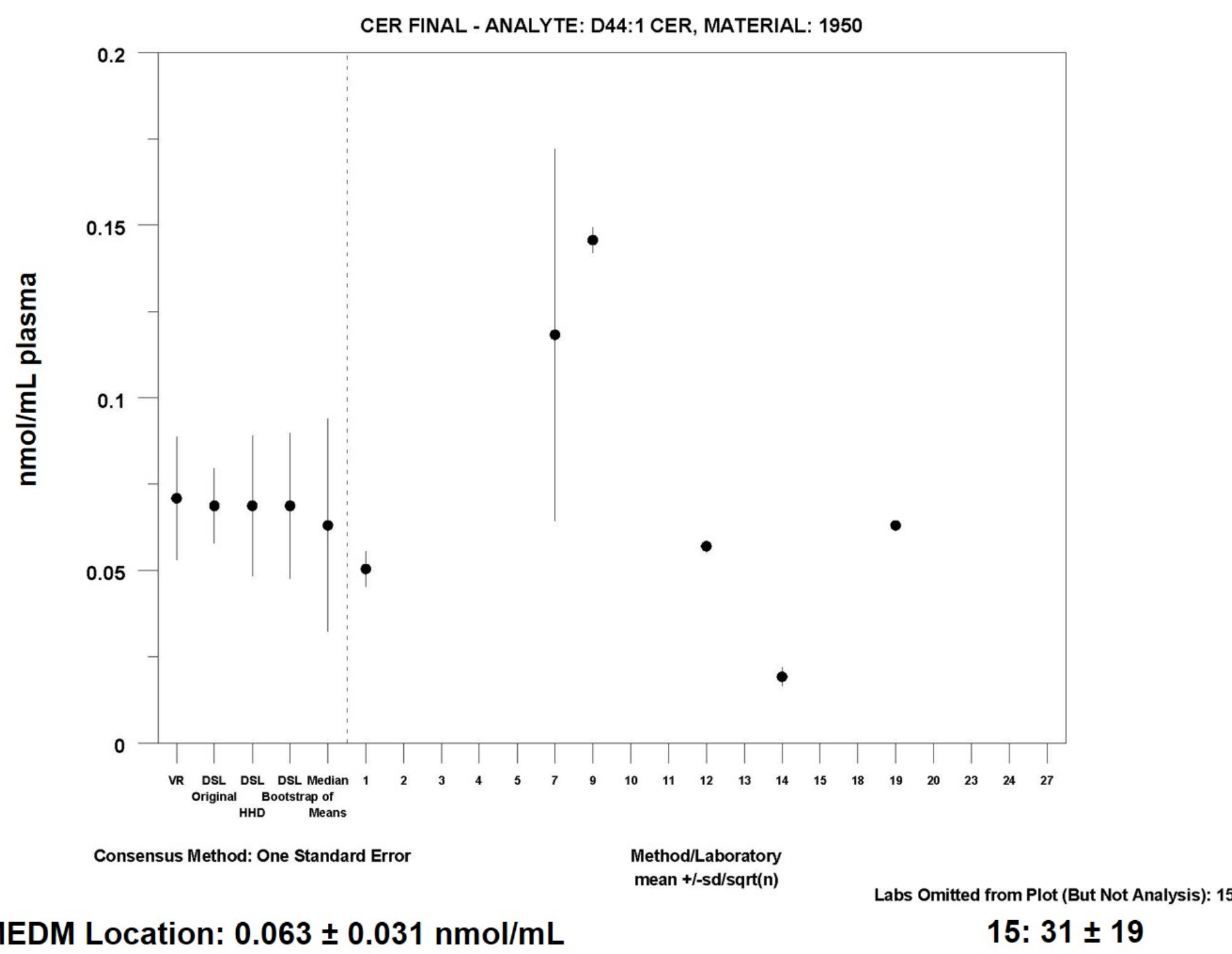




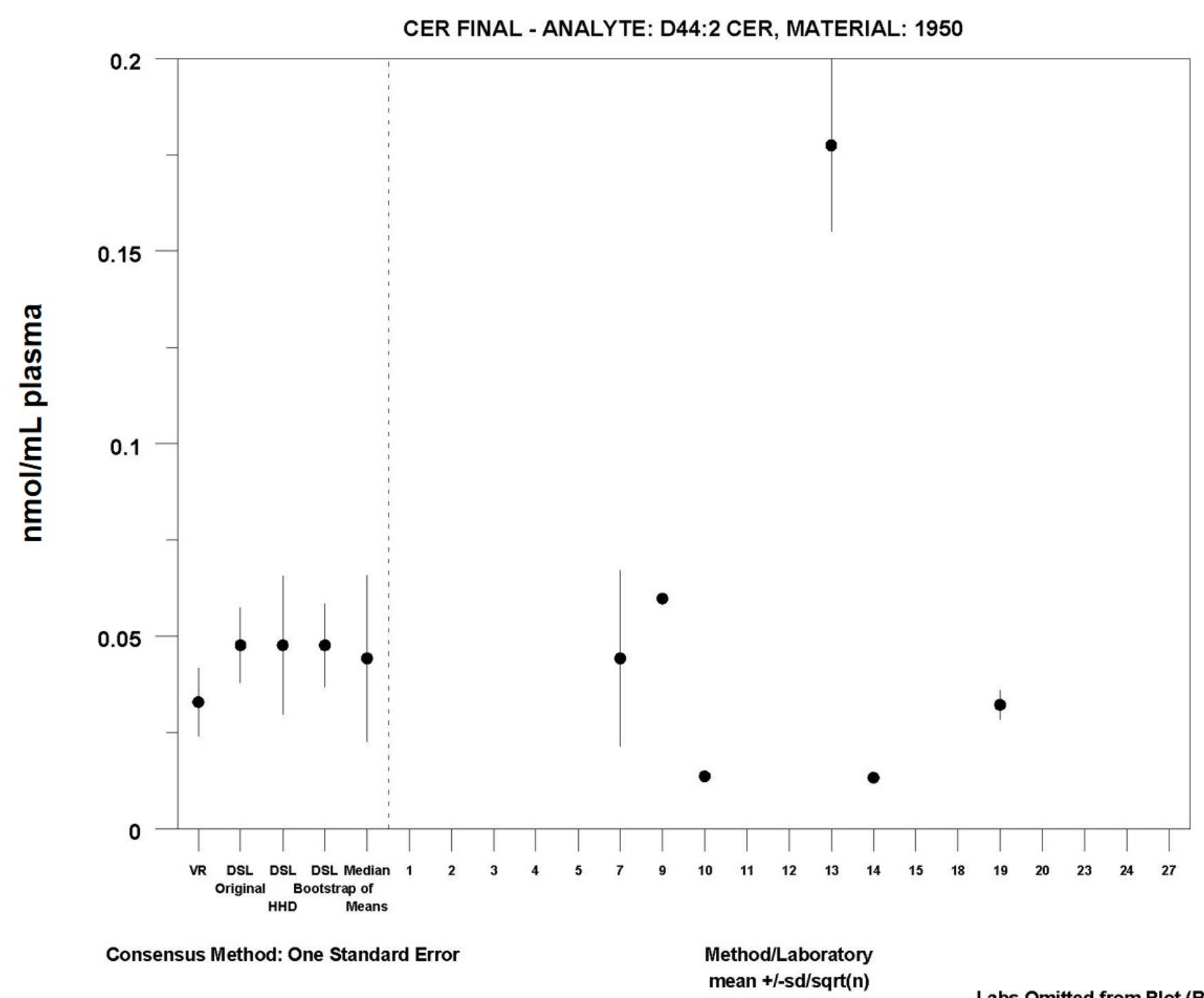

MEDM Location: $0.044 \pm 0.022 \mathrm{nmol} / \mathrm{mL}$

Labs Omitted from Plot (But Not Analysis): 15

15: $2.6 \pm 0.6$ 


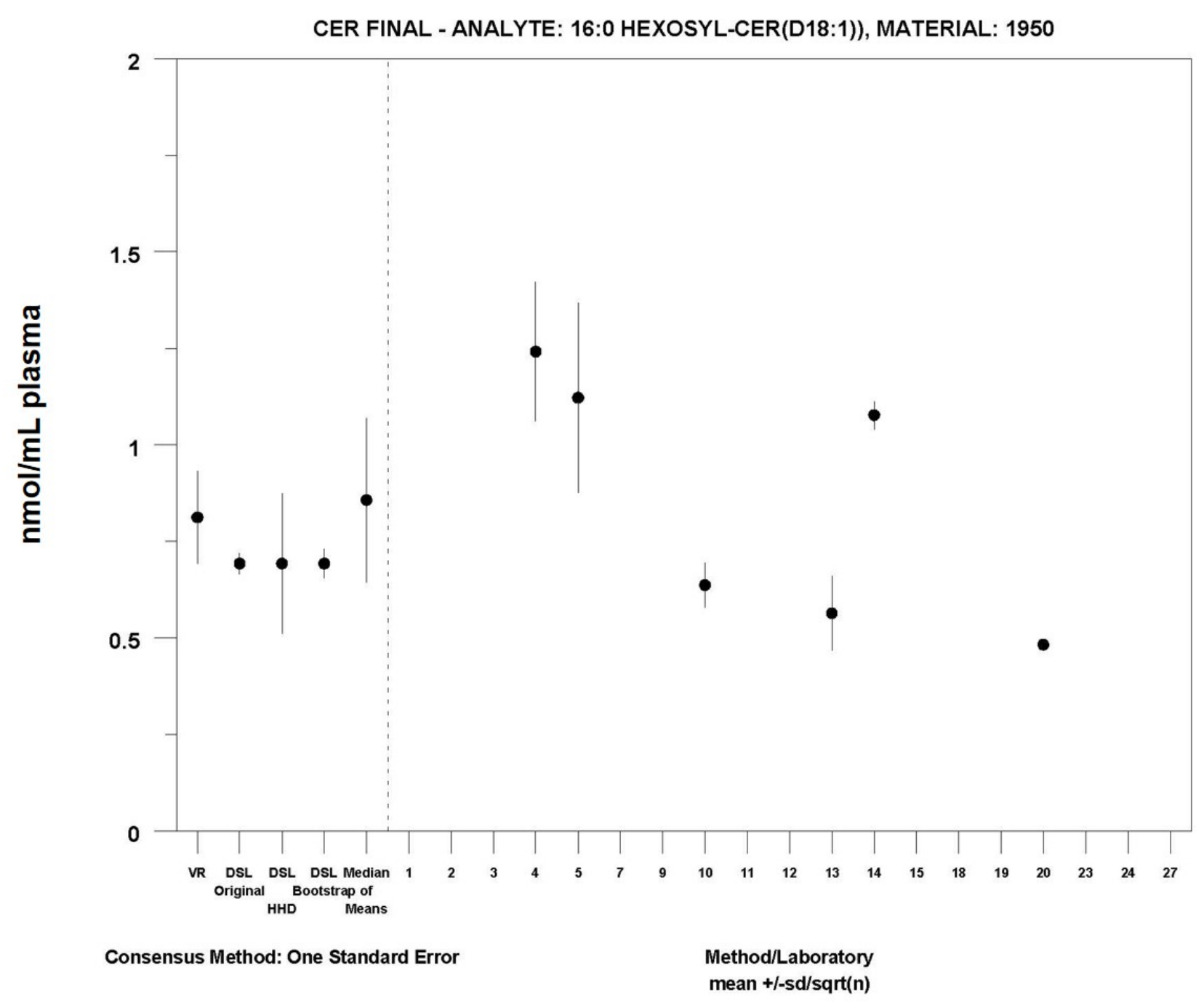

MEDM Location: $0.86 \pm 0.21 \mathrm{nmol} / \mathrm{mL}$ 


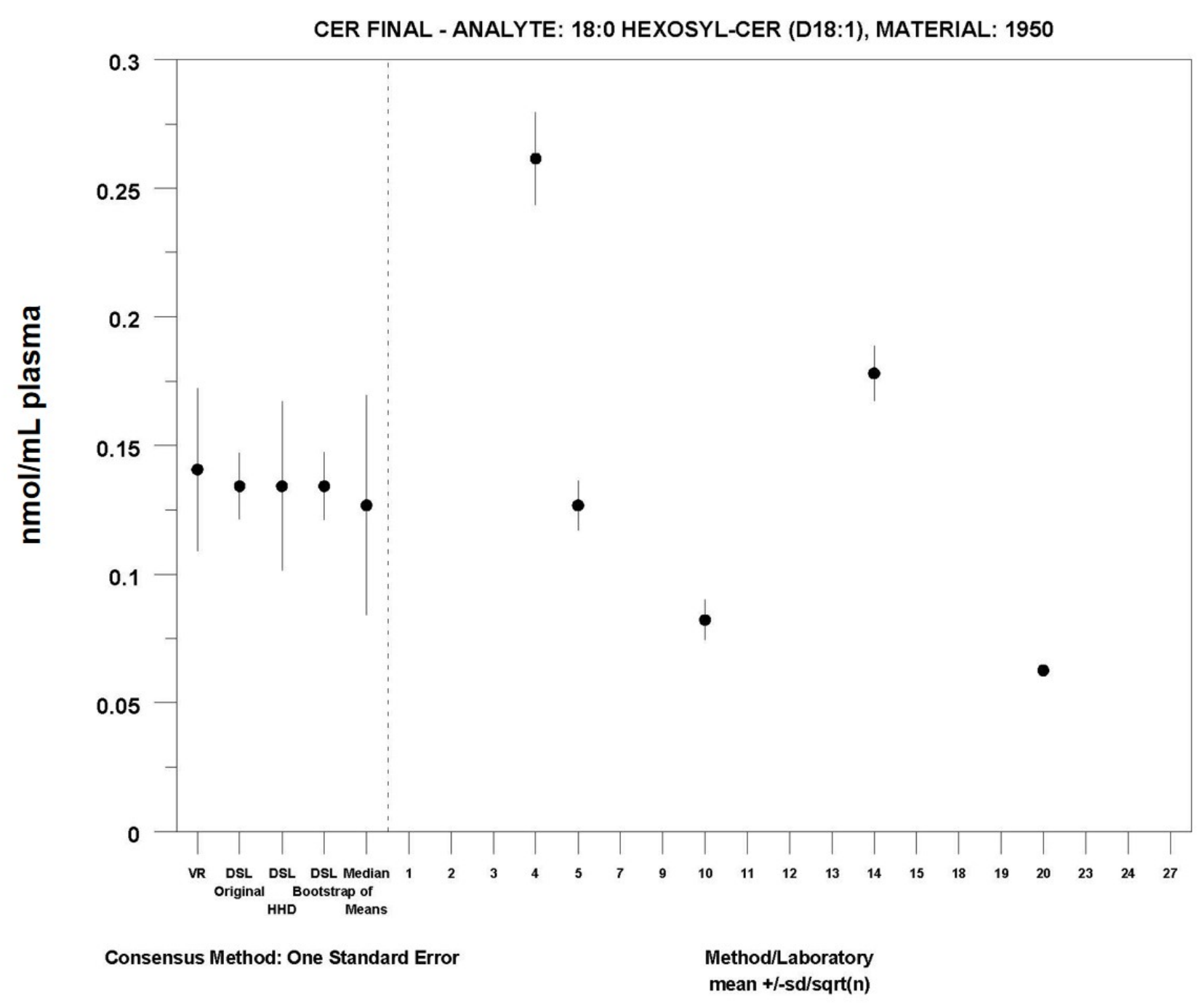

MEDM Location: $0.13 \pm 0.043 \mathrm{nmol} / \mathrm{mL}$ 


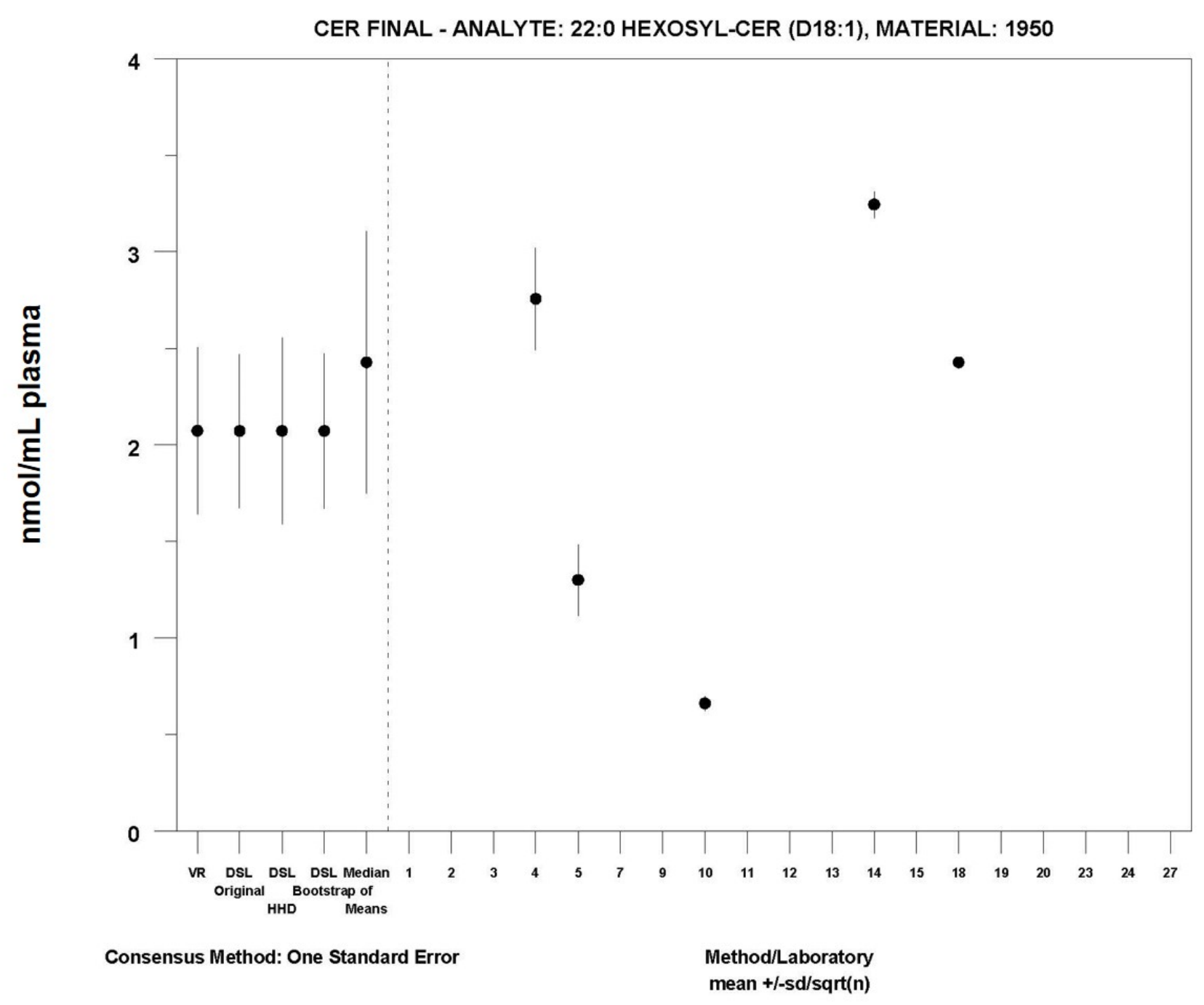

MEDM Location: $2.4 \pm 0.68 \mathrm{nmol} / \mathrm{mL}$ 


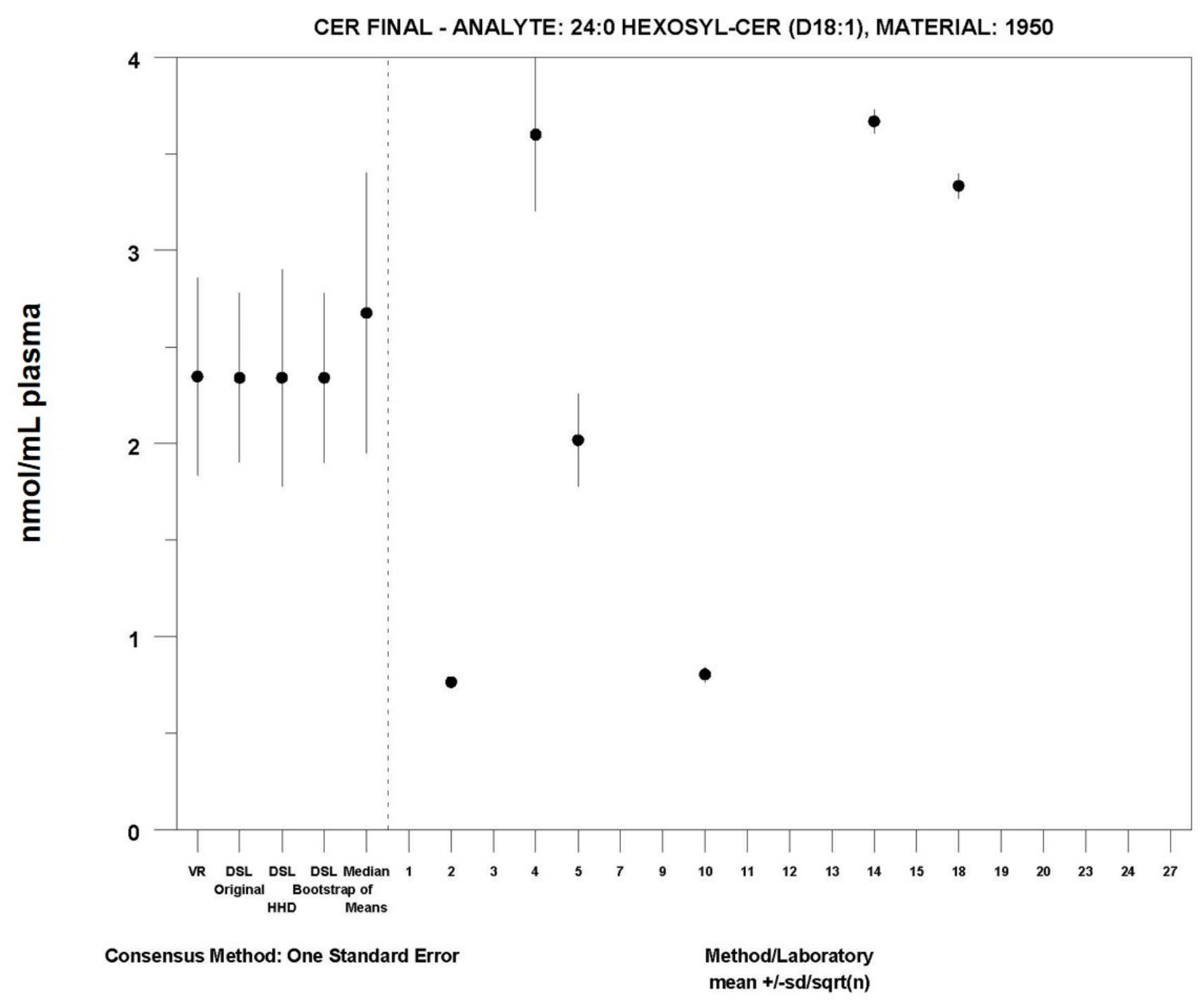

MEDM Location: $2.7 \pm 0.73 \mathrm{nmol} / \mathrm{mL}$ 


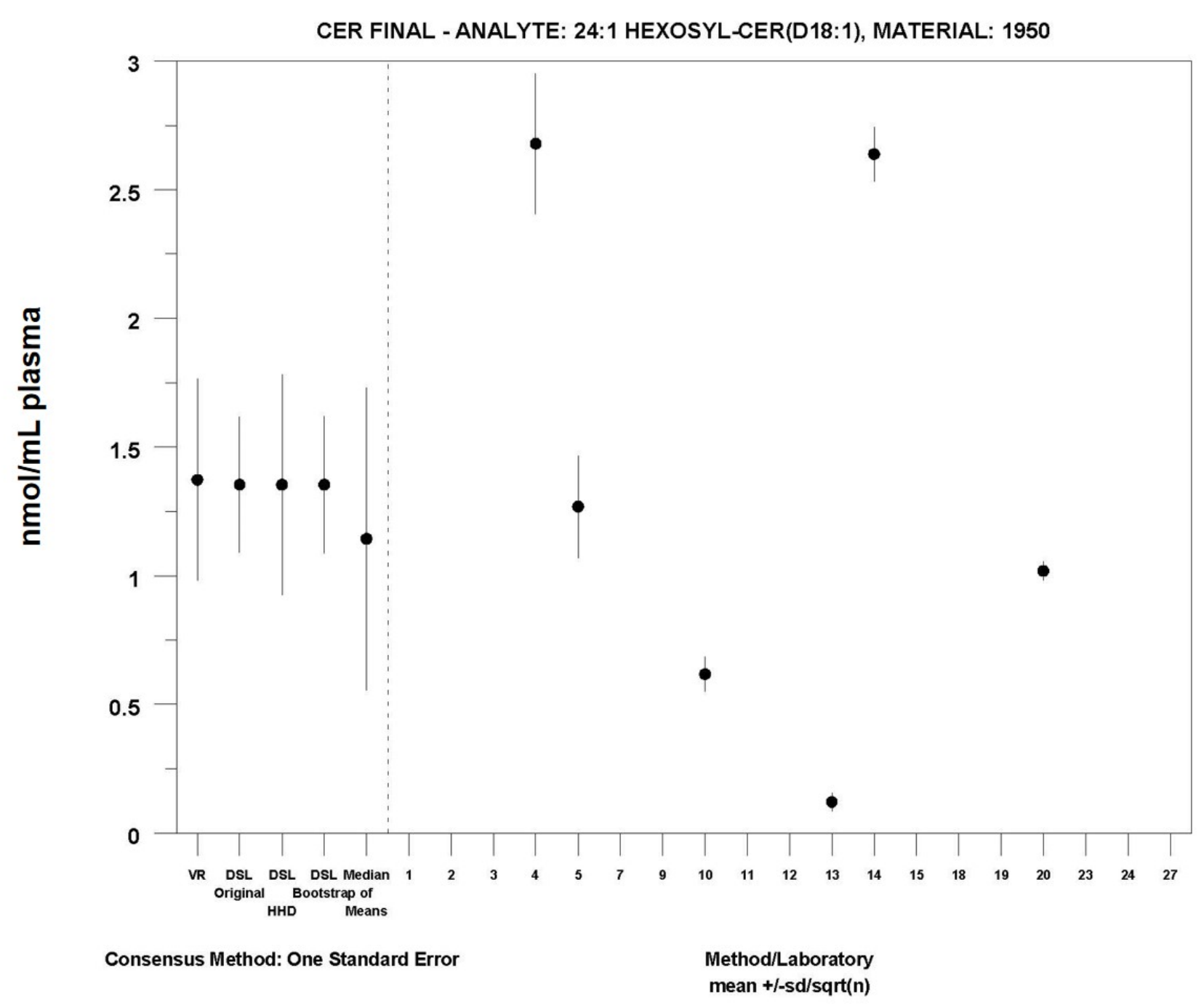

MEDM Location: $1.14 \pm 1.17 \mathrm{nmol} / \mathrm{mL}$ 


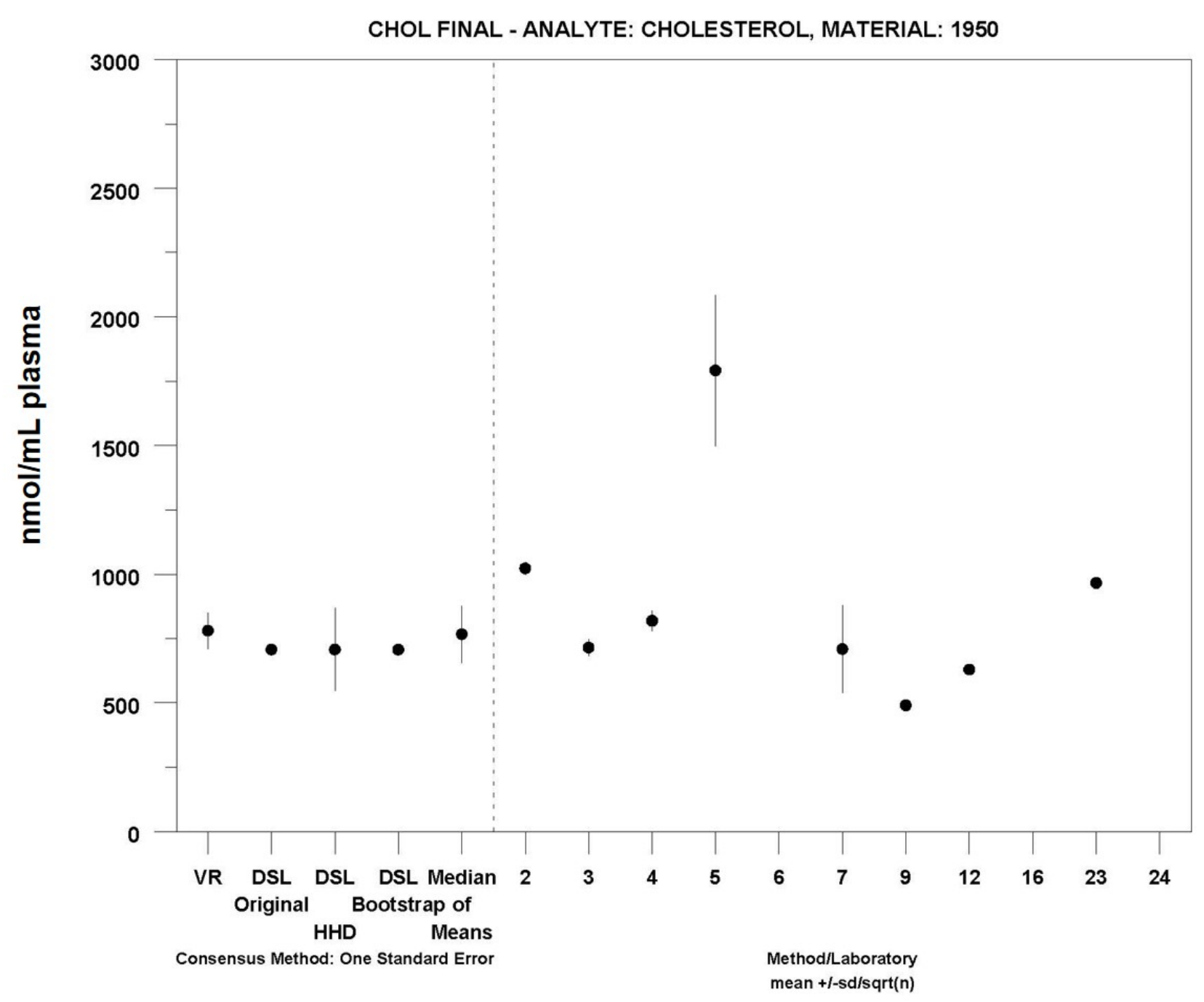

MEDM Location: $770 \pm 110 \mathrm{nmol} / \mathrm{mL}$ 


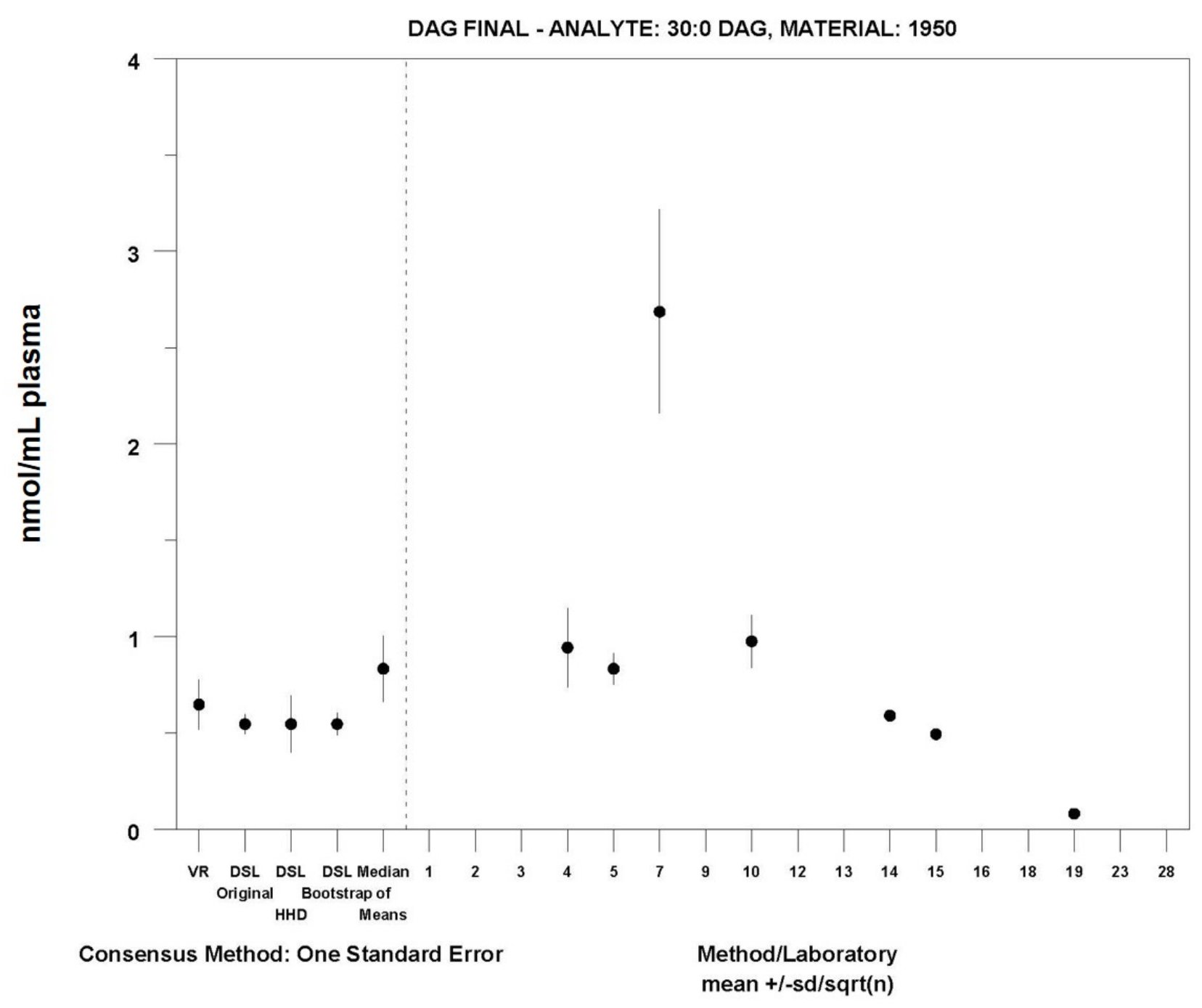

MEDM Location: $0.83 \pm 0.17 \mathrm{nmol} / \mathrm{mL}$ 


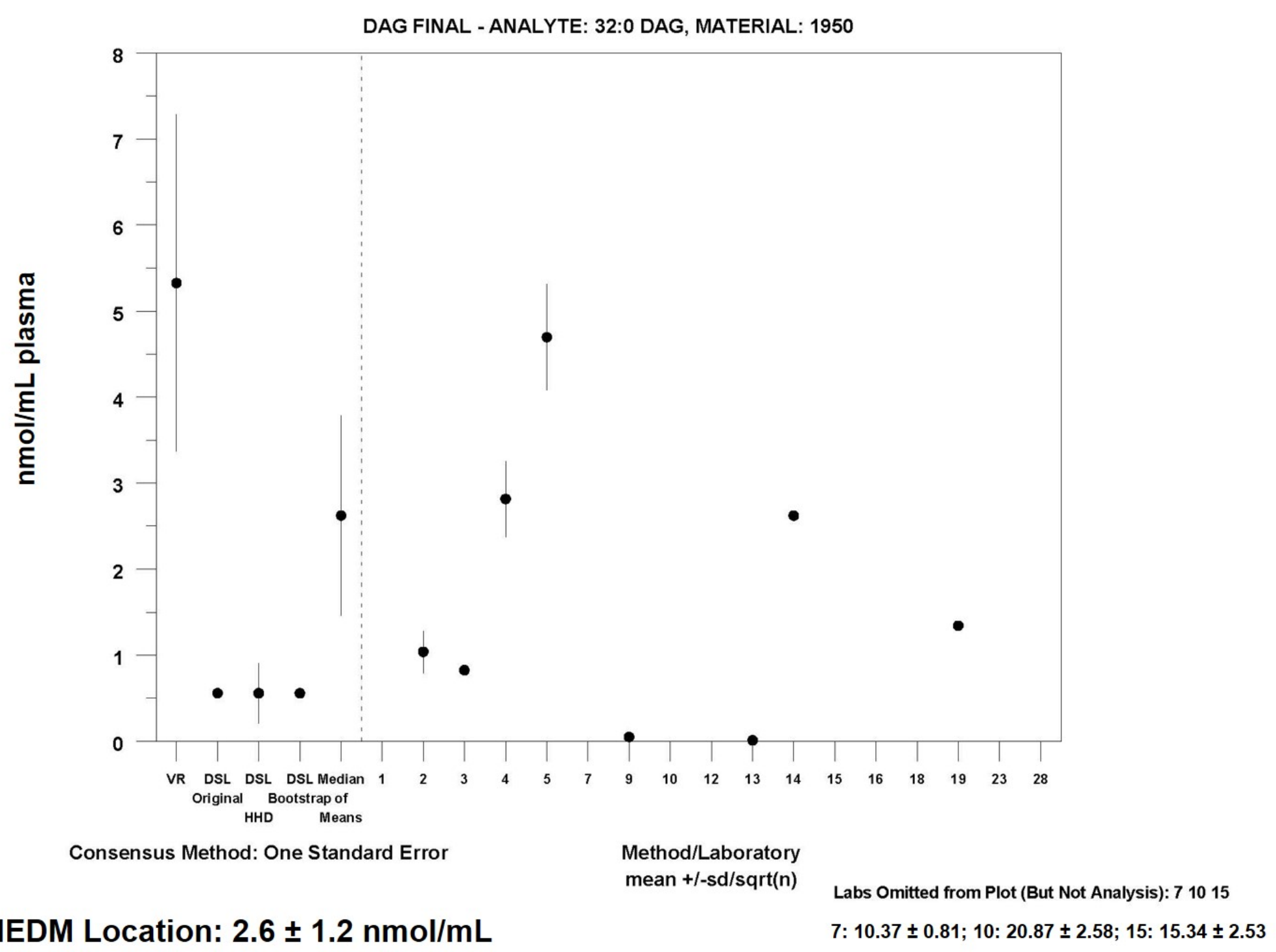




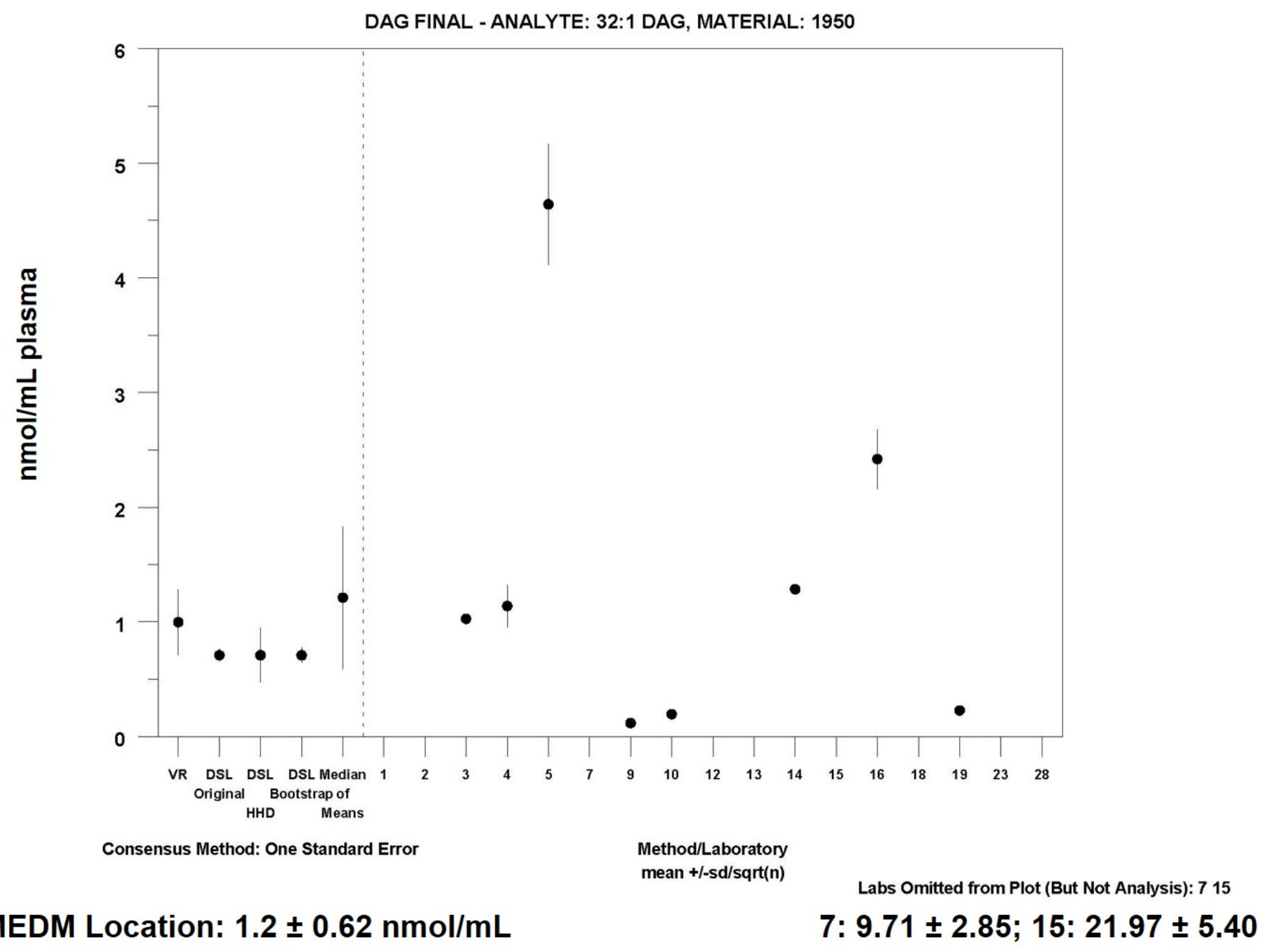




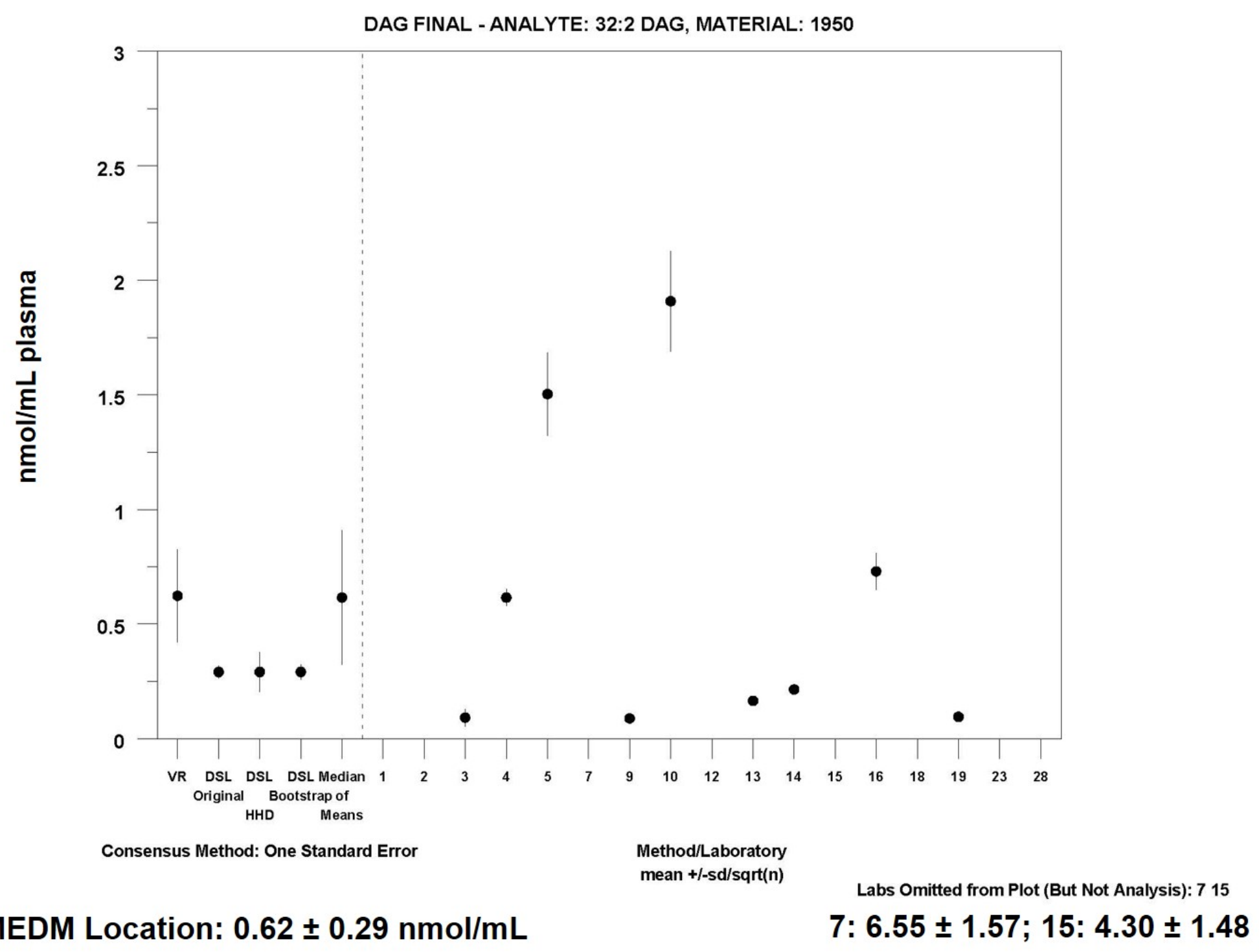




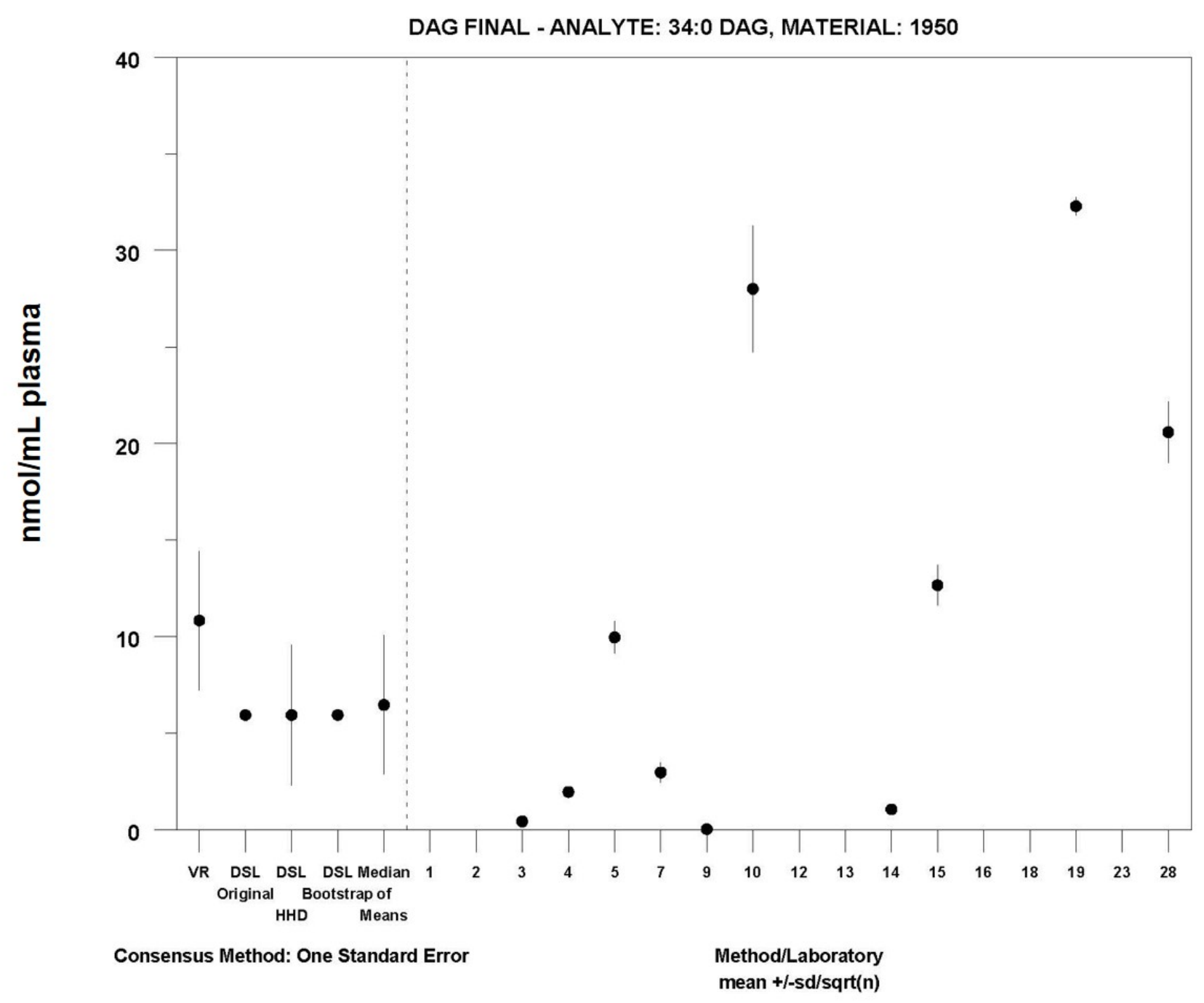

MEDM Location: $6.5 \pm 3.6 \mathrm{nmol} / \mathrm{mL}$ 


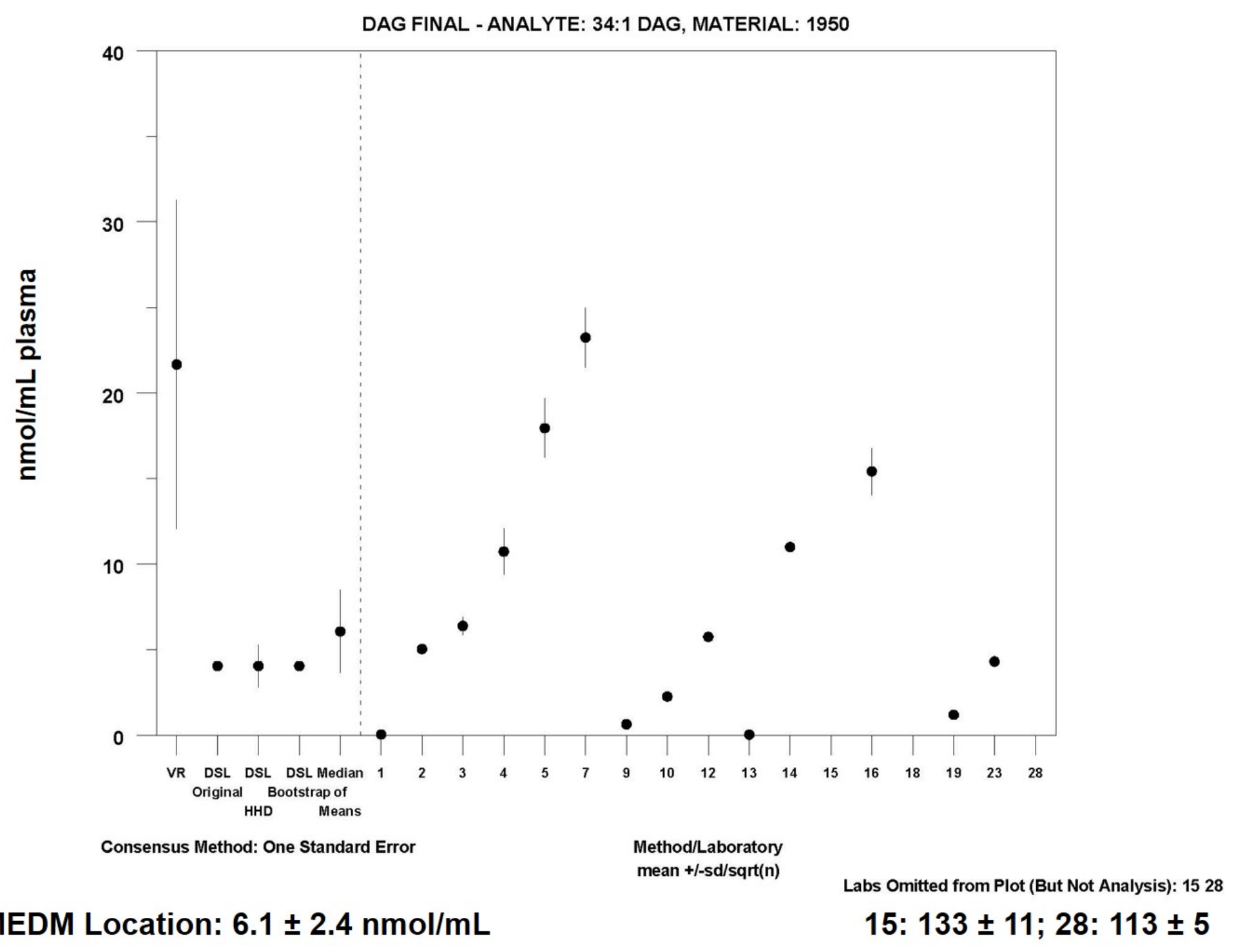




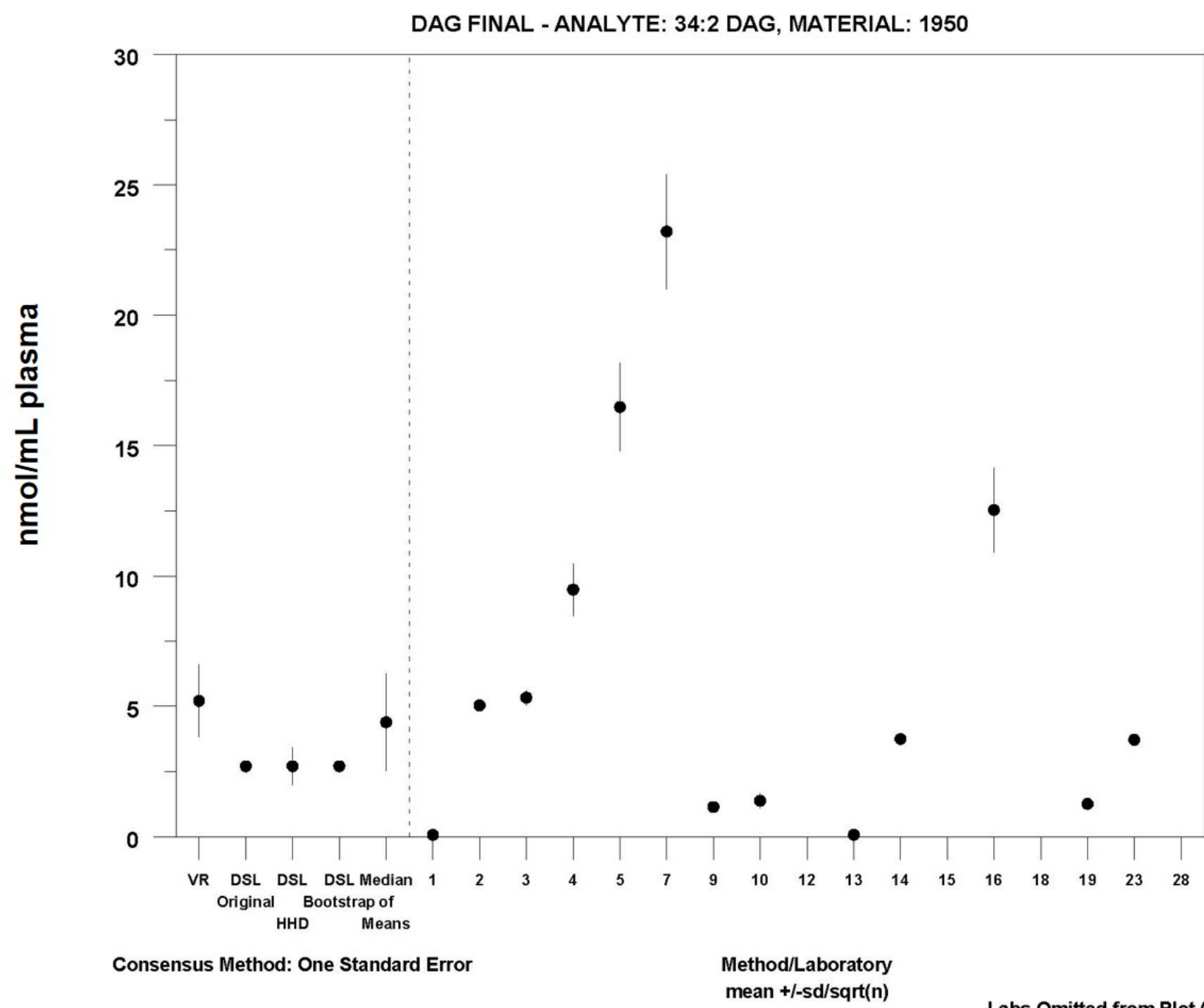

MEDM Location: $4.4 \pm 1.9 \mathrm{nmol} / \mathrm{mL}$

15: $126 \pm 104$ 


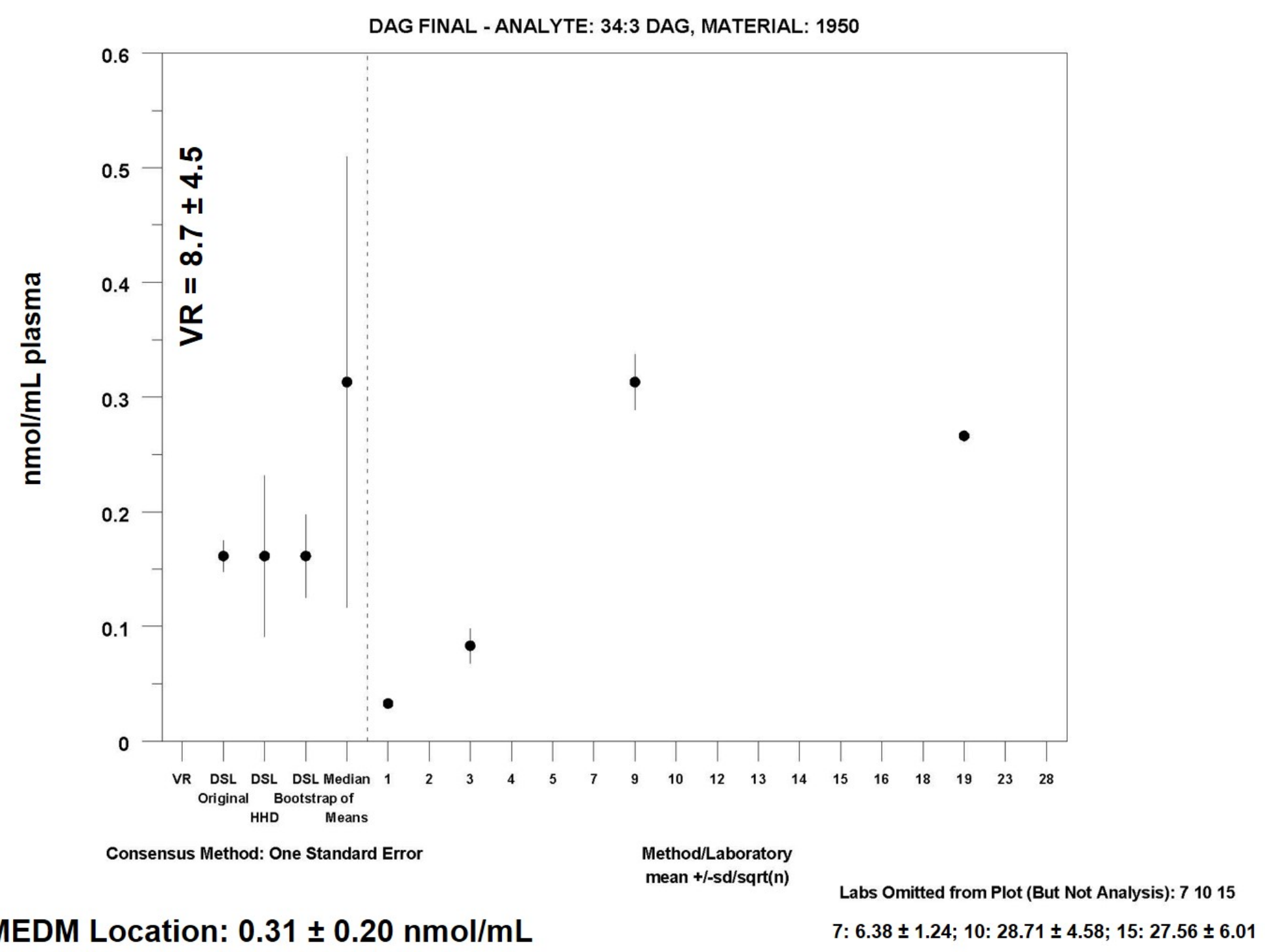




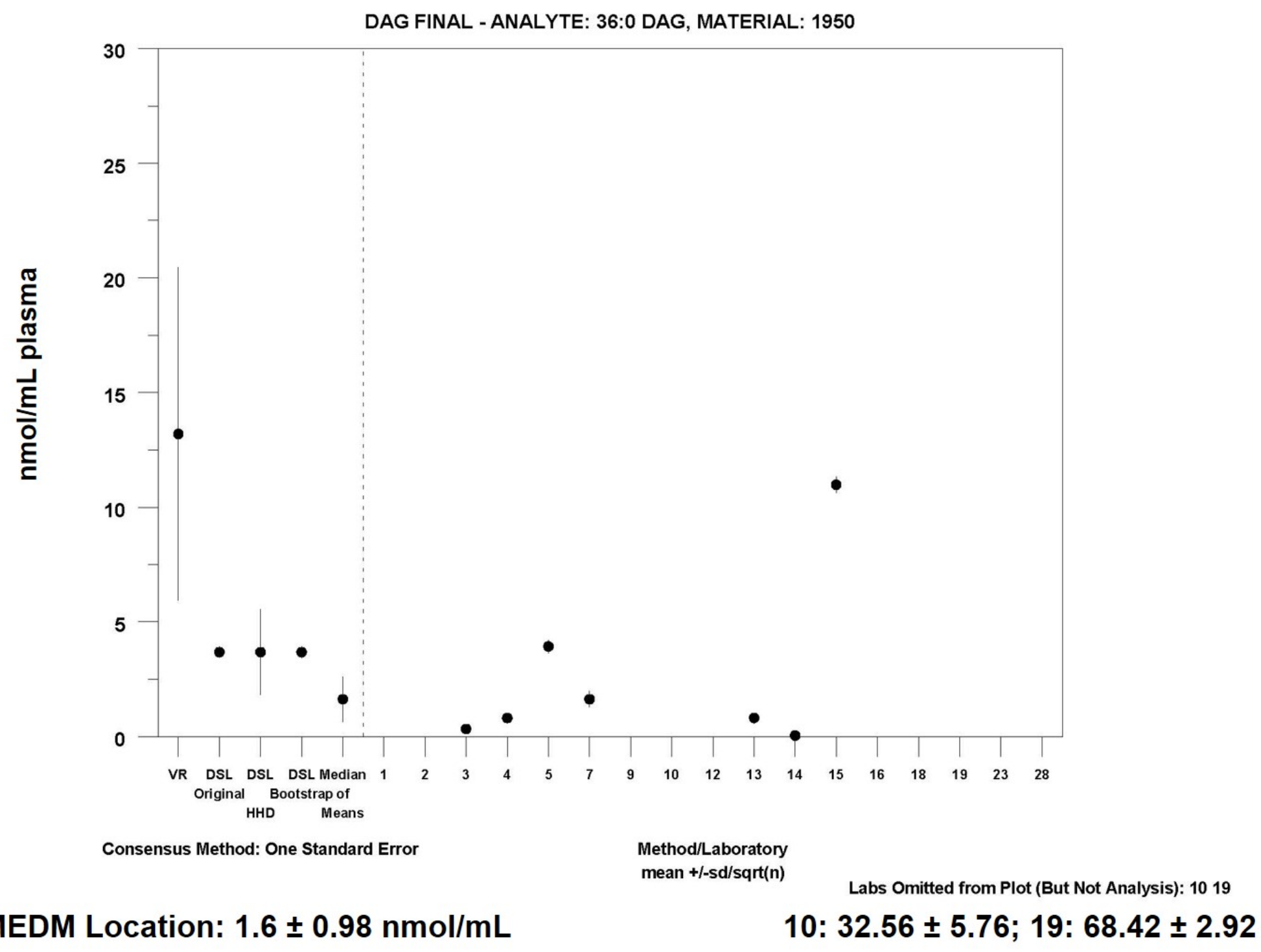




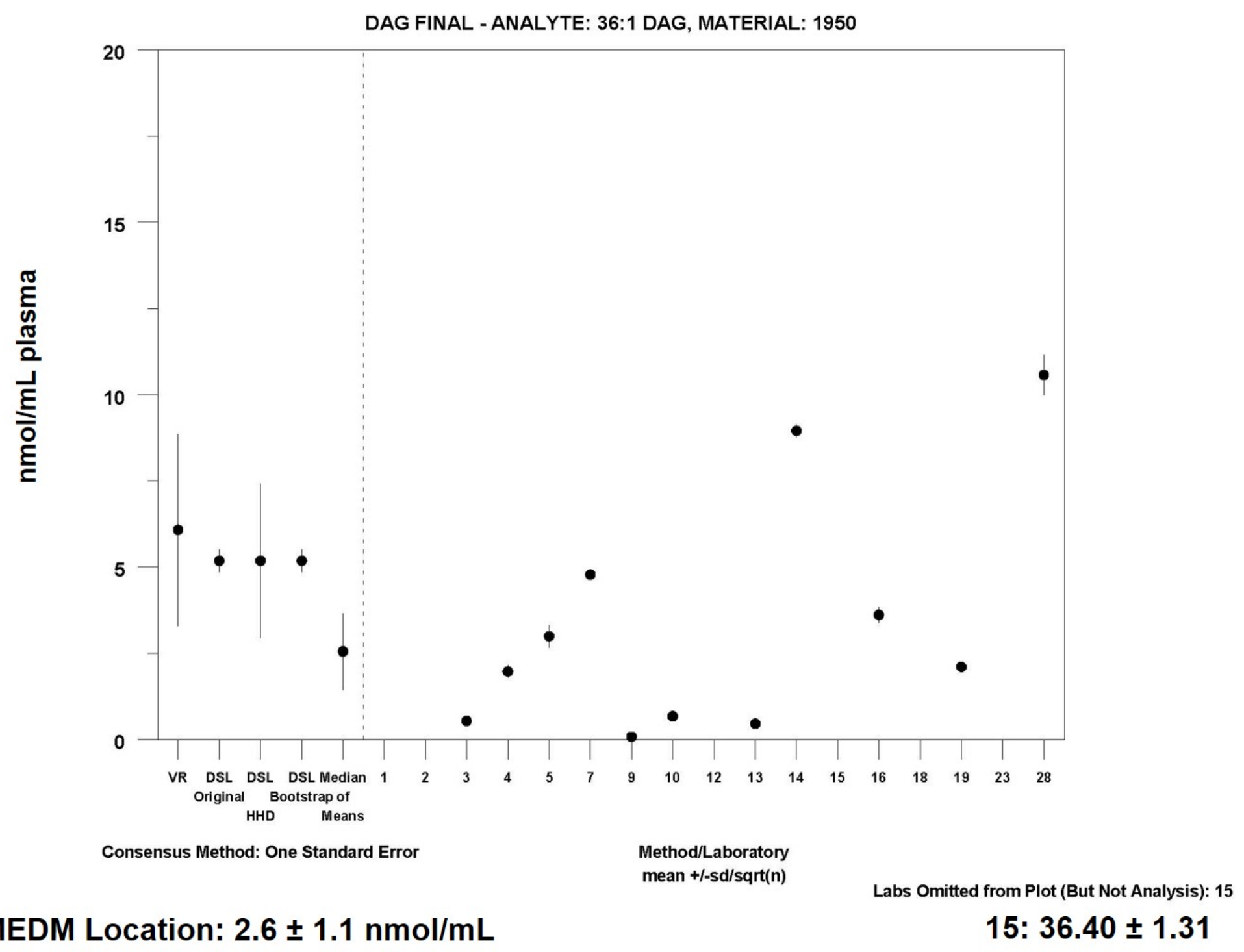




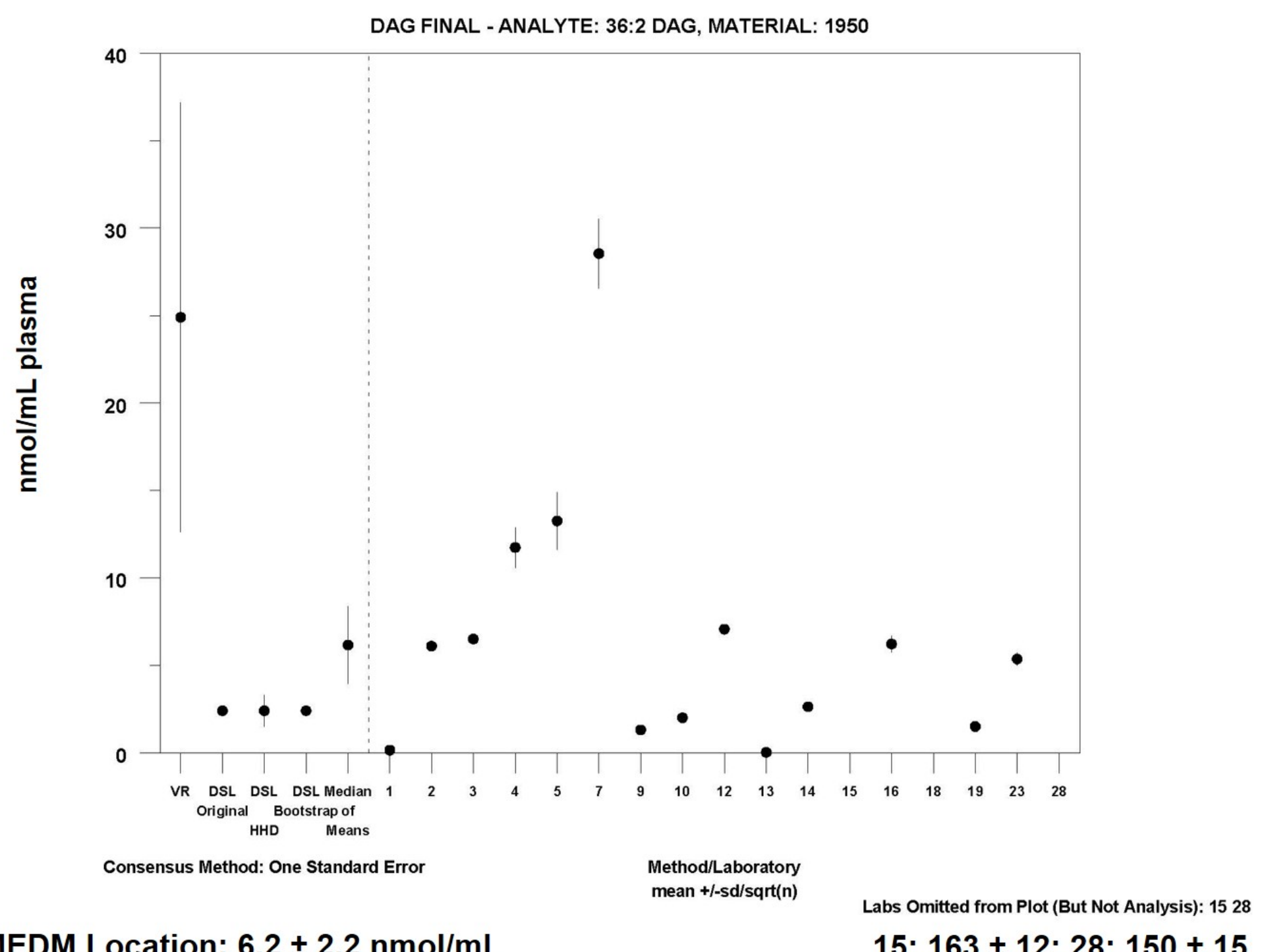

MEDM Location: $6.2 \pm 2.2 \mathrm{nmol} / \mathrm{mL}$

15: $163 \pm 12 ; 28: 150 \pm 15$ 


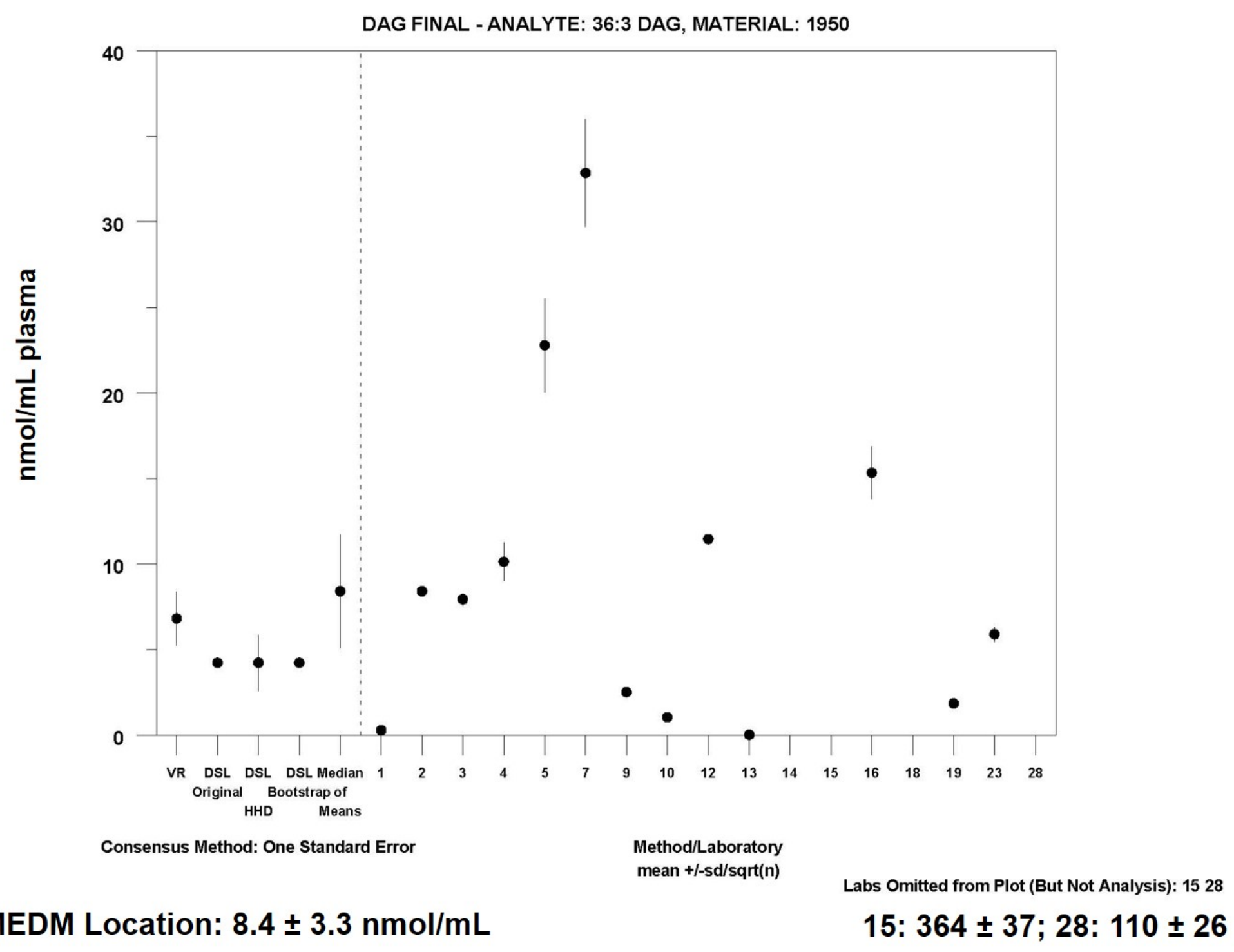




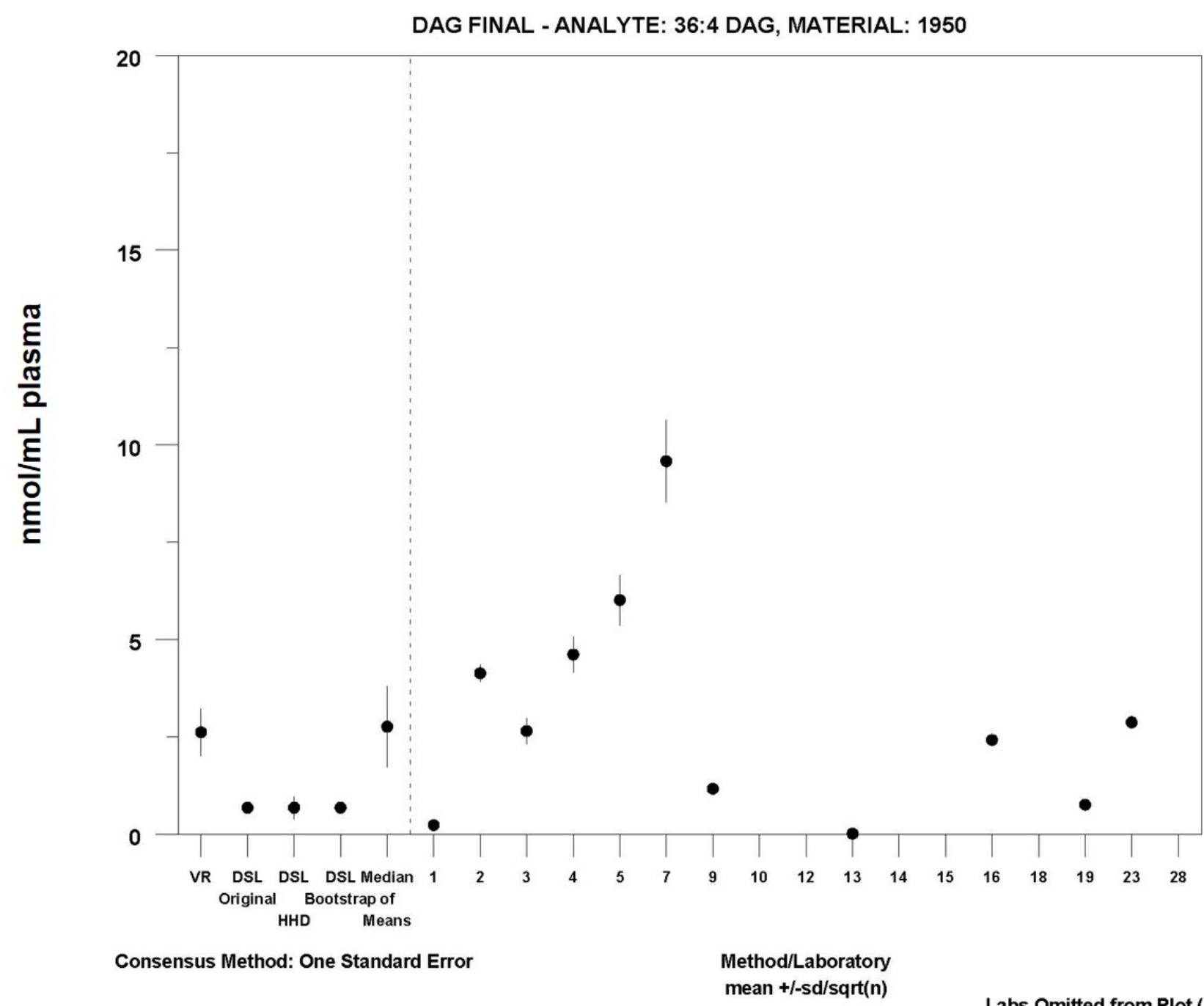

MEDM Location: $2.8 \pm 1.0 \mathrm{nmol} / \mathrm{mL}$

Labs Omitted from Plot (But Not Analysis): 15

15: $134 \pm 25$ 


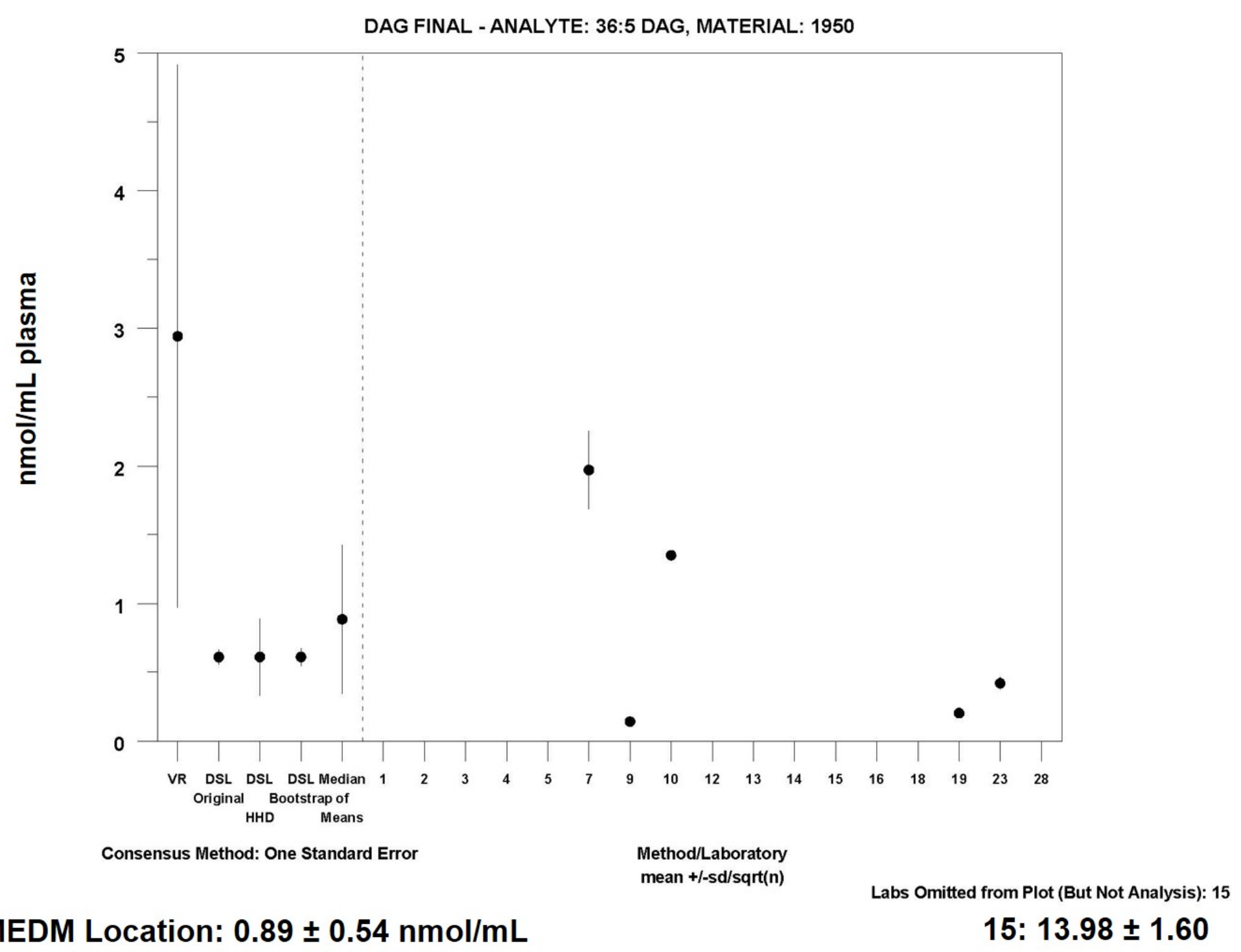




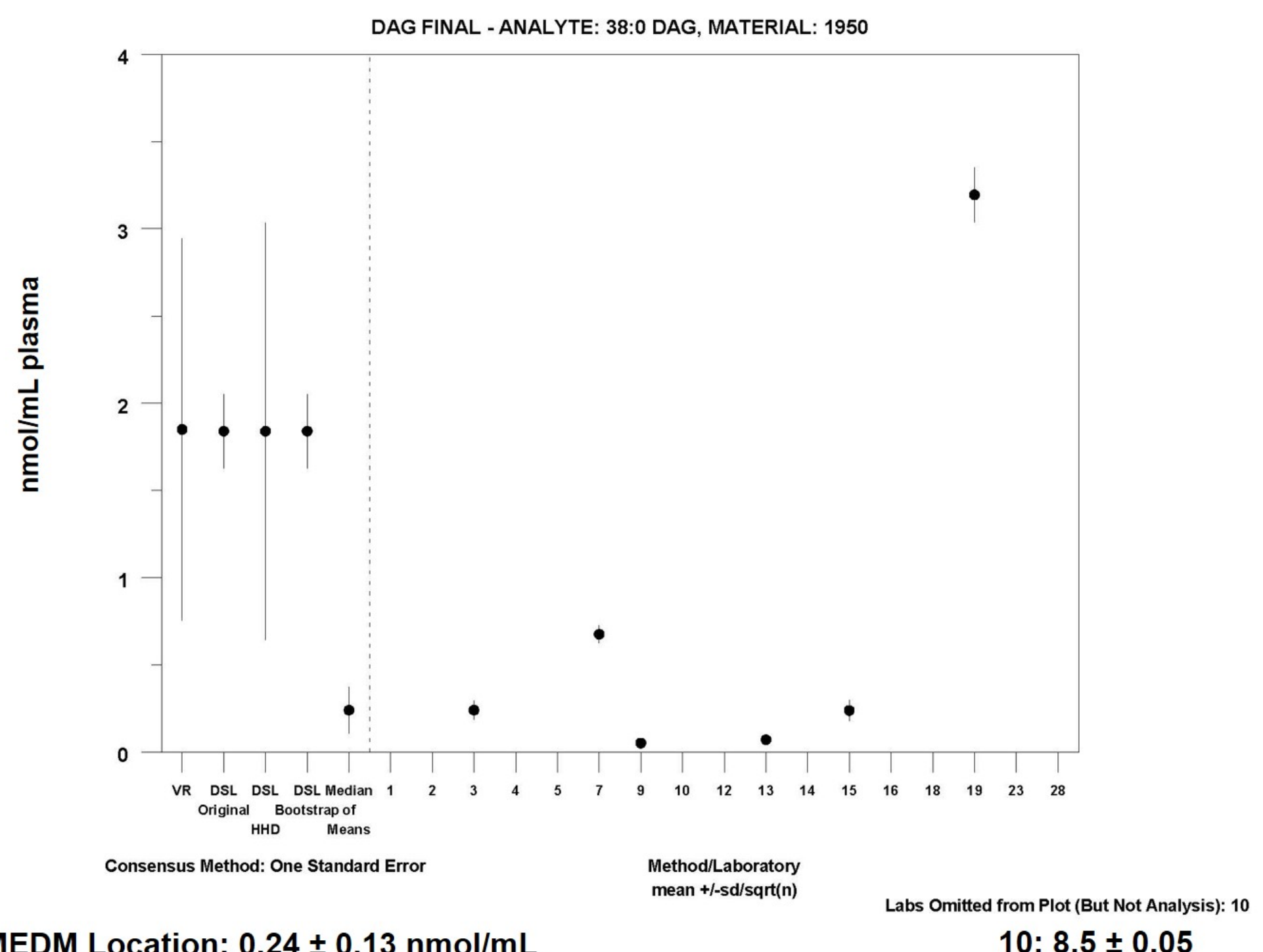

MEDM Location: $0.24 \pm 0.13 \mathrm{nmol} / \mathrm{mL}$ 


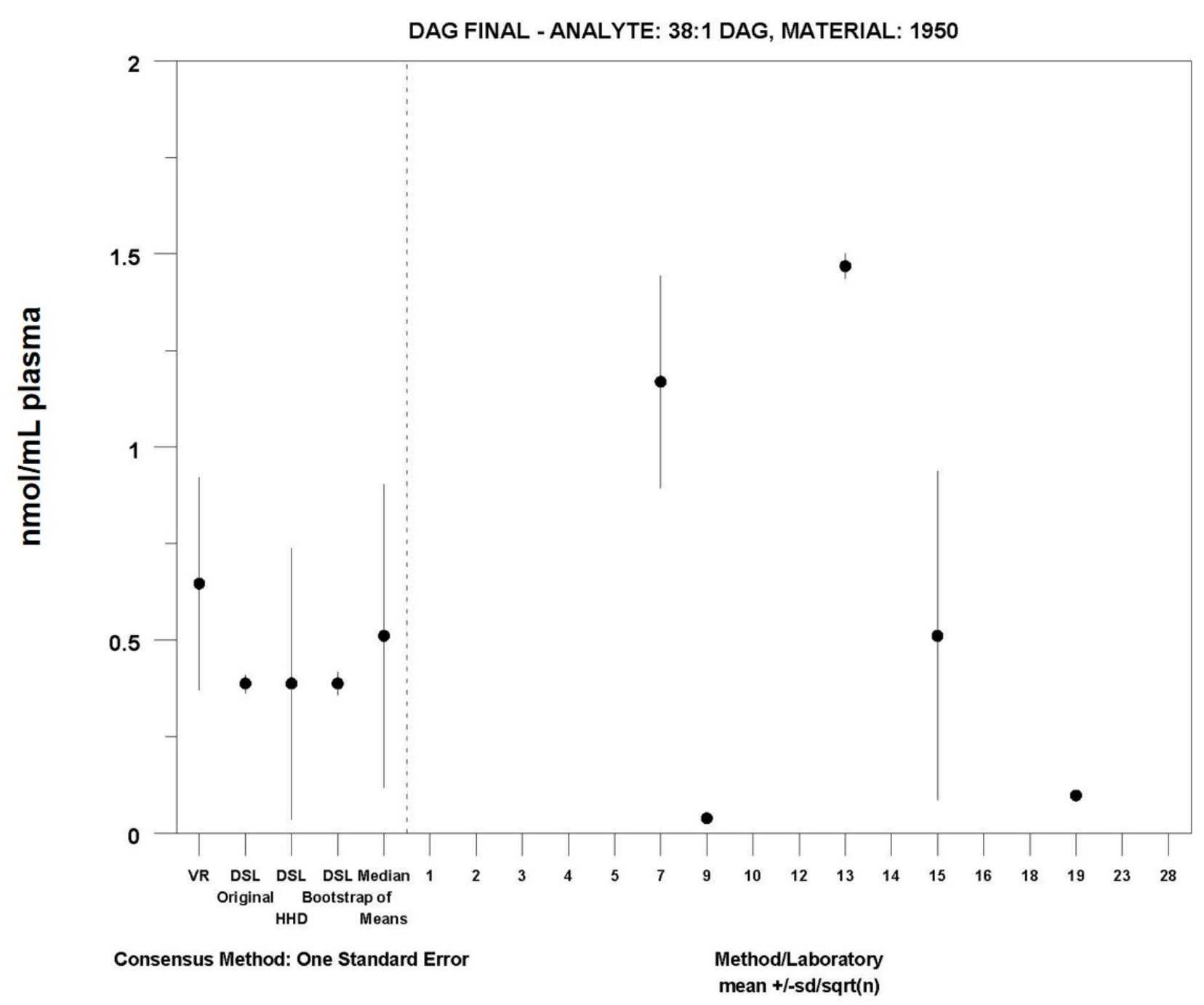

MEDM Location: $0.51 \pm 0.39 \mathrm{nmol} / \mathrm{mL}$ 


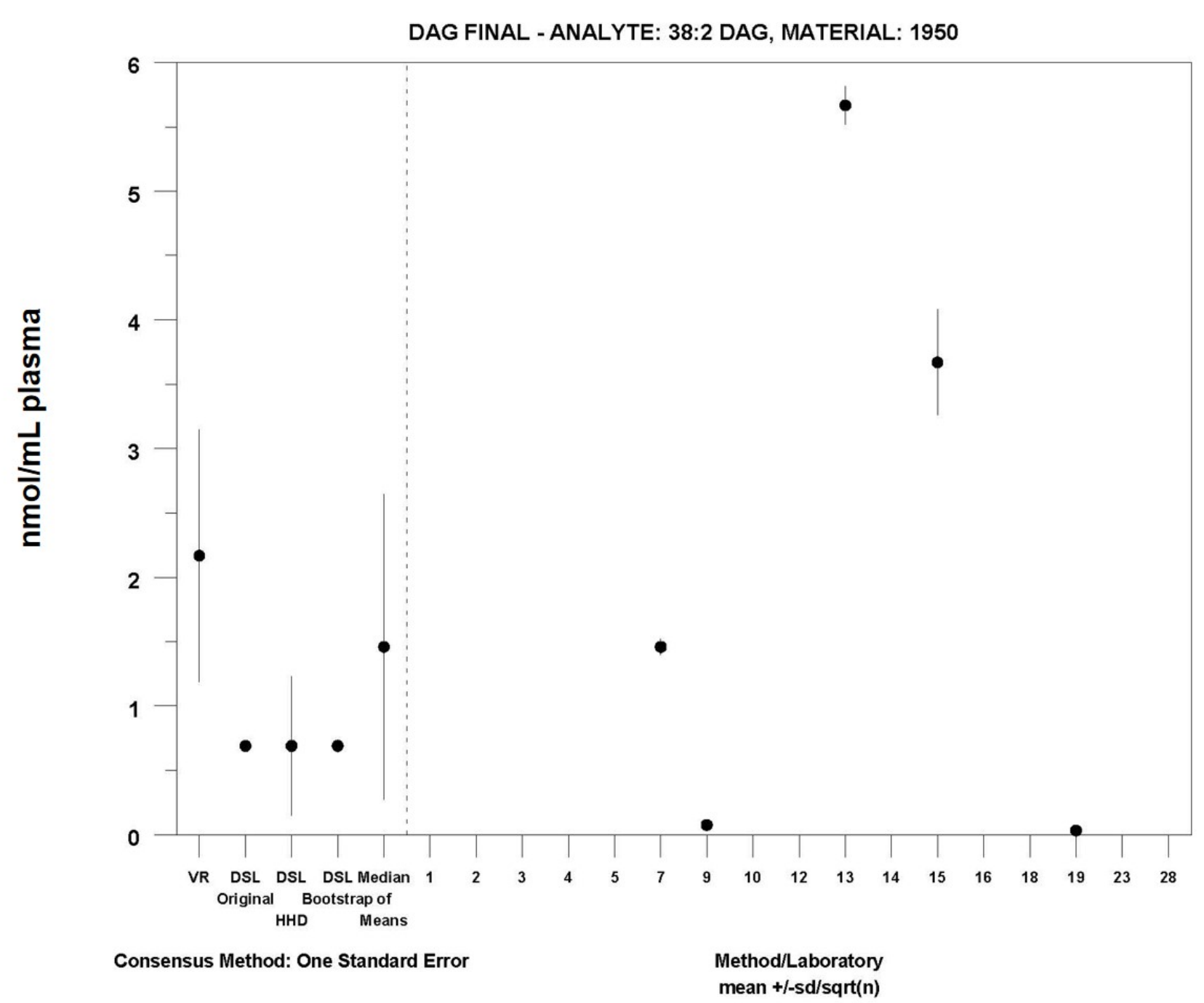

MEDM Location: $1.5 \pm 1.2 \mathrm{nmol} / \mathrm{mL}$ 


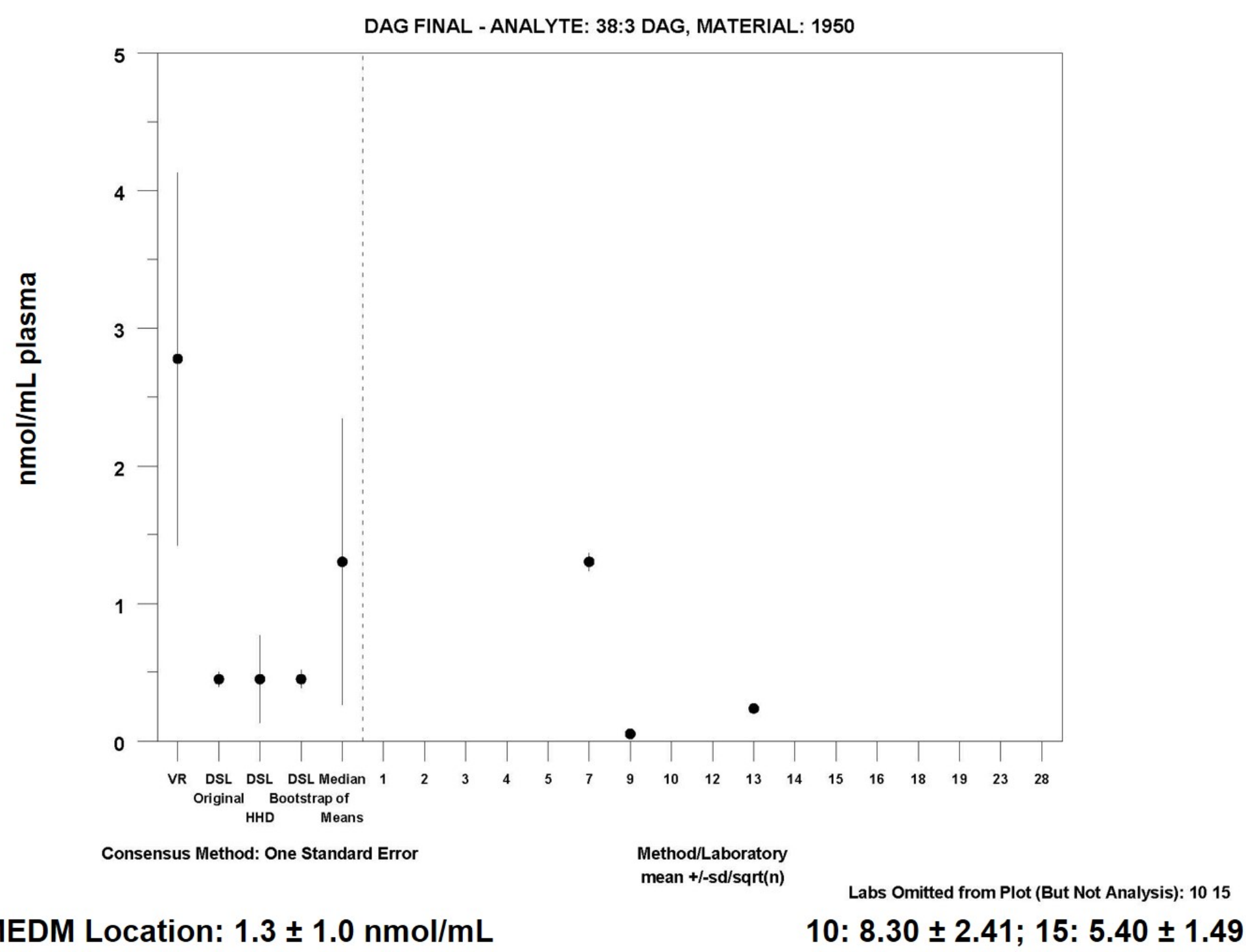




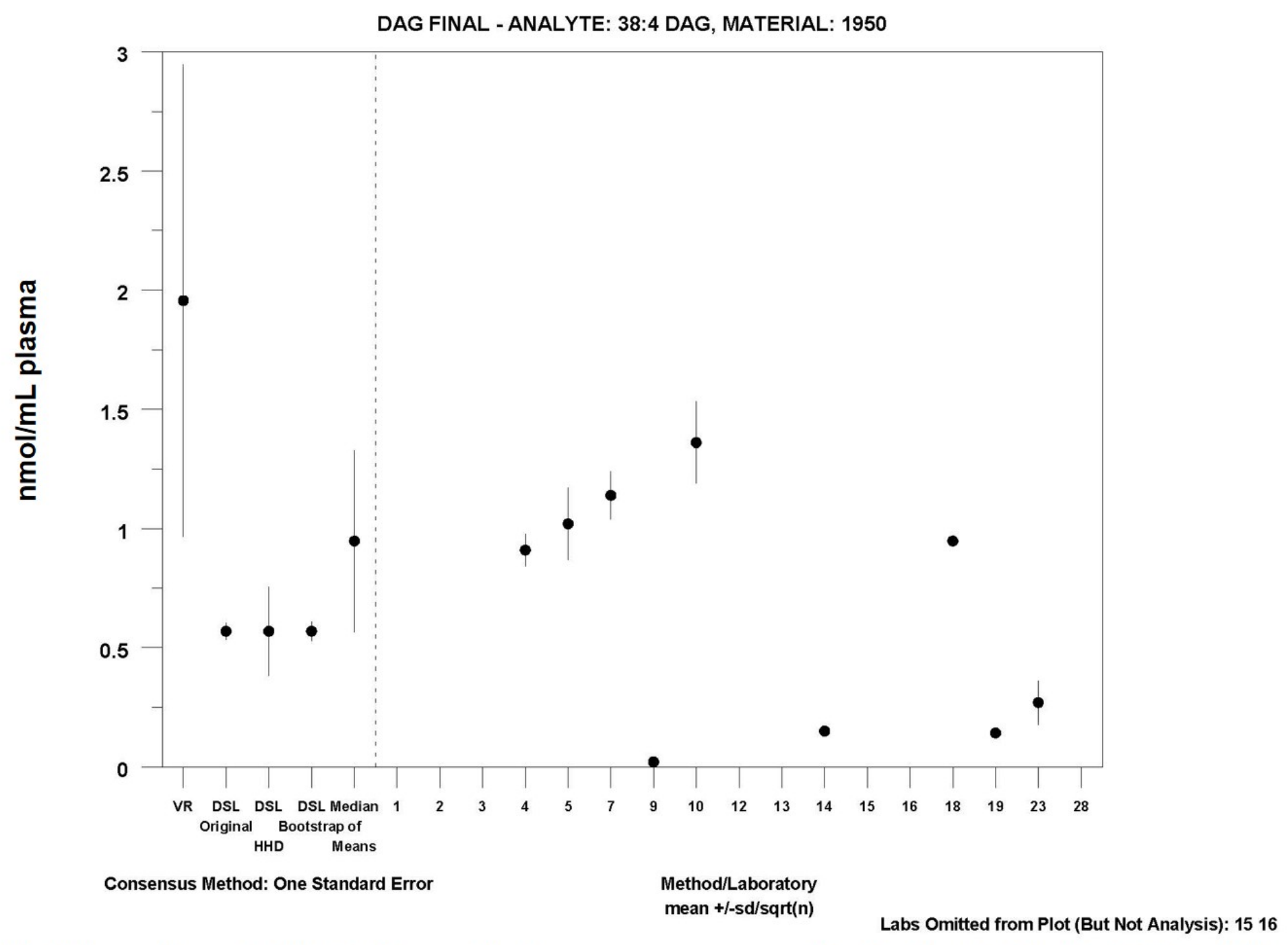

MEDM Location: $0.95 \pm 0.38 \mathrm{nmol} / \mathrm{mL}$ 15: $12.01 \pm 0.84 ; 16: 3.86 \pm 1.19$ 


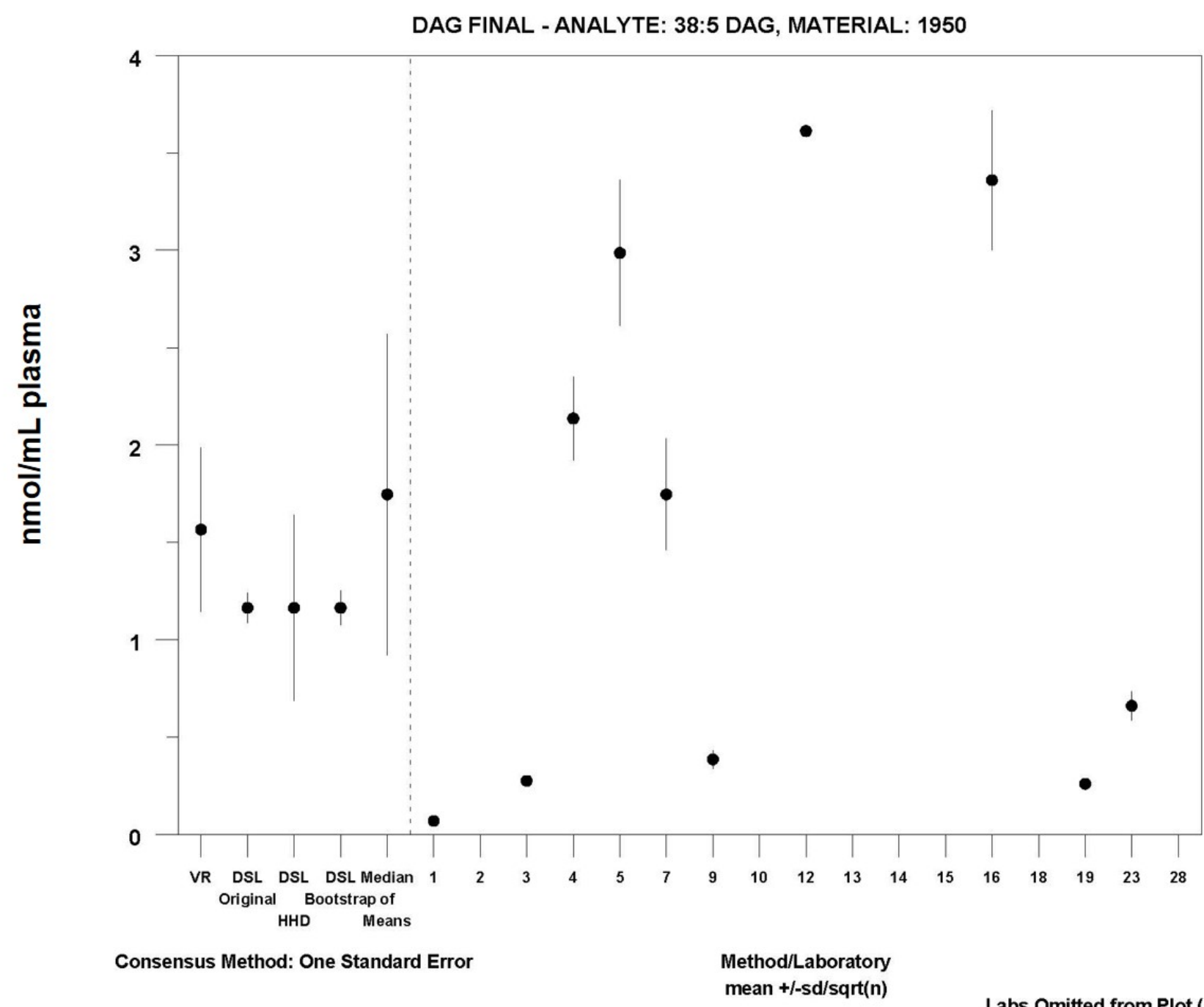

MEDM Location: $1.8 \pm 0.82 \mathrm{nmol} / \mathrm{mL}$

Labs Omitted from Plot (But Not Analysis): 15

15: $8.75 \pm 7.51$ 


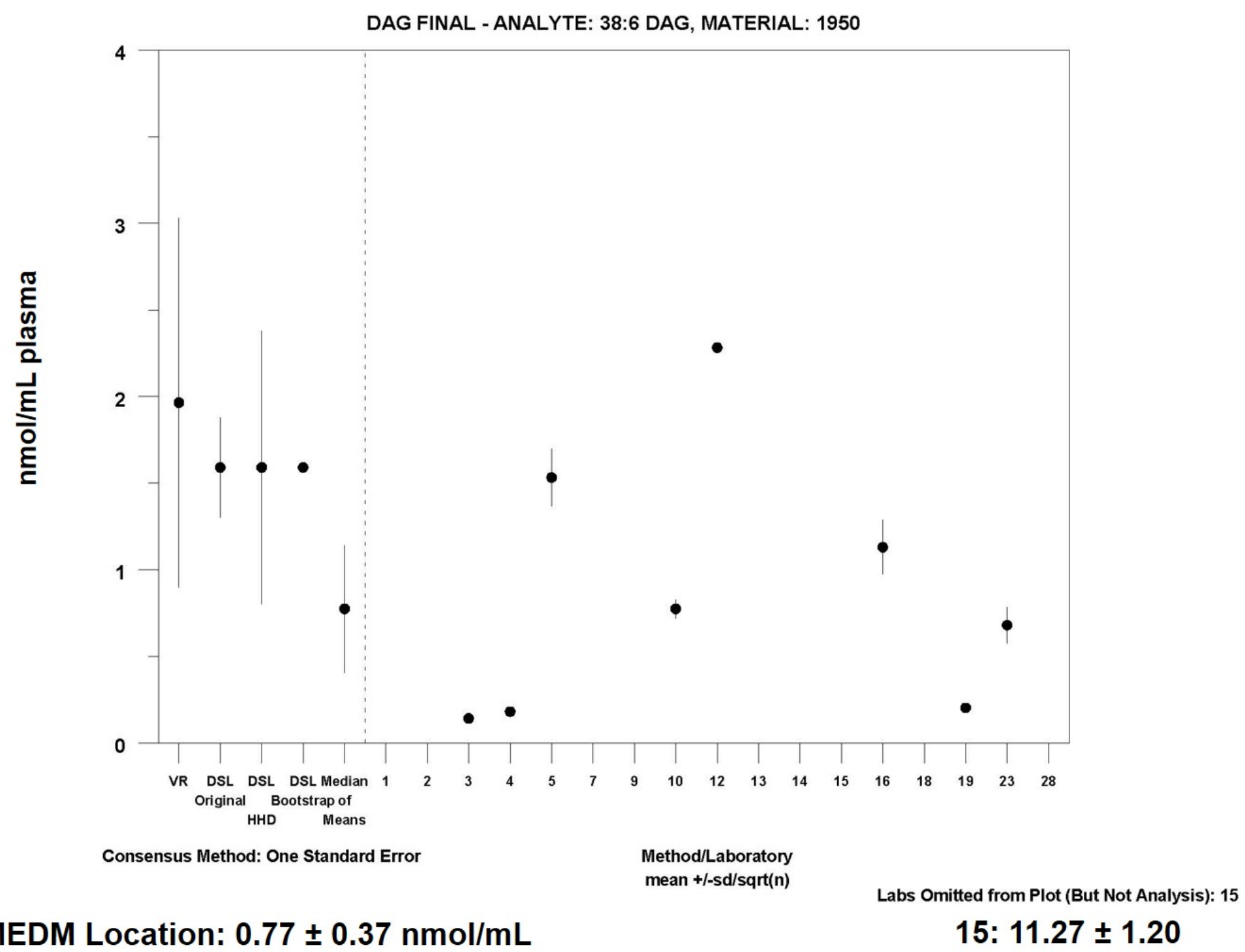




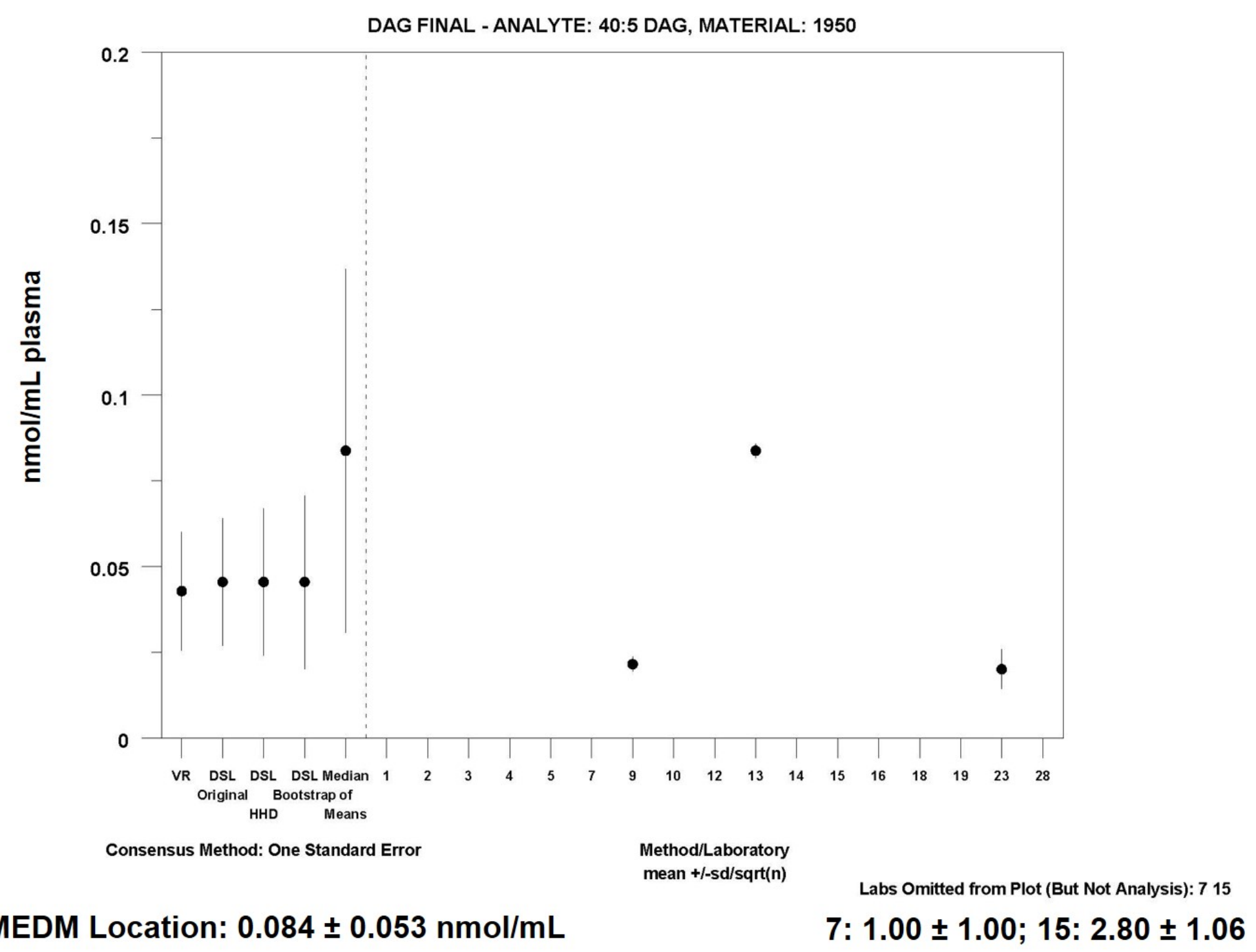




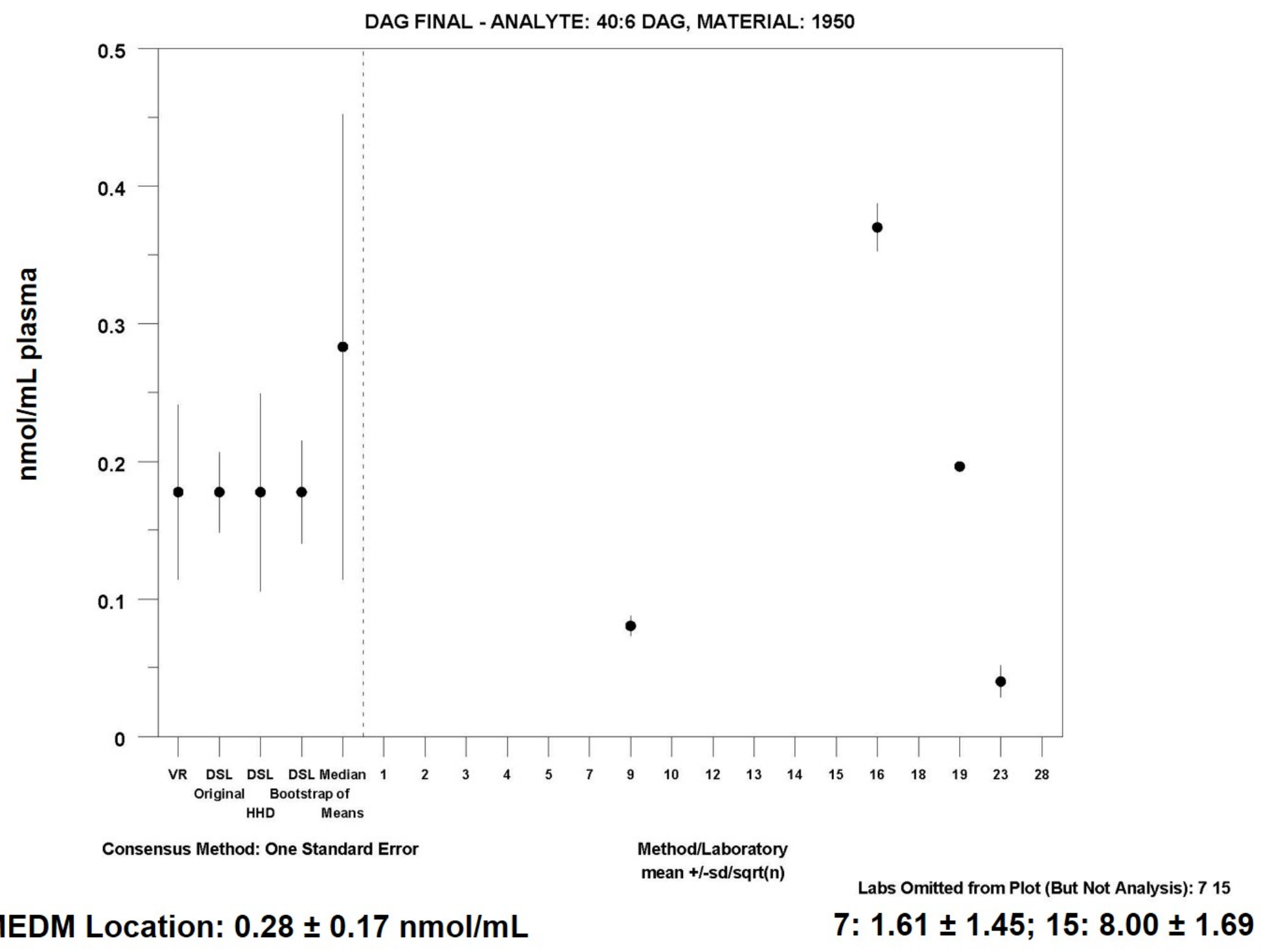




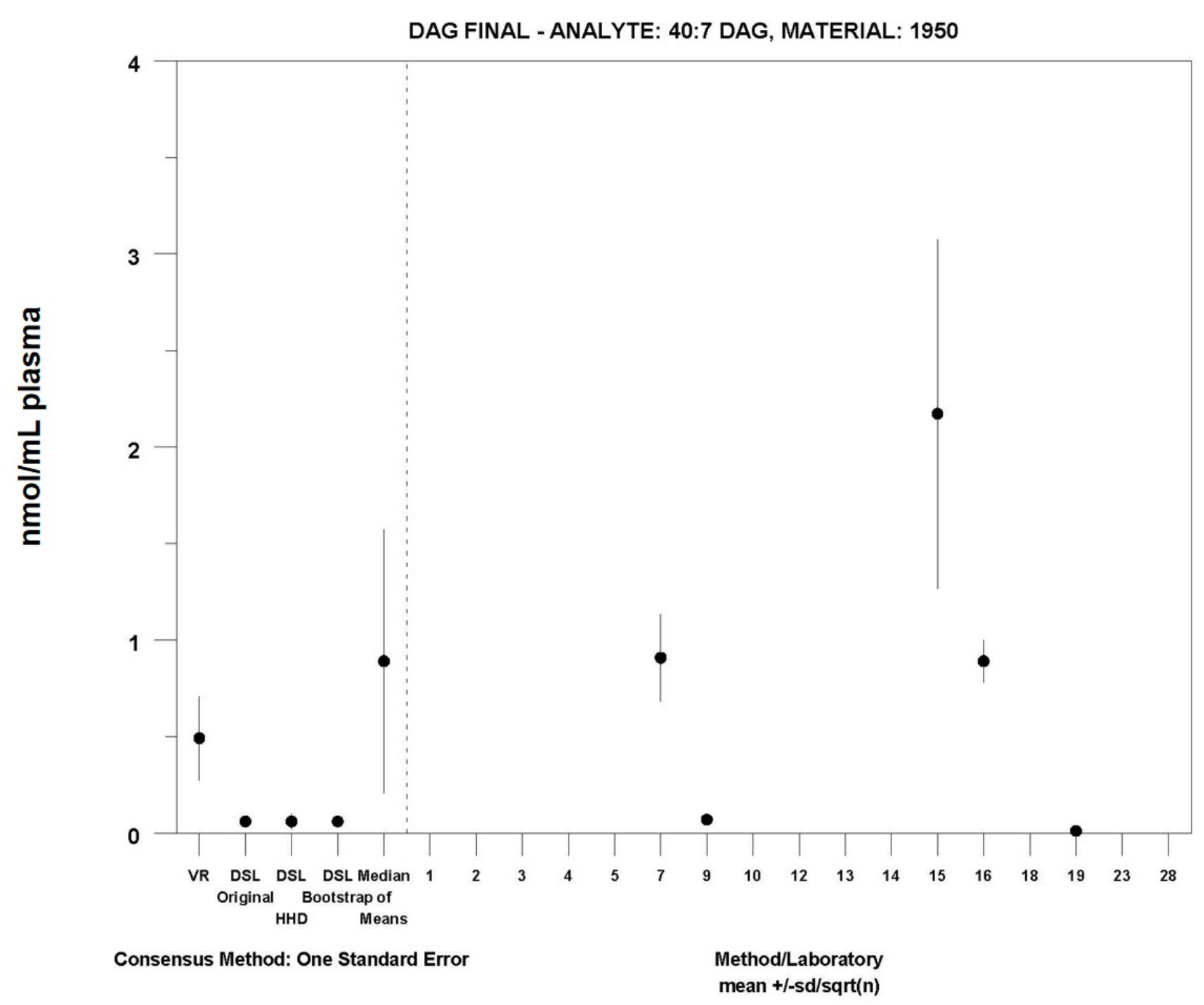

MEDM Location: $0.89 \pm 0.68 \mathrm{nmol} / \mathrm{mL}$ 


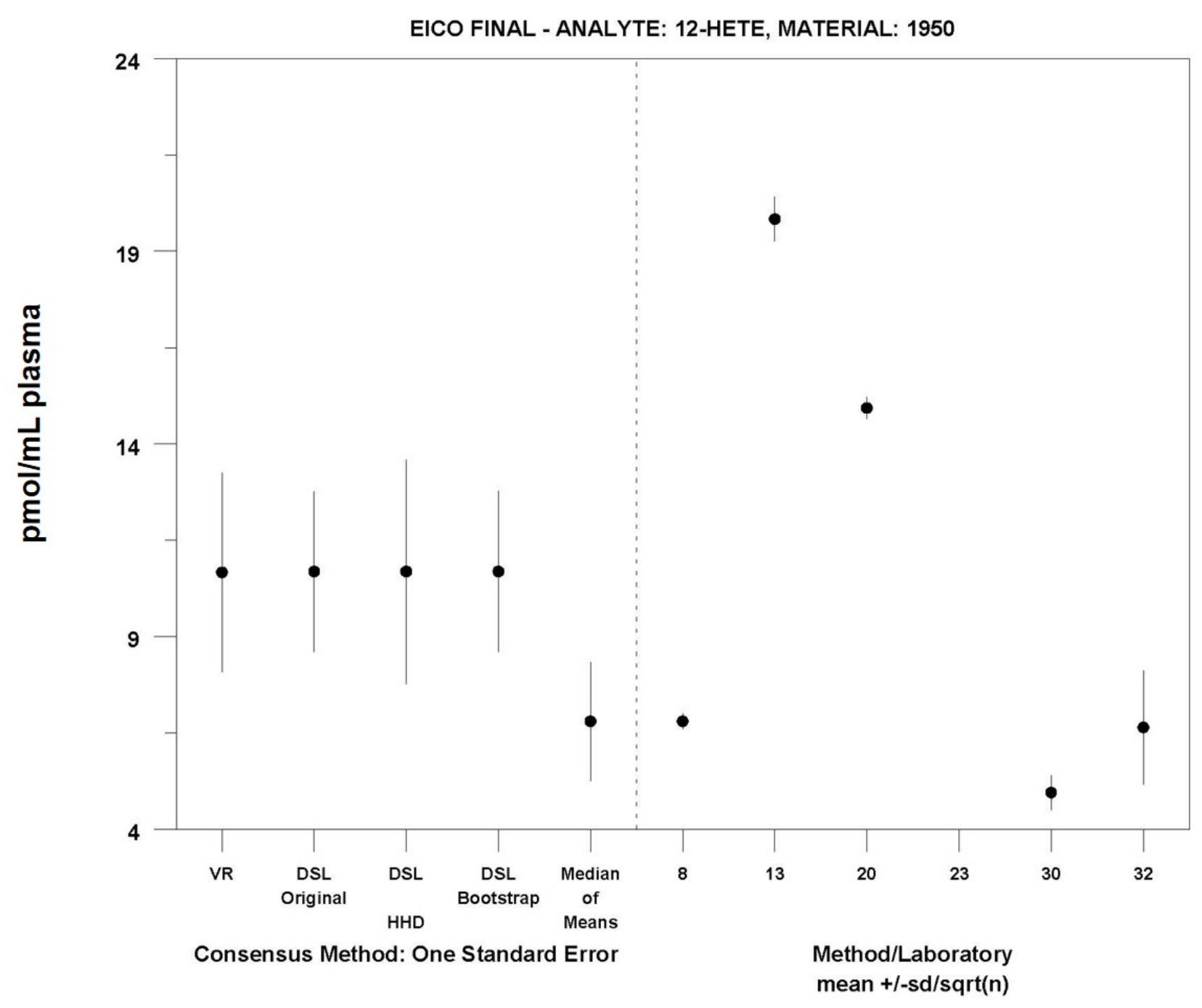

MEDM Location: $6.8 \pm 1.5 \mathrm{pmol} / \mathrm{mL}$ 


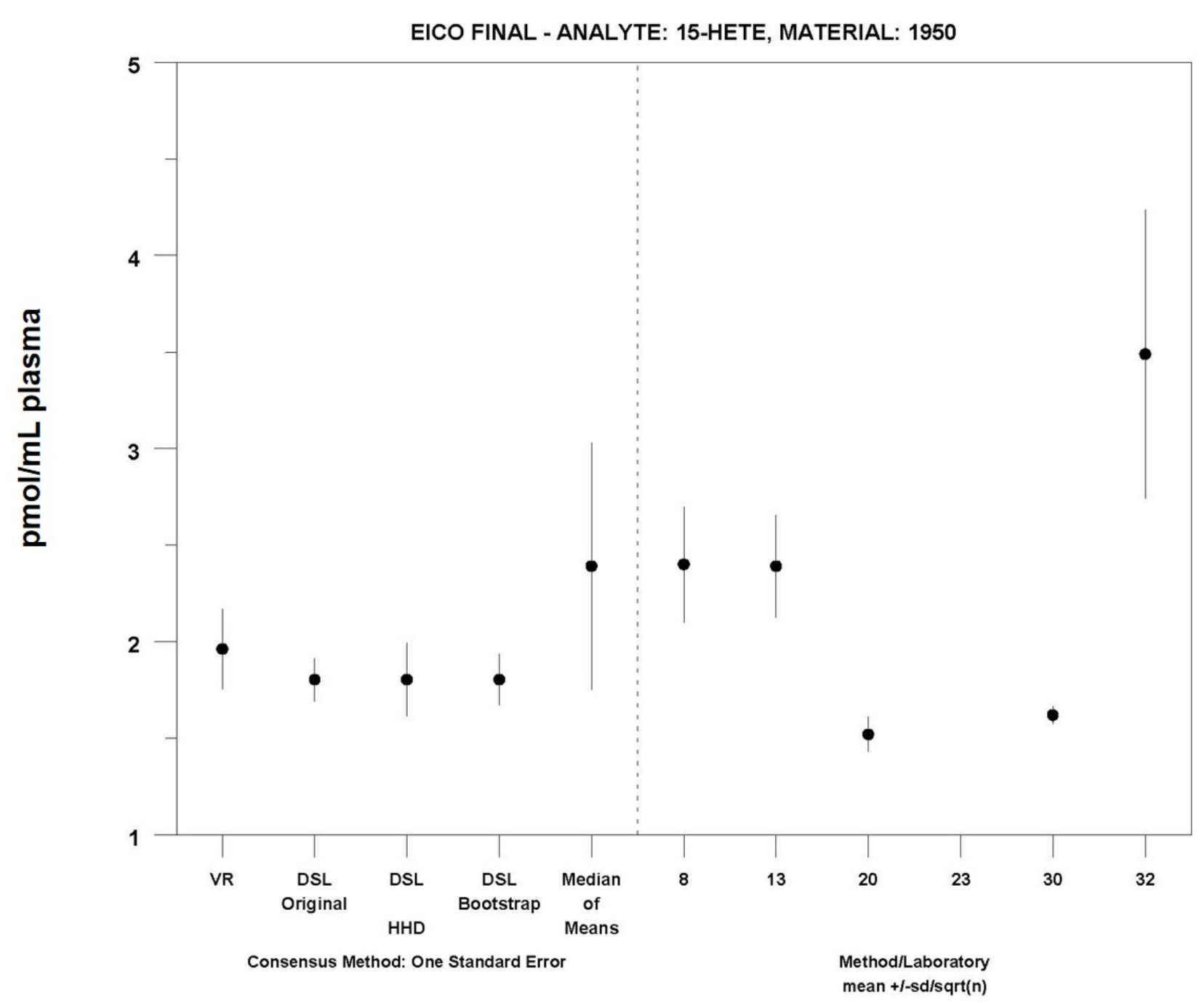

MEDM Location: $2.4 \pm 0.64 \mathrm{pmol} / \mathrm{mL}$ 


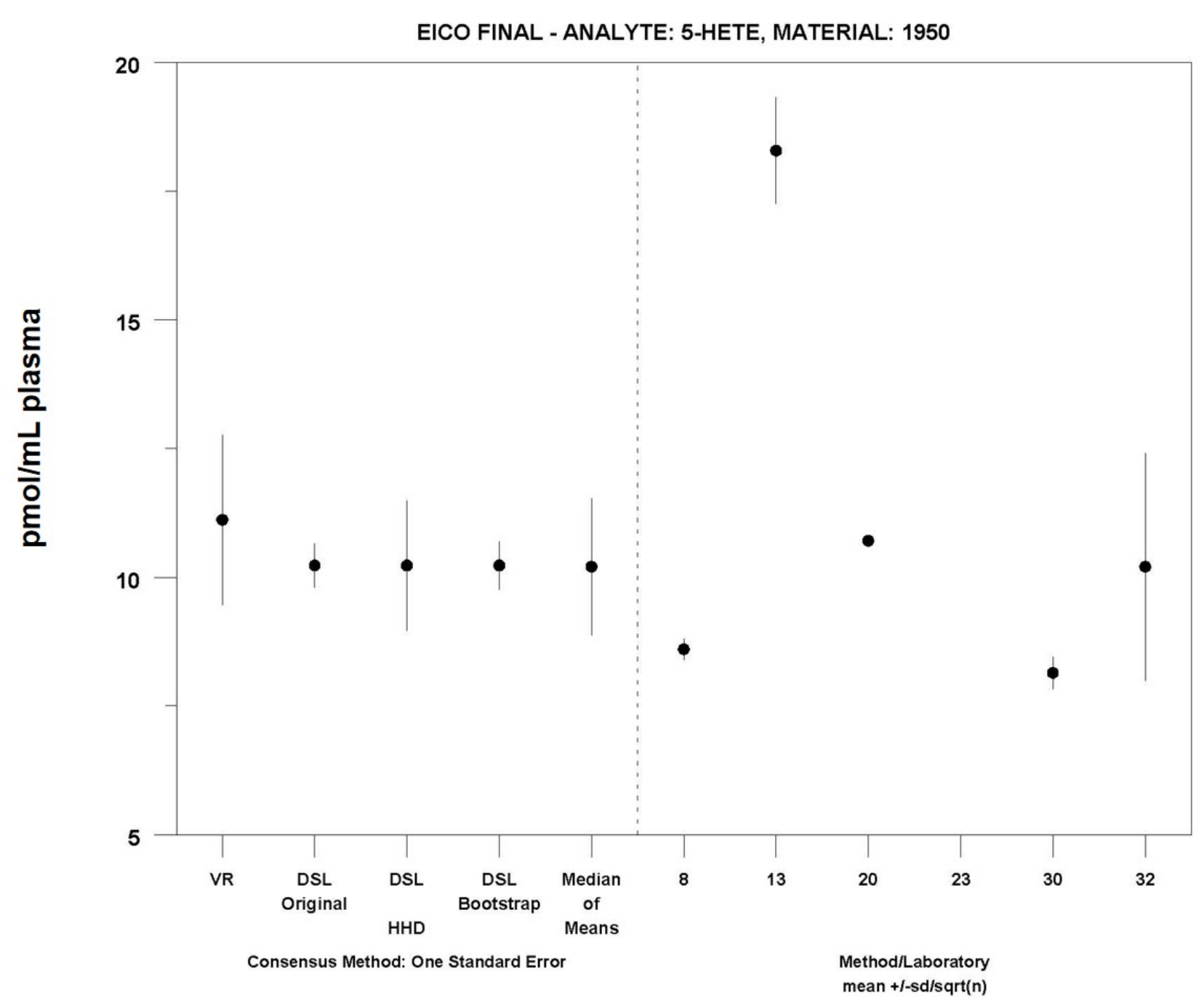

MEDM Location: $10 \pm 1.3 \mathrm{pmol} / \mathrm{mL}$ 


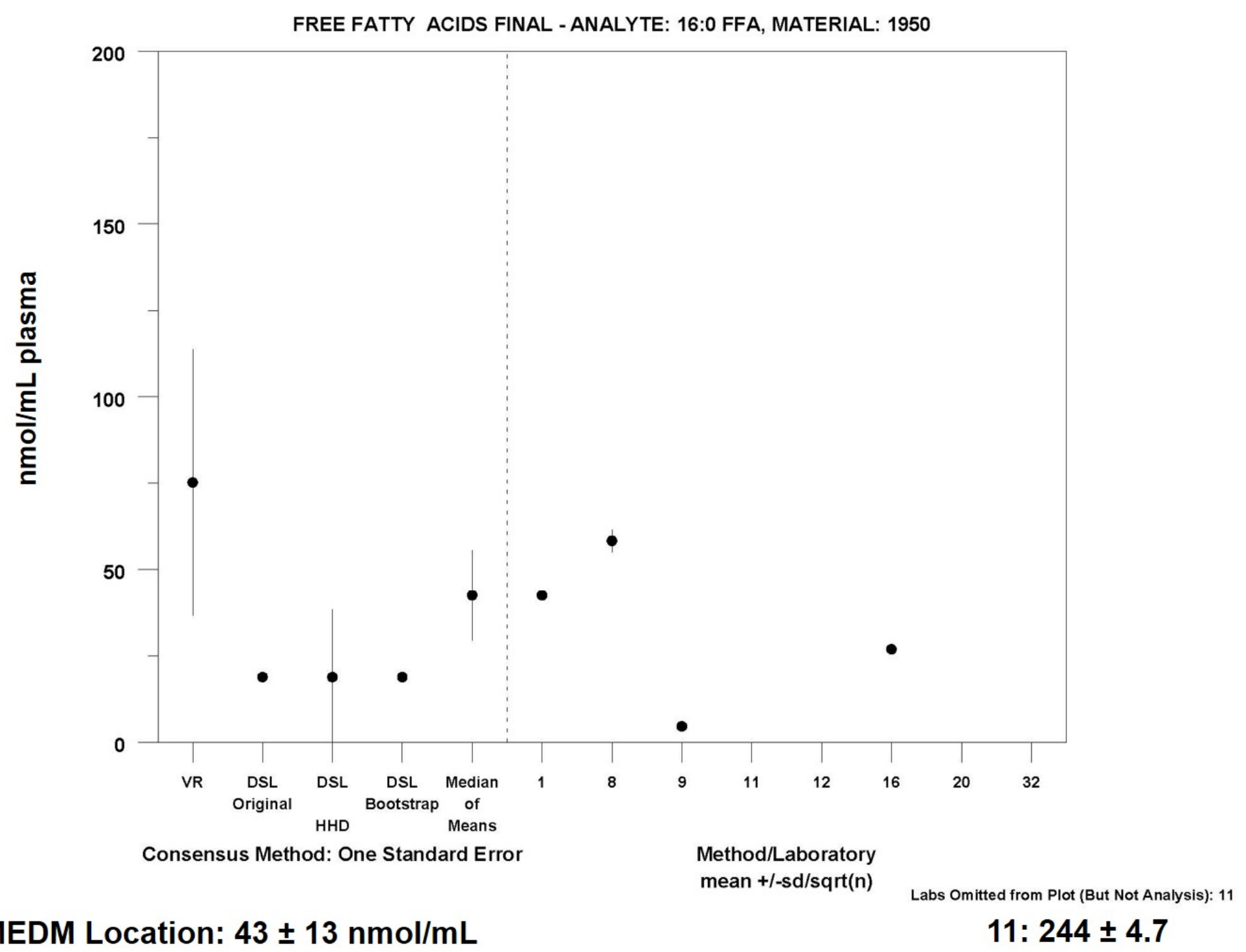




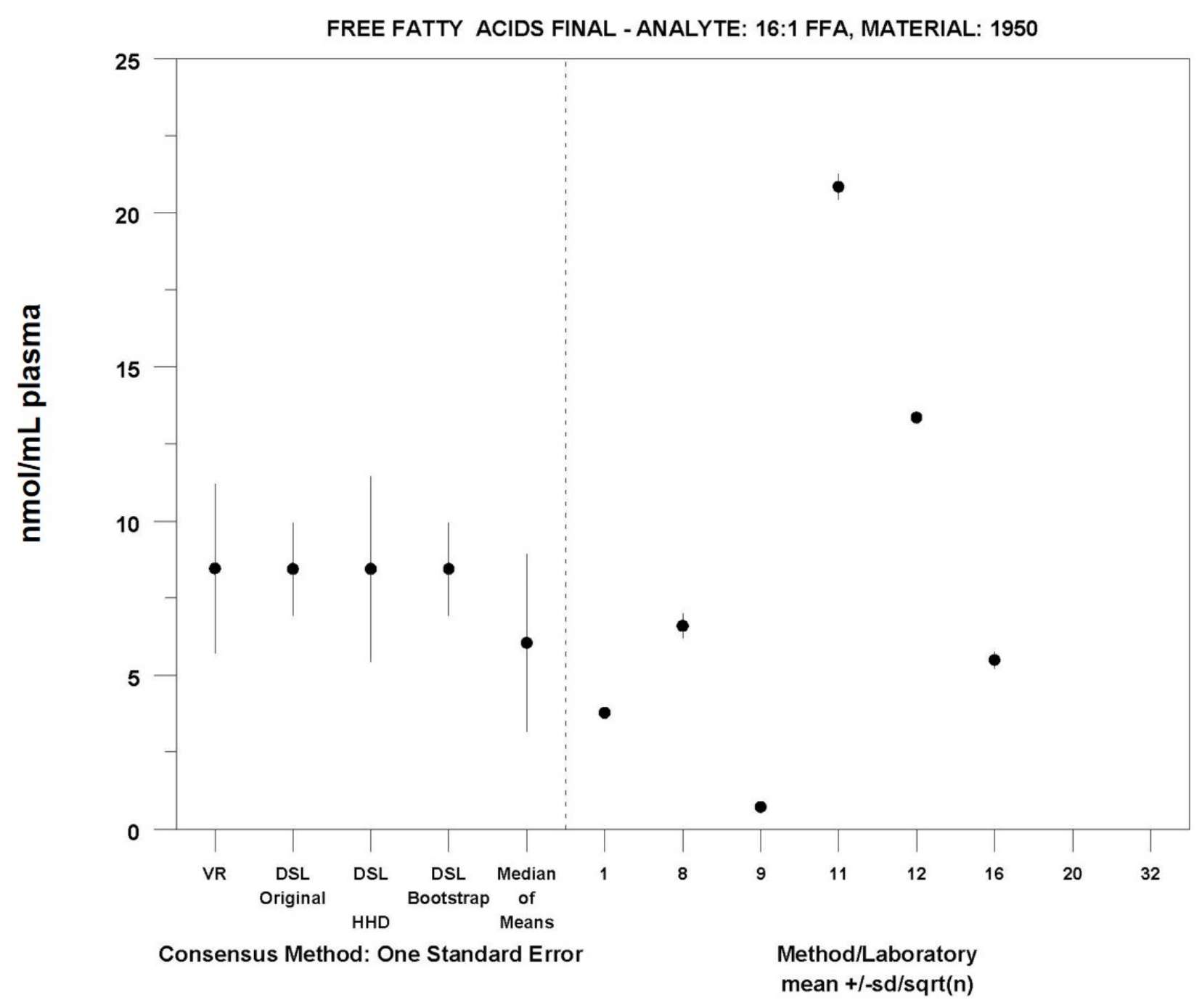

MEDM Location: $6.1 \pm 2.9 \mathrm{nmol} / \mathrm{mL}$ 


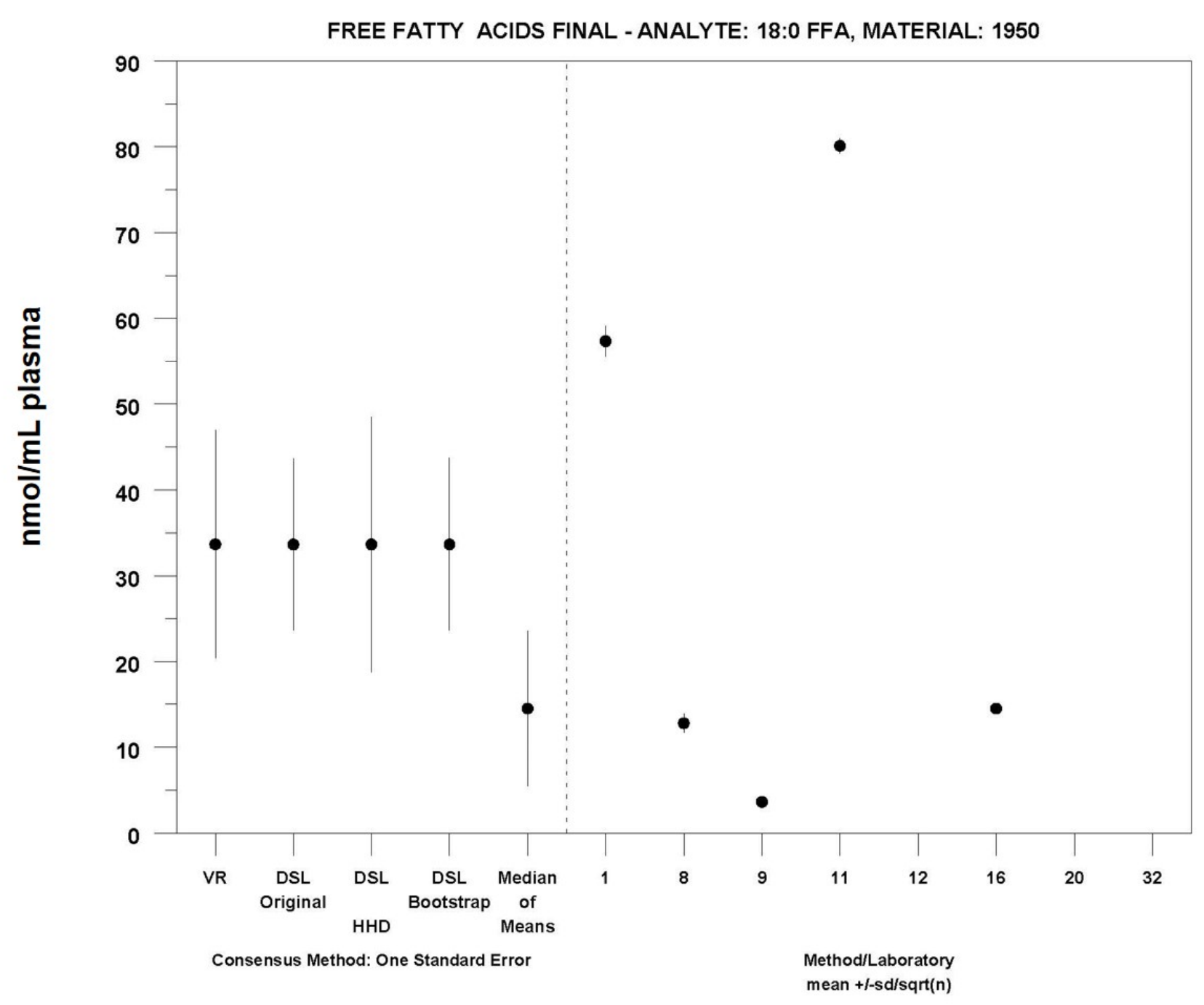

MEDM Location: $15 \pm 9.0 \mathrm{nmol} / \mathrm{mL}$ 


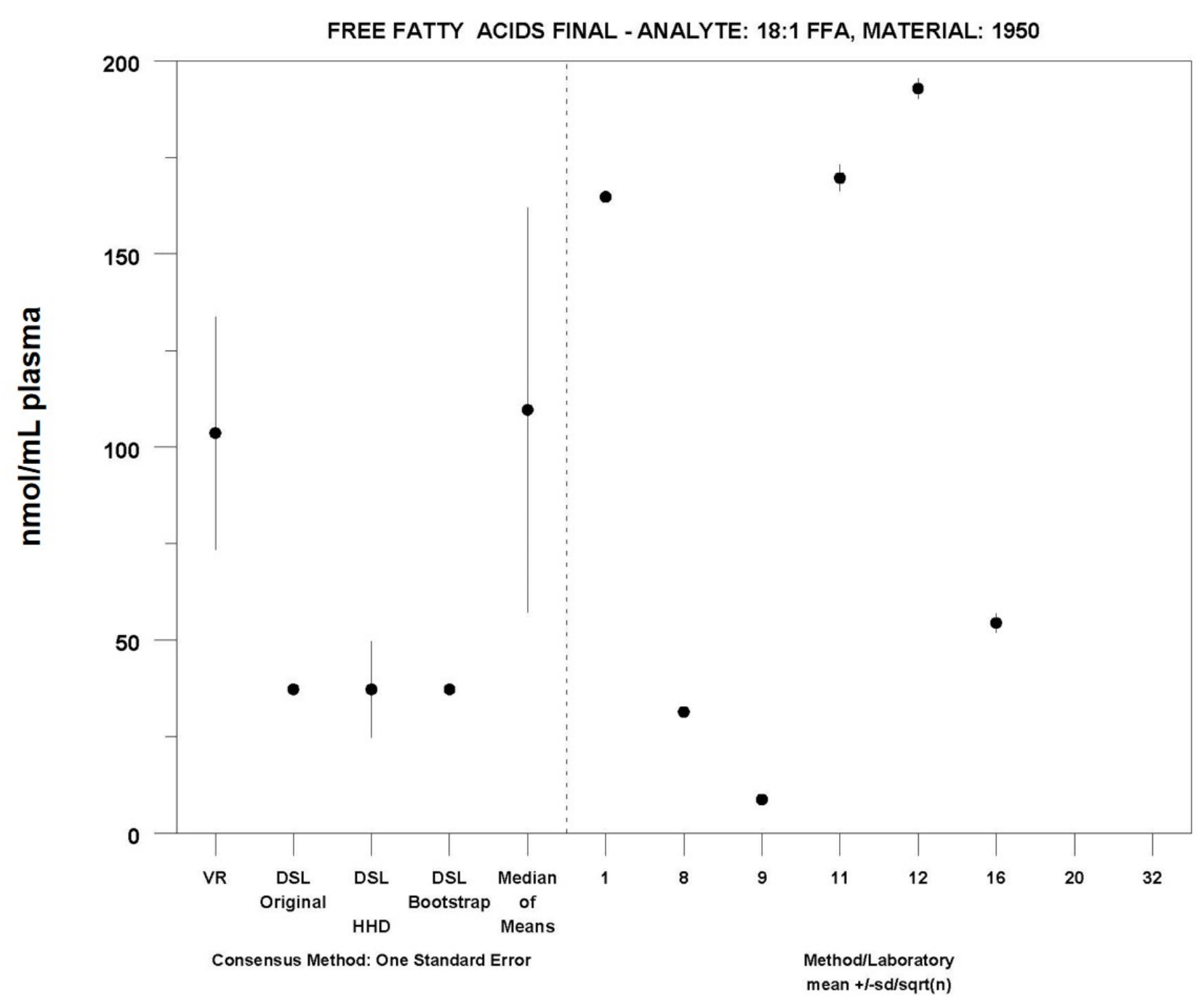

MEDM Location: $110 \pm 105 \mathrm{nmol} / \mathrm{mL}$ 


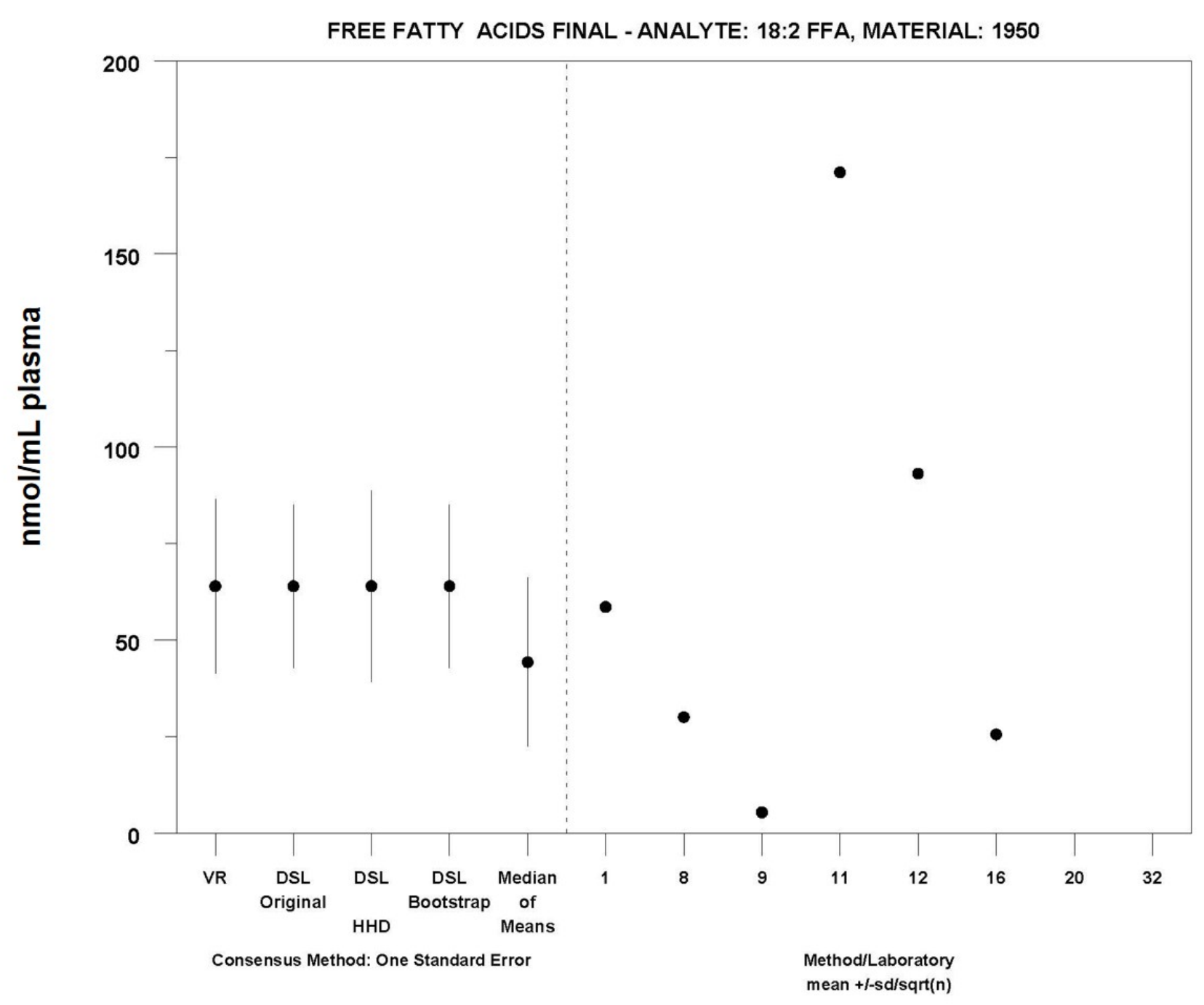

MEDM Location: $44 \pm 22 \mathrm{nmol} / \mathrm{mL}$ 


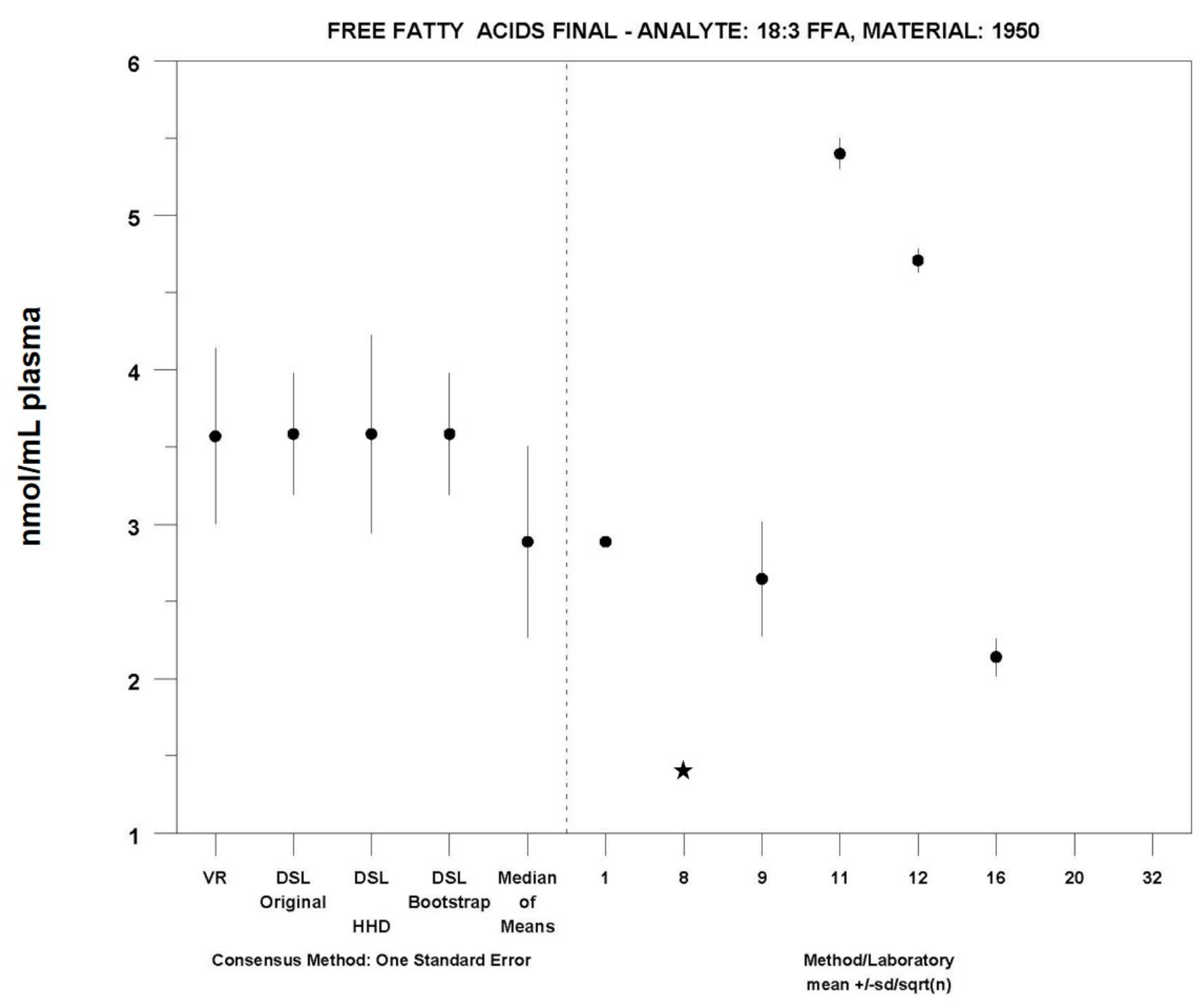

MEDM Location: $2.9 \pm 0.62 \mathrm{nmol} / \mathrm{mL}$ 


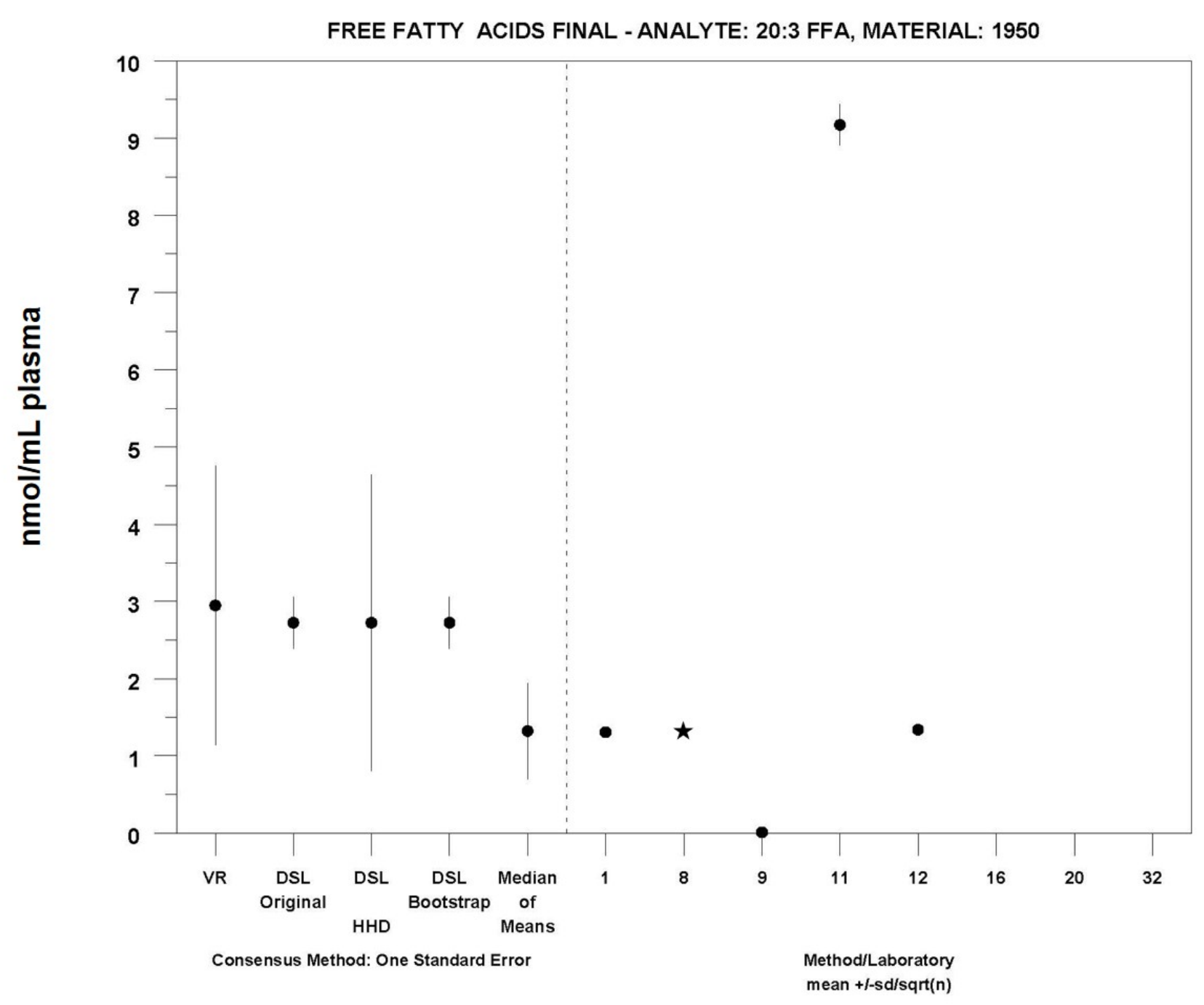

MEDM Location: $1.3 \pm 0.62 \mathrm{nmol} / \mathrm{mL}$ 


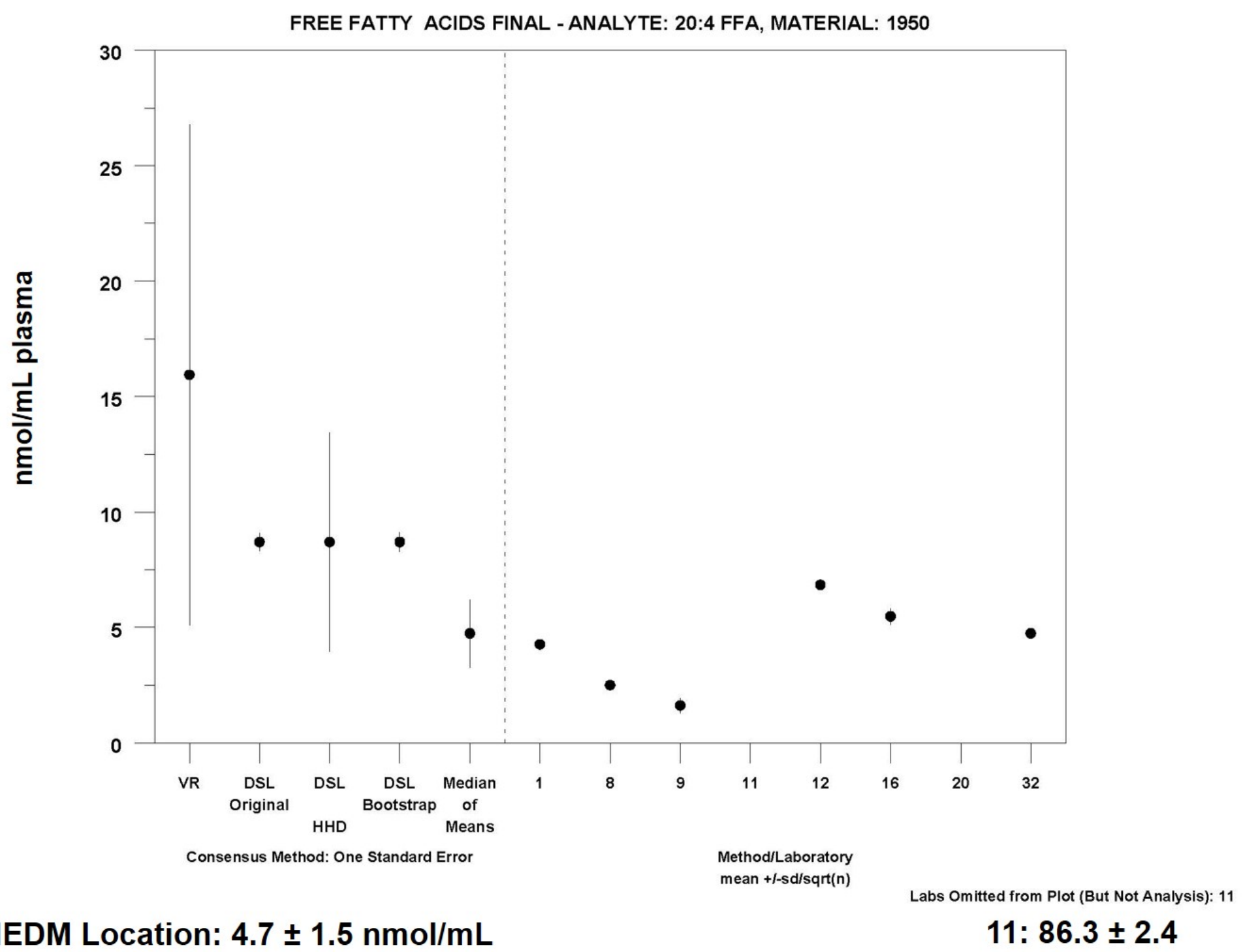




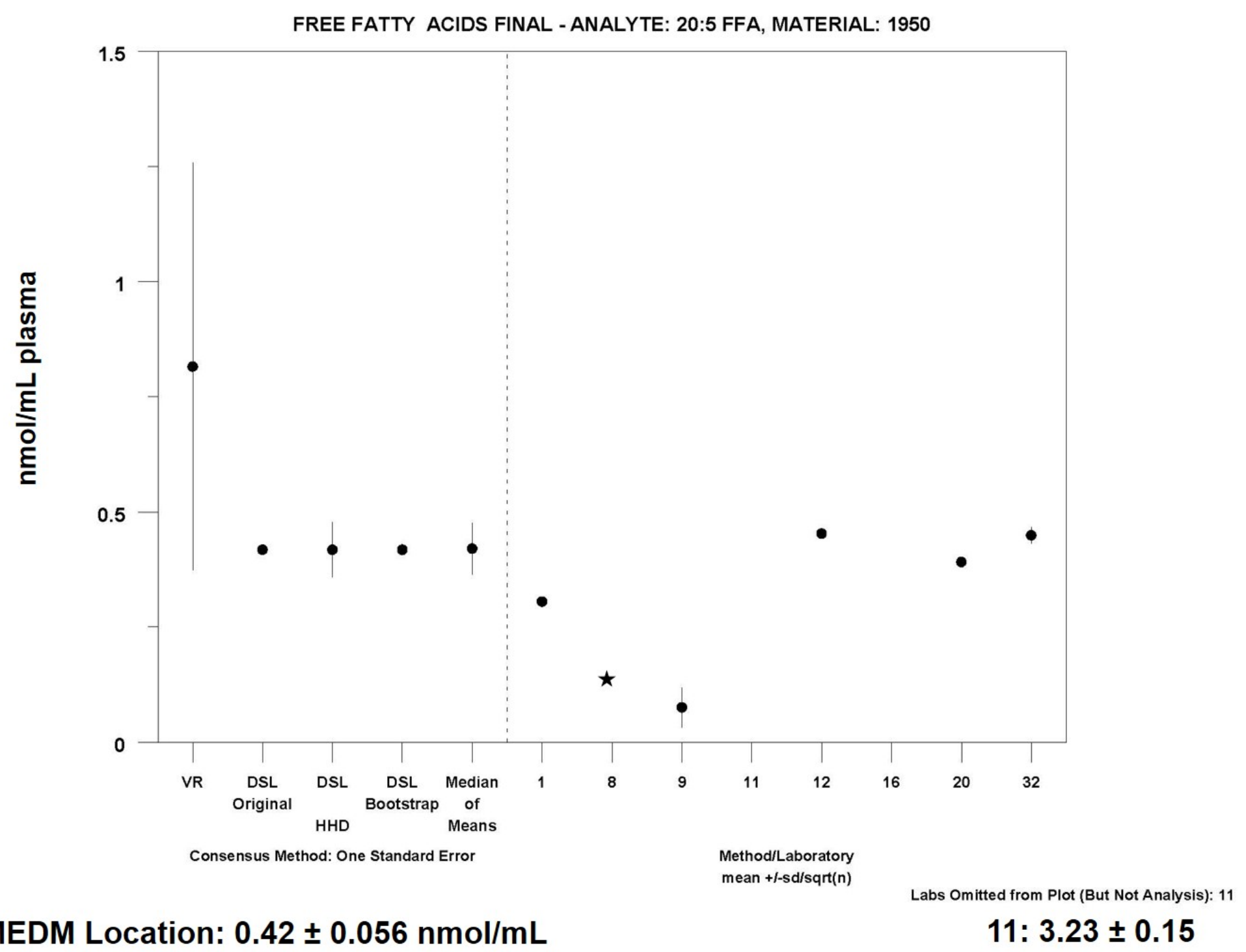




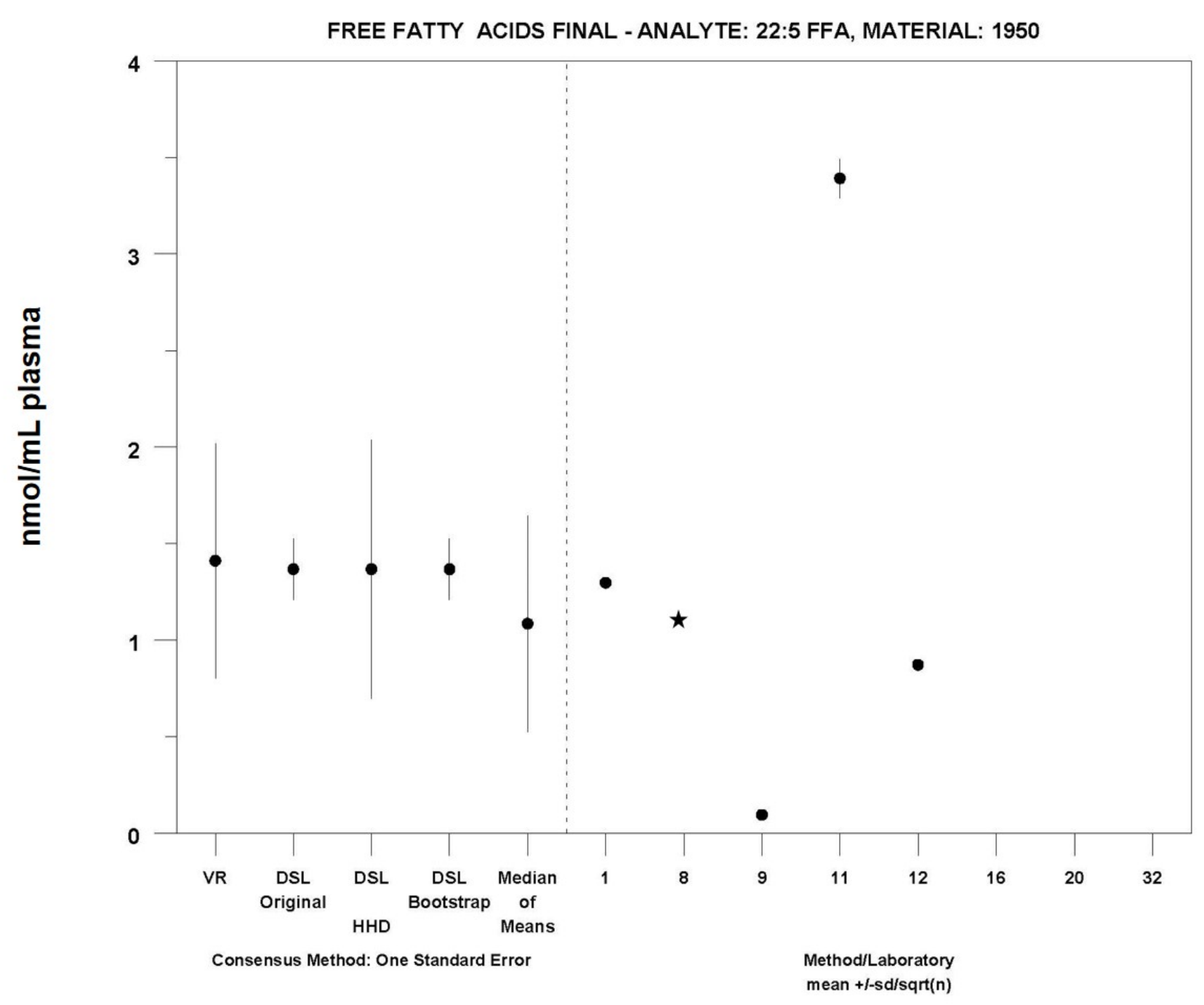

MEDM Location: $1.1 \pm 0.56 \mathrm{nmol} / \mathrm{mL}$ 


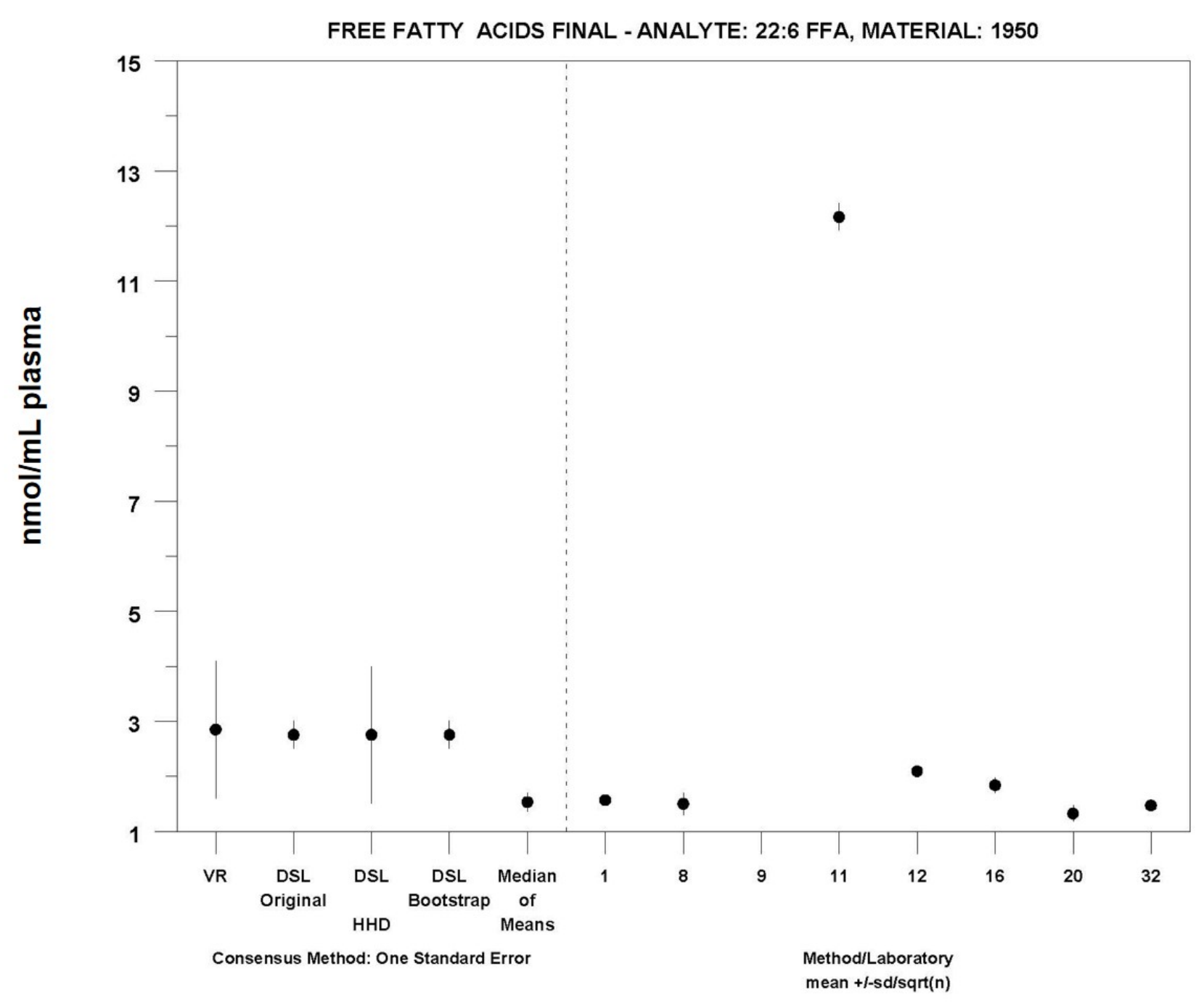

MEDM Location: $1.5 \pm 0.17 \mathrm{nmol} / \mathrm{mL}$ 


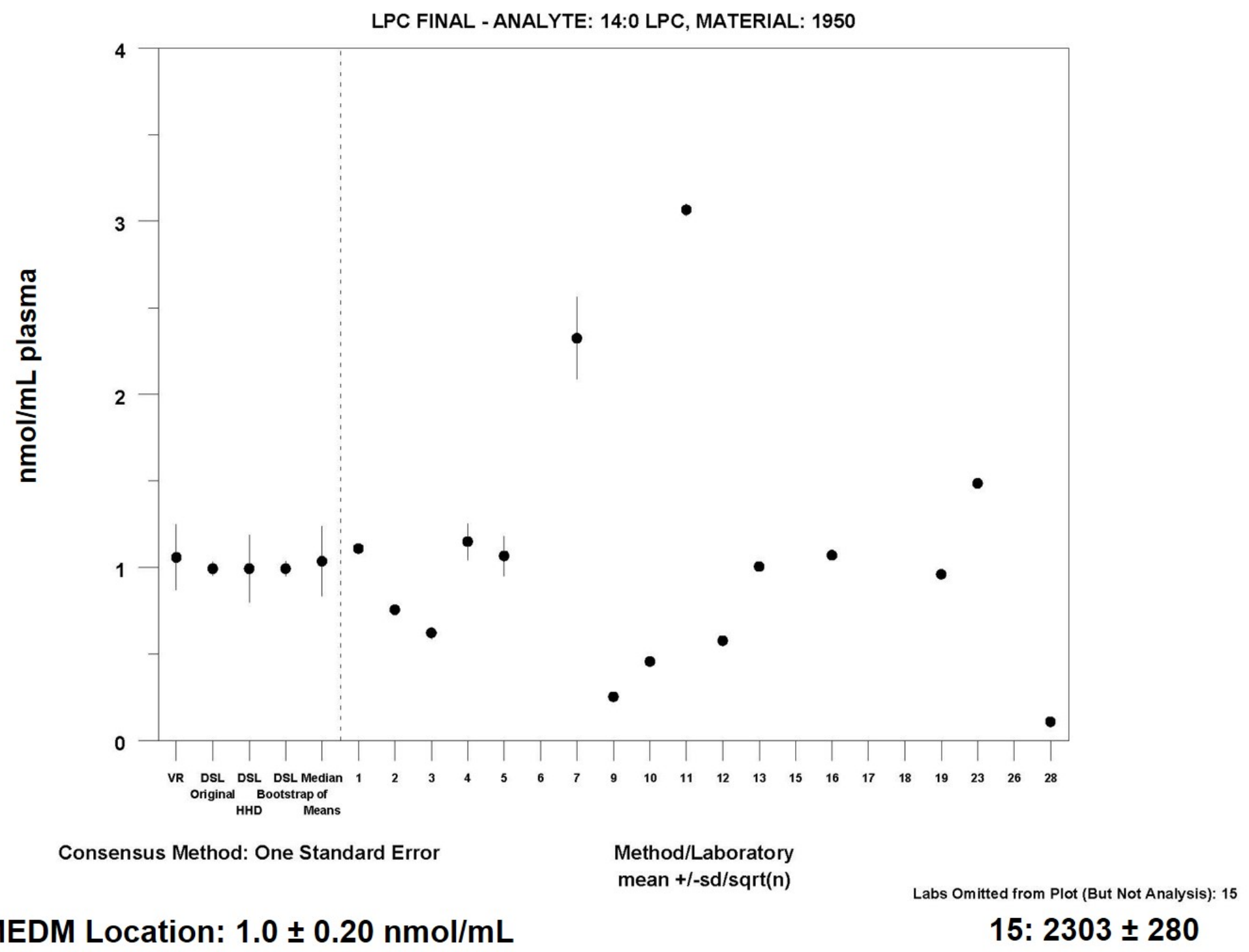




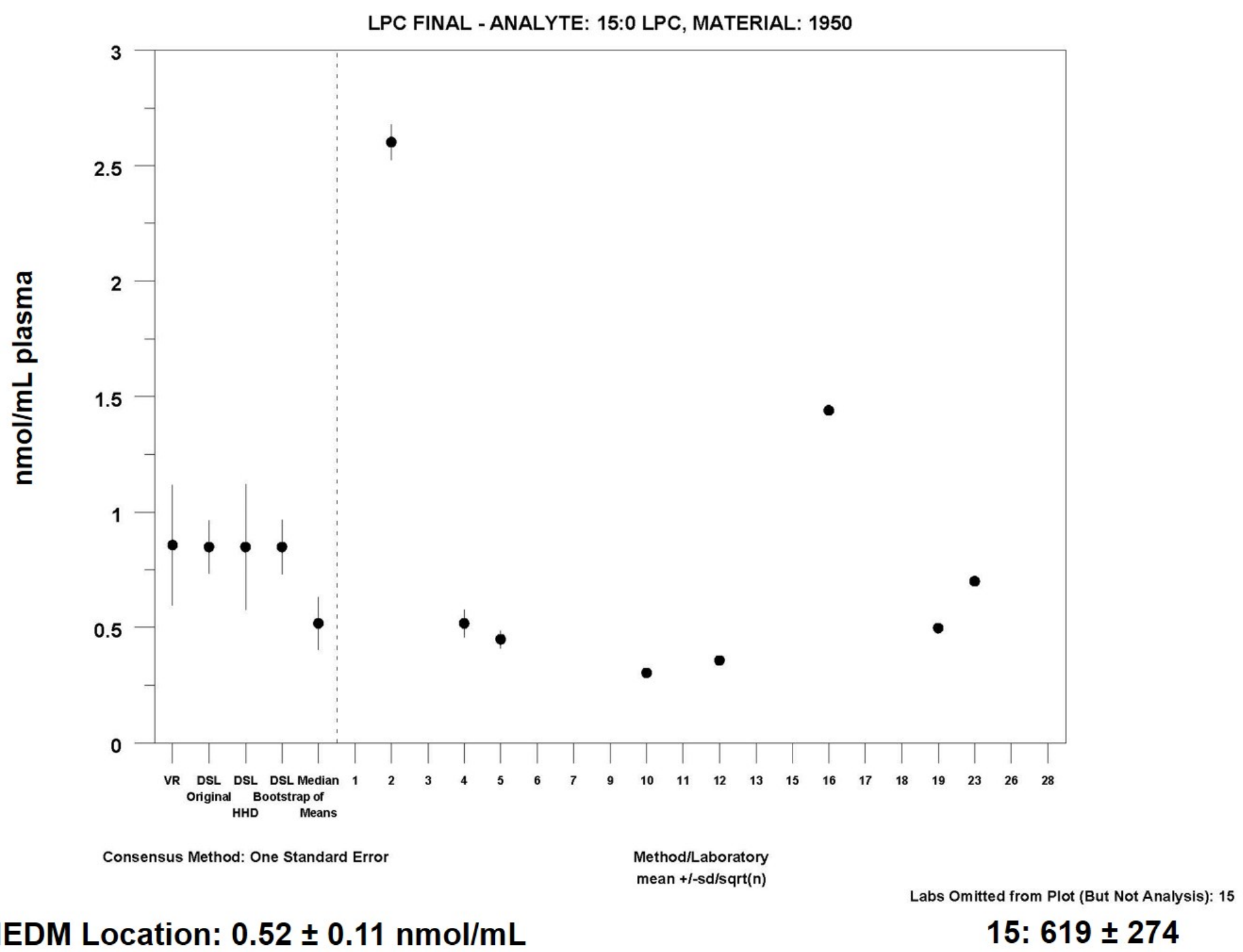




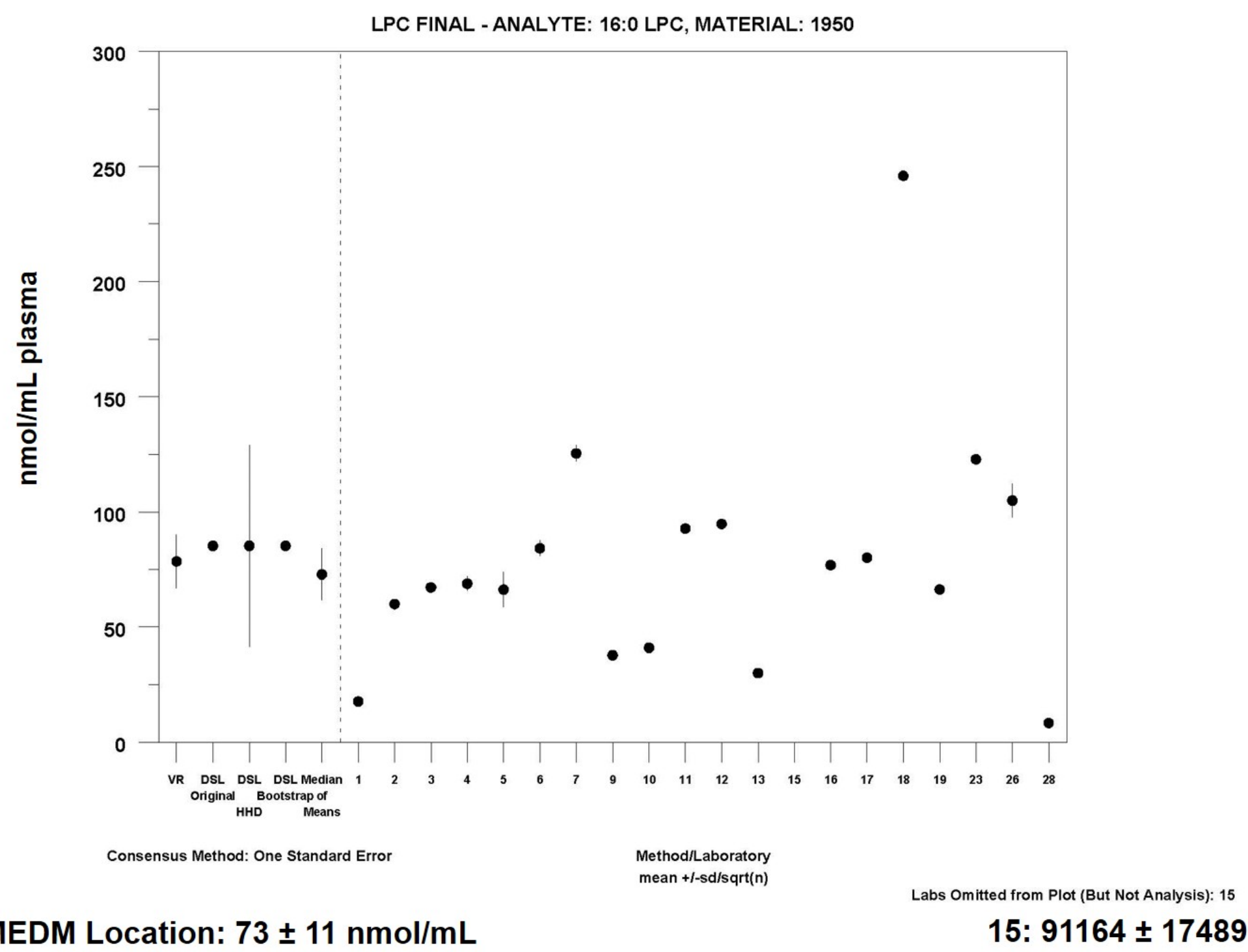




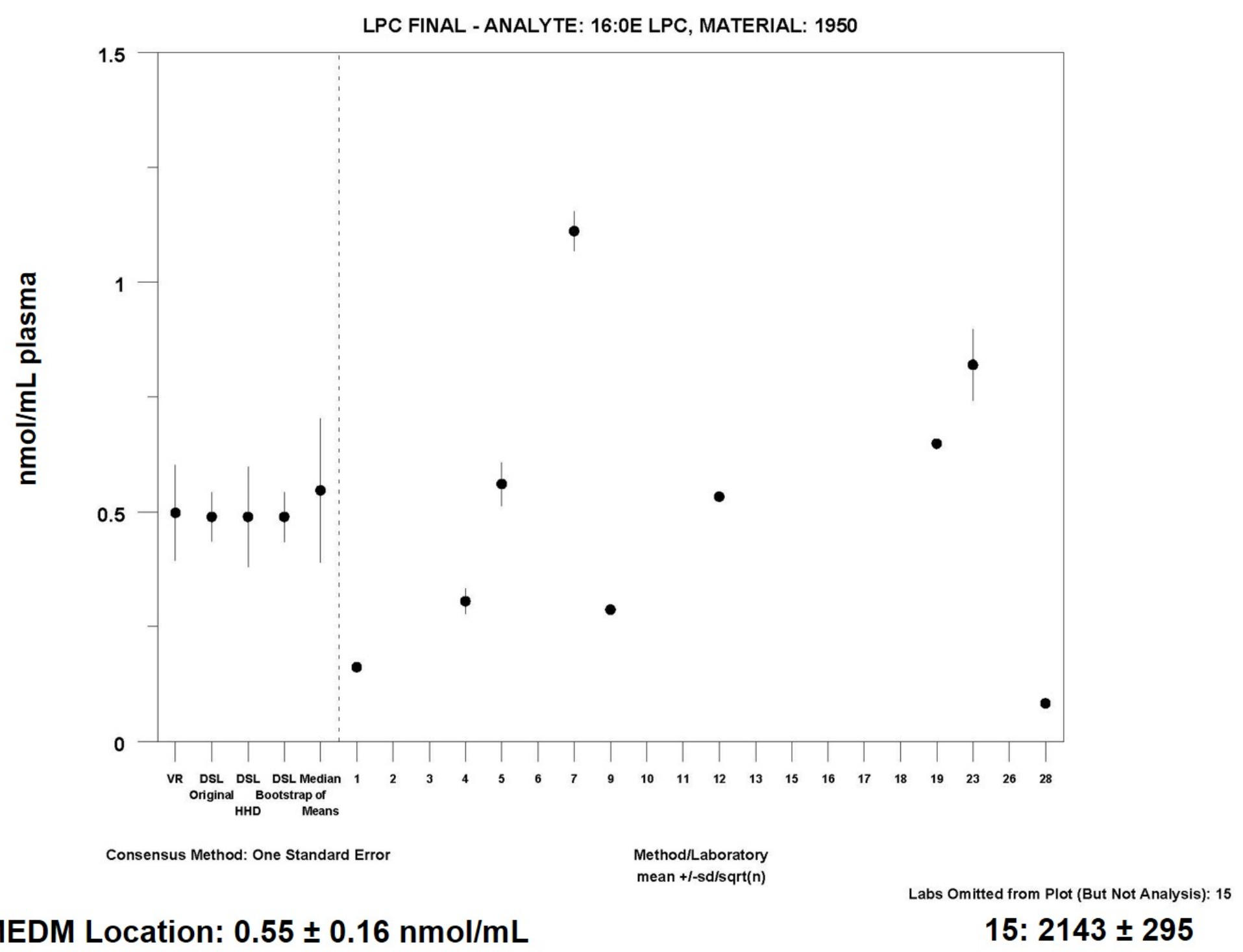




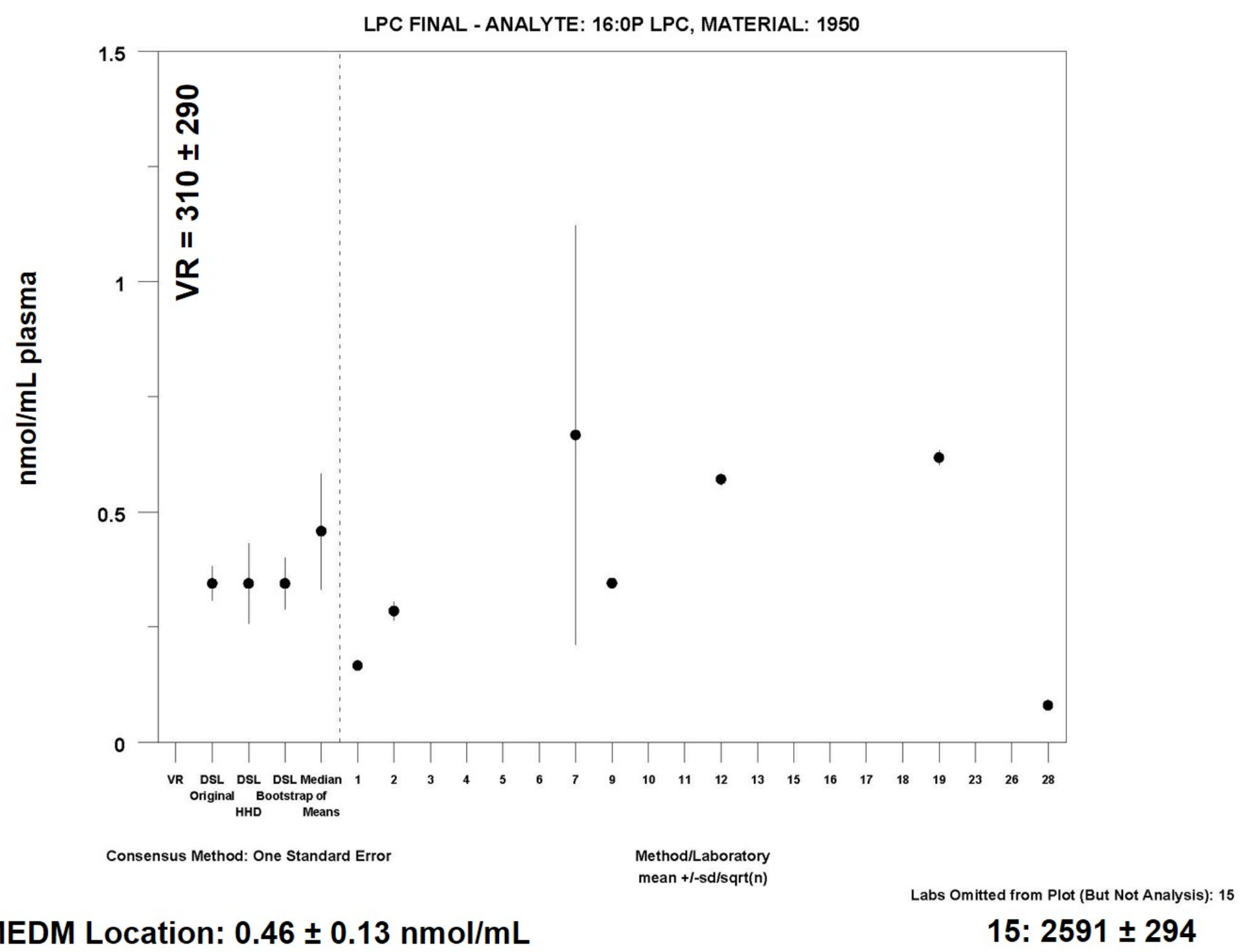




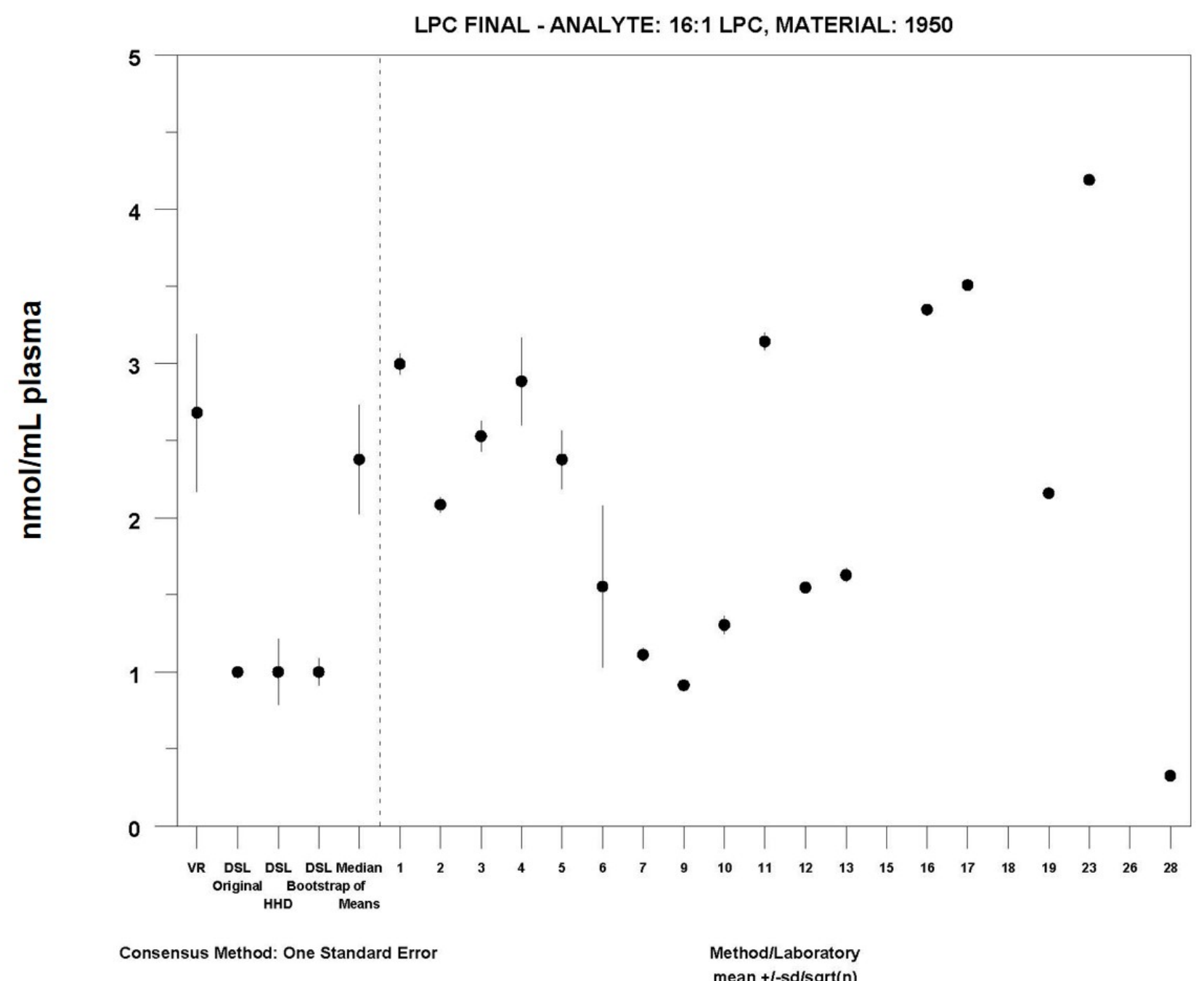

MEDM Location: $2.4 \pm 0.35 \mathrm{nmol} / \mathrm{mL}$

15: $4574 \pm 609 ; 18: 10.6 \pm 0.08$ 


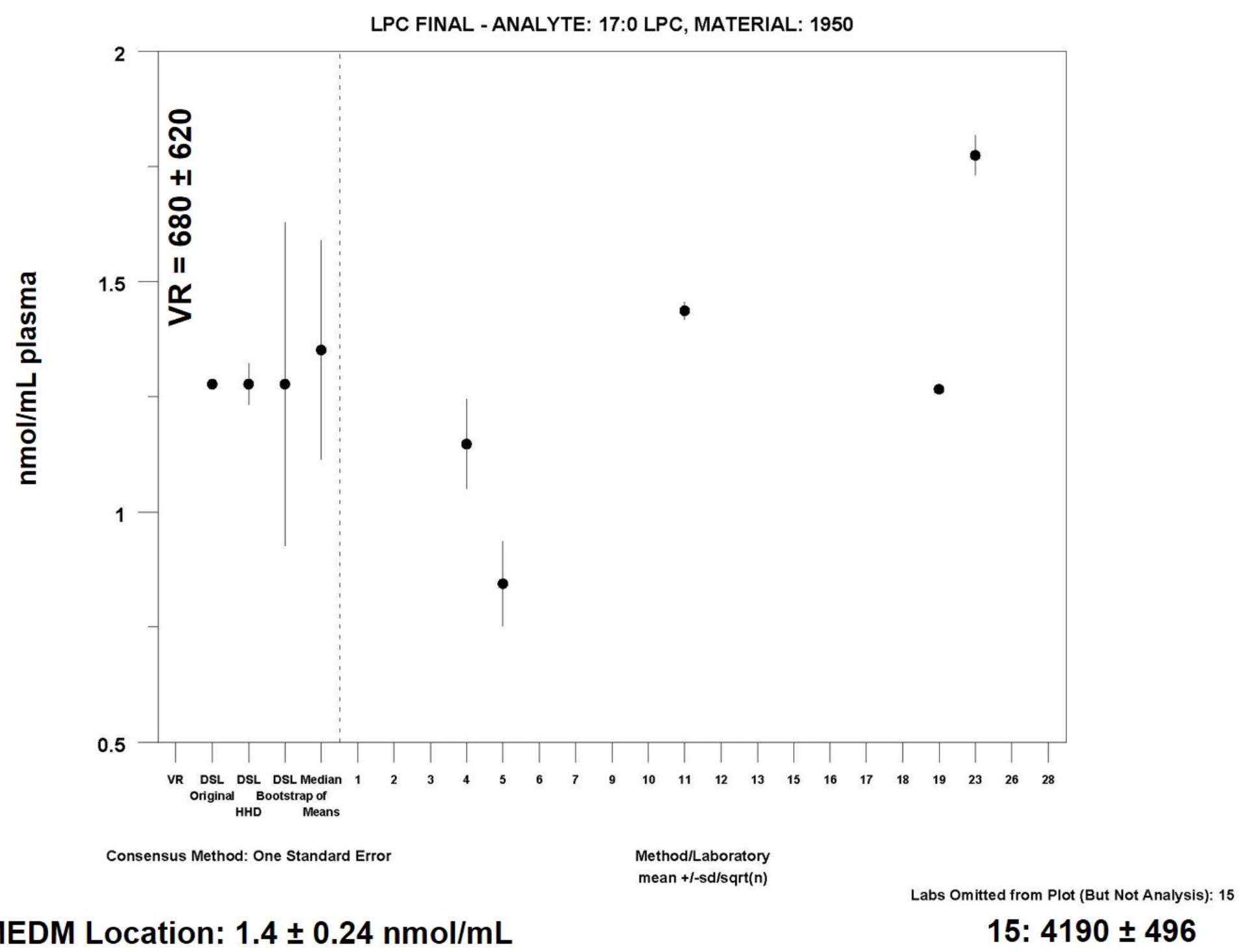




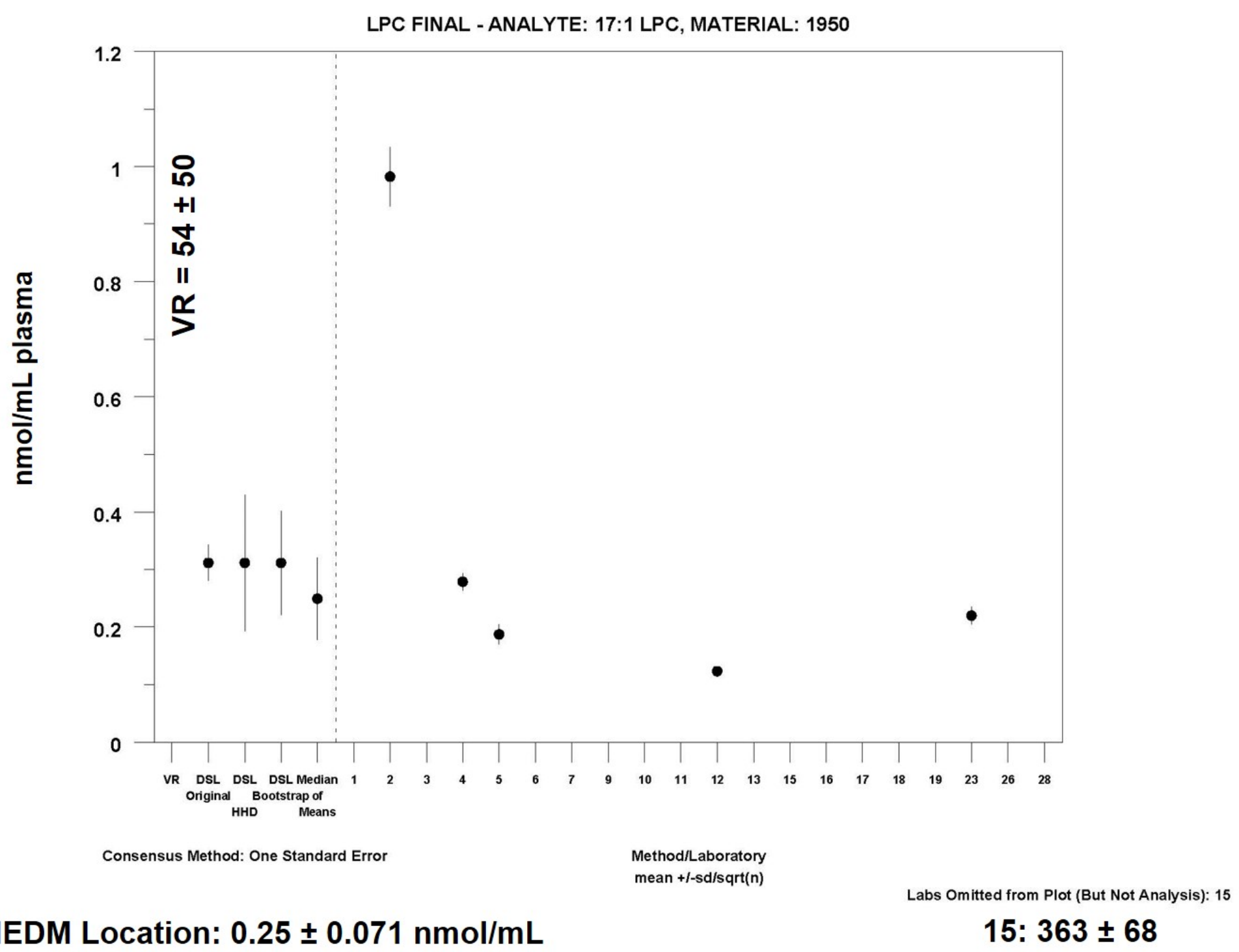




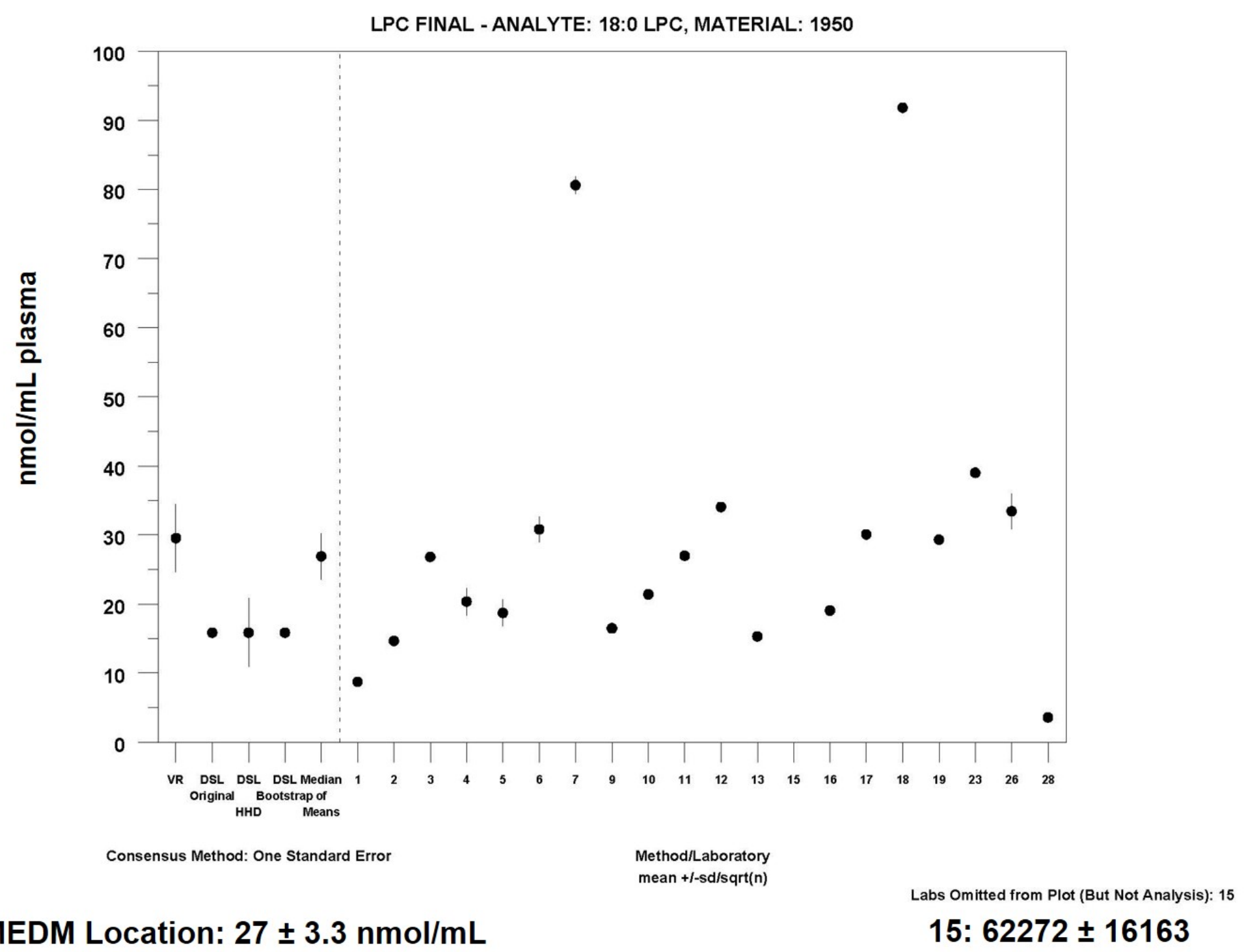




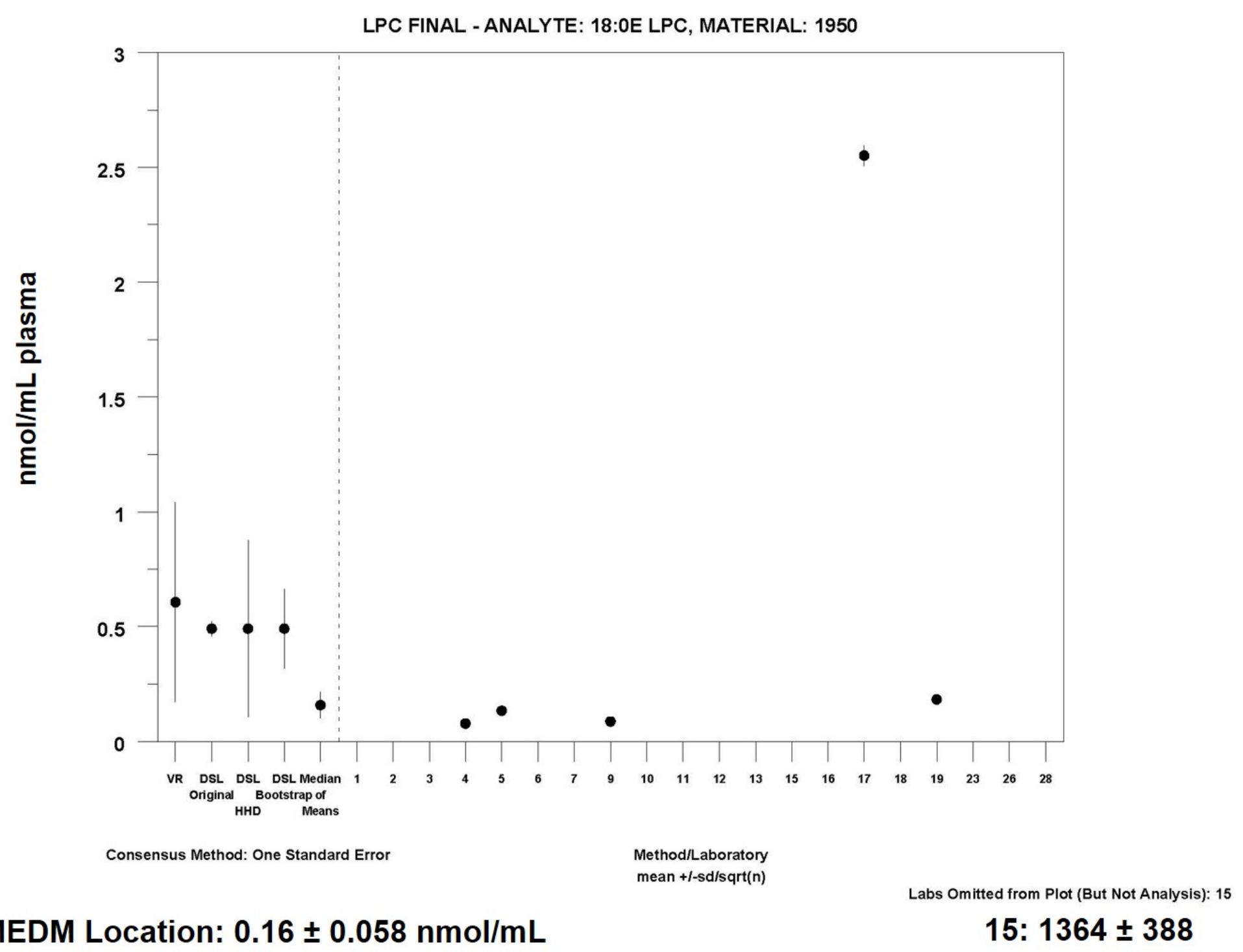




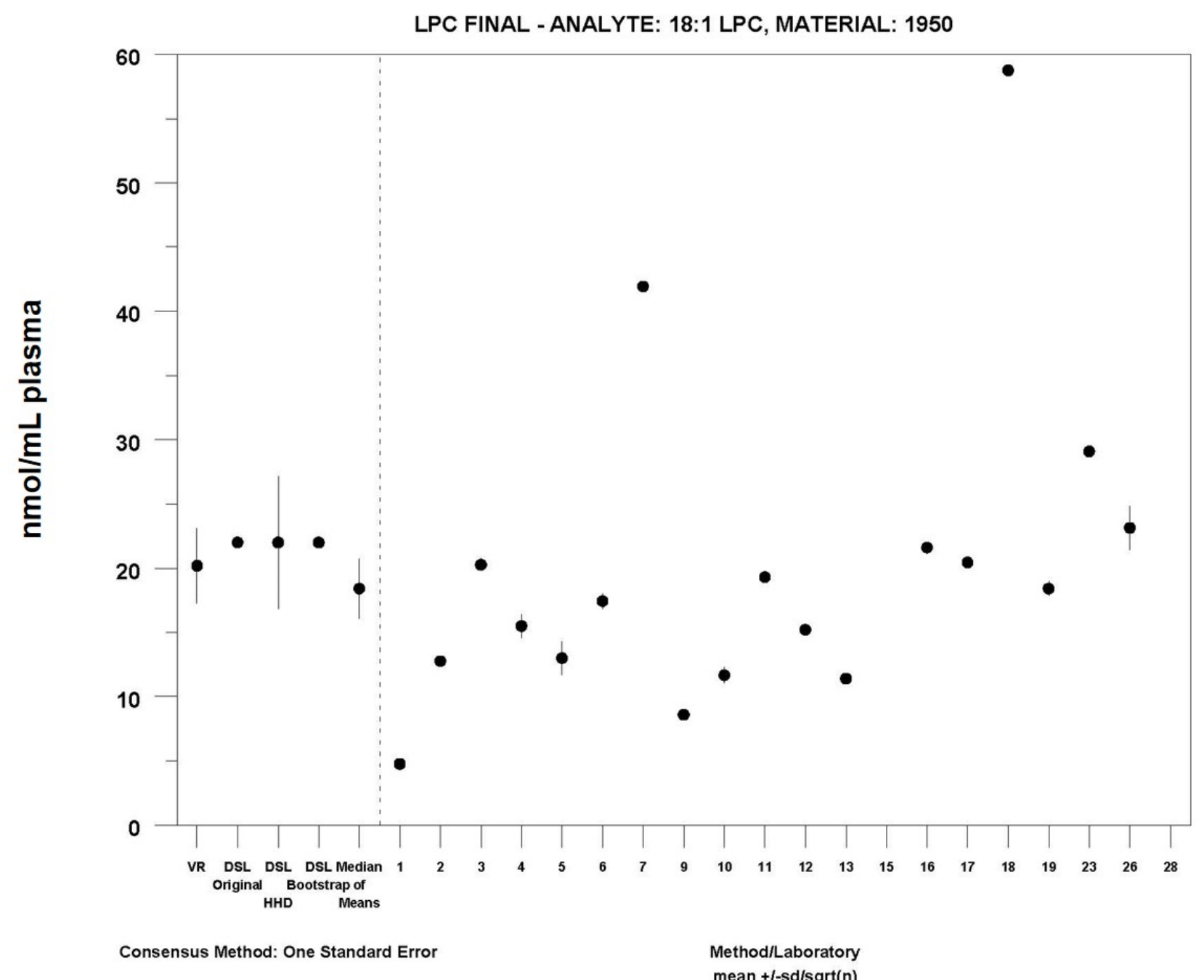

MEDM Location: $18 \pm 2.3 \mathrm{nmol} / \mathrm{mL}$

Labs Omitted from Plot (But Not Analysis): 15

15: $76657 \pm 11374$ 


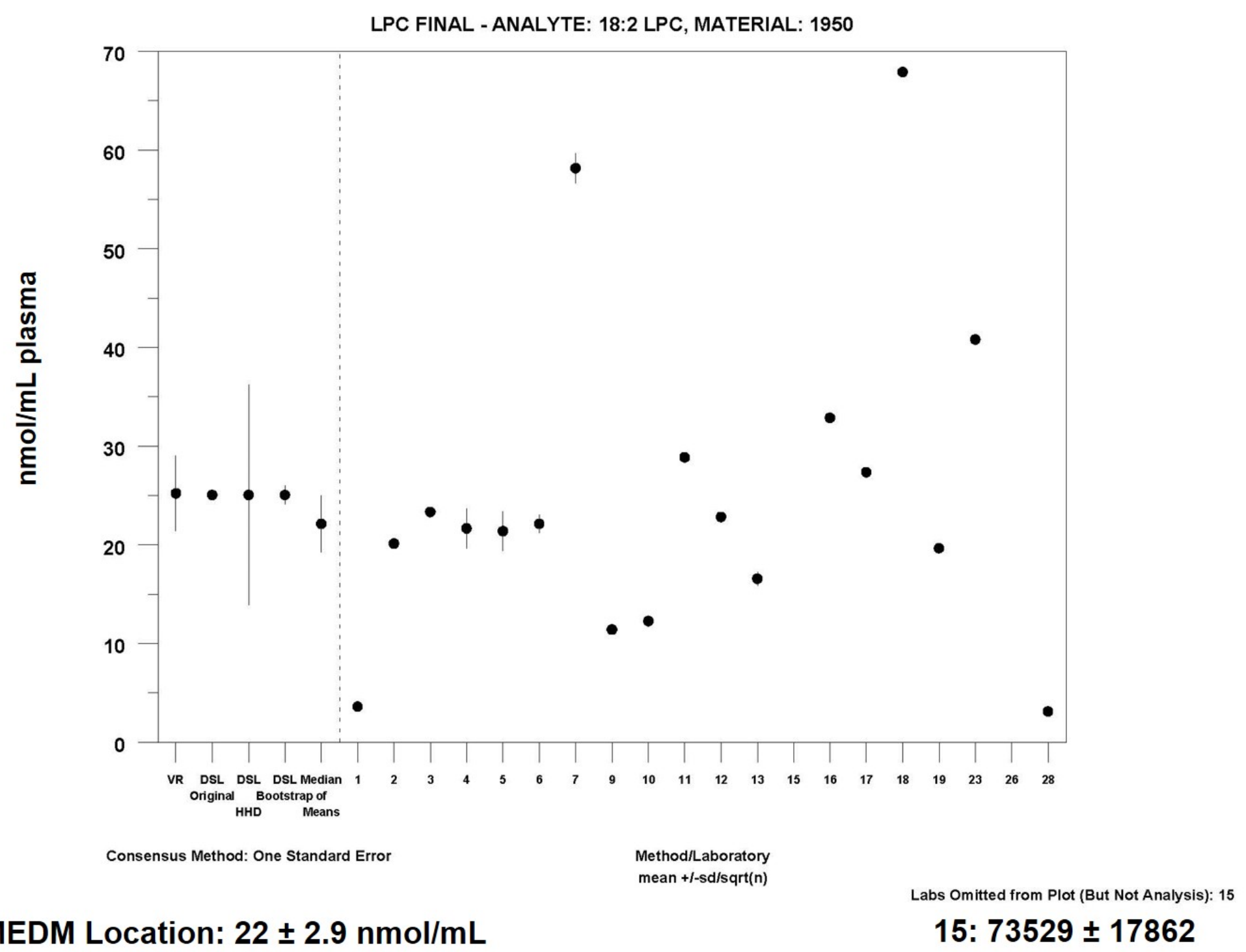




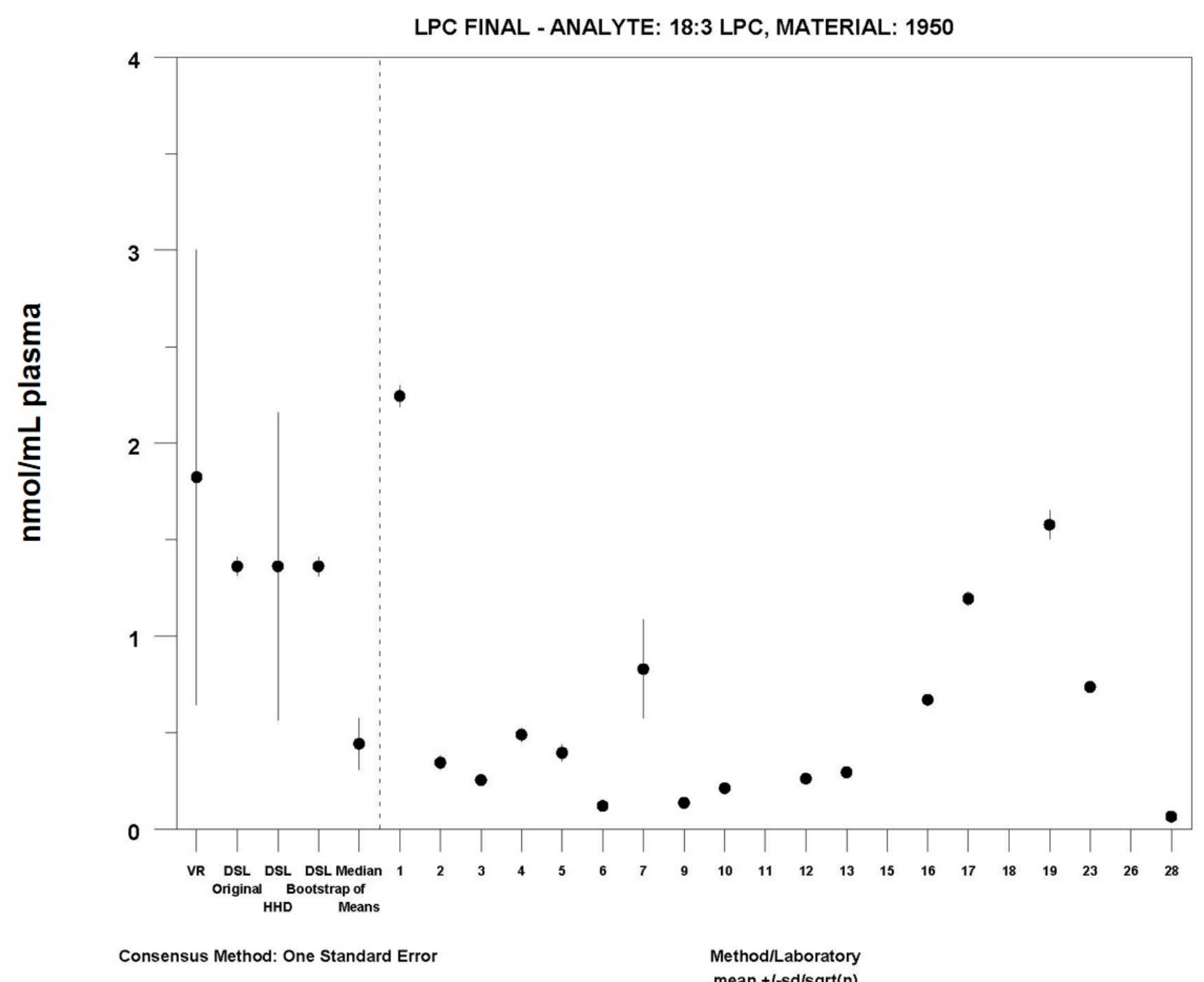

MEDM Location: $0.44 \pm 0.13 \mathrm{nmol} / \mathrm{mL}$

Labs Omitted from Plot (But Not Analysis): 1518

15: $2027 \pm 873 ; 18: 21 \pm 0.3$ 


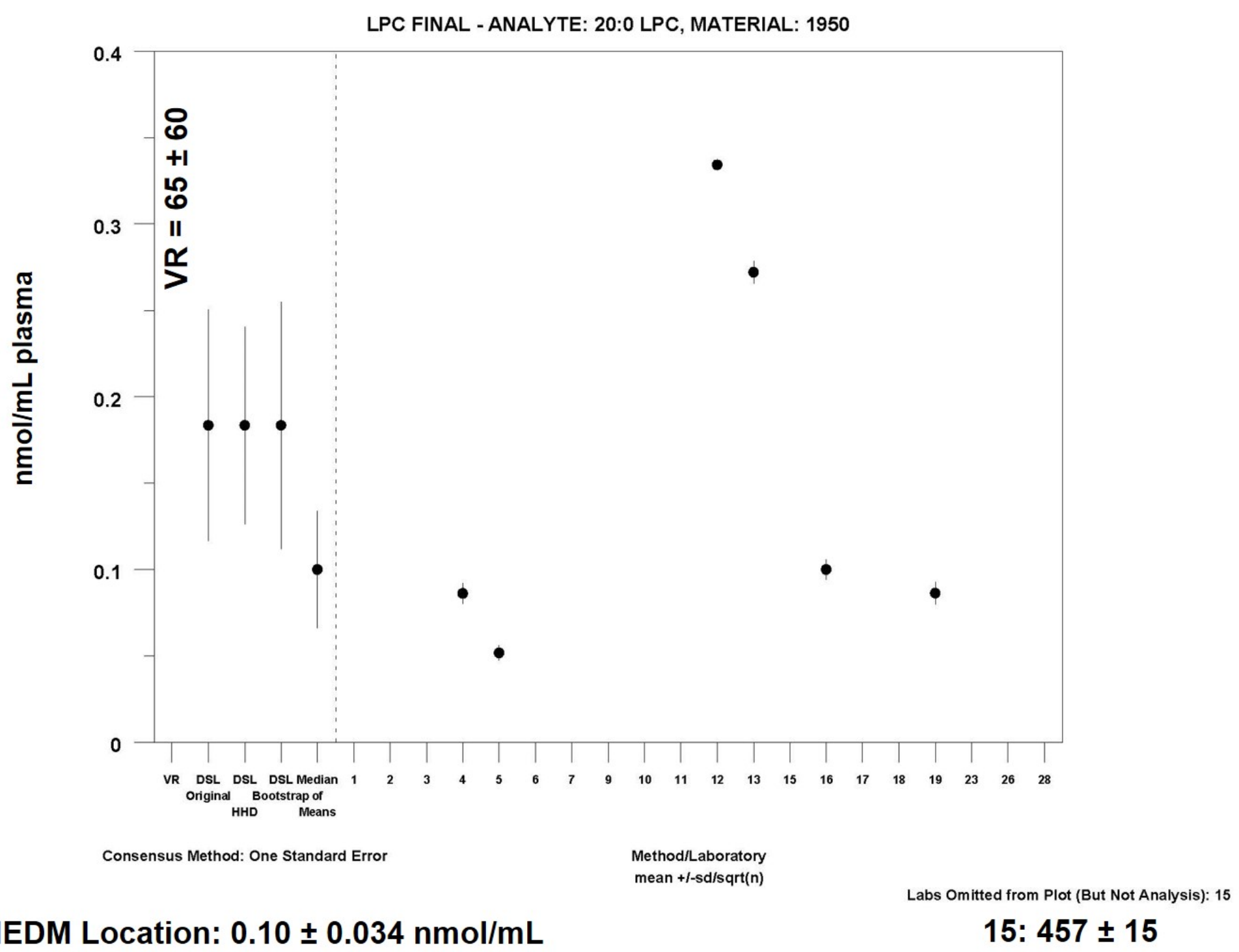




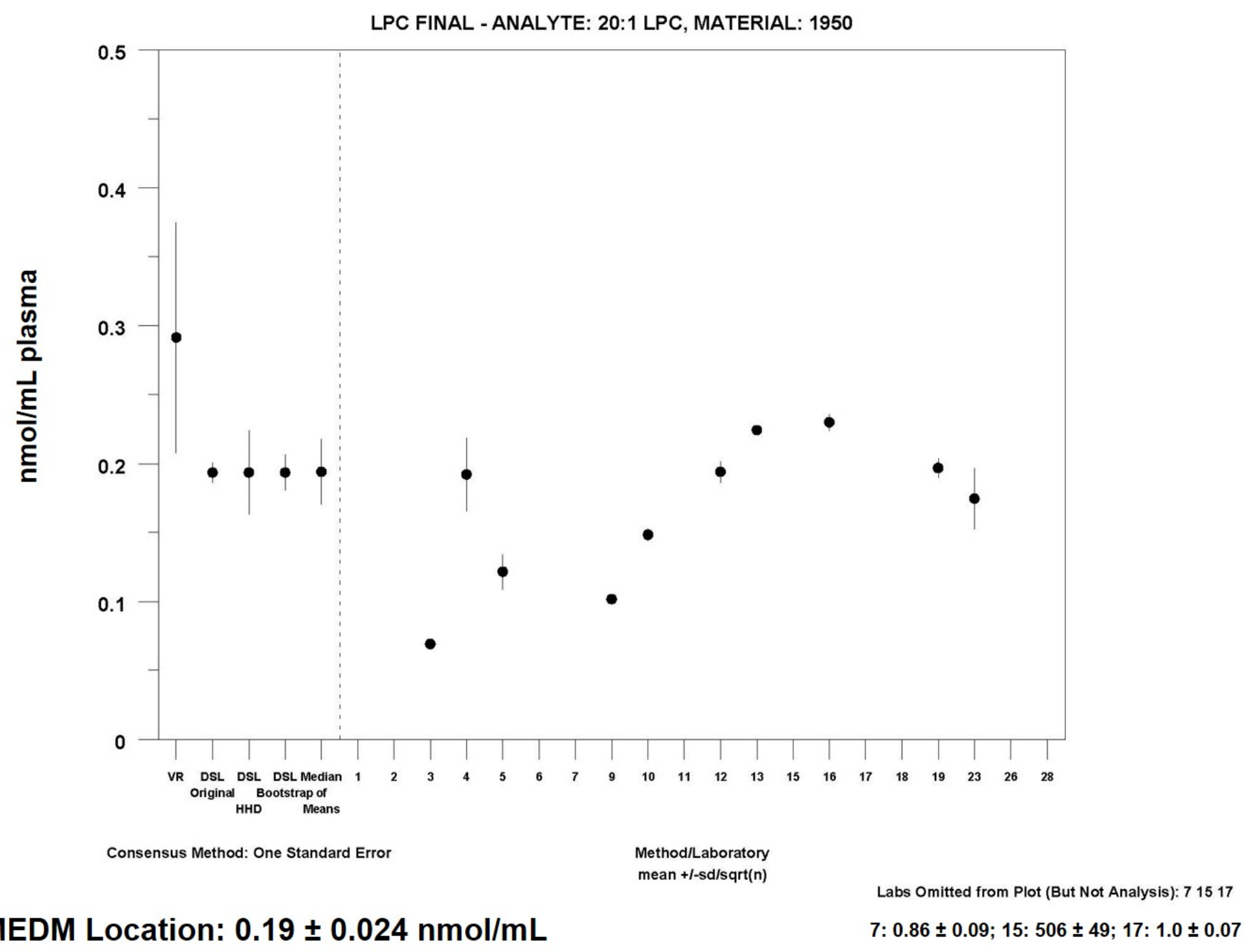




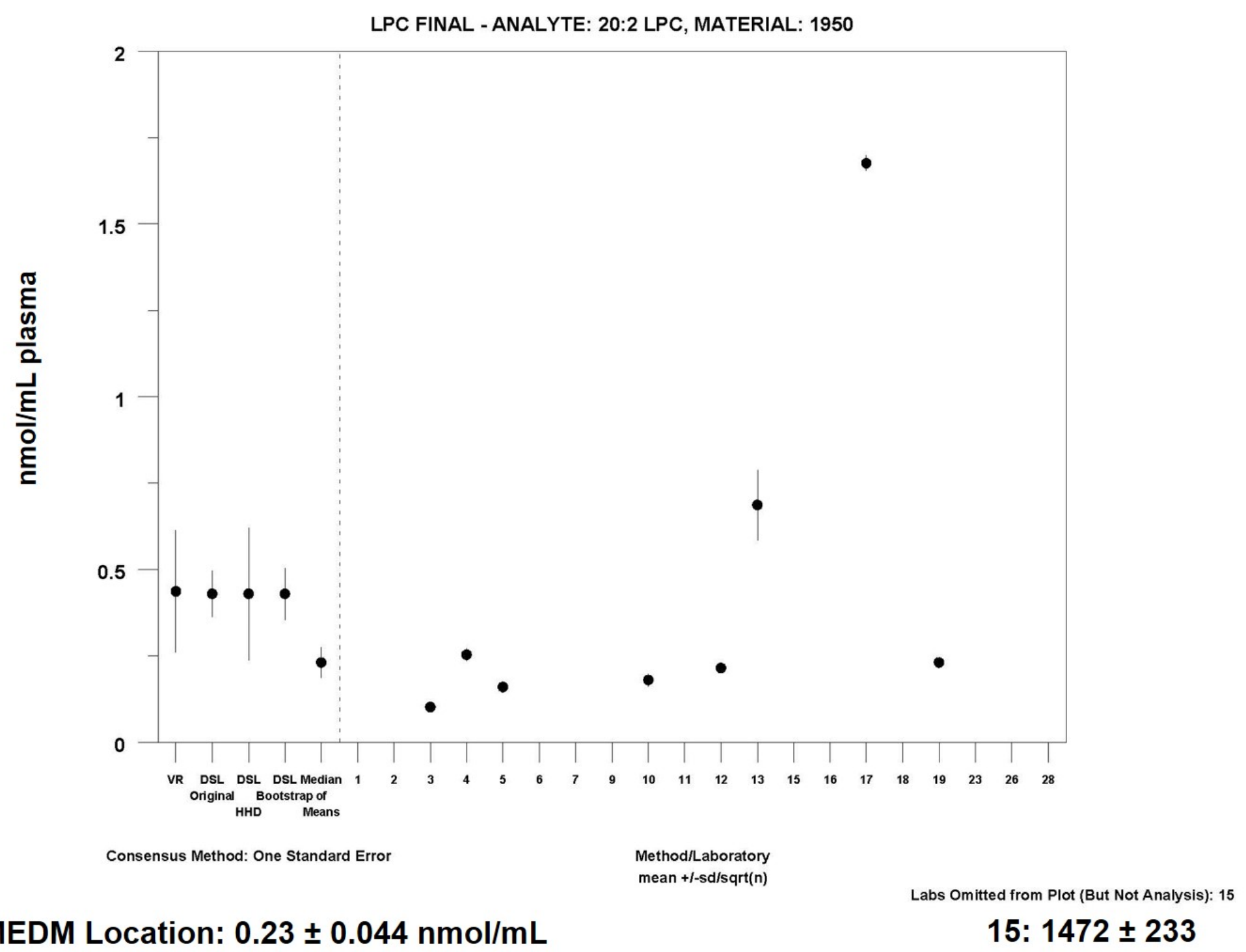




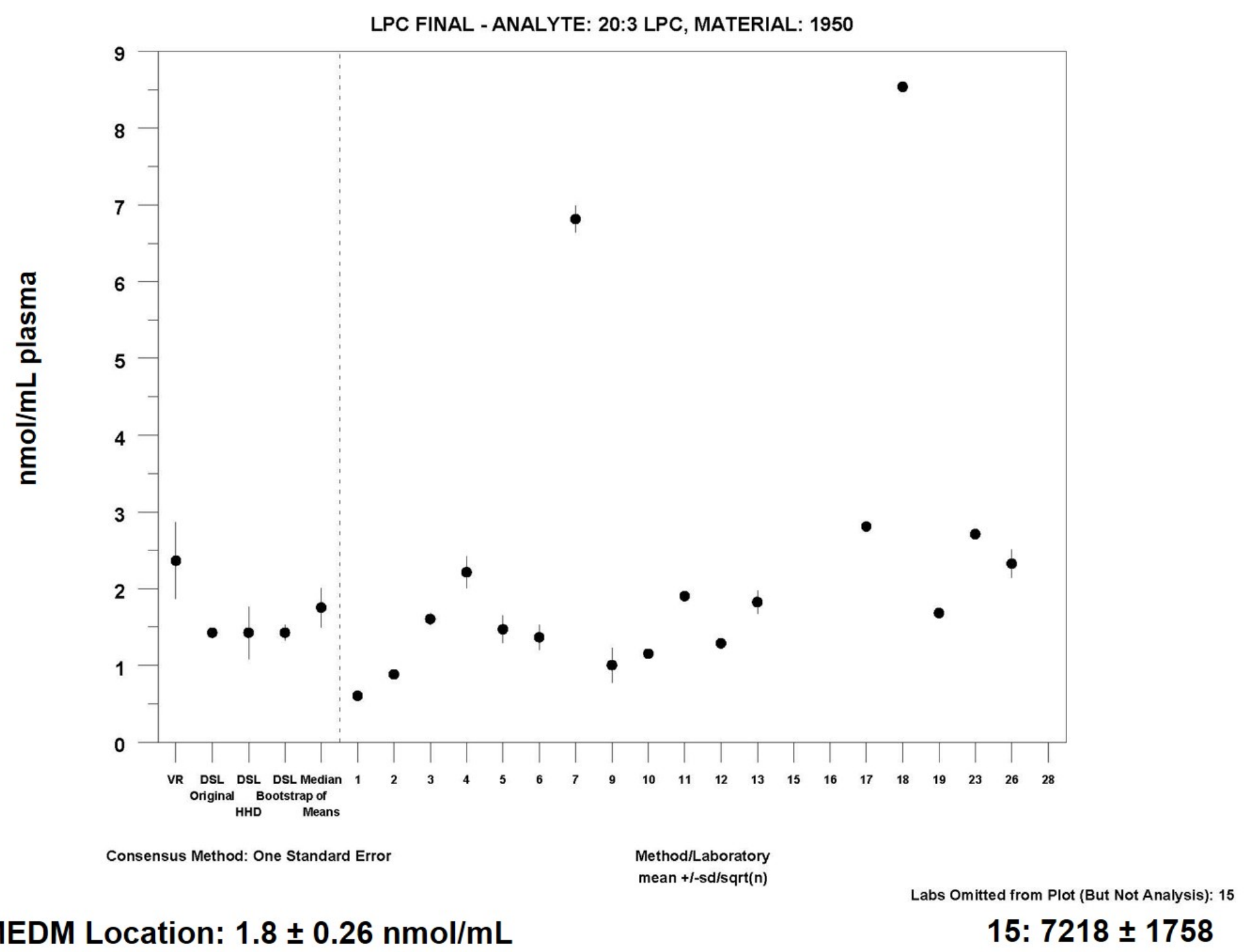




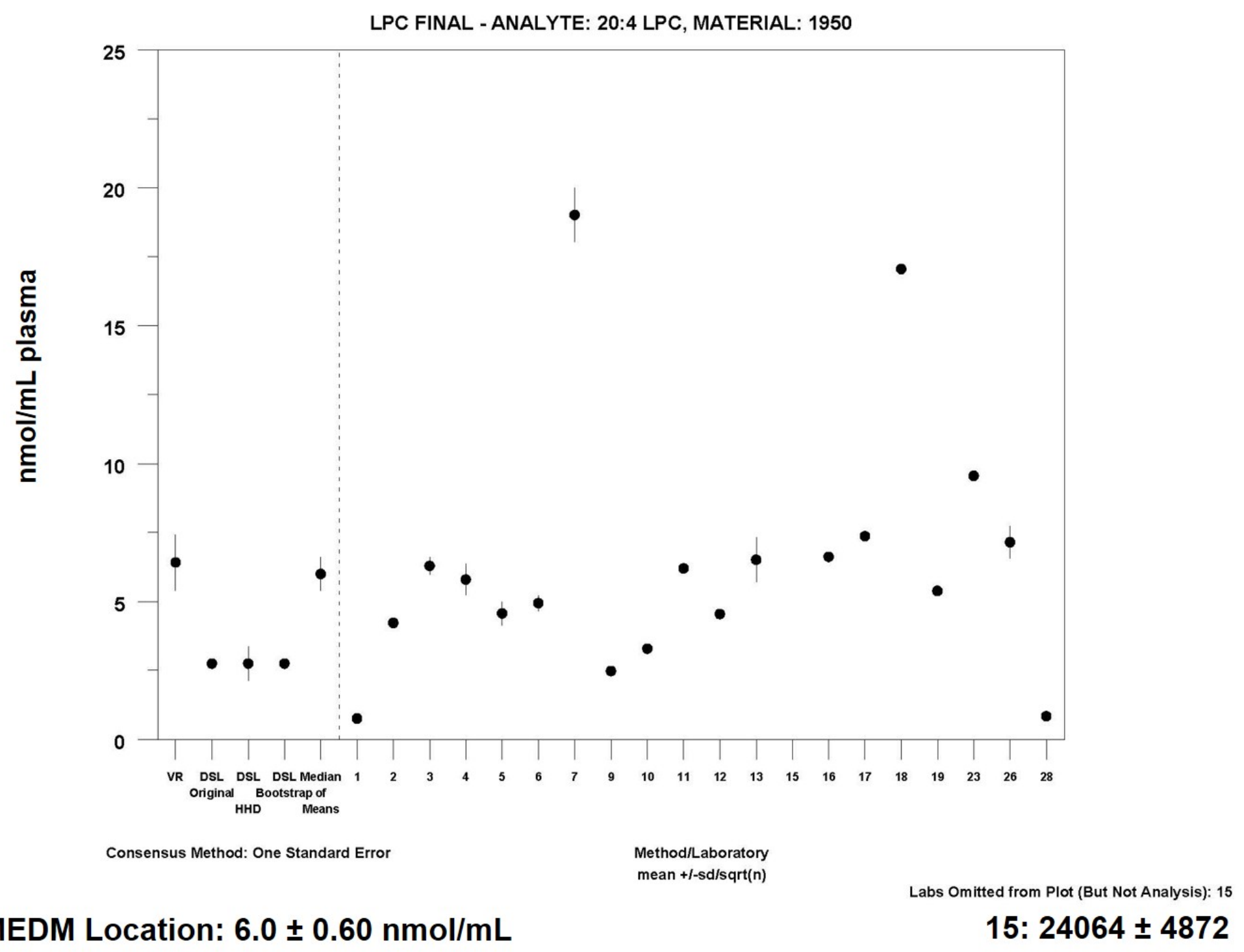




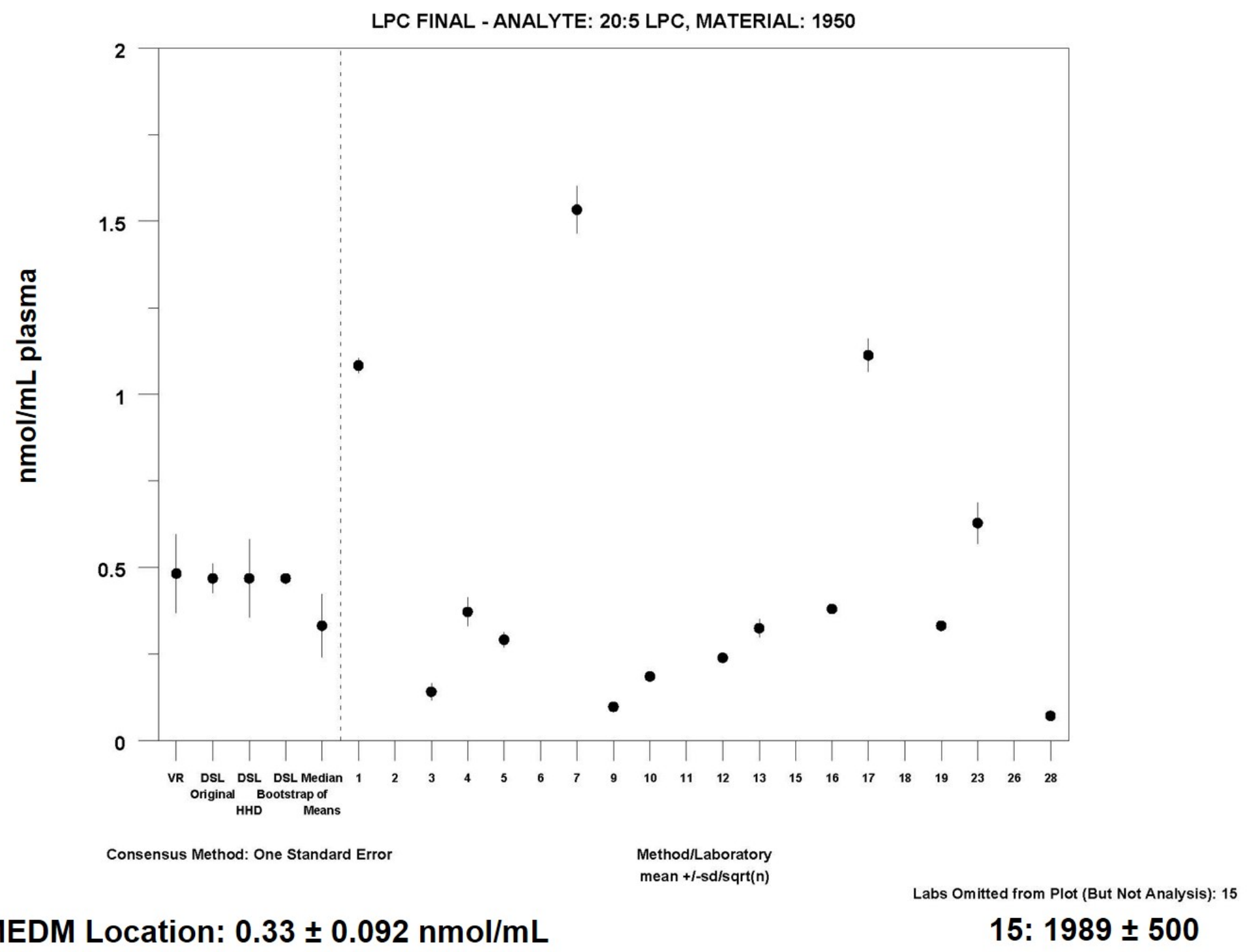




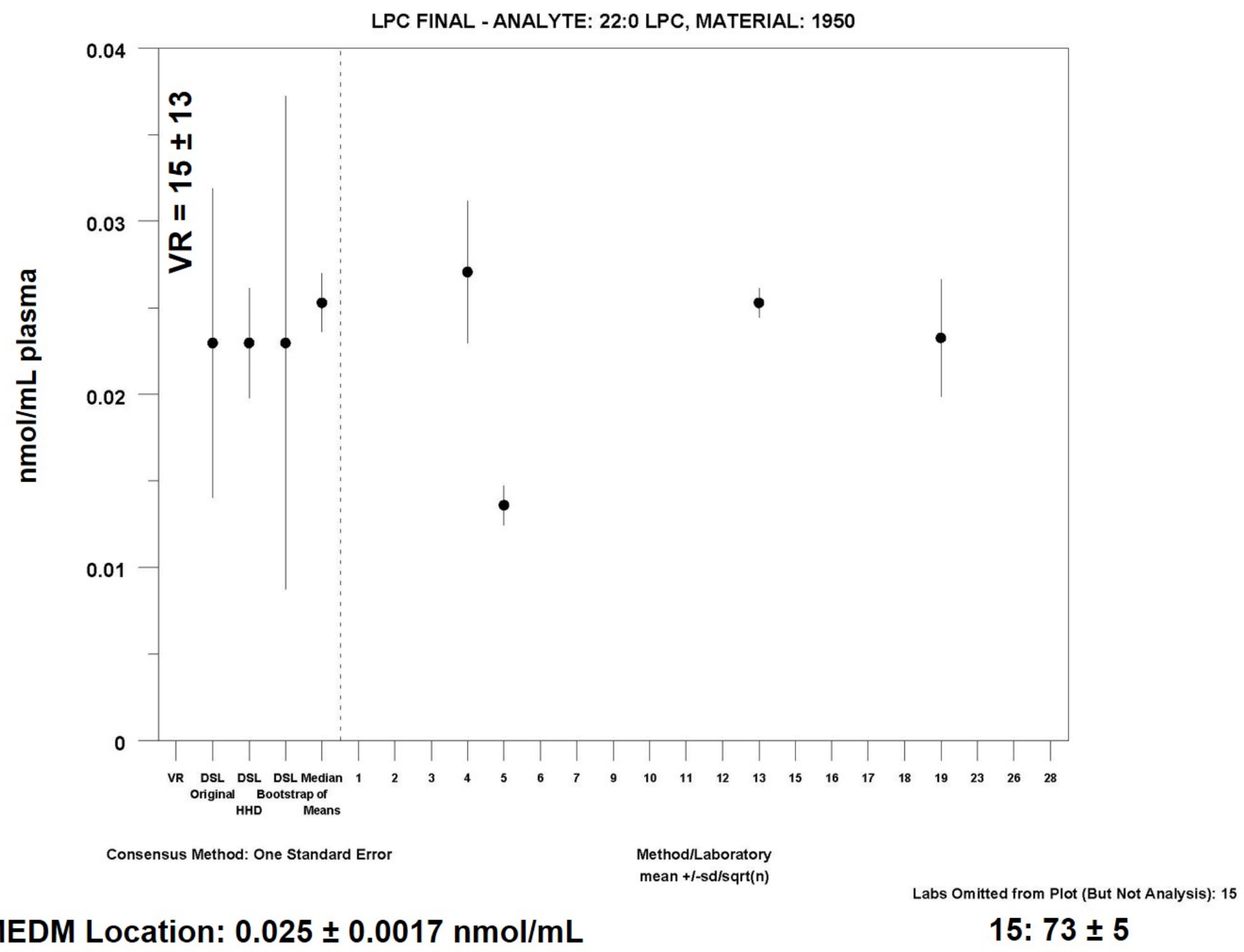




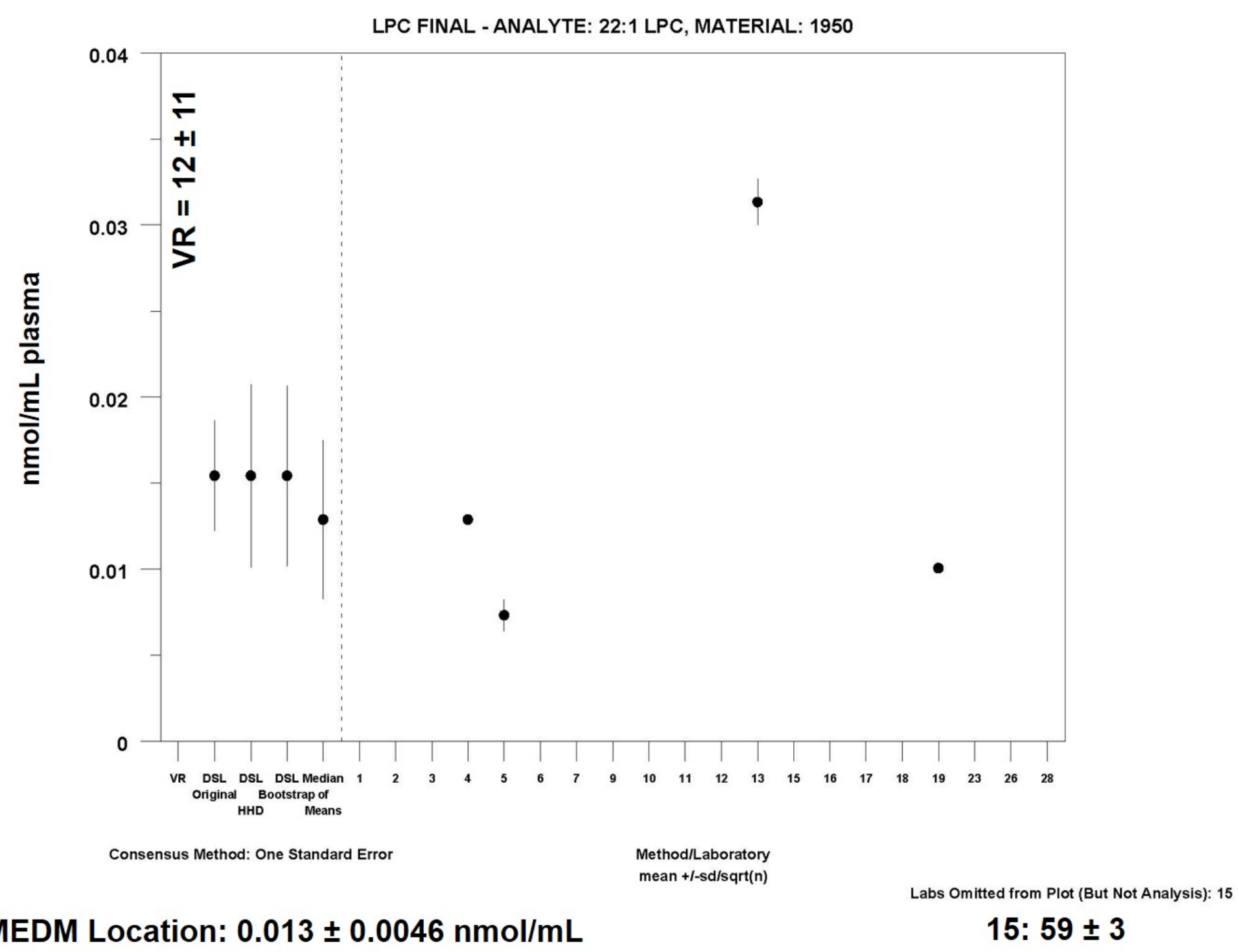




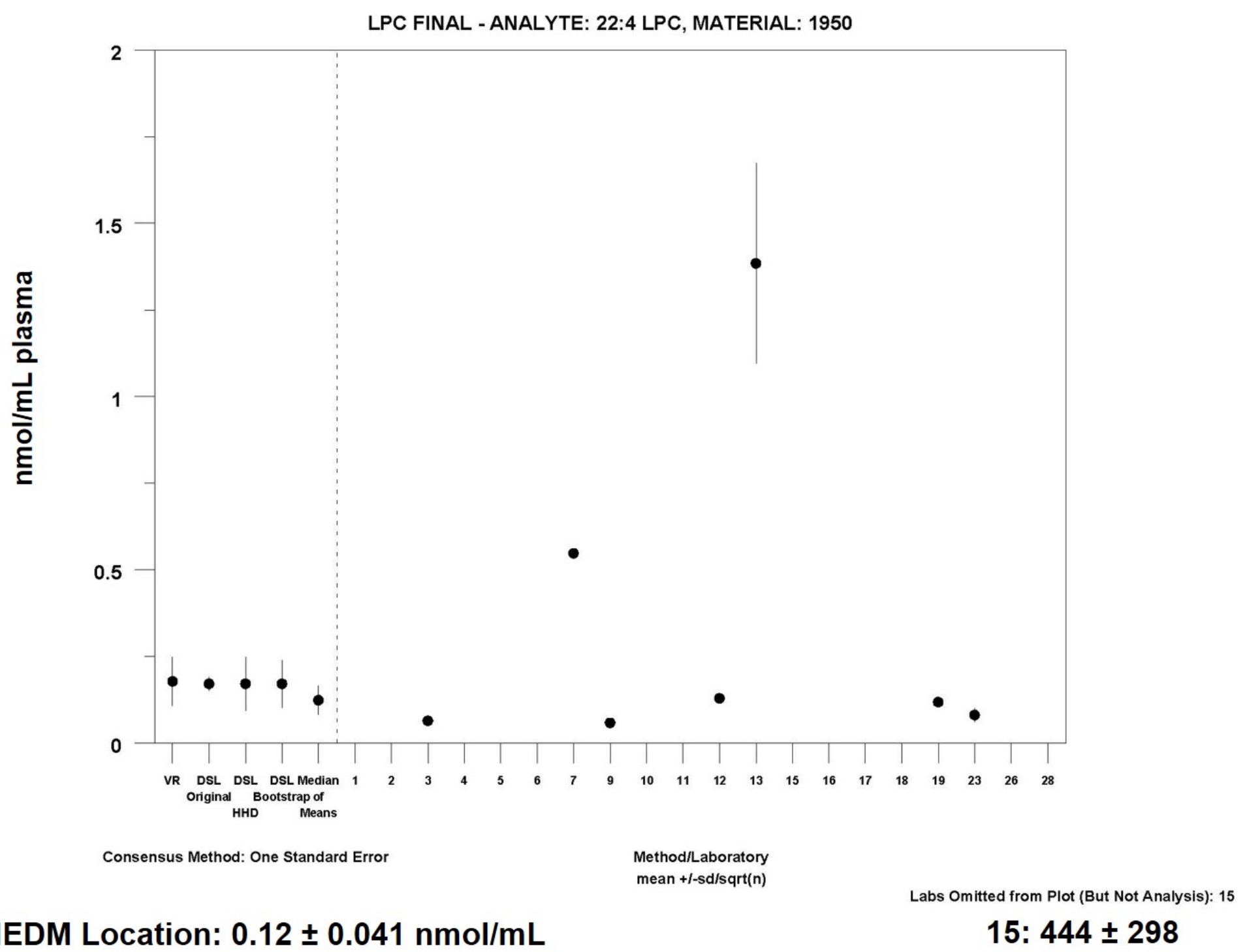




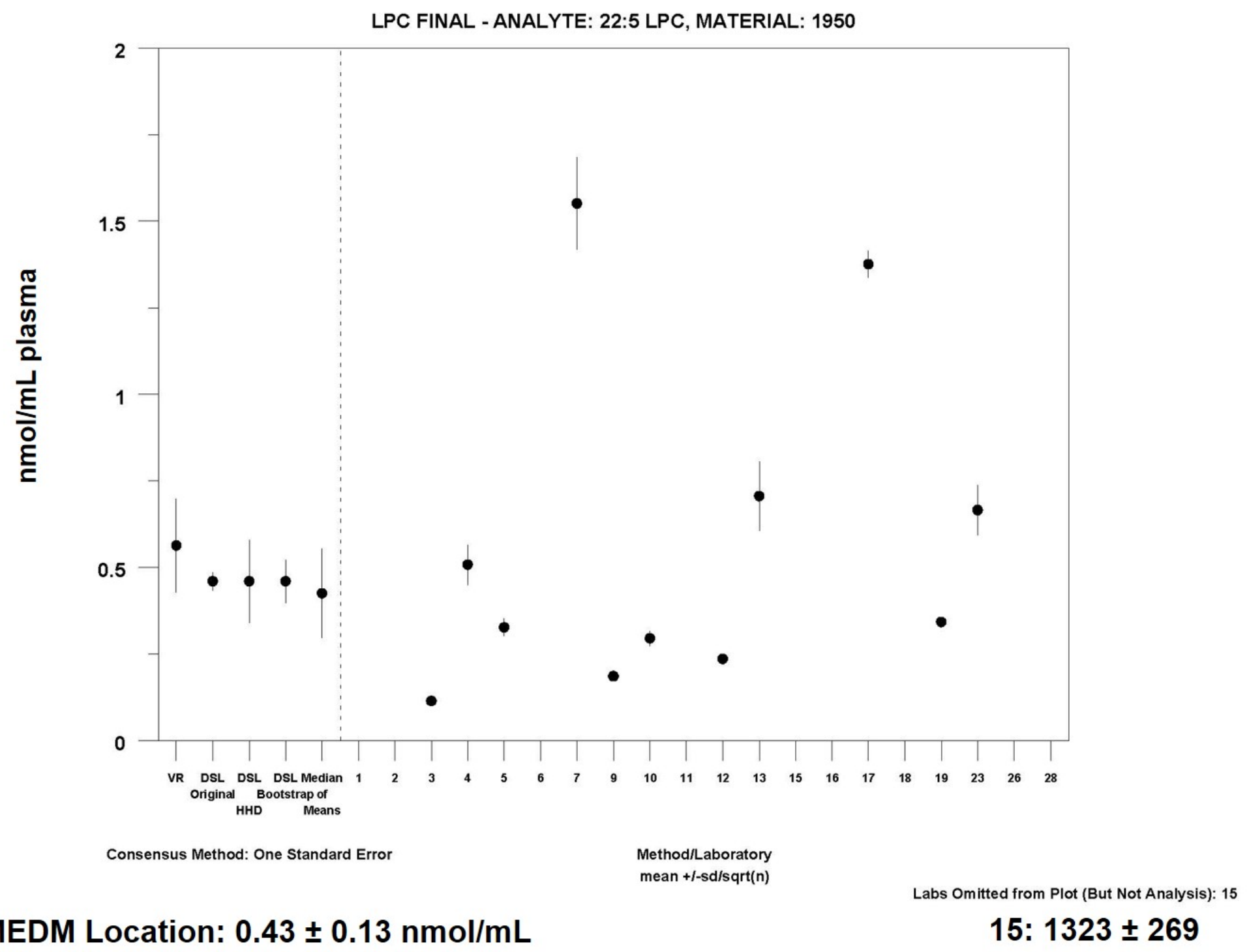




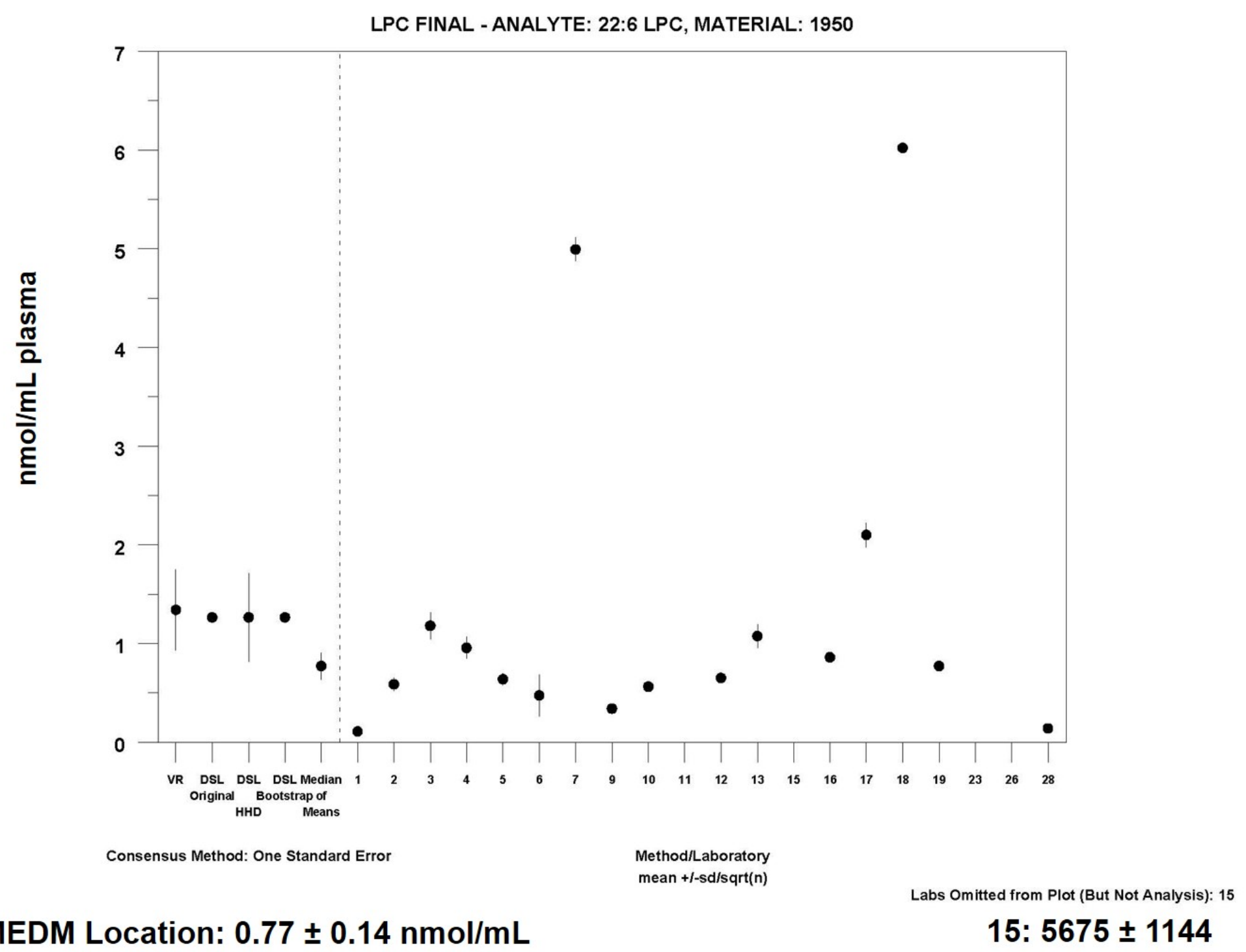




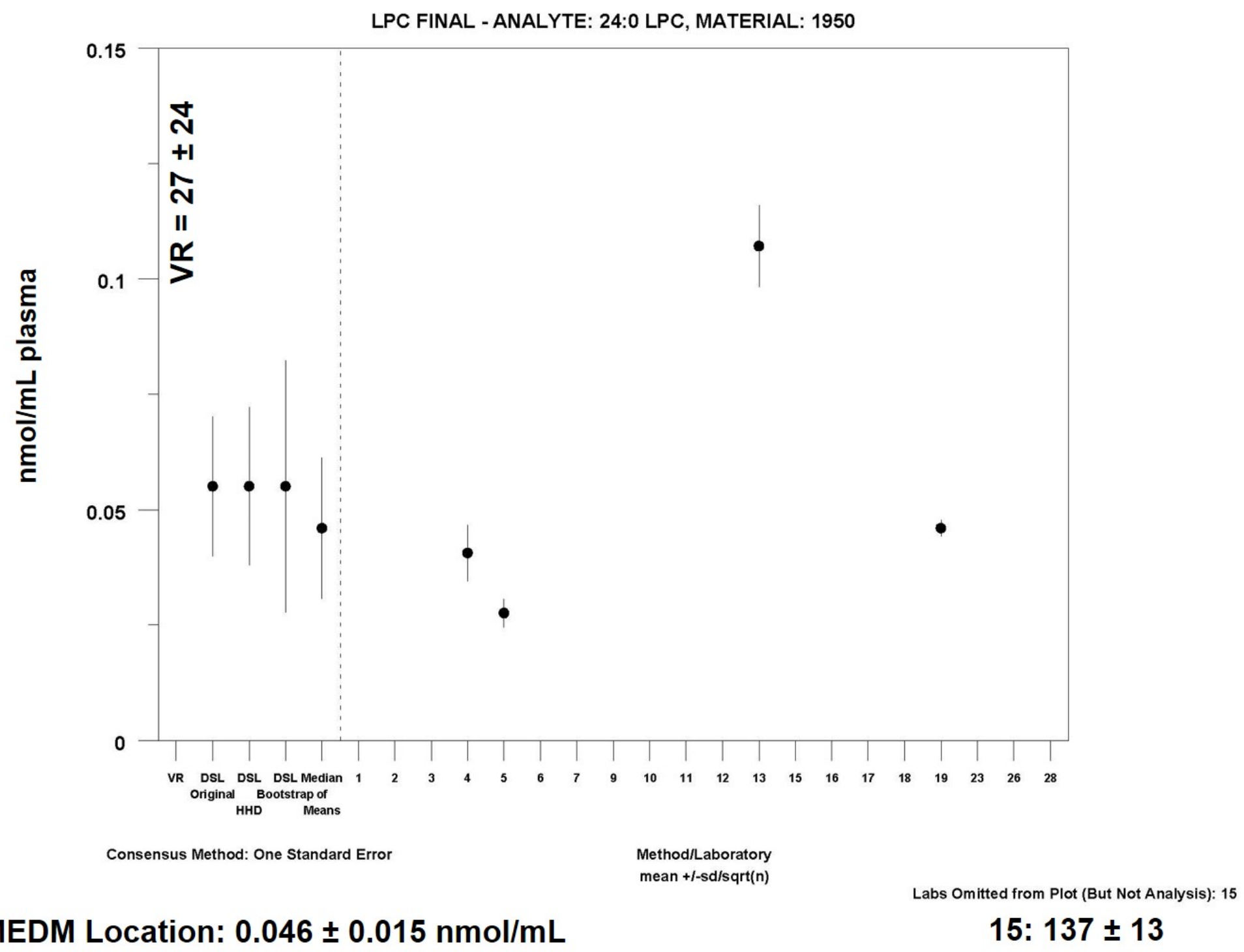




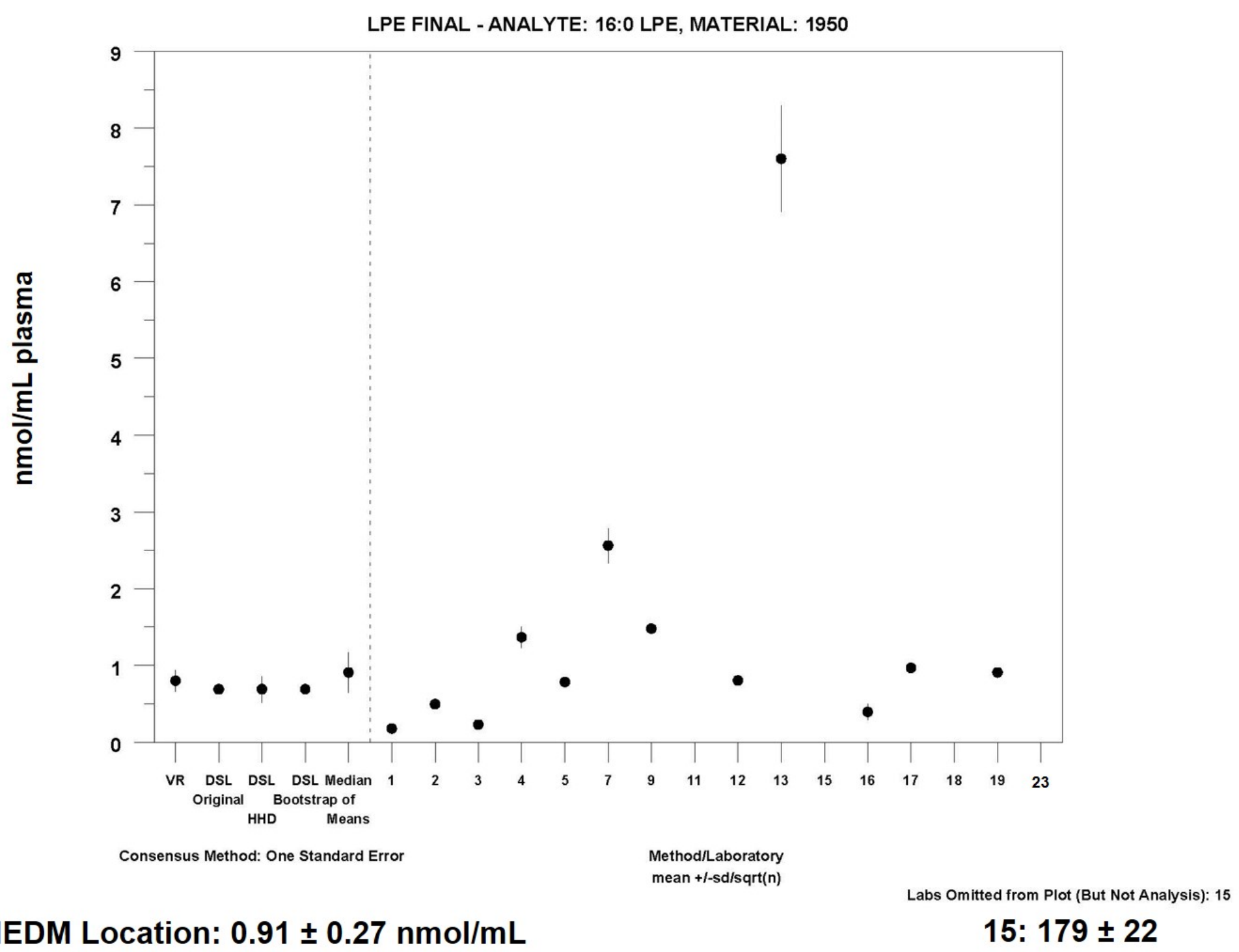




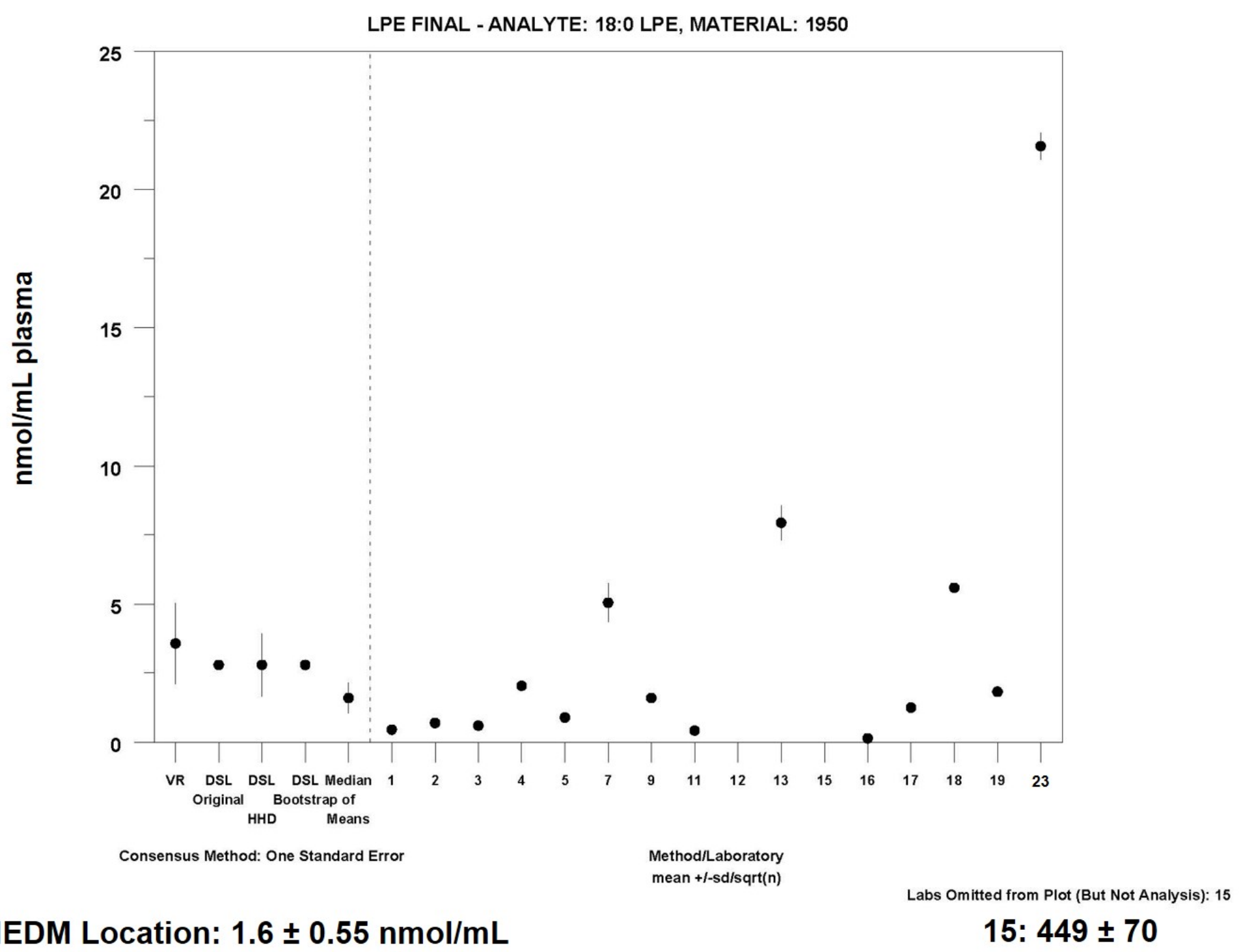




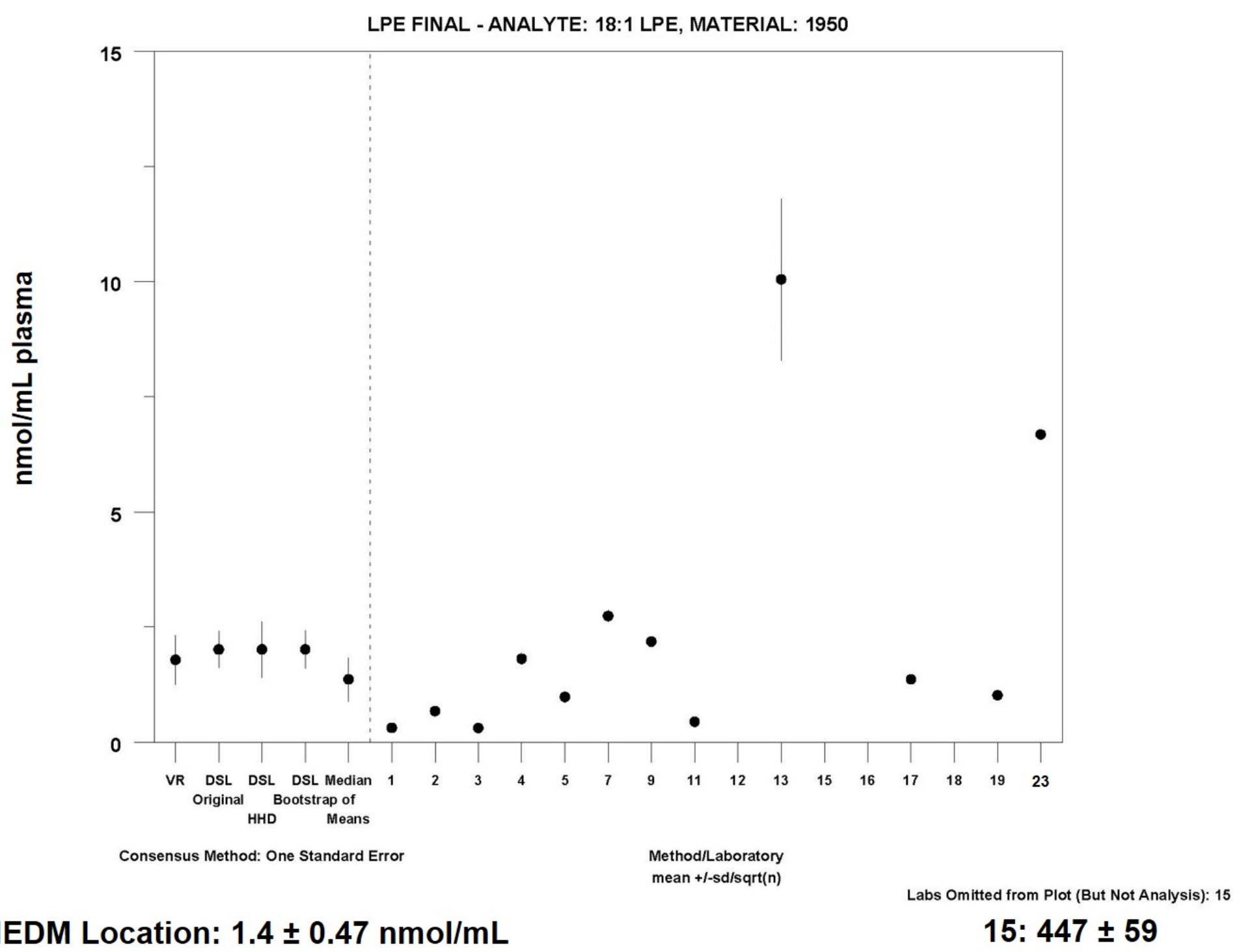




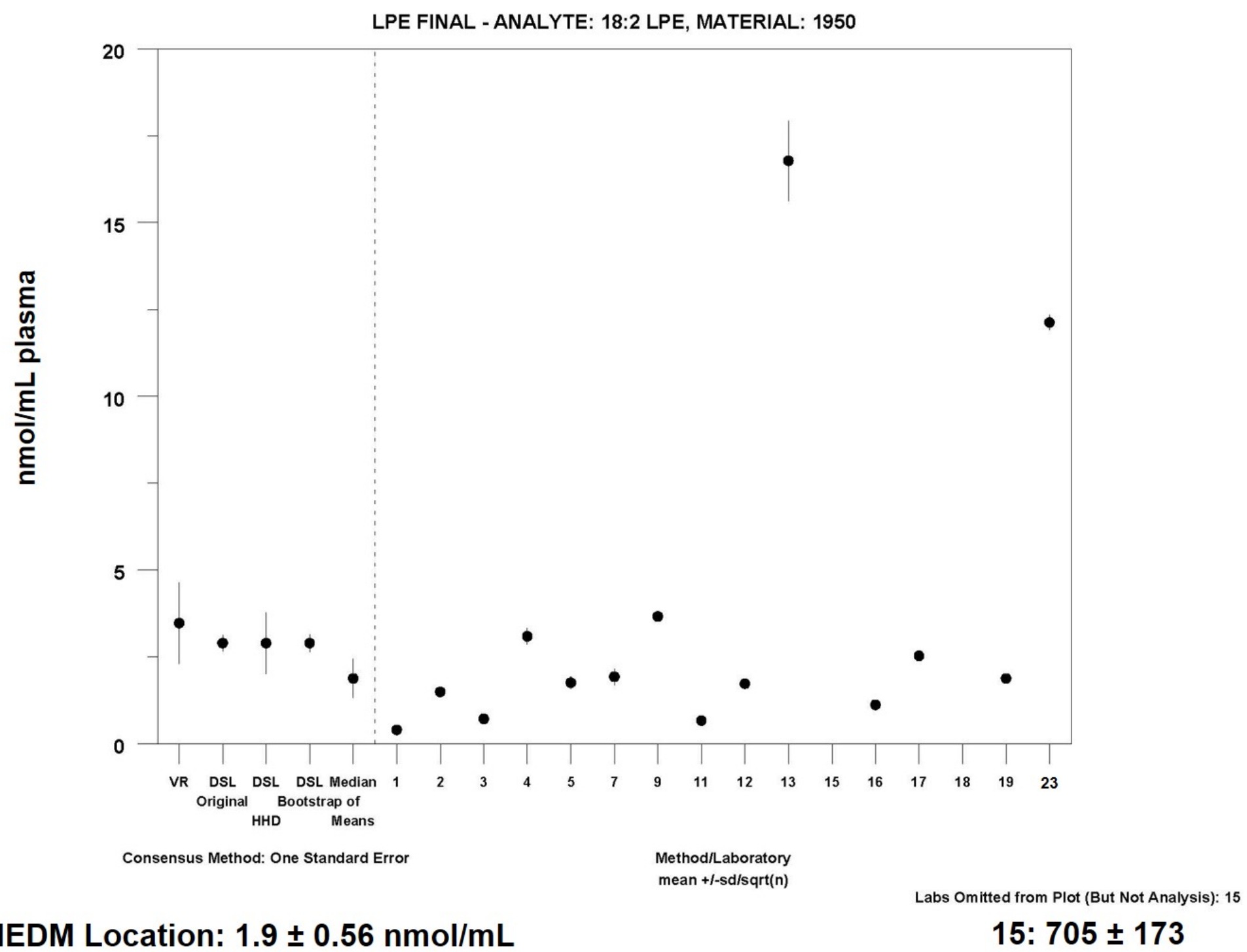




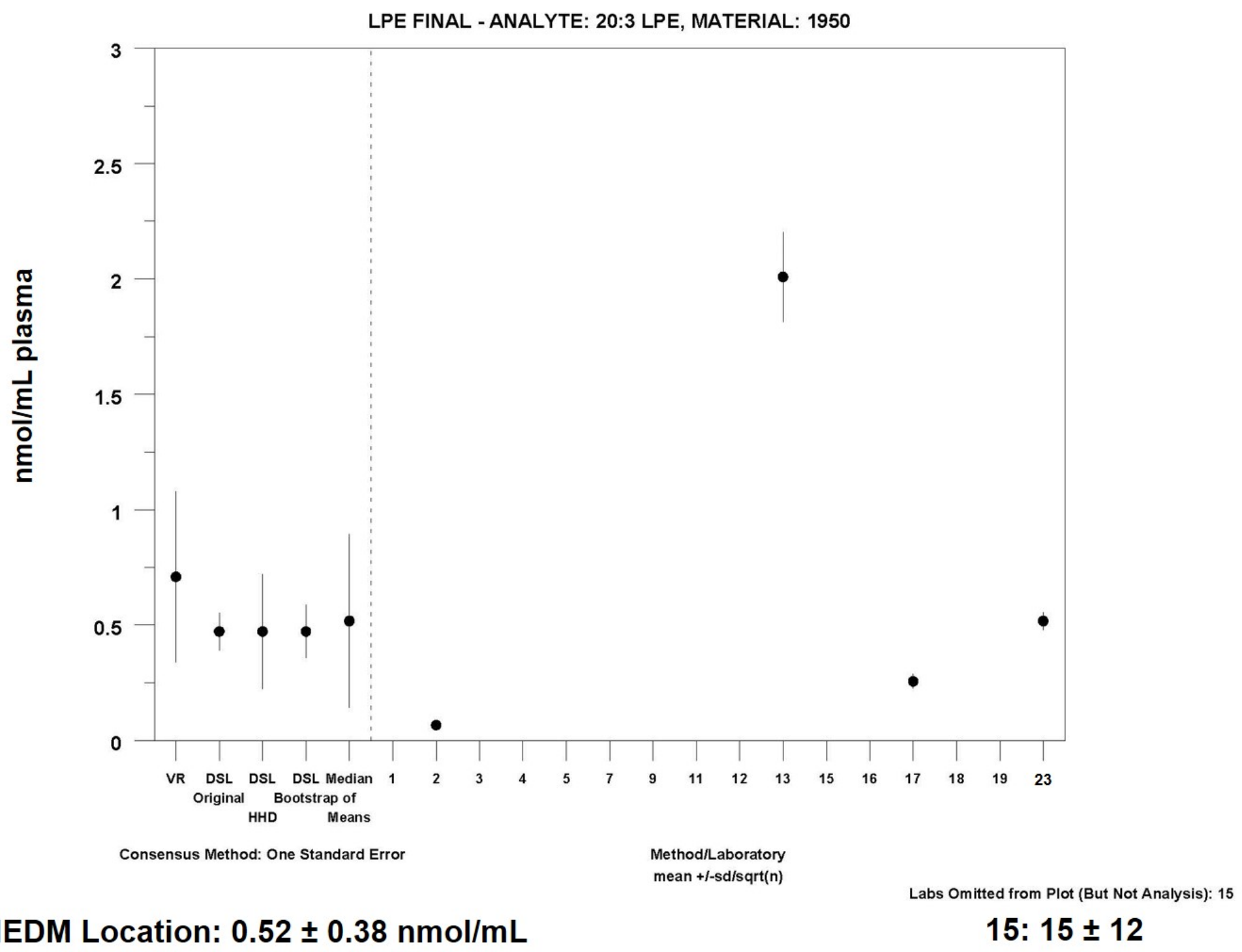




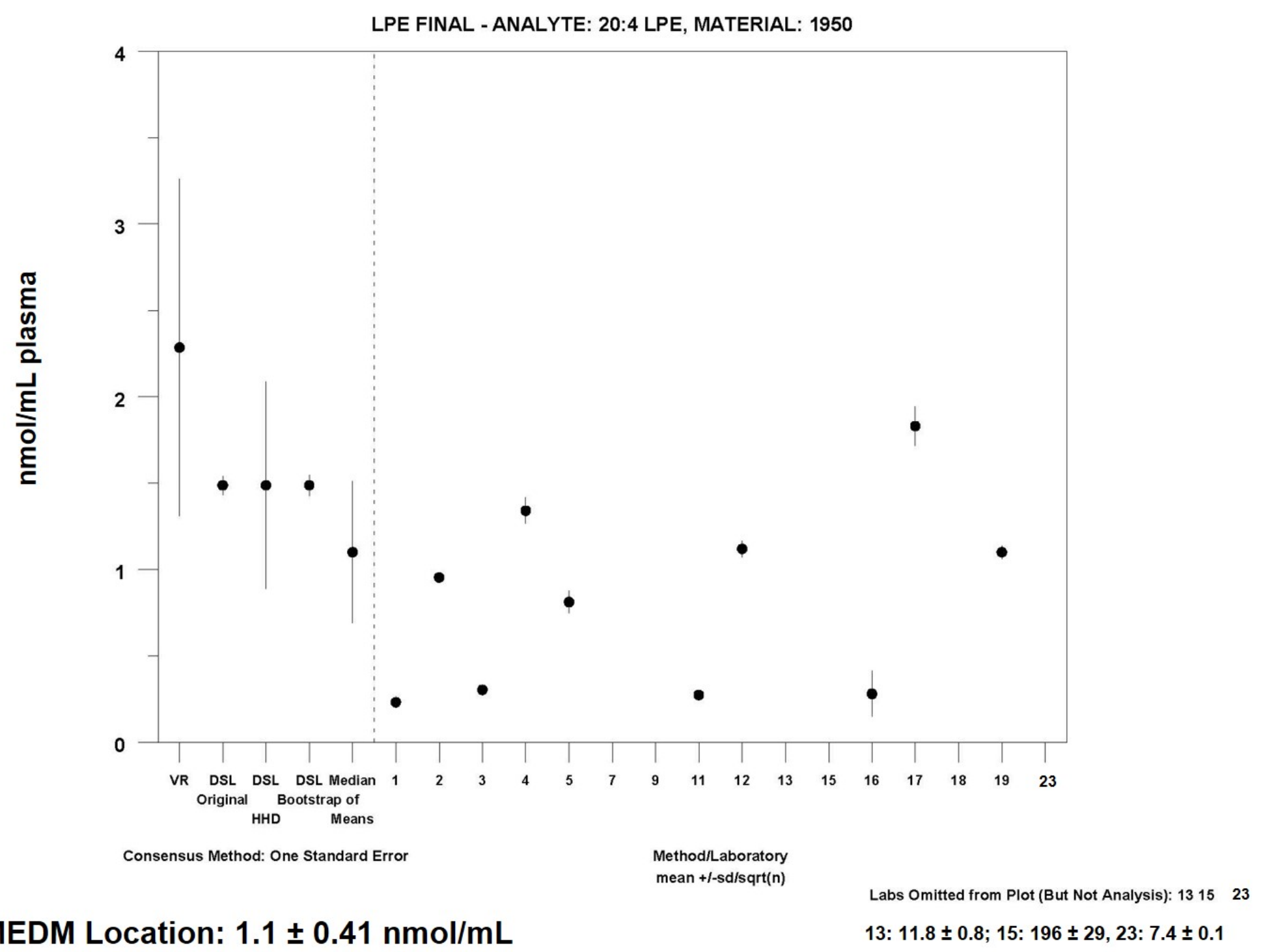




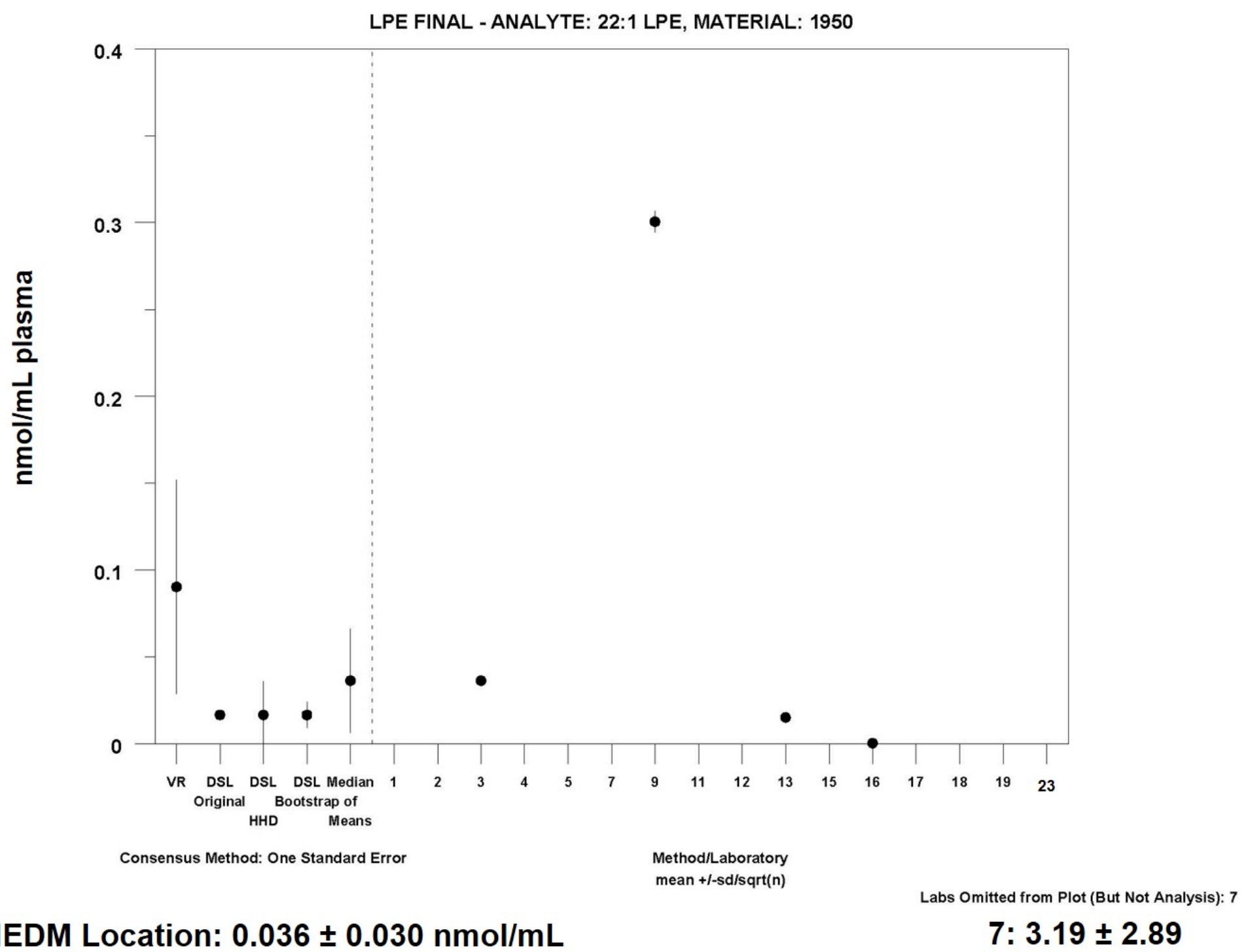




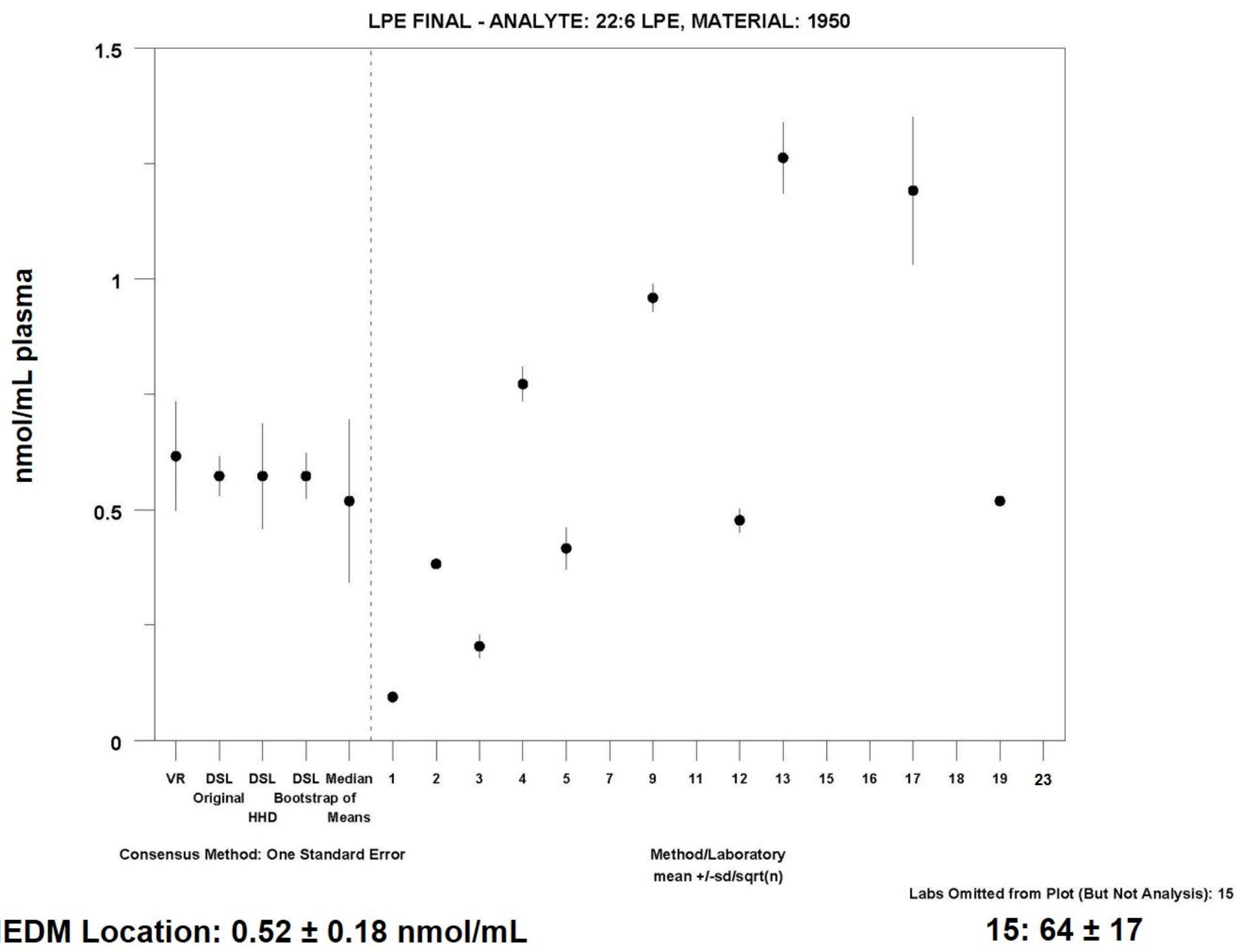




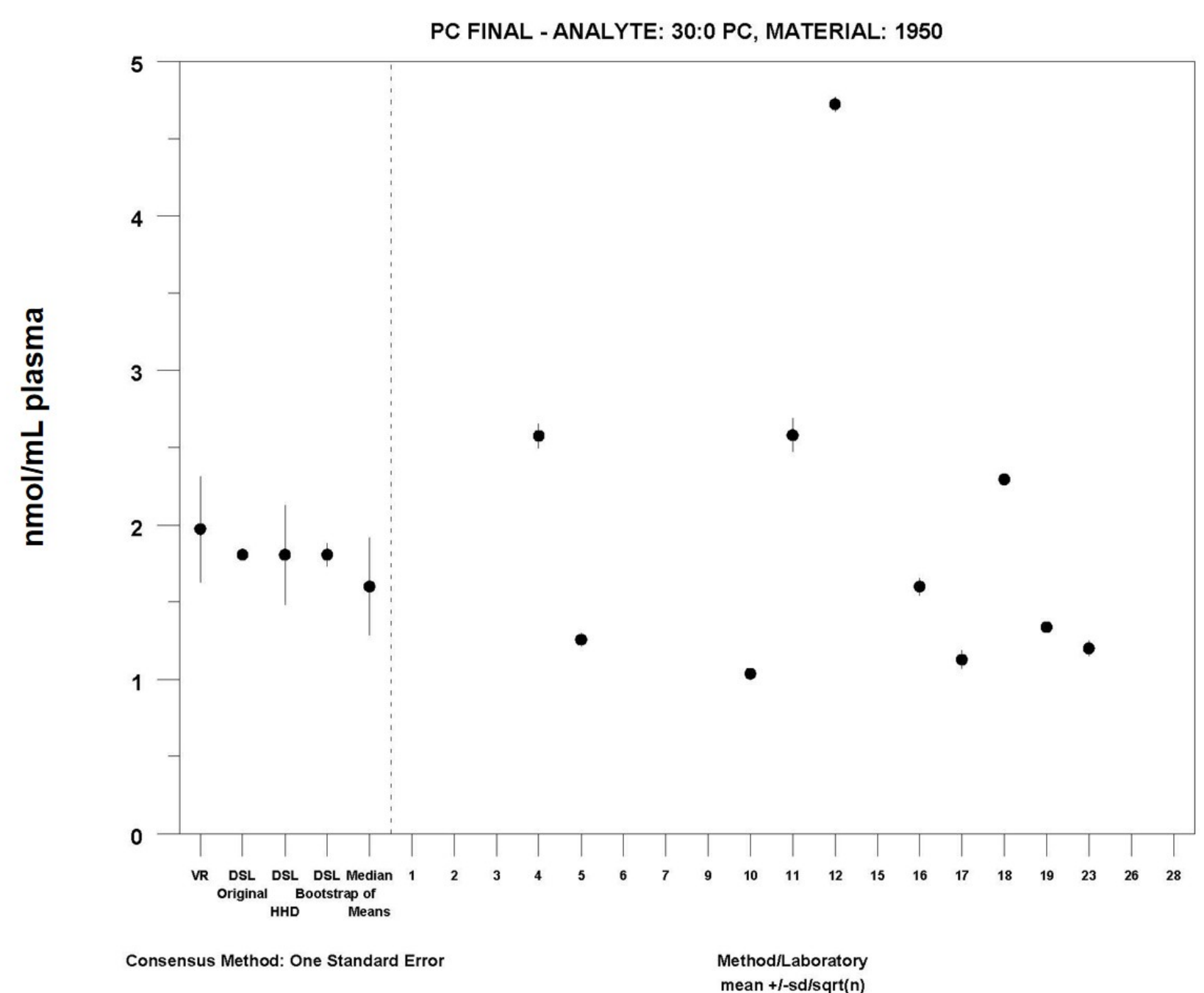

MEDM Location: $1.6 \pm 0.32 \mathrm{nmol} / \mathrm{mL}$

Labs Omitted from Plot (But Not Analysis): 15

15: $738 \pm 159$ 


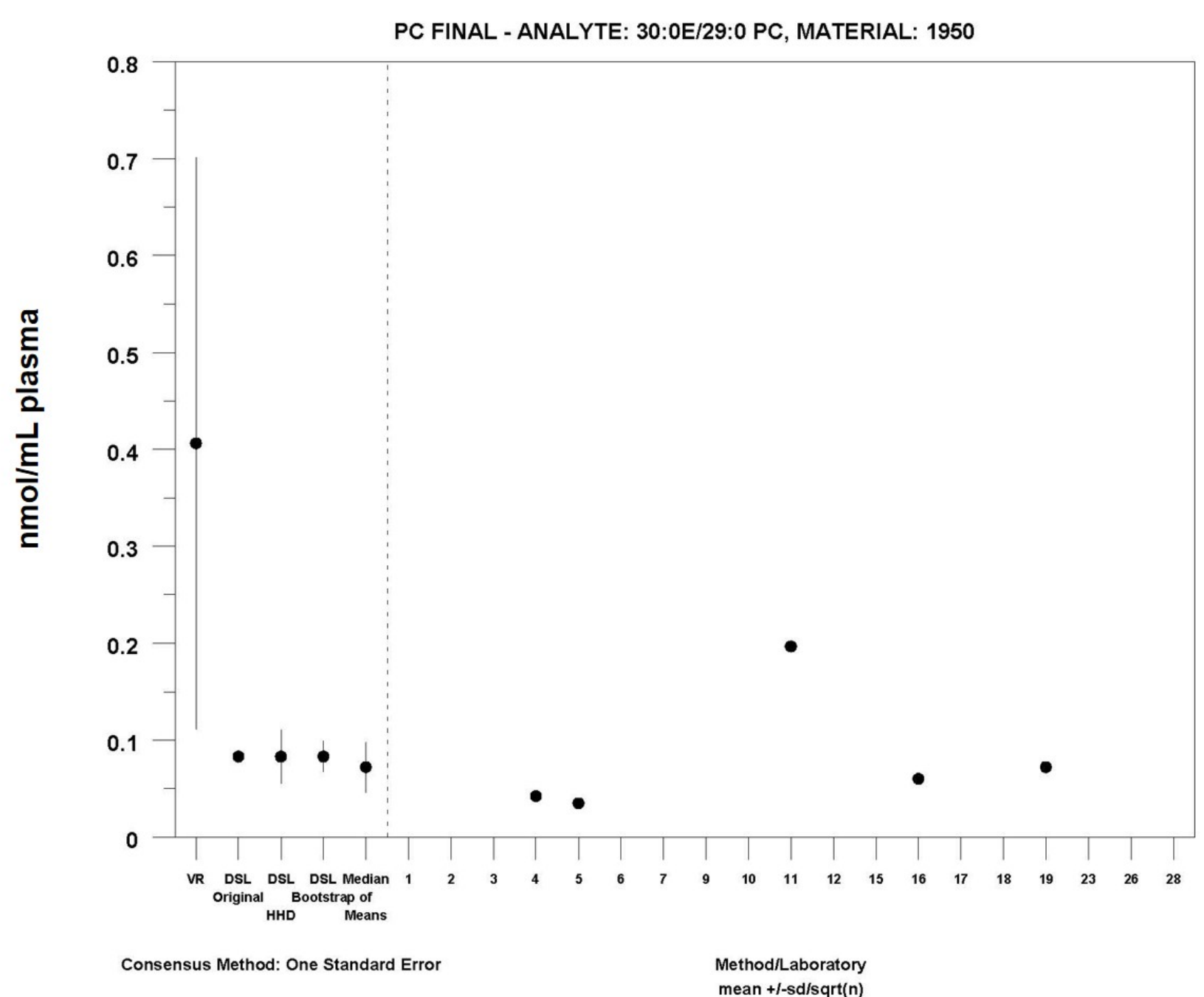

MEDM Location: $0.072 \pm 0.026 \mathrm{nmol} / \mathrm{mL}$

Labs Omitted from Plot (But Not Analysis): 215

15: $51 \pm 13$ 


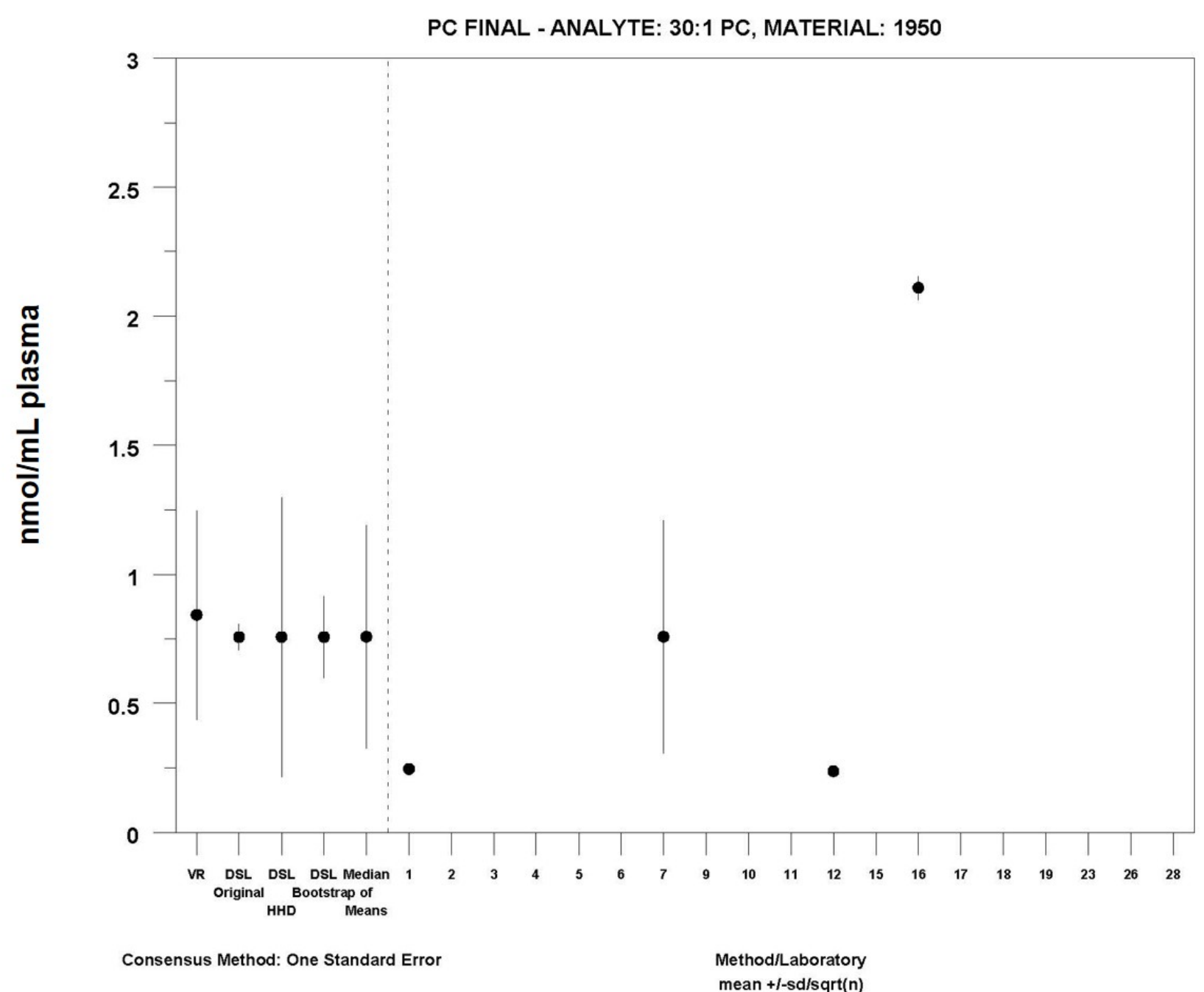

MEDM Location: $0.76 \pm 0.43 \mathrm{nmol} / \mathrm{mL}$

Labs Omitted from Plot (But Not Analysis): 15

15: $363 \pm 107$ 


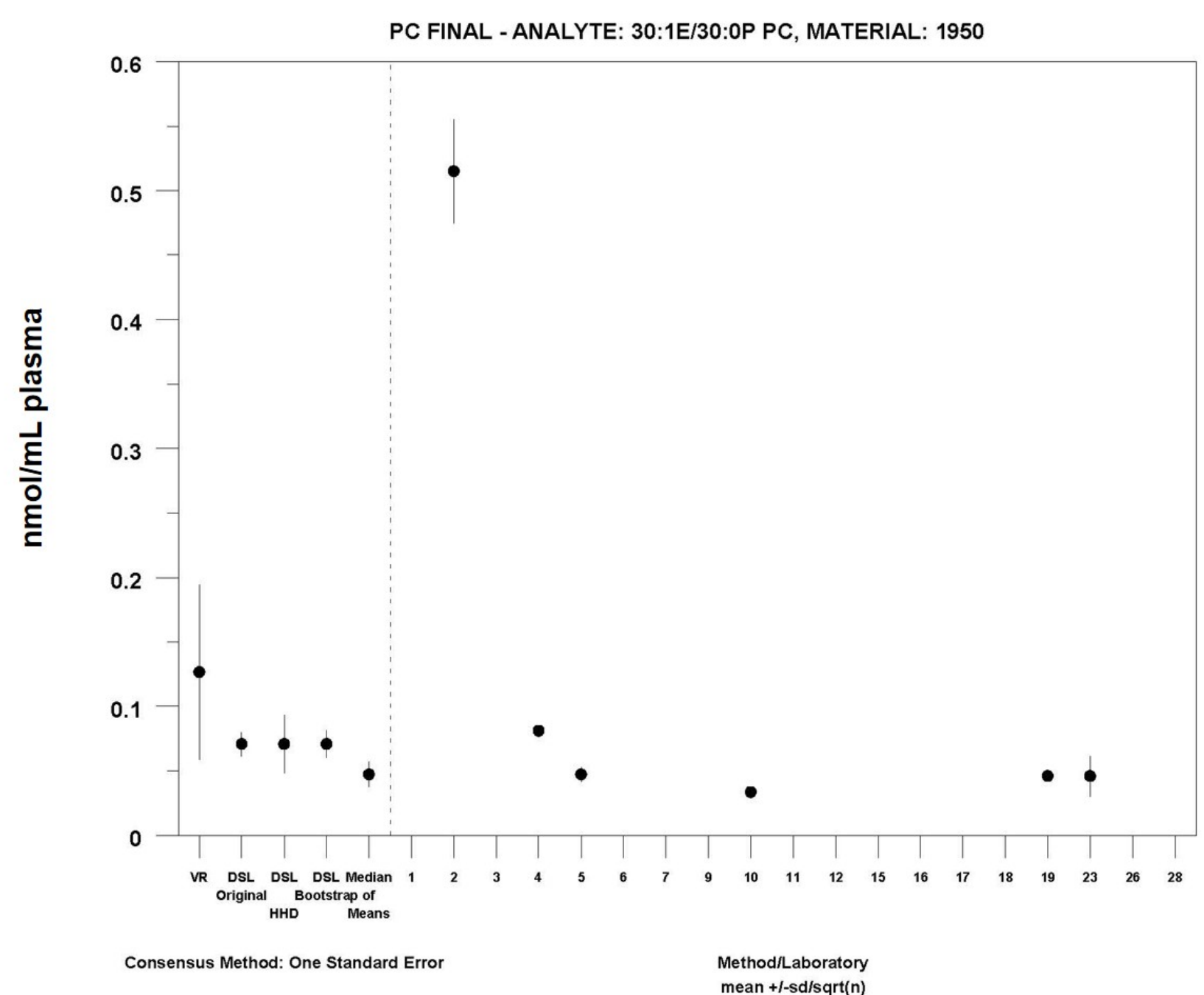

MEDM Location: $0.047 \pm 0.0096 \mathrm{nmol} / \mathrm{mL}$

Labs Omitted from Plot (But Not Analysis): 15

15: $5.3 \pm 1.5$ 


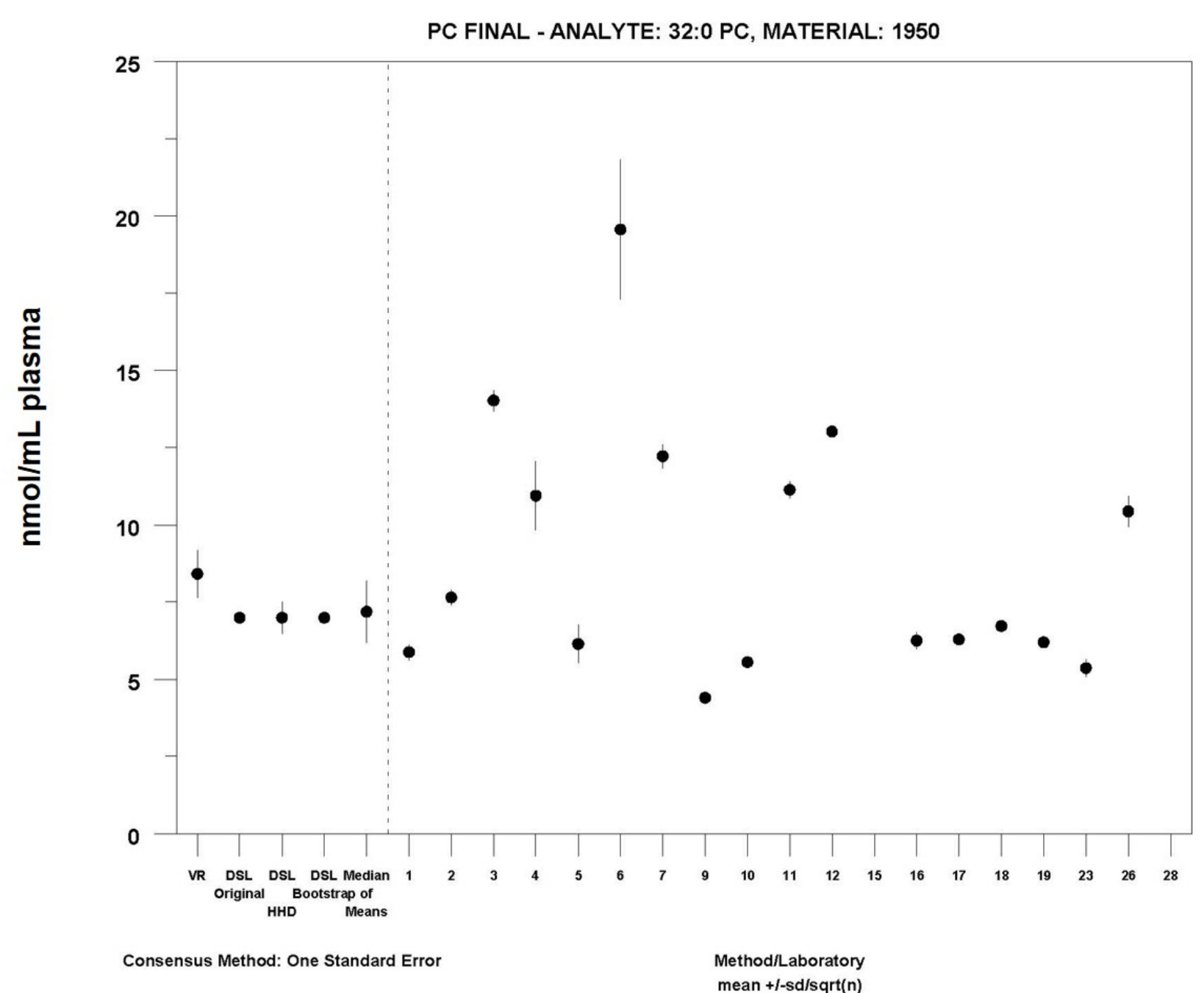

MEDM Location: $7.2 \pm 1.0 \mathrm{nmol} / \mathrm{mL}$

Labs Omitted from Plot (But Not Analysis): 15 15: $1618 \pm 150$ 


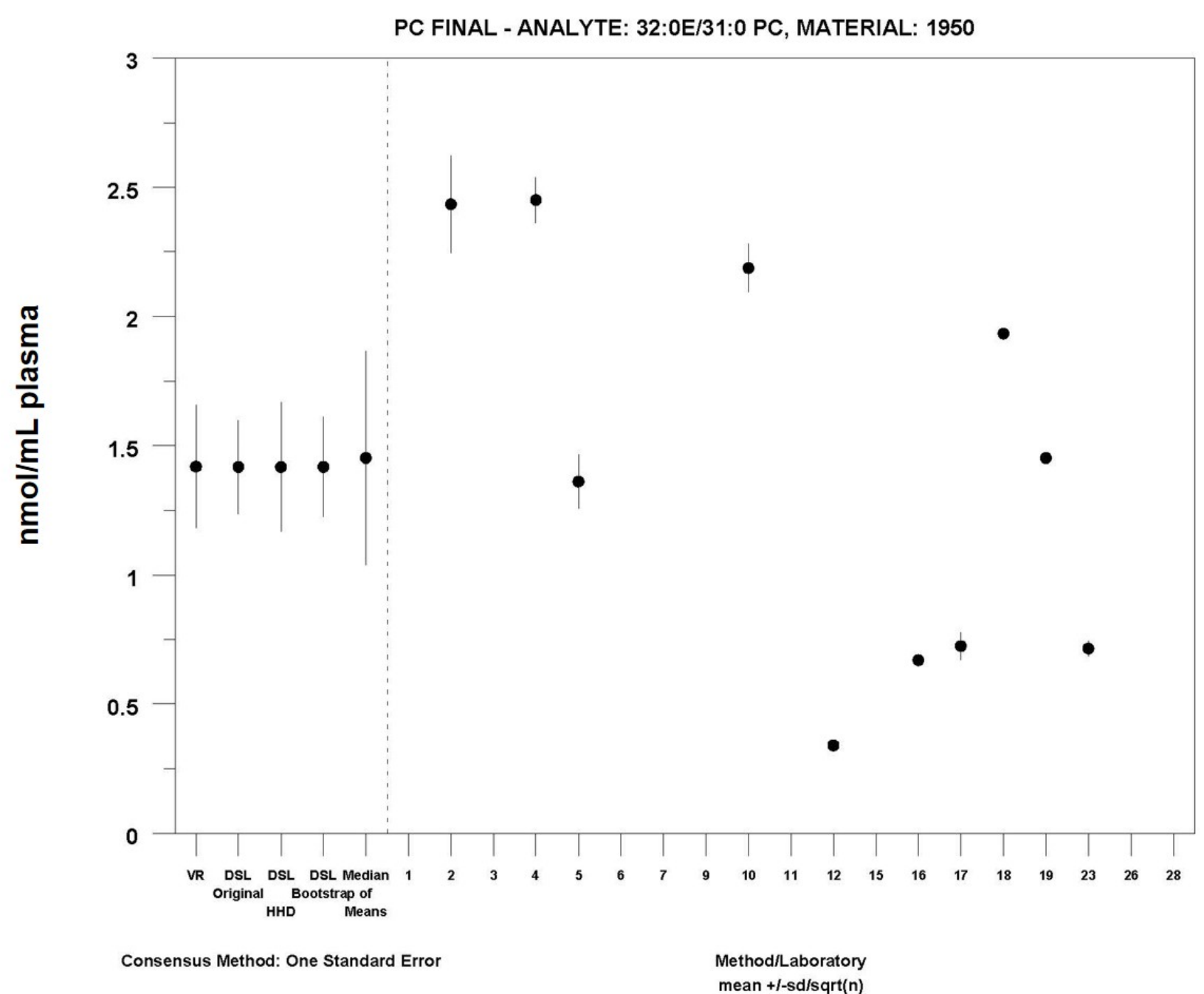

MEDM Location: $1.5 \pm 0.41 \mathrm{nmol} / \mathrm{mL}$

Labs Omitted from Plot (But Not Analysis): 15

15: $1010 \pm 159$ 


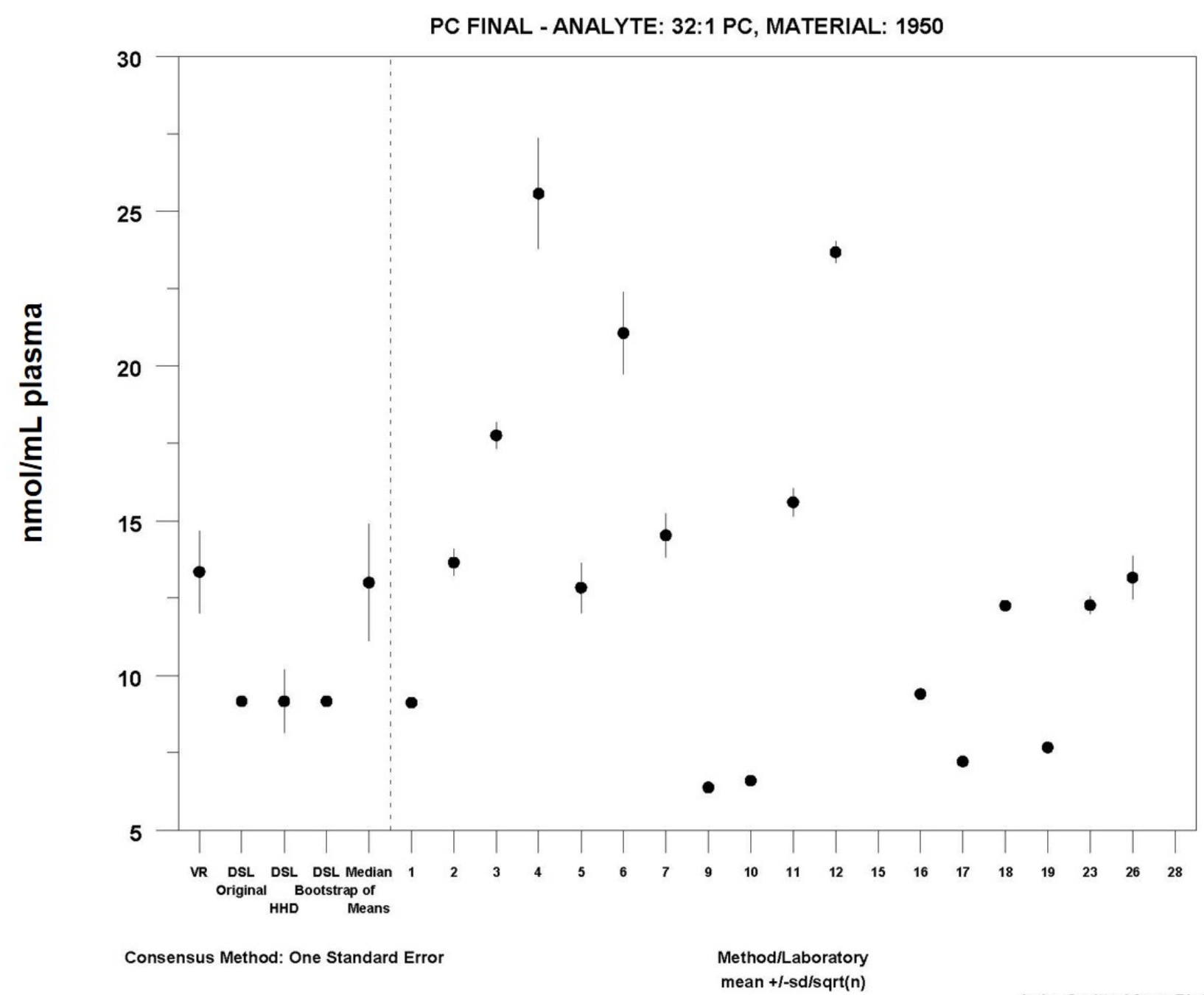

MEDM Location: $13 \pm 1.9 \mathrm{nmol} / \mathrm{mL}$

Labs Omitted from Plot (But Not Analysis): 15

15: $2495 \pm 98$ 


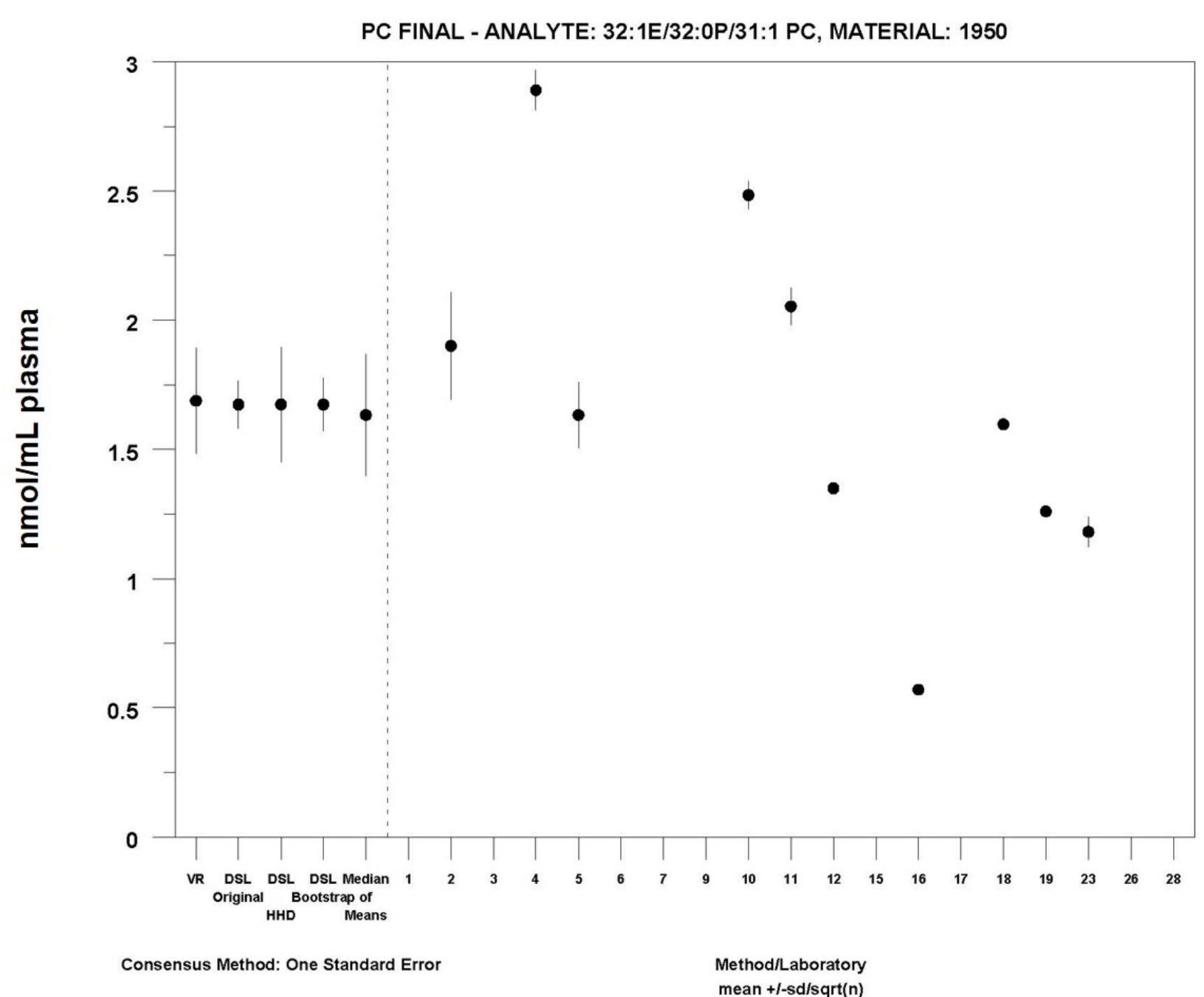

MEDM Location: $1.6 \pm 0.24 \mathrm{nmol} / \mathrm{mL}$

Labs Omitted from Plot (But Not Analysis): 15

15: $842 \pm 205$ 


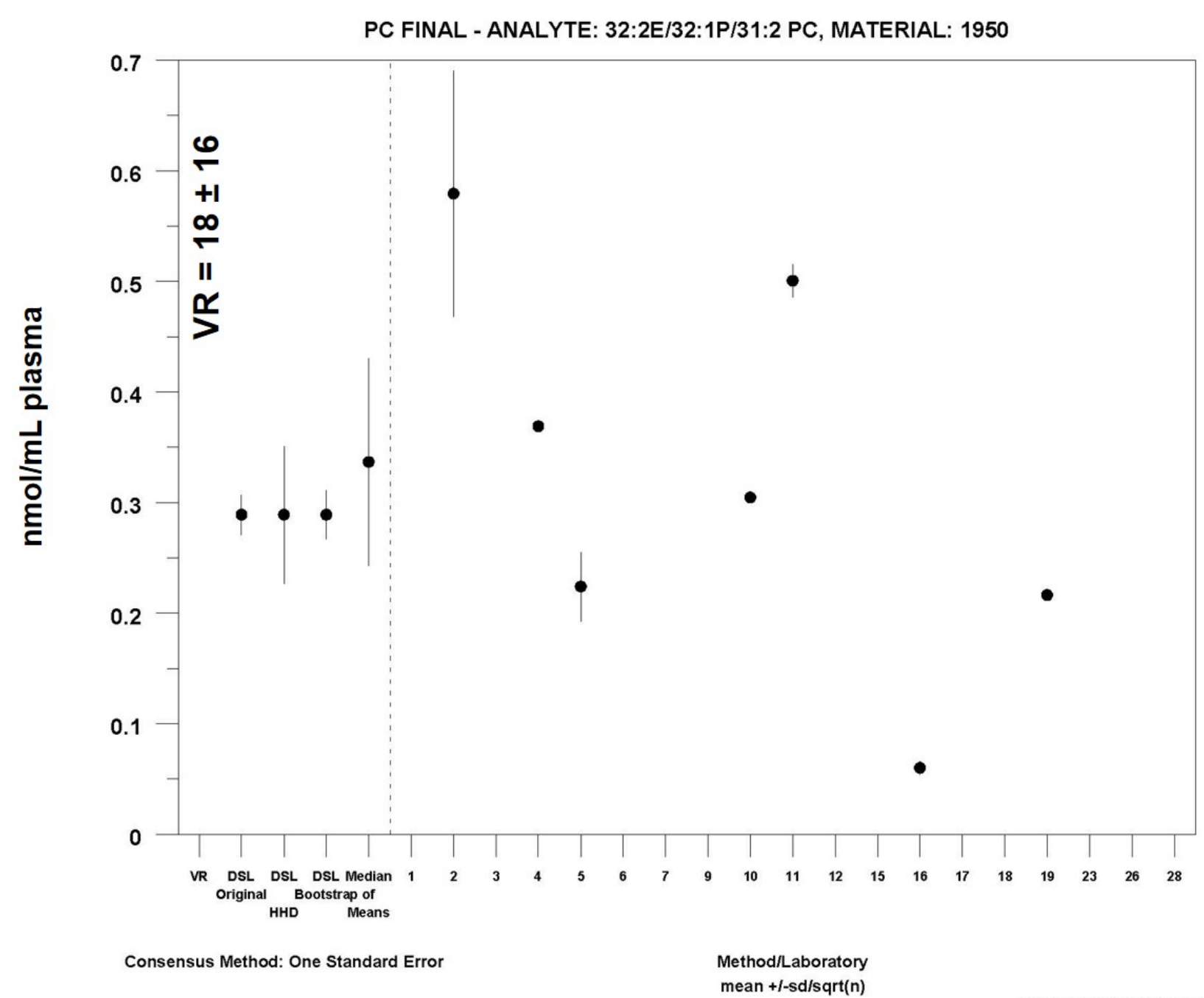

MEDM Location: $0.34 \pm 0.093 \mathrm{nmol} / \mathrm{mL}$

Labs Omitted from Plot (But Not Analysis): 15

15: $141 \pm 6$ 


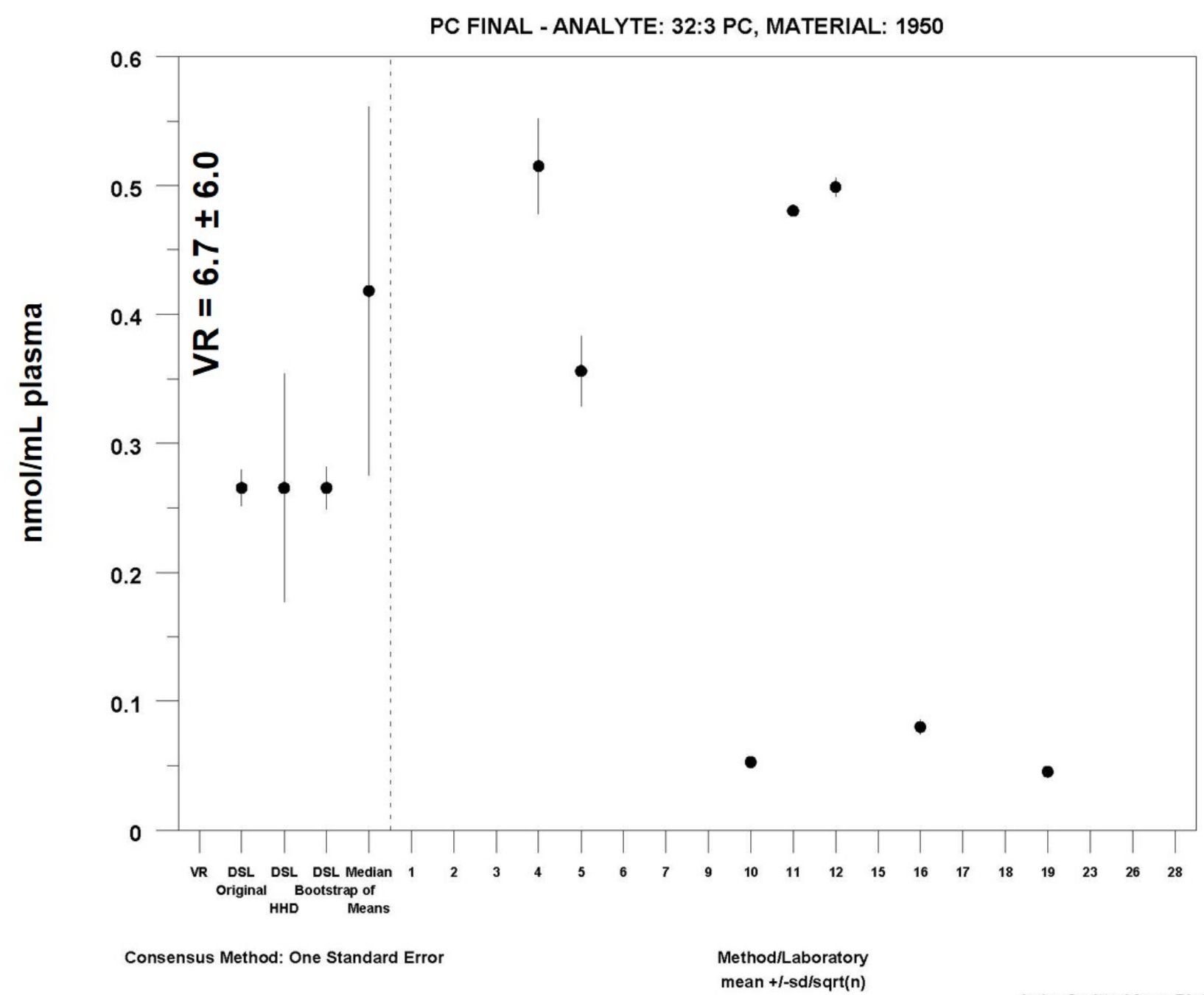

MEDM Location: $0.42 \pm 0.14 \mathrm{nmol} / \mathrm{mL}$

Labs Omitted from Plot (But Not Analysis): 15

15: $53 \pm 5$ 


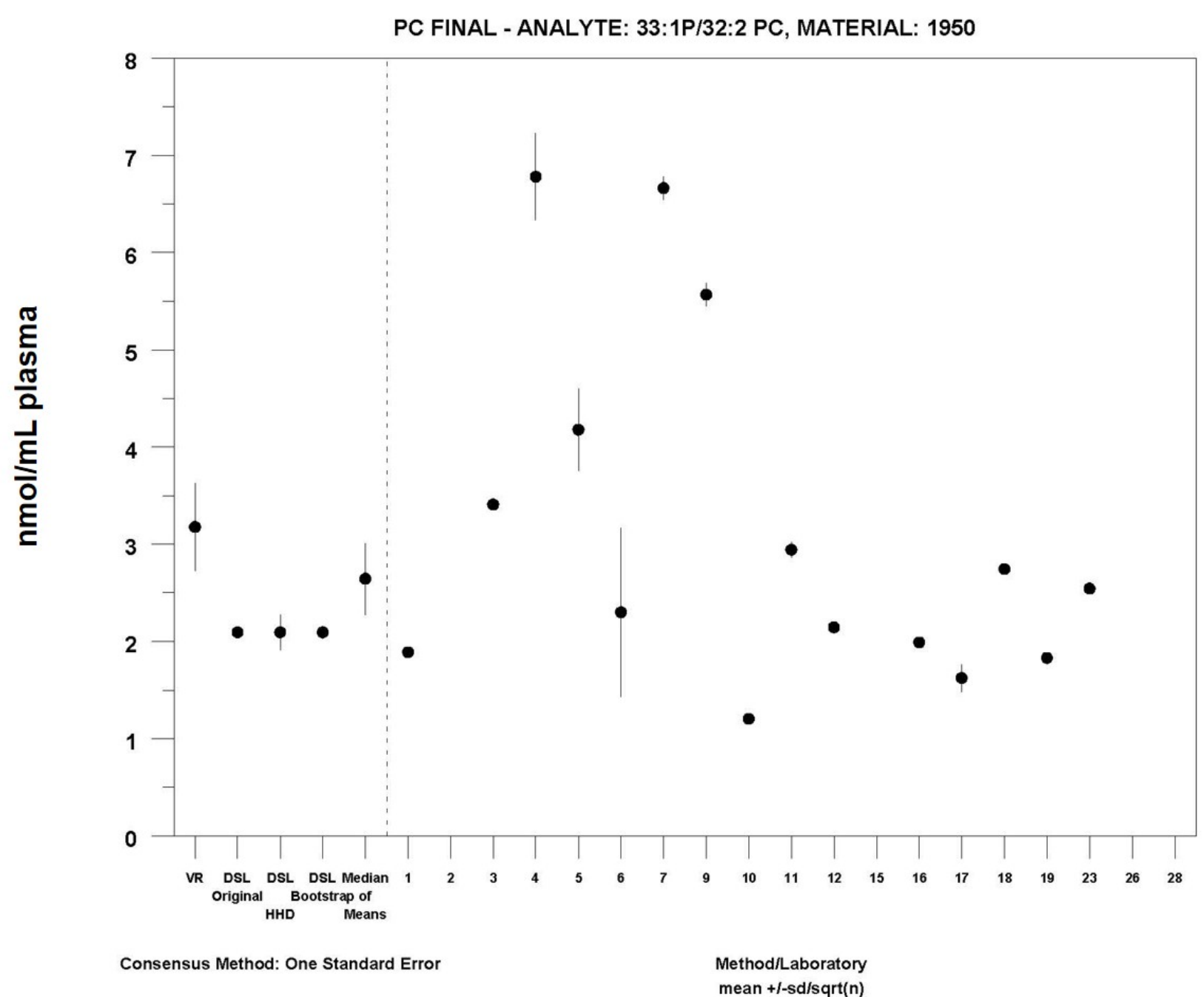

MEDM Location: $2.6 \pm 0.37 \mathrm{nmol} / \mathrm{mL}$

Labs Omitted from Plot (But Not Analysis): 15

15: $2435 \pm 1463$ 


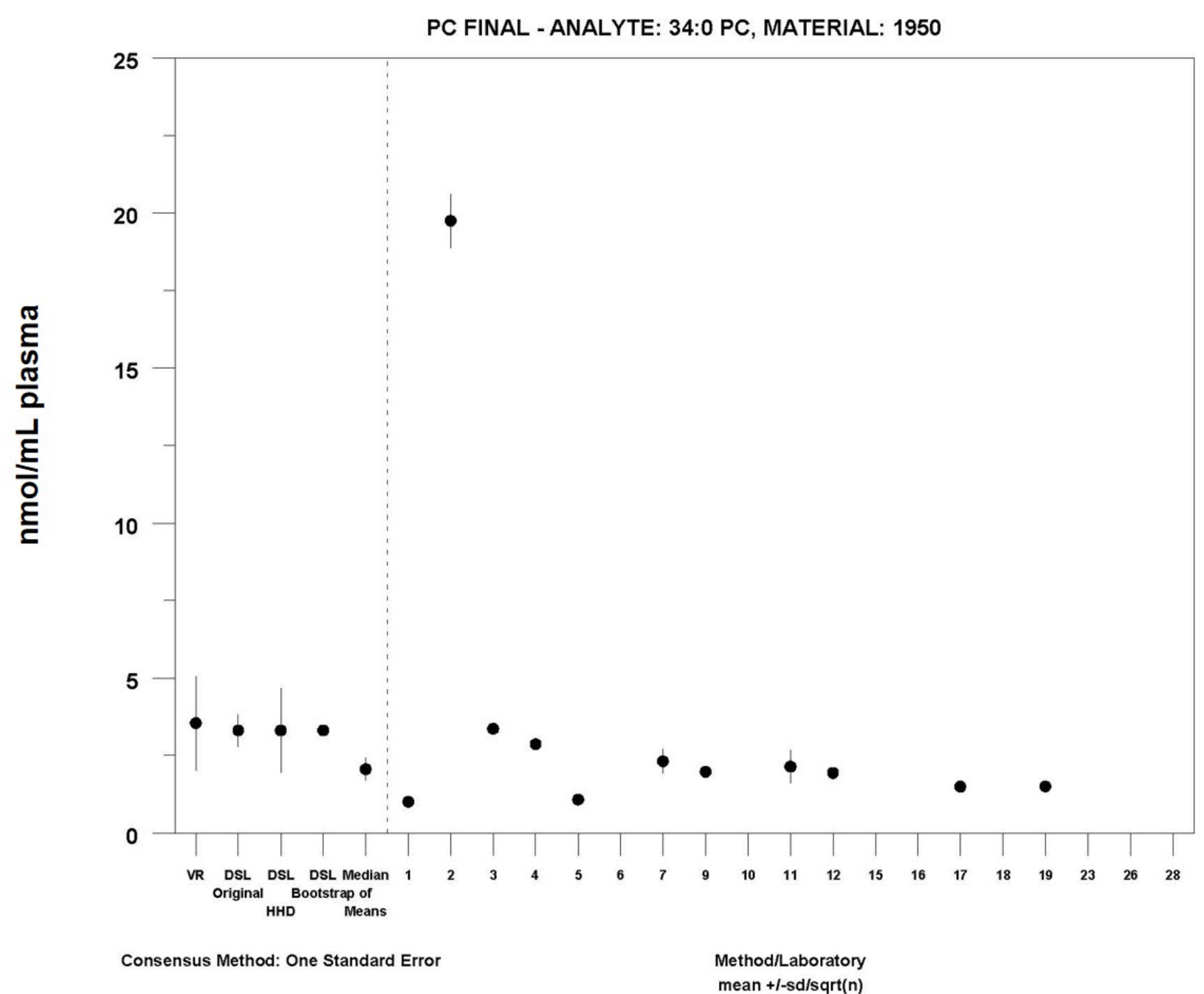

MEDM Location: $2.1 \pm 0.37 \mathrm{nmol} / \mathrm{mL}$

Labs Omitted from Plot (But Not Analysis): 15

15: $690 \pm 200$ 


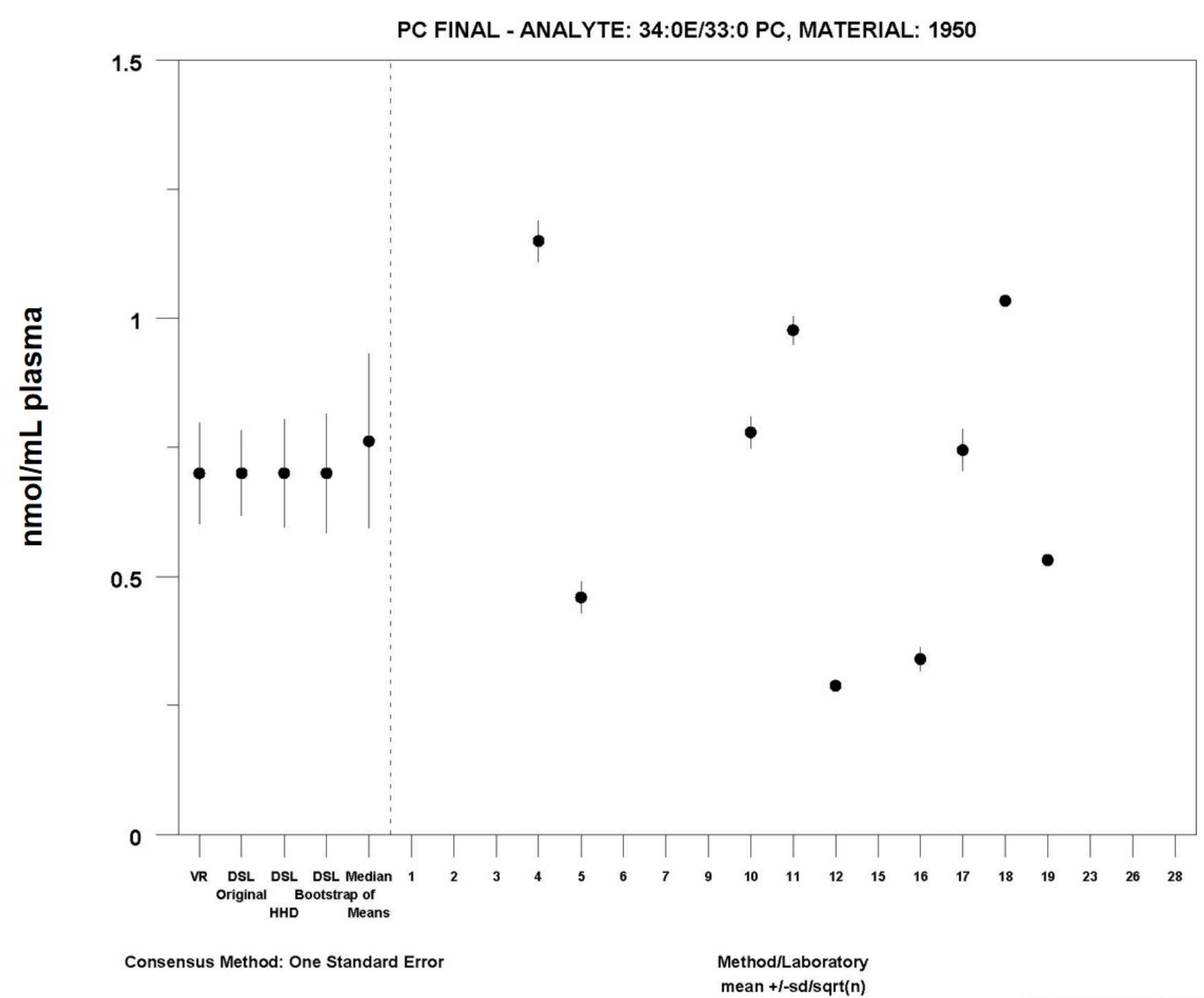

MEDM Location: $0.76 \pm 0.17 \mathrm{nmol} / \mathrm{mL}$

Labs Omitted from Plot (But Not Analysis): 15

15: $532 \pm 117$ 


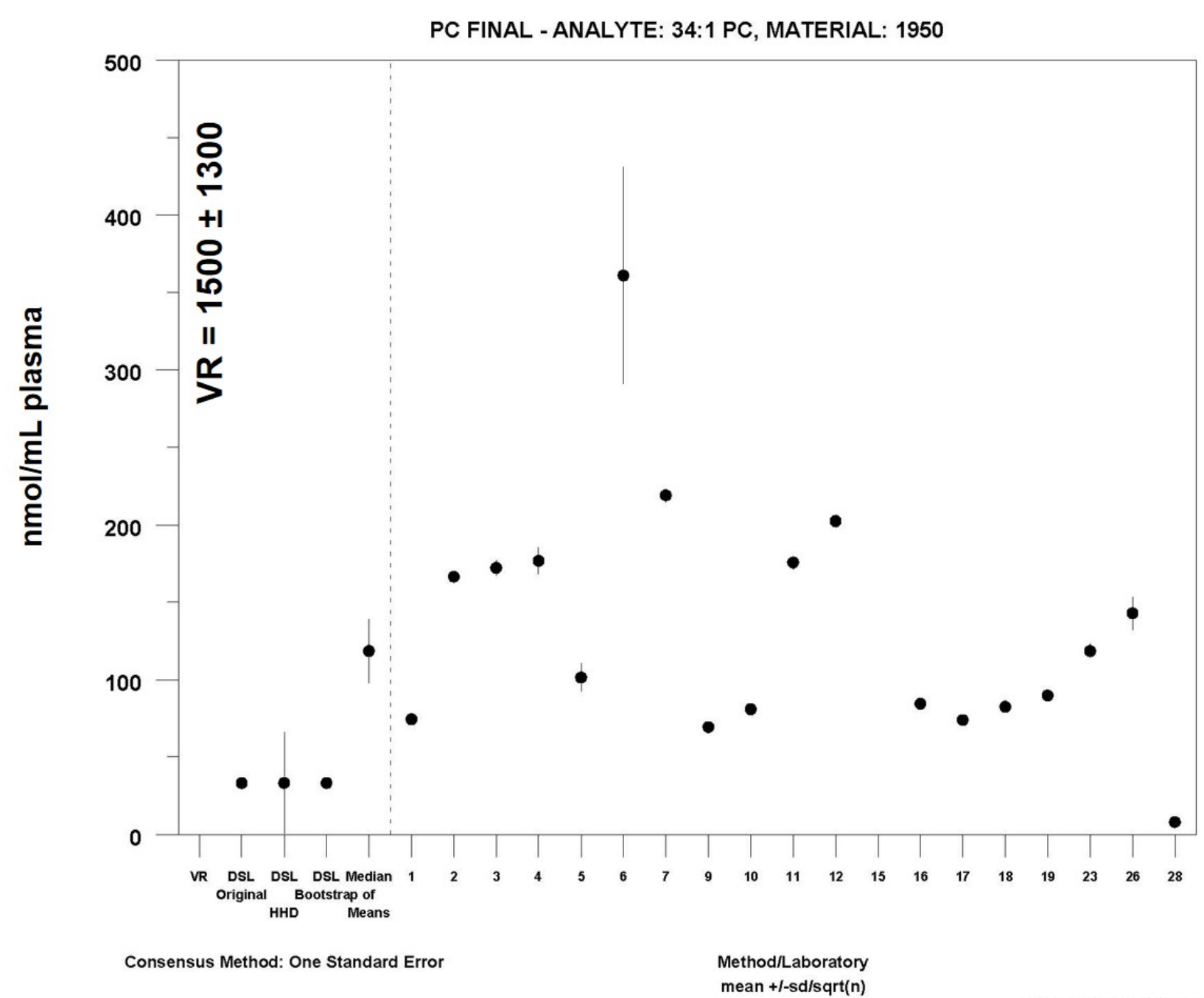

MEDM Location: $120 \pm 21 \mathrm{nmol} / \mathrm{mL}$

Labs Omitted from Plot (But Not Analysis): 15

15: $25437 \pm 612$ 


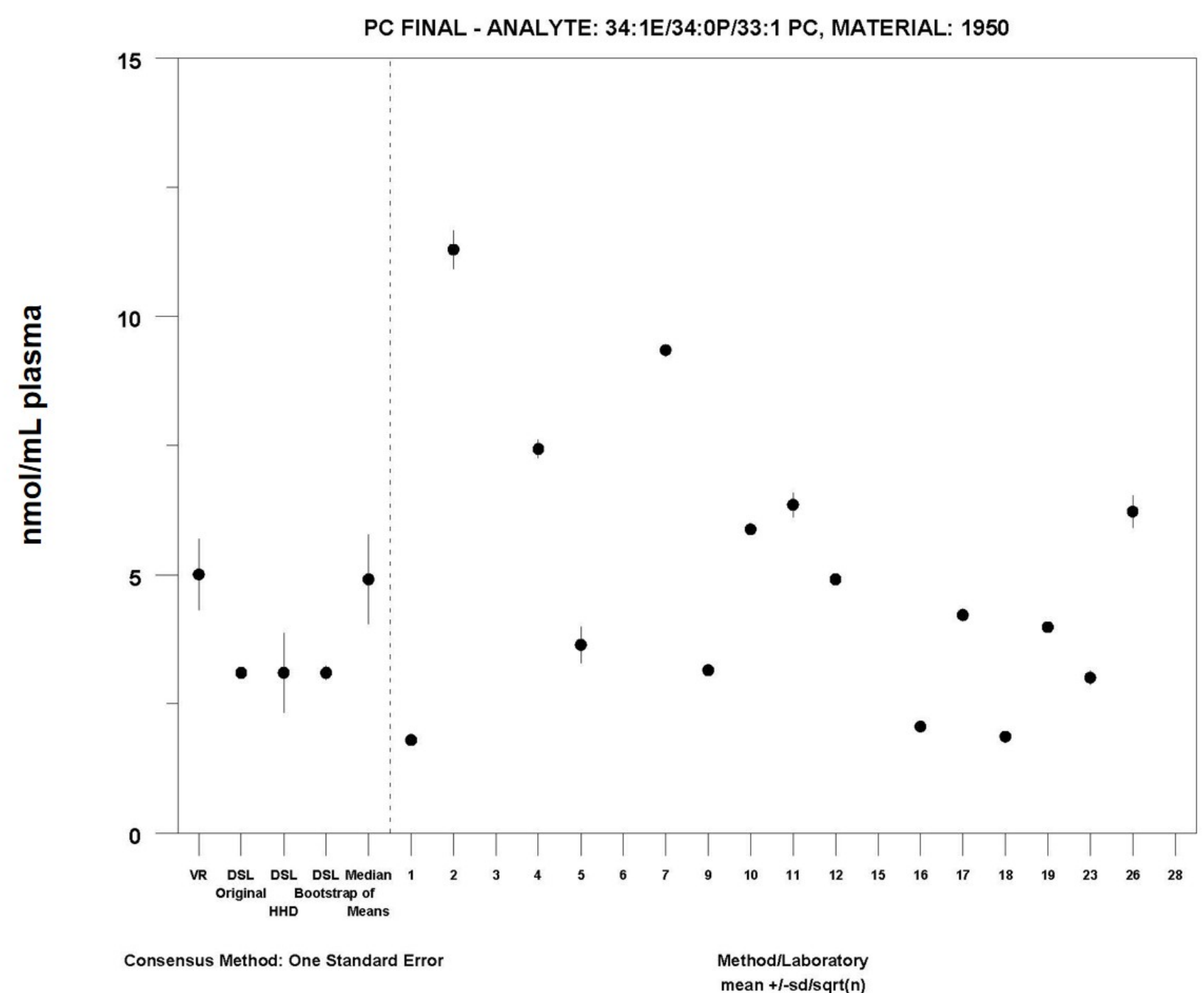

MEDM Location: $4.9 \pm 0.86 \mathrm{nmol} / \mathrm{mL}$

Labs Omitted from Plot (But Not Analysis): 615

6: $662 \pm 67 ; 15: 3144 \pm 540$ 


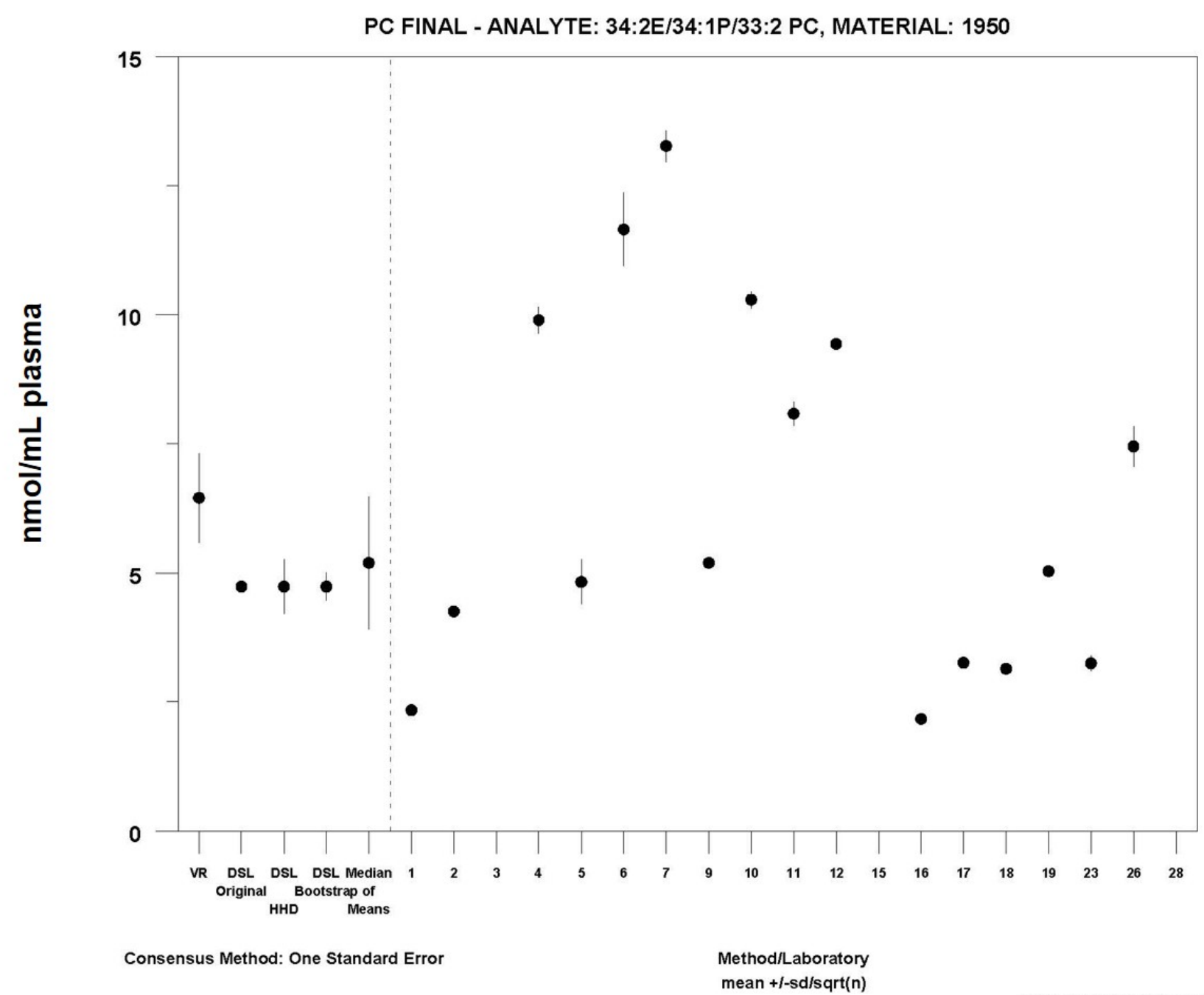

MEDM Location: $5.2 \pm 1.3 \mathrm{nmol} / \mathrm{mL}$

Labs Omitted from Plot (But Not Analysis): 15

15: $4154 \pm 665$ 


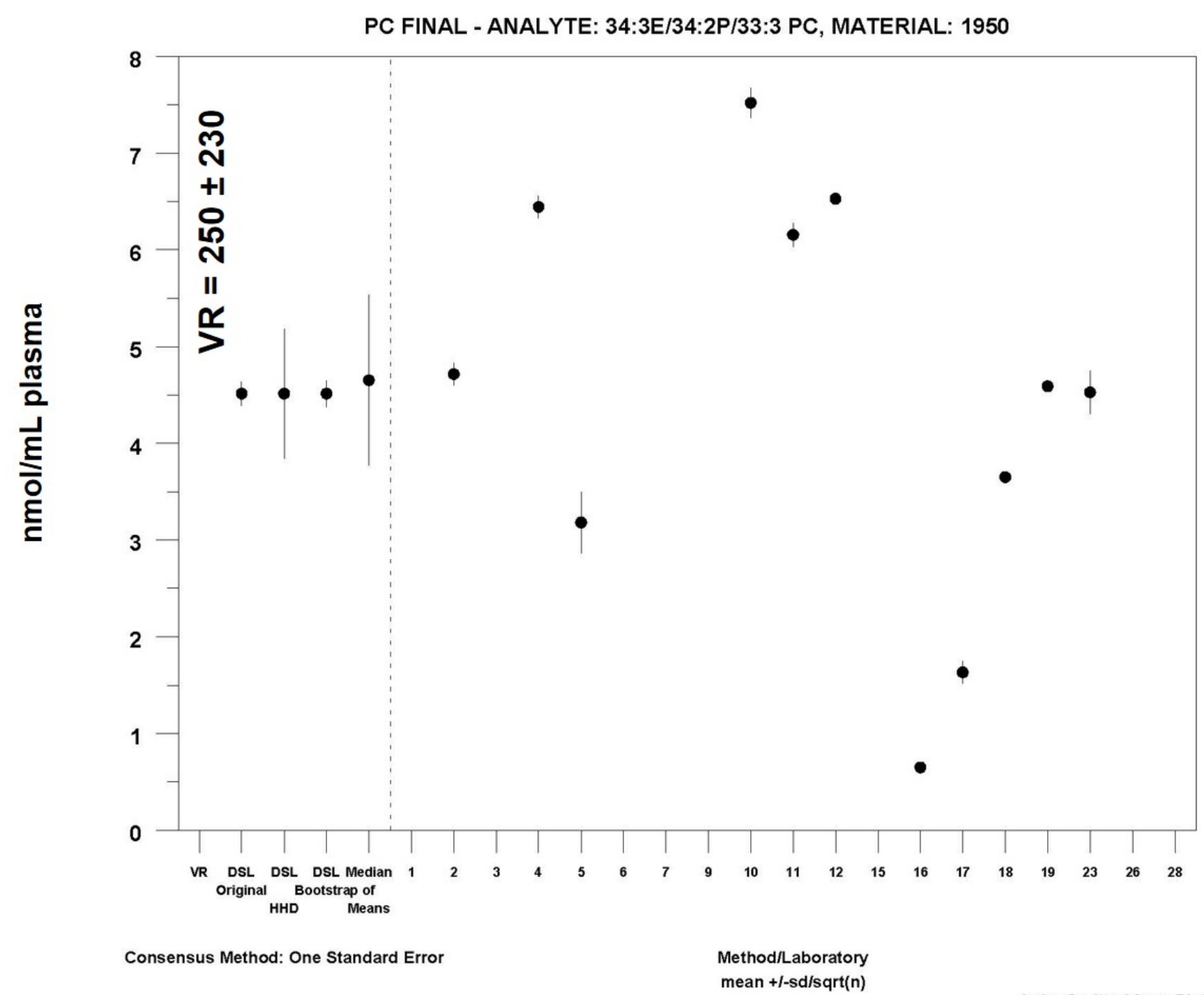

MEDM Location: $4.7 \pm 0.88 \mathrm{nmol} / \mathrm{mL}$

Labs Omitted from Plot (But Not Analysis): 15

15: $2957 \pm 150$ 


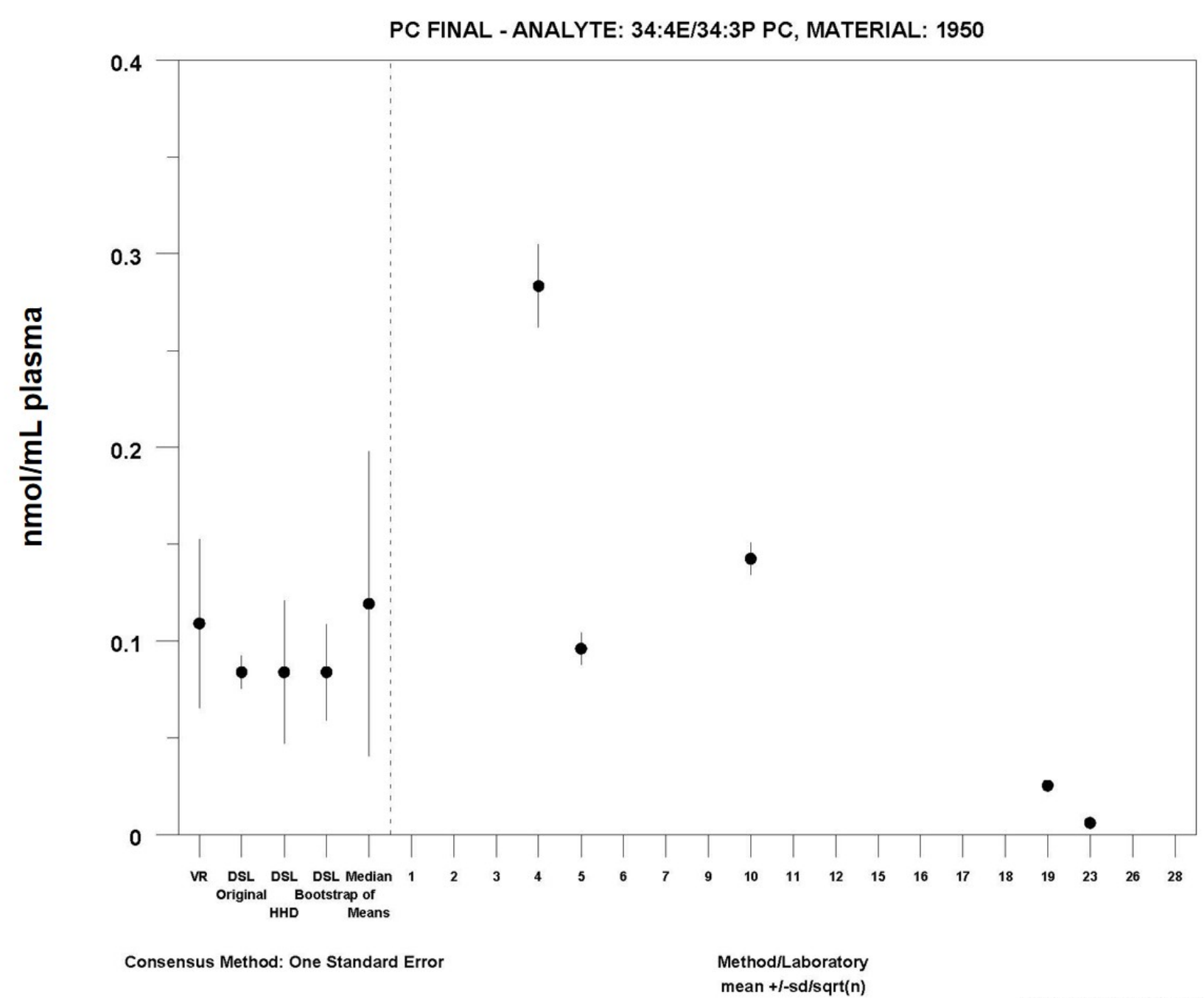

MEDM Location: $0.12 \pm 0.079 \mathrm{nmol} / \mathrm{mL}$

Labs Omitted from Plot (But Not Analysis): 15

15: $33 \pm 26$ 


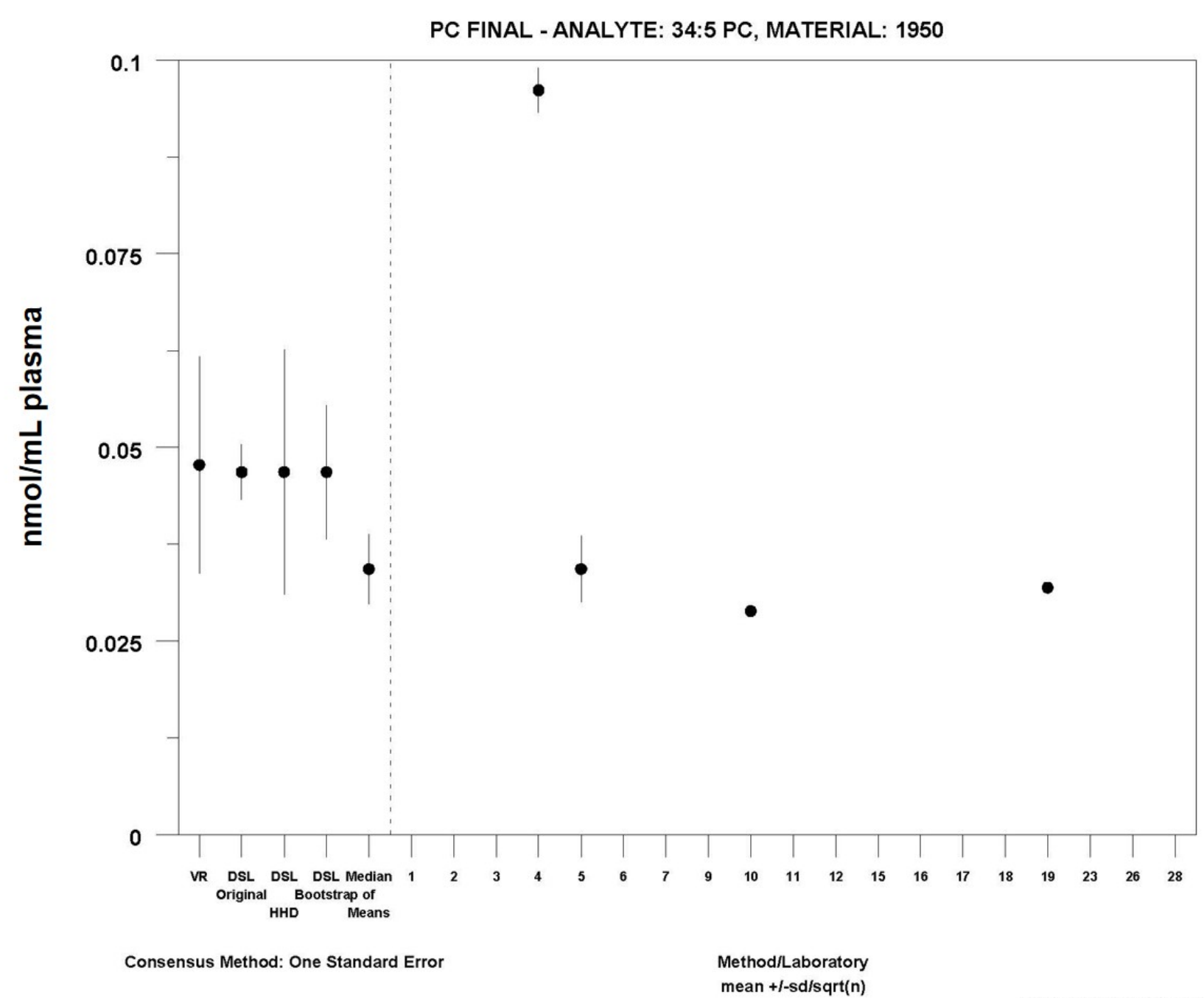

MEDM Location: $0.034 \pm 0.0045 \mathrm{nmol} / \mathrm{mL}$

Labs Omitted from Plot (But Not Analysis): 15

15: $81 \pm 49$ 


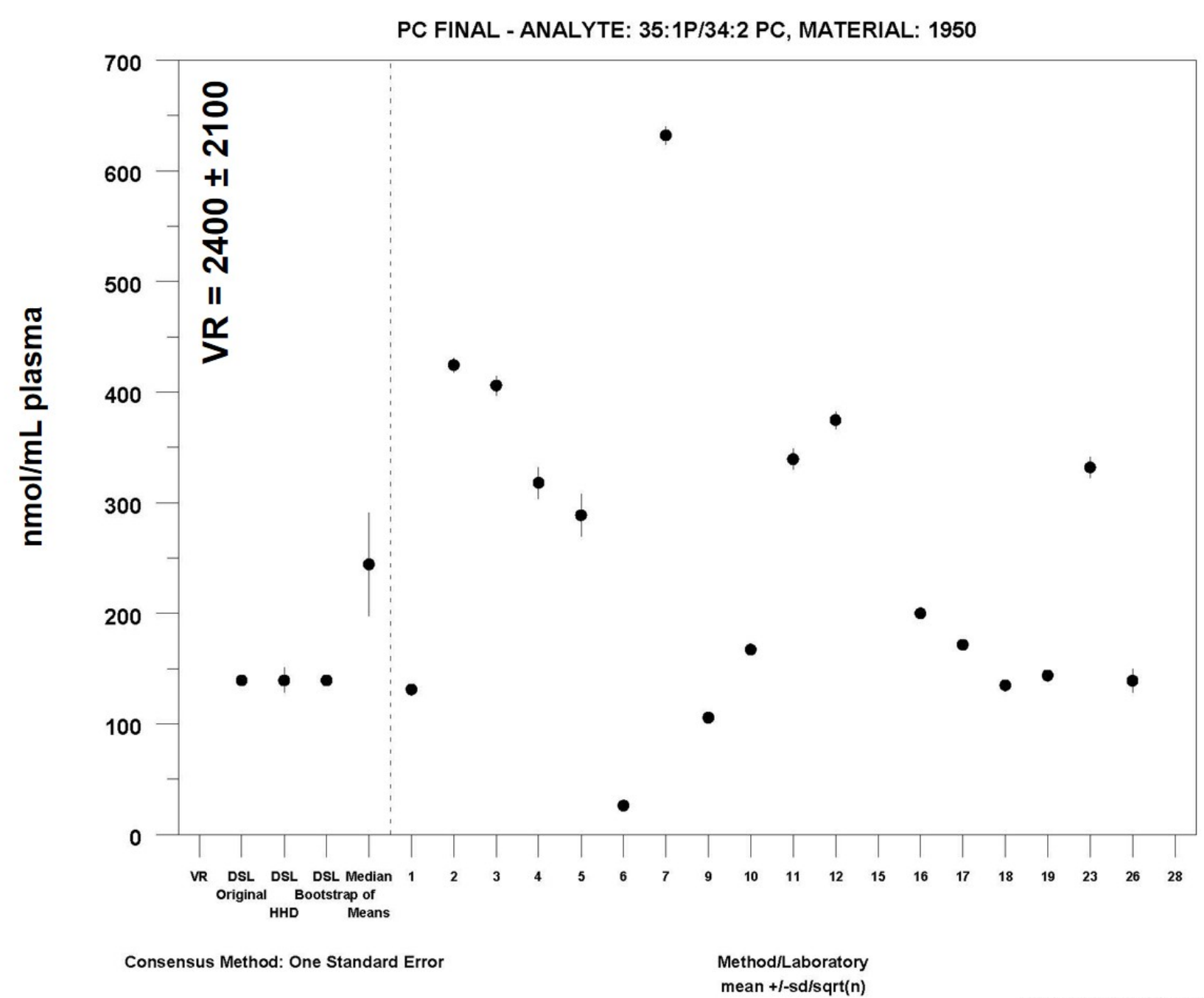

MEDM Location: $240 \pm 47 \mathrm{nmol} / \mathrm{mL}$

Labs Omitted from Plot (But Not Analysis): 15 15: $39210 \pm 1051$ 


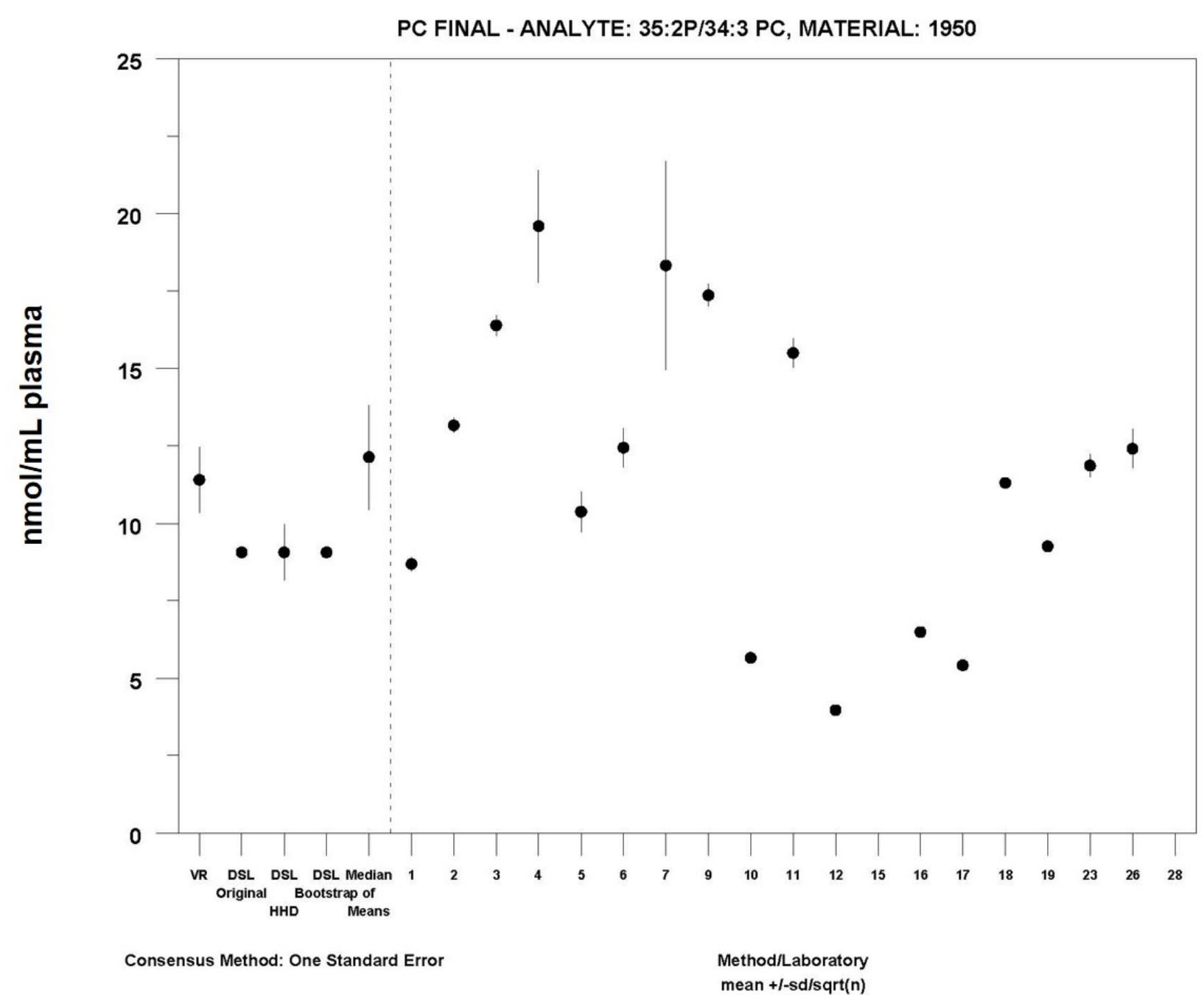

MEDM Location: $12 \pm 1.7 \mathrm{nmol} / \mathrm{mL}$

Labs Omitted from Plot (But Not Analysis): 15

15: $9196 \pm 2657$ 


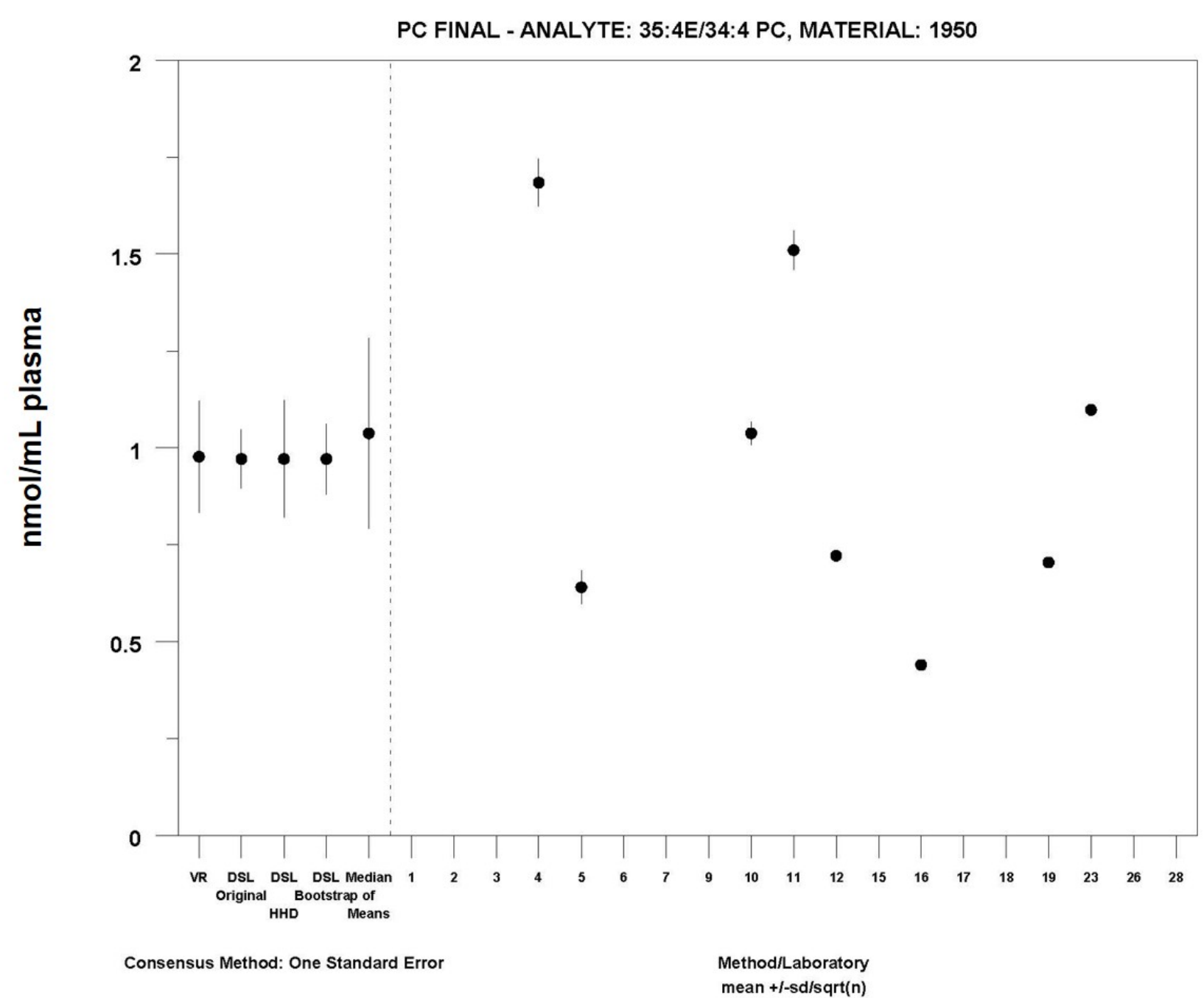

MEDM Location: $1.0 \pm 0.25 \mathrm{nmol} / \mathrm{mL}$

Labs Omitted from Plot (But Not Analysis): 15

15: $1132 \pm 69$ 


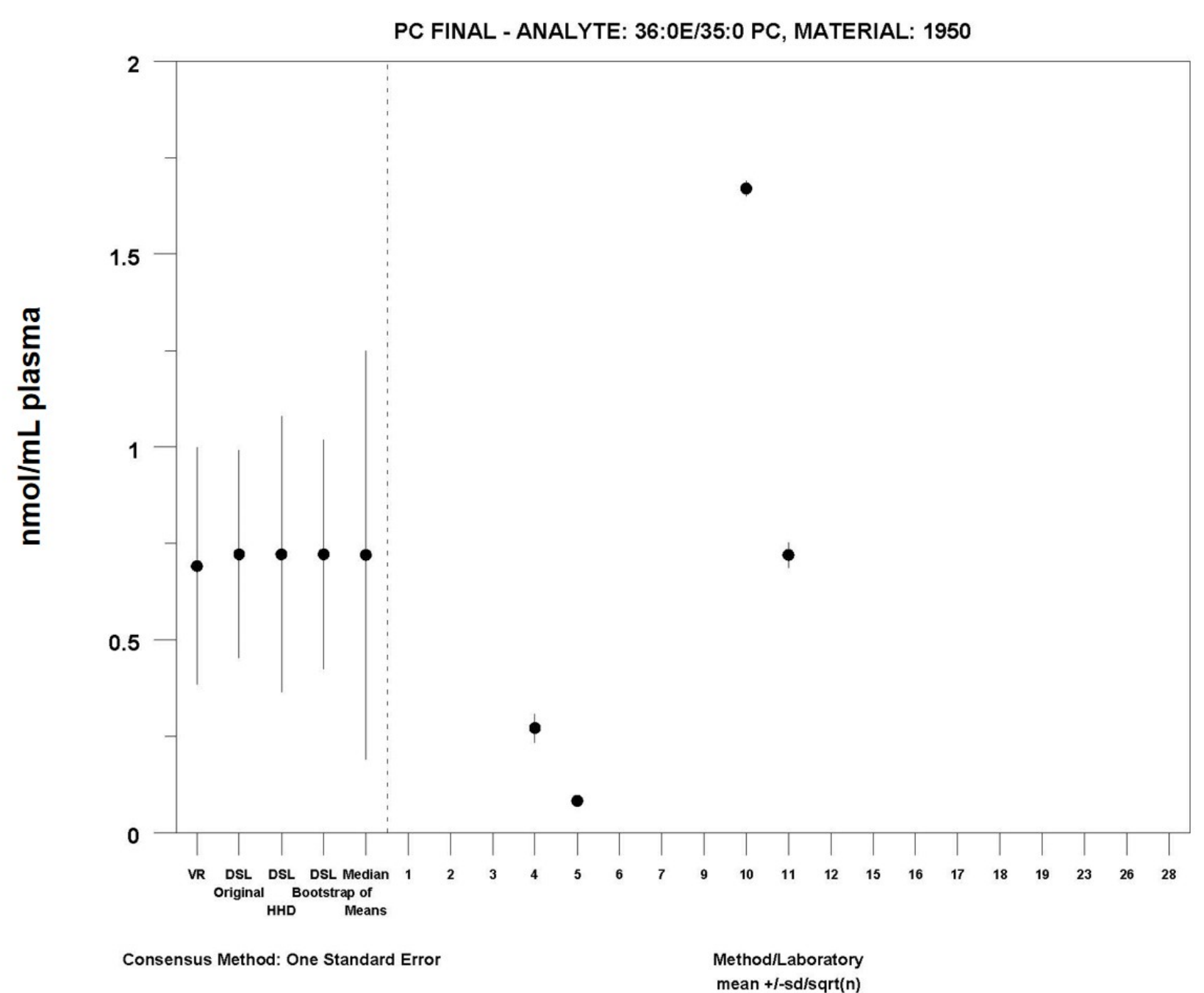

MEDM Location: $0.72 \pm 0.53 \mathrm{nmol} / \mathrm{mL}$

Labs Omitted from Plot (But Not Analysis): 15

15: $51 \pm 17$ 


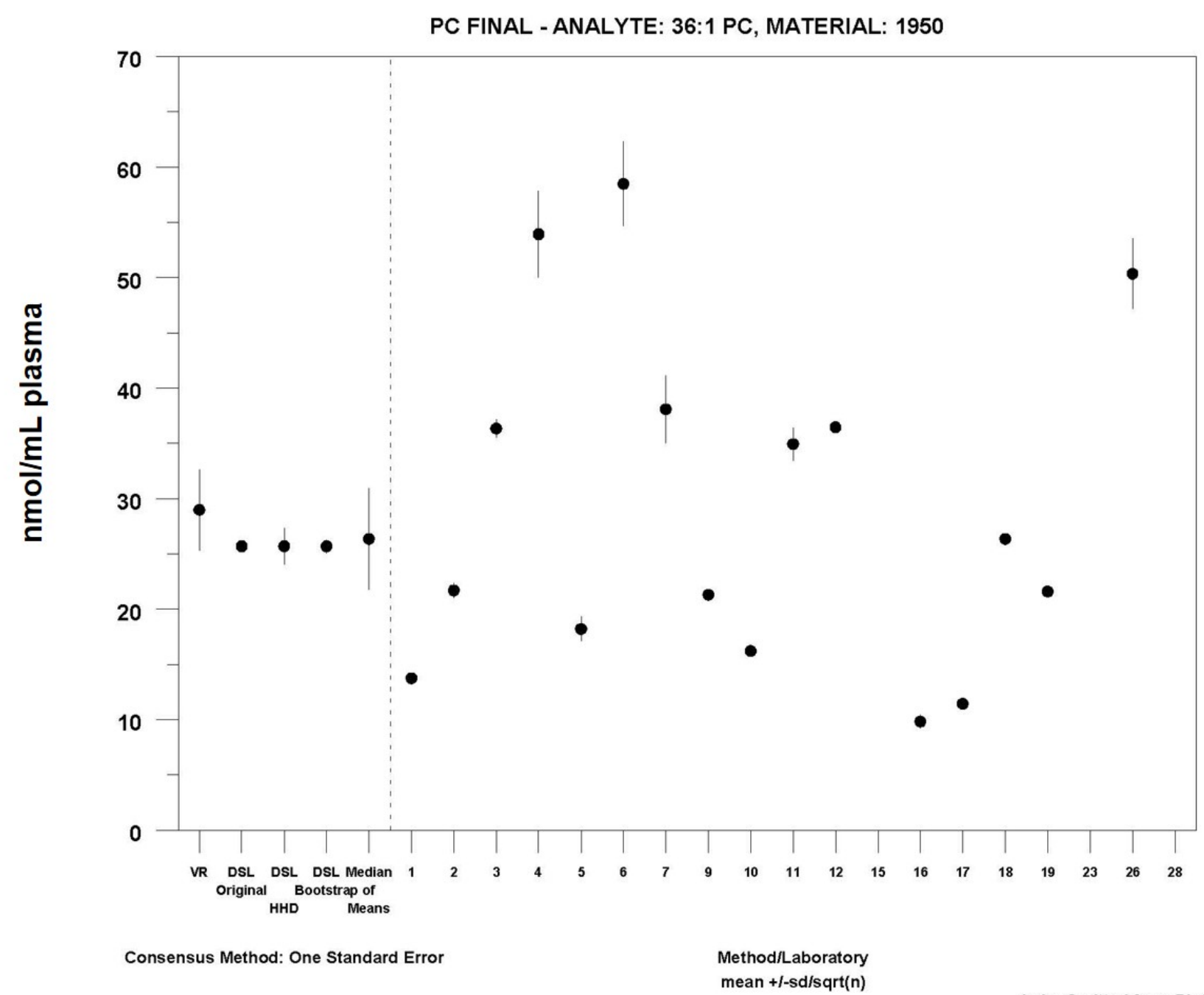

MEDM Location: $26 \pm 4.6 \mathrm{nmol} / \mathrm{mL}$

Labs Omitted from Plot (But Not Analysis): 15

15: $8146 \pm 6637$ 


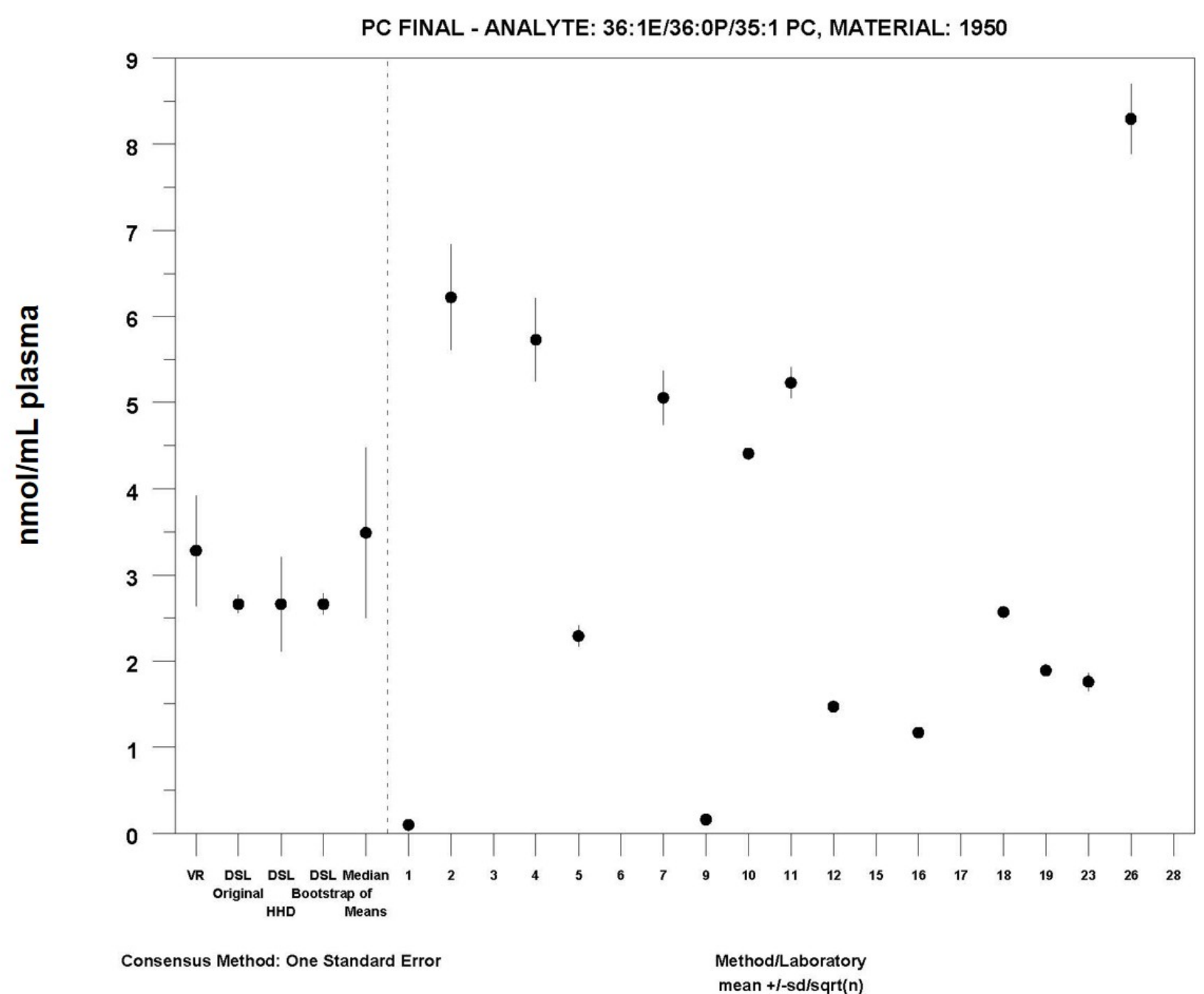

mean $+/$-sd/sqrt(n)

Labs Omitted from Plot (But Not Analysis): 615

MEDM Location: $3.5 \pm 0.99 \mathrm{nmol} / \mathrm{mL}$

6: $406 \pm 32 ; 15: 928 \pm 337$ 


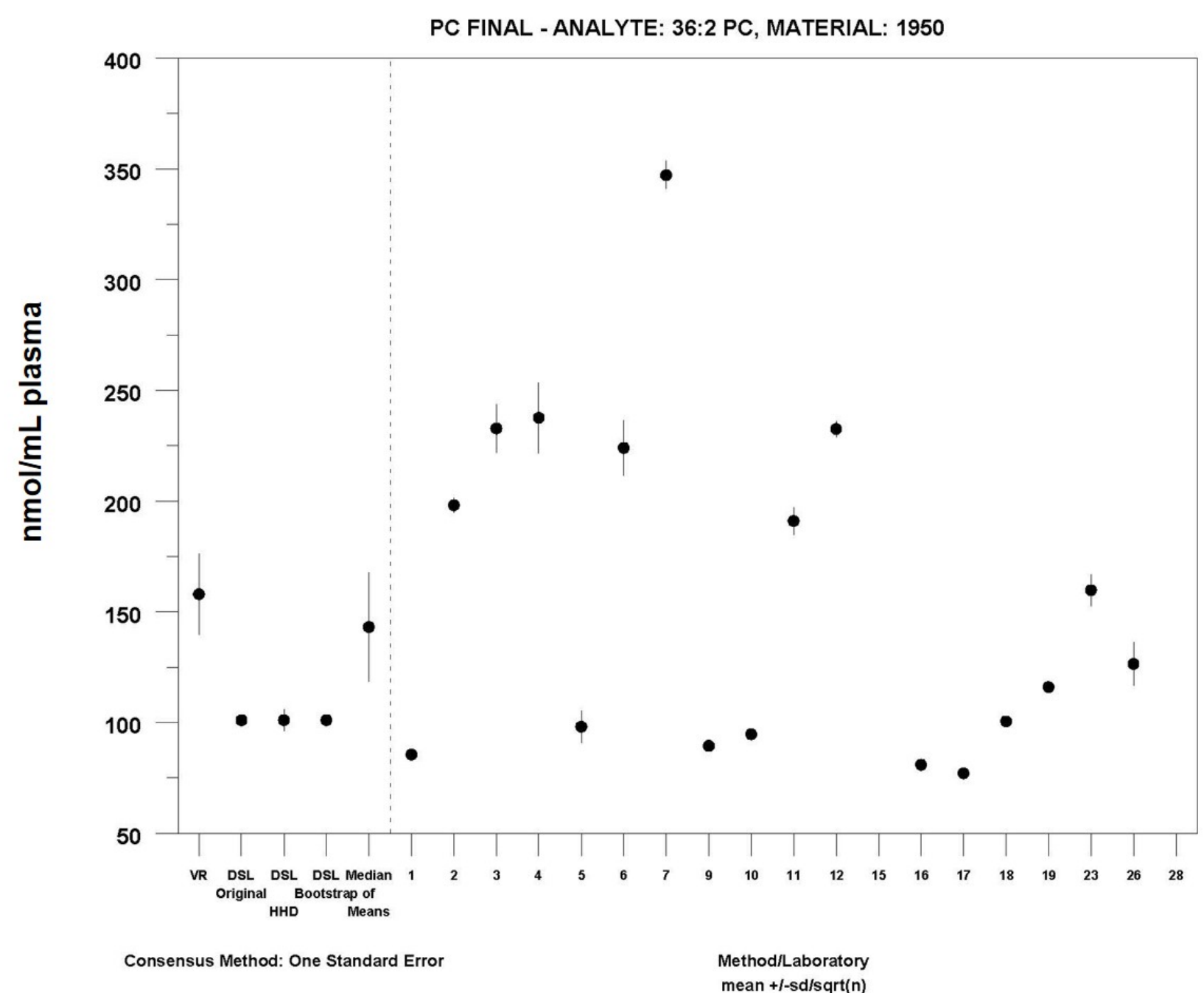

MEDM Location: $140 \pm 25 \mathrm{nmol} / \mathrm{mL}$

Labs Omitted from Plot (But Not Analysis): 15

15: $30108 \pm 14540$ 


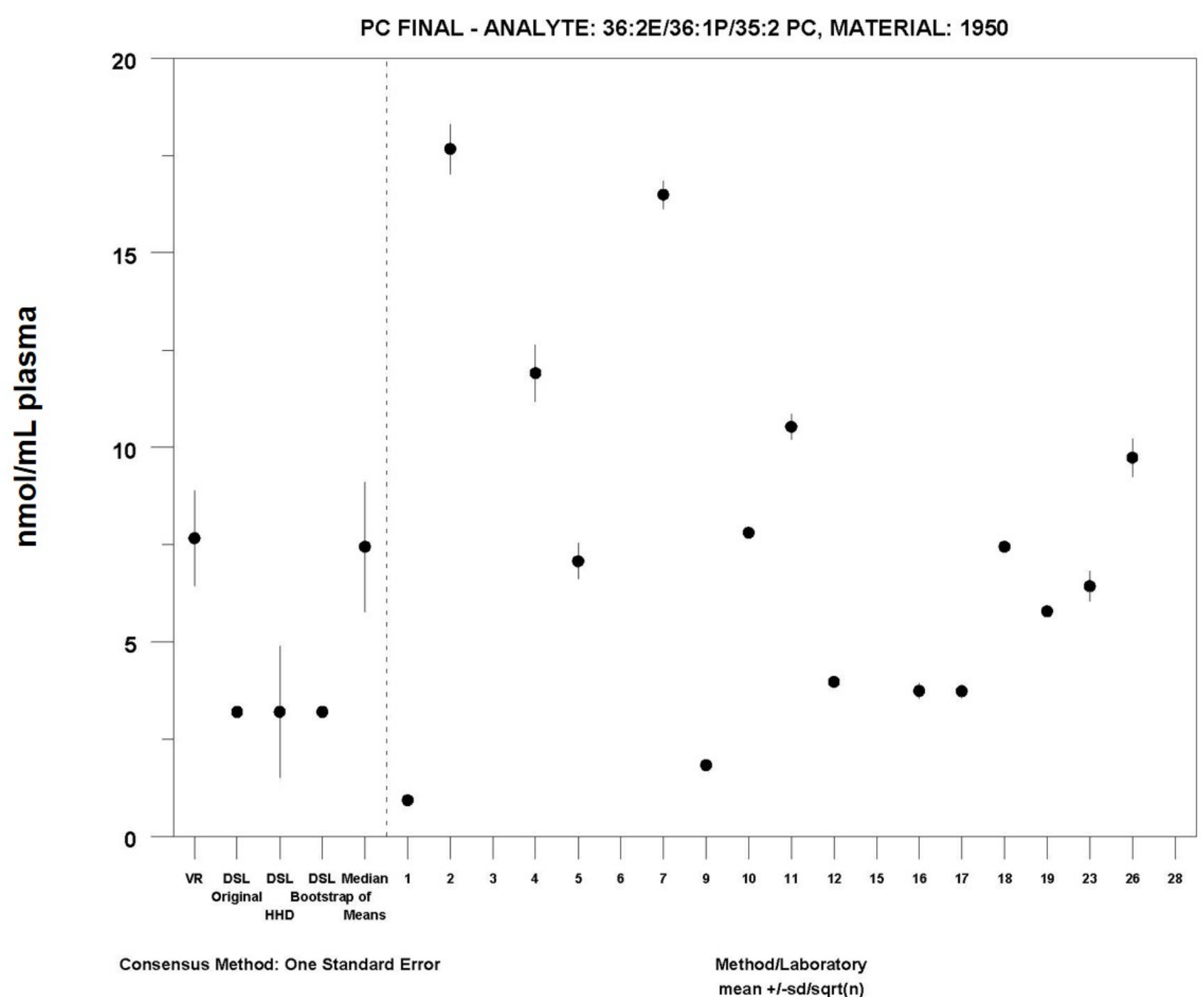

MEDM Location: $7.4 \pm 1.7 \mathrm{nmol} / \mathrm{mL}$

Labs Omitted from Plot (But Not Analysis): 615

6: $305 \pm 27 ; 15: 4496 \pm 448$ 


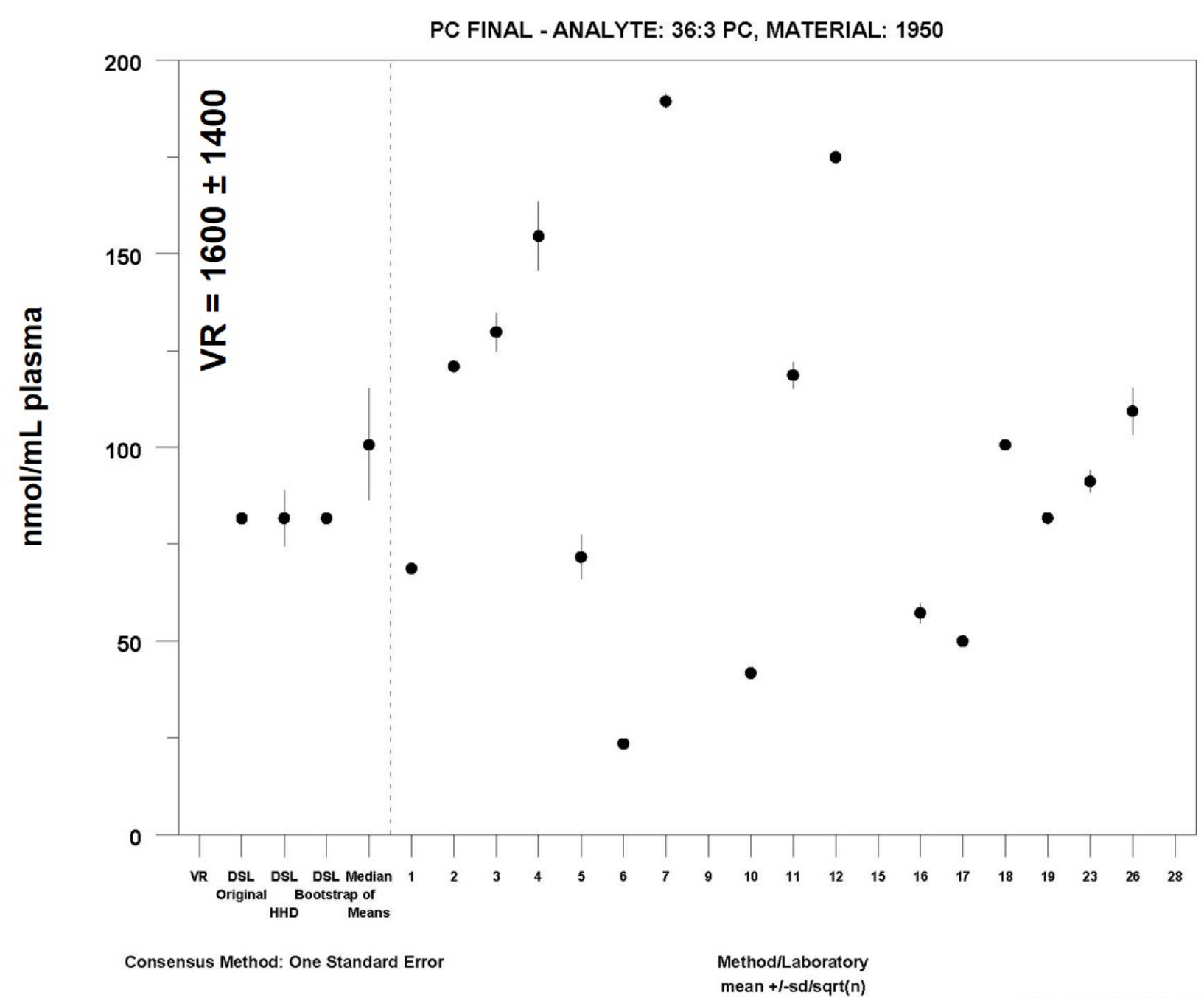

MEDM Location: $100 \pm 14 \mathrm{nmol} / \mathrm{mL}$

Labs Omitted from Plot (But Not Analysis): 15

15: $24813 \pm 539$ 


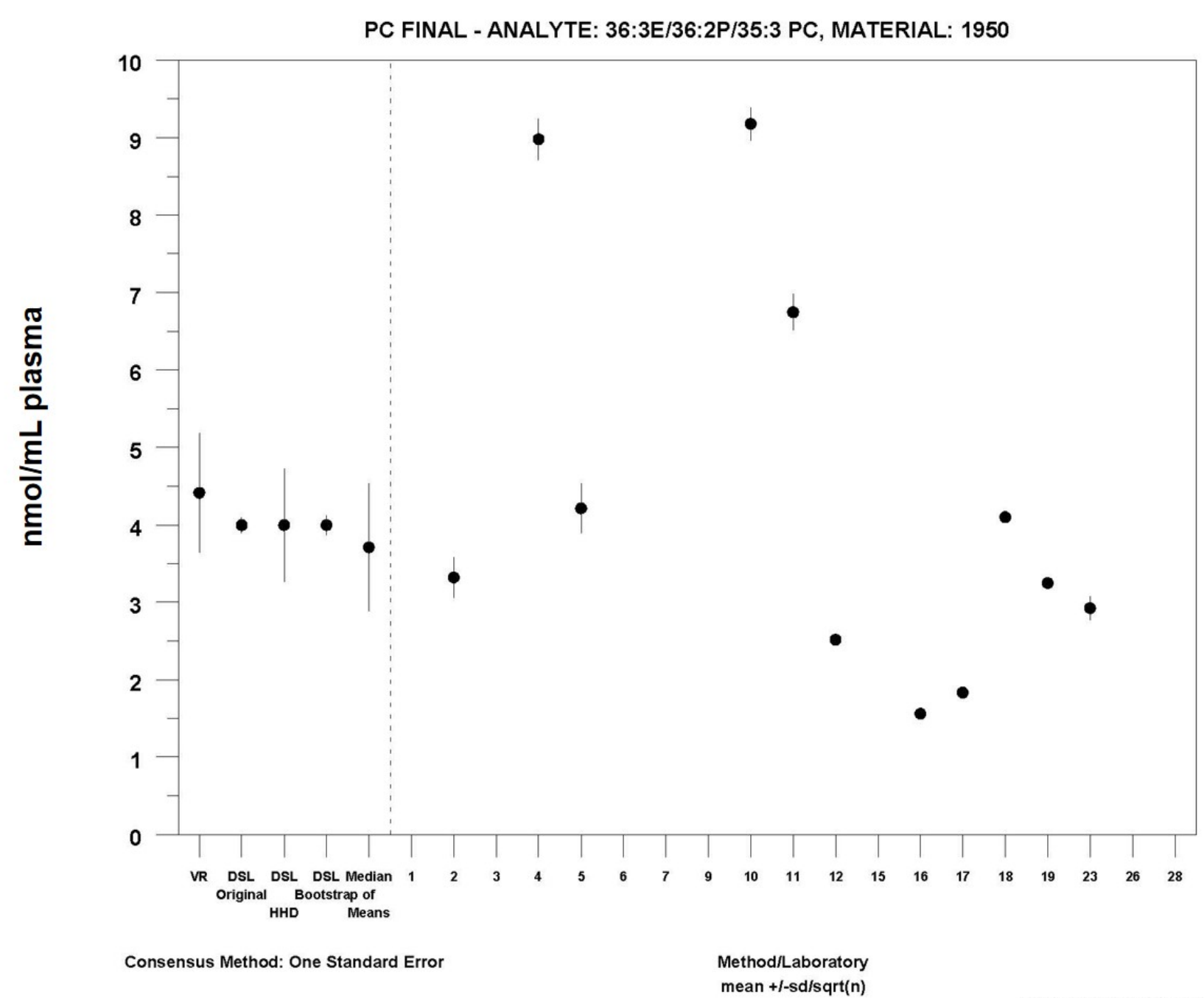

MEDM Location: $3.7 \pm 0.82 \mathrm{nmol} / \mathrm{mL}$

Labs Omitted from Plot (But Not Analysis): 15

15: $2300 \pm 235$ 


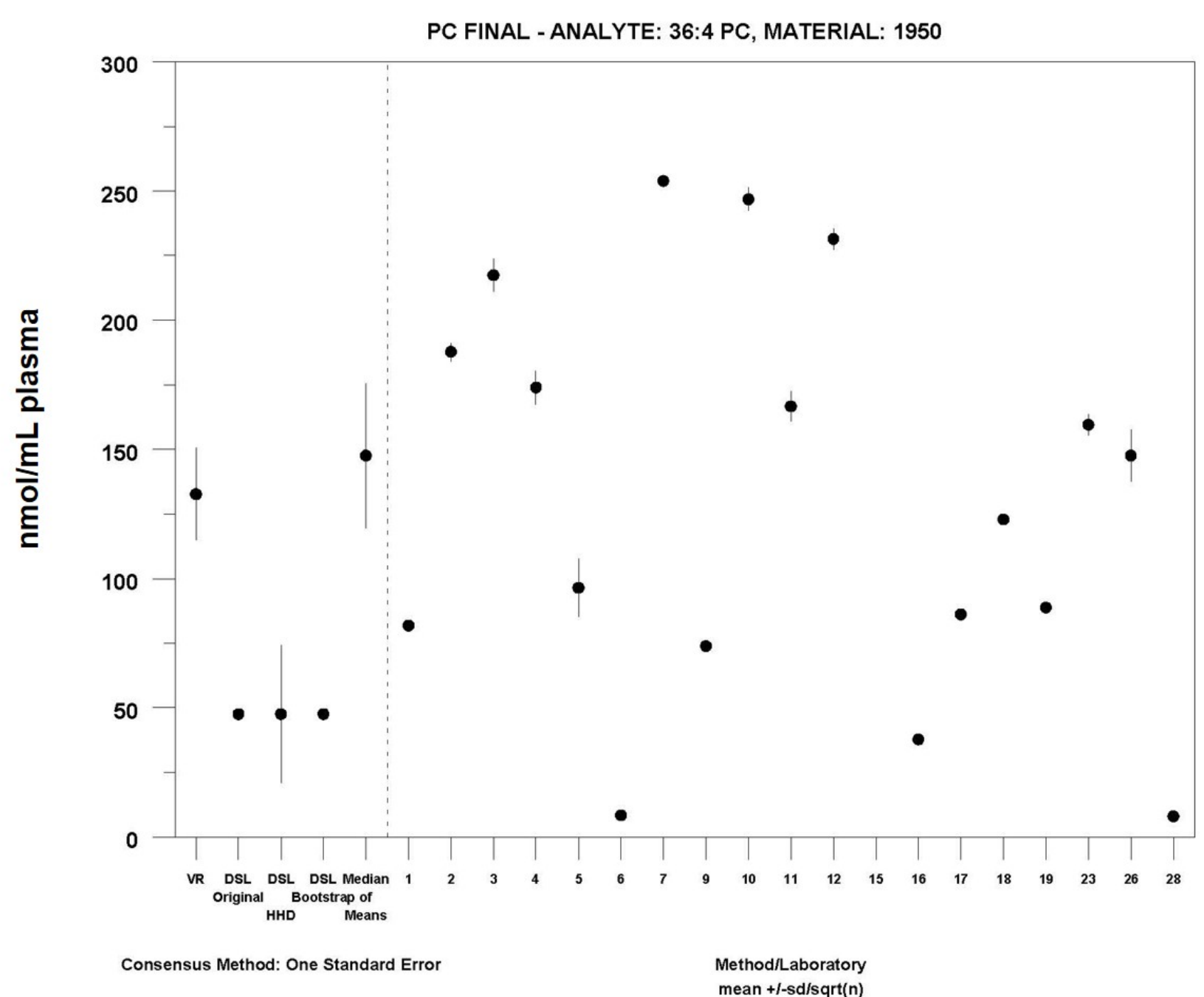

MEDM Location: $150 \pm 28 \mathrm{nmol} / \mathrm{mL}$

Labs Omitted from Plot (But Not Analysis): 15

15: $33029 \pm 3652$ 


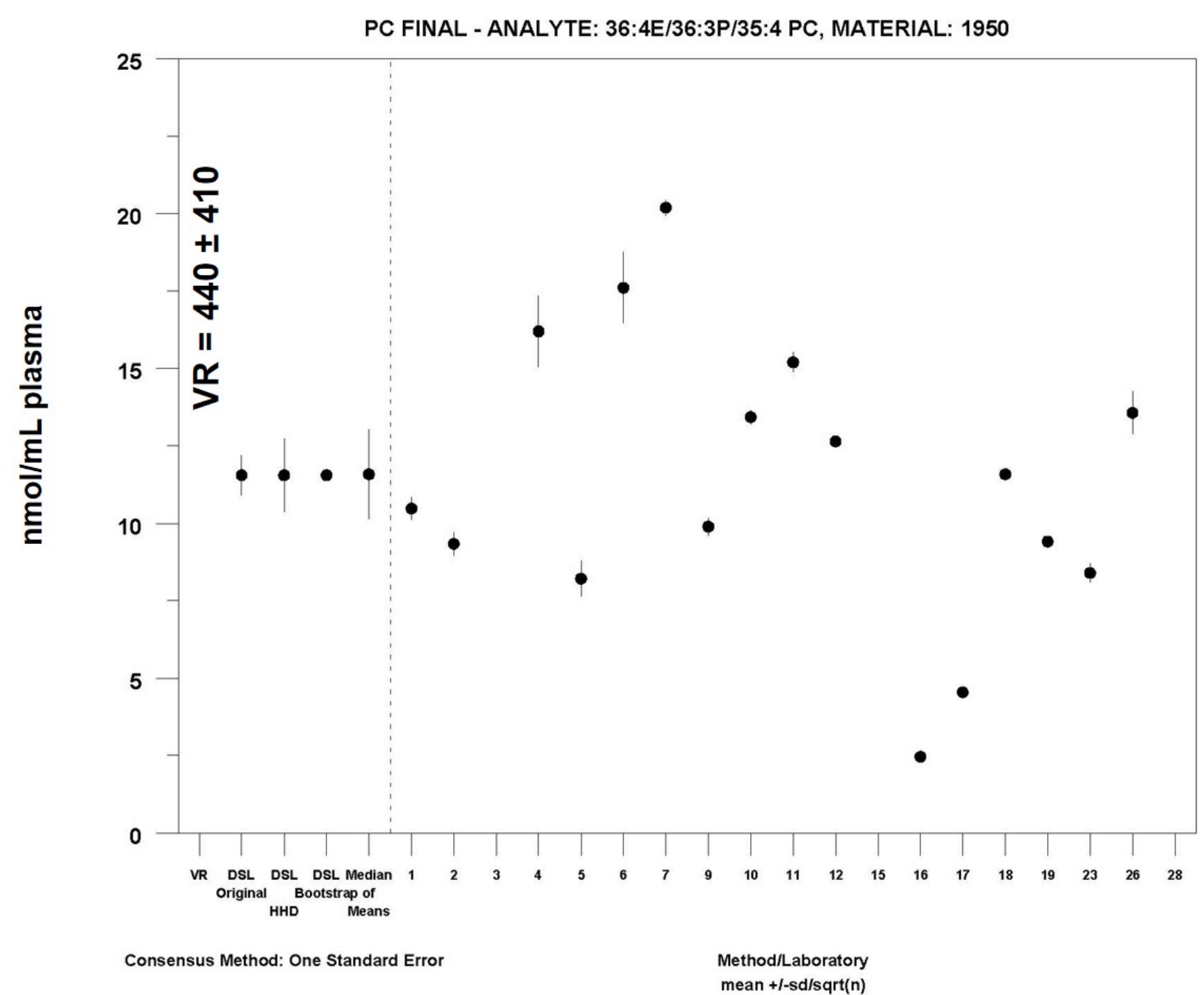

MEDM Location: $12 \pm 1.4 \mathrm{nmol} / \mathrm{mL}$

Labs Omitted from Plot (But Not Analysis): 15

15: $7267 \pm 198$ 


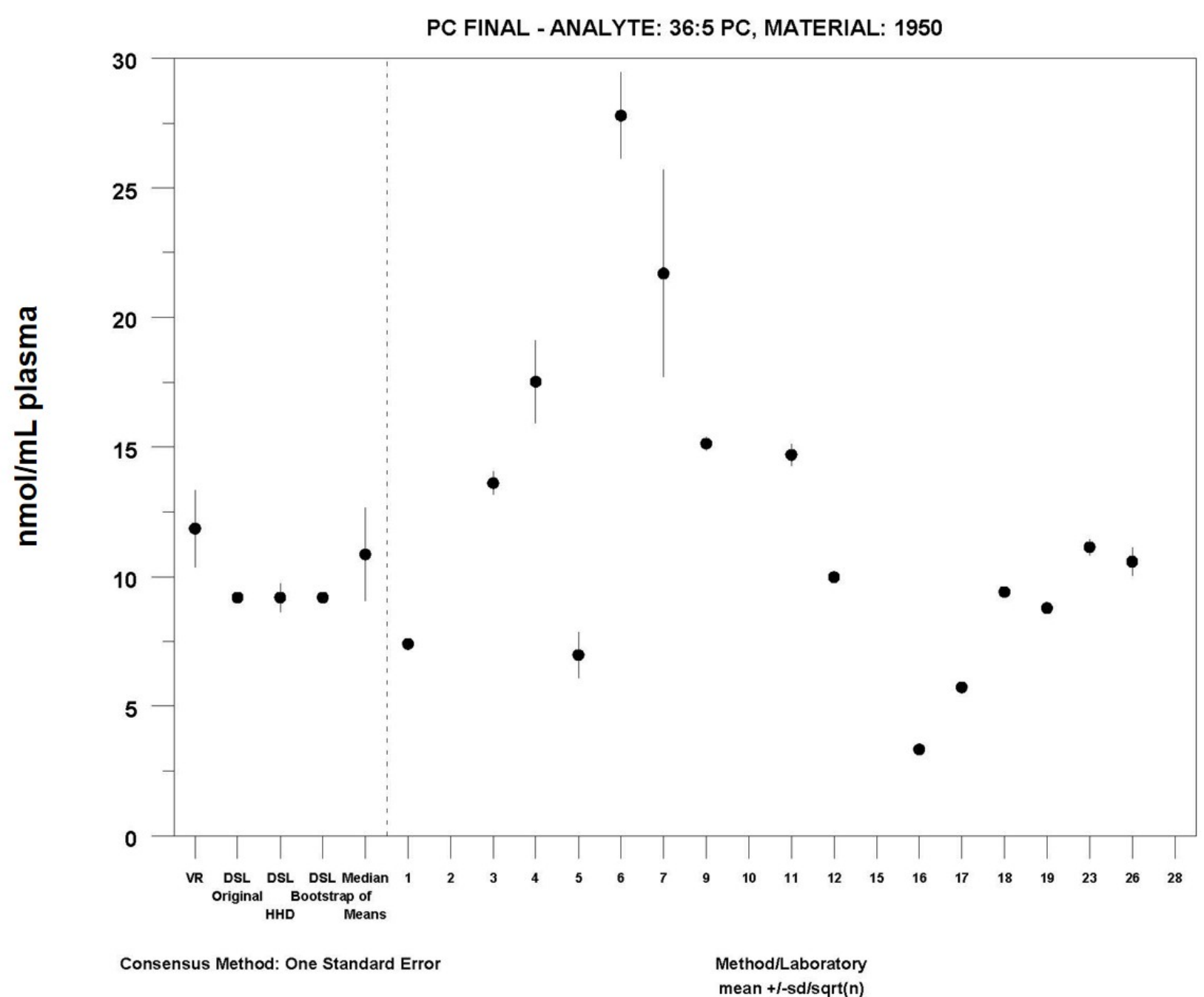

MEDM Location: $11 \pm 1.8 \mathrm{nmol} / \mathrm{mL}$

Labs Omitted from Plot (But Not Analysis): 15

15: $8151 \pm 880$ 


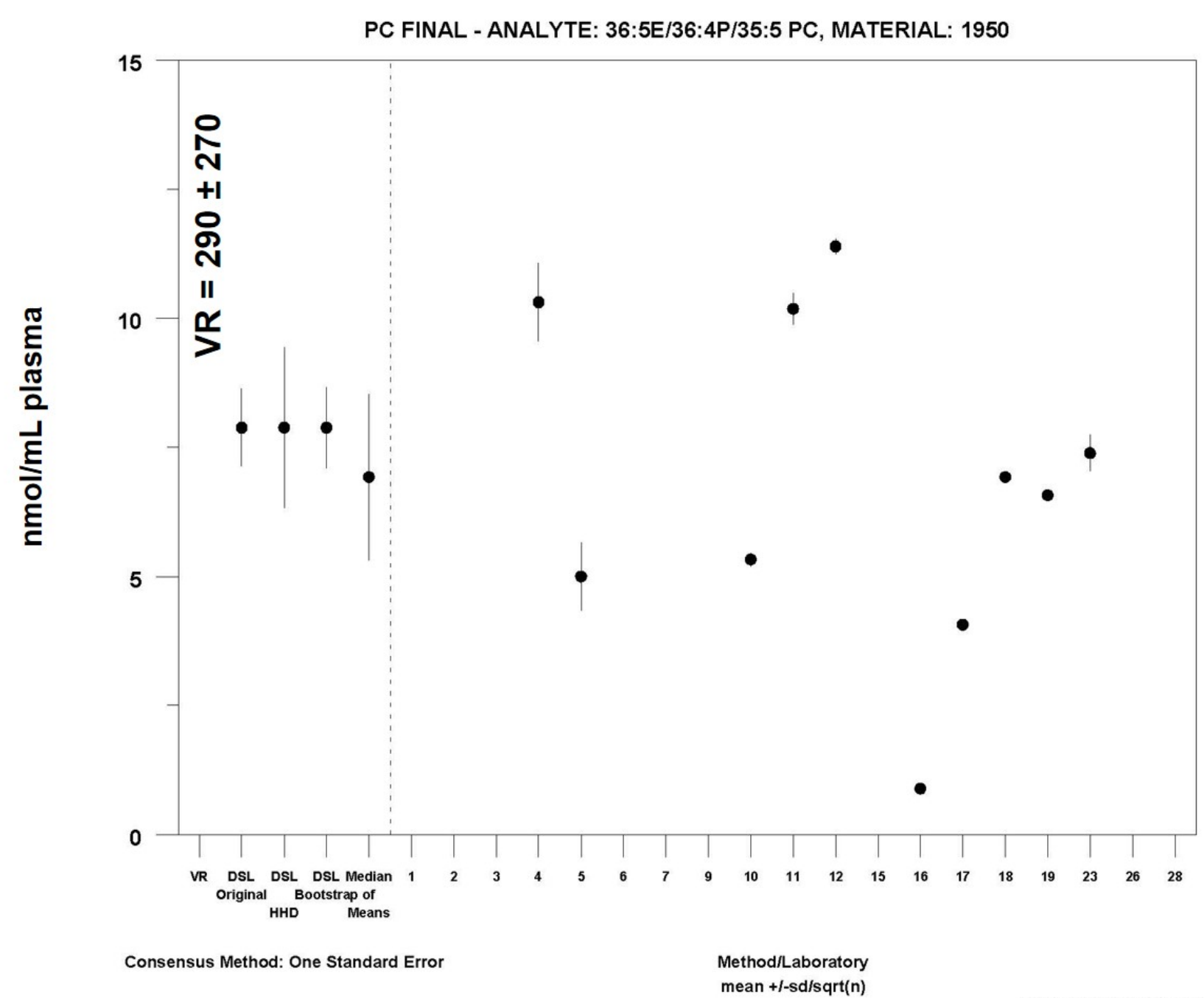

MEDM Location: $6.9 \pm 1.6 \mathrm{nmol} / \mathrm{mL}$

Labs Omitted from Plot (But Not Analysis): 15

15: $3105 \pm 68$ 


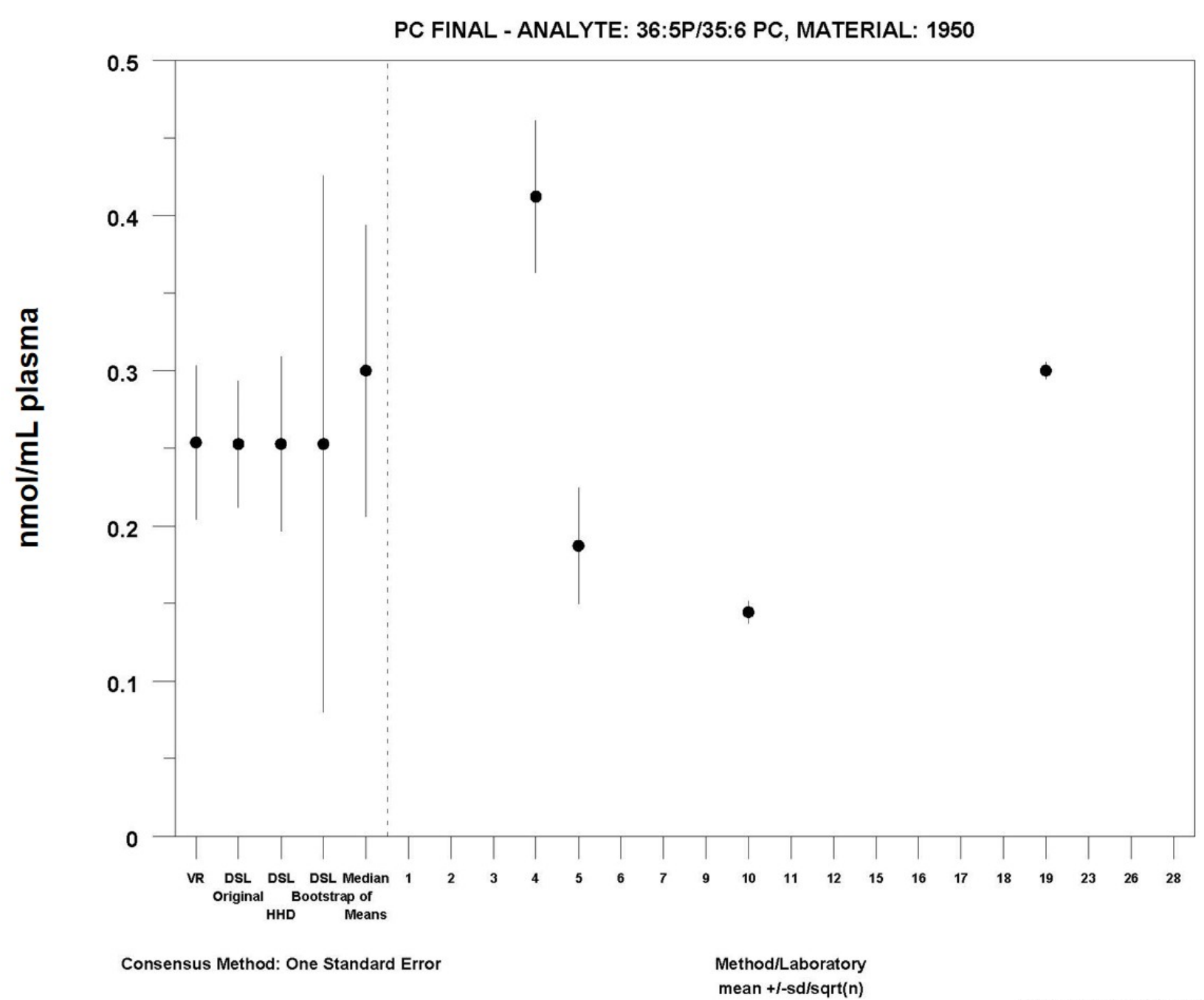

MEDM Location: $0.30 \pm 0.094 \mathrm{nmol} / \mathrm{mL}$

Labs Omitted from Plot (But Not Analysis): 15

15: $186 \pm 129$ 


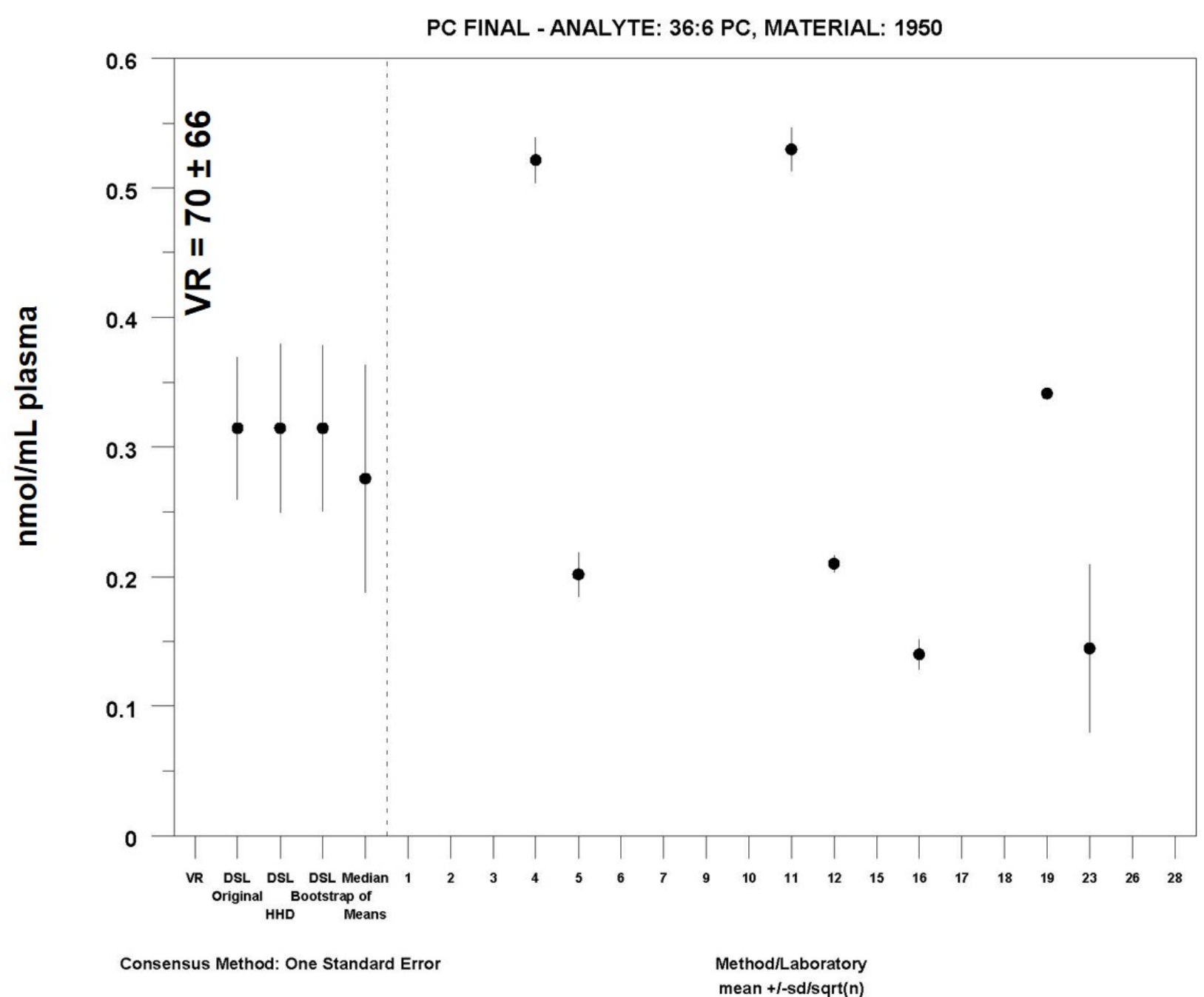

MEDM Location: $0.28 \pm 0.088 \mathrm{nmol} / \mathrm{mL}$

Labs Omitted from Plot (But Not Analysis): 15

\section{5: $563 \pm 20$}




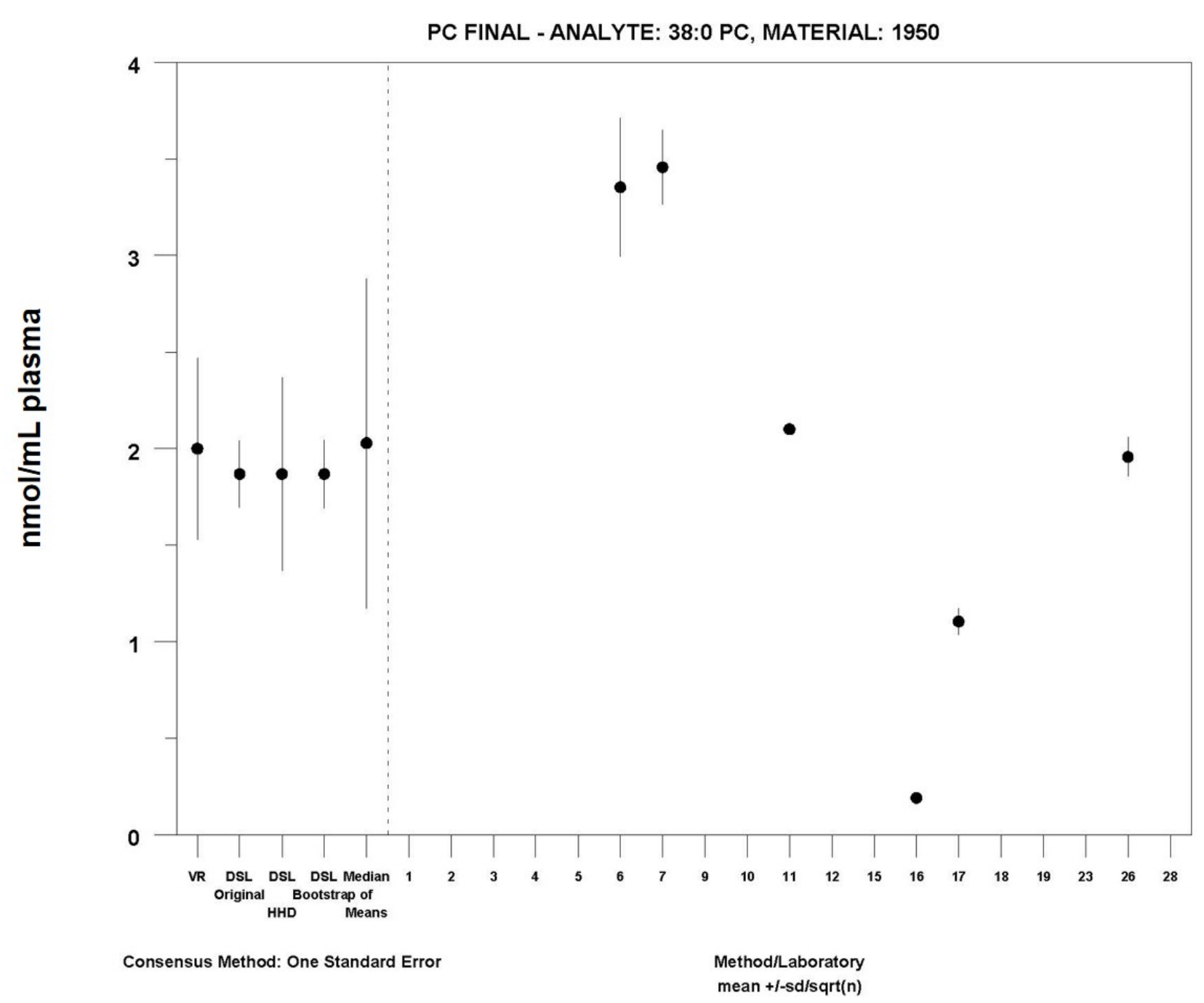

MEDM Location: $2.0 \pm 0.85 \mathrm{nmol} / \mathrm{mL}$ 


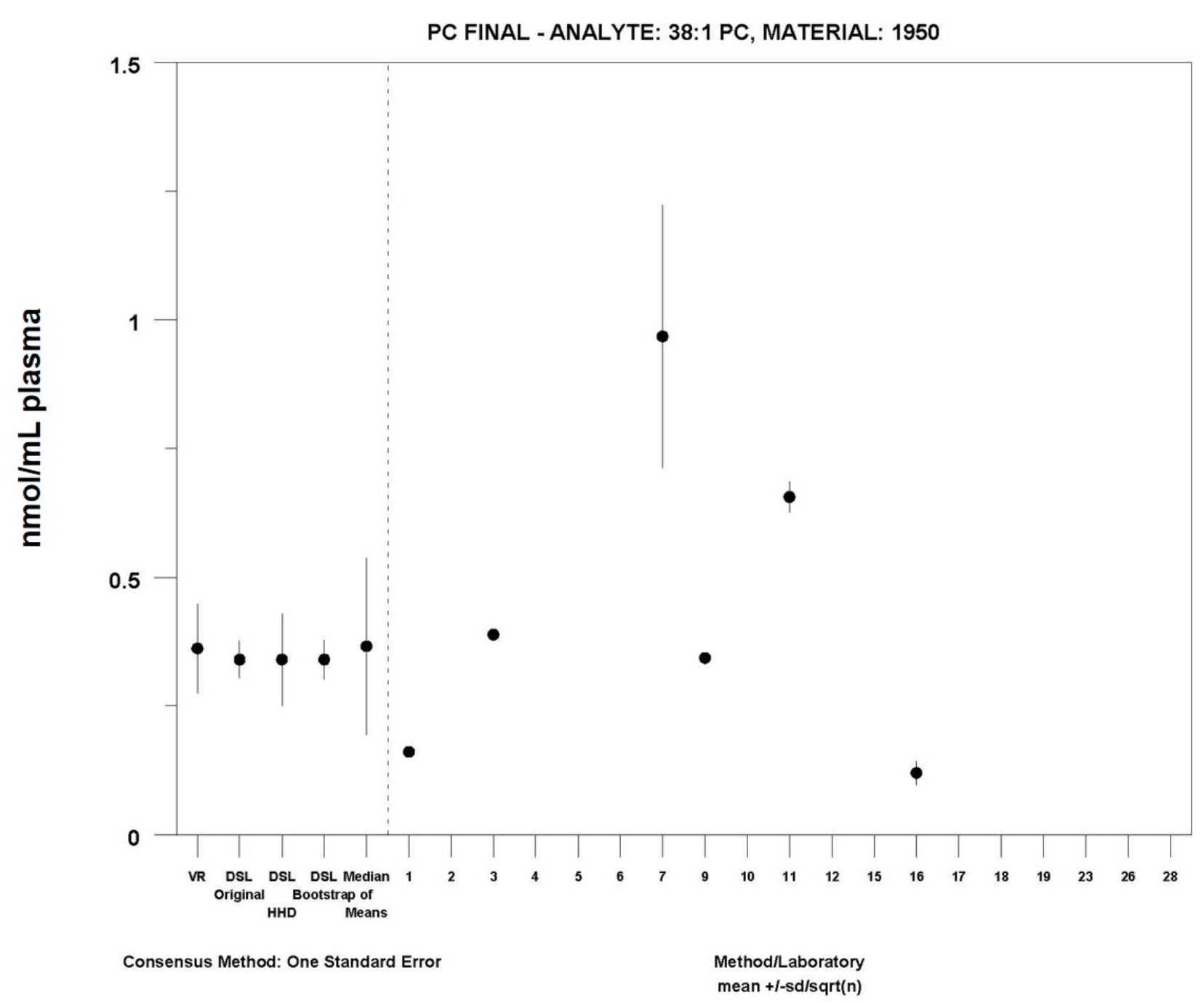

MEDM Location: $0.37 \pm 0.17 \mathrm{nmol} / \mathrm{mL}$ 


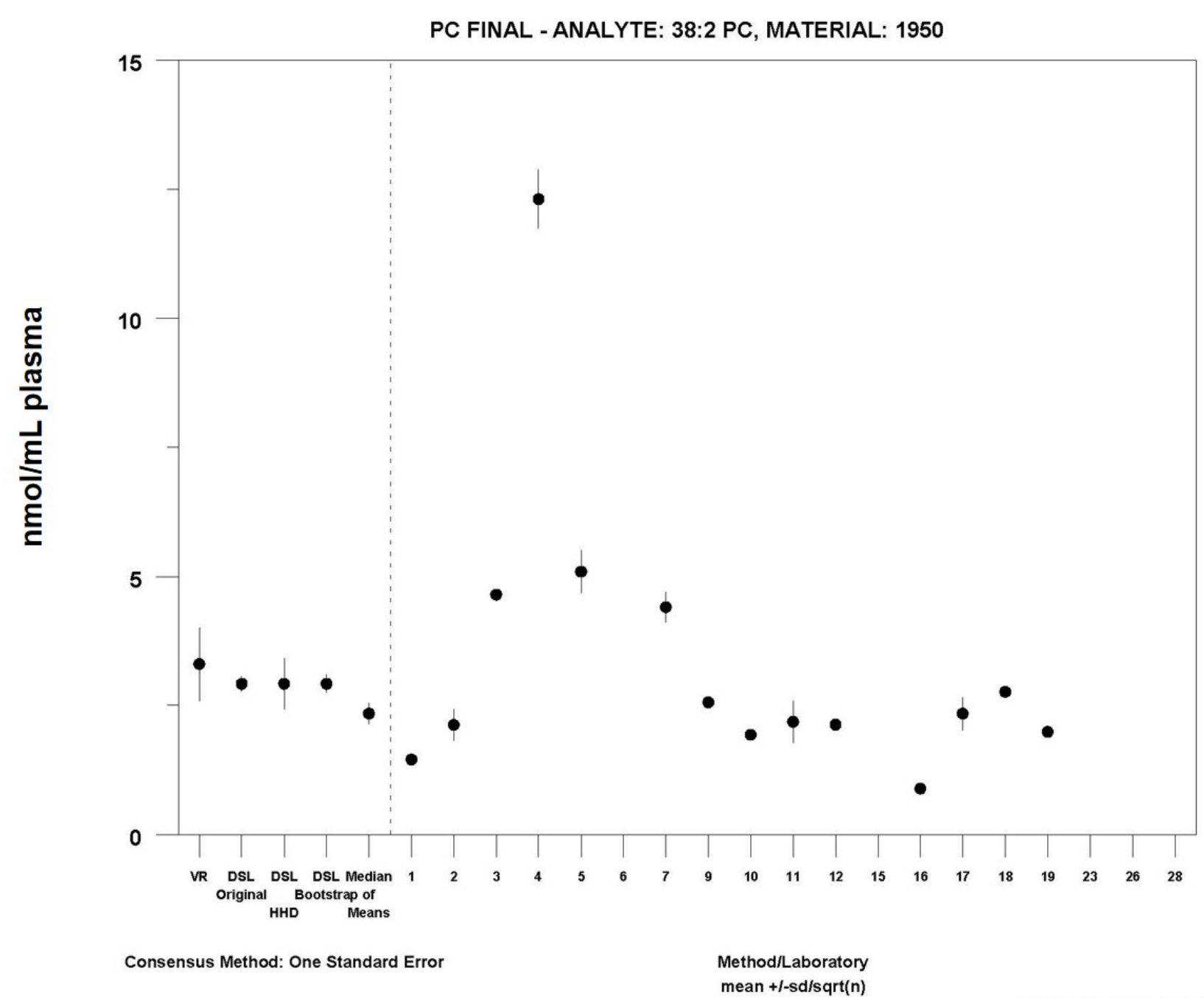

MEDM Location: $2.3 \pm 0.20 \mathrm{nmol} / \mathrm{mL}$

Labs Omitted from Plot (But Not Analysis): 15

15: $1468 \pm 247$ 


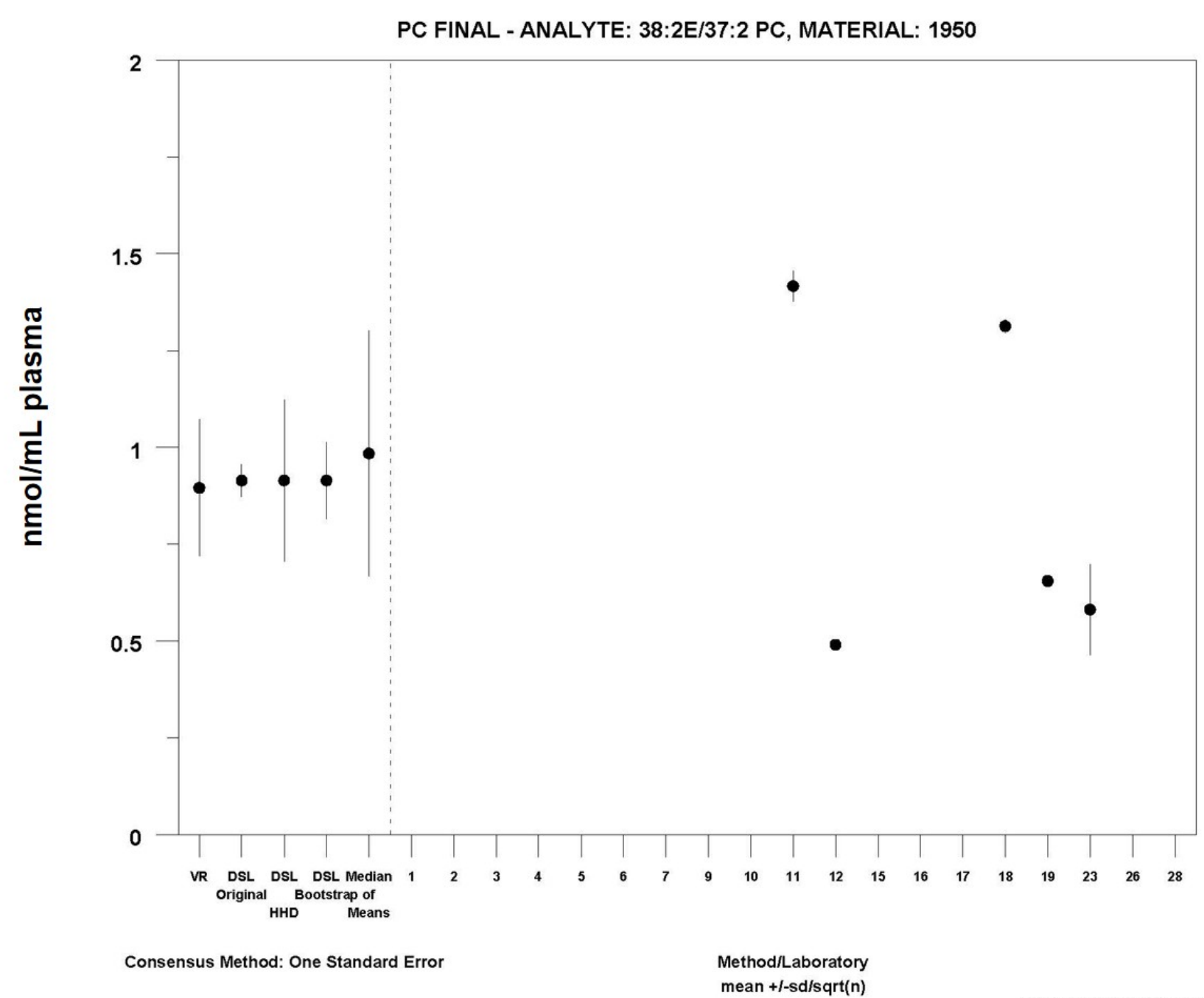

MEDM Location: $0.98 \pm 0.32 \mathrm{nmol} / \mathrm{mL}$

Labs Omitted from Plot (But Not Analysis): 15

15: $249 \pm 182$ 


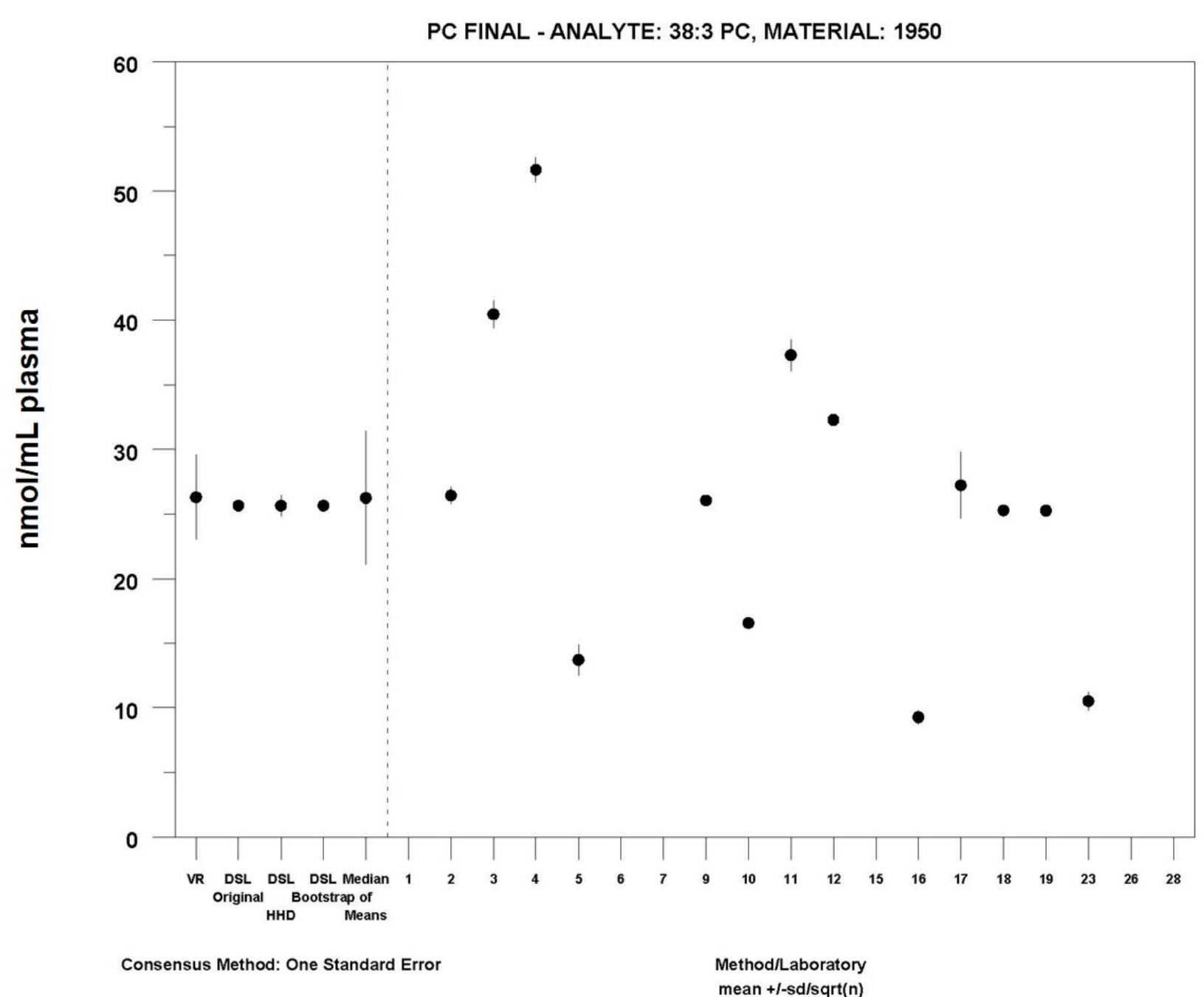

MEDM Location: $26 \pm 5.2 \mathrm{nmol} / \mathrm{mL}$

Labs Omitted from Plot (But Not Analysis): 15

15: $3956 \pm 2608$ 


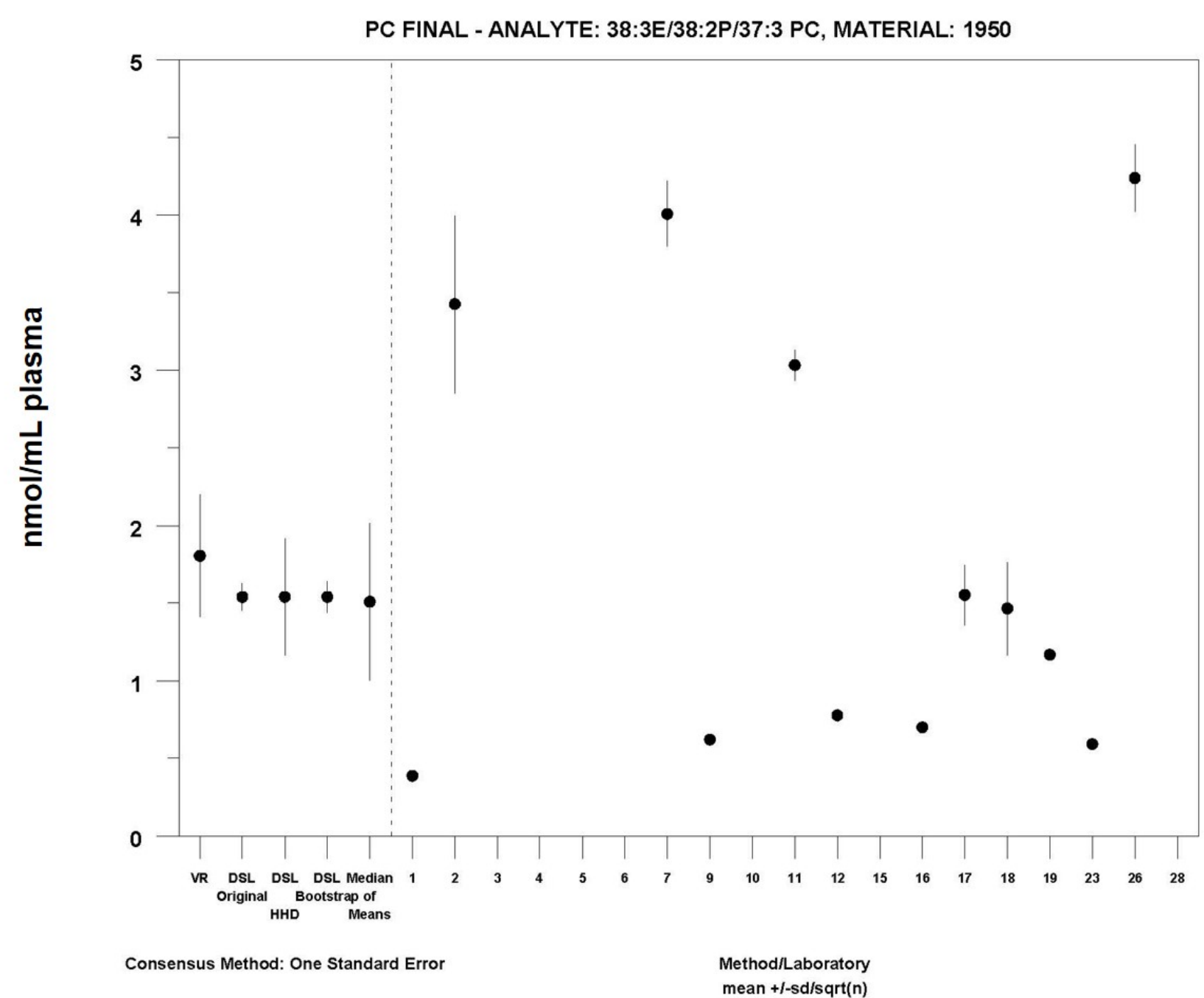

MEDM Location: $1.5 \pm 0.51 \mathrm{nmol} / \mathrm{mL}$

Labs Omitted from Plot (But Not Analysis): 615

6: $194 \pm 21 ; 15: 585 \pm 67$ 


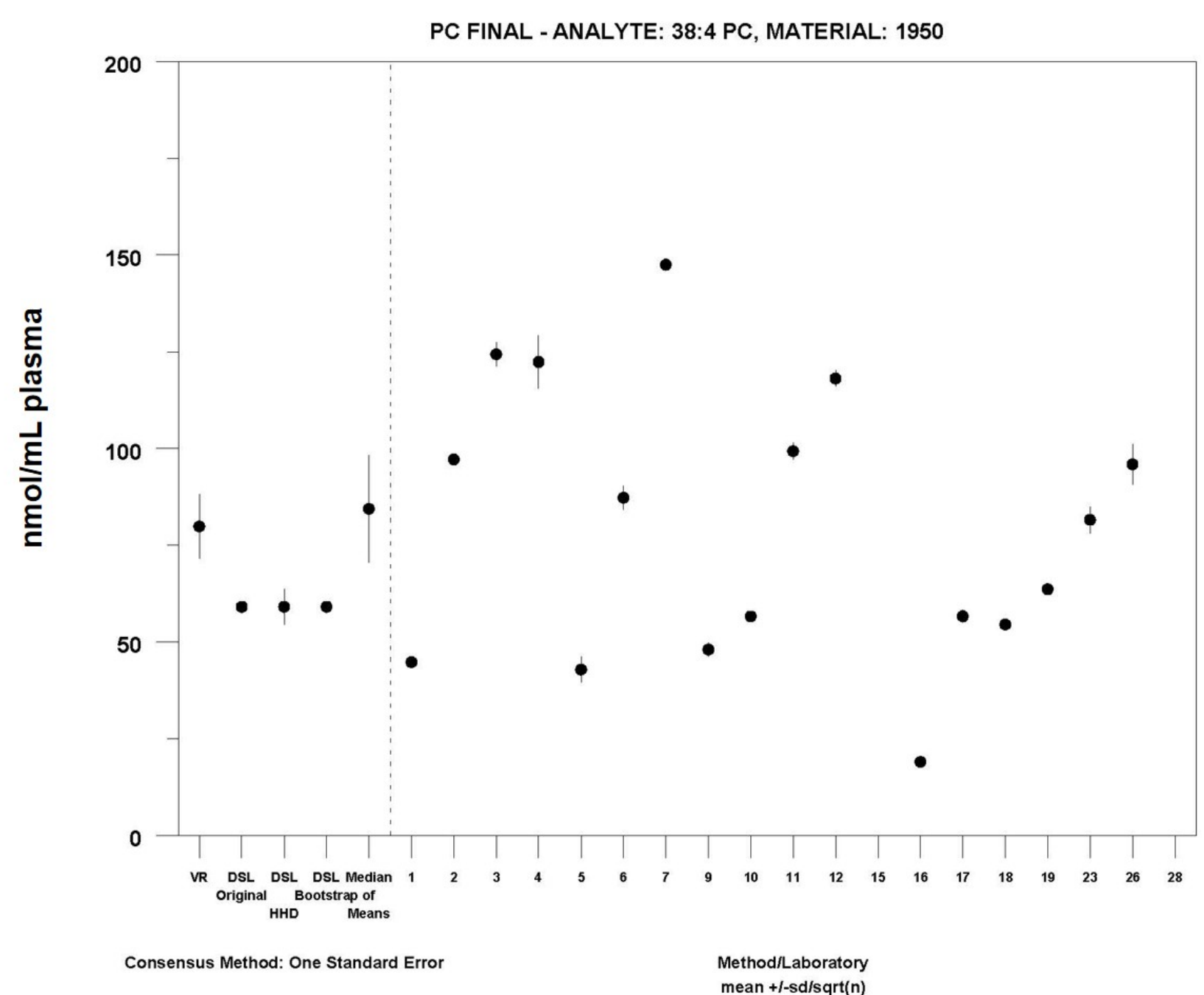

MEDM Location: $84 \pm 14 \mathrm{nmol} / \mathrm{mL}$

Labs Omitted from Plot (But Not Analysis): 15

15: $14779 \pm 971$ 


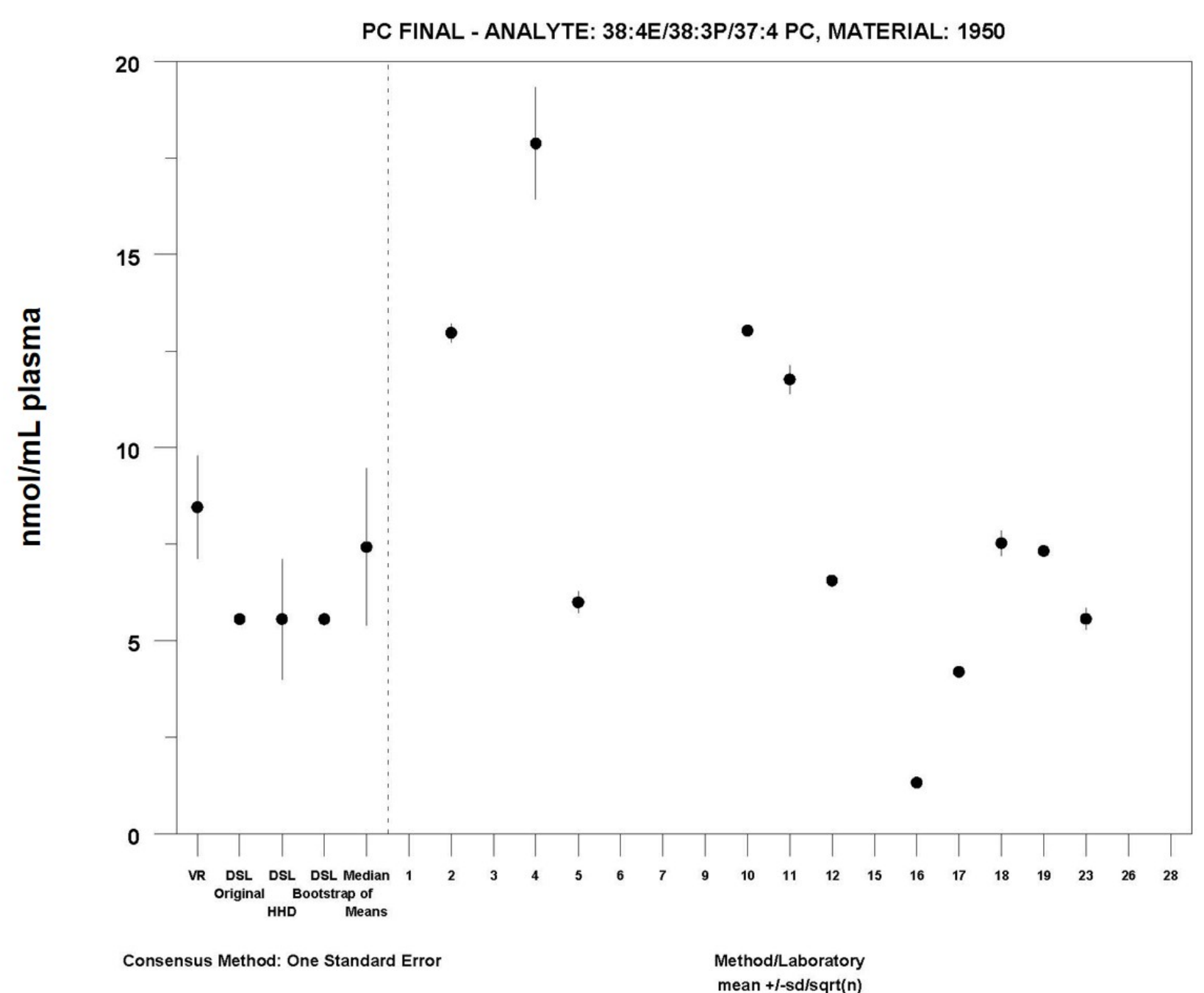

MEDM Location: $7.4 \pm 2.0 \mathrm{nmol} / \mathrm{mL}$

Labs Omitted from Plot (But Not Analysis): 15

15: $3336 \pm 1223$ 


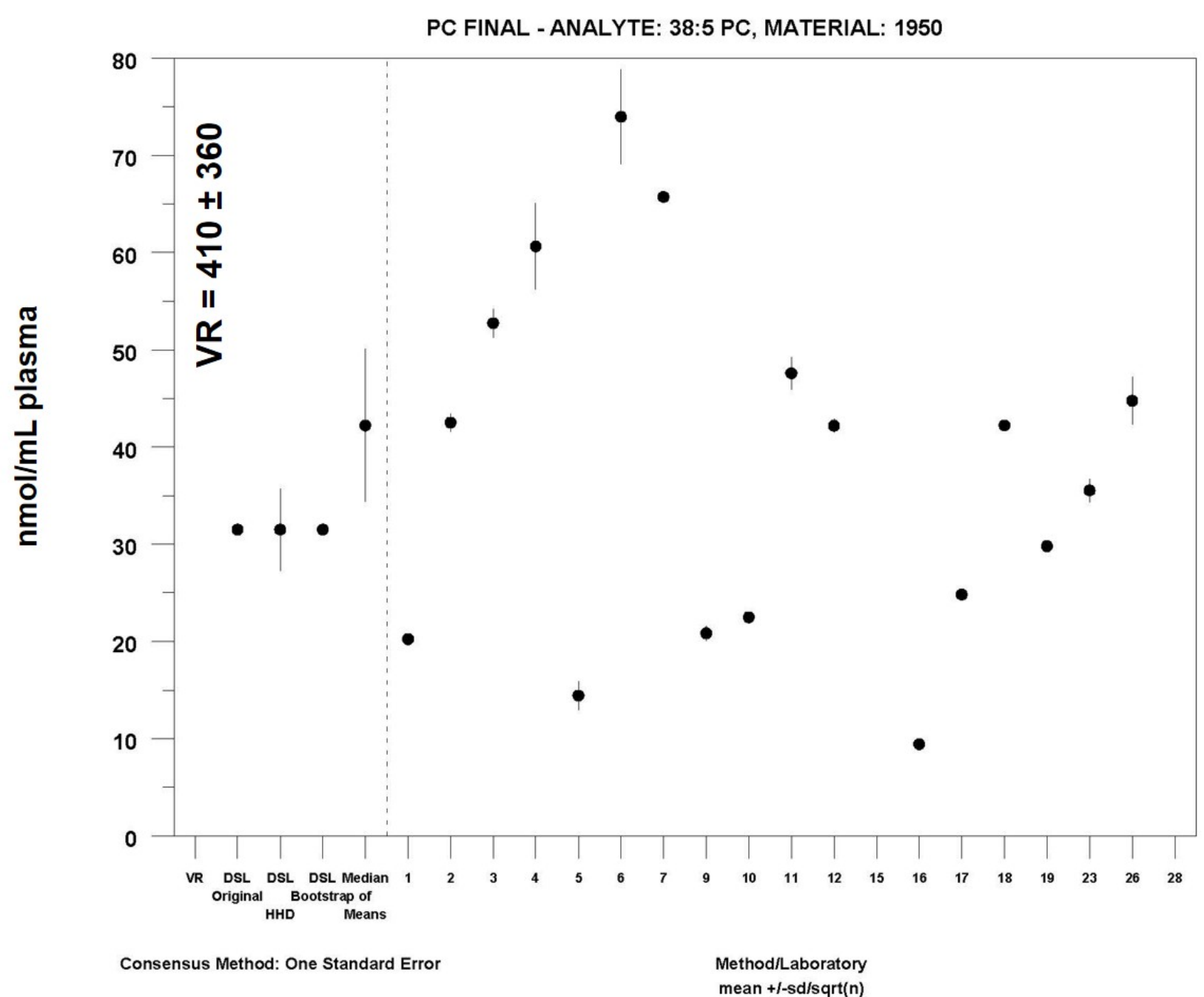

MEDM Location: $42 \pm 7.9 \mathrm{nmol} / \mathrm{mL}$

Labs Omitted from Plot (But Not Analysis): 15

15: $6748 \pm 86$ 


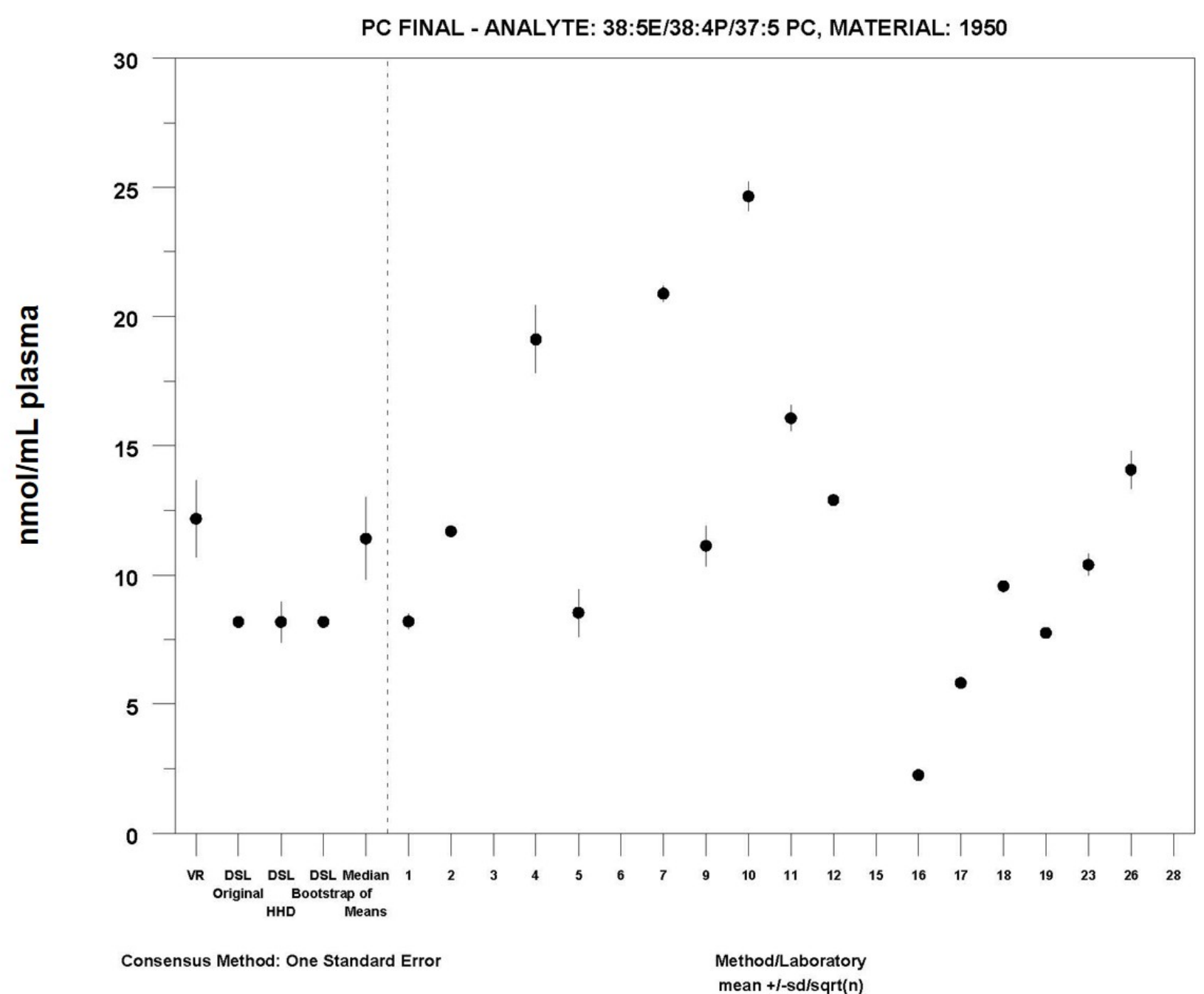

MEDM Location: $11 \pm 1.6 \mathrm{nmol} / \mathrm{mL}$

Labs Omitted from Plot (But Not Analysis): 15

15: $8931 \pm 5618$ 


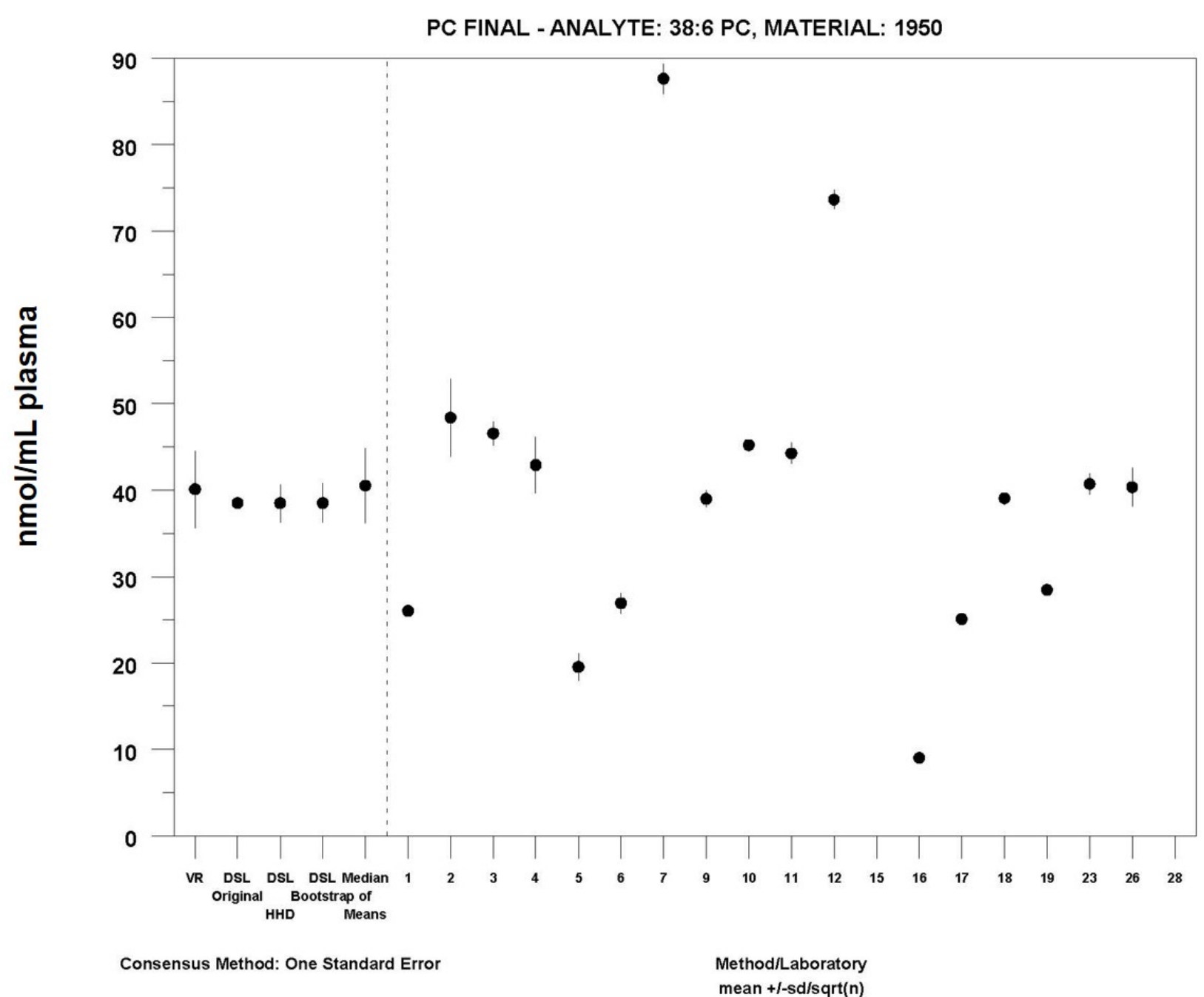

MEDM Location: $41 \pm 4.4 \mathrm{nmol} / \mathrm{mL}$

Labs Omitted from Plot (But Not Analysis): 15

15: $12844 \pm 9649$ 


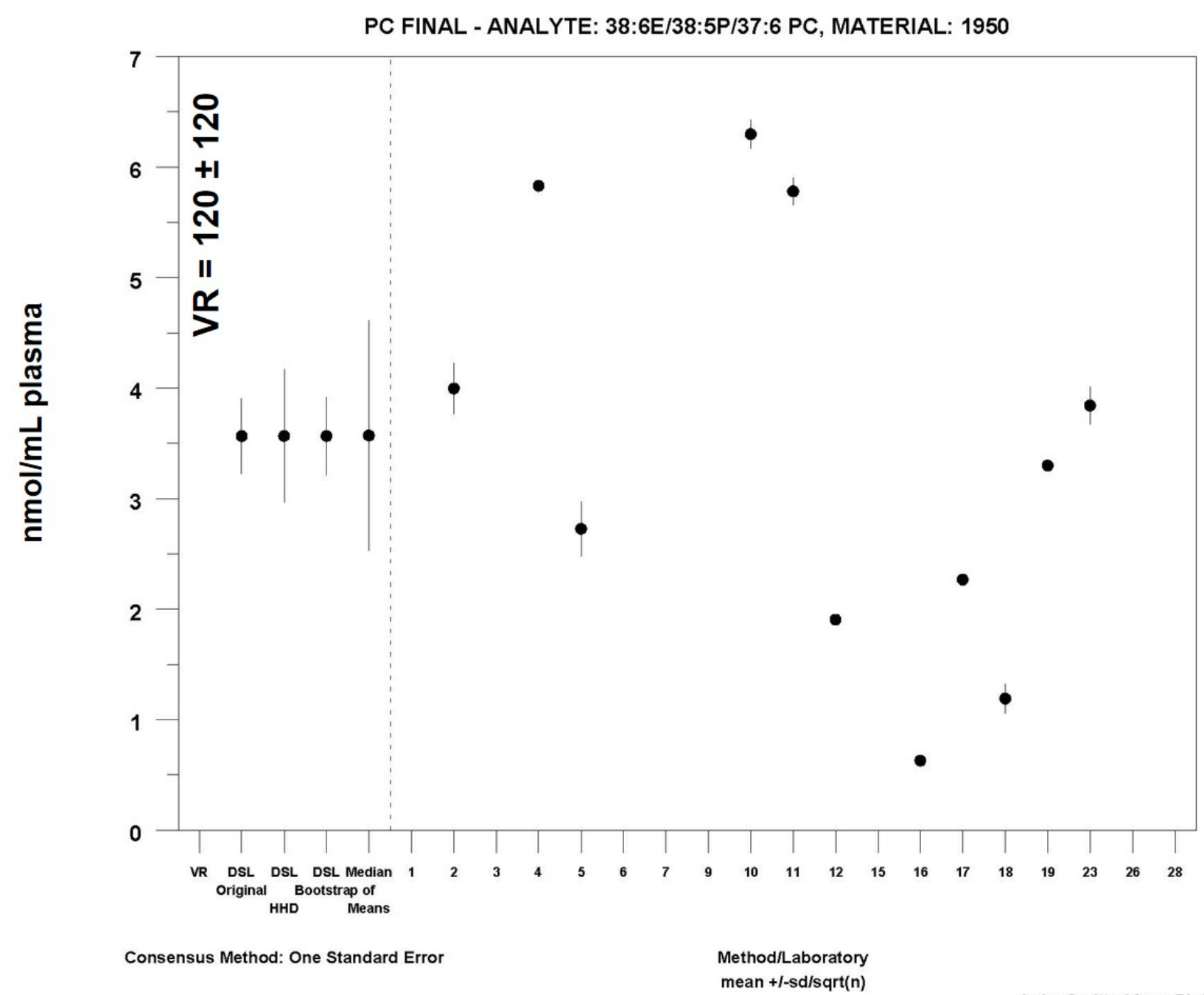

MEDM Location: $3.6 \pm 1.0 \mathrm{nmol} / \mathrm{mL}$

Labs Omitted from Plot (But Not Analysis): 15

15: $14.63 \pm 61$ 


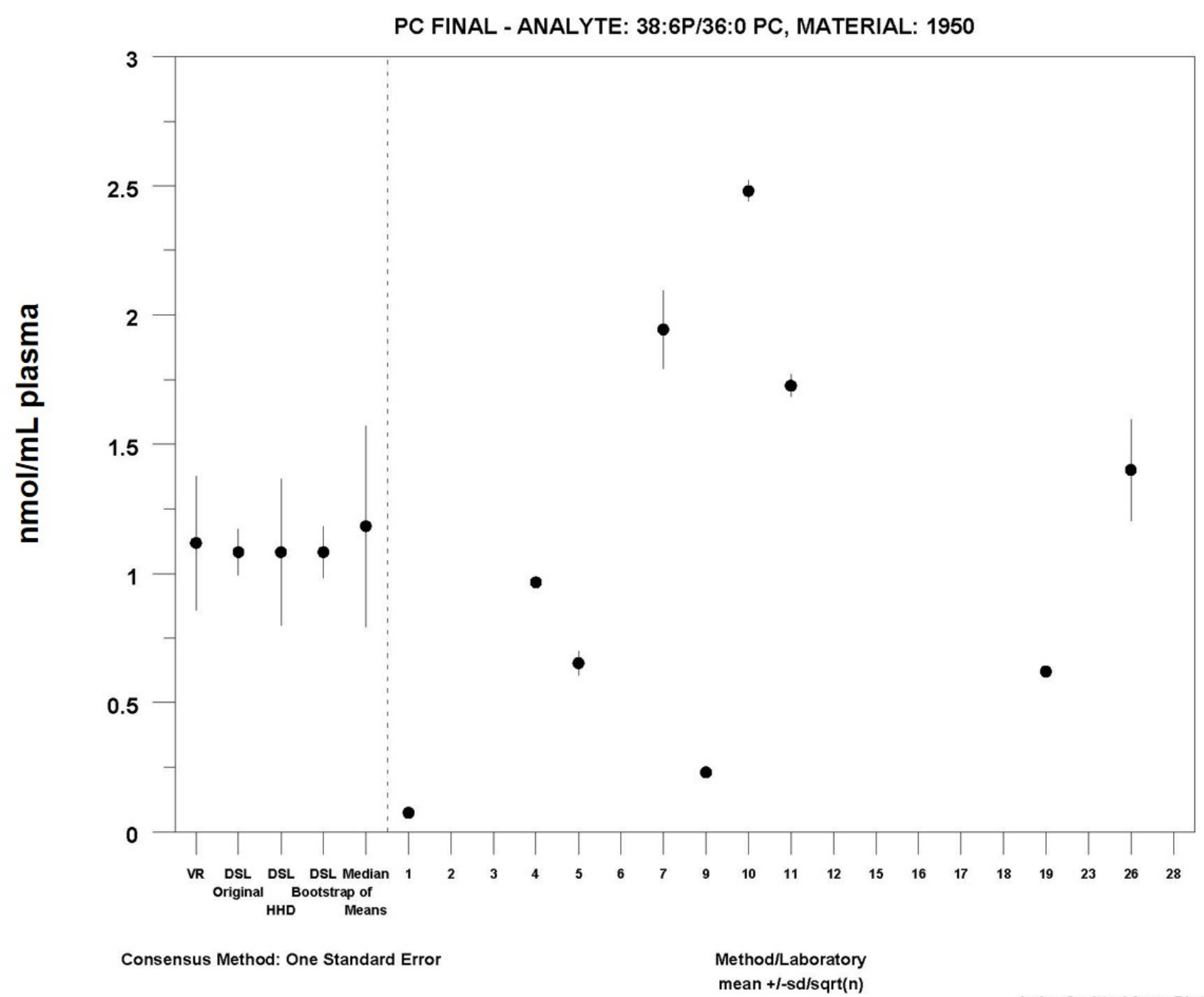

MEDM Location: $1.2 \pm 0.39 \mathrm{nmol} / \mathrm{mL}$

Labs Omitted from Plot (But Not Analysis): 15

15: $86 \pm 29$ 


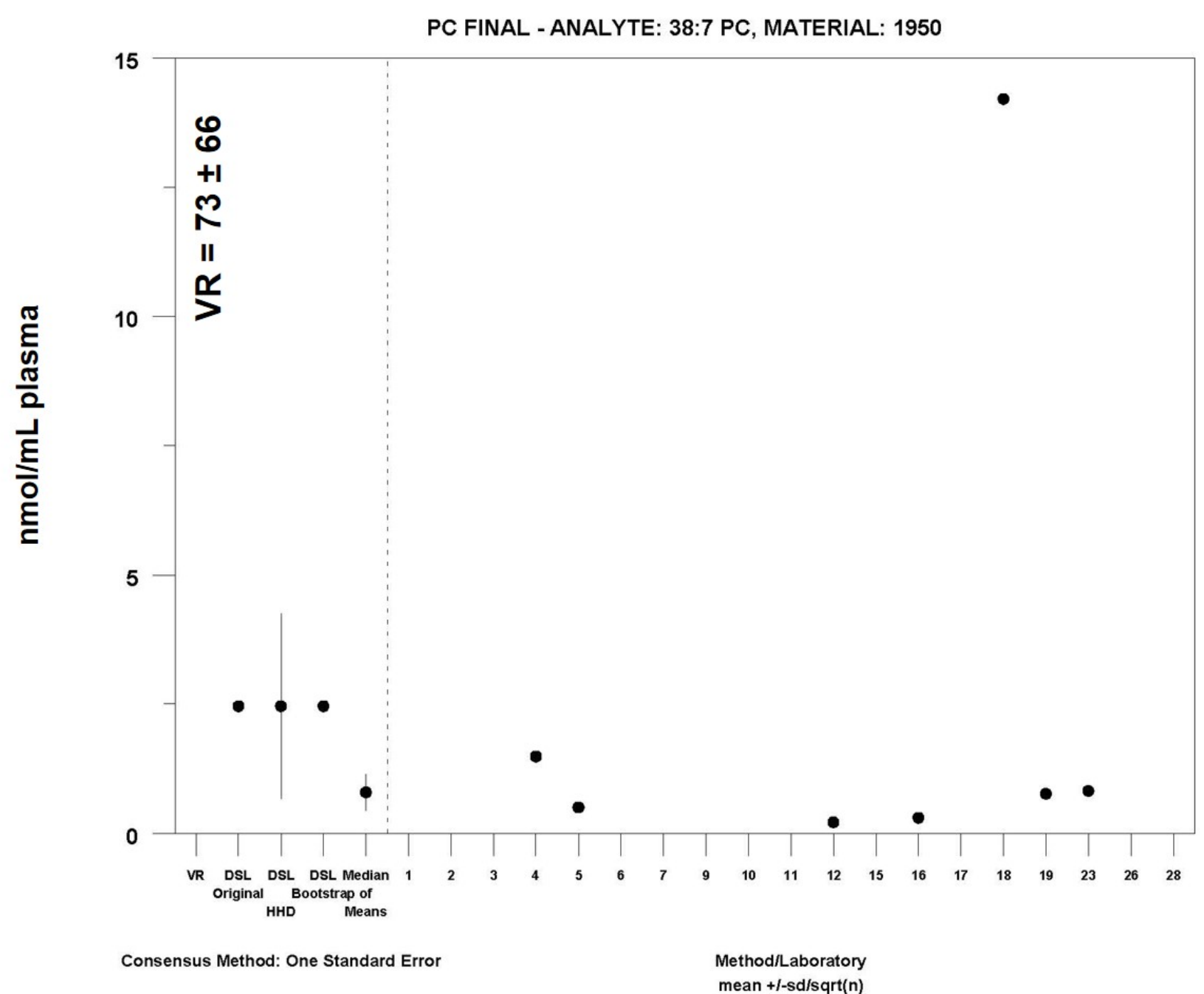

MEDM Location: $0.79 \pm 0.35 \mathrm{nmol} / \mathrm{mL}$

Labs Omitted from Plot (But Not Analysis): 15

\section{5: $567 \pm 21$}




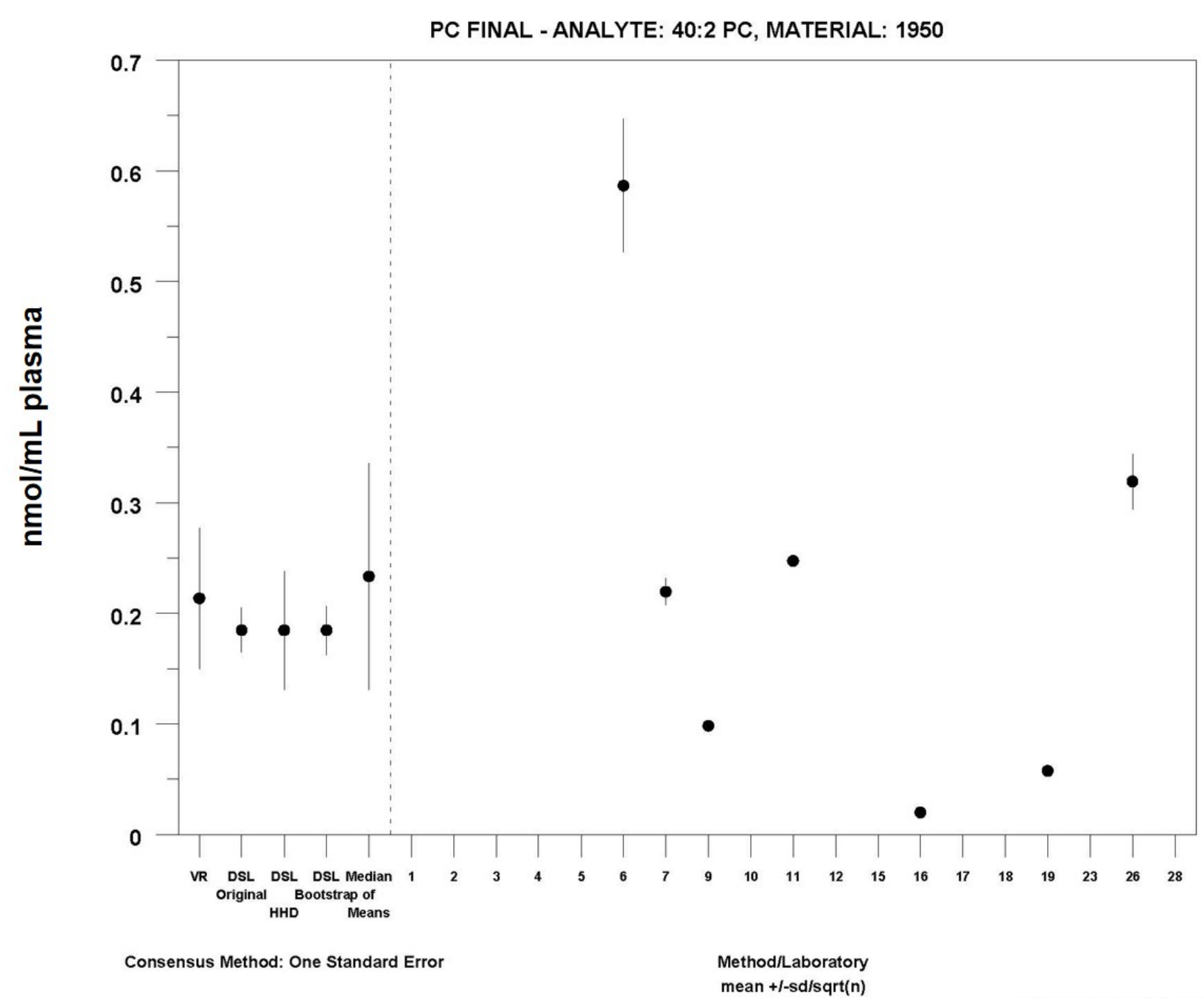

MEDM Location: $0.23 \pm 0.10 \mathrm{nmol} / \mathrm{mL}$

Labs Omitted from Plot (But Not Analysis): 15

15: $20 \pm 4$ 


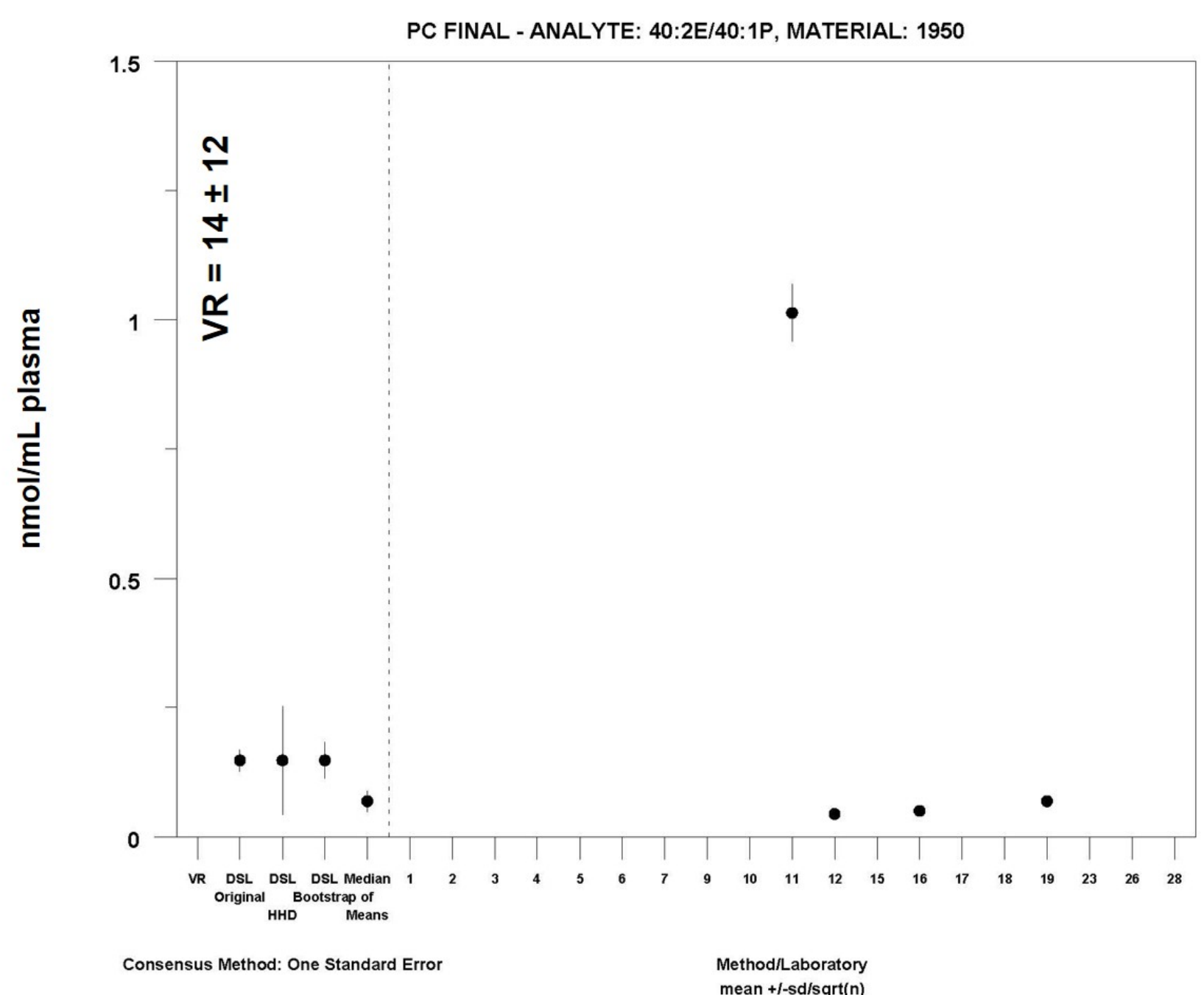

MEDM Location: $0.069 \pm 0.021 \mathrm{nmol} / \mathrm{mL}$

Labs Omitted from Plot (But Not Analysis): 15 15: $71 \pm 7$ 


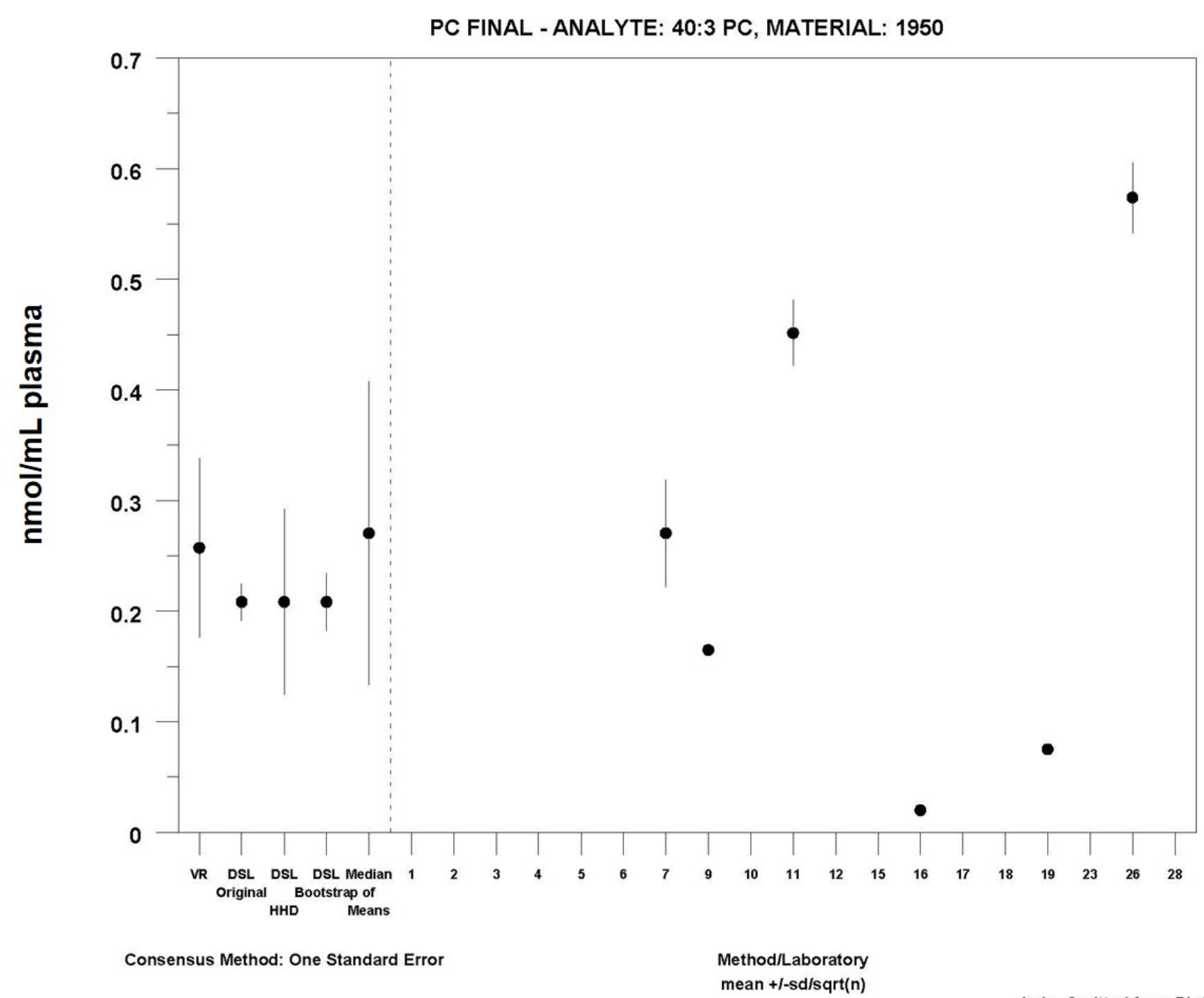

MEDM Location: $0.27 \pm 0.14 \mathrm{nmol} / \mathrm{mL}$

Labs Omitted from Plot (But Not Analysis): 15

15: $105 \pm 61$ 


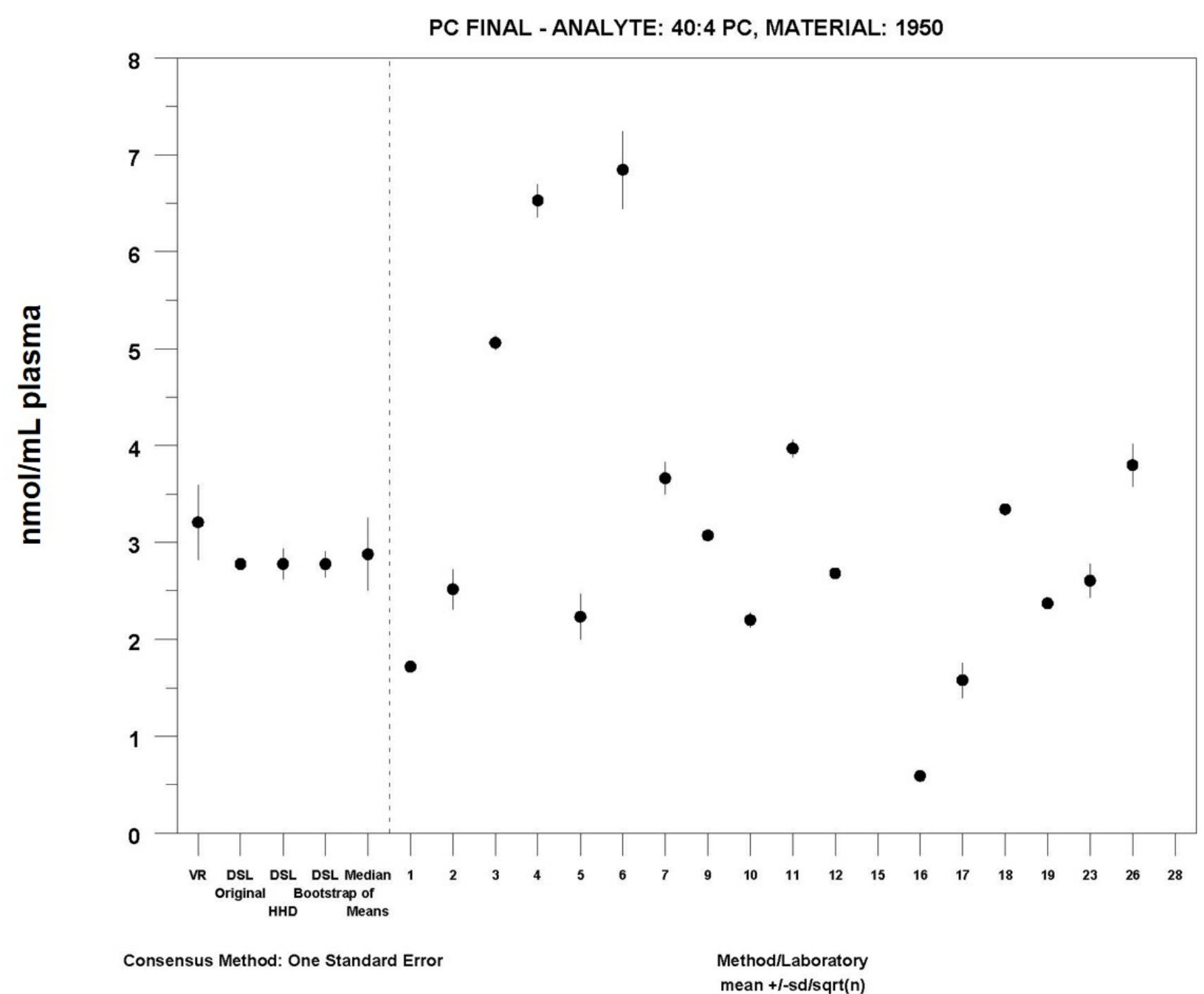

MEDM Location: $2.9 \pm 0.37 \mathrm{nmol} / \mathrm{mL}$

Labs Omitted from Plot (But Not Analysis): 15

\section{5: $15.76 \pm 757$}




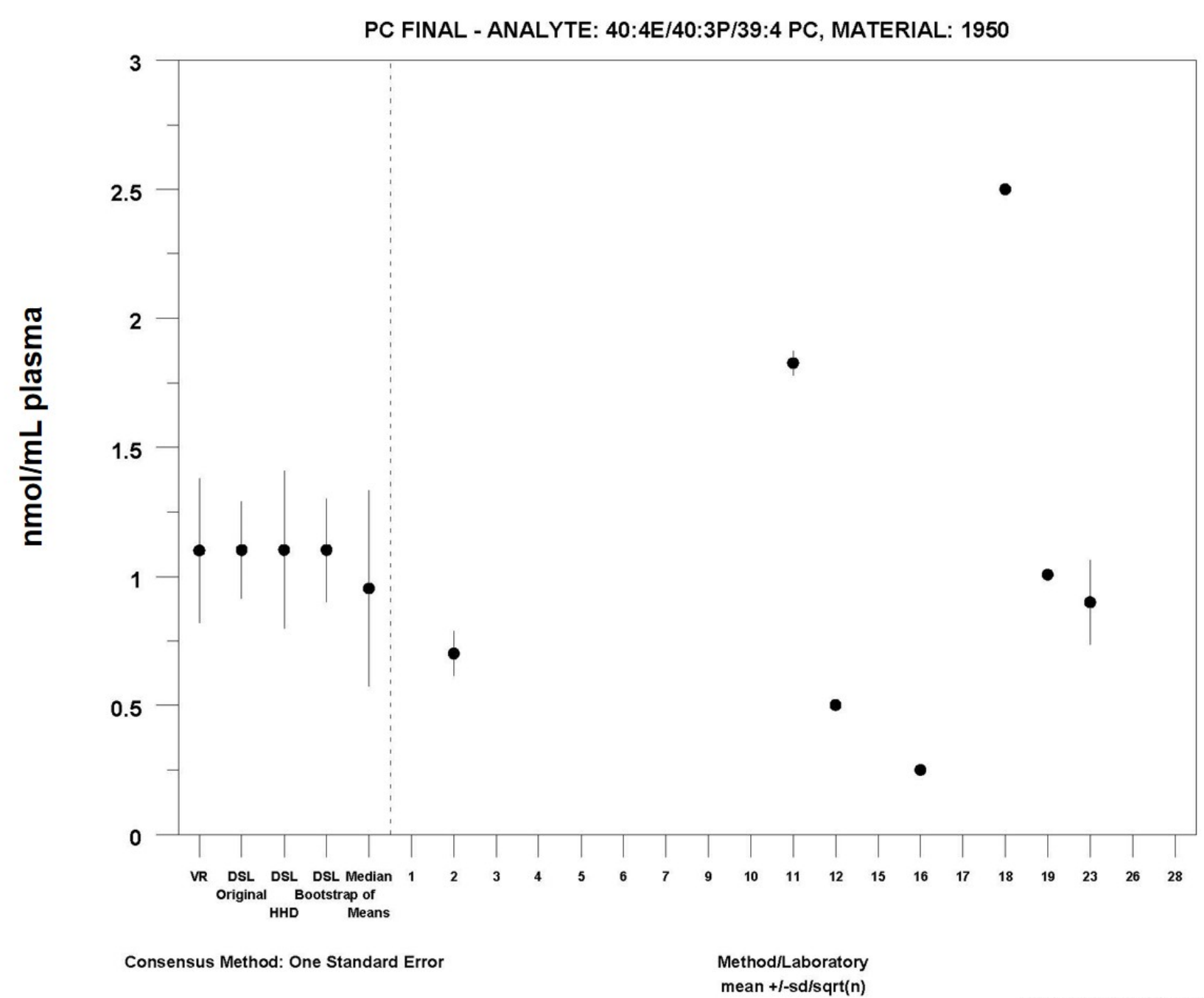

MEDM Location: $0.95 \pm 0.38 \mathrm{nmol} / \mathrm{mL}$

Labs Omitted from Plot (But Not Analysis): 15

15: $491 \pm 273$ 


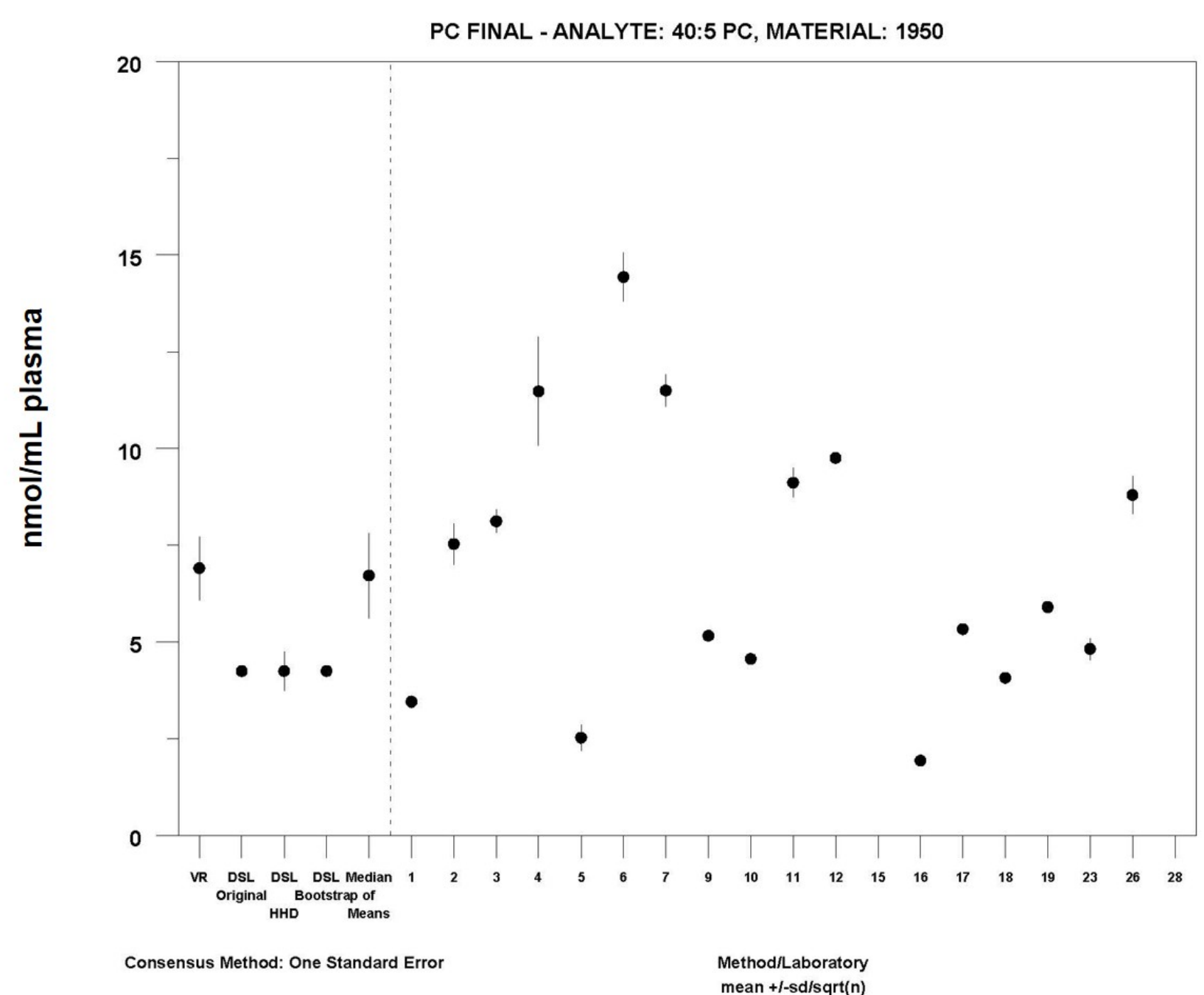

MEDM Location: $6.7 \pm 1.1 \mathrm{nmol} / \mathrm{mL}$

Labs Omitted from Plot (But Not Analysis): 15

15: $1811 \pm 423$ 


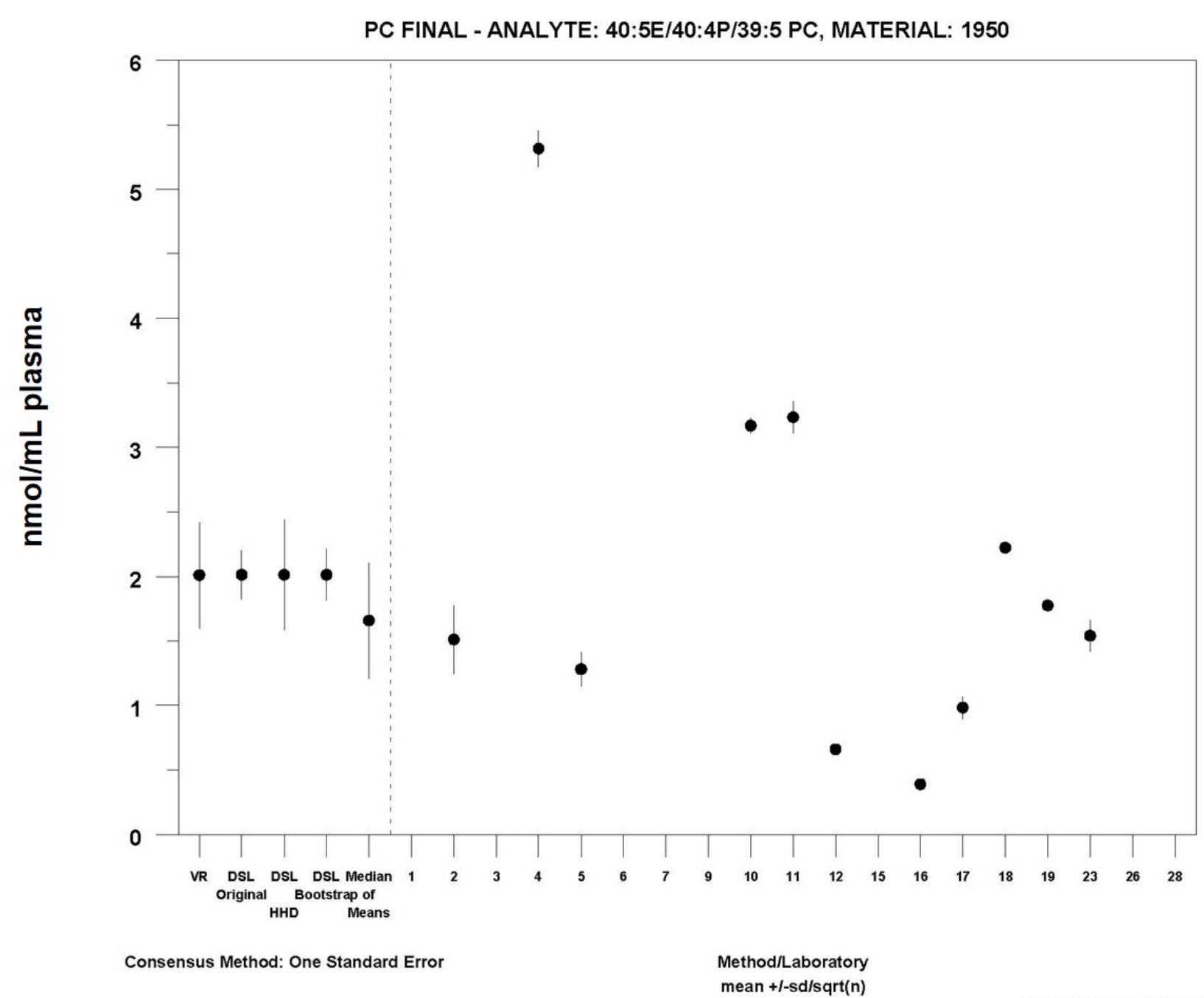

MEDM Location: $1.7 \pm 0.45 \mathrm{nmol} / \mathrm{mL}$

Labs Omitted from Plot (But Not Analysis): 15

15: $129 \pm 34$ 


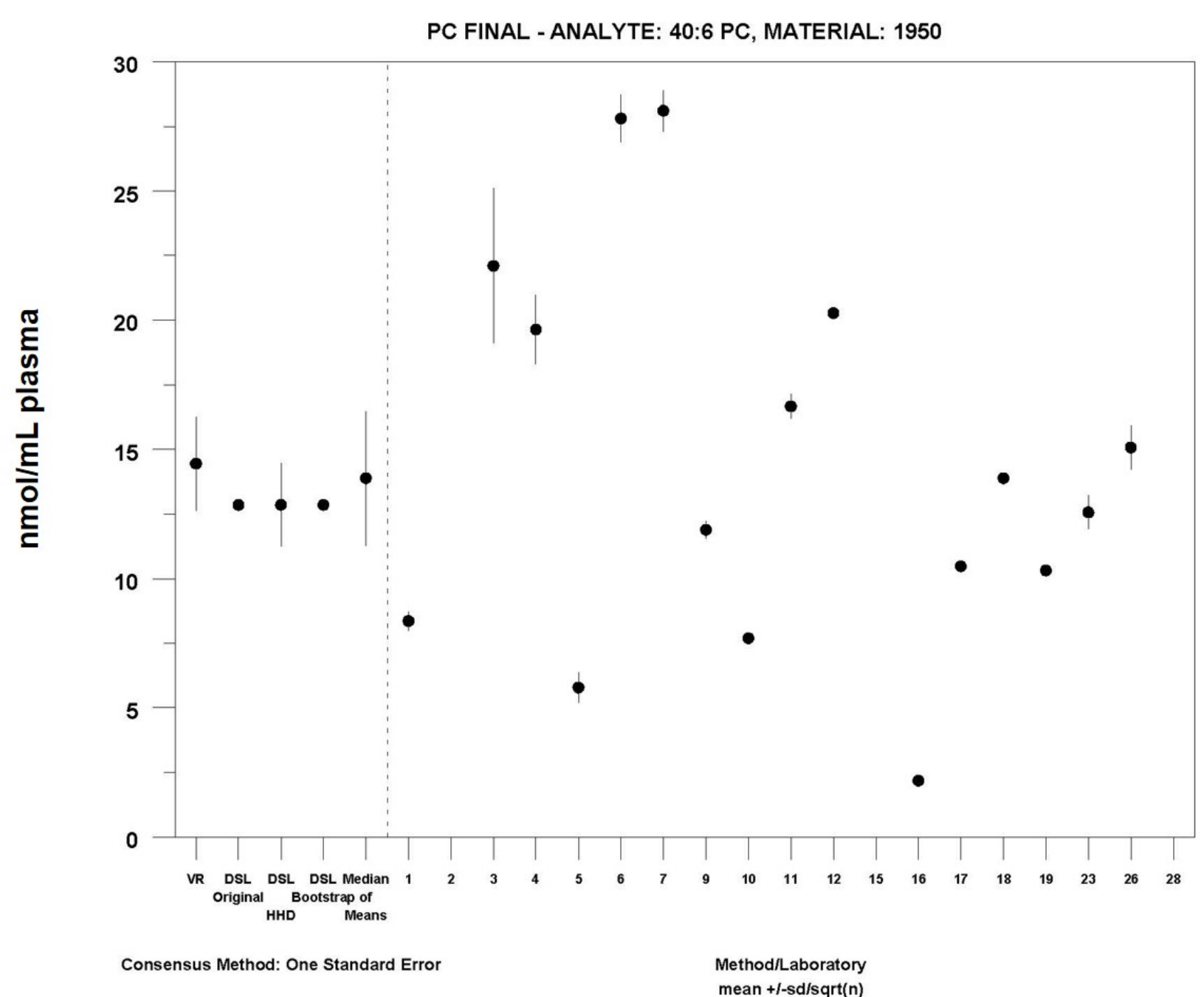

MEDM Location: $14 \pm 2.6 \mathrm{nmol} / \mathrm{mL}$

Labs Omitted from Plot (But Not Analysis): 15

15: $6251 \pm 369$ 


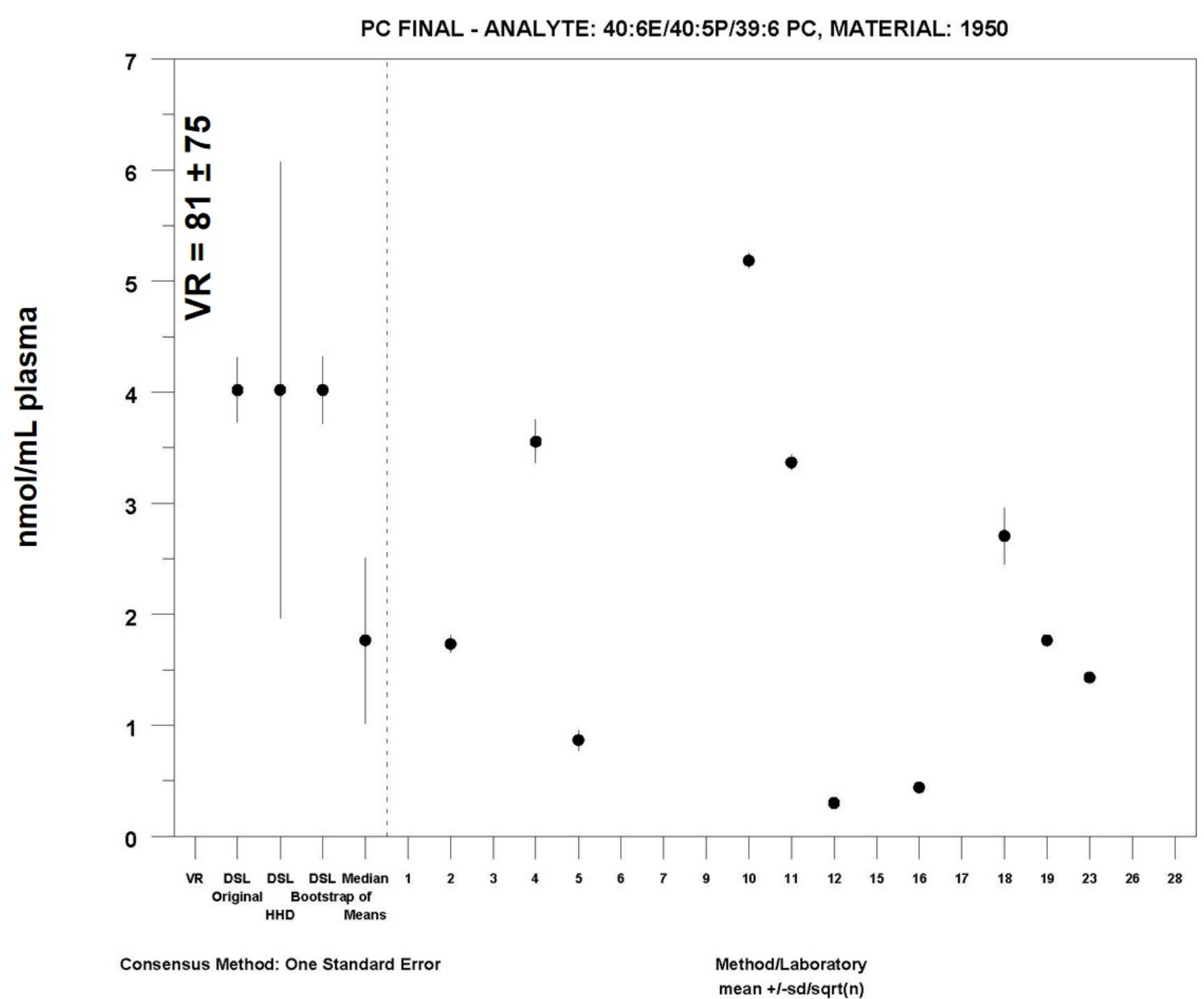

MEDM Location: $1.8 \pm 0.74 \mathrm{nmol} / \mathrm{mL}$

Labs Omitted from Plot (But Not Analysis): 15

15: $869 \pm 11$ 


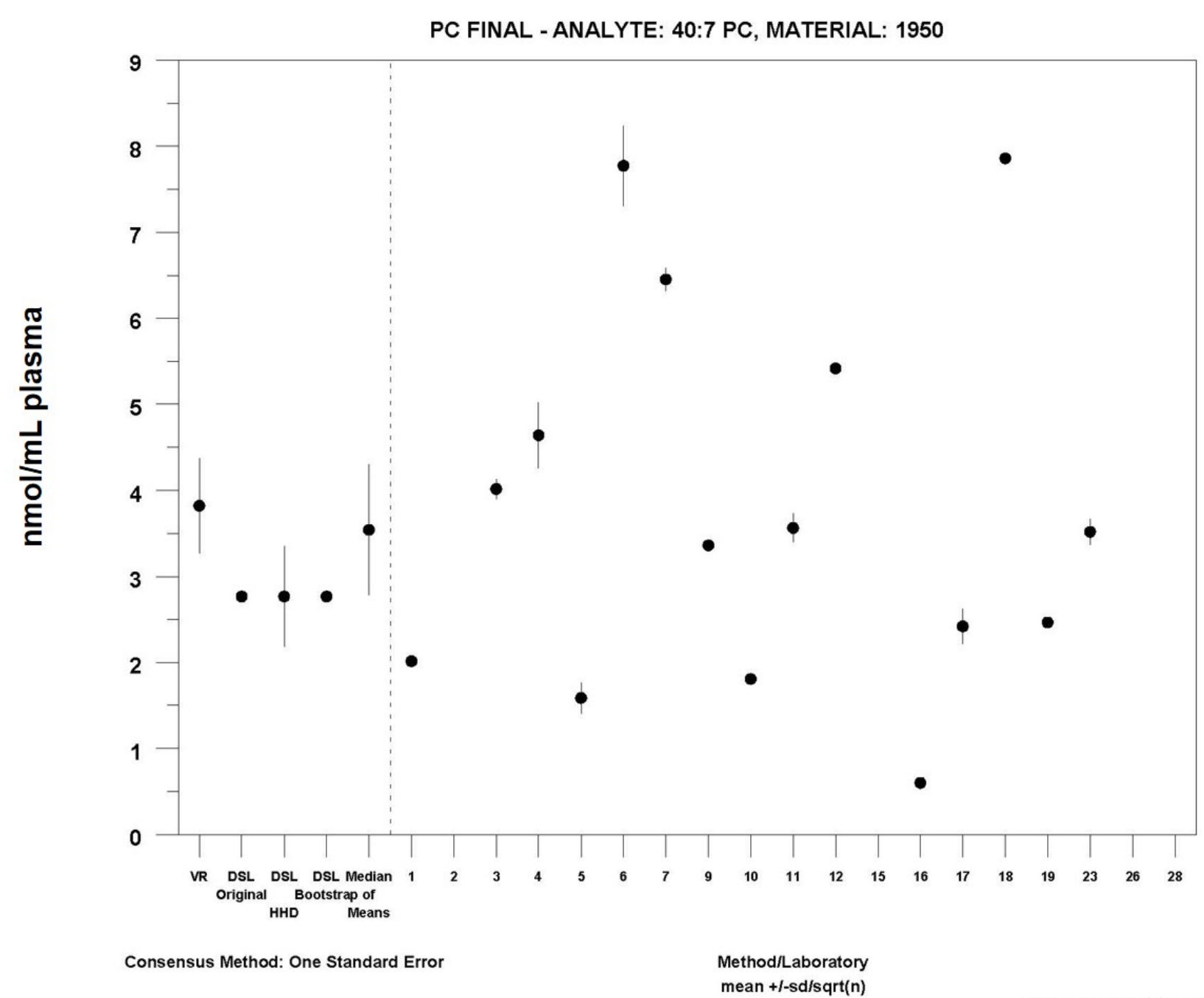

MEDM Location: $3.5 \pm 0.76 \mathrm{nmol} / \mathrm{mL}$

Labs Omitted from Plot (But Not Analysis): 15

15: $1014 \pm 578$ 


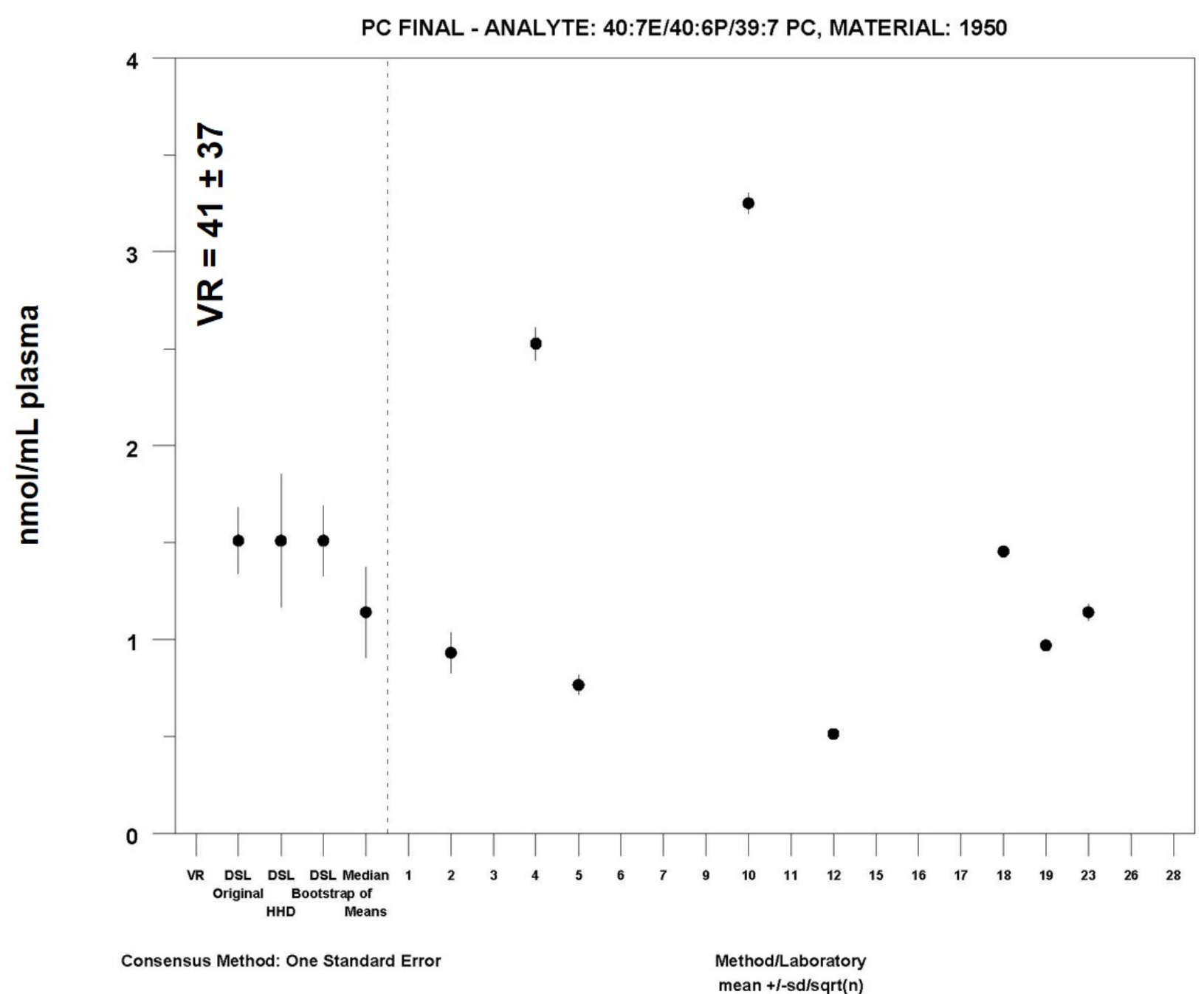

MEDM Location: $1.1 \pm 0.23 \mathrm{nmol} / \mathrm{mL}$

Labs Omitted from Plot (But Not Analysis): 15

15: $359 \pm 21$ 


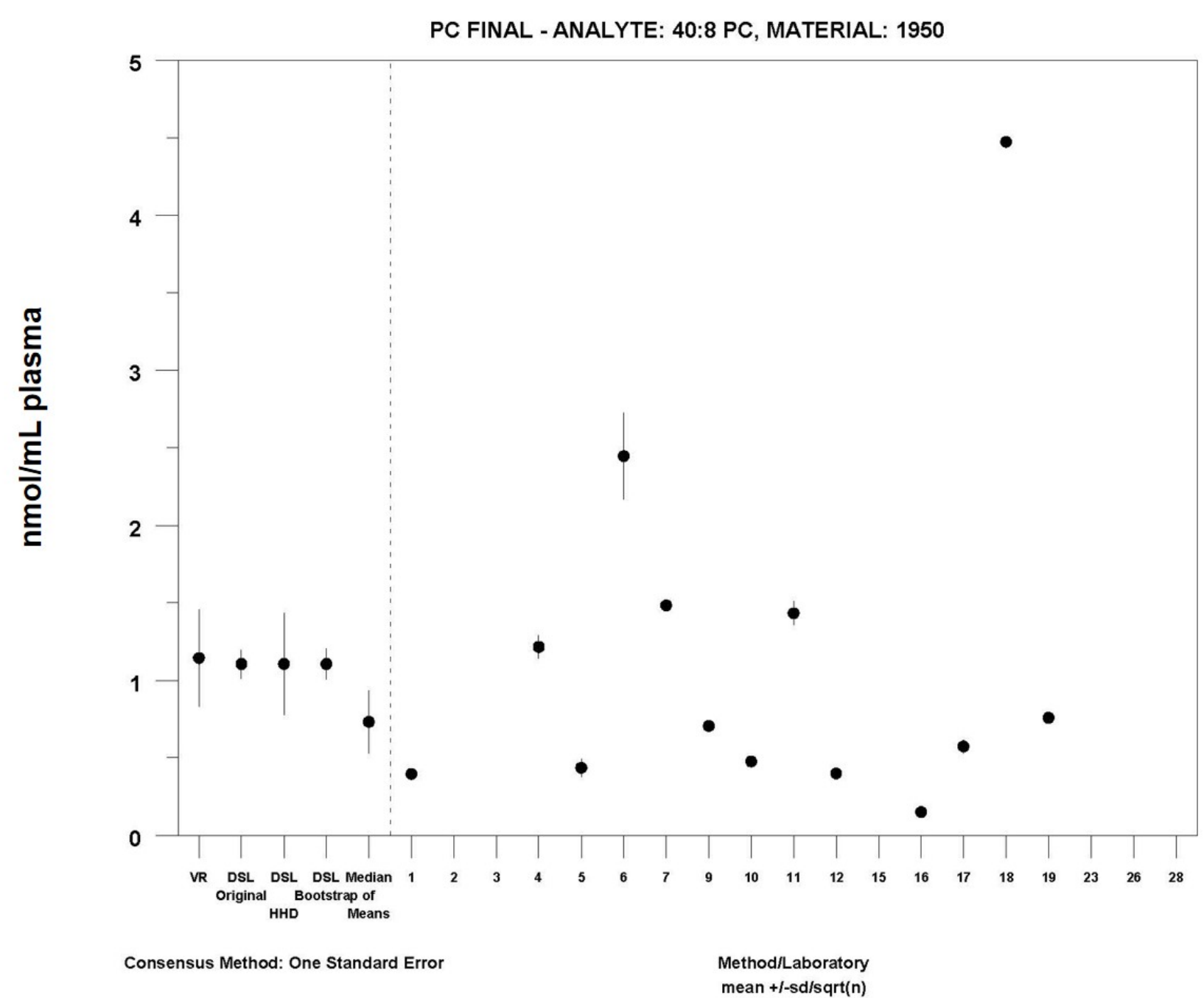

MEDM Location: $0.73 \pm 0.20 \mathrm{nmol} / \mathrm{mL}$

Labs Omitted from Plot (But Not Analysis): 15

15: $614 \pm 404$ 


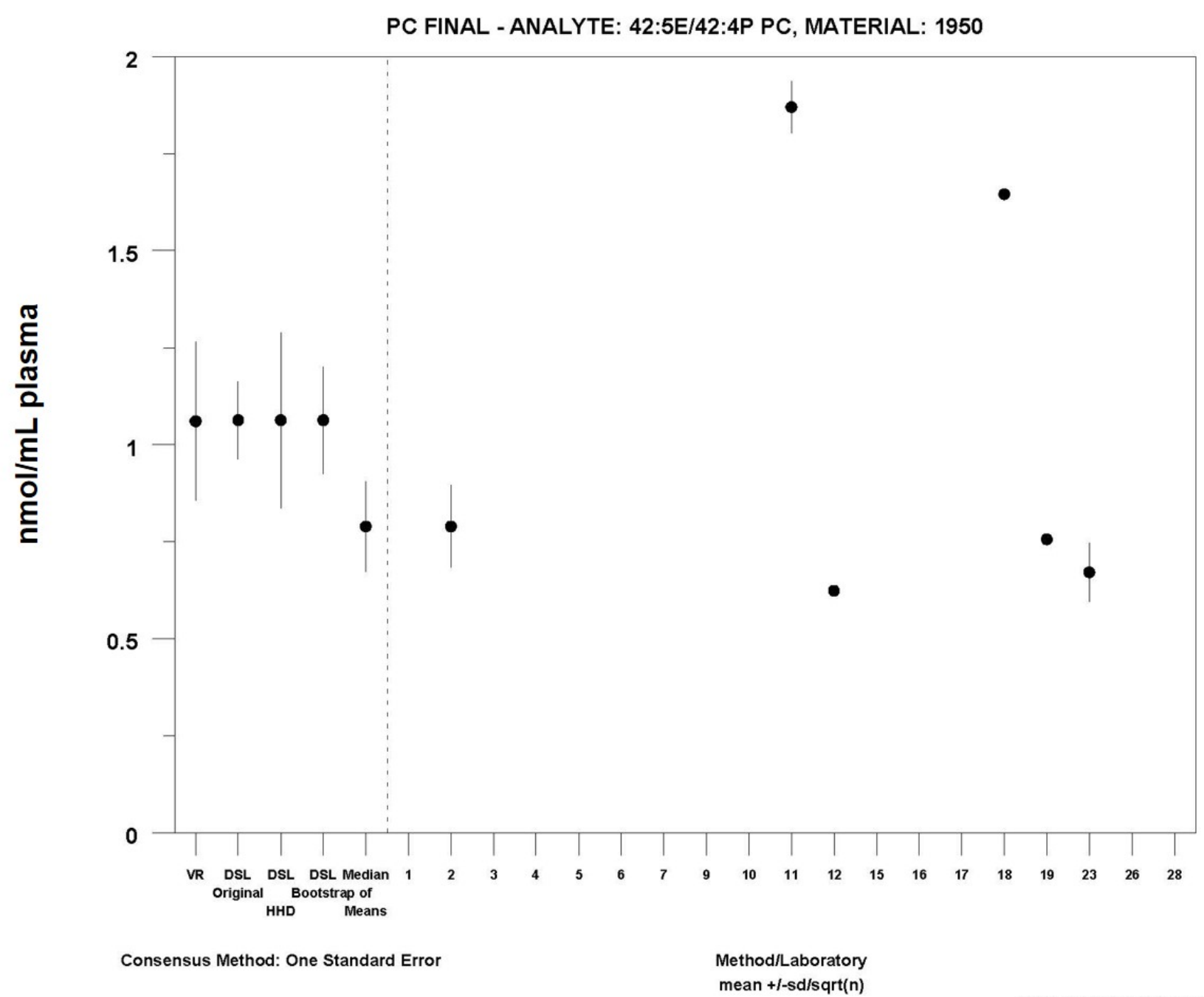

MEDM Location: $0.79 \pm 0.12 \mathrm{nmol} / \mathrm{mL}$

Labs Omitted from Plot (But Not Analysis): 15

15: $885 \pm 597$ 


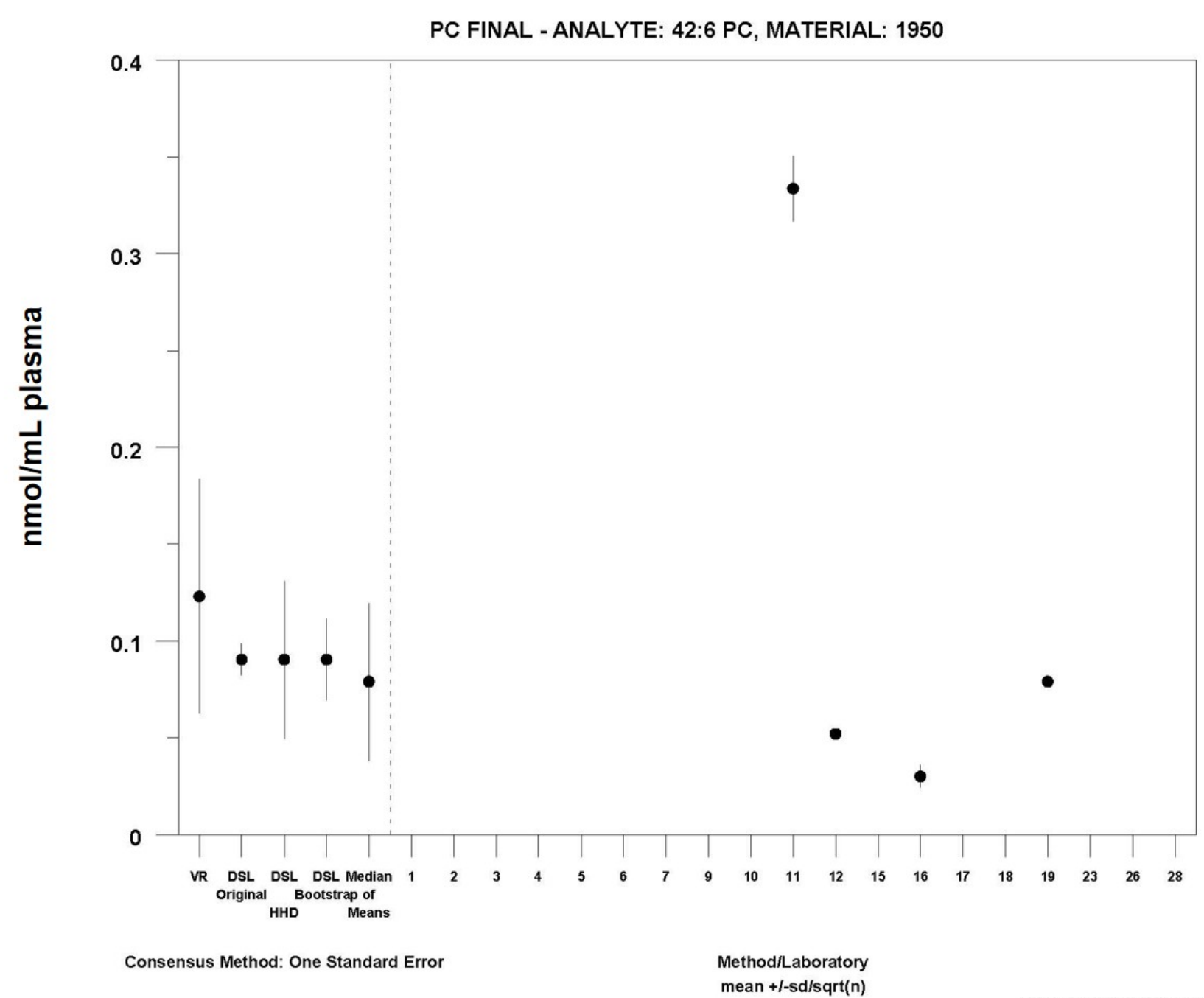

MEDM Location: $0.079 \pm 0.041 \mathrm{nmol} / \mathrm{mL}$

Labs Omitted from Plot (But Not Analysis): 15

15: $32 \pm 12$ 


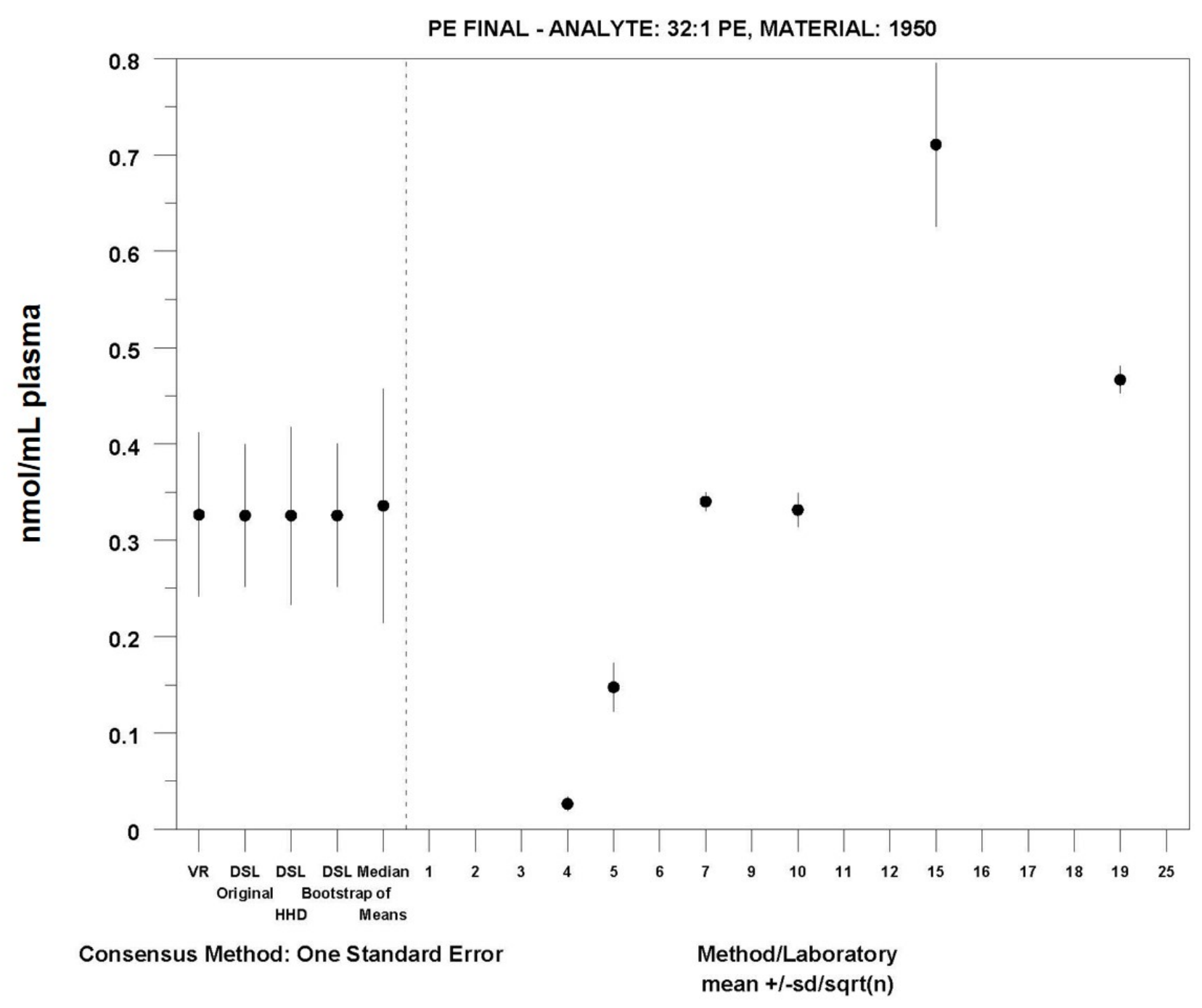

MEDM Location: $0.34 \pm 0.12 \mathrm{nmol} / \mathrm{mL}$ 


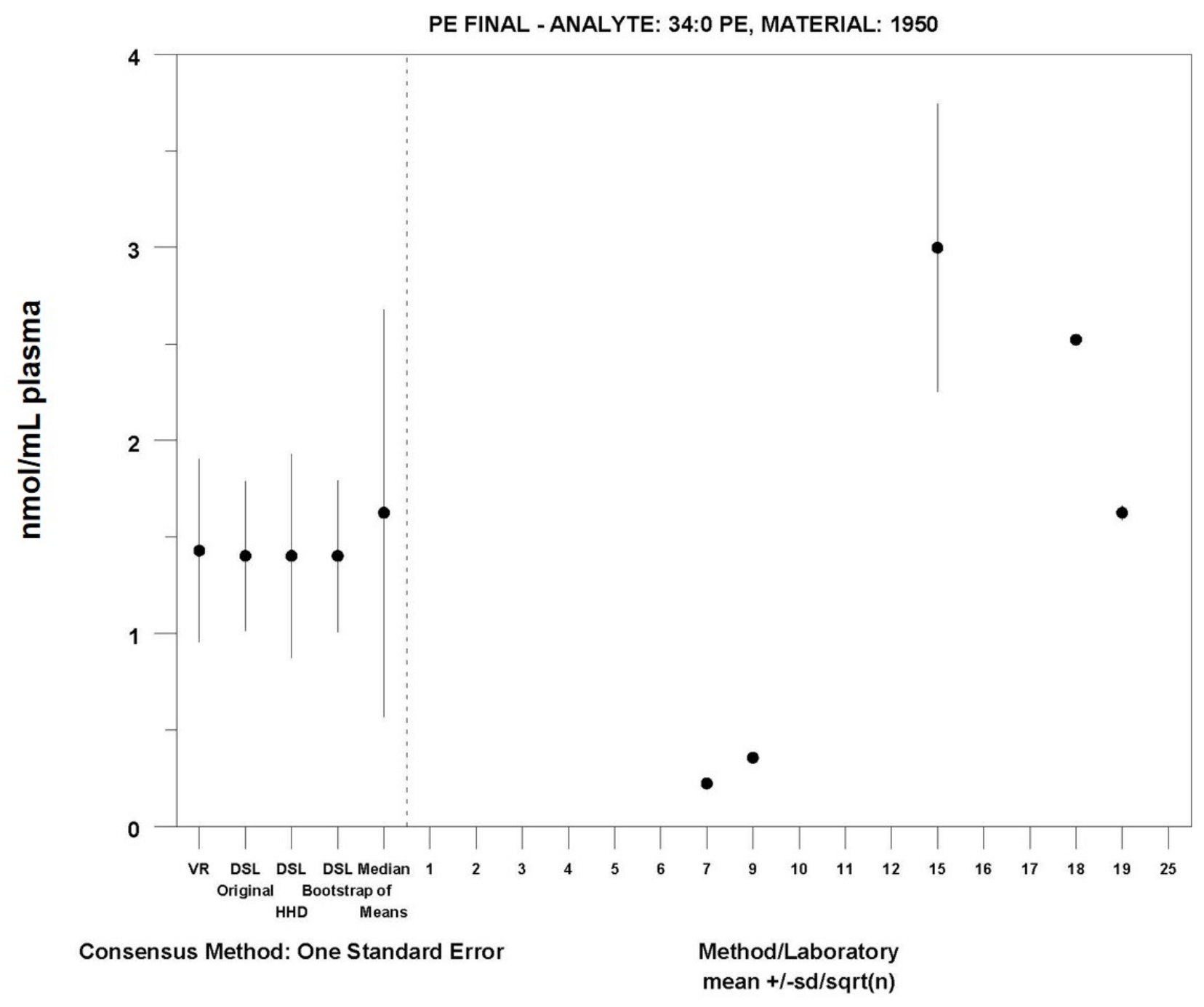

MEDM Location: $1.6 \pm 1.1 \mathrm{nmol} / \mathrm{mL}$ 


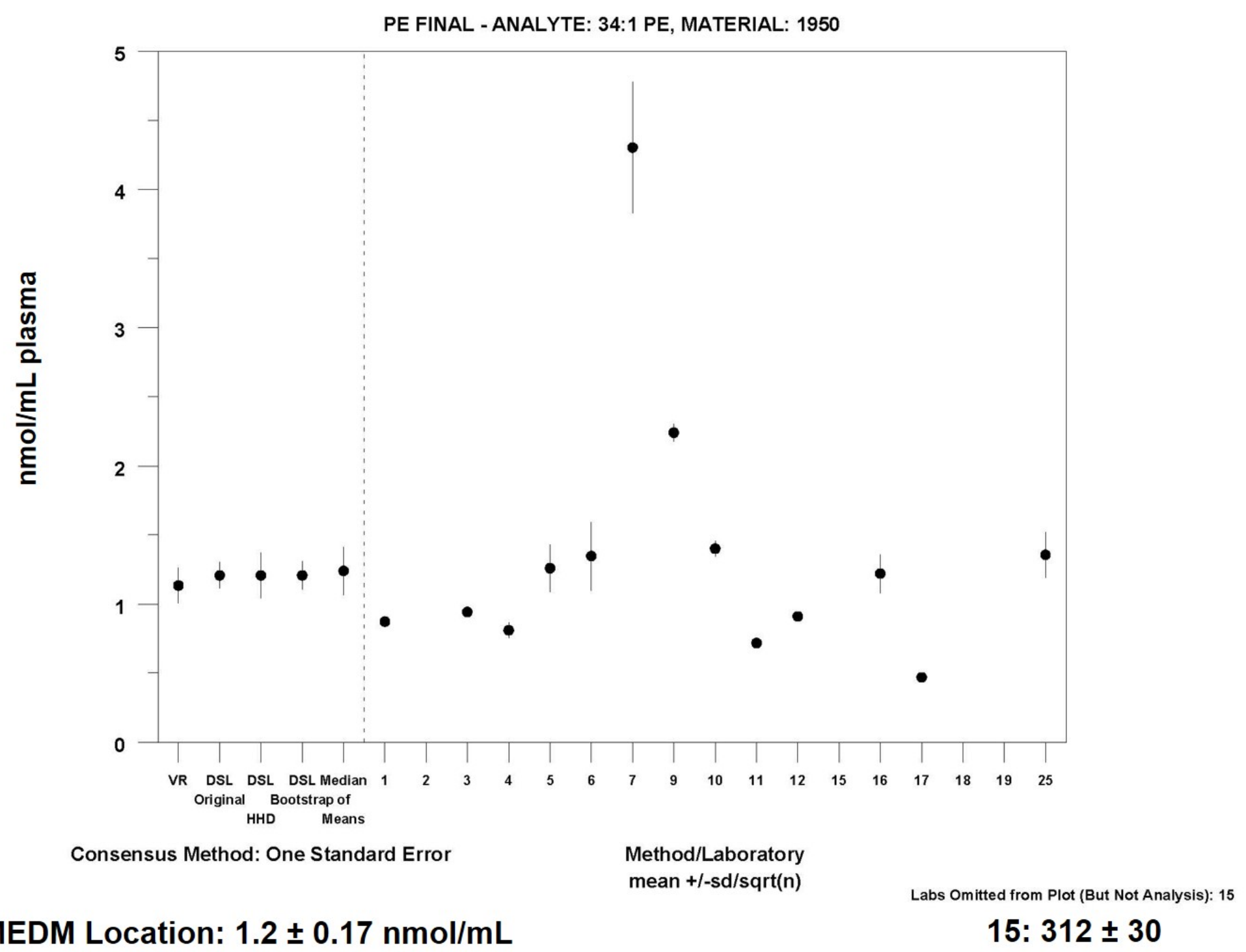




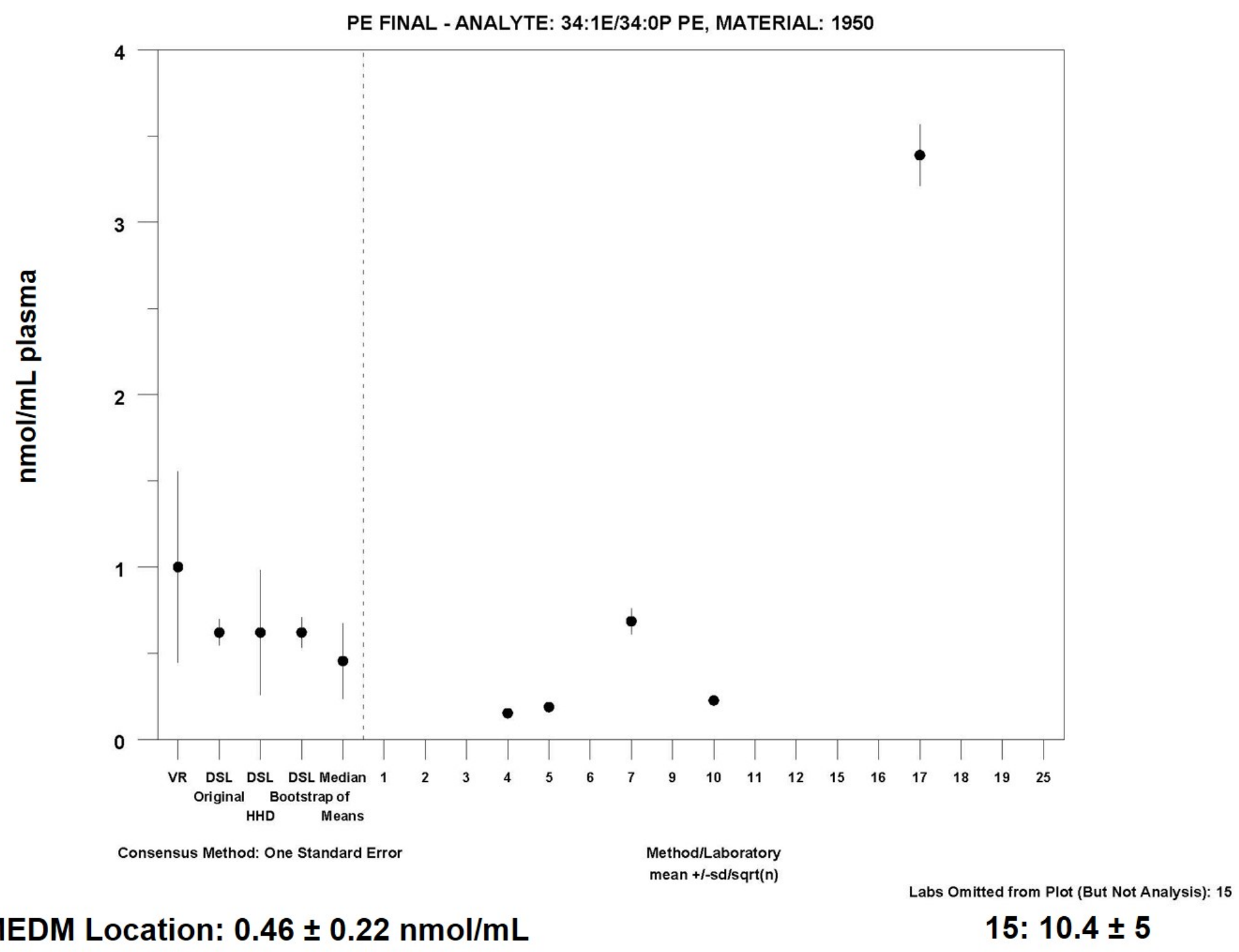




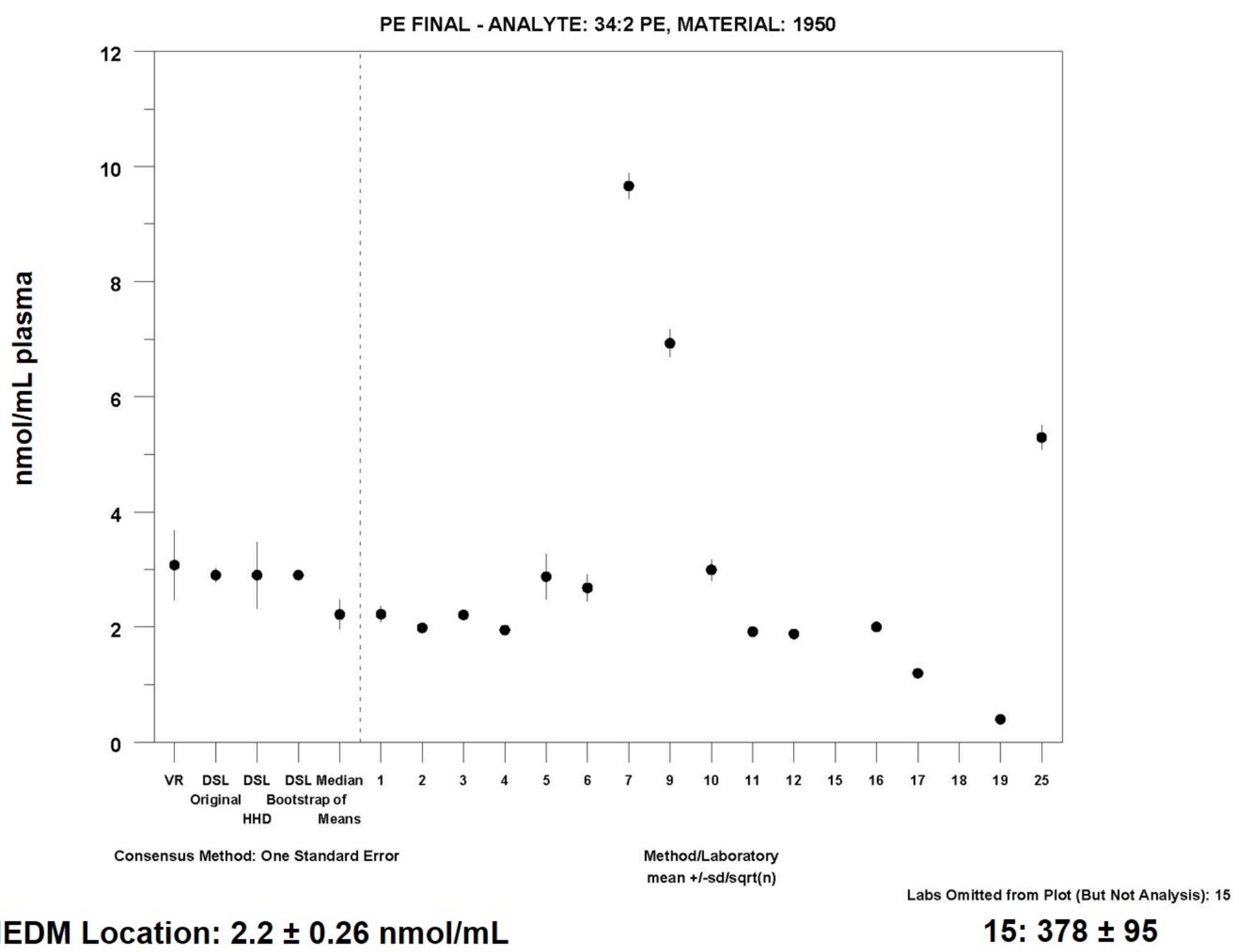




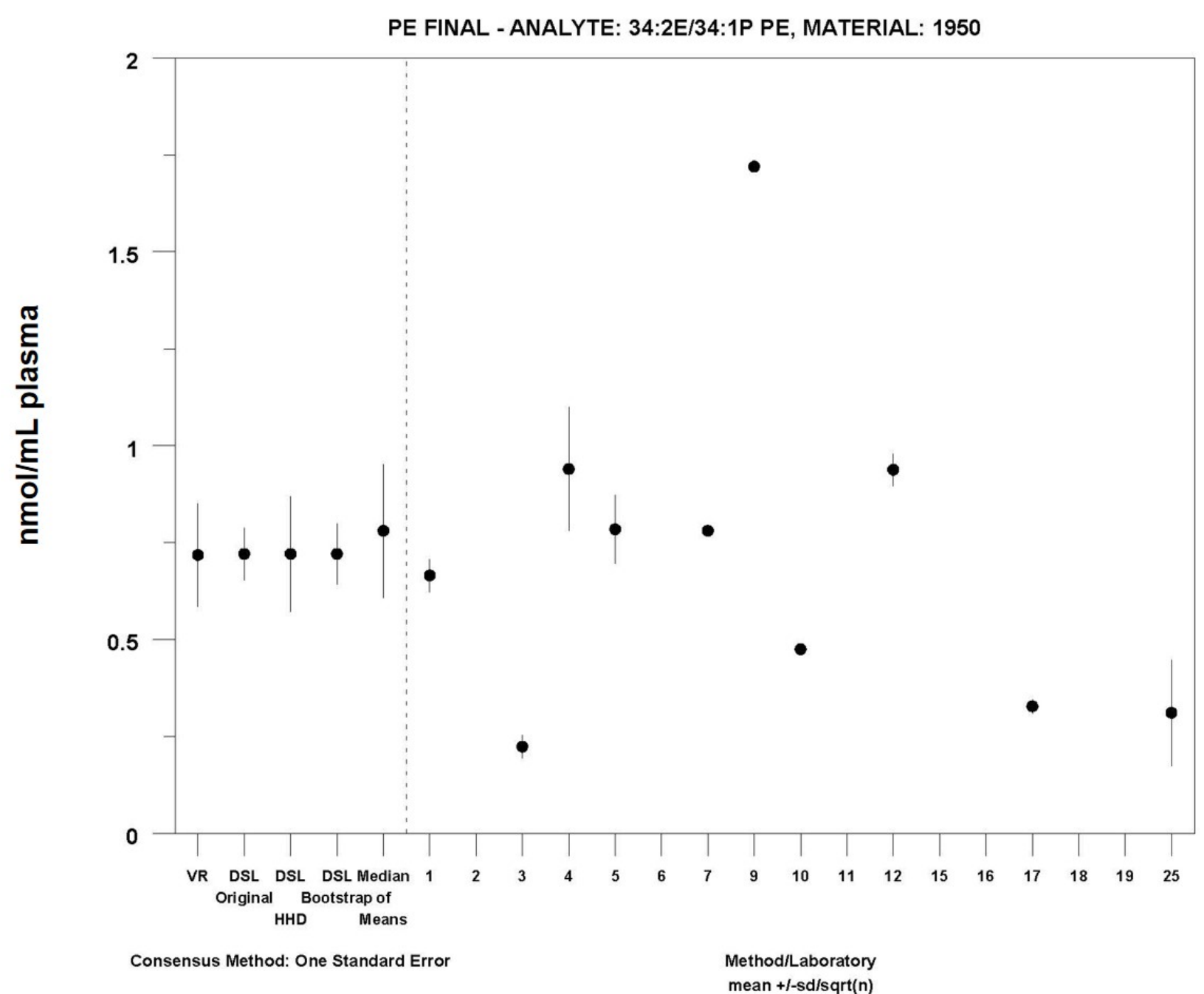

MEDM Location: $0.78 \pm 0.17 \mathrm{nmol} / \mathrm{mL}$

Labs Omitted from Plot (But Not Analysis): 15

15: $0.103 \pm 55$ 


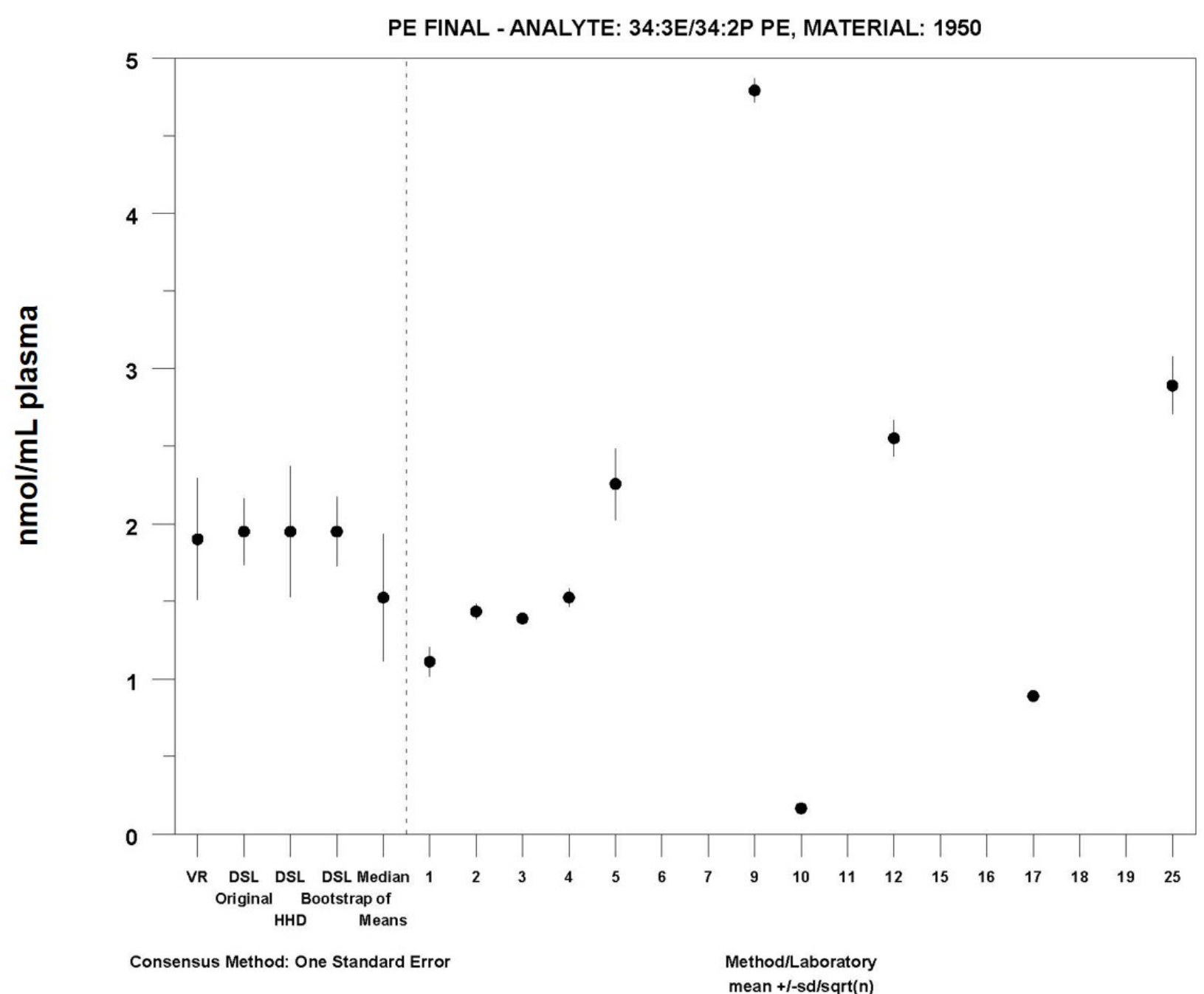

MEDM Location: $1.5 \pm 0.41 \mathrm{nmol} / \mathrm{mL}$

Labs Omitted from Plot (But Not Analysis): 15

15: $99 \pm 14$ 


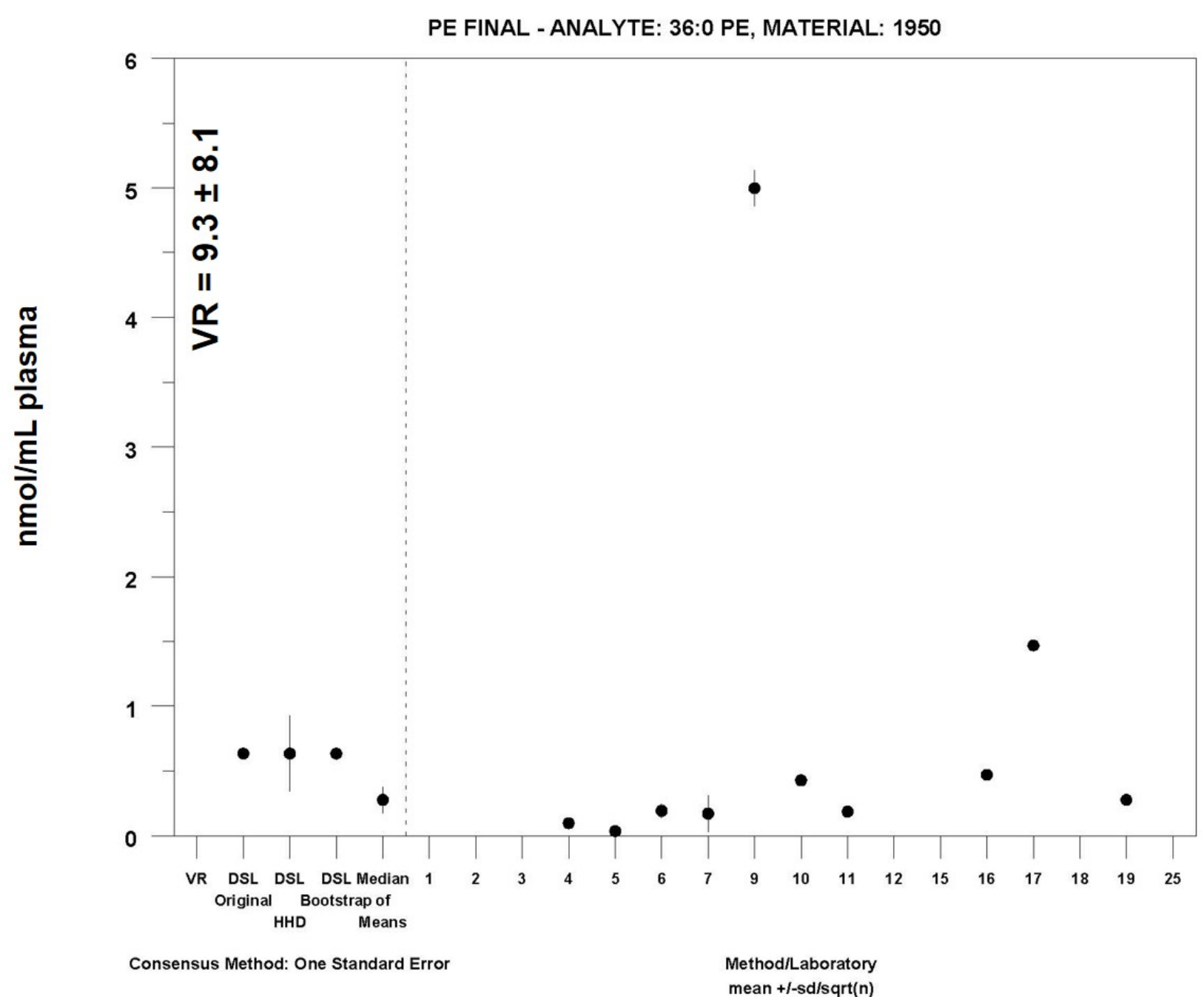

MEDM Location: $0.28 \pm 0.10 \mathrm{nmol} / \mathrm{mL}$

Labs Omitted from Plot (But Not Analysis): 15

15: $95 \pm 7$ 


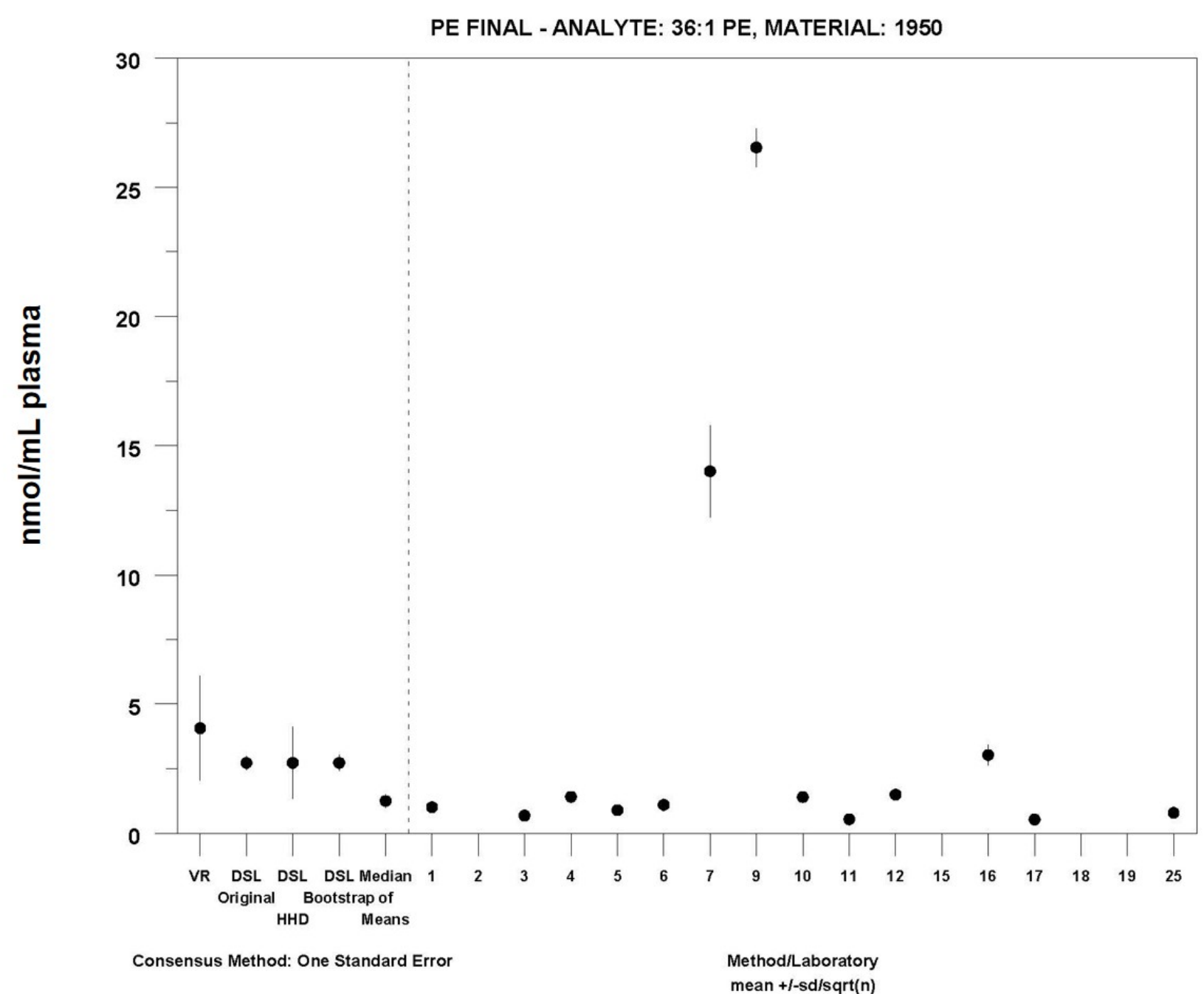

MEDM Location: $1.3 \pm 0.26 \mathrm{nmol} / \mathrm{mL}$

Labs Omitted from Plot (But Not Analysis): 15

15: $669 \pm 211$ 


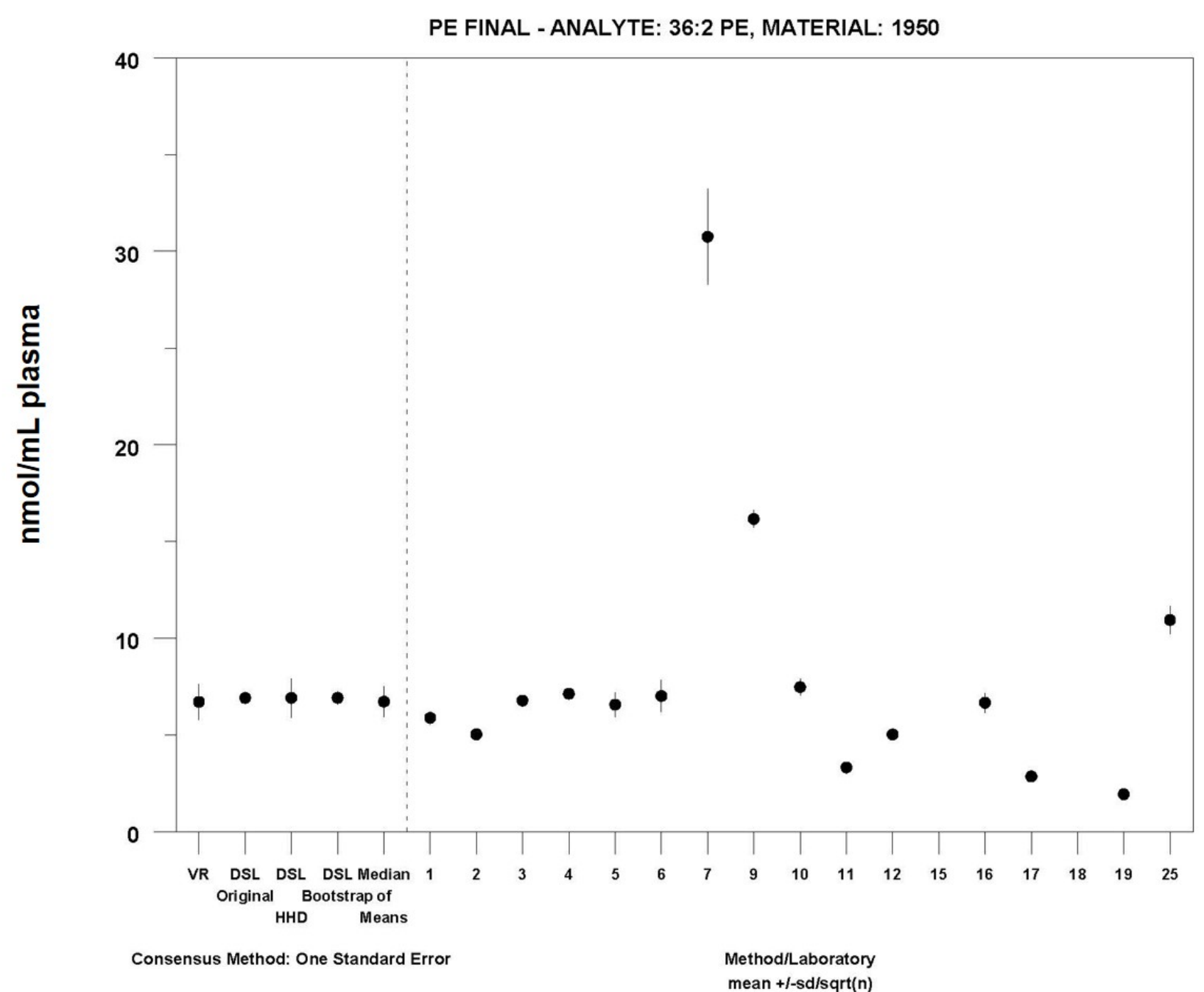

MEDM Location: $6.7 \pm 0.79 \mathrm{nmol} / \mathrm{mL}$

Labs Omitted from Plot (But Not Analysis): 15

15: $665 \pm 37$ 


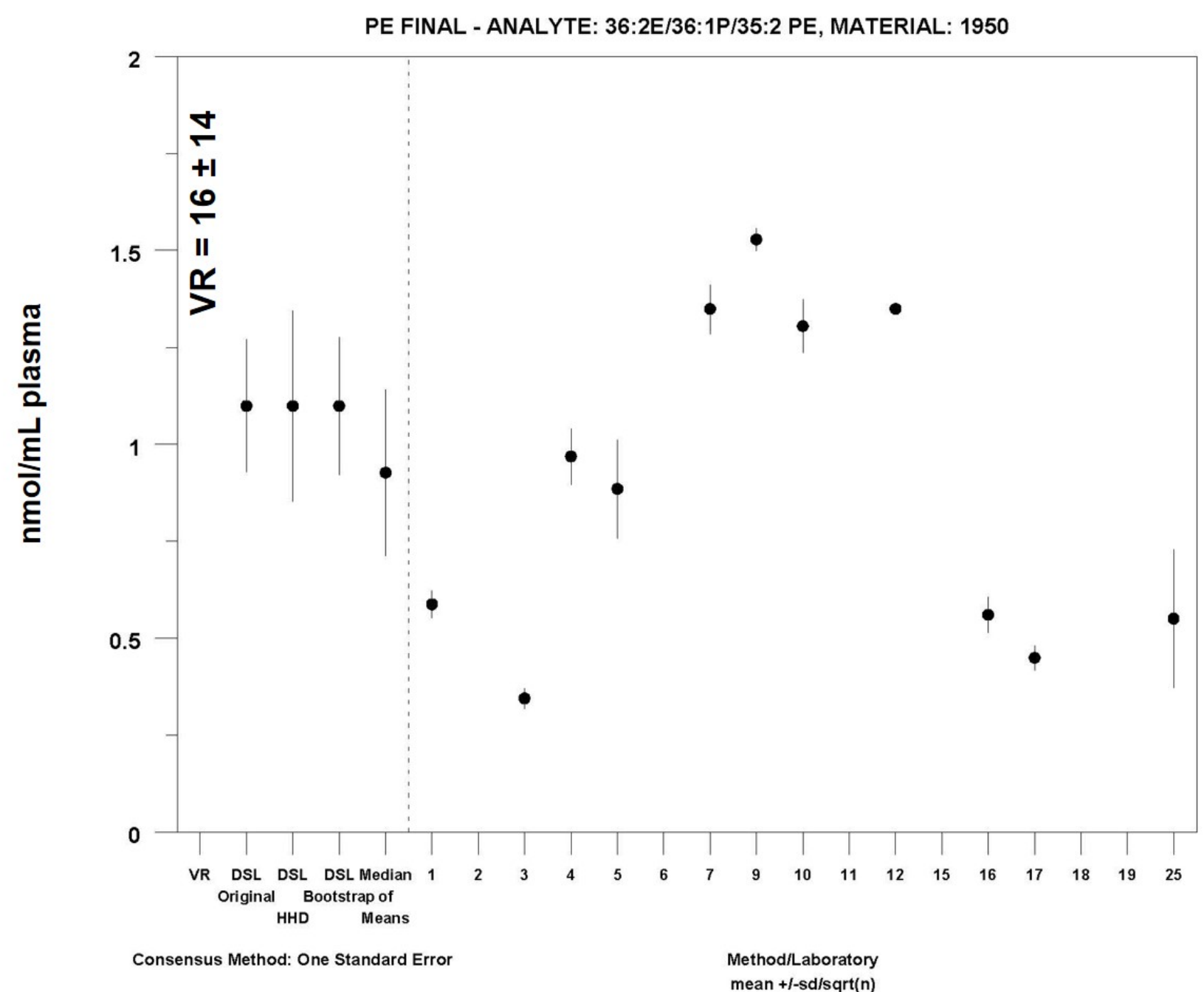

MEDM Location: $0.93 \pm 0.22 \mathrm{nmol} / \mathrm{mL}$

Labs Omitted from Plot (But Not Analysis): 15

$$
\text { 15: } 179 \pm 9
$$




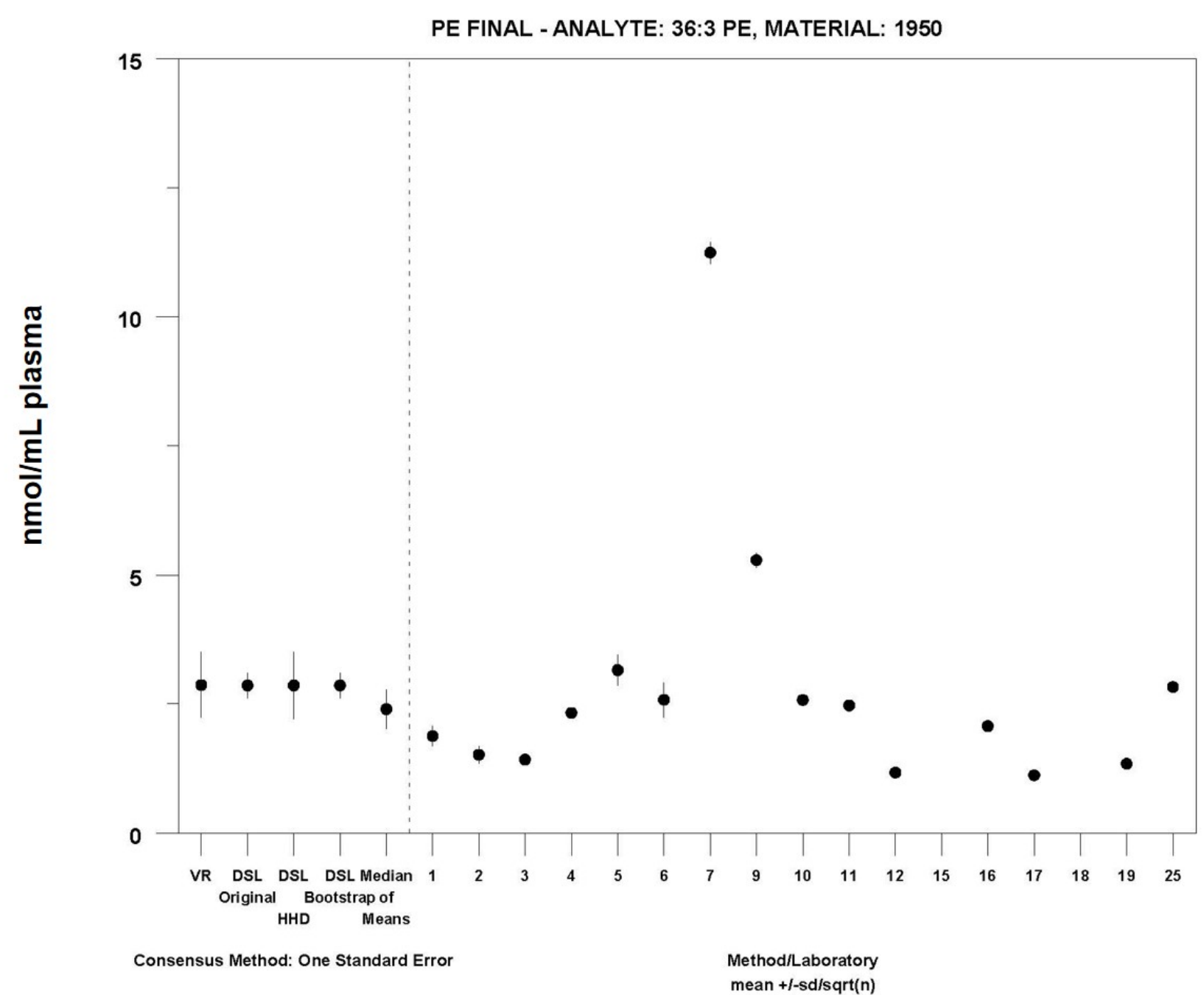

MEDM Location: $2.4 \pm 0.38 \mathrm{nmol} / \mathrm{mL}$

Labs Omitted from Plot (But Not Analysis): 15

15: $182 \pm 41$ 


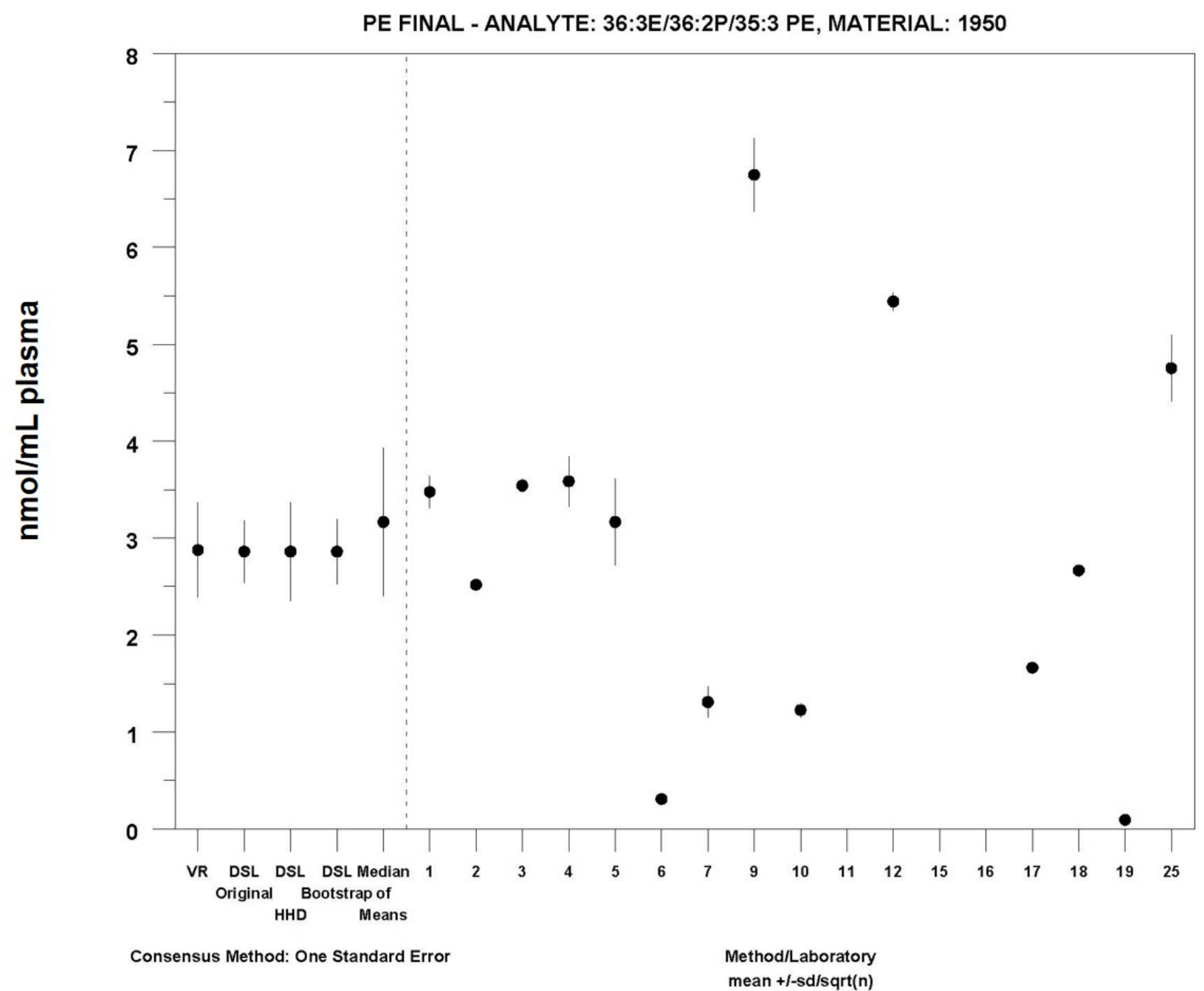

MEDM Location: $3.2 \pm 0.76 \mathrm{nmol} / \mathrm{mL}$

Labs Omitted from Plot (But Not Analysis): 15

15: $201 \pm 81$ 


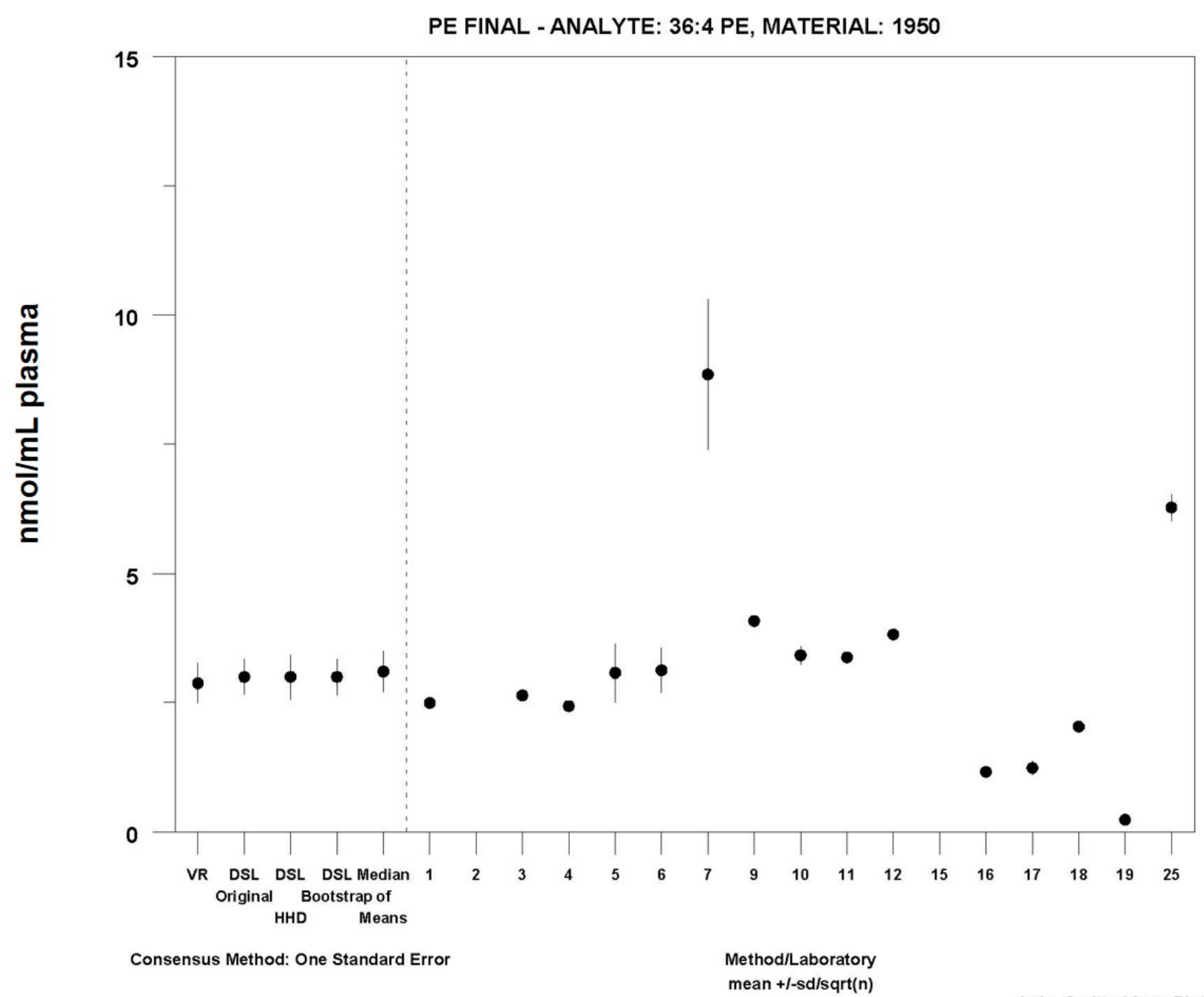

MEDM Location: $3.1 \pm 0.39 \mathrm{nmol} / \mathrm{mL}$

Labs Omitted from Plot (But Not Analysis): 15

15: $226 \pm 74$ 


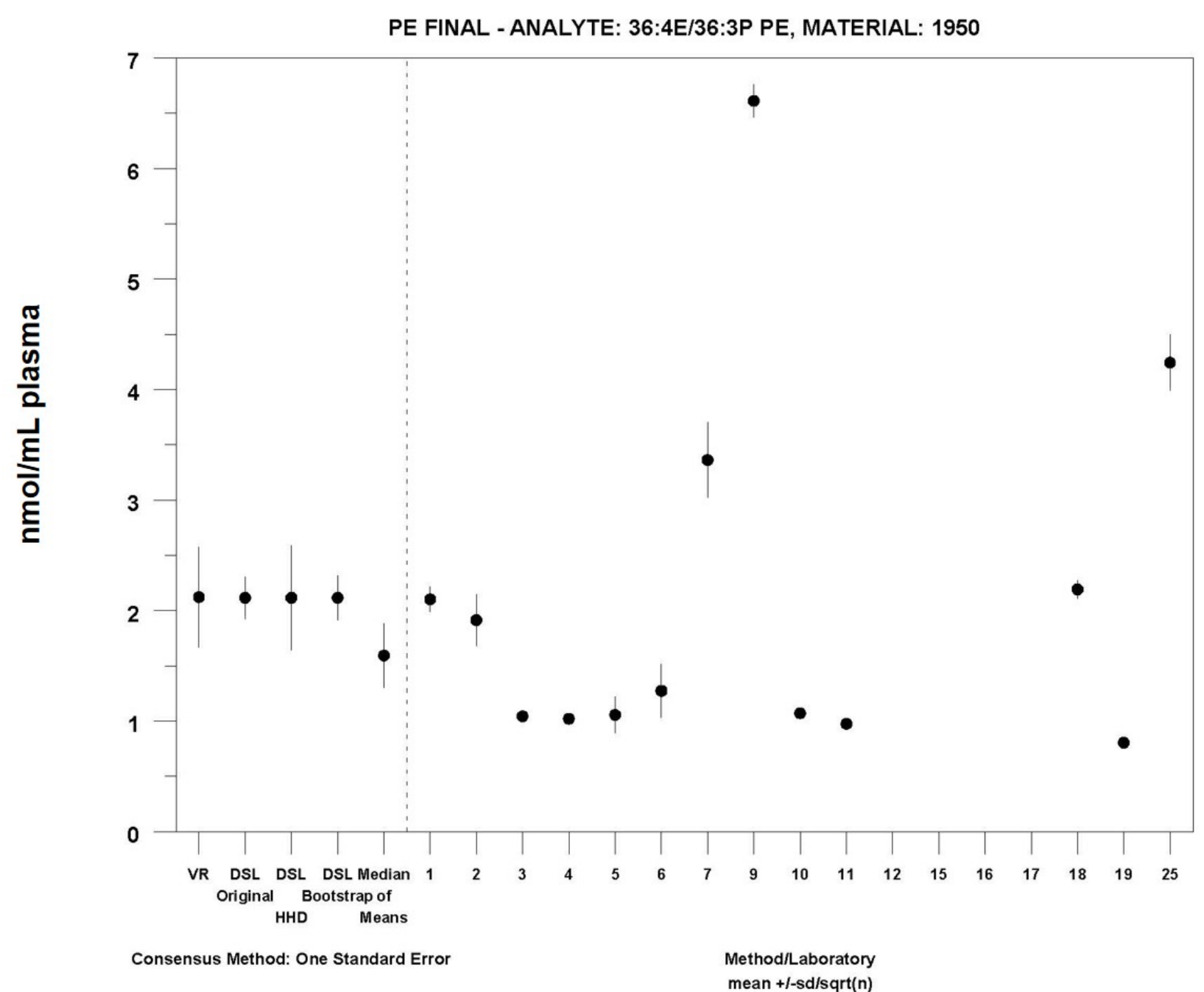

MEDM Location: $1.6 \pm 0.29 \mathrm{nmol} / \mathrm{mL}$

Labs Omitted from Plot (But Not Analysis): 15

15: $367 \pm 37$ 


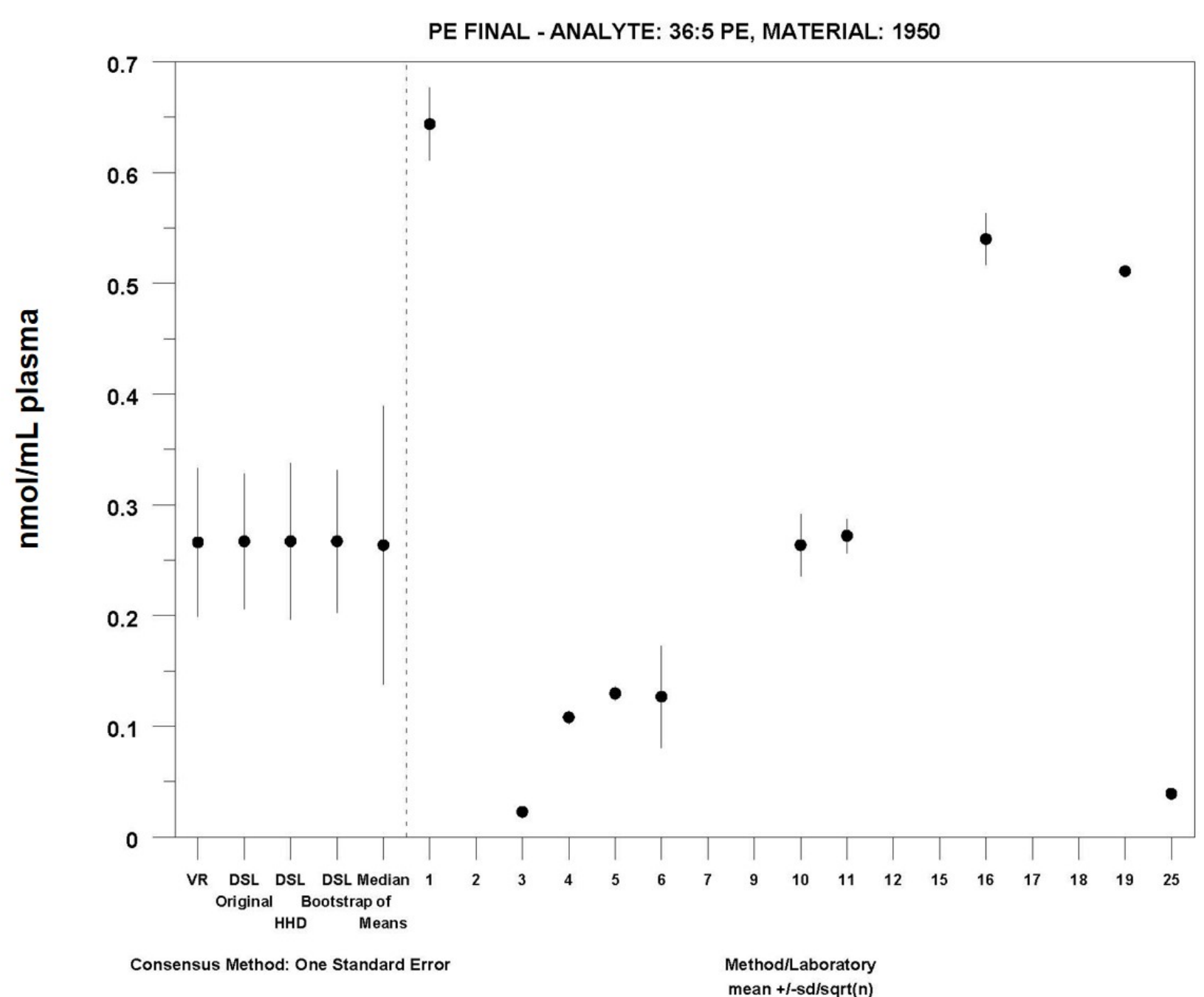

MEDM Location: $0.26 \pm 0.13 \mathrm{nmol} / \mathrm{mL}$

Labs Omitted from Plot (But Not Analysis): 15

15: $9 \pm 7$ 


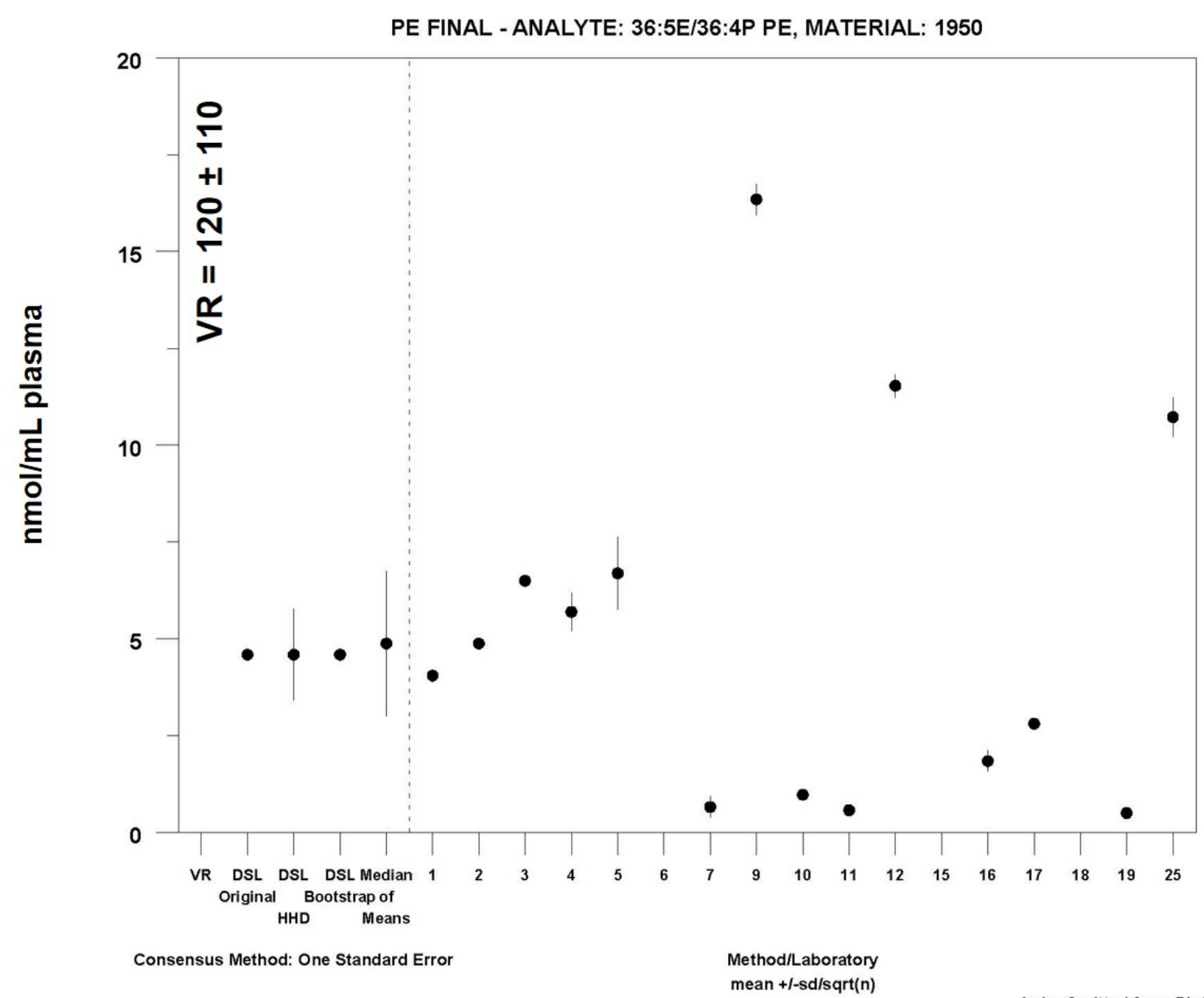

MEDM Location: $4.9 \pm 1.9 \mathrm{nmol} / \mathrm{mL}$

Labs Omitted from Plot (But Not Analysis): 15

15: $1707 \pm 54$ 


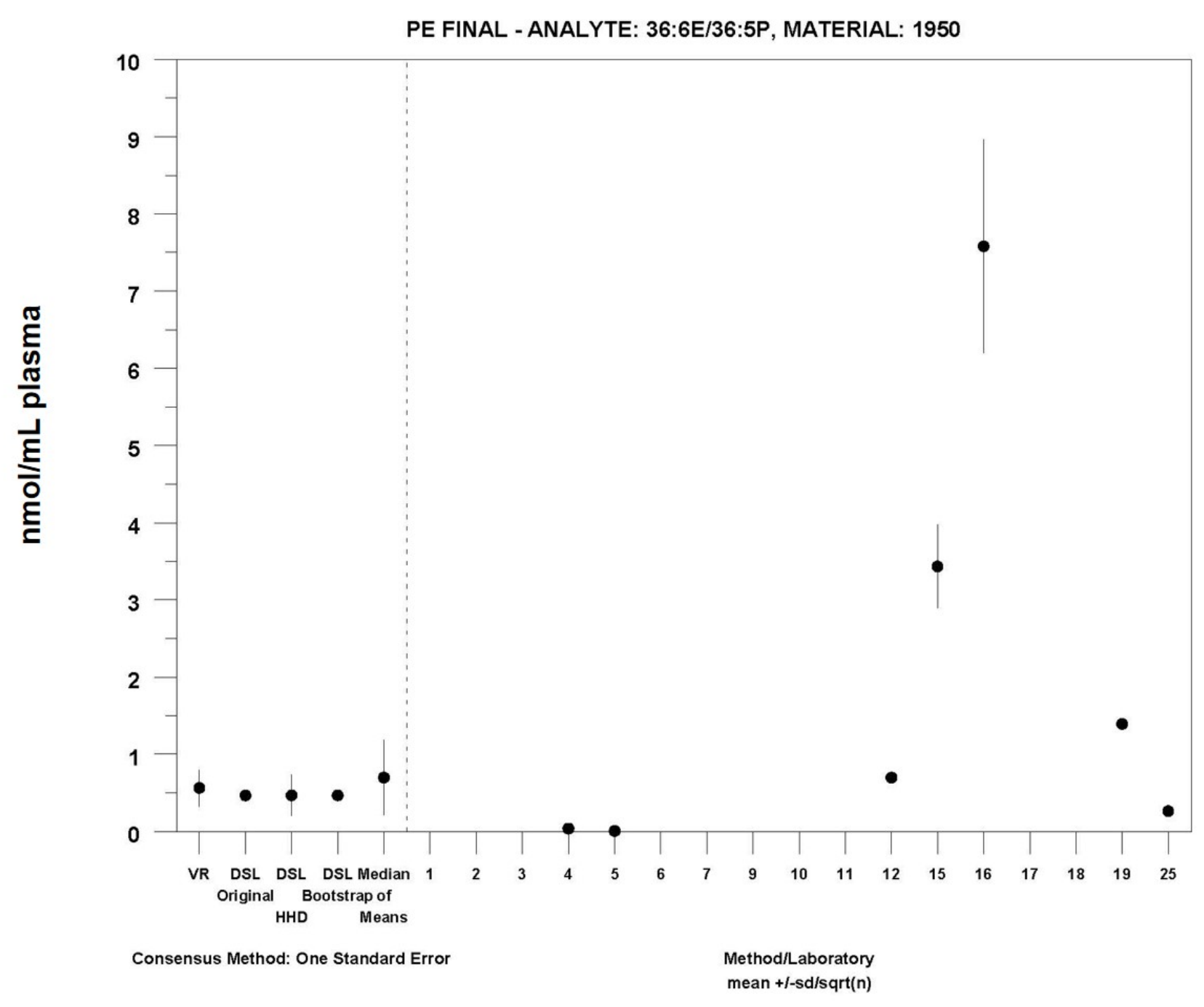

MEDM Location: $0.70 \pm 0.49 \mathrm{nmol} / \mathrm{mL}$ 


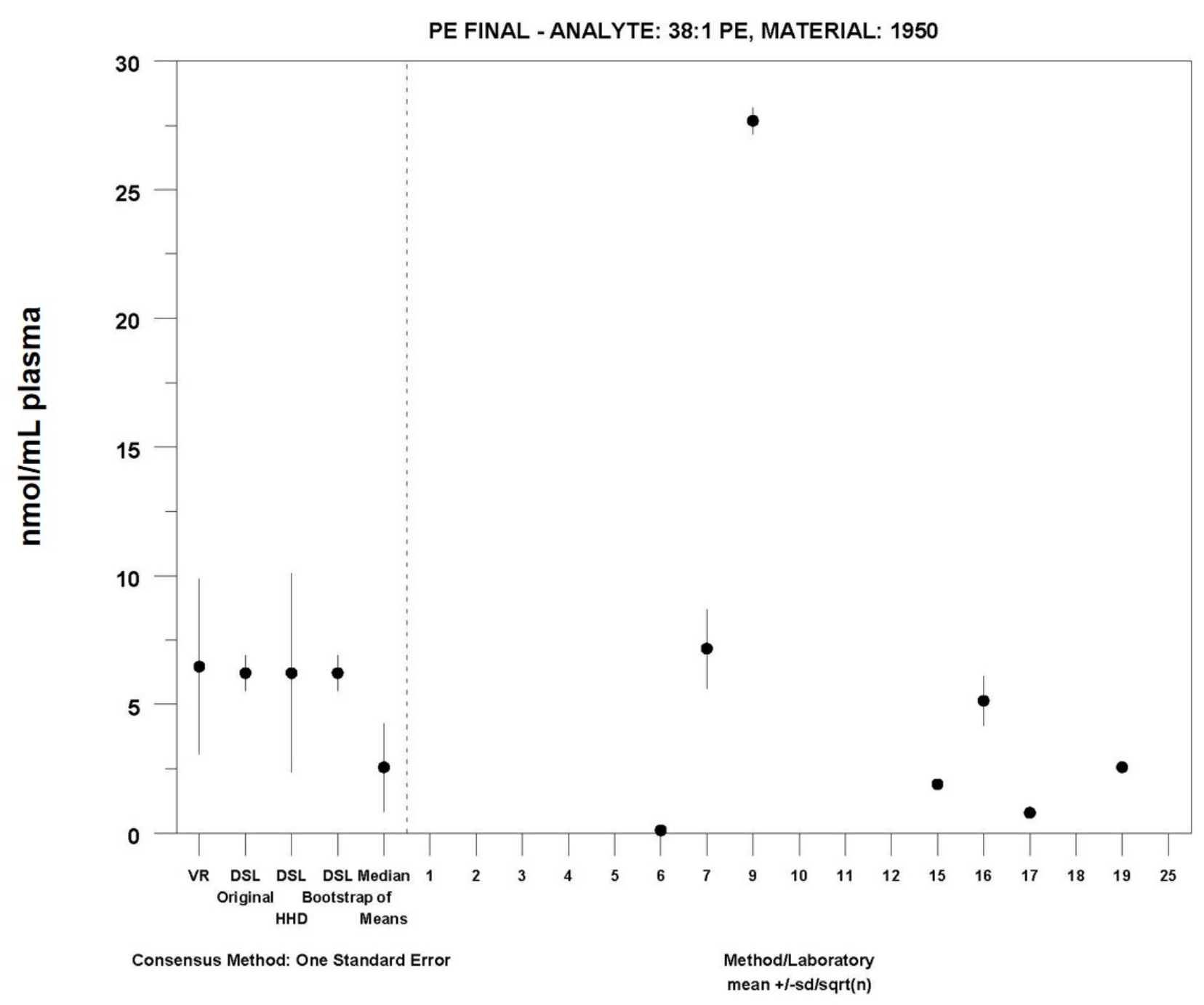

MEDM Location: $2.6 \pm 1.7 \mathrm{nmol} / \mathrm{mL}$ 


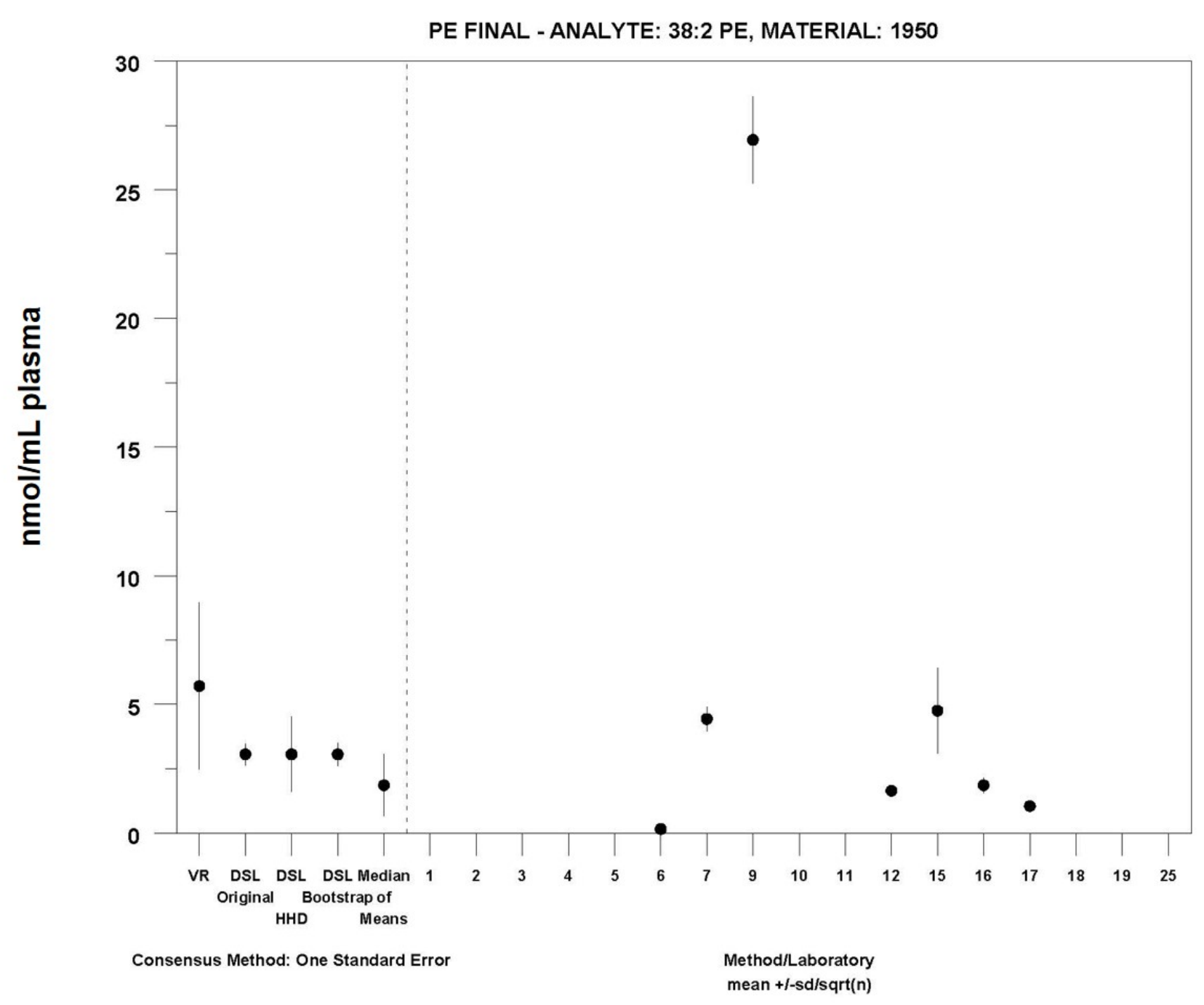

MEDM Location: $1.9 \pm 1.2 \mathrm{nmol} / \mathrm{mL}$ 


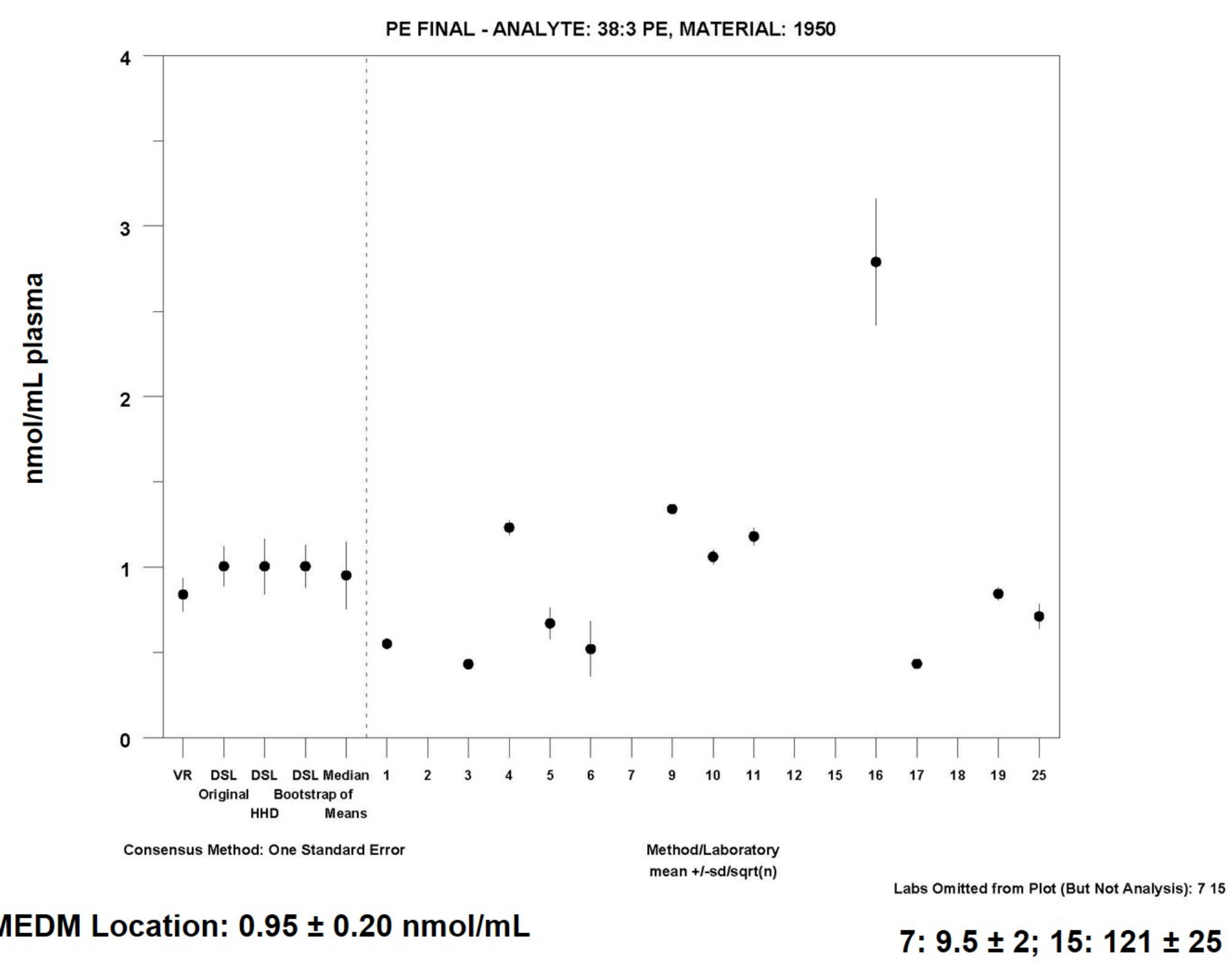




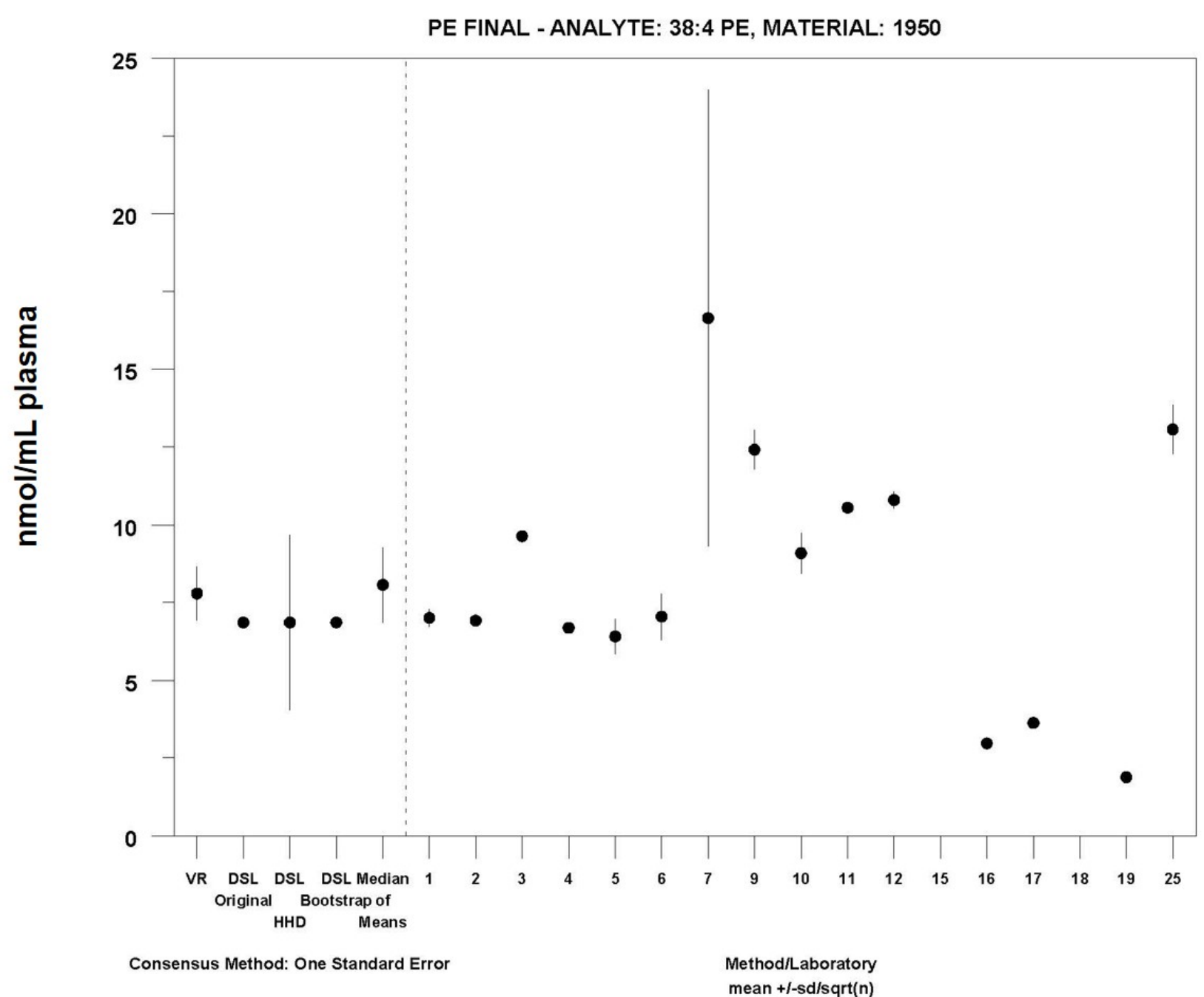

MEDM Location: $8.1 \pm 1.2 \mathrm{nmol} / \mathrm{mL}$

Labs Omitted from Plot (But Not Analysis): 15

15: $767 \pm 185$ 


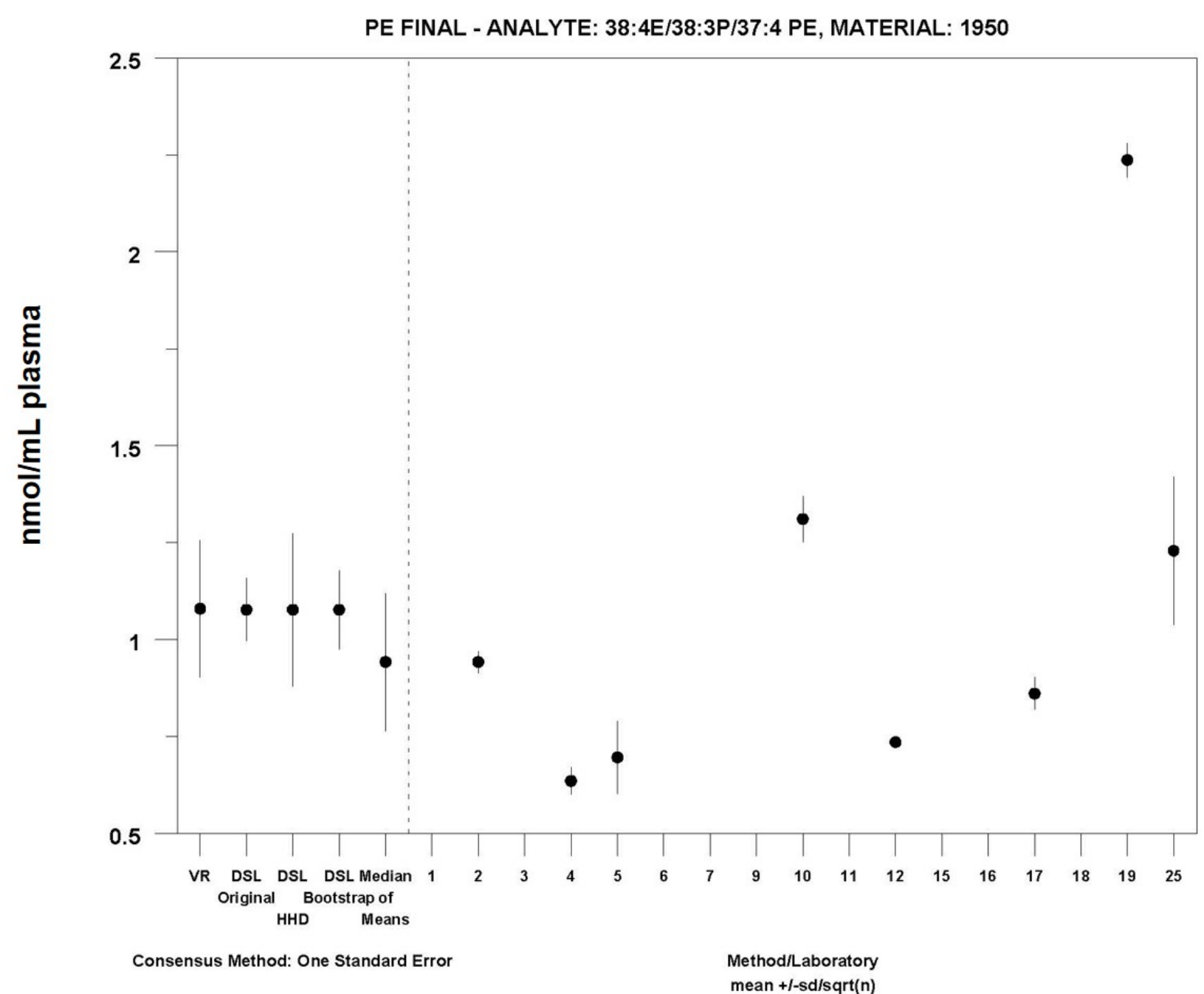

MEDM Location: $0.94 \pm 0.18 \mathrm{nmol} / \mathrm{mL}$

Labs Omitted from Plot (But Not Analysis): 15

15: $119 \pm 31$ 


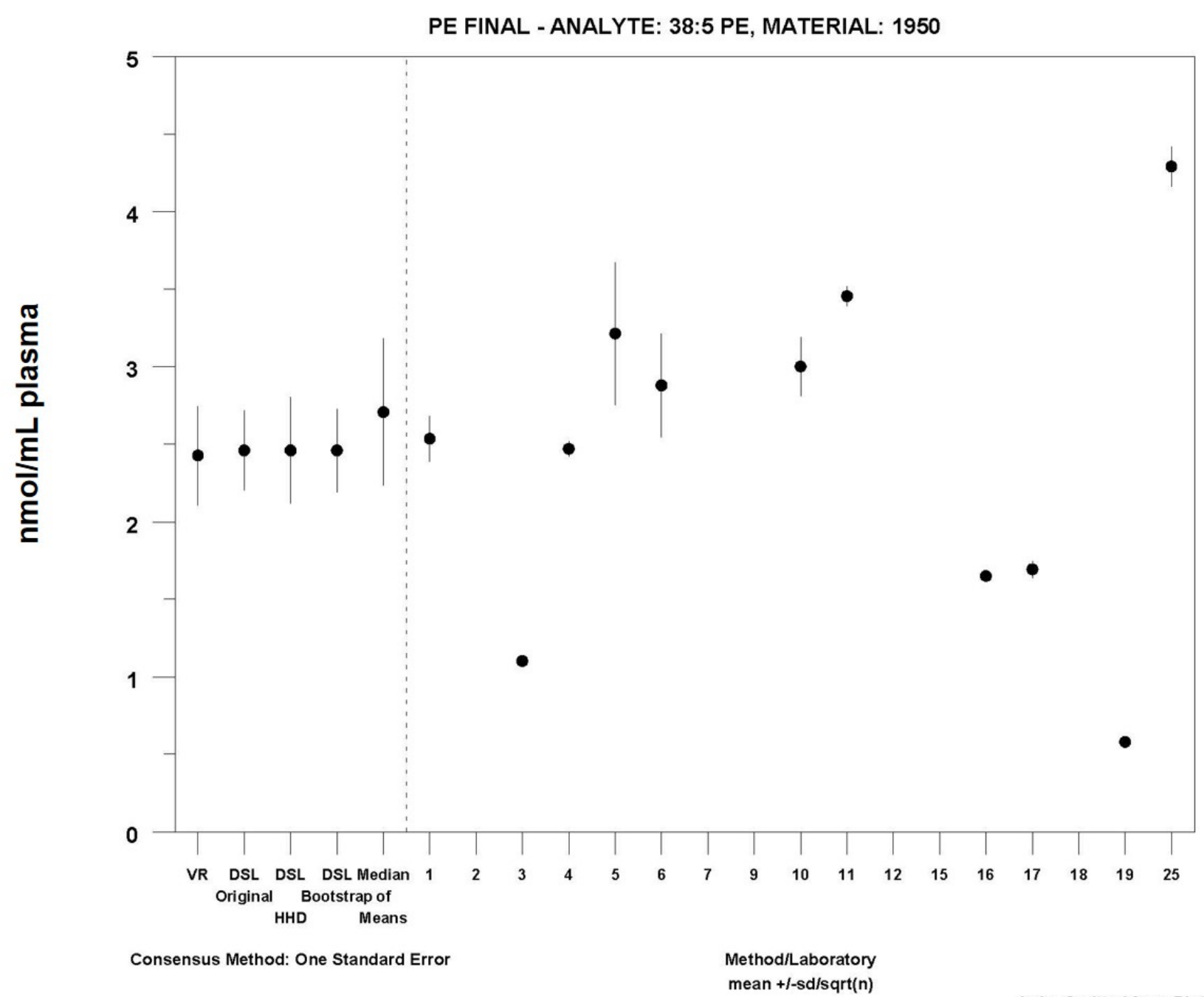

MEDM Location: $2.7 \pm 0.47 \mathrm{nmol} / \mathrm{mL}$

Labs Omitted from Plot (But Not Analysis): 15

15: $74 \pm 18$ 


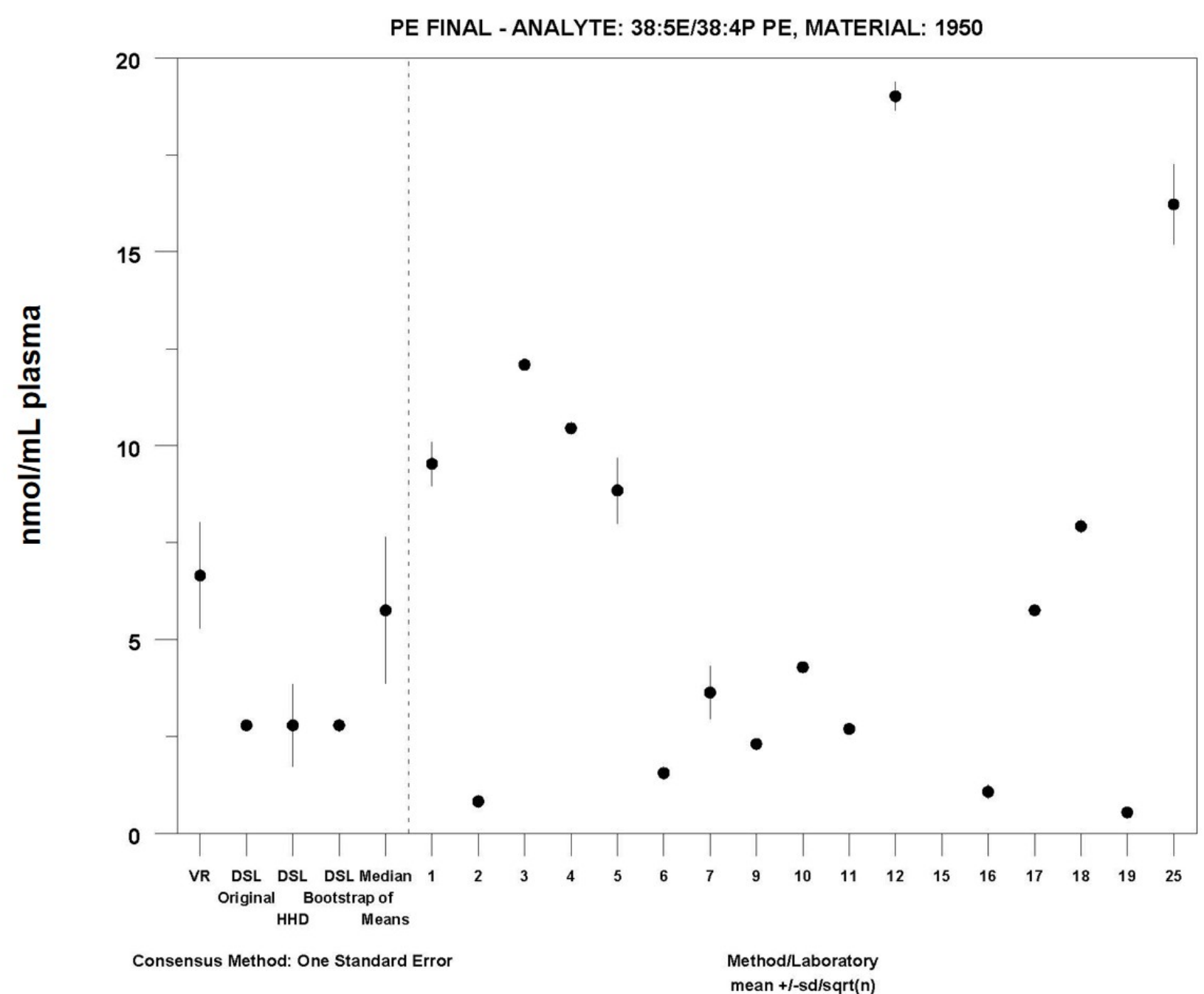

MEDM Location: $5.8 \pm 1.9 \mathrm{nmol} / \mathrm{mL}$

Labs Omitted from Plot (But Not Analysis): 15

15: $986 \pm 892$ 


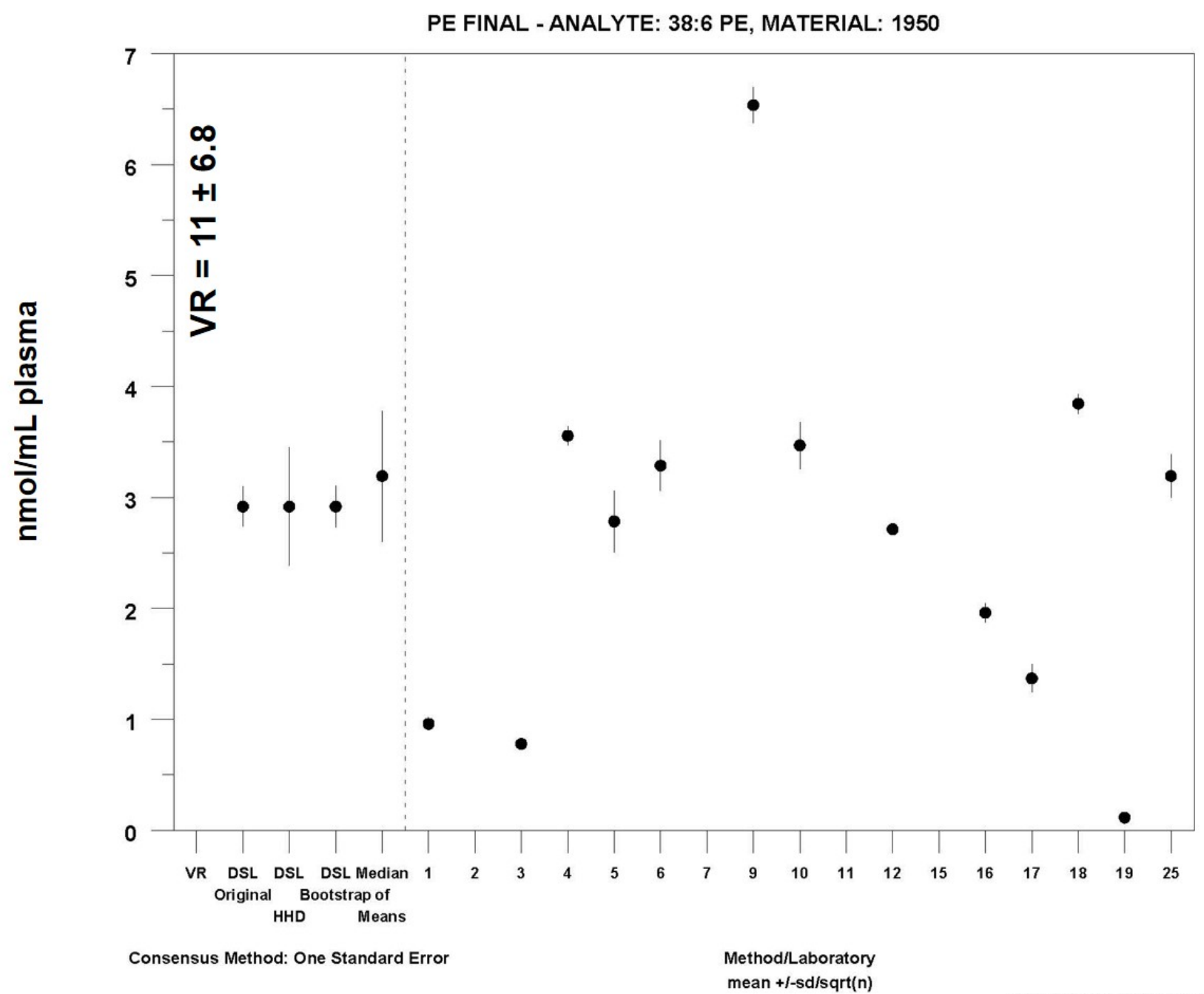

MEDM Location: $3.2 \pm 0.59 \mathrm{nmol} / \mathrm{mL}$

Labs Omitted from Plot (But Not Analysis): 715

$7: 16 \pm 4 ; 15: 110 \pm 7$ 


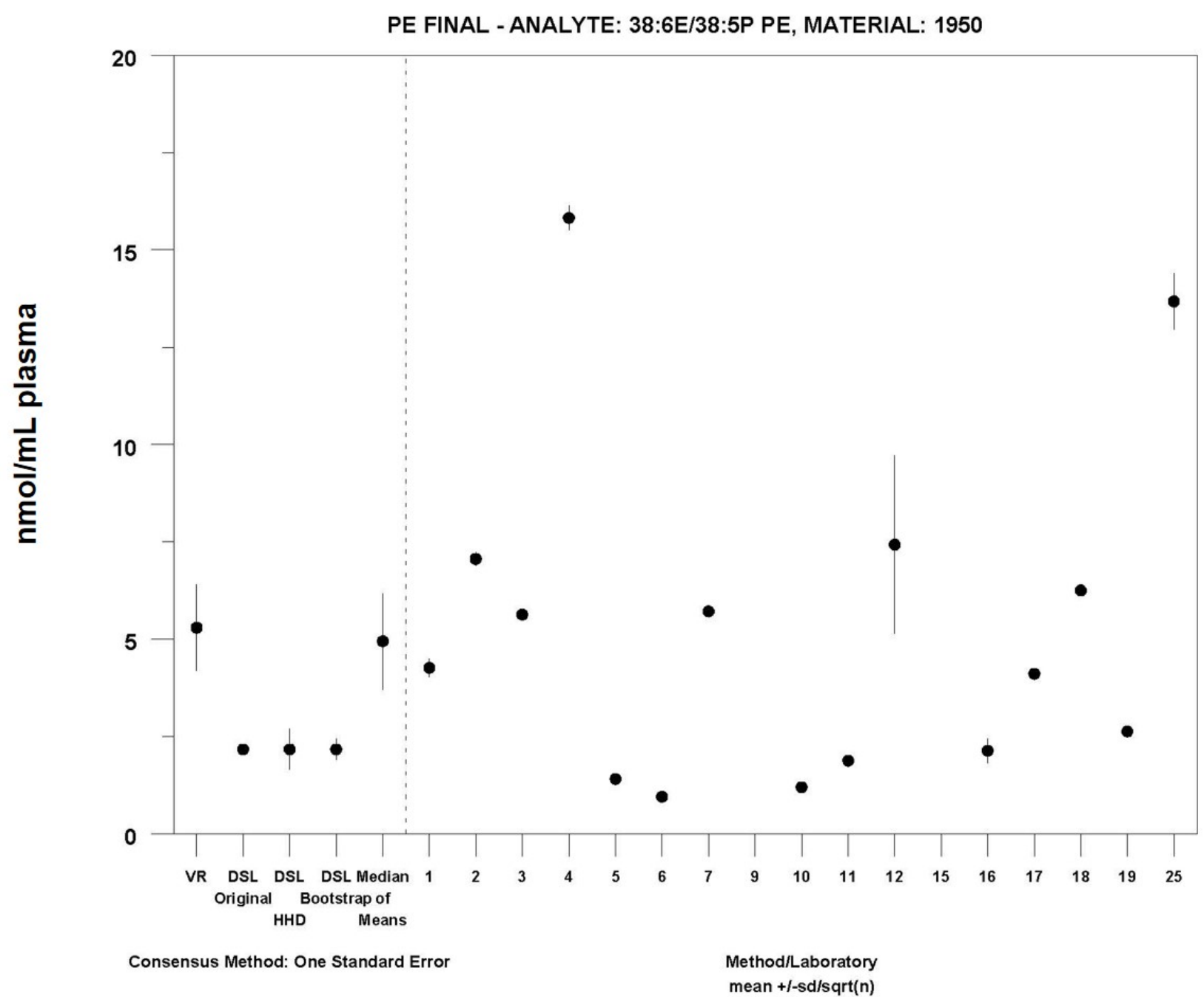

MEDM Location: $4.9 \pm 1.2 \mathrm{nmol} / \mathrm{mL}$

Labs Omitted from Plot (But Not Analysis): 15

15: $2693 \pm 651$ 


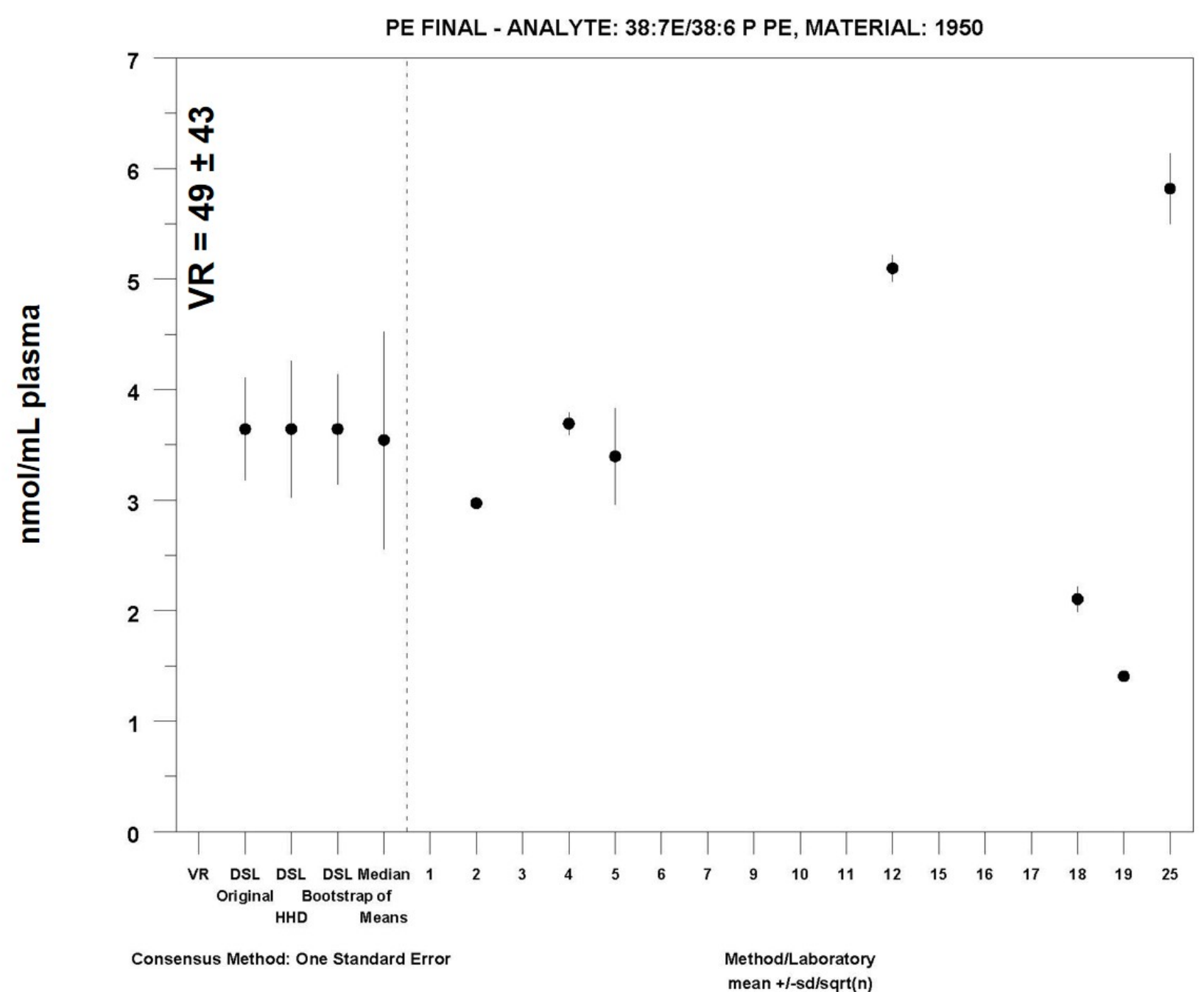

MEDM Location: $3.5 \pm 0.98 \mathrm{nmol} / \mathrm{mL}$

Labs Omitted from Plot (But Not Analysis): 15

15: $382 \pm 38$ 


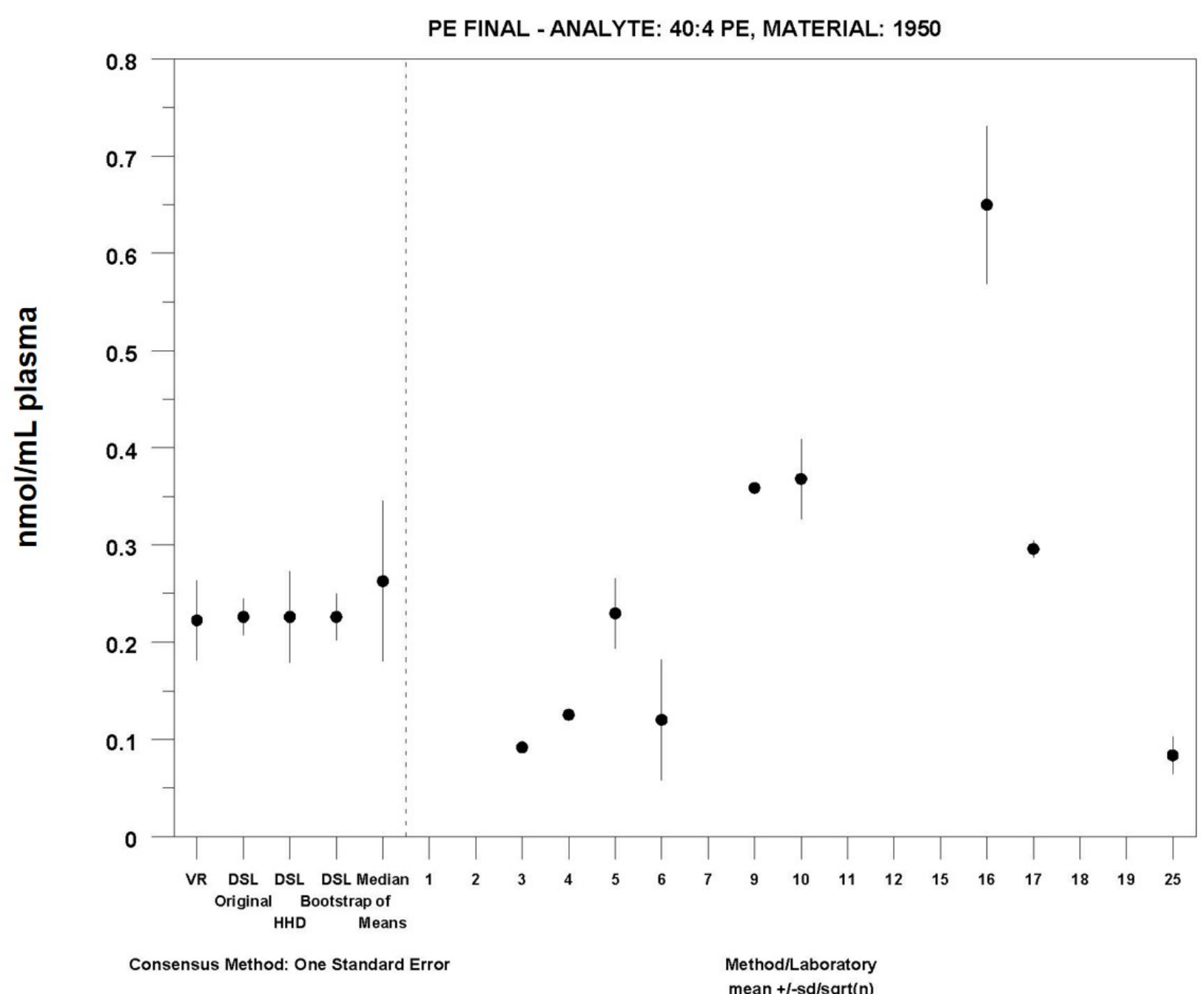

MEDM Location: $0.26 \pm 0.082 \mathrm{nmol} / \mathrm{mL}$

Labs Omitted from Plot (But Not Analysis): 15

15: $27 \pm 10$ 


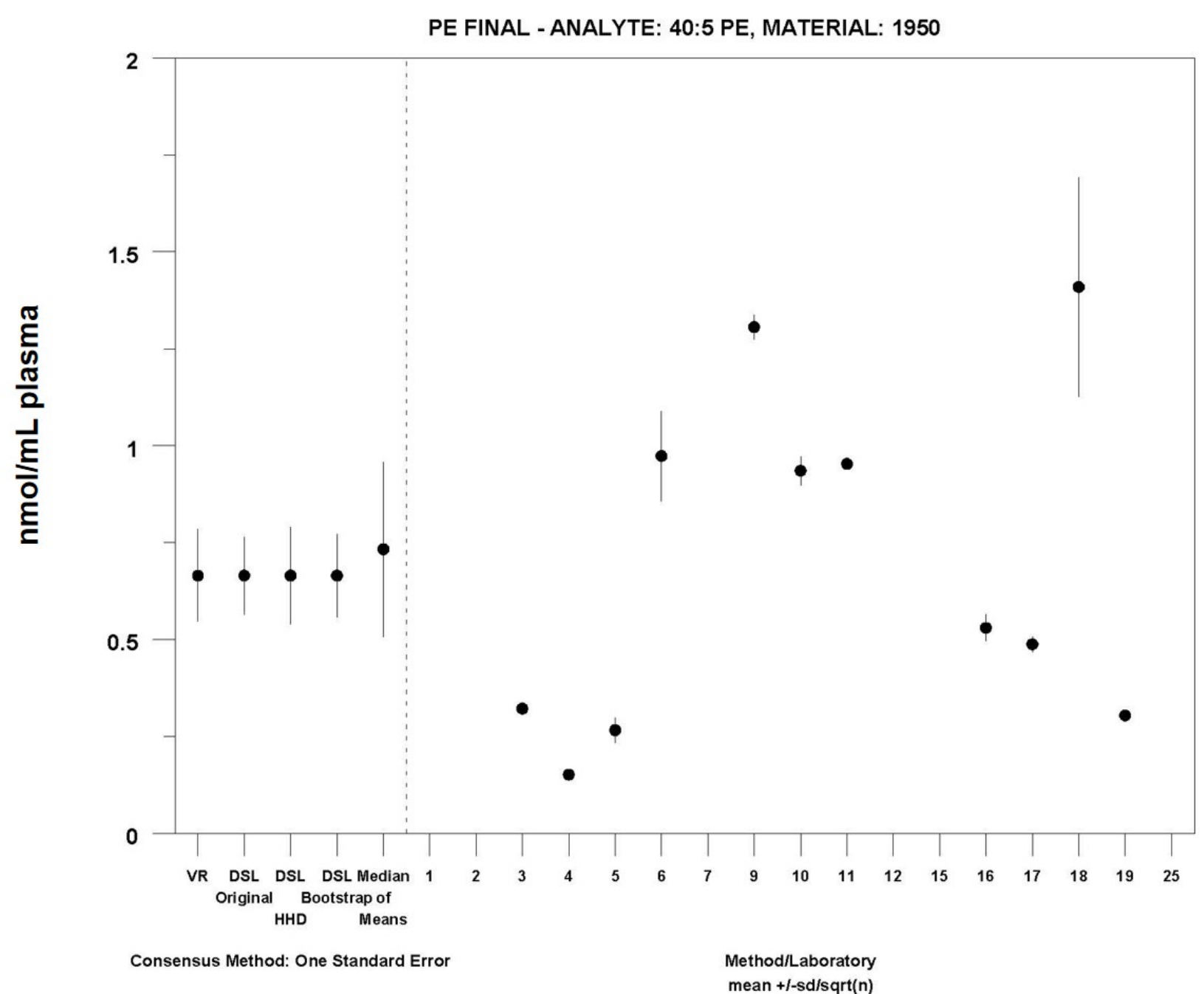

MEDM Location: $0.73 \pm 0.23 \mathrm{nmol} / \mathrm{mL}$

Labs Omitted from Plot (But Not Analysis): 15

15: $35 \pm 17$ 


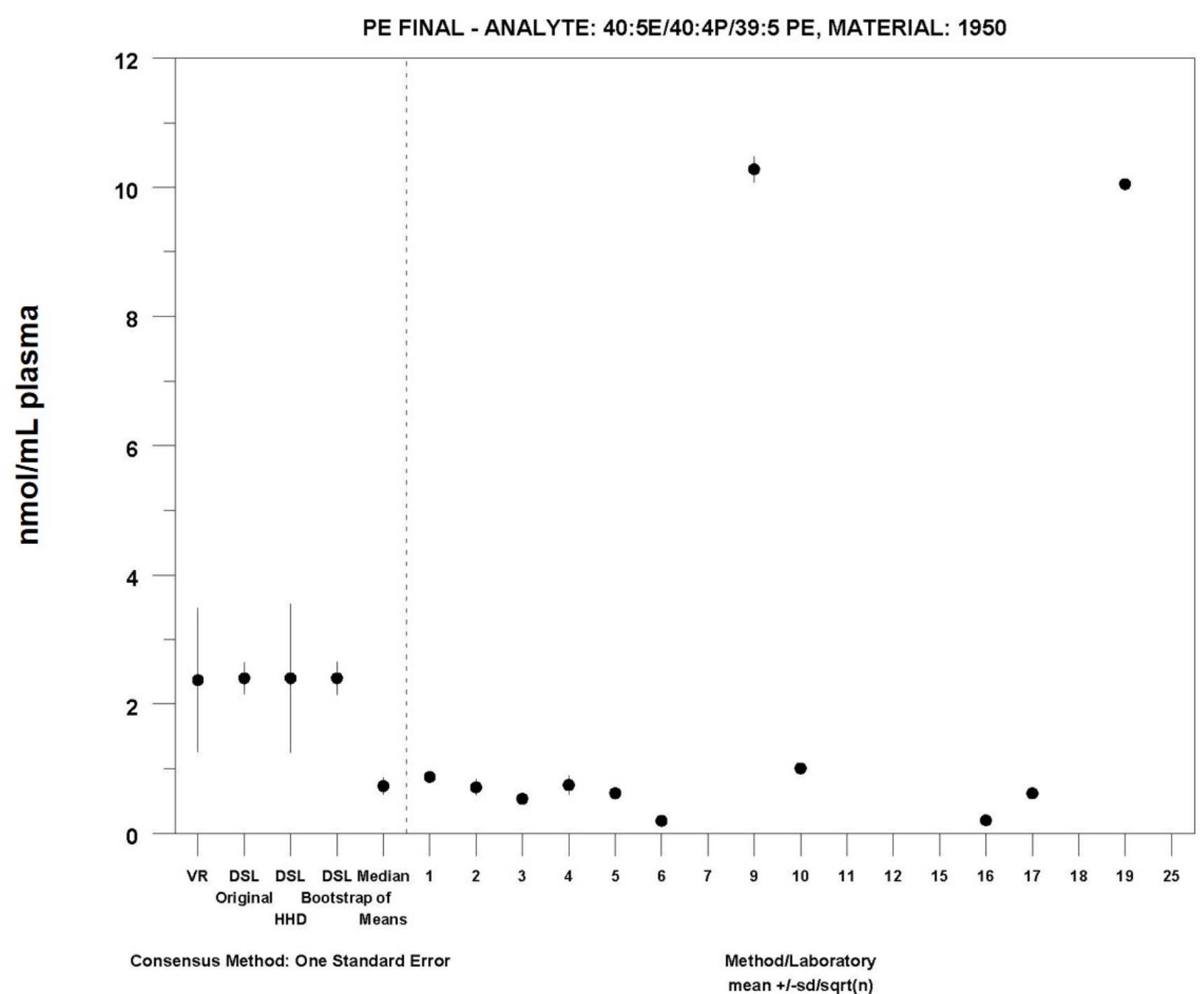

MEDM Location: $0.73 \pm 0.13 \mathrm{nmol} / \mathrm{mL}$

Labs Omitted from Plot (But Not Analysis): 15

15: $157 \pm 17$ 


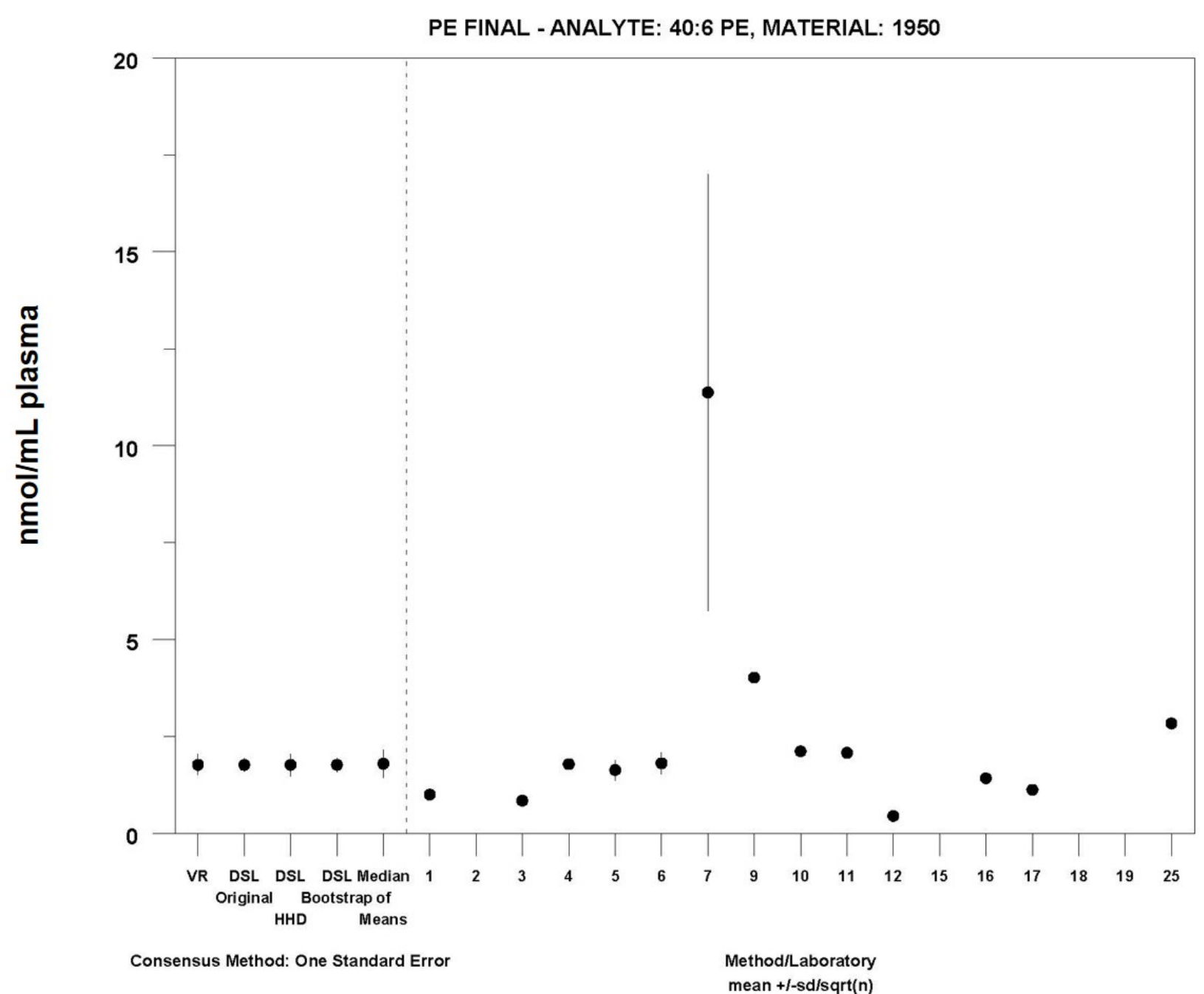

MEDM Location: $1.8 \pm 0.36 \mathrm{nmol} / \mathrm{mL}$

Labs Omitted from Plot (But Not Analysis): 15

15: $82 \pm 25$ 


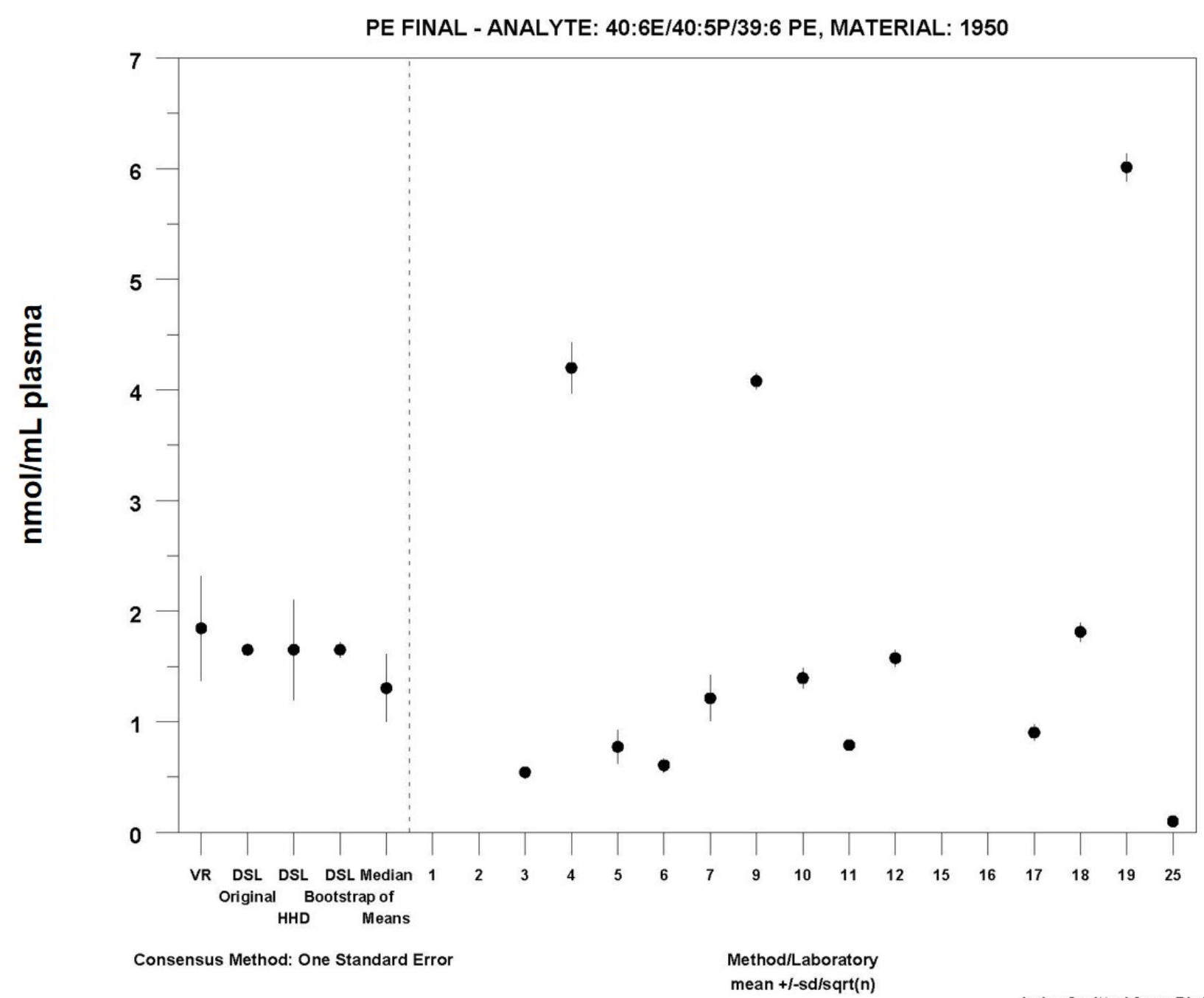

MEDM Location: $1.3 \pm 0.31 \mathrm{nmol} / \mathrm{mL}$

Labs Omitted from Plot (But Not Analysis): 15

15: $430 \pm 70$ 


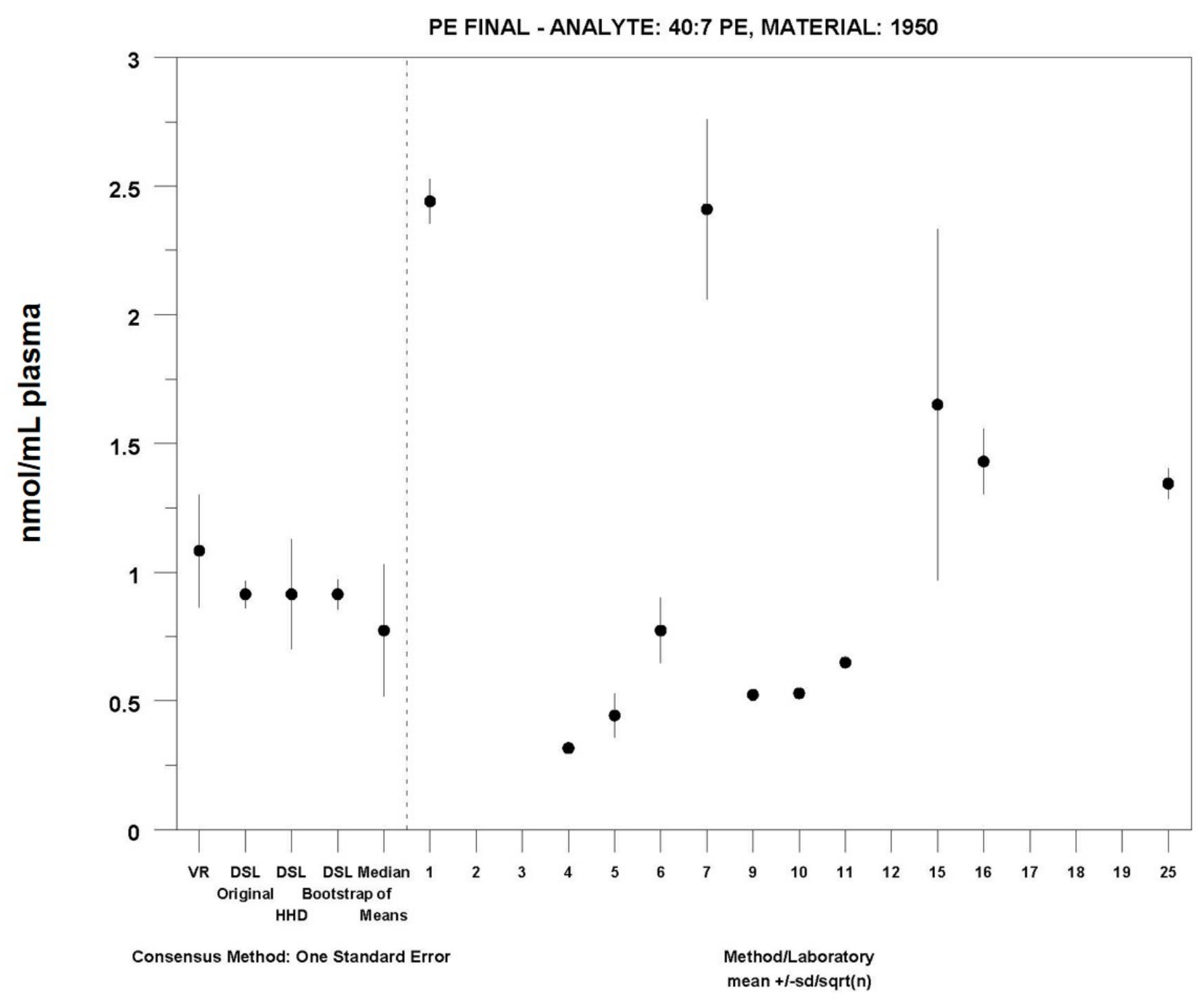

MEDM Location: $0.77 \pm 0.26 \mathrm{nmol} / \mathrm{mL}$ 


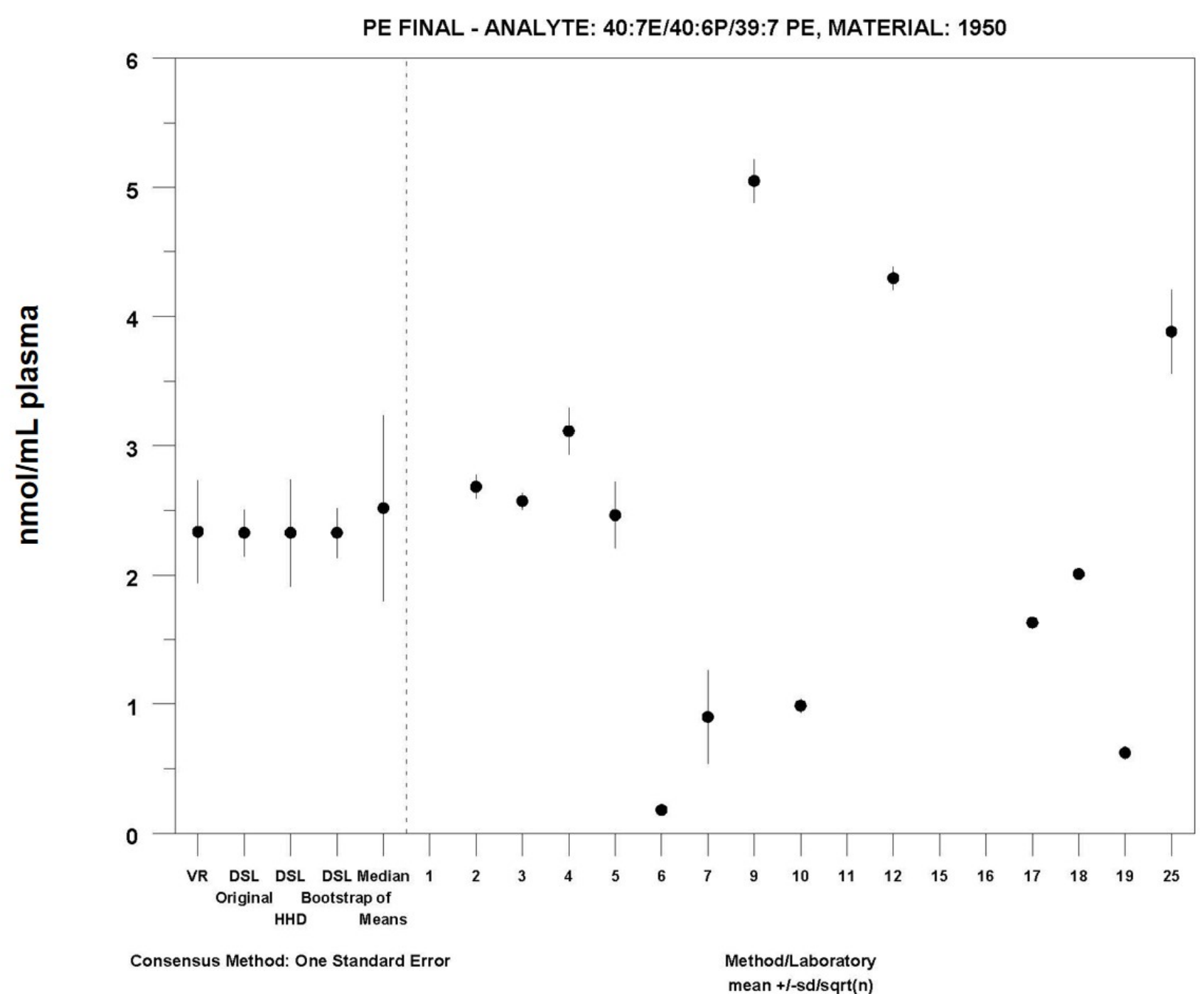

MEDM Location: $2.5 \pm 0.72 \mathrm{nmol} / \mathrm{mL}$

Labs Omitted from Plot (But Not Analysis): 15

15: $429 \pm 77$ 


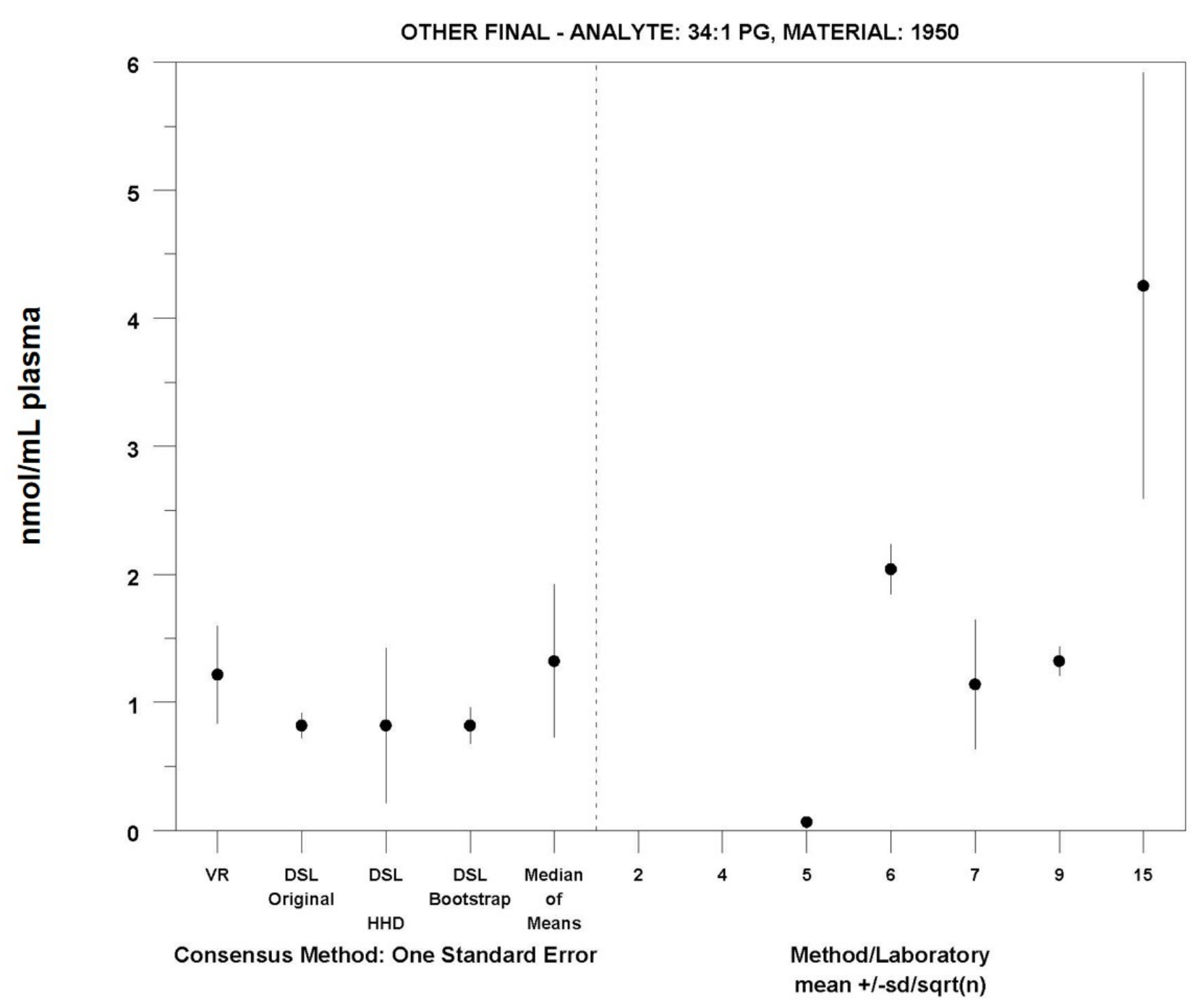

MEDM Location: $1.3 \pm 0.60 \mathrm{nmol} / \mathrm{mL}$ 


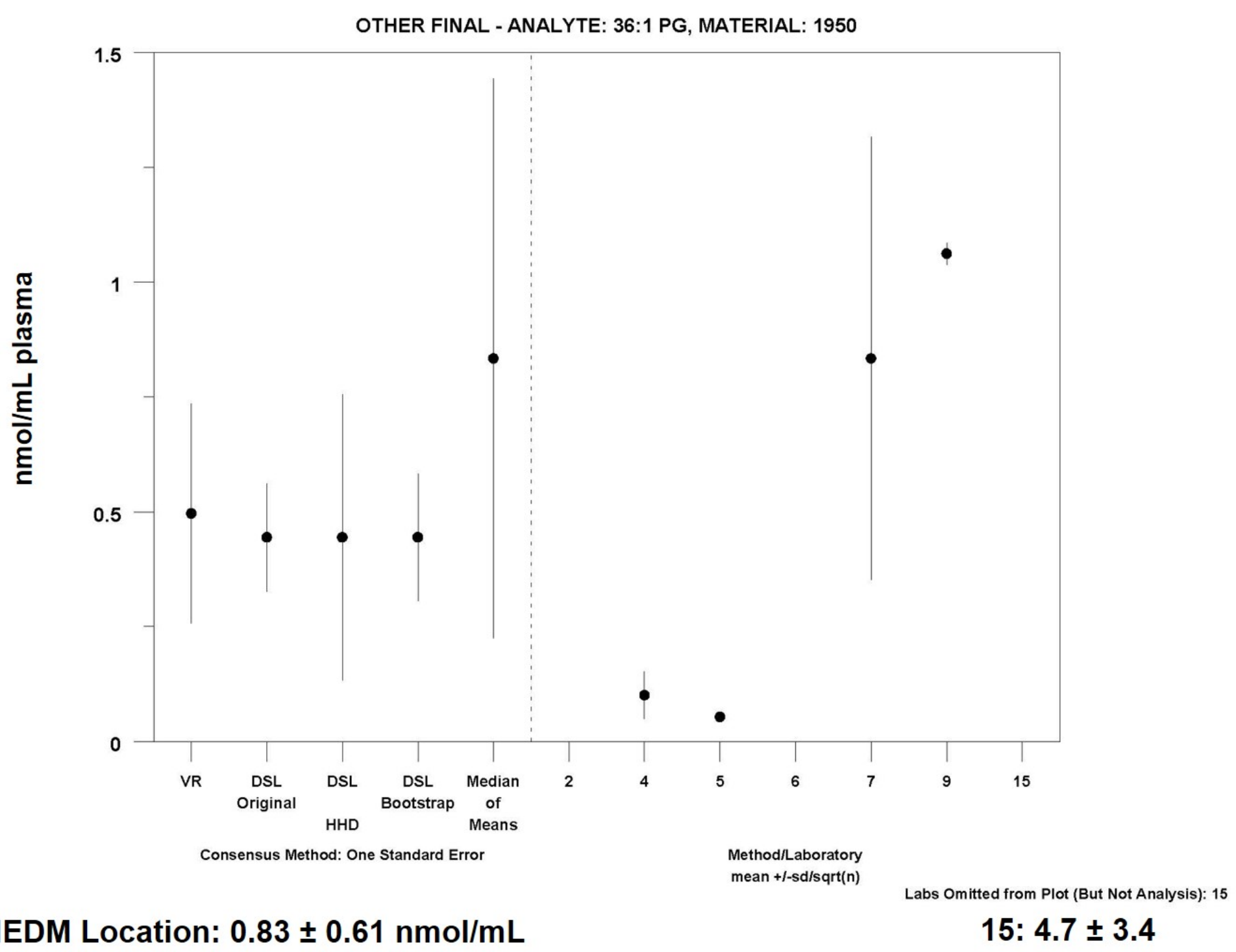




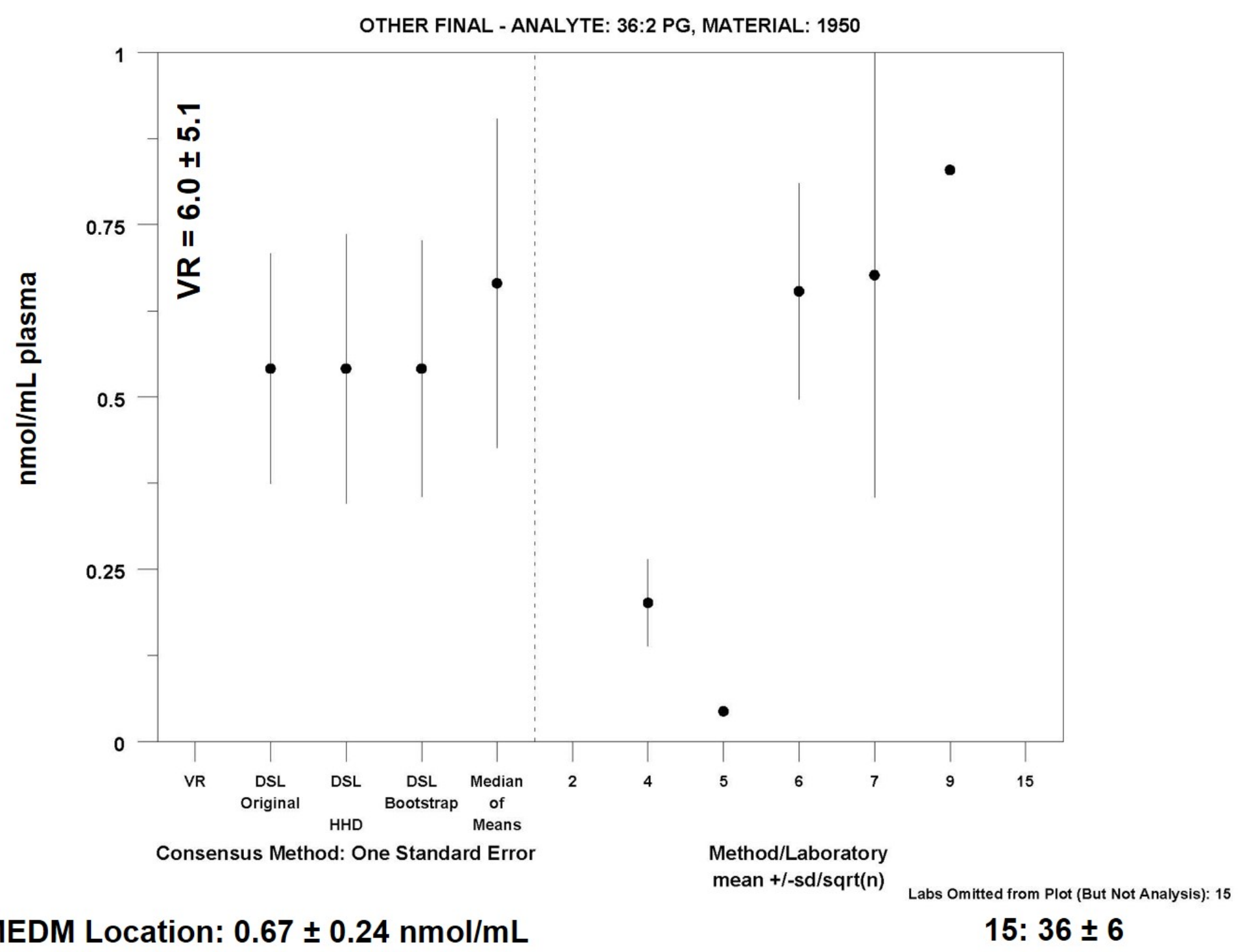




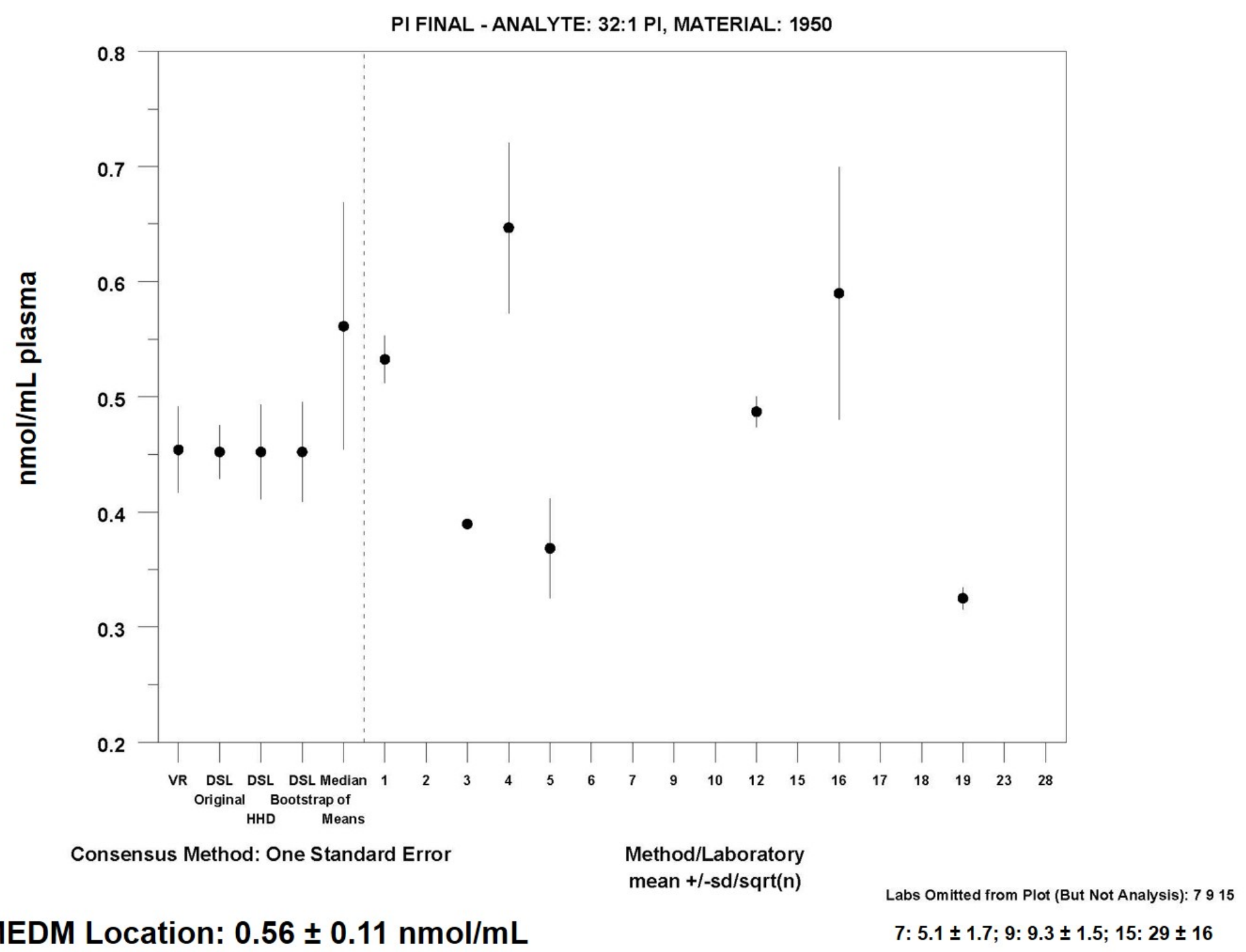




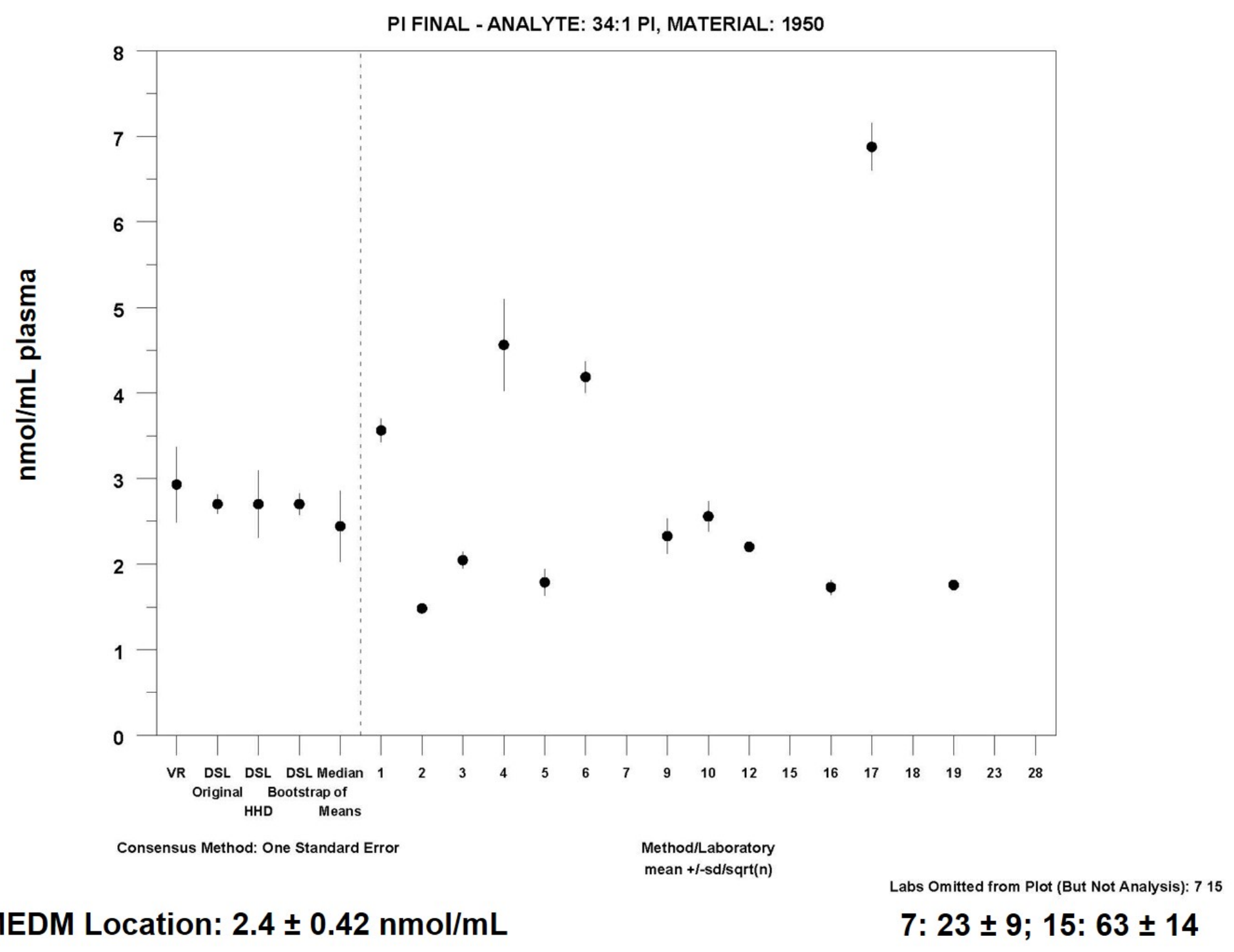




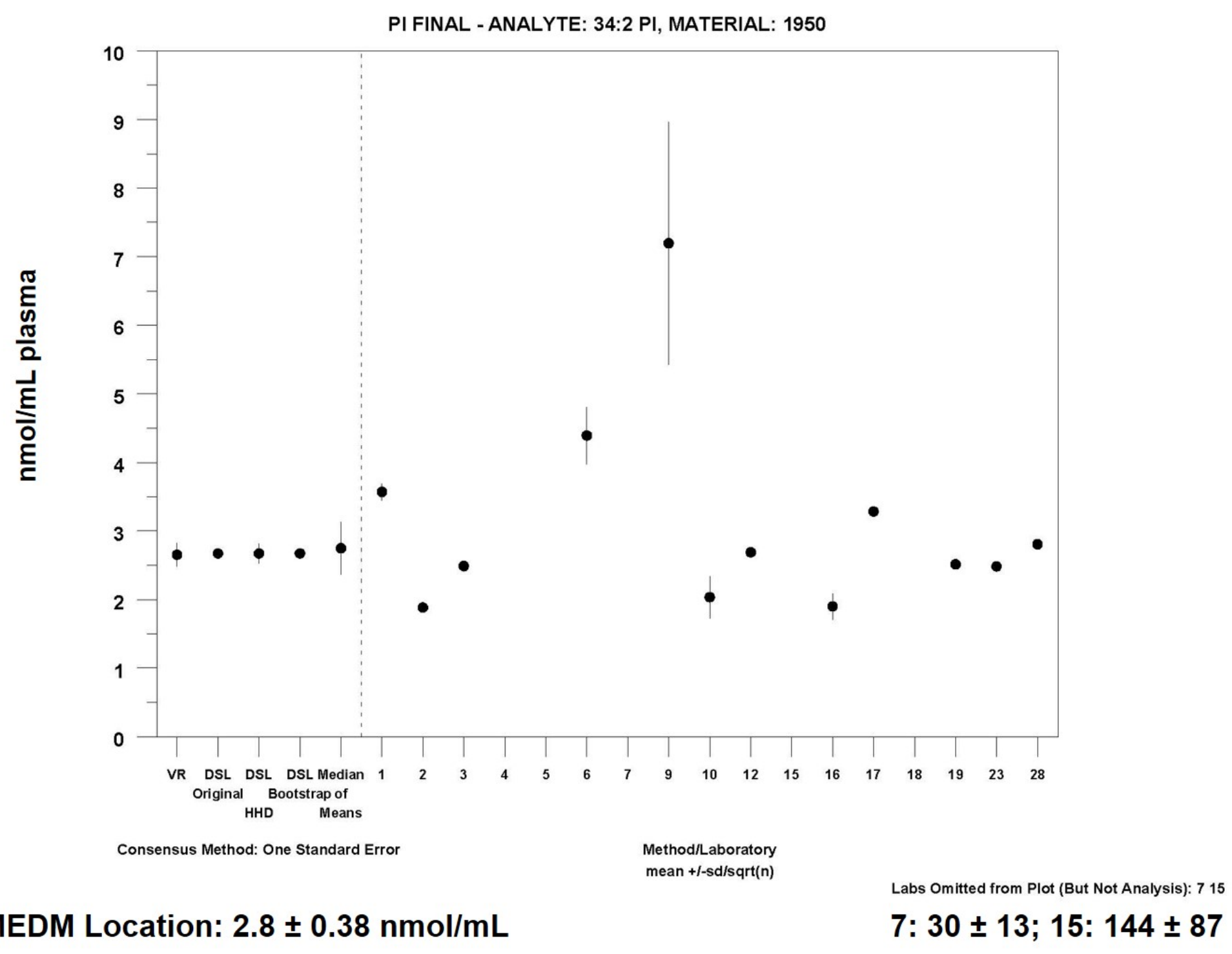




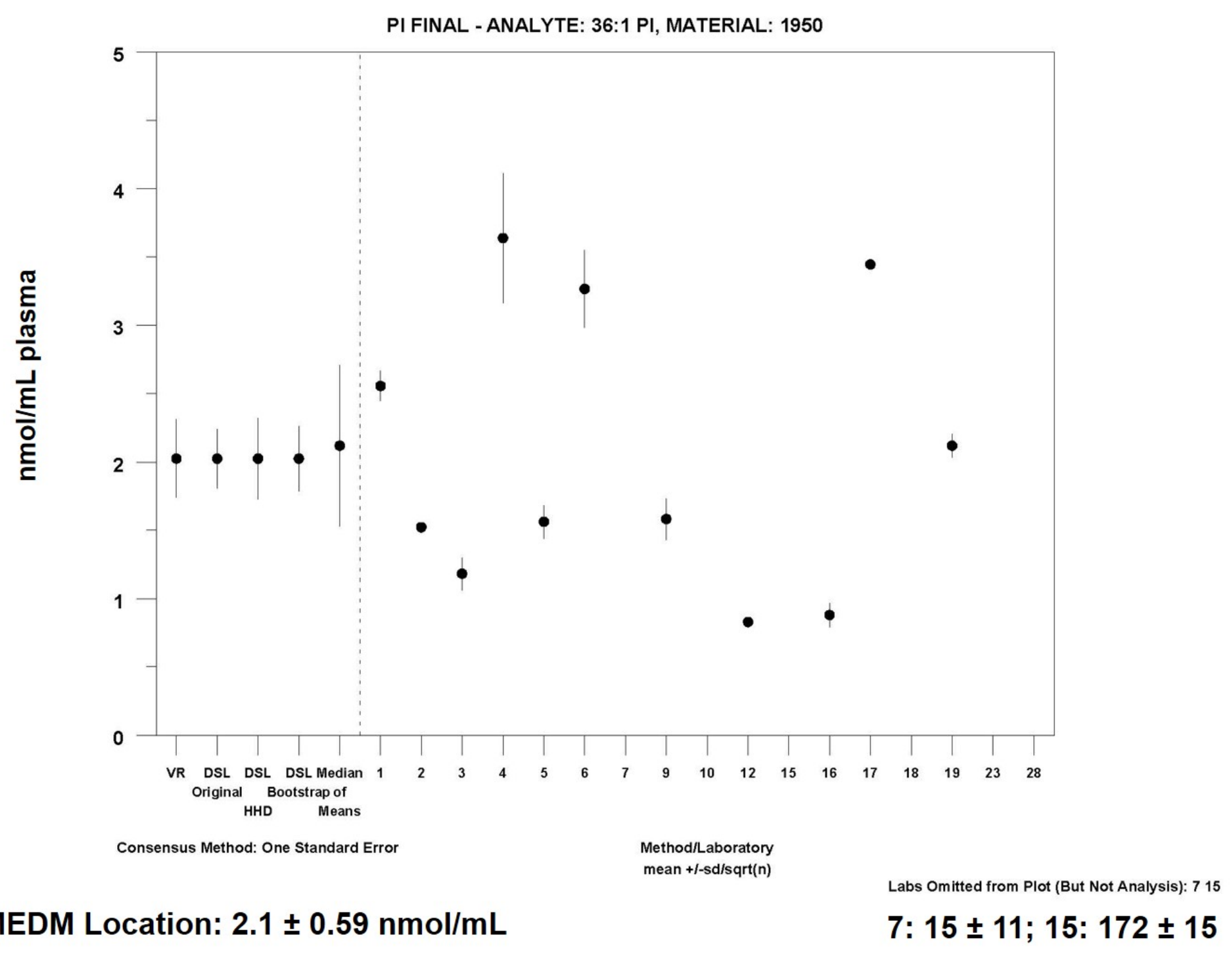




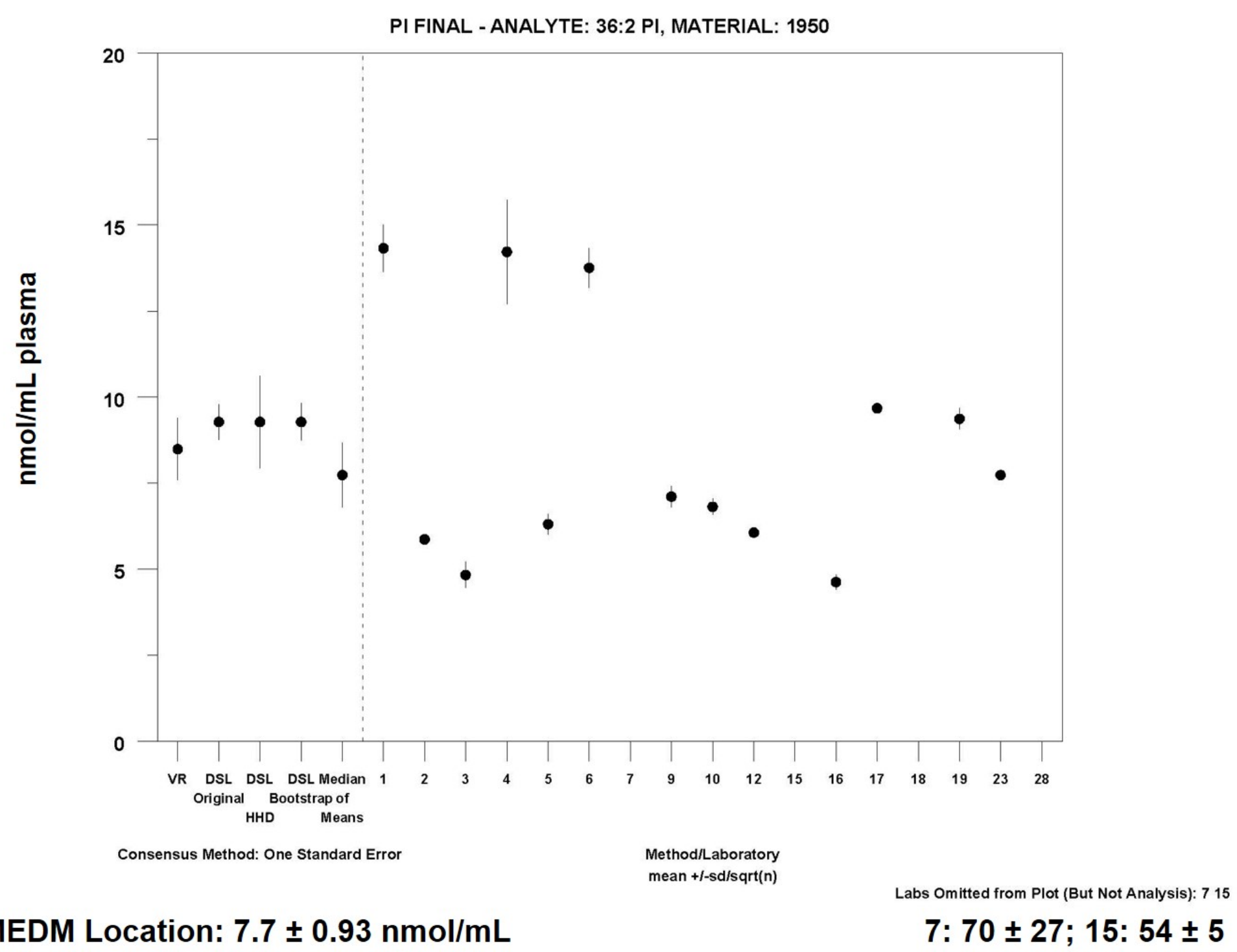




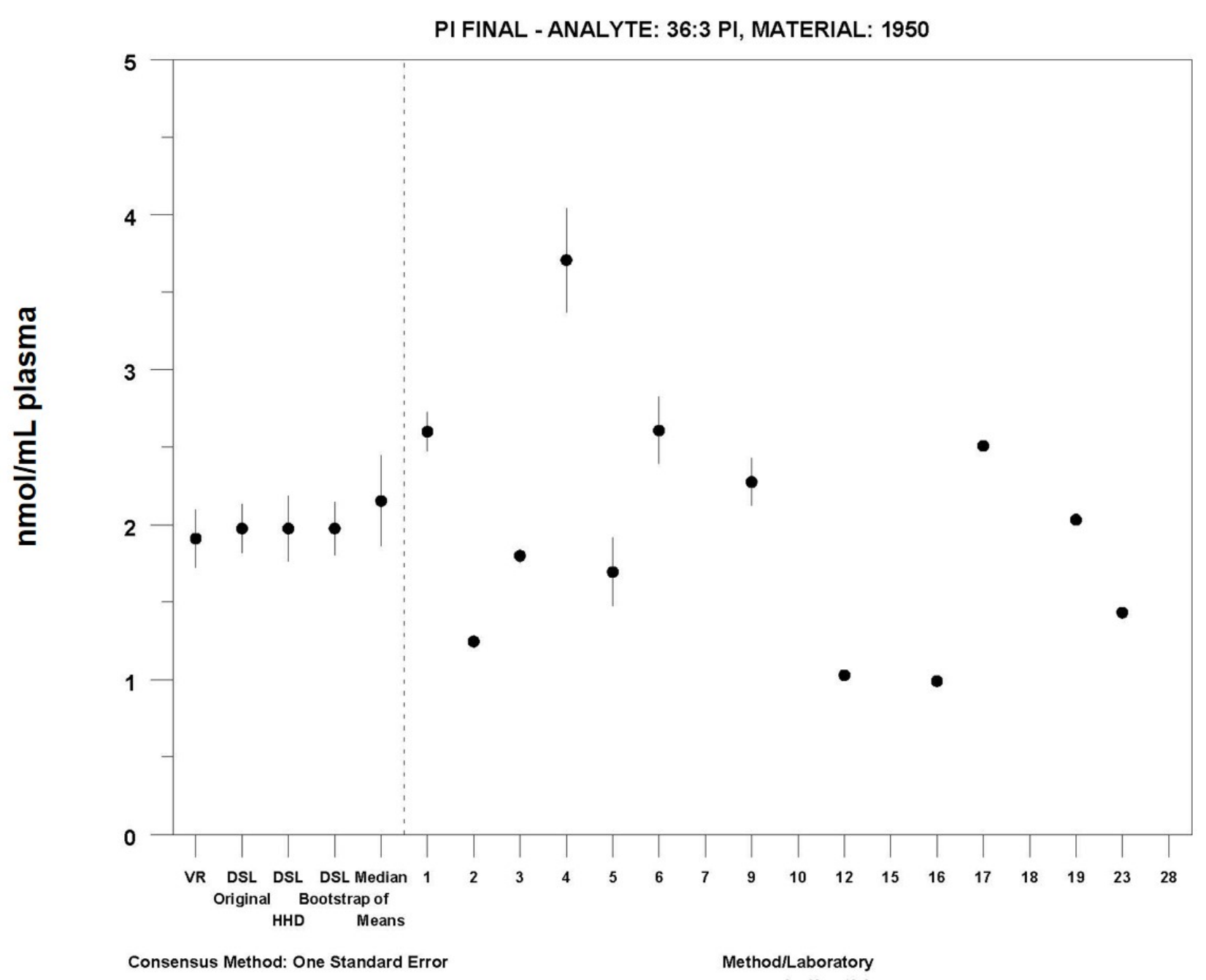

MEDM Location: $2.2 \pm 0.29 \mathrm{nmol} / \mathrm{mL}$

Labs Omitted from Plot (But Not Analysis): 715

$7: 14 \pm 7 ; 15: 136 \pm 27$ 


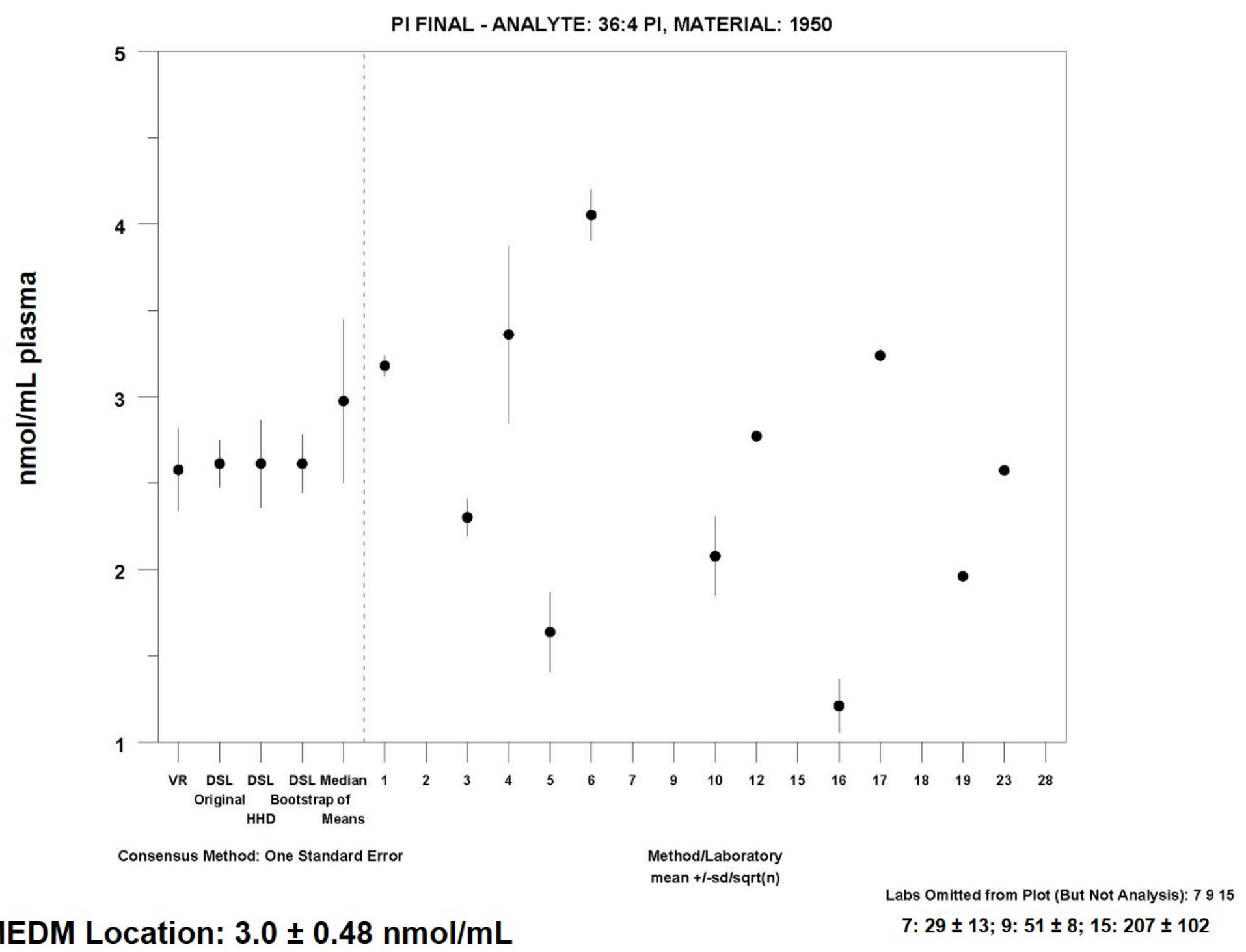




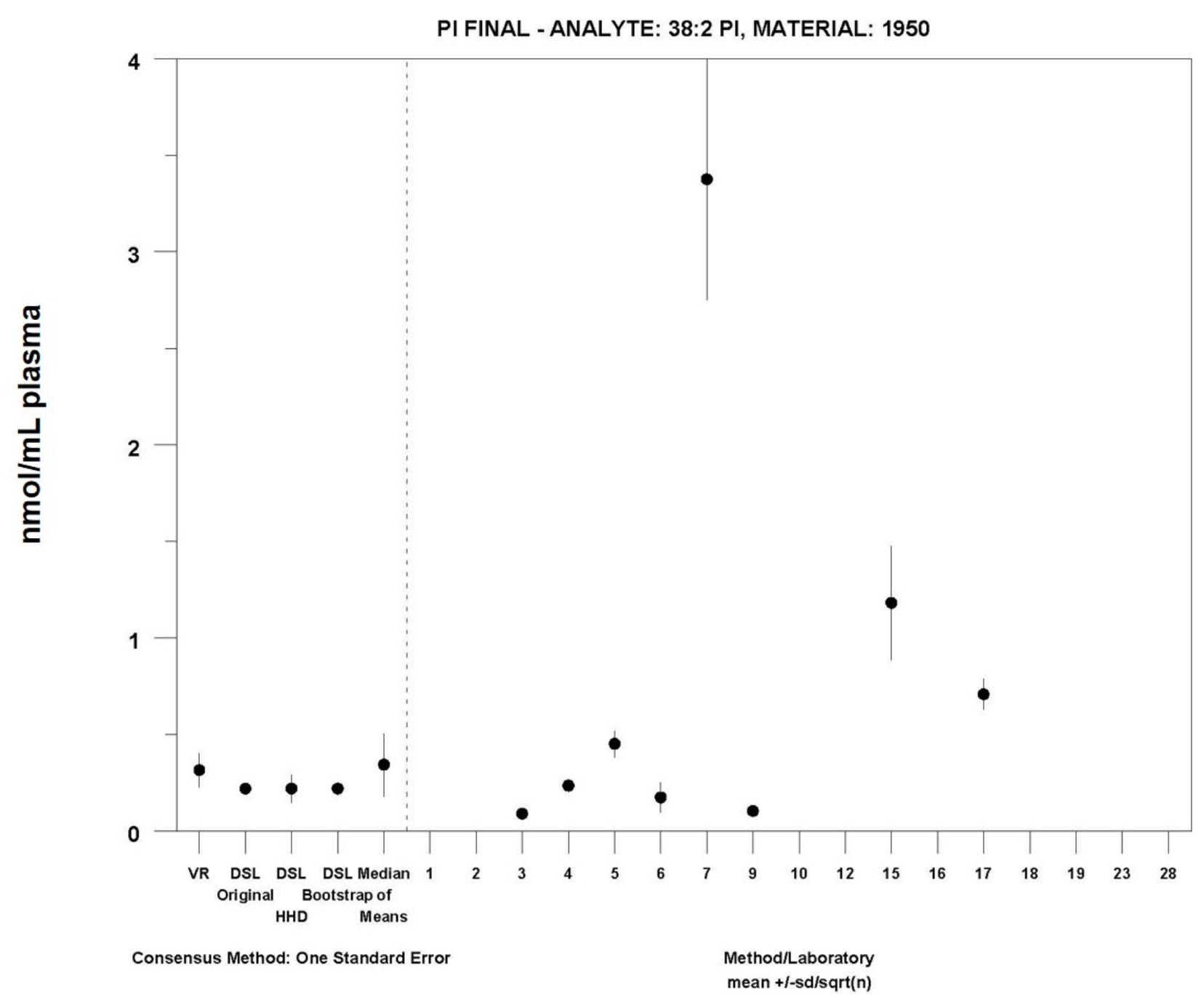

MEDM Location: $0.34 \pm 0.16 \mathrm{nmol} / \mathrm{mL}$ 


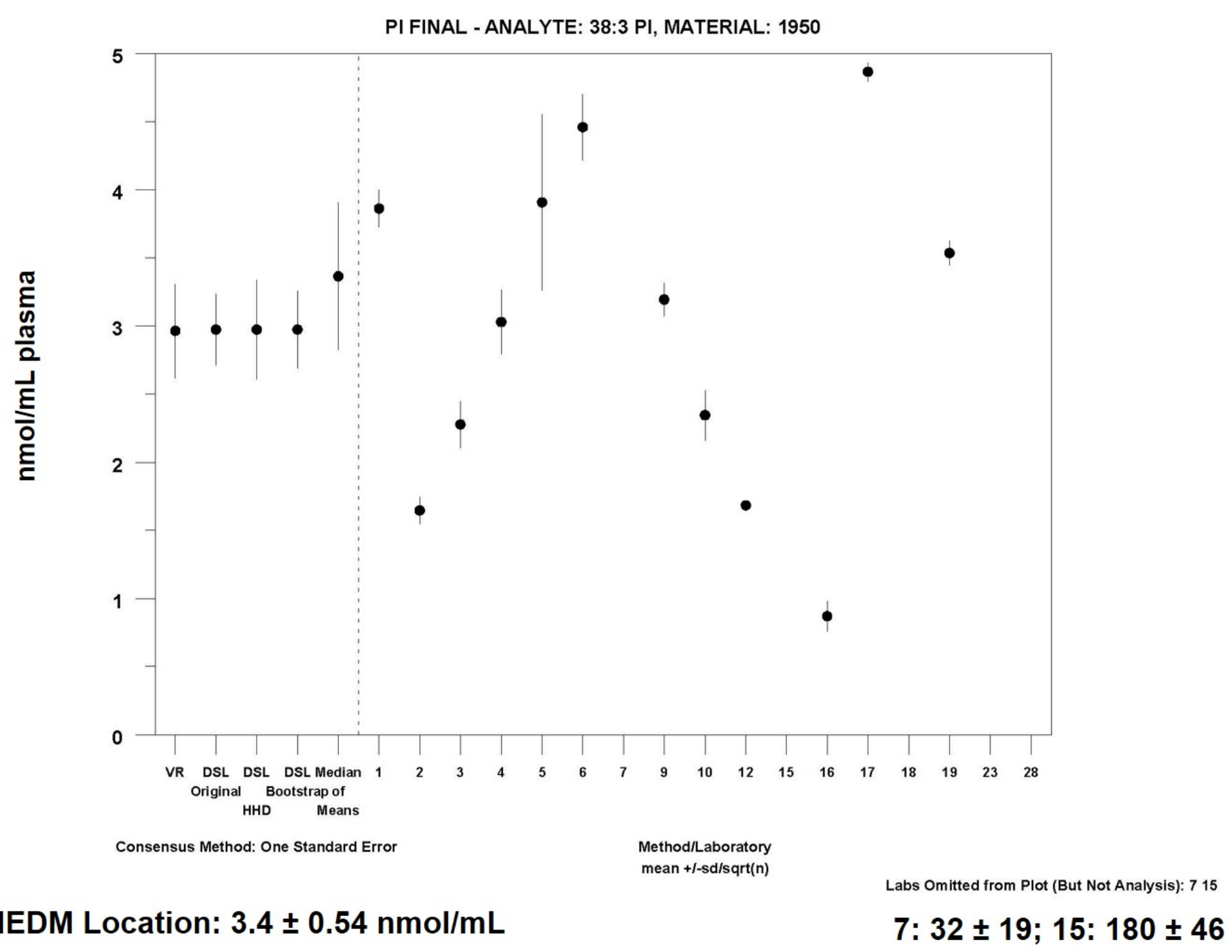




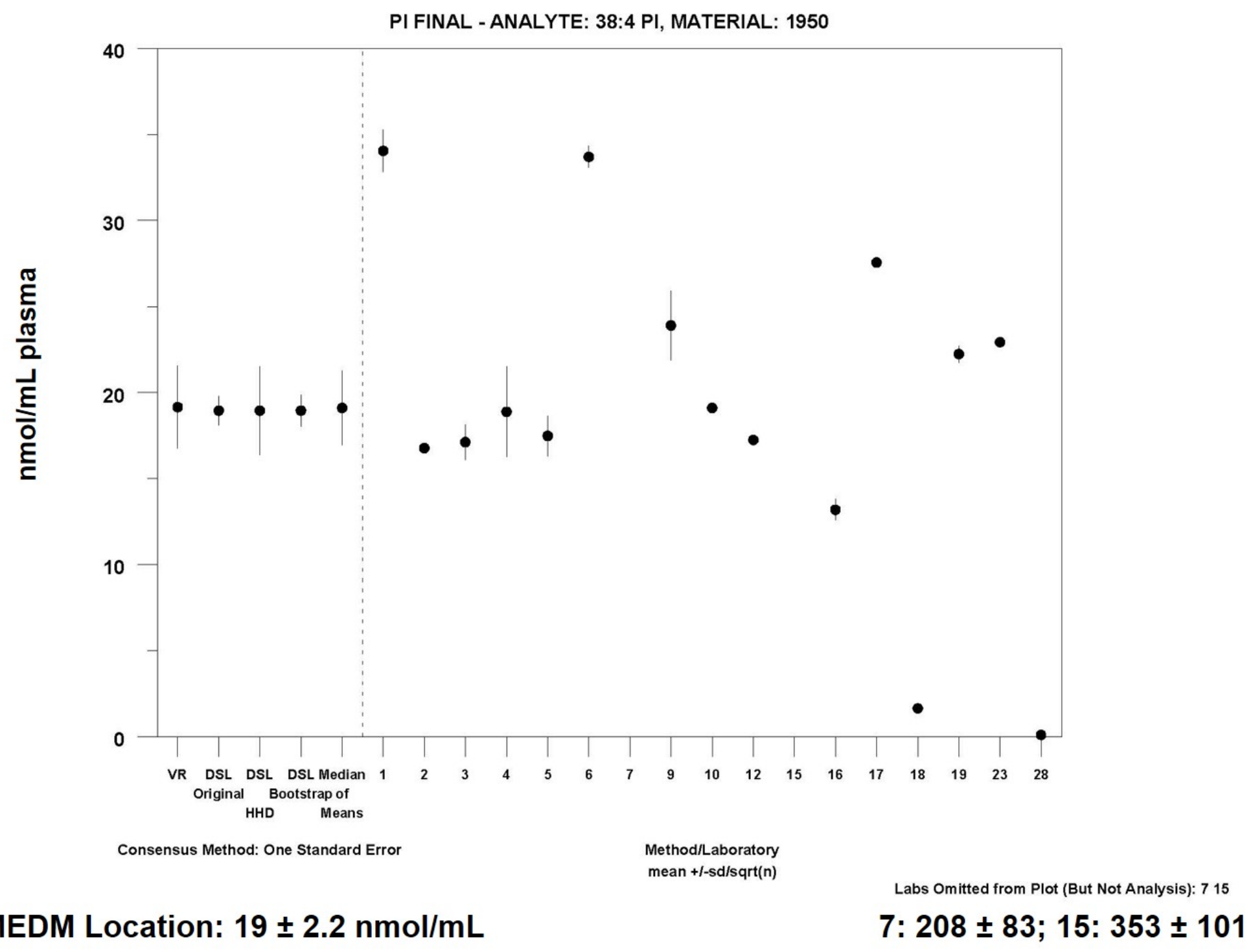




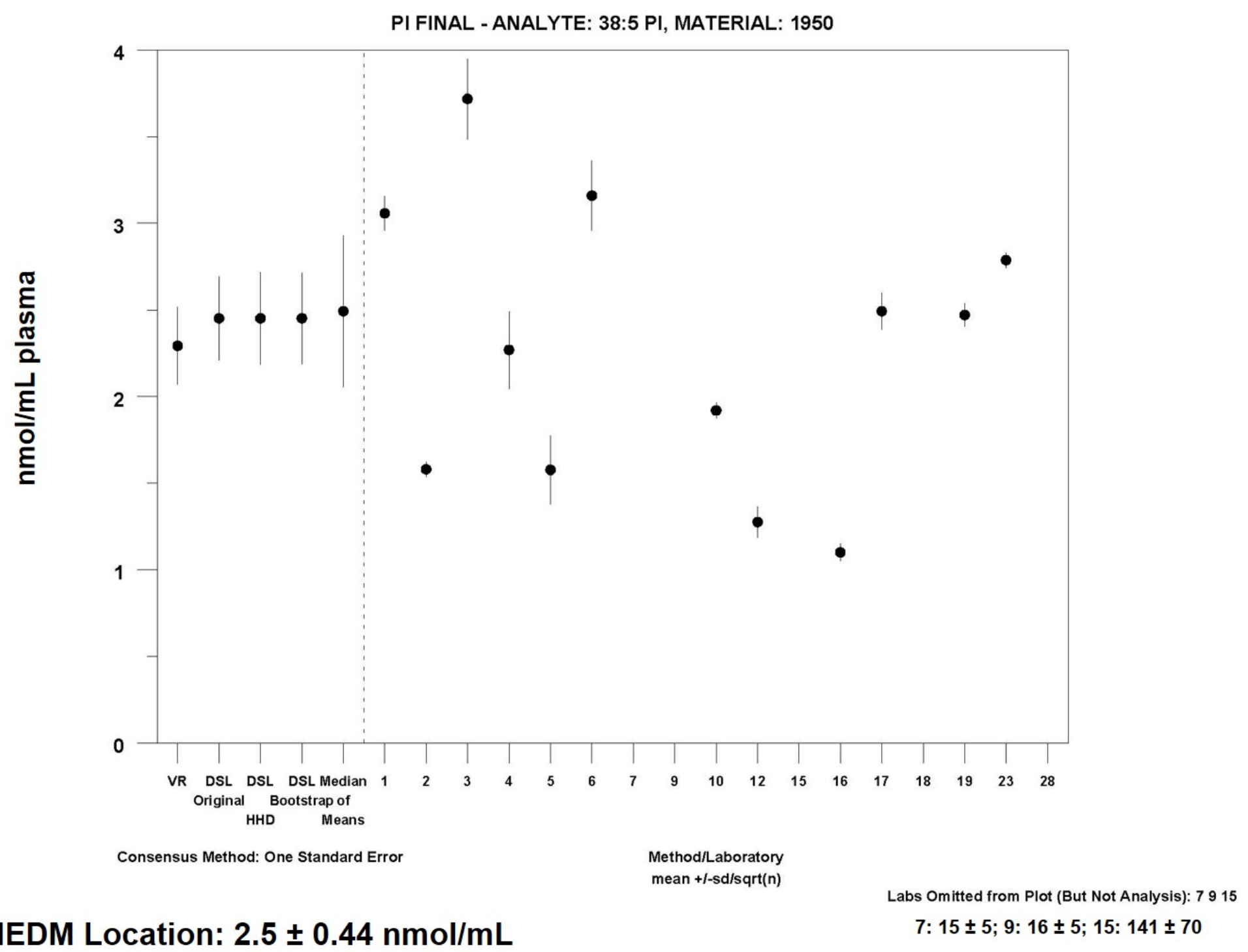




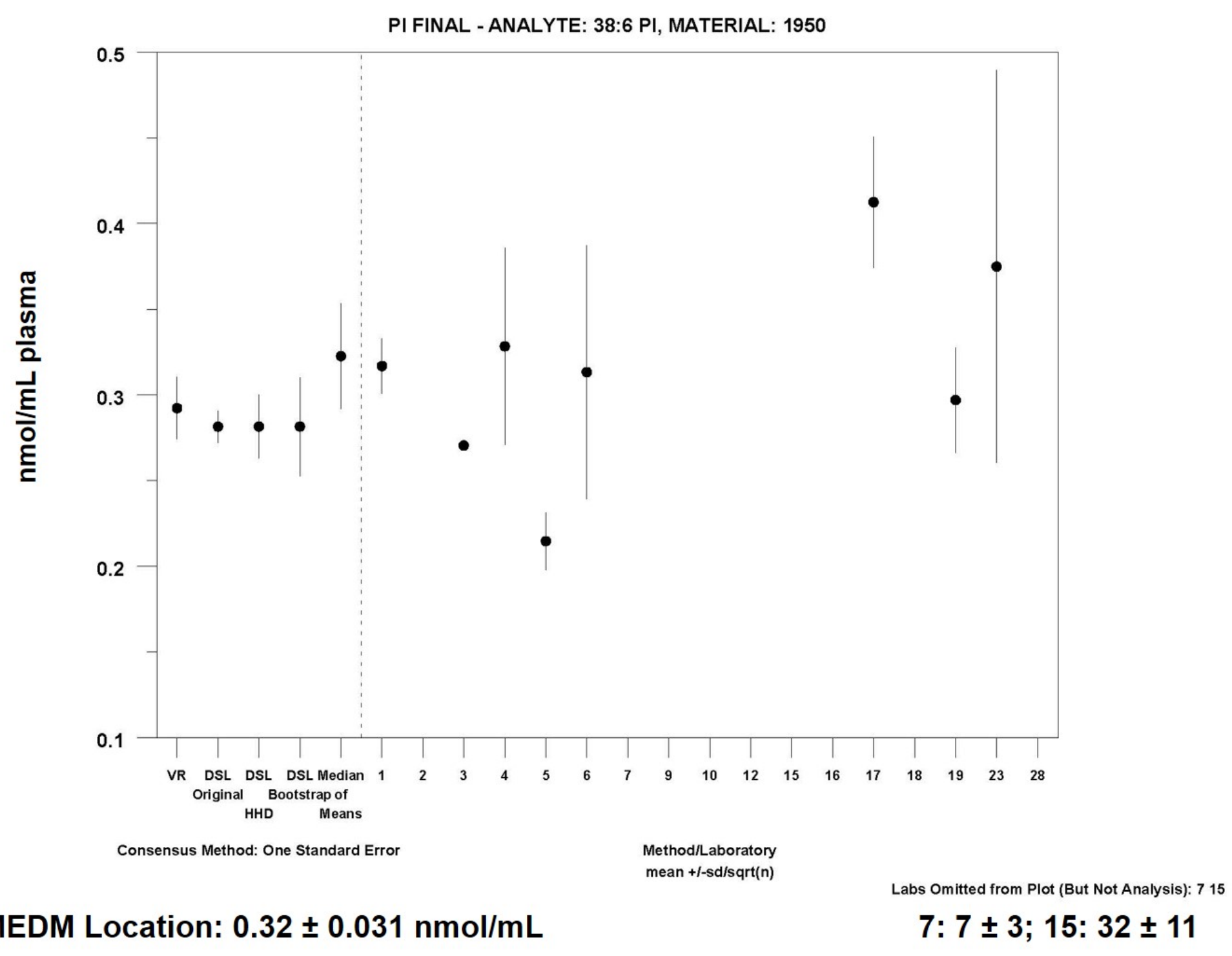




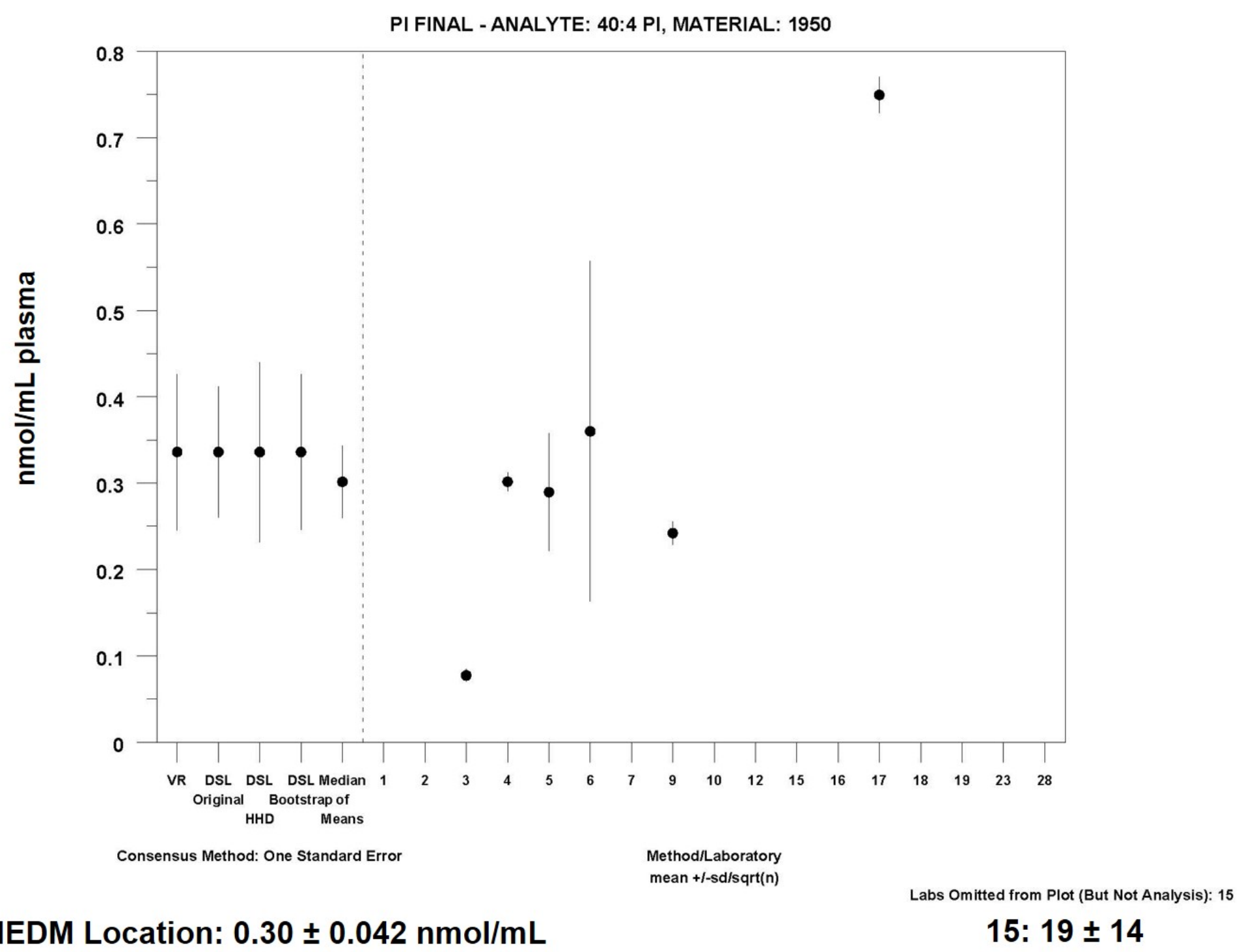




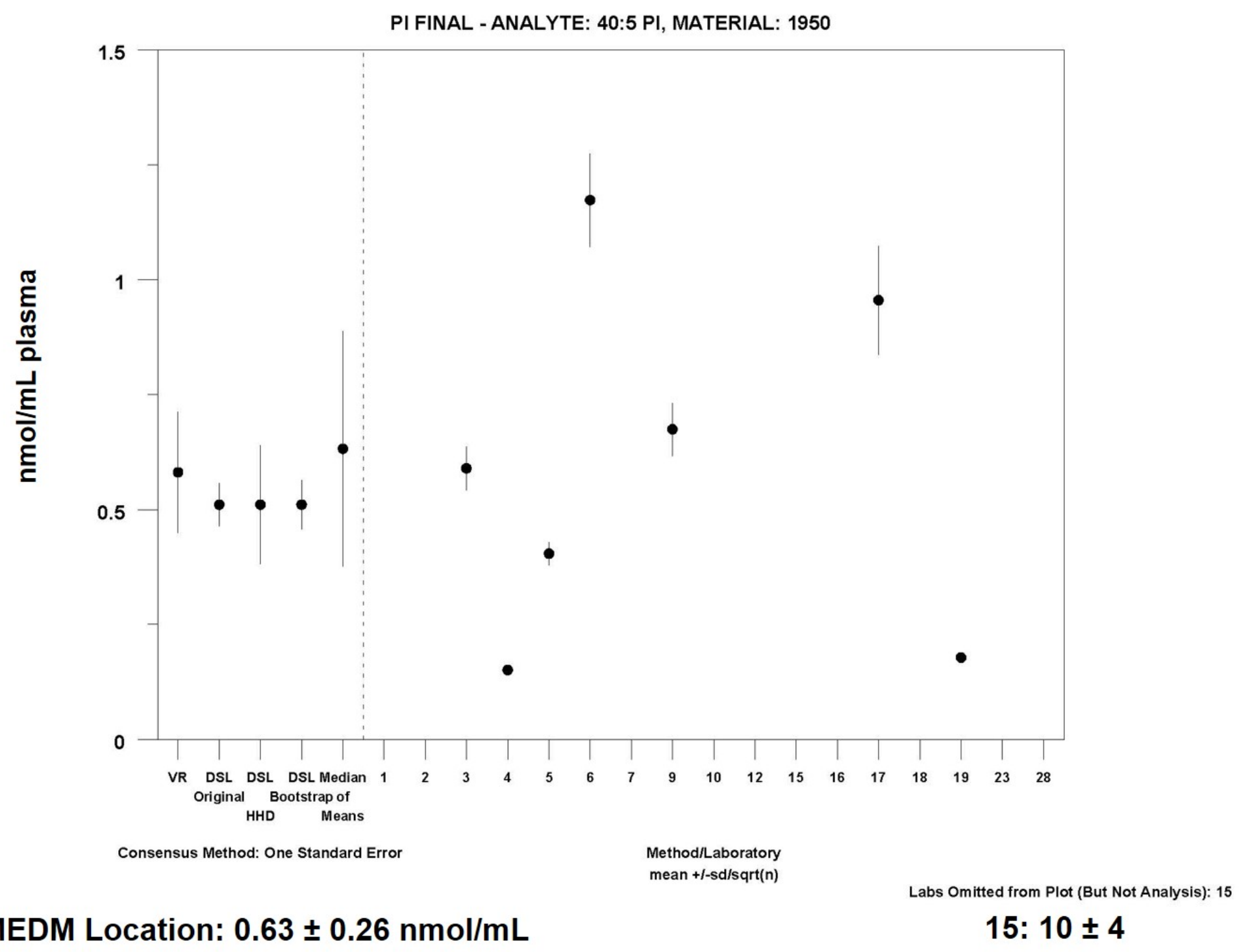




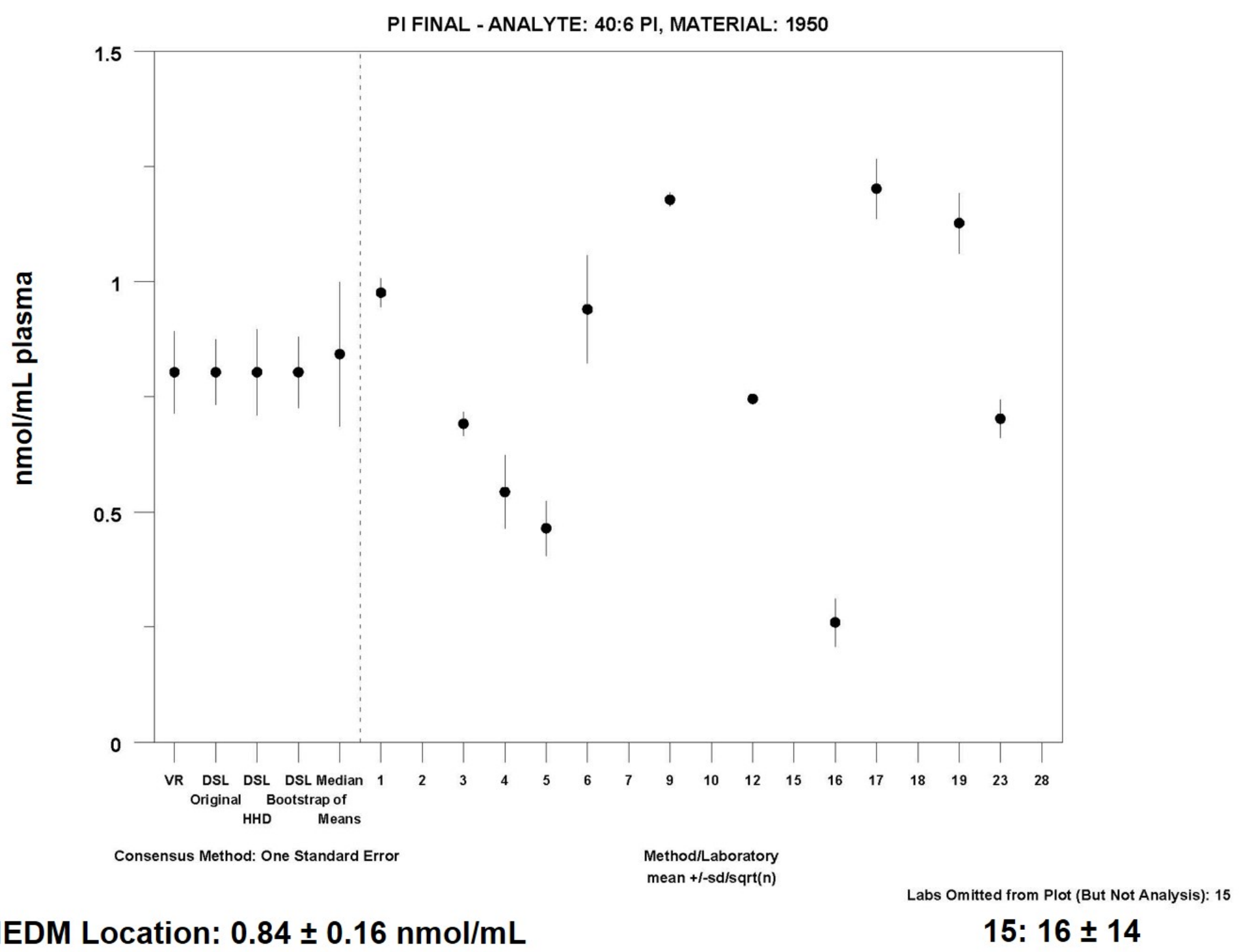




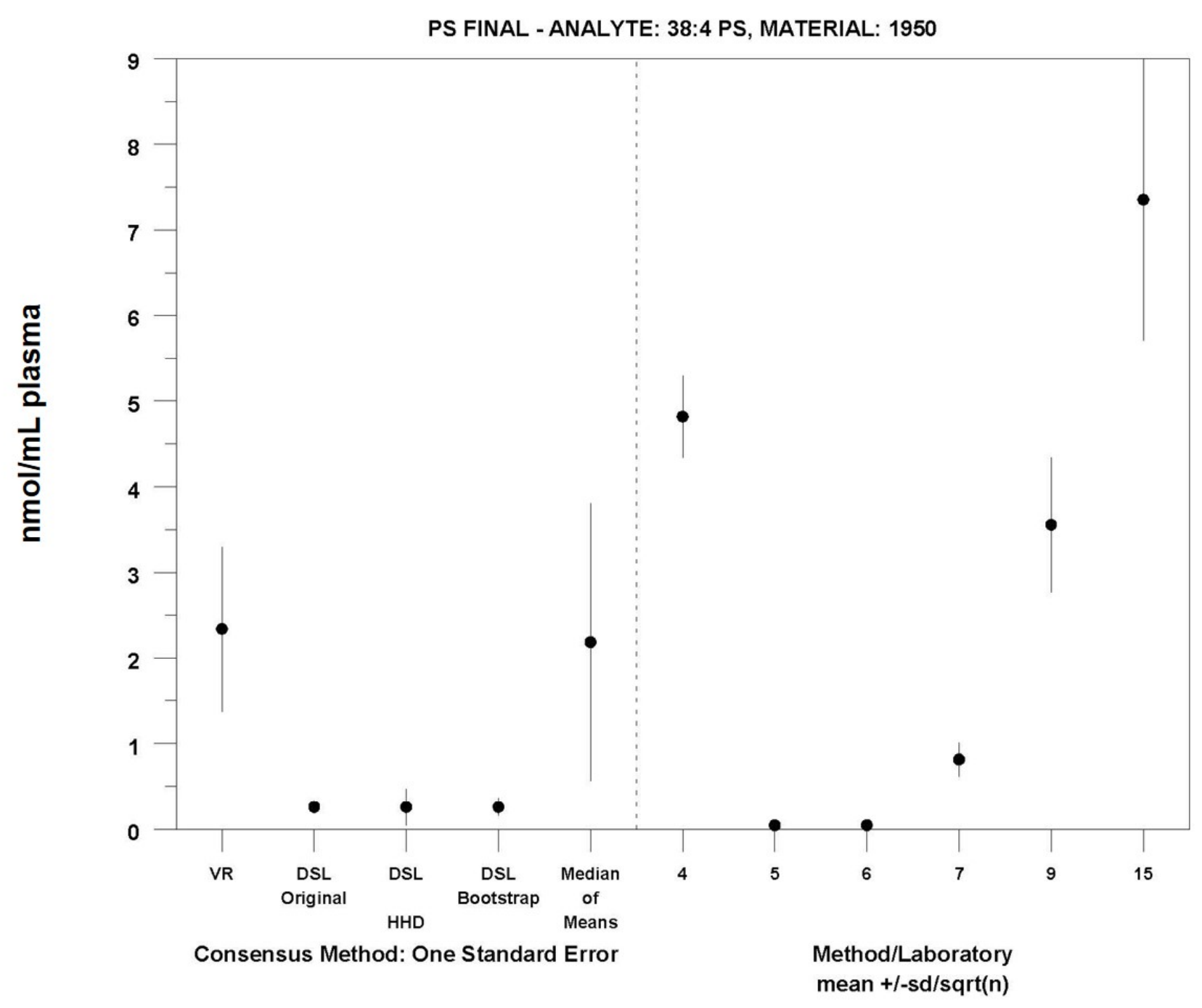

MEDM Location: $2.2 \pm 1.6 \mathrm{nmol} / \mathrm{mL}$ 


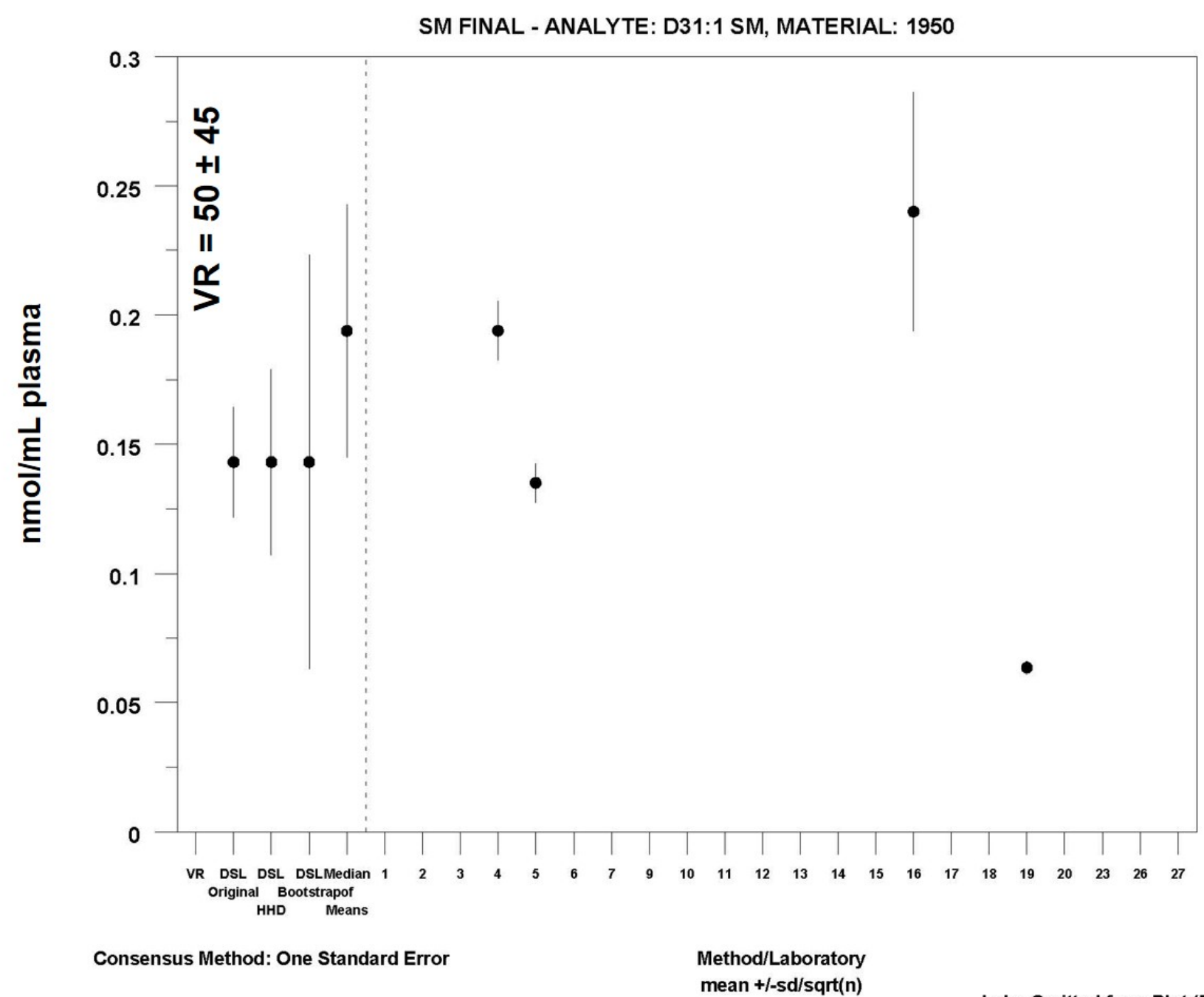

MEDM Location: $0.19 \pm 0.049 \mathrm{nmol} / \mathrm{mL}$

15: $268 \pm 50$ 


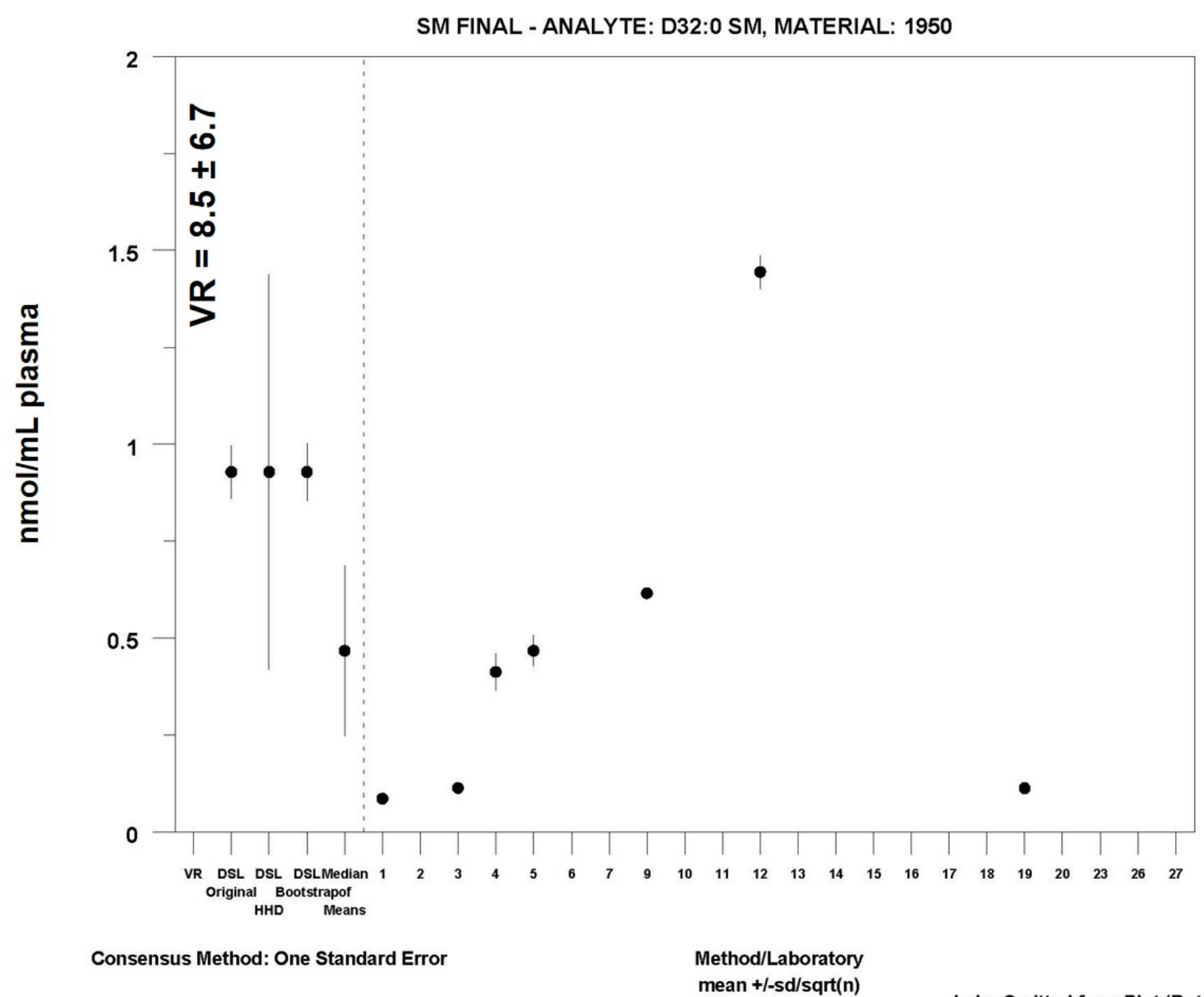

MEDM Location: $0.47 \pm 0.22 \mathrm{nmol} / \mathrm{mL}$ 13: $9 \pm 0.4 ; 15: 70 \pm 9$ 


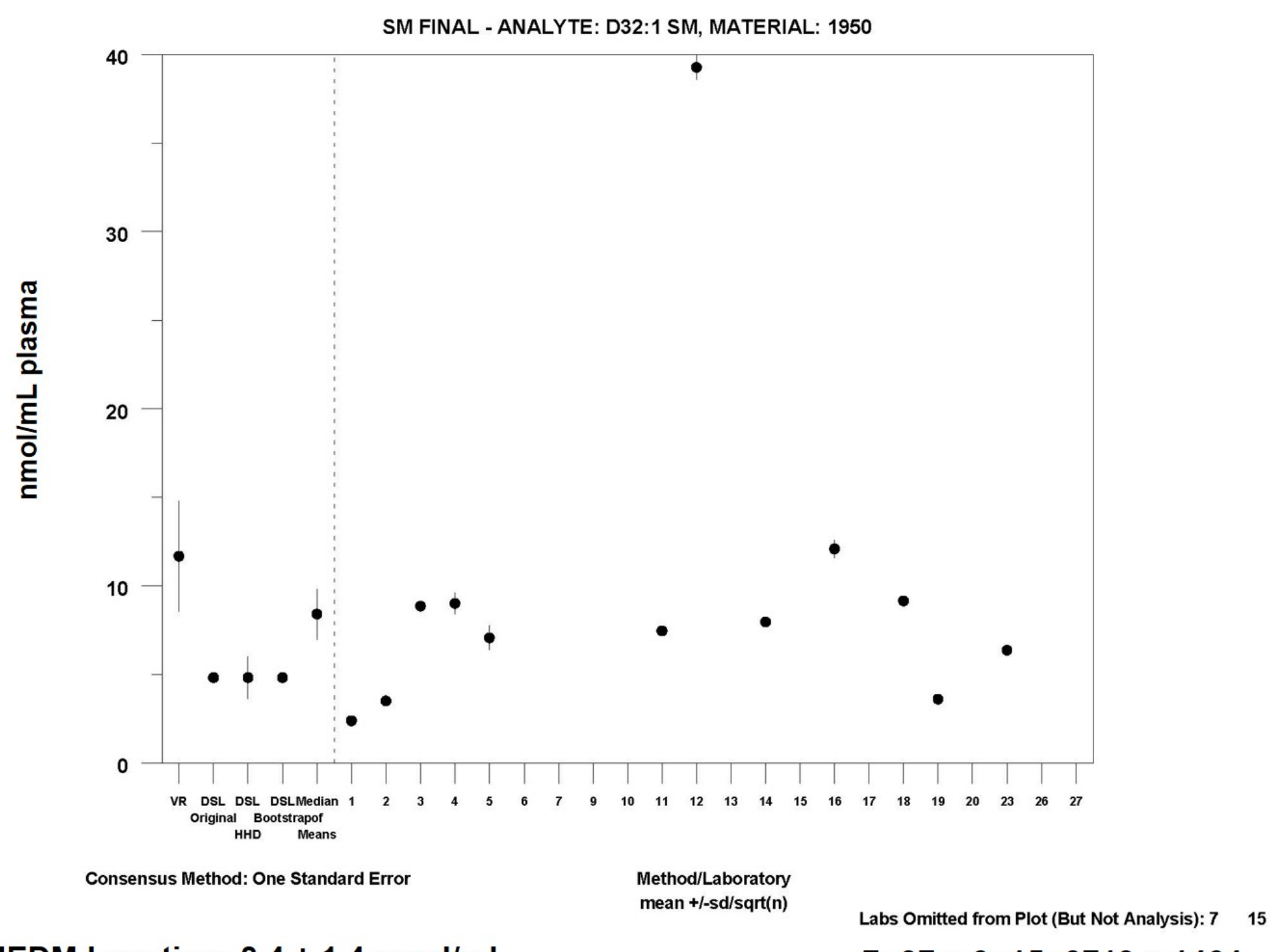

MEDM Location: $8.4 \pm 1.4 \mathrm{nmol} / \mathrm{mL}$

$7: 37 \pm 6 ; 15: 3719 \pm 1464$ 


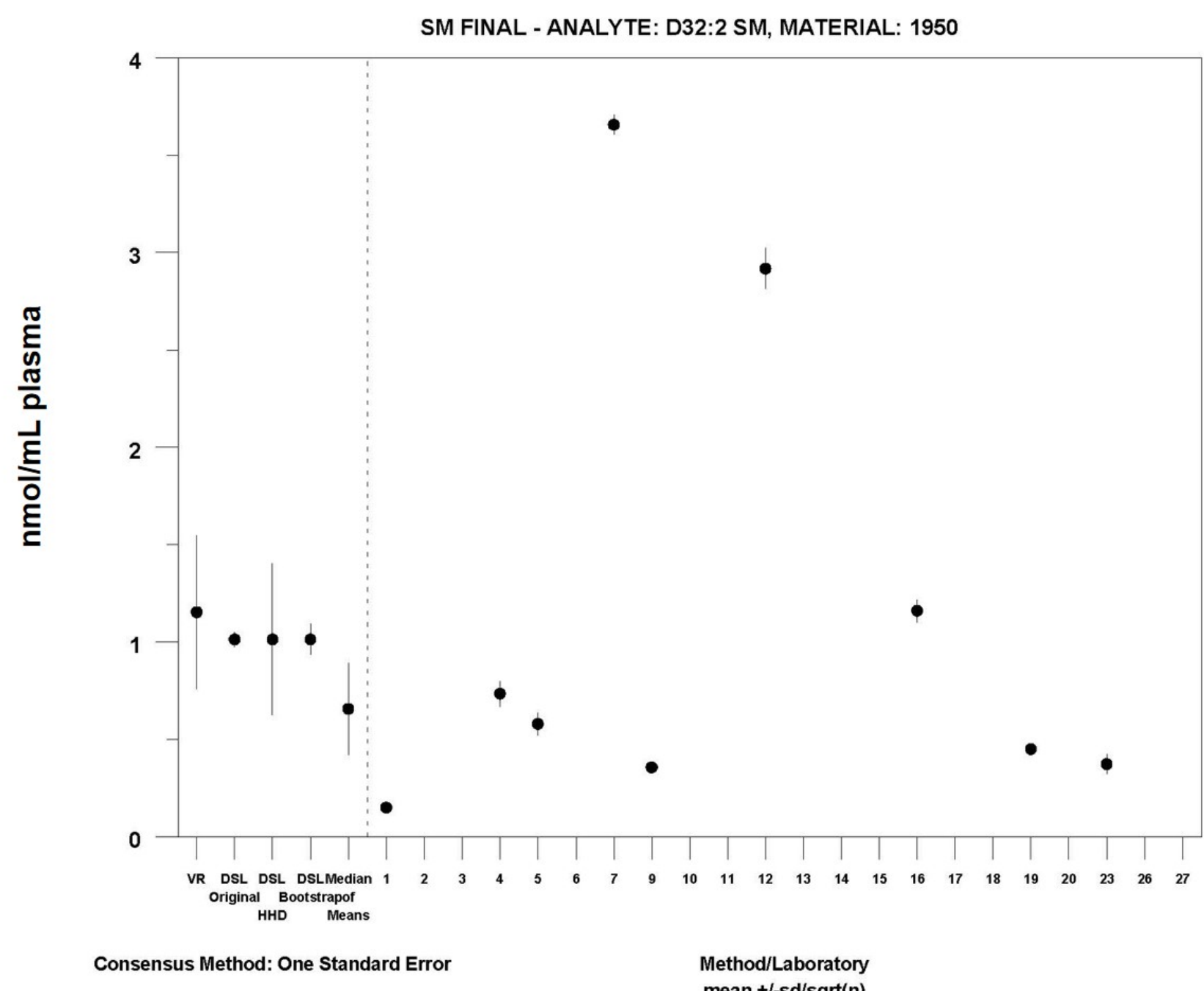

MEDM Location: $0.66 \pm 0.24 \mathrm{nmol} / \mathrm{mL}$

Labs Omitted from Plot (But Not Analysis): 15

15: $191 \pm 94$ 


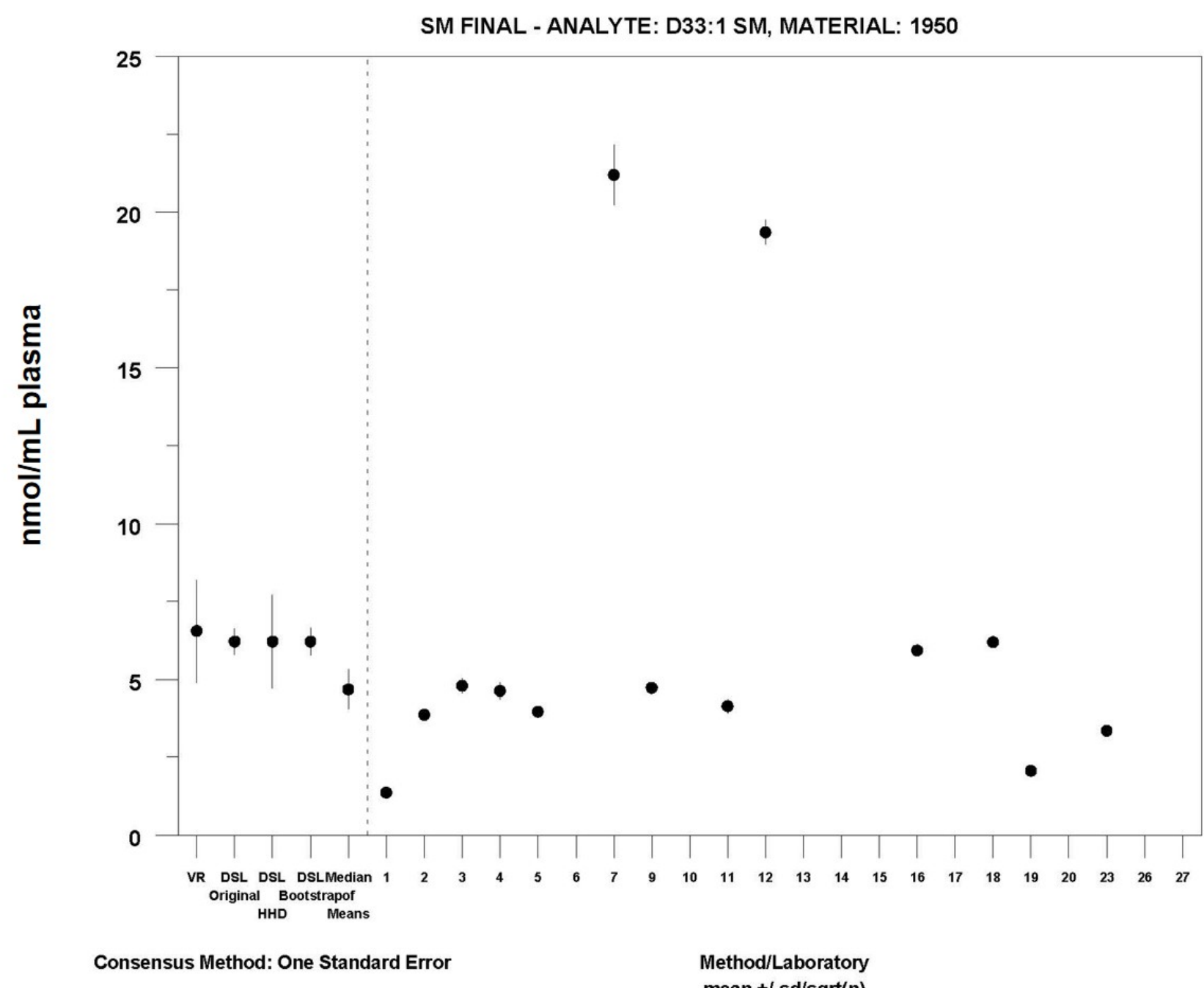

MEDM Location: $4.7 \pm 0.64 \mathrm{nmol} / \mathrm{mL}$

Labs Omitted from Plot (But Not Analysis): 15

15: $1659 \pm 152$ 


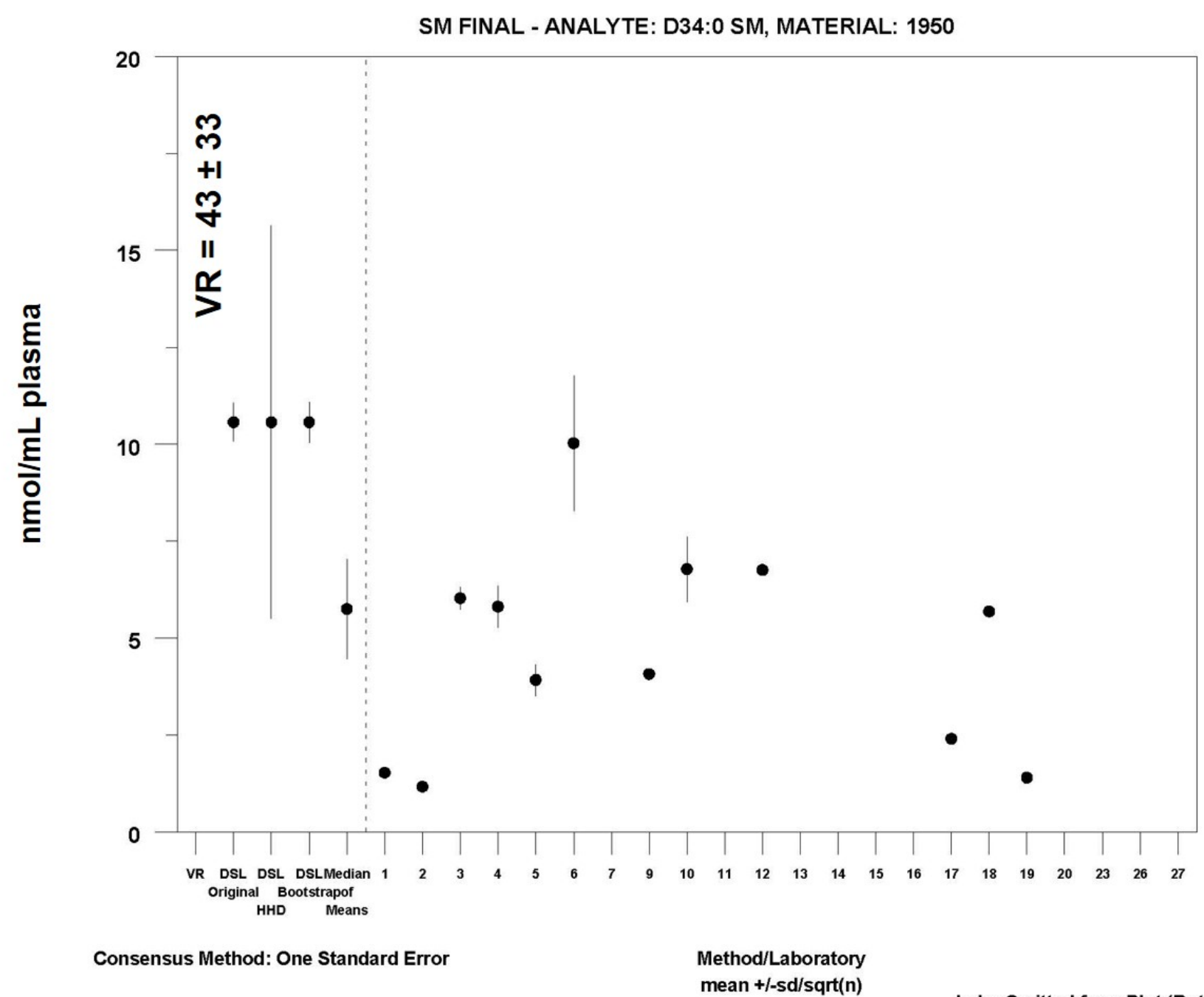

MEDM Location: $5.8 \pm 1.3 \mathrm{nmol} / \mathrm{mL}$

Labs Omitted from Plot (But Not Analysis): 1315 13: $65 \pm 0.6 ; 15: 481 \pm 16$ 


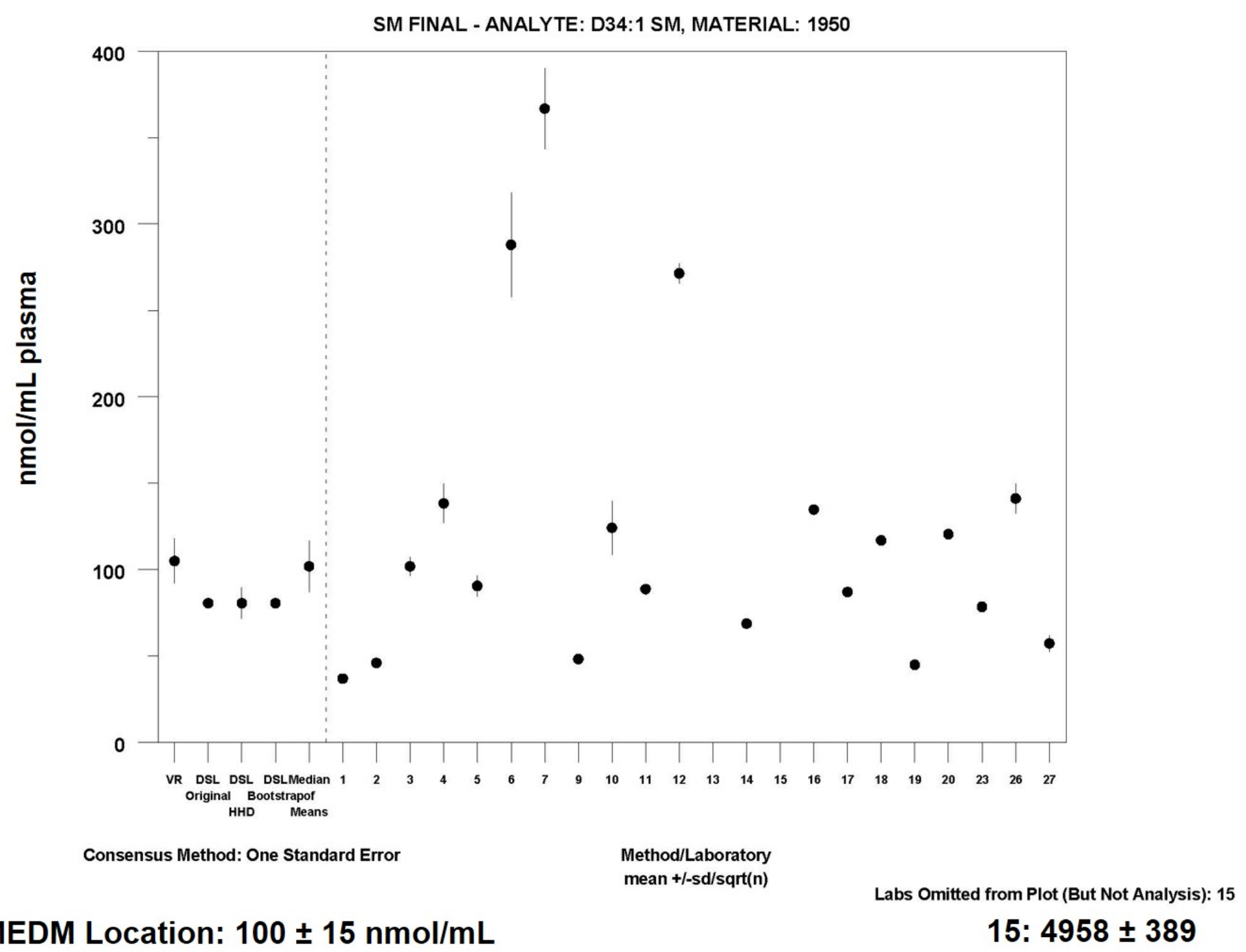




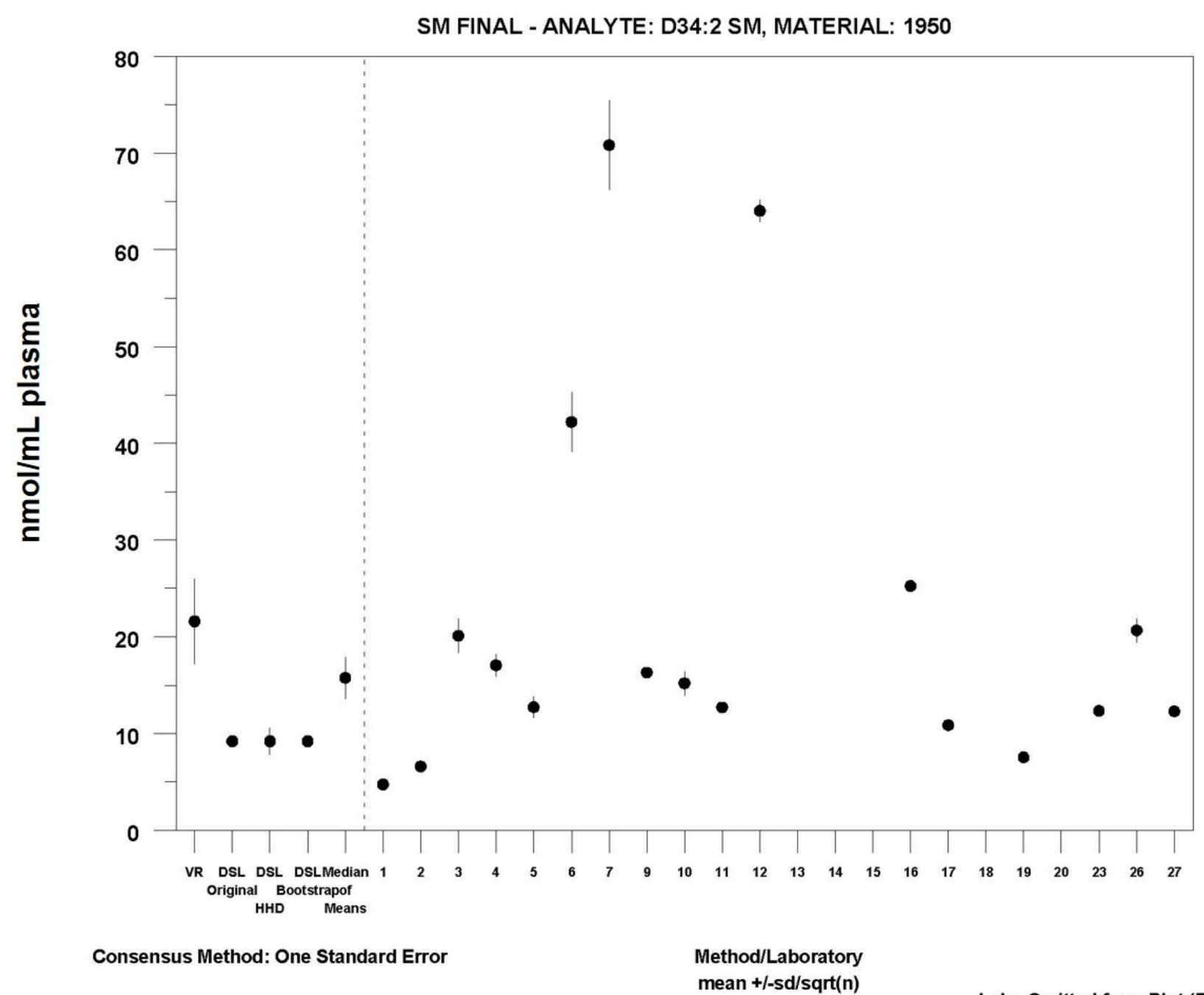

MEDM Location: $16 \pm 2.2 \mathrm{nmol} / \mathrm{mL}$ 15: $3215 \pm 2116$ 


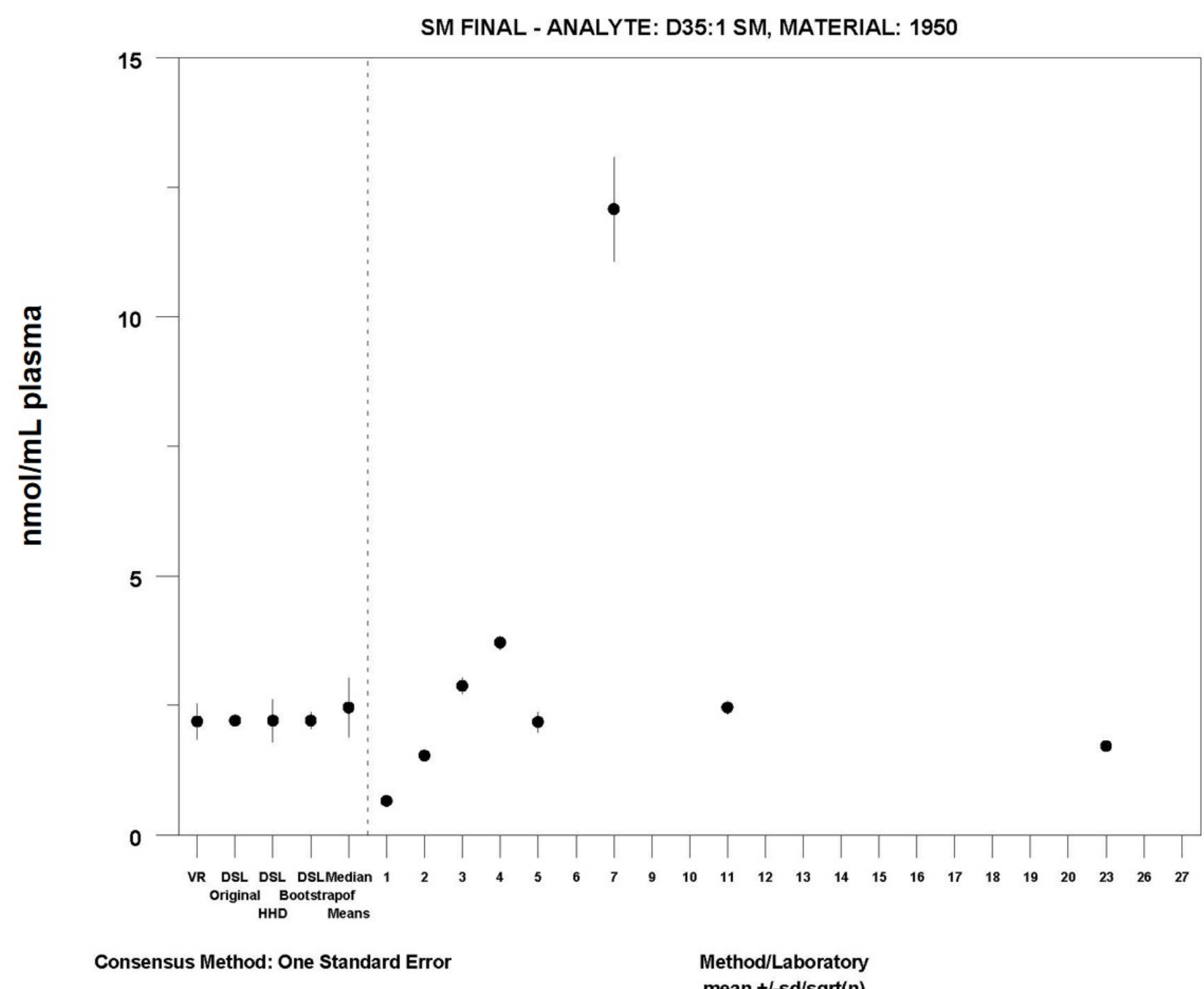

MEDM Location: $2.5 \pm 0.58 \mathrm{nmol} / \mathrm{mL}$

Labs Omitted from Plot (But Not Analysis): 15

15: $421 \pm 59$ 


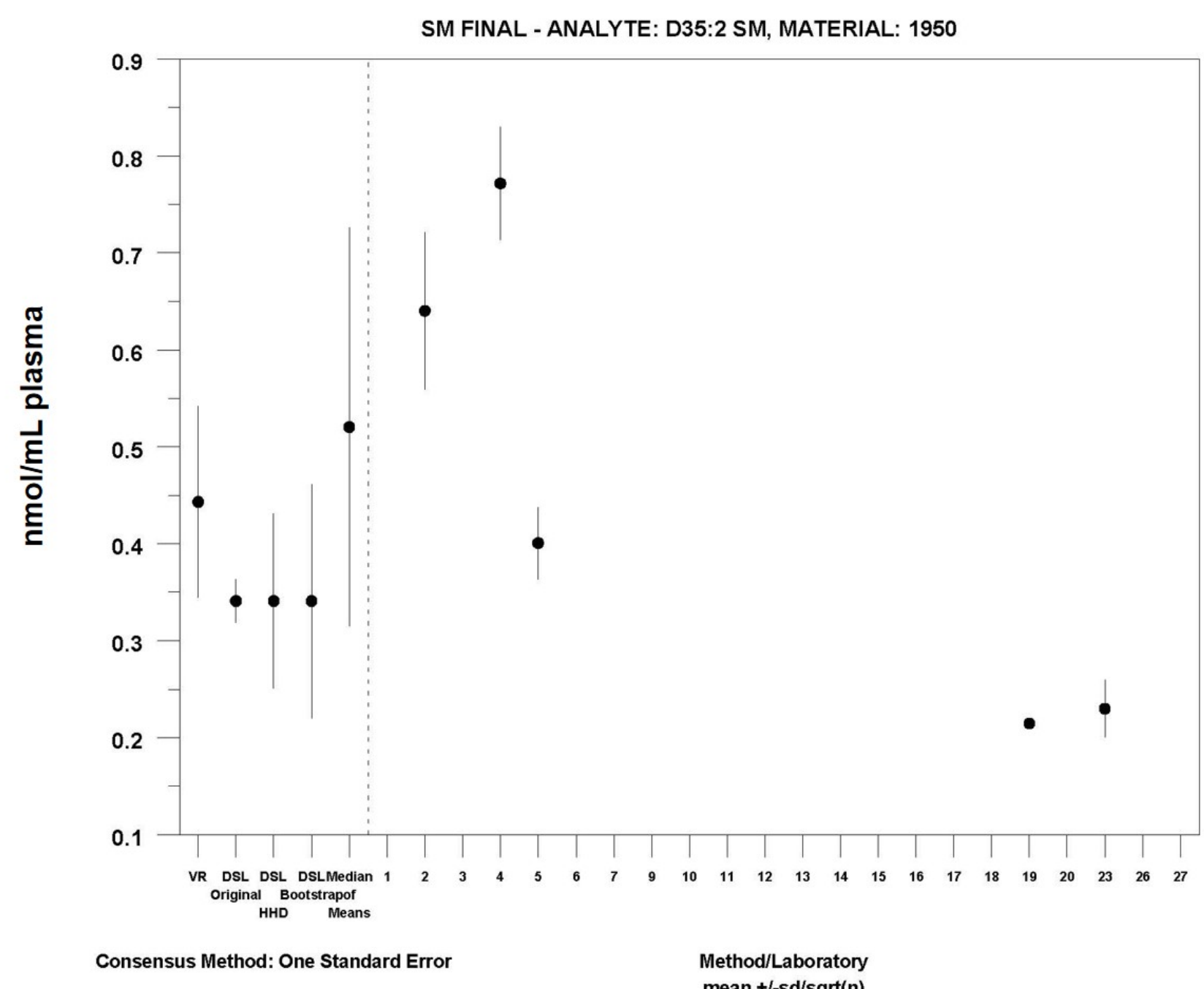

MEDM Location: $0.52 \pm 0.21 \mathrm{nmol} / \mathrm{mL}$

Labs Omitted from Plot (But Not Analysis): 15

15: $134 \pm 45$ 


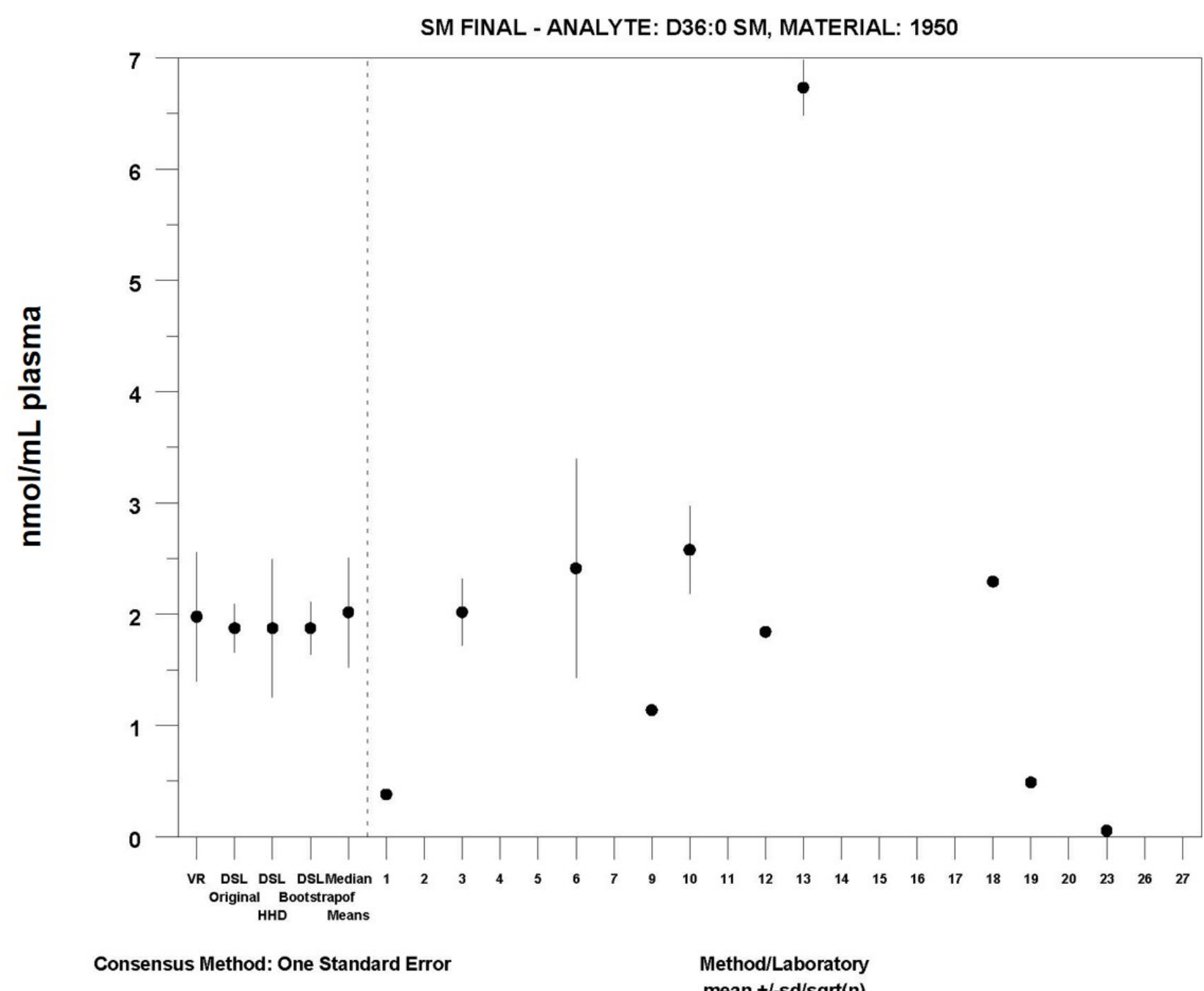

MEDM Location: $2.0 \pm 0.49 \mathrm{nmol} / \mathrm{mL}$

Labs Omitted from Plot (But Not Analysis): 15

15: $181 \pm 74$ 


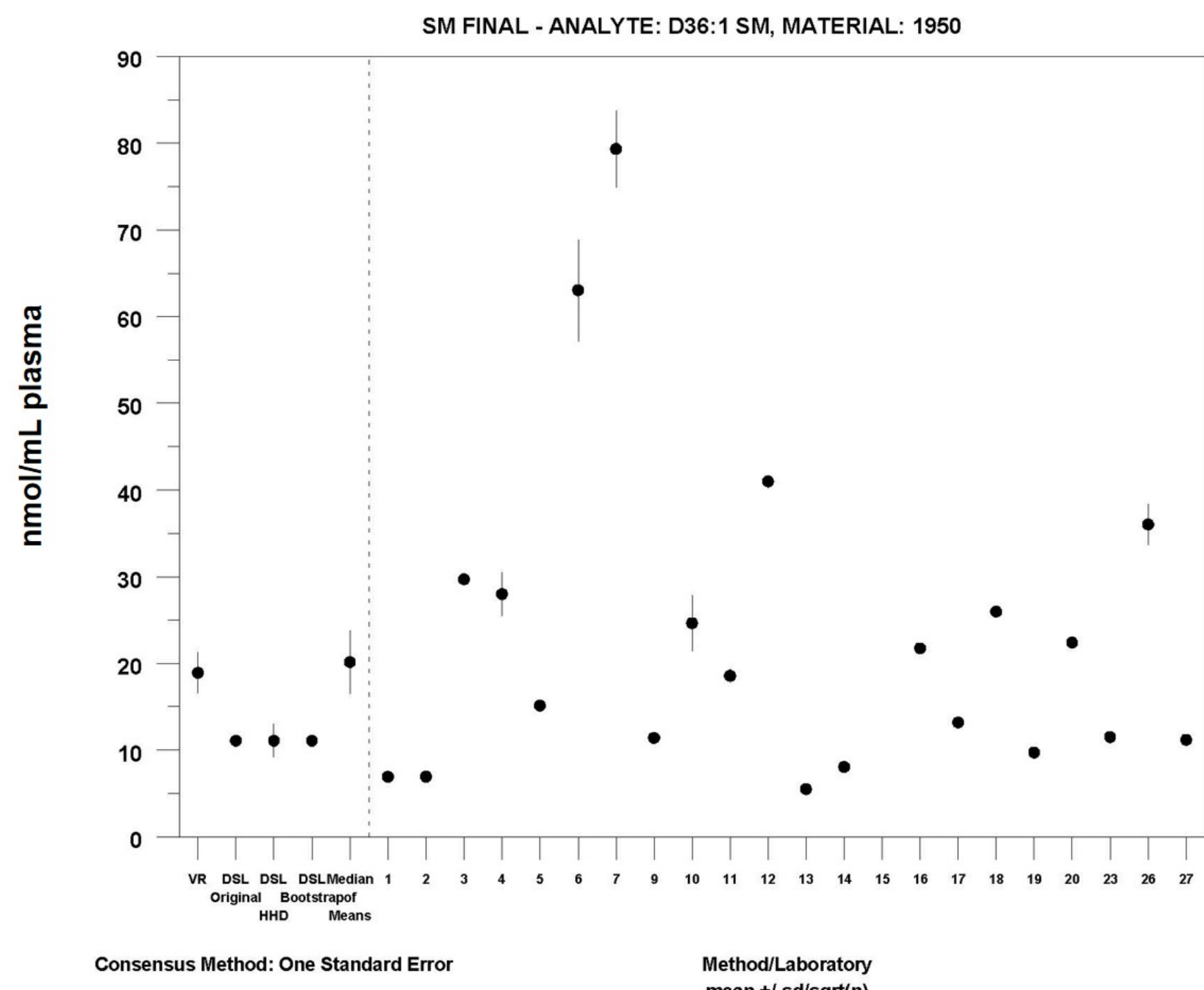

MEDM Location: $20 \pm 3.7 \mathrm{nmol} / \mathrm{mL}$

Labs Omitted from Plot (But Not Analysis): 15

15: $1044 \pm 59$ 


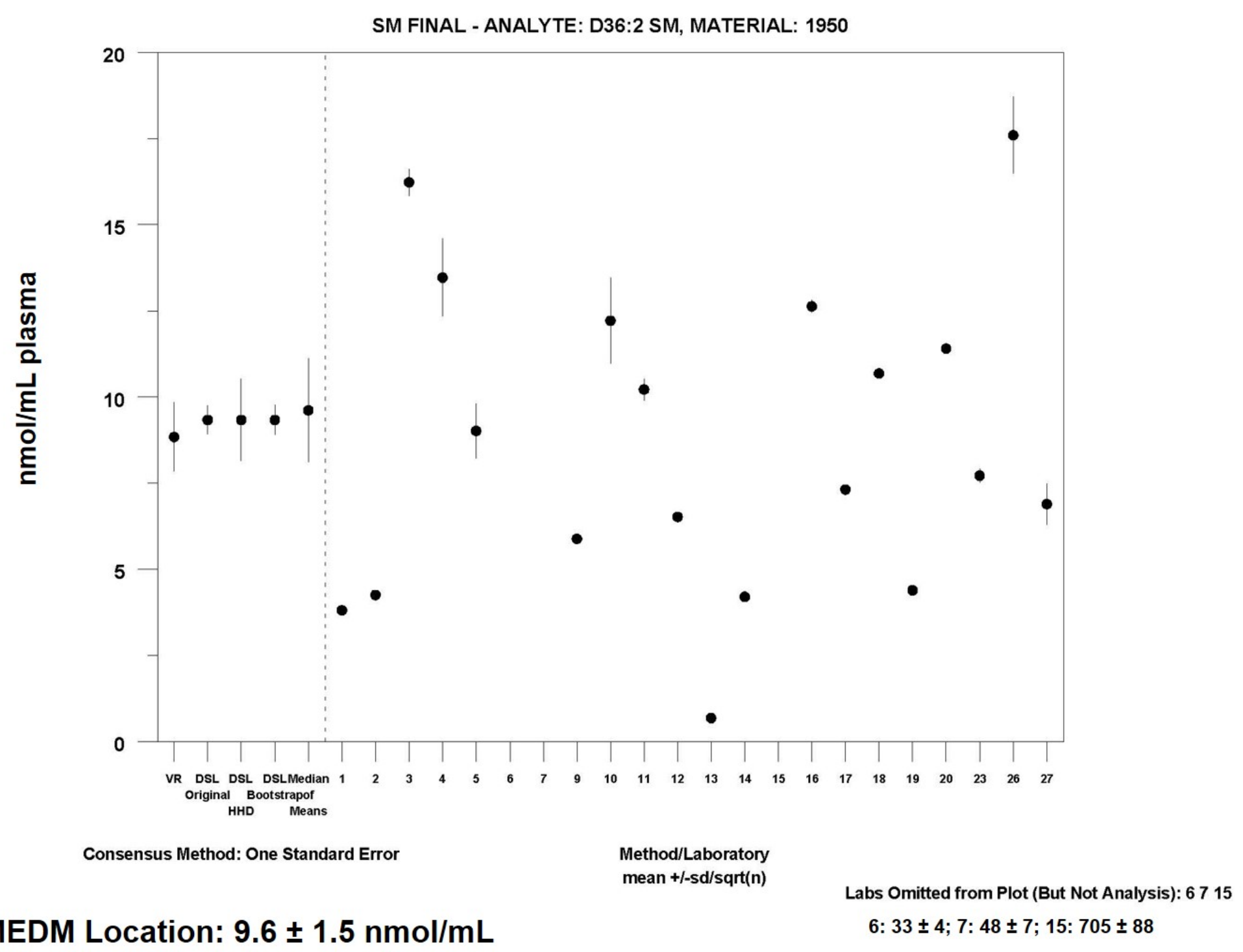




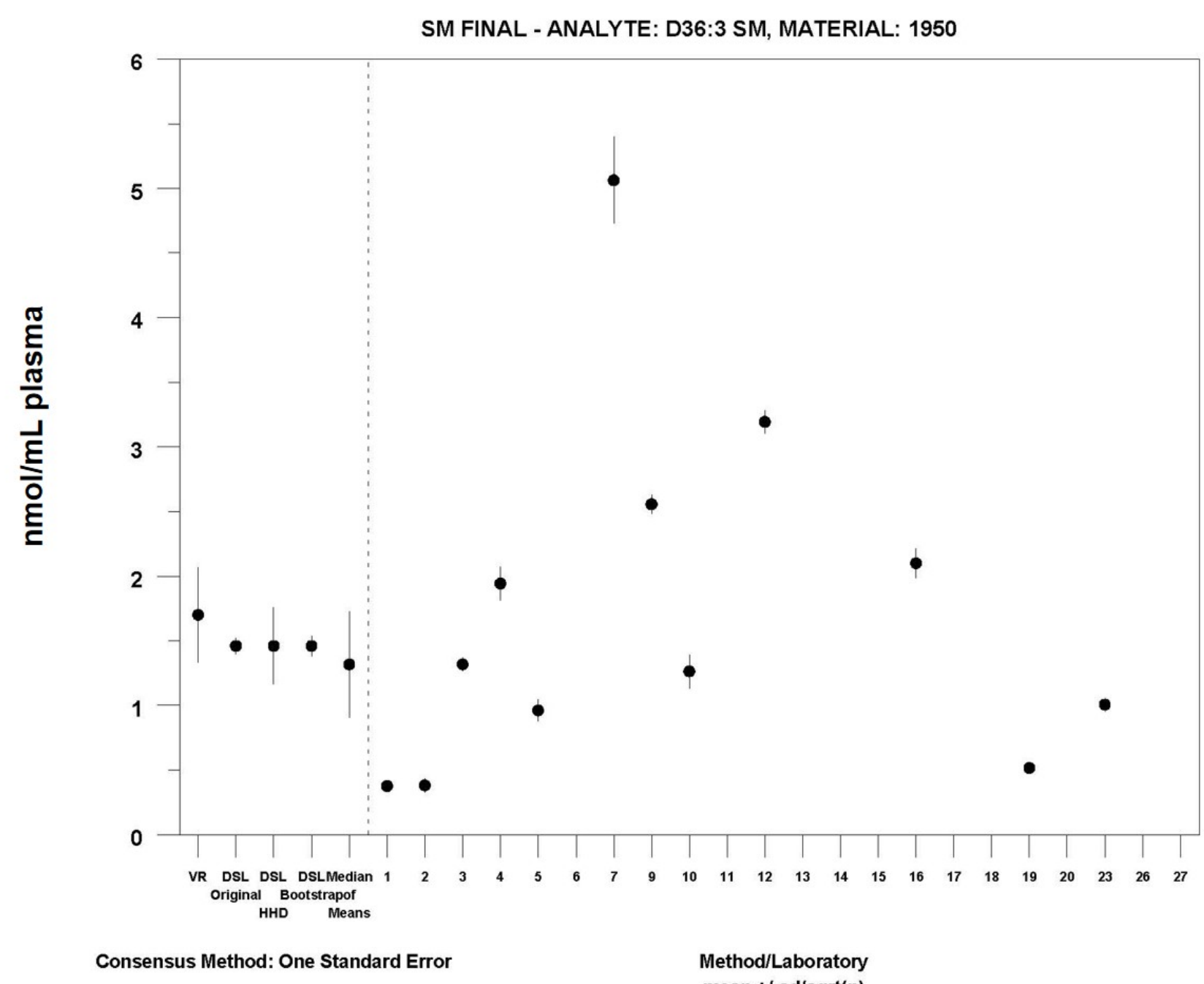

MEDM Location: $1.3 \pm 0.41 \mathrm{nmol} / \mathrm{mL}$

Labs Omitted from Plot (But Not Analysis): 15

15: $280 \pm 98$ 


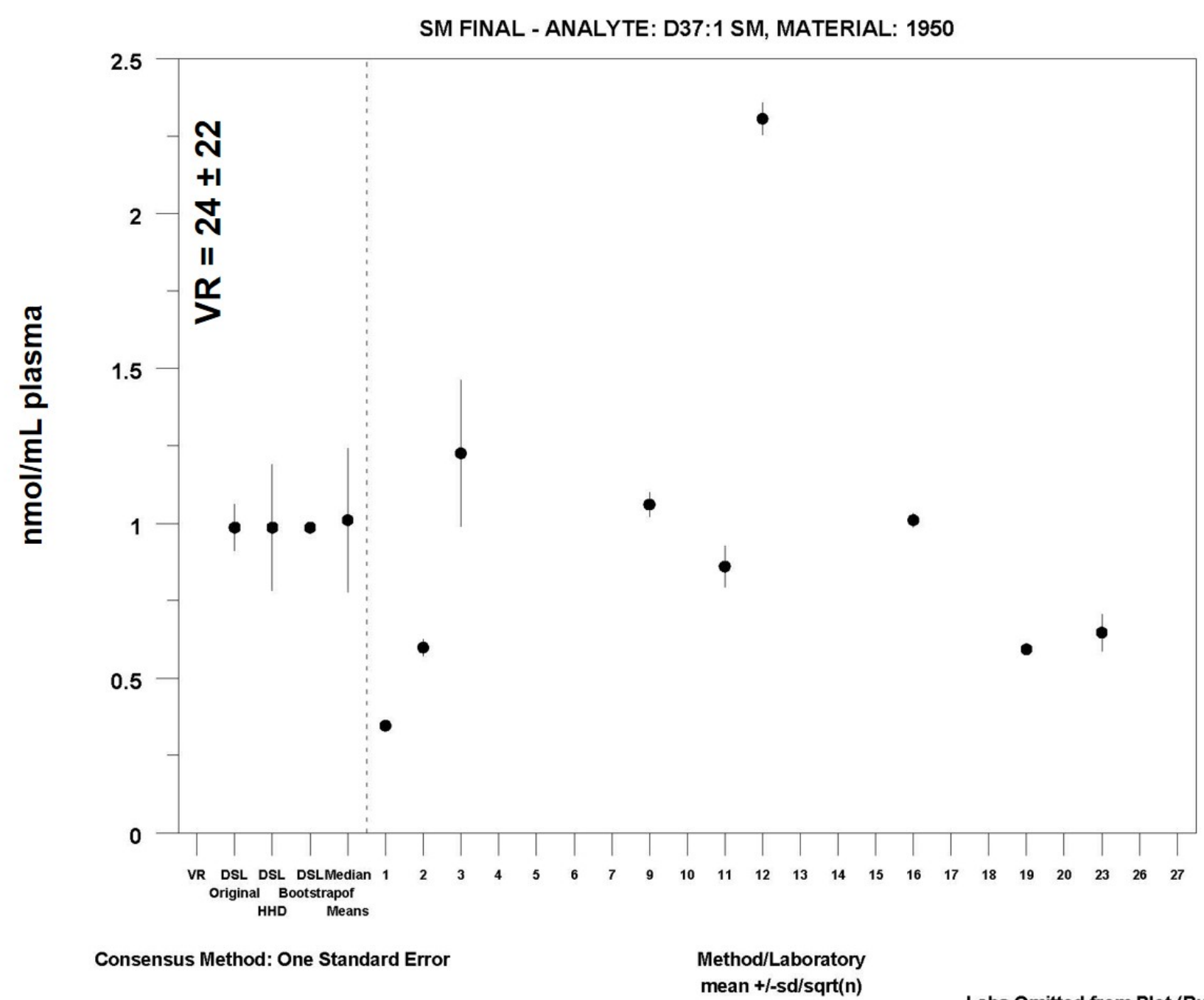

MEDM Location: $1.0 \pm 0.23 \mathrm{nmol} / \mathrm{mL}$

Labs Omitted from Plot (But Not Analysis): 715

7: $9 \pm 2 ; 15: 258 \pm 21$ 


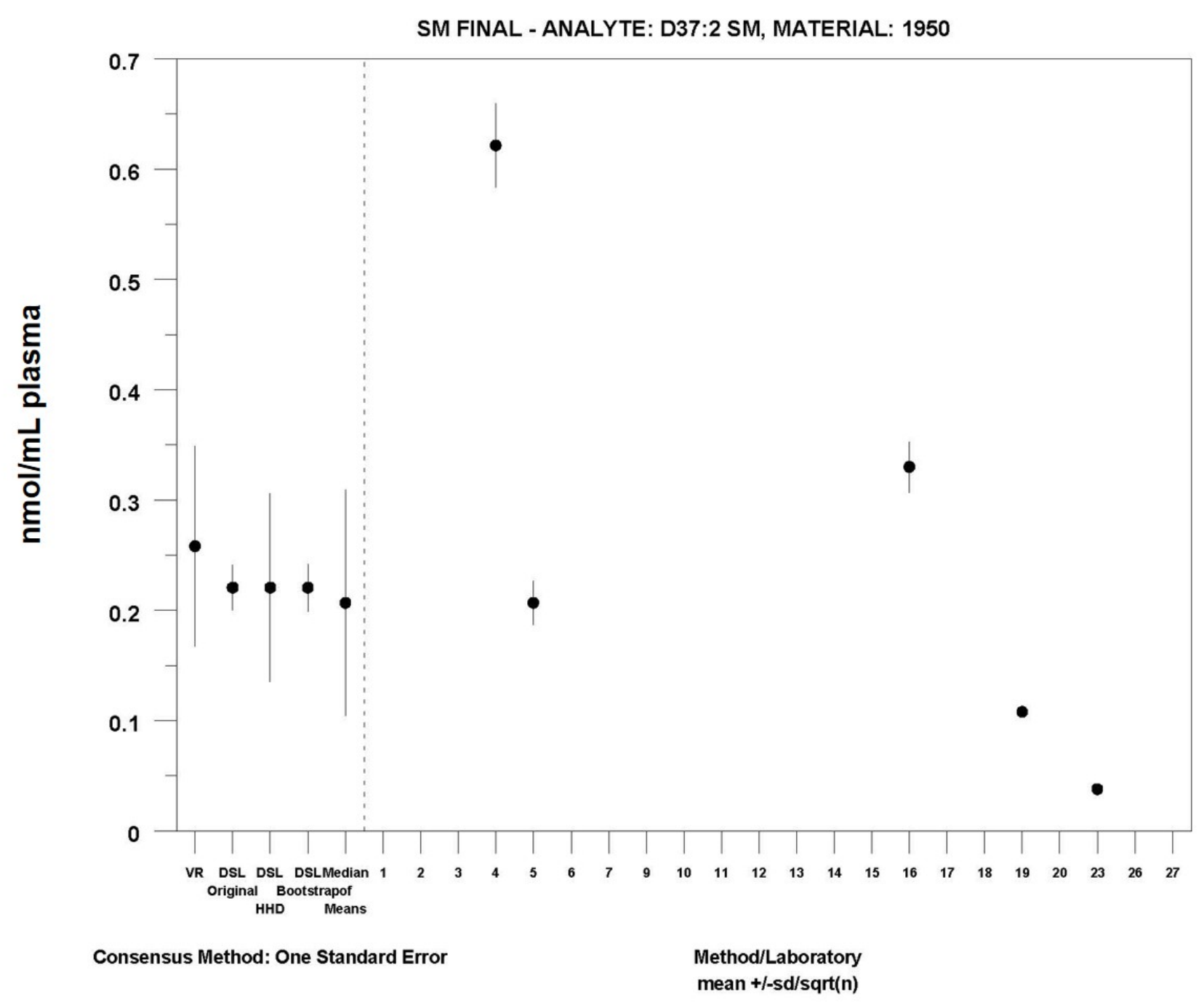

MEDM Location: $0.21 \pm 0.10 \mathrm{nmol} / \mathrm{mL}$ 


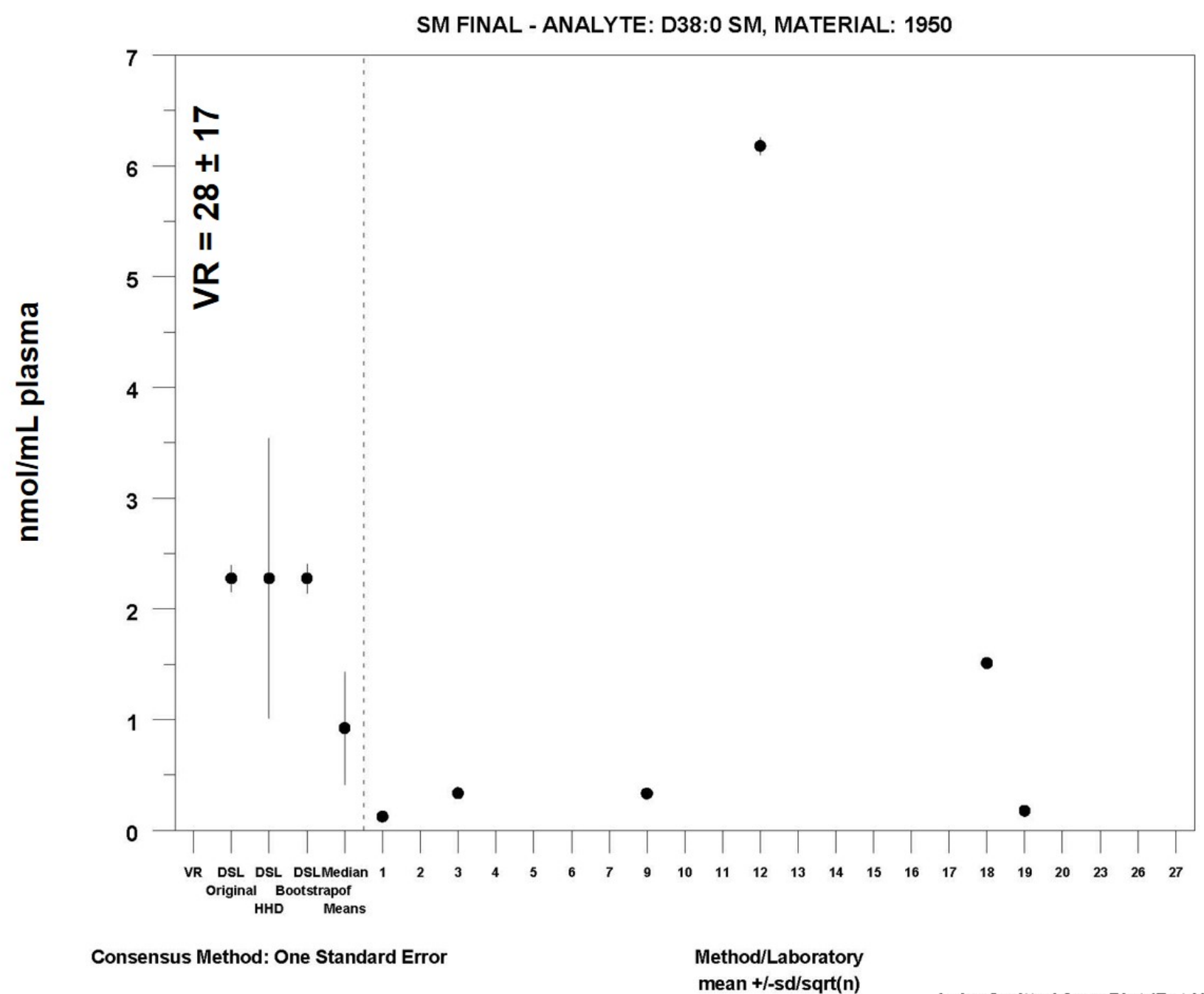

MEDM Location: $0.92 \pm 0.51 \mathrm{nmol} / \mathrm{mL}$

Labs Omitted from Plot (But Not Analysis): 1315 13: $83 \pm 2 ; 15: 130 \pm 6$ 


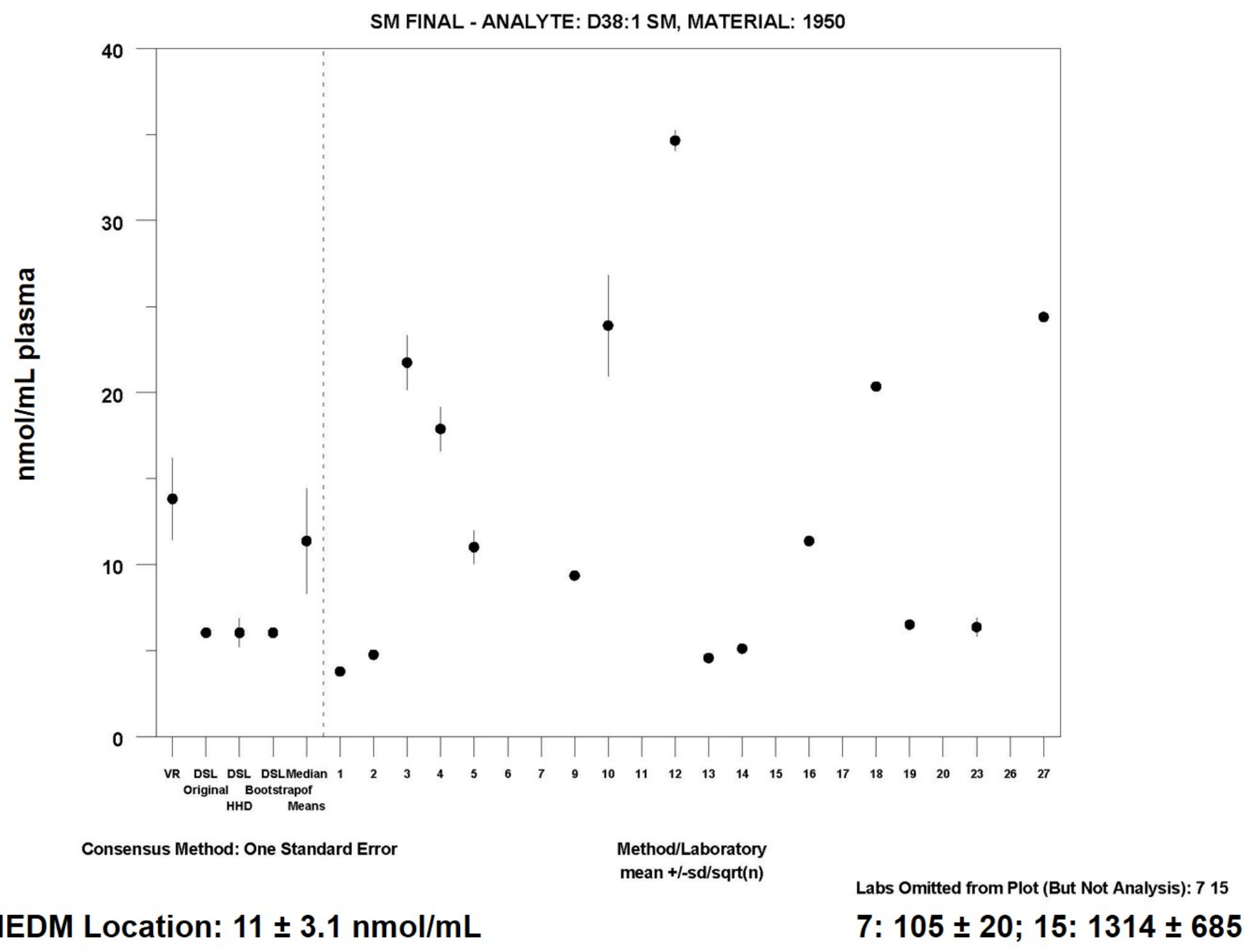




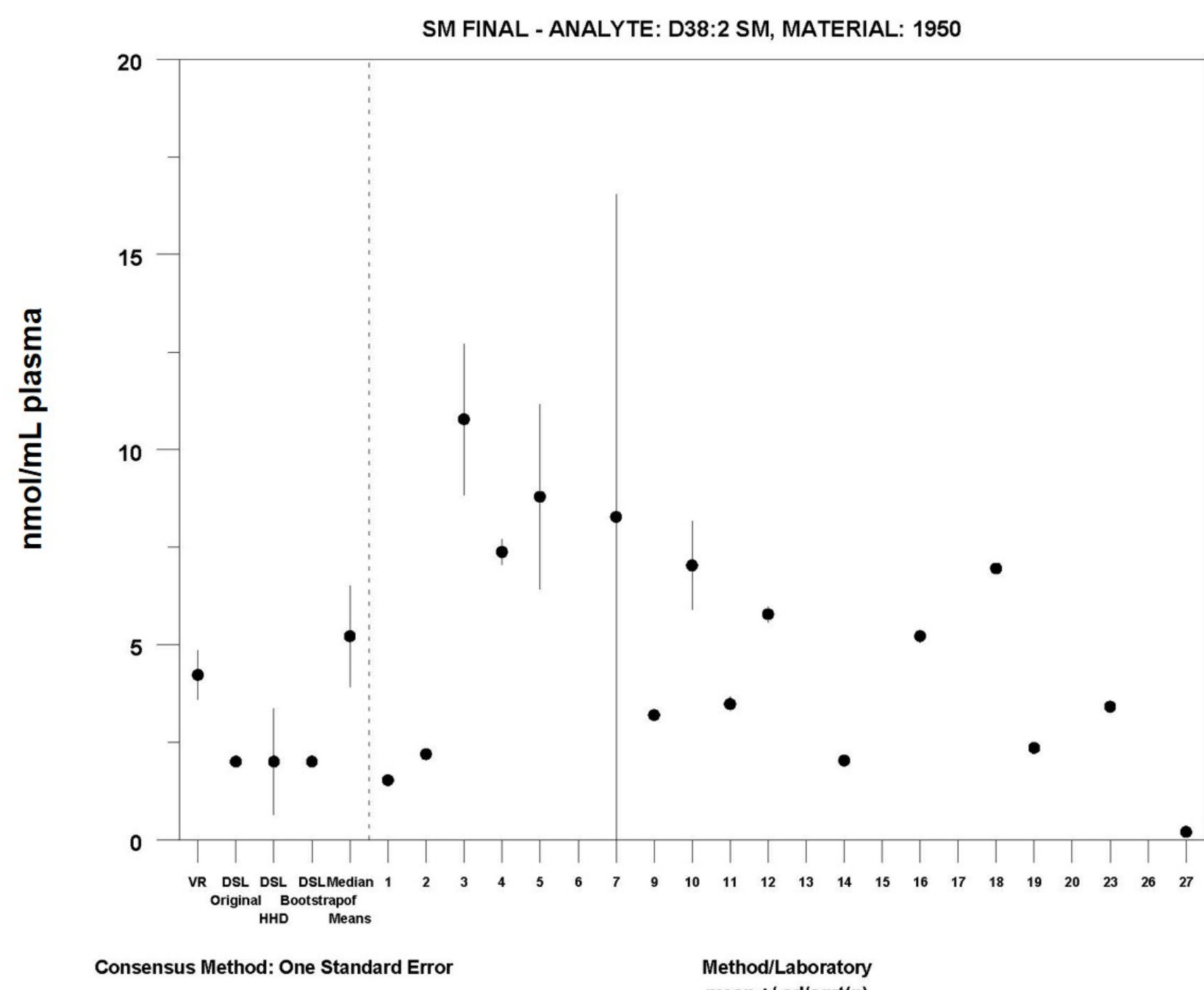

MEDM Location: $5.2 \pm 1.3 \mathrm{nmol} / \mathrm{mL}$

Labs Omitted from Plot (But Not Analysis): 15

15: $444 \pm 153$ 


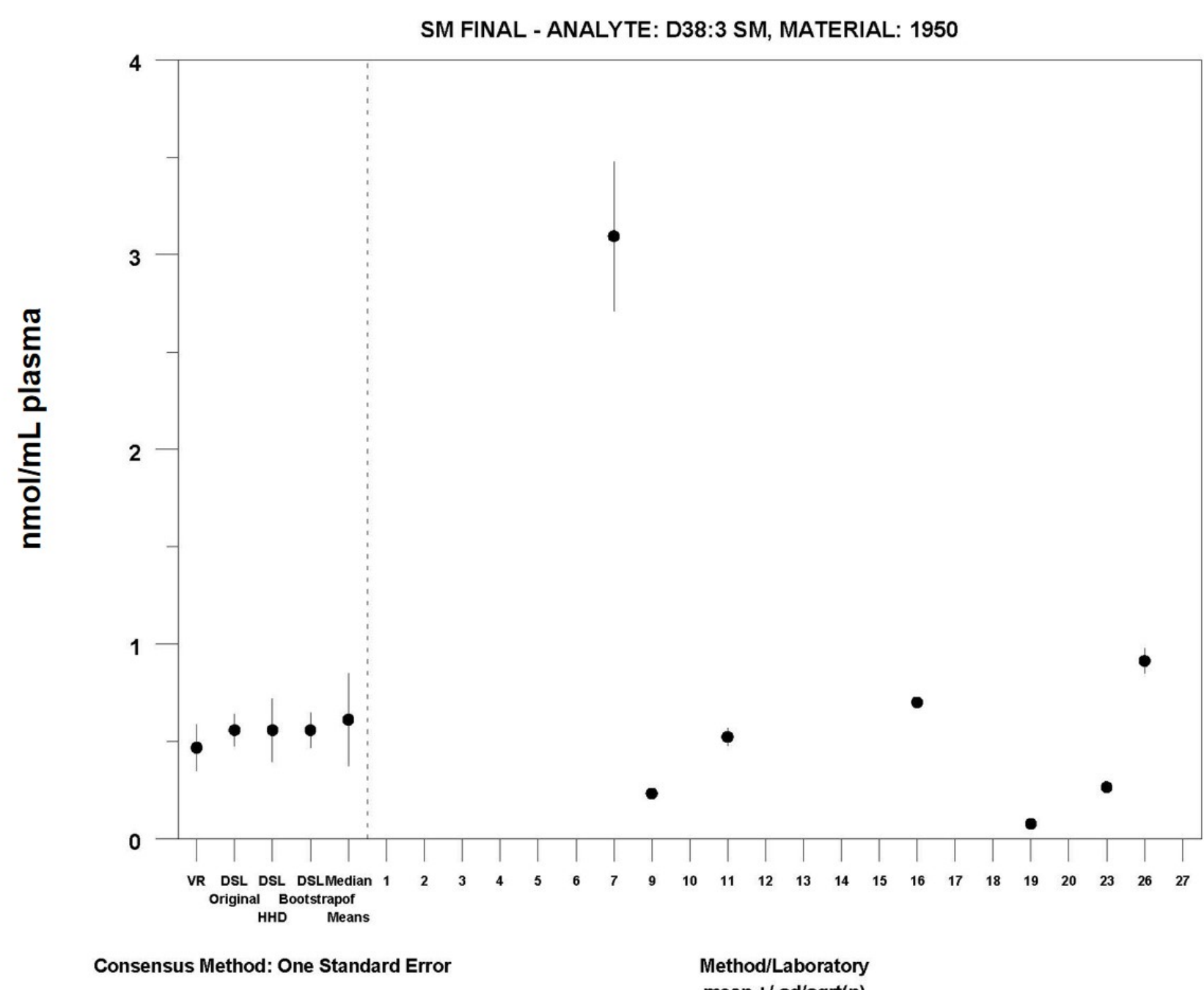

MEDM Location: $0.61 \pm 0.24 \mathrm{nmol} / \mathrm{mL}$

Labs Omitted from Plot (But Not Analysis): 15

15: $10 \pm 3$ 


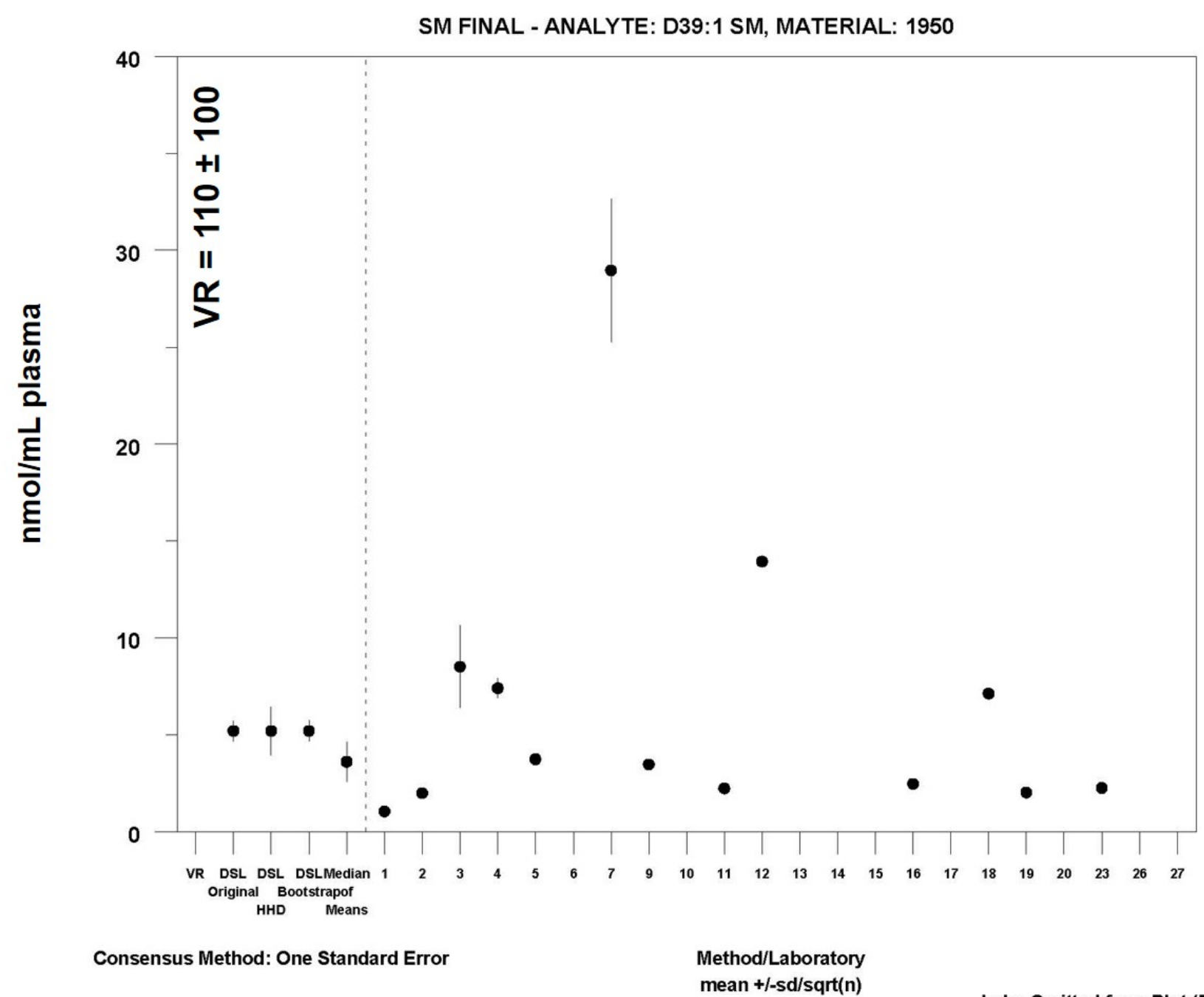

MEDM Location: $3.6 \pm 1.0 \mathrm{nmol} / \mathrm{mL}$

Labs Omitted from Plot (But Not Analysis): 15

15: $1527 \pm 59$ 


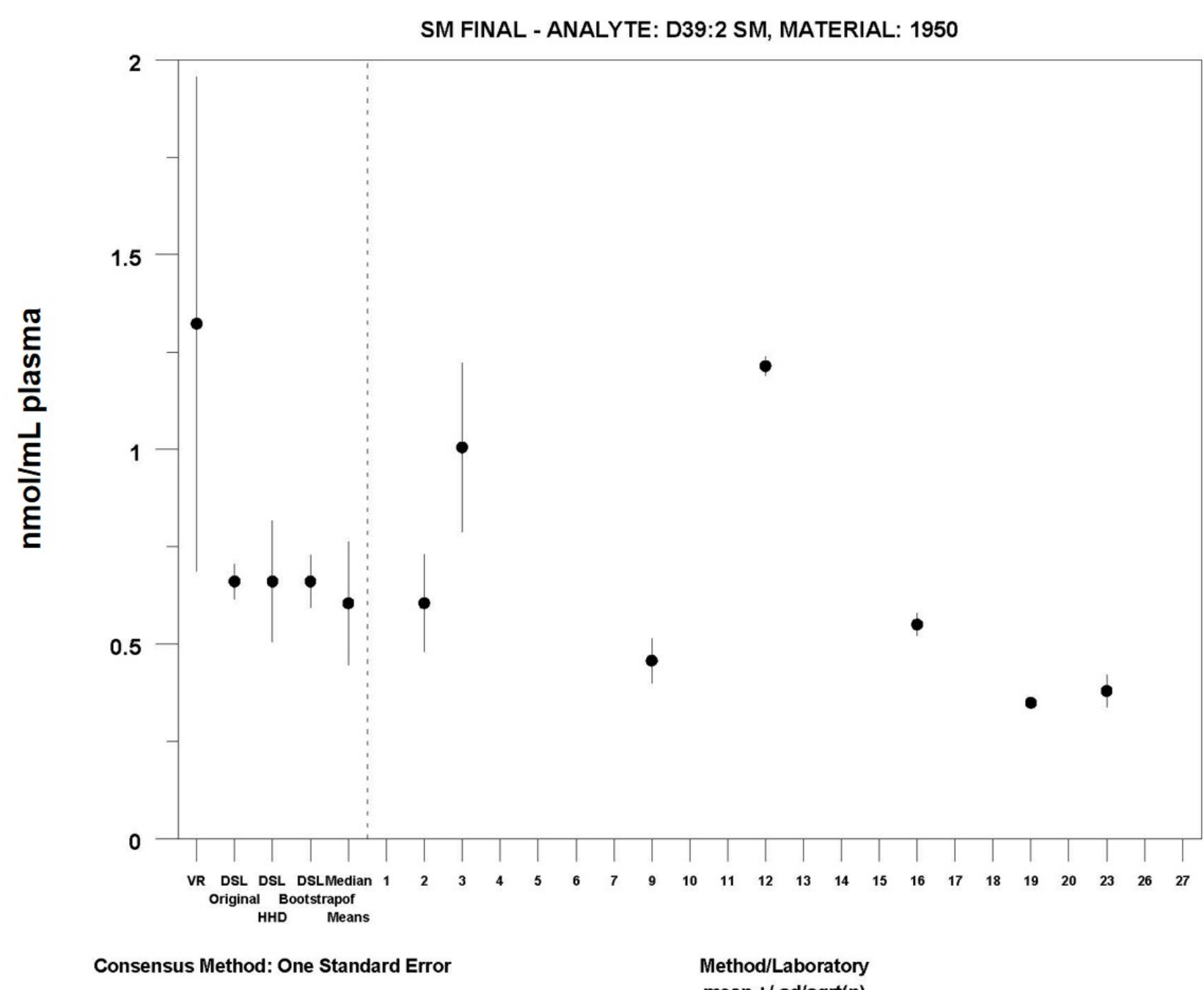

MEDM Location: $0.61 \pm 0.16 \mathrm{nmol} / \mathrm{mL}$

Labs Omitted from Plot (But Not Analysis): 715

7: $6.4 \pm 0.8 ; 15: 107 \pm 27$ 


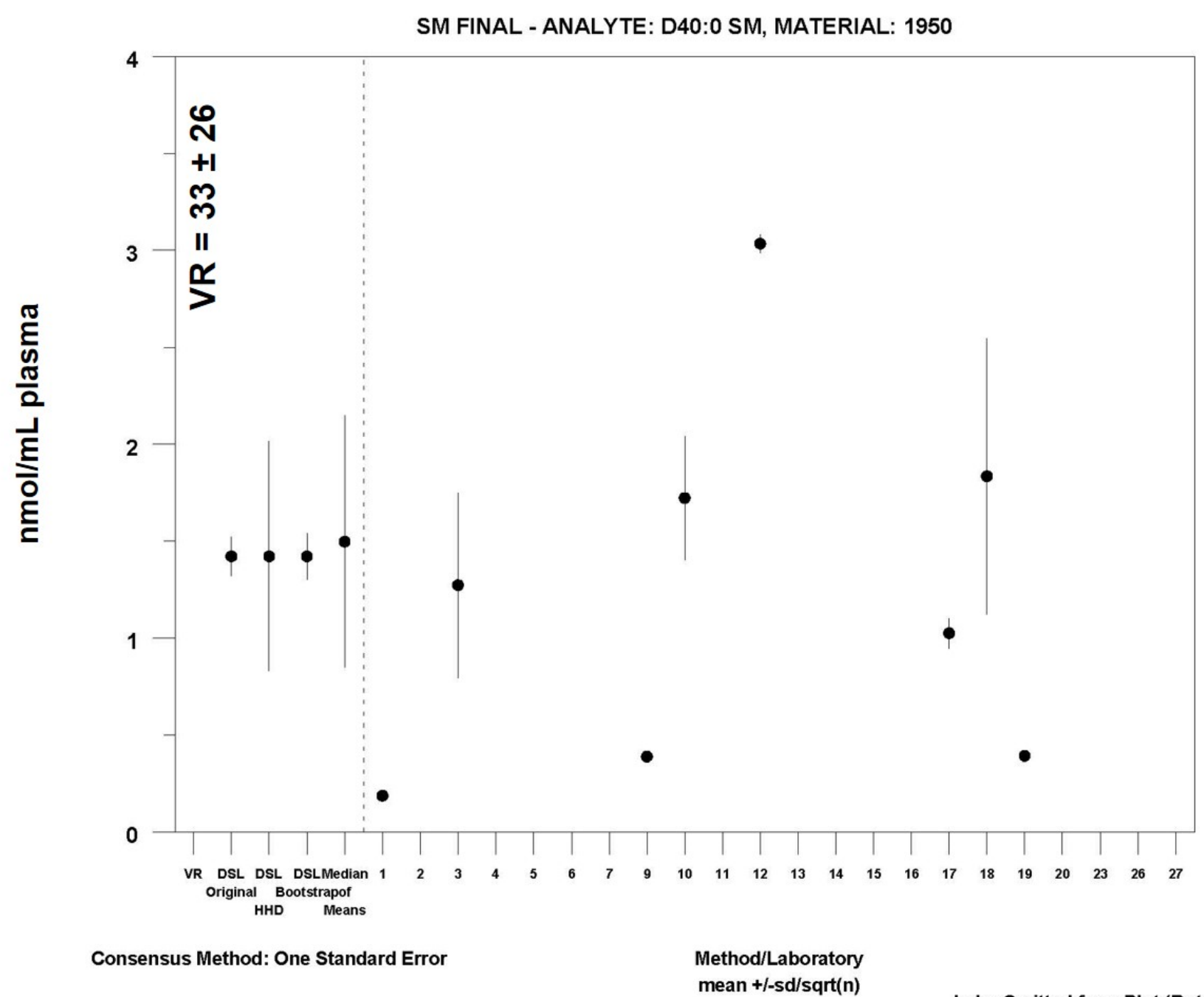

MEDM Location: $1.5 \pm 0.65 \mathrm{nmol} / \mathrm{mL}$

Labs Omitted from Plot (But Not Analysis): 1315 13: $44 \pm 2 ; 15: 276 \pm 15$ 


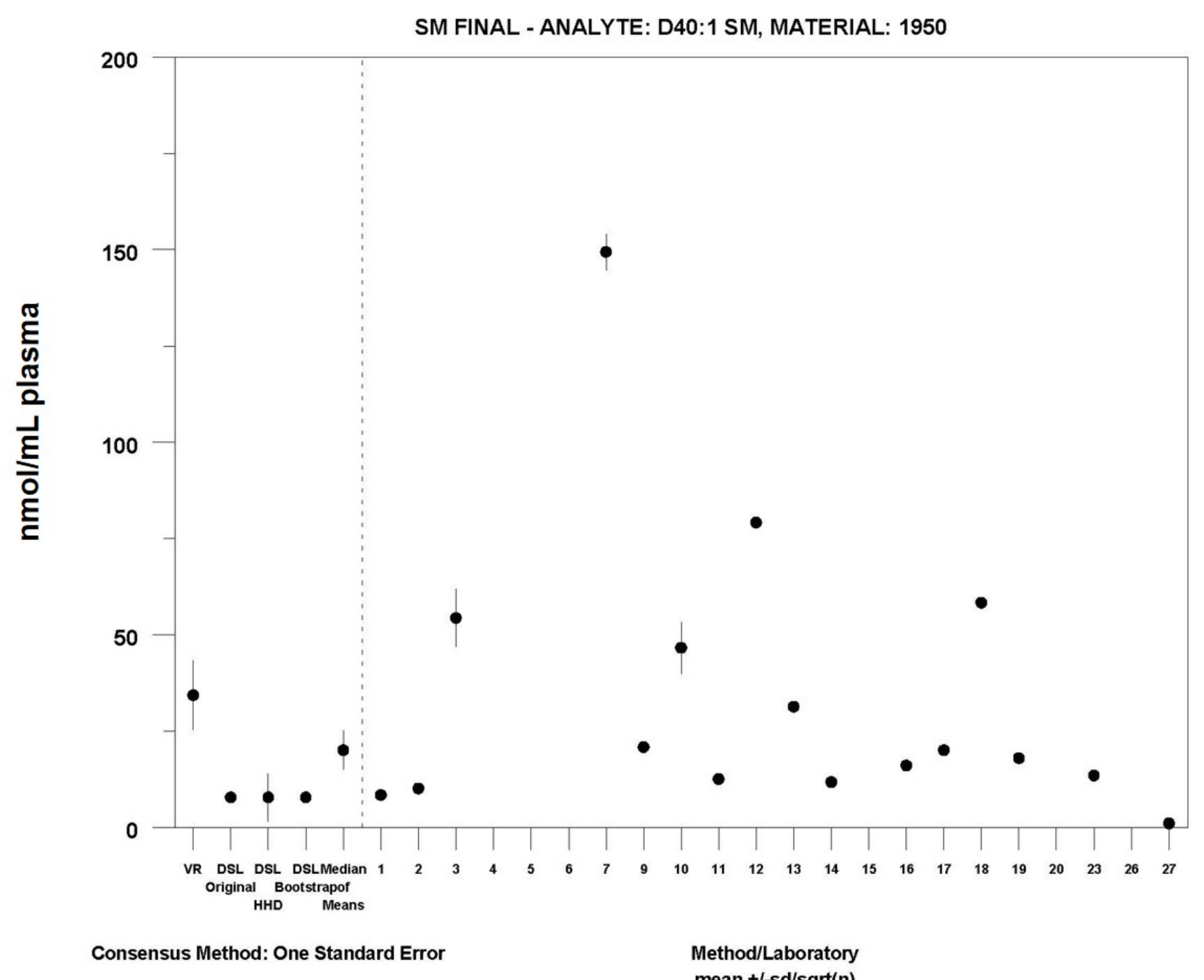

MEDM Location: $20 \pm 5.1 \mathrm{nmol} / \mathrm{mL}$

Labs Omitted from Plot (But Not Analysis): 15

15: $4560 \pm 214$ 


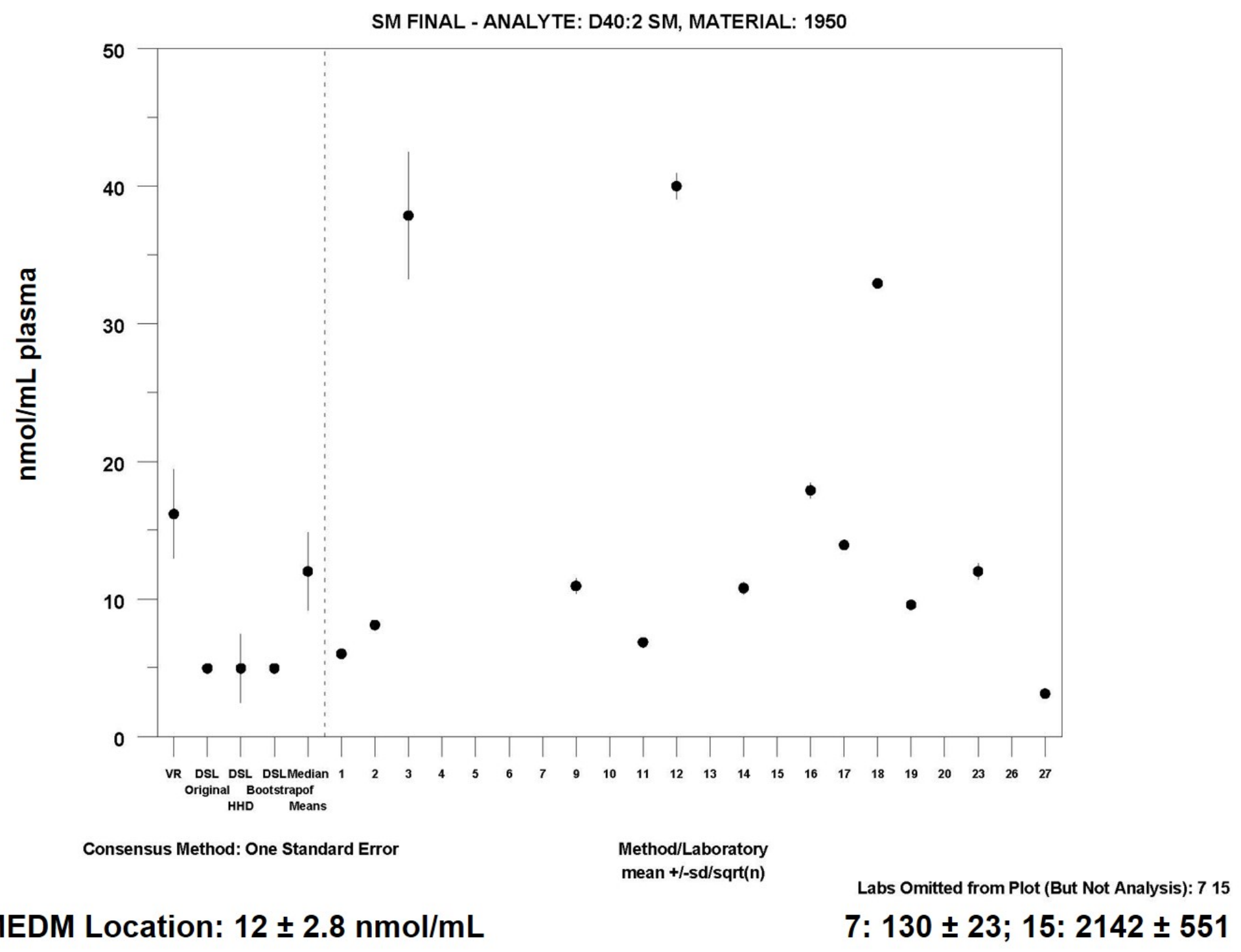




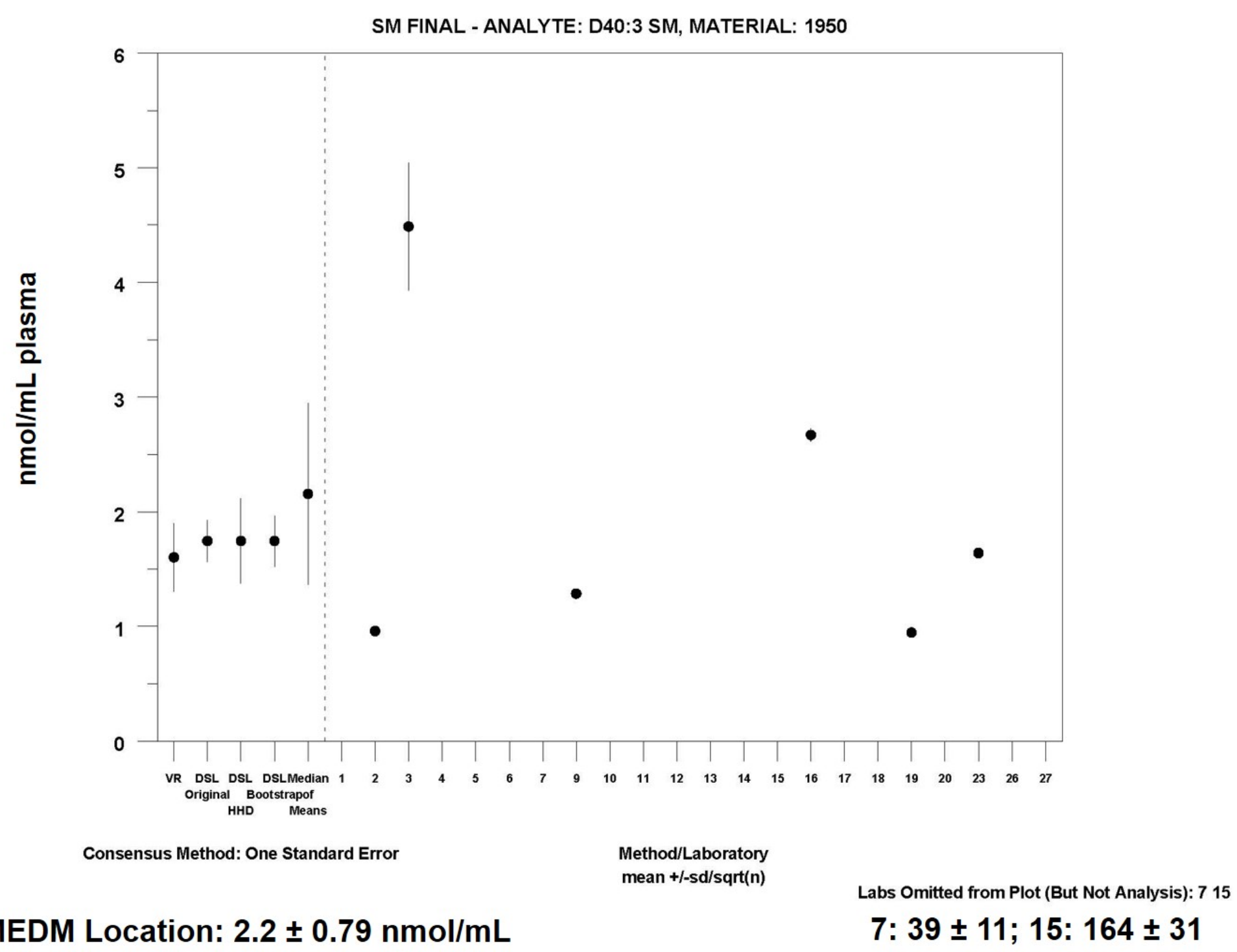




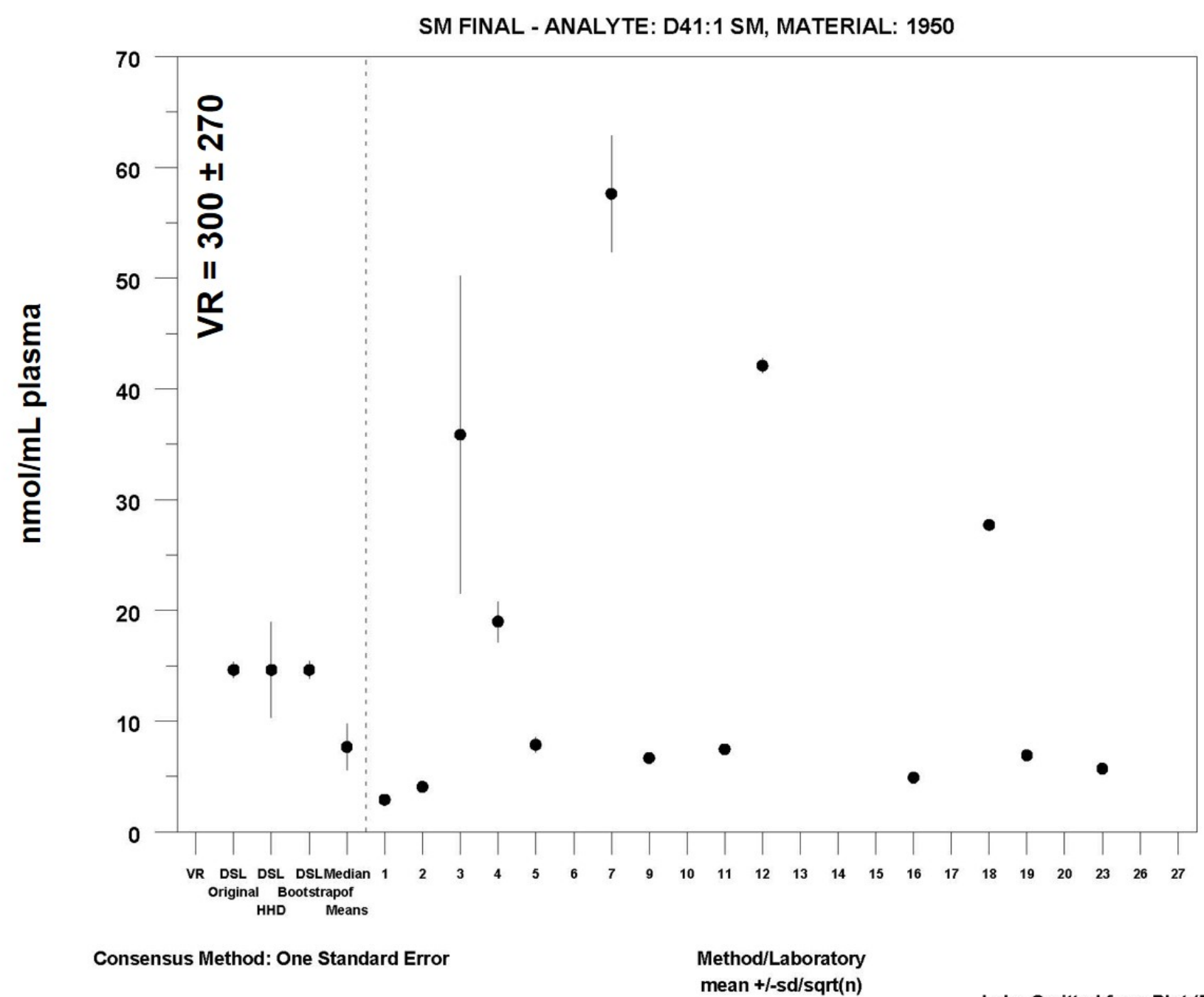

MEDM Location: $7.7 \pm 2.1 \mathrm{nmol} / \mathrm{mL}$

15: $3917 \pm 55$ 


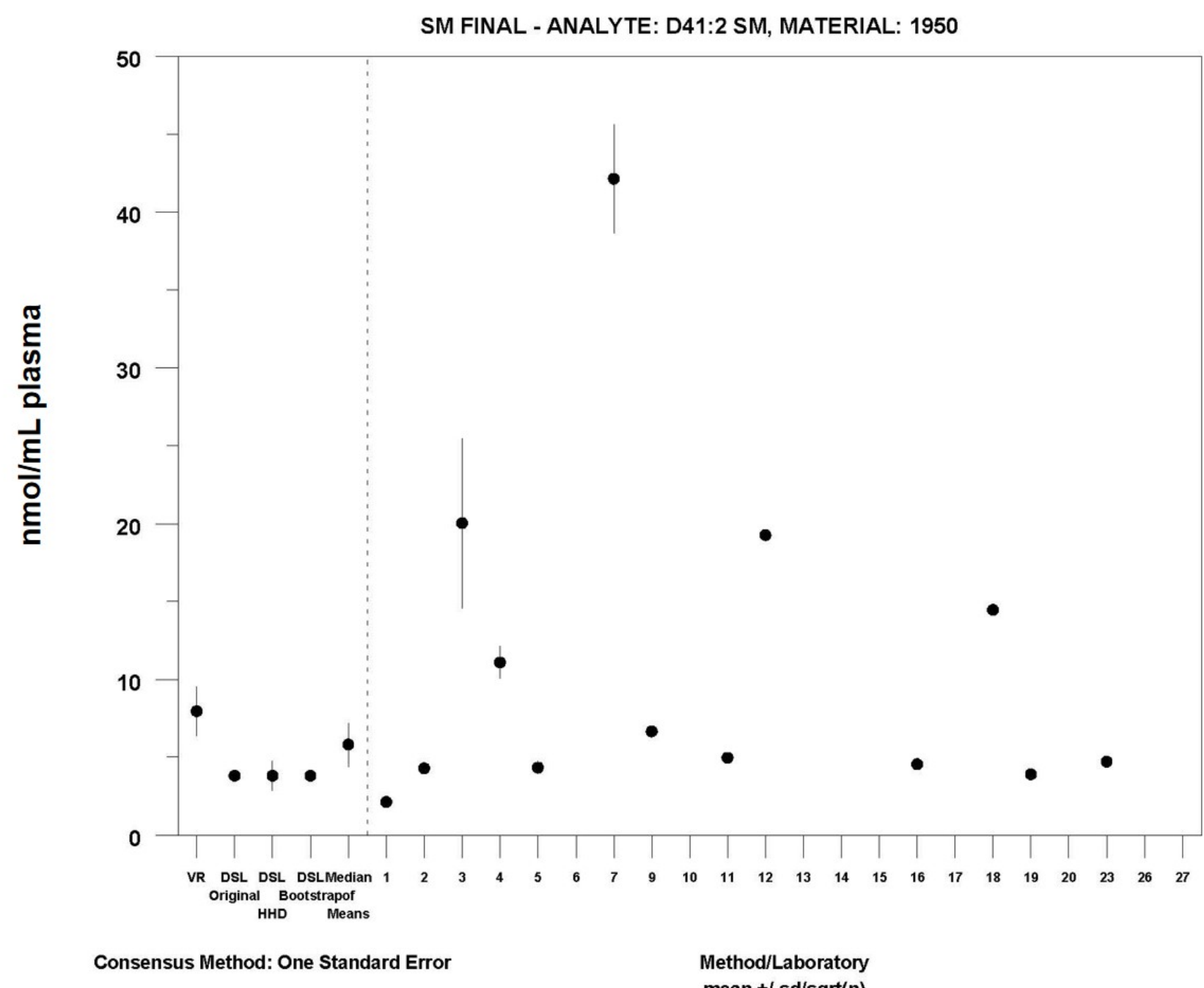

MEDM Location: $5.8 \pm 1.4 \mathrm{nmol} / \mathrm{mL}$

Labs Omitted from Plot (But Not Analysis): 15

15: $1553 \pm 501$ 


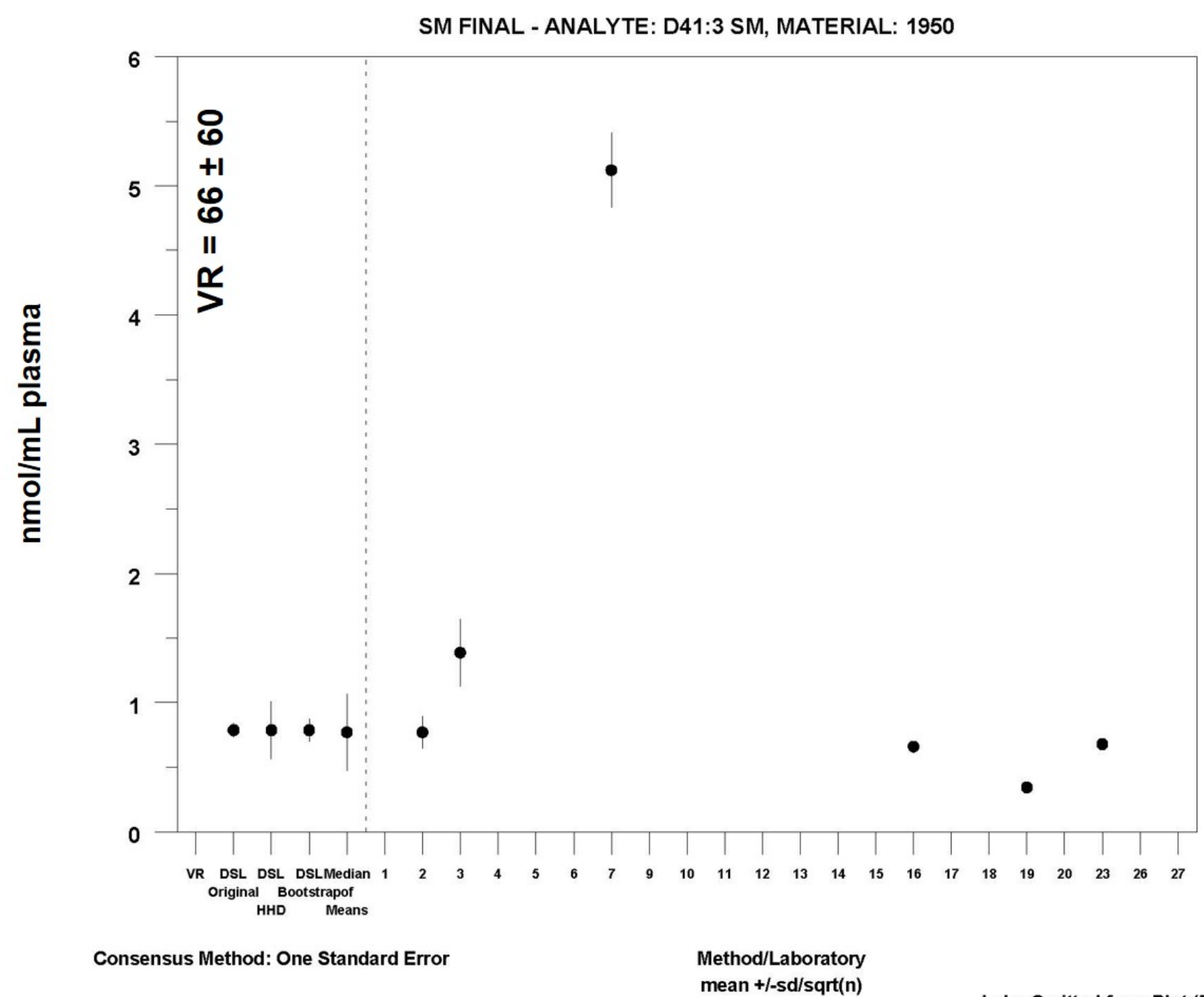

MEDM Location: $0.77 \pm 0.30 \mathrm{nmol} / \mathrm{mL}$

15: $453 \pm 14$ 


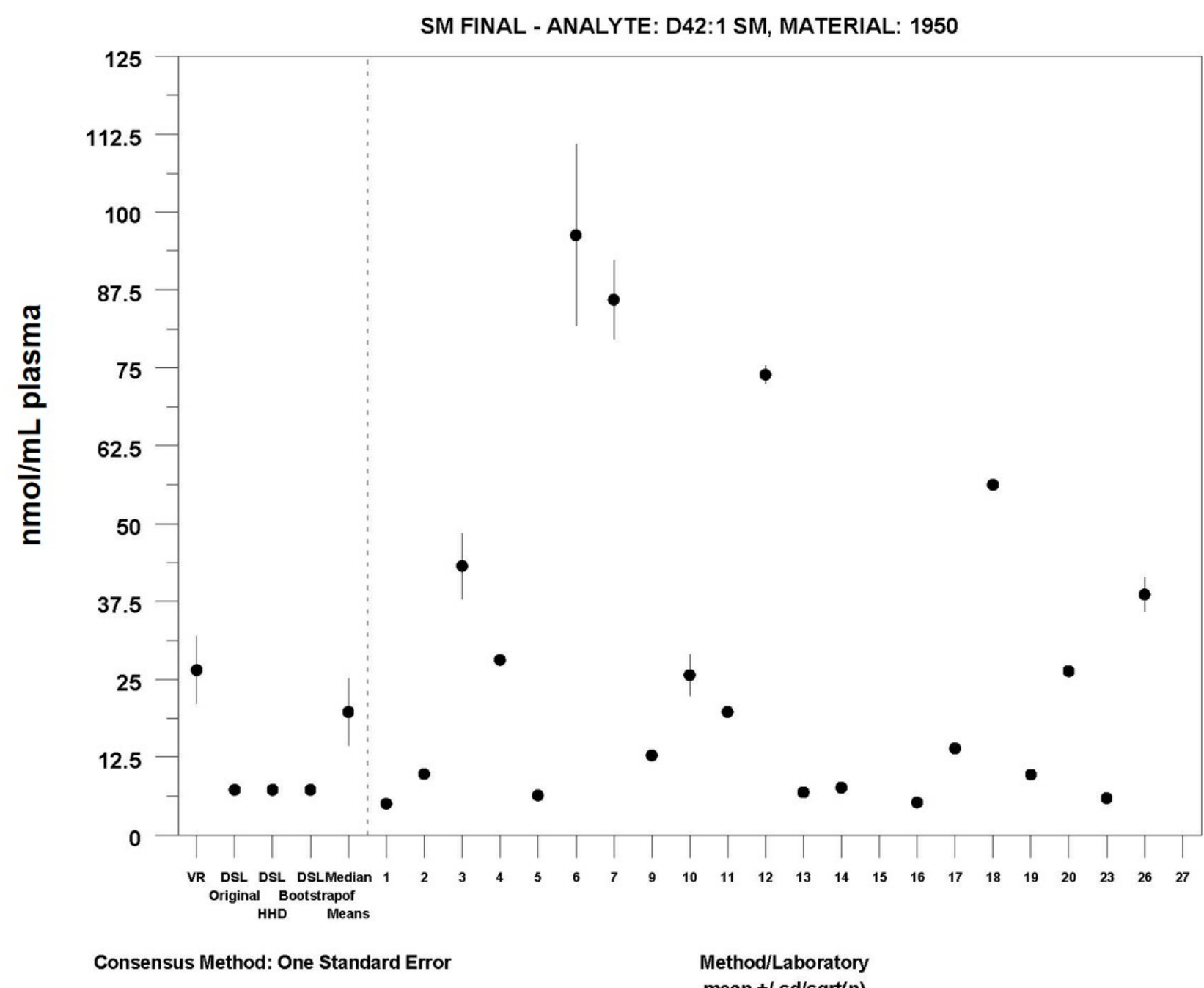

MEDM Location: $20 \pm 5.4 \mathrm{nmol} / \mathrm{mL}$

Labs Omitted from Plot (But Not Analysis): 15

15: $5189 \pm 362$ 


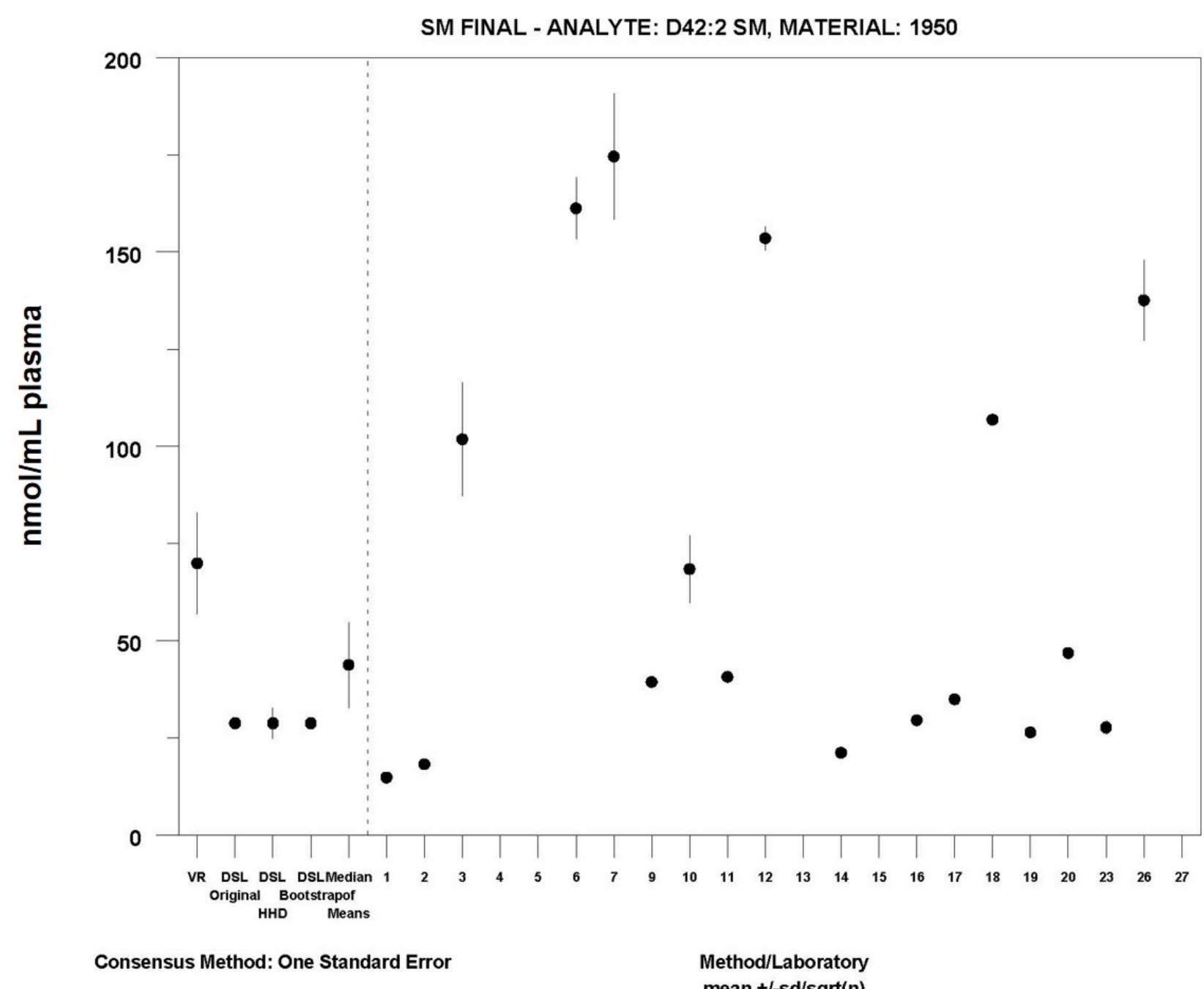

MEDM Location: $44 \pm 11 \mathrm{nmol} / \mathrm{mL}$

Labs Omitted from Plot (But Not Analysis): 15

15: $4407 \pm 473$ 


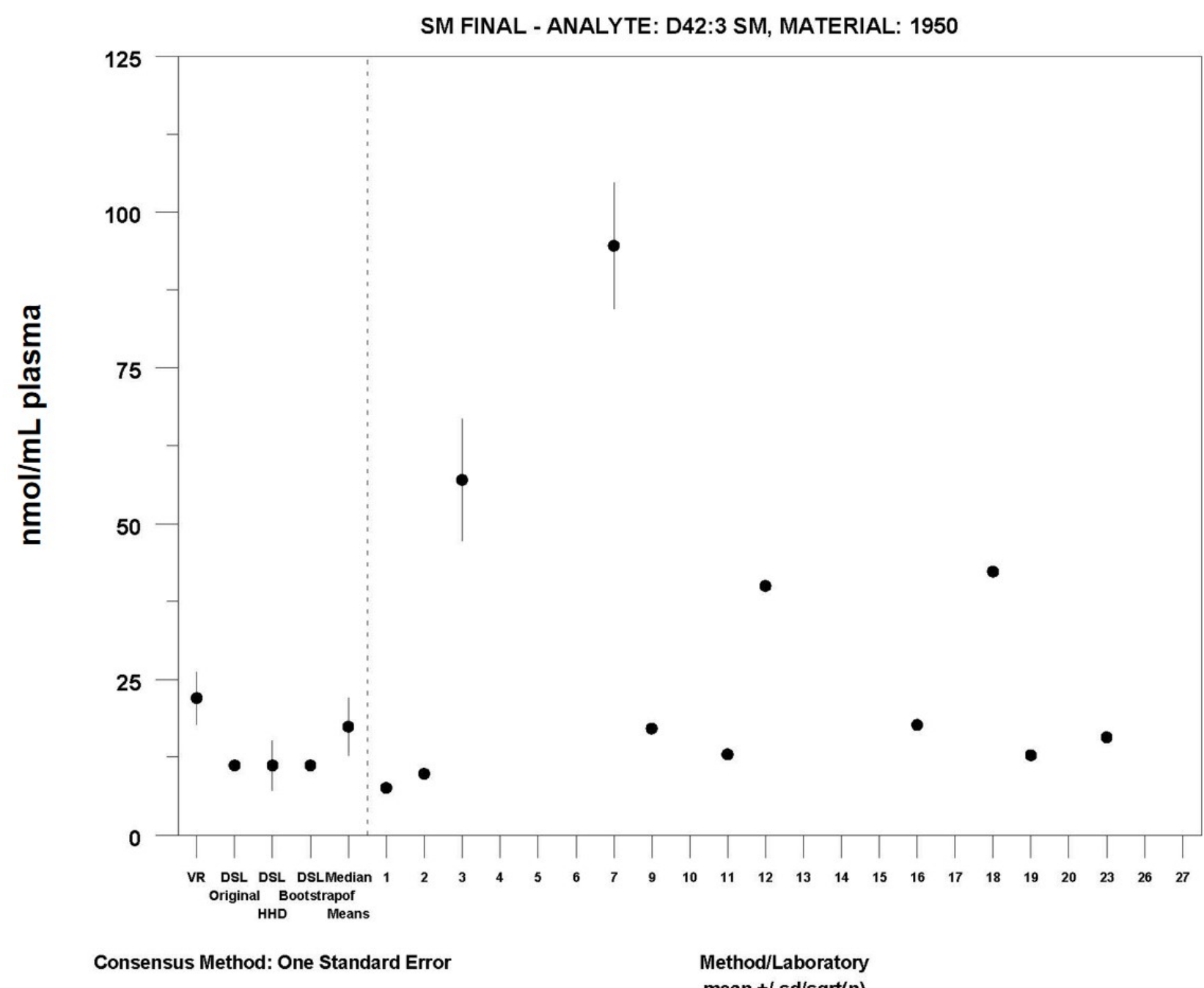

MEDM Location: $17 \pm 4.7 \mathrm{nmol} / \mathrm{mL}$

Labs Omitted from Plot (But Not Analysis): 15

15: $2161 \pm 1016$ 


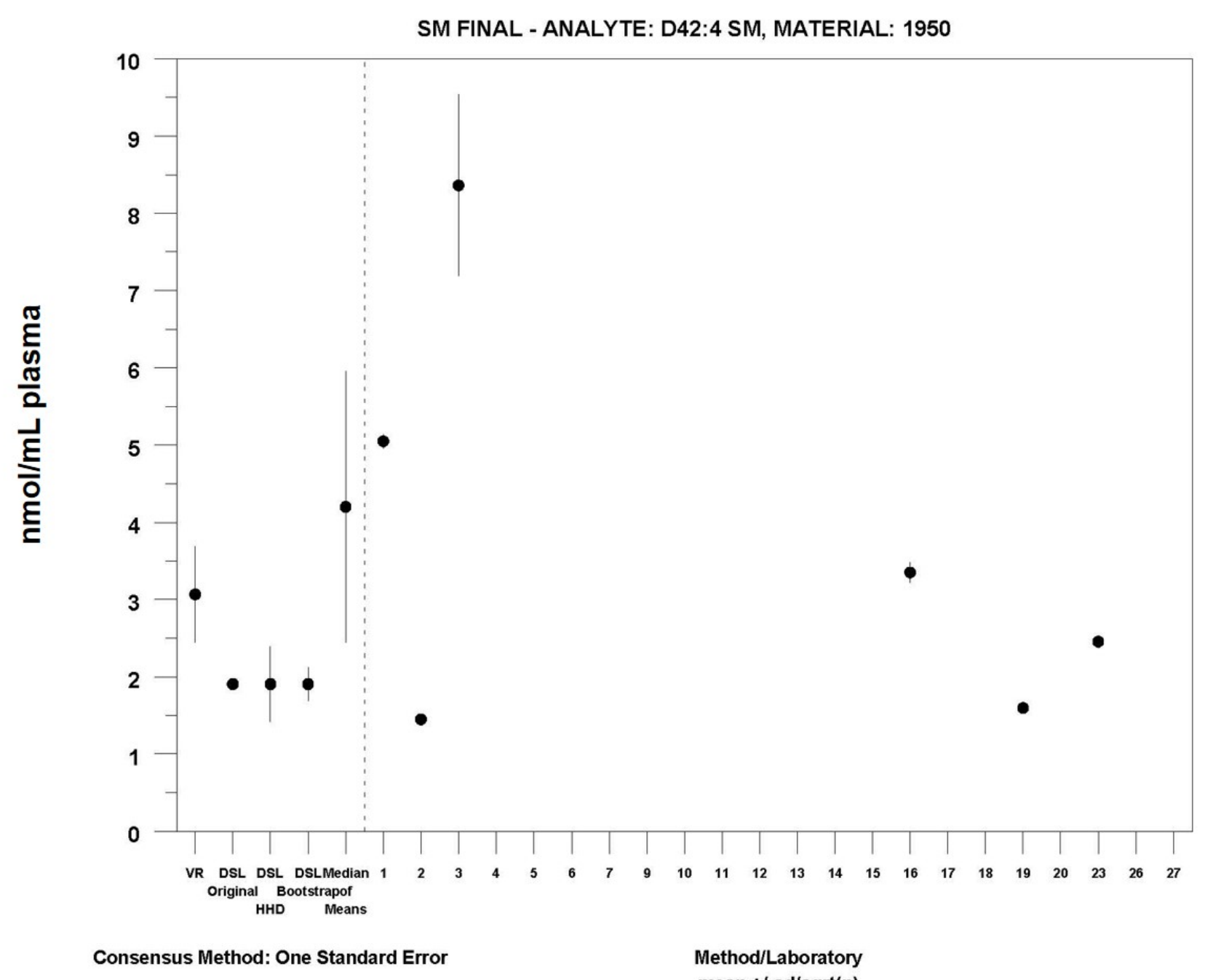

MEDM Location: $4.2 \pm 1.8 \mathrm{nmol} / \mathrm{mL}$

Labs Omitted from Plot (But Not Analysis): 715

7: $27 \pm 8 ; 15: 235 \pm 97$ 


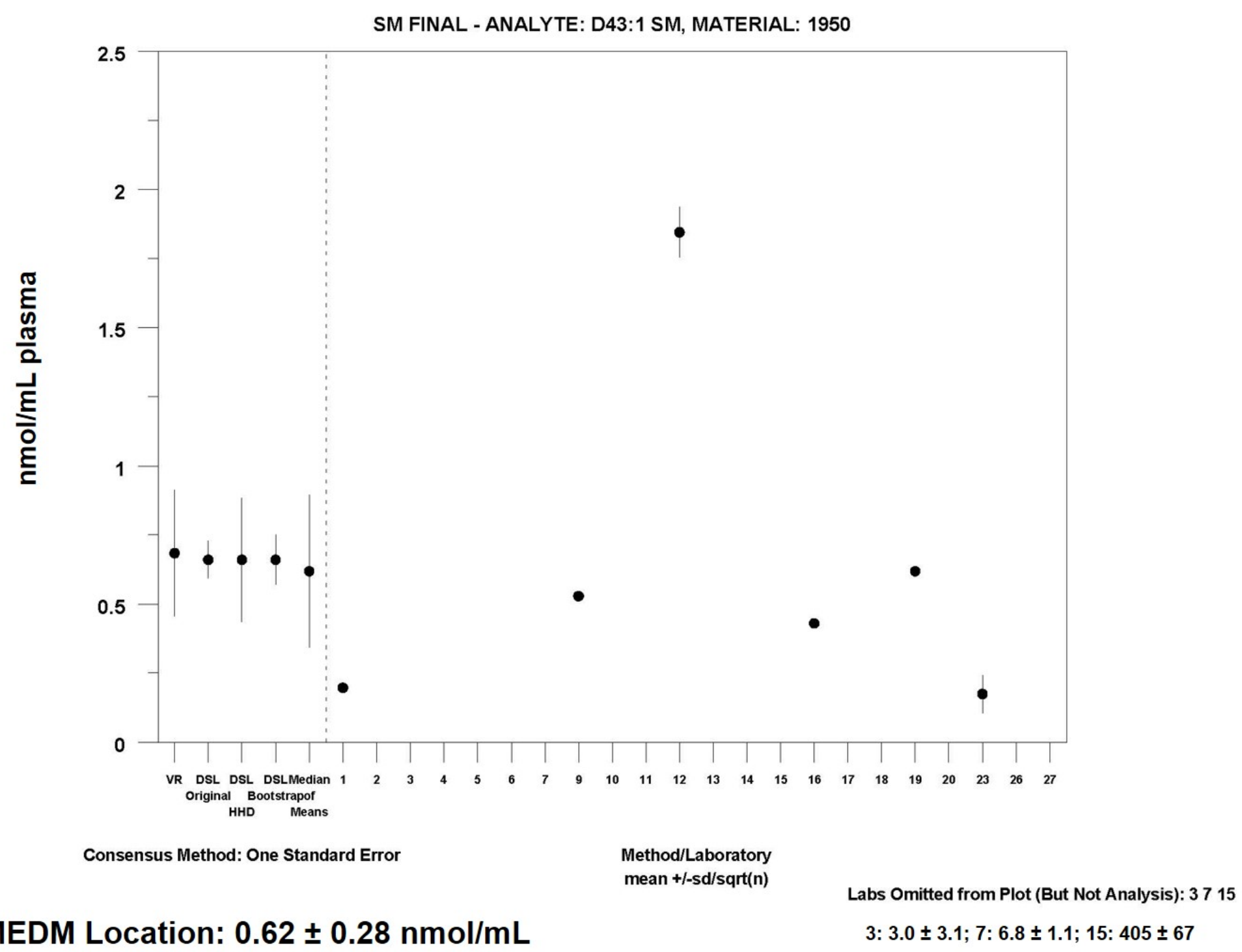




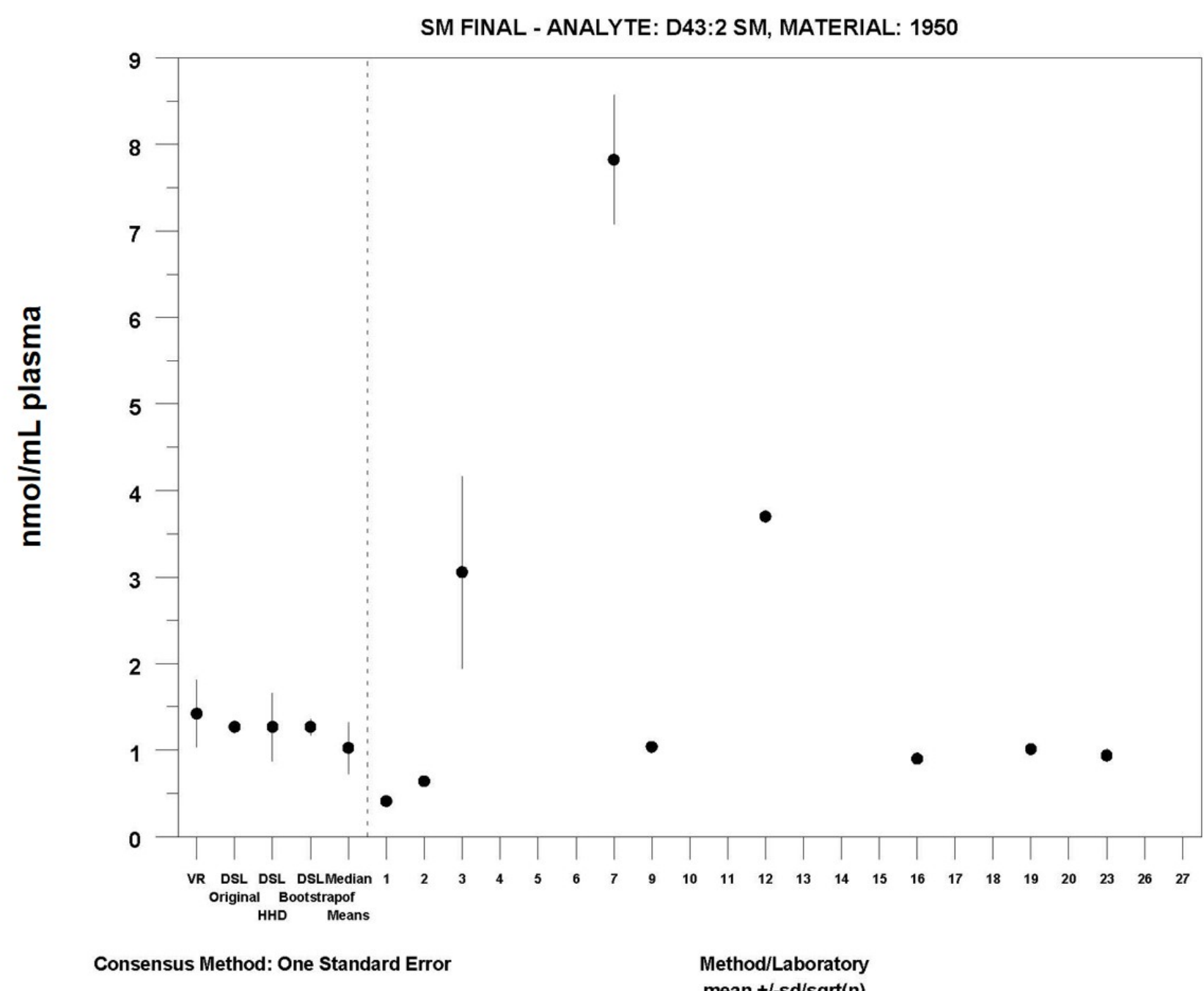

MEDM Location: $1.03 \pm 0.588 \mathrm{nmol} / \mathrm{mL}$

Labs Omitted from Plot (But Not Analysis): 15

15: $257 \pm 218$ 


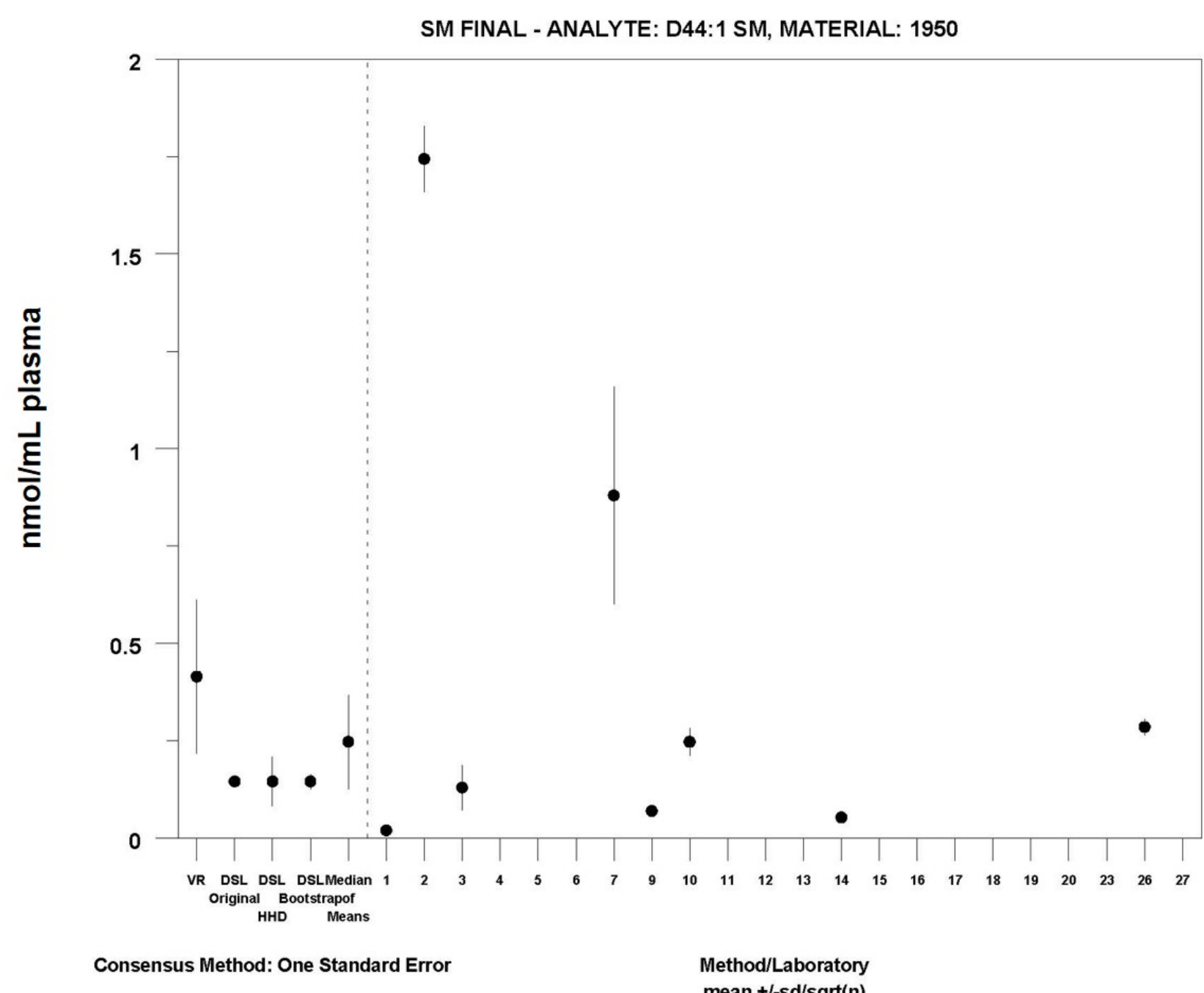

MEDM Location: $0.25 \pm 0.12 \mathrm{nmol} / \mathrm{mL}$

Labs Omitted from Plot (But Not Analysis): 15

15: $86 \pm 31$ 


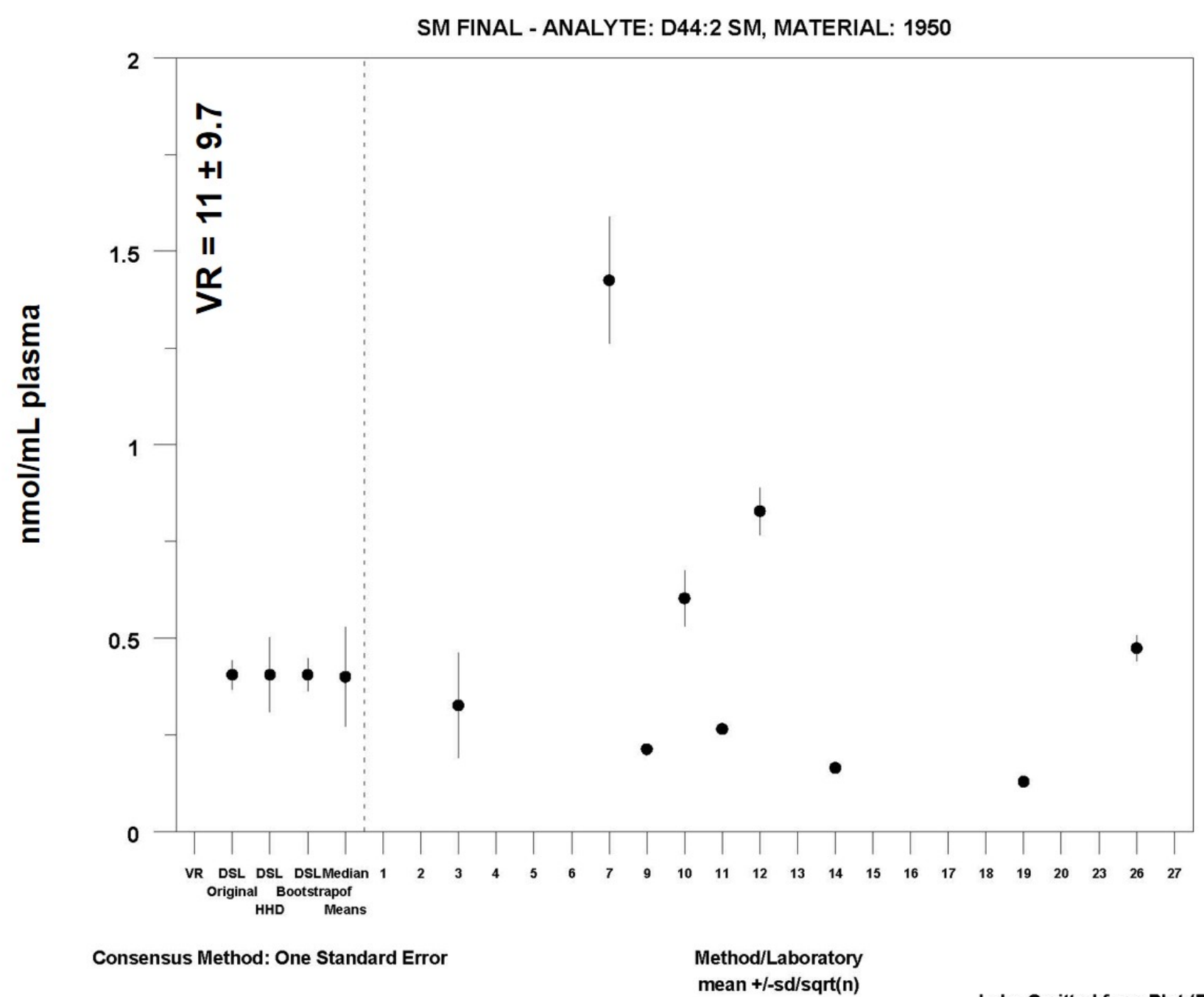

MEDM Location: $0.40 \pm 0.13 \mathrm{nmol} / \mathrm{mL}$

15: $103 \pm 6$ 


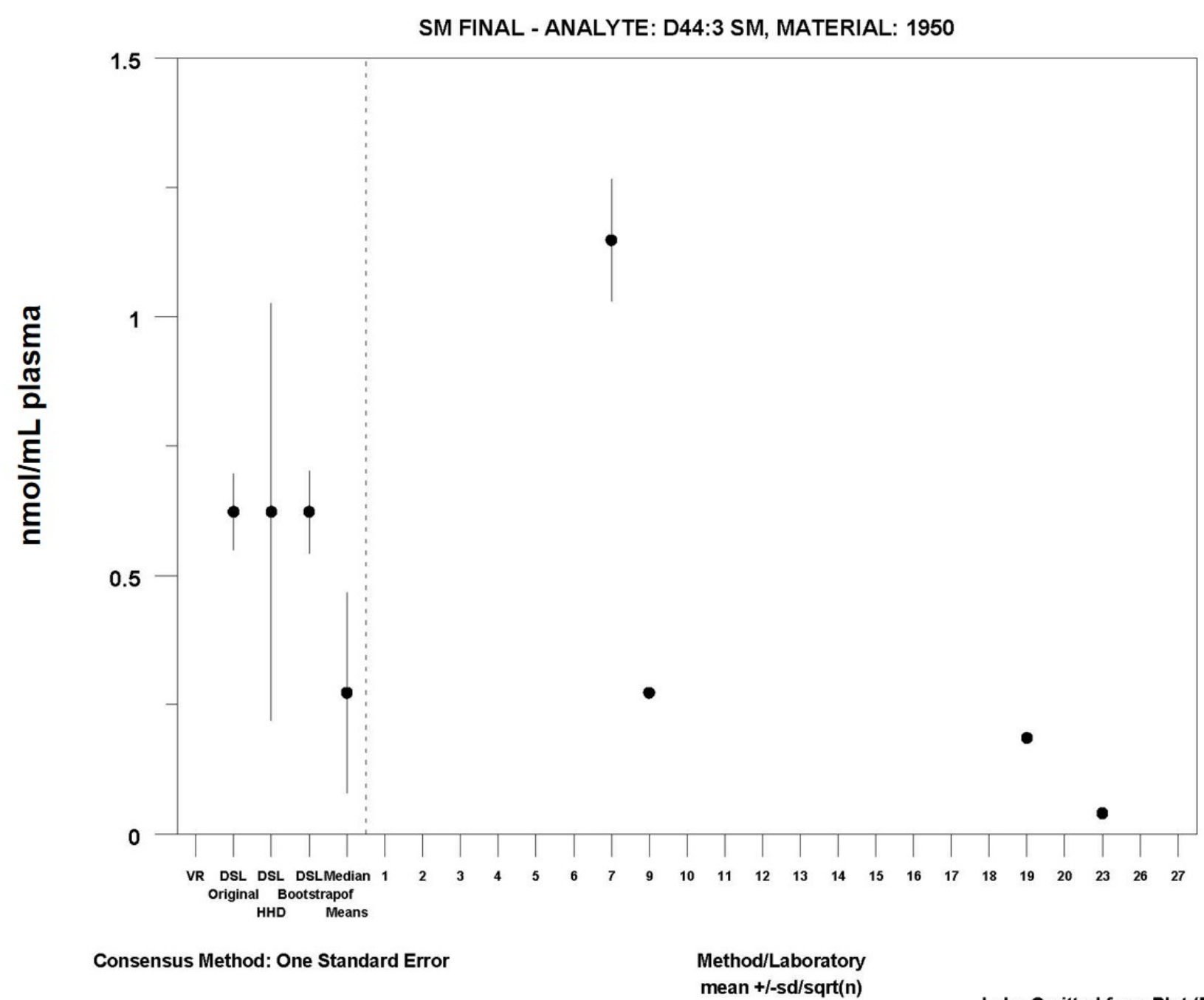

MEDM Location: $0.27 \pm 0.19 \mathrm{nmol} / \mathrm{mL}$

15: $20 \pm 1$ 


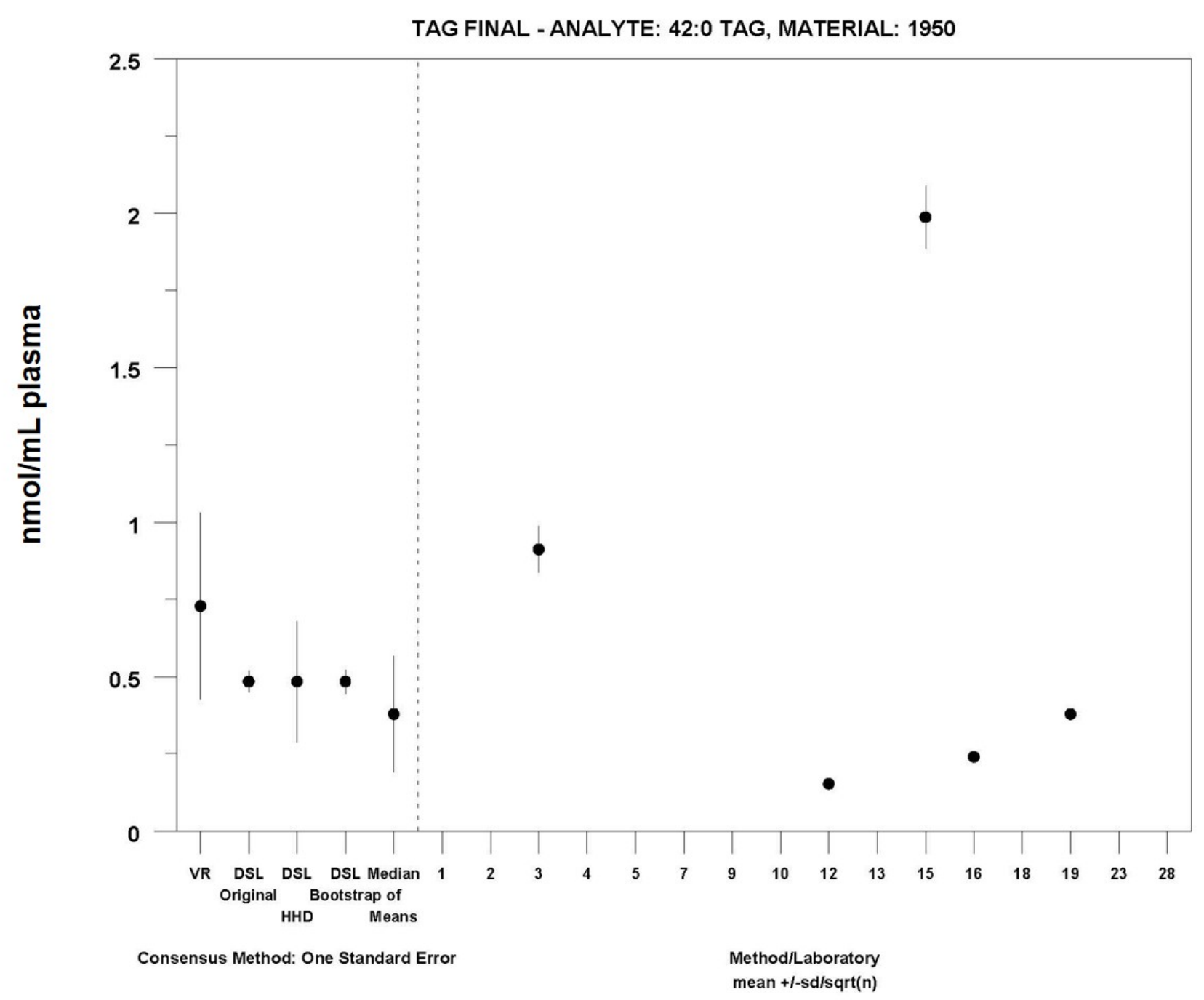

MEDM Location: $0.38 \pm 0.19 \mathrm{nmol} / \mathrm{mL}$ 


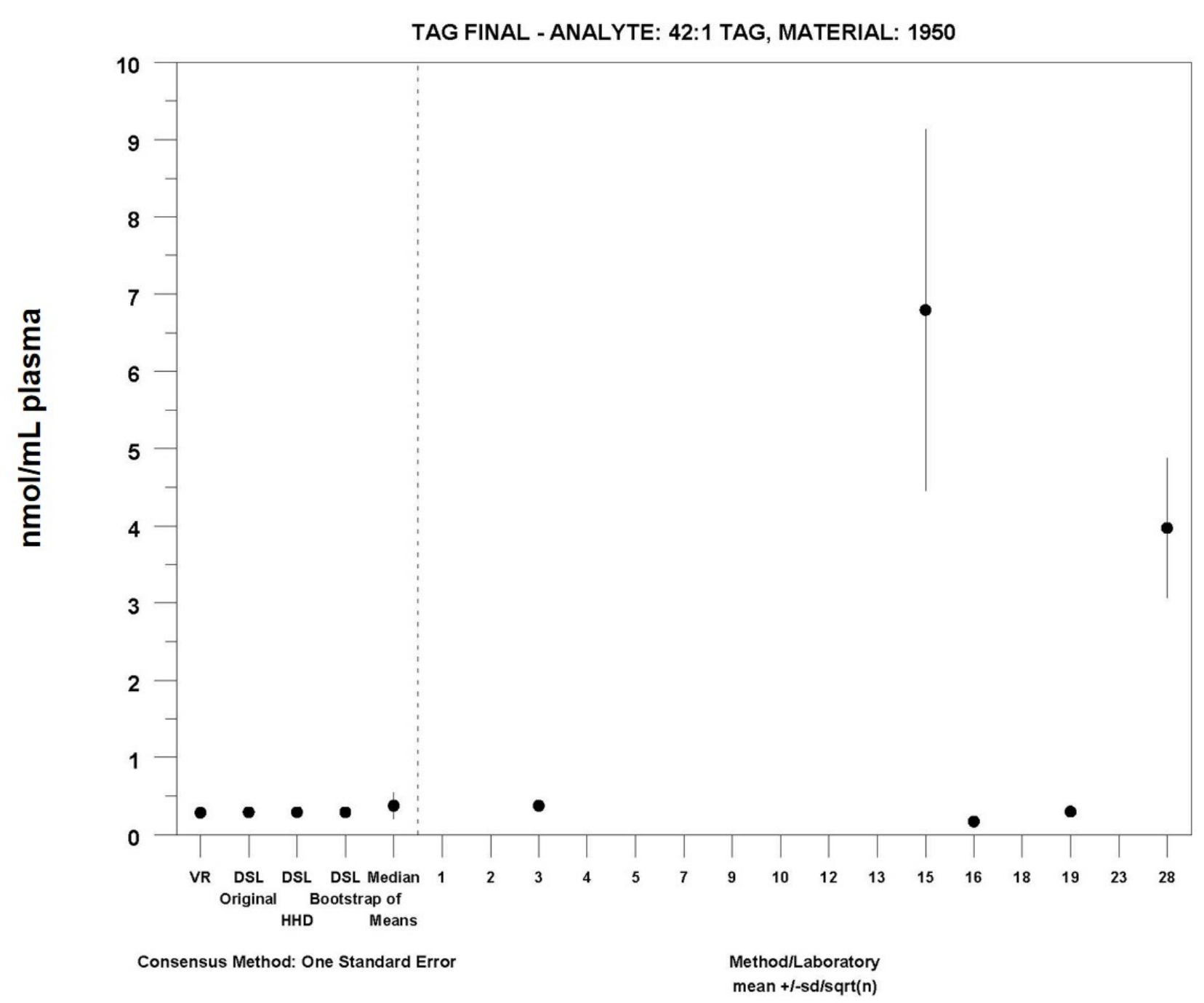

MEDM Location: $0.37 \pm 0.17 \mathrm{nmol} / \mathrm{mL}$ 


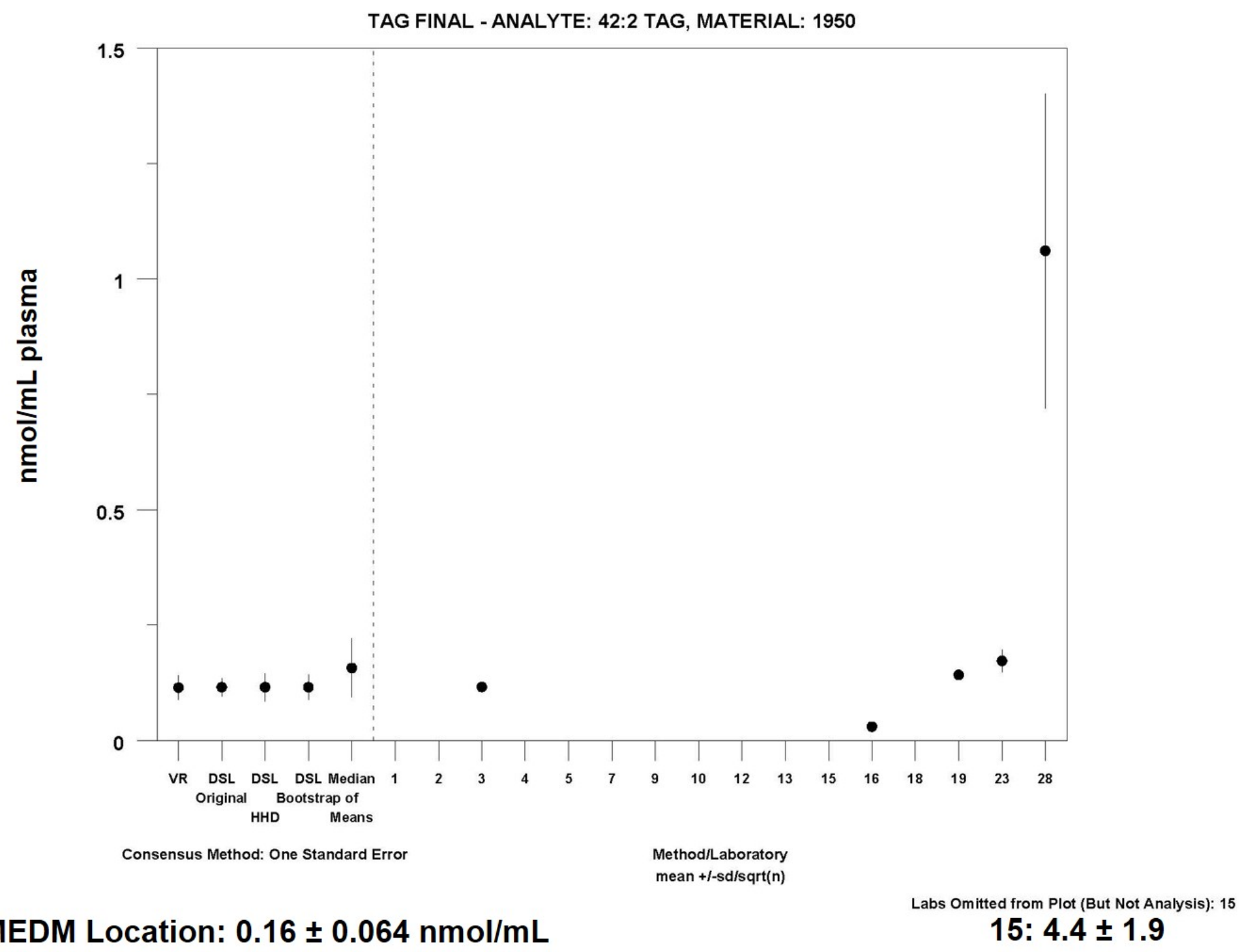




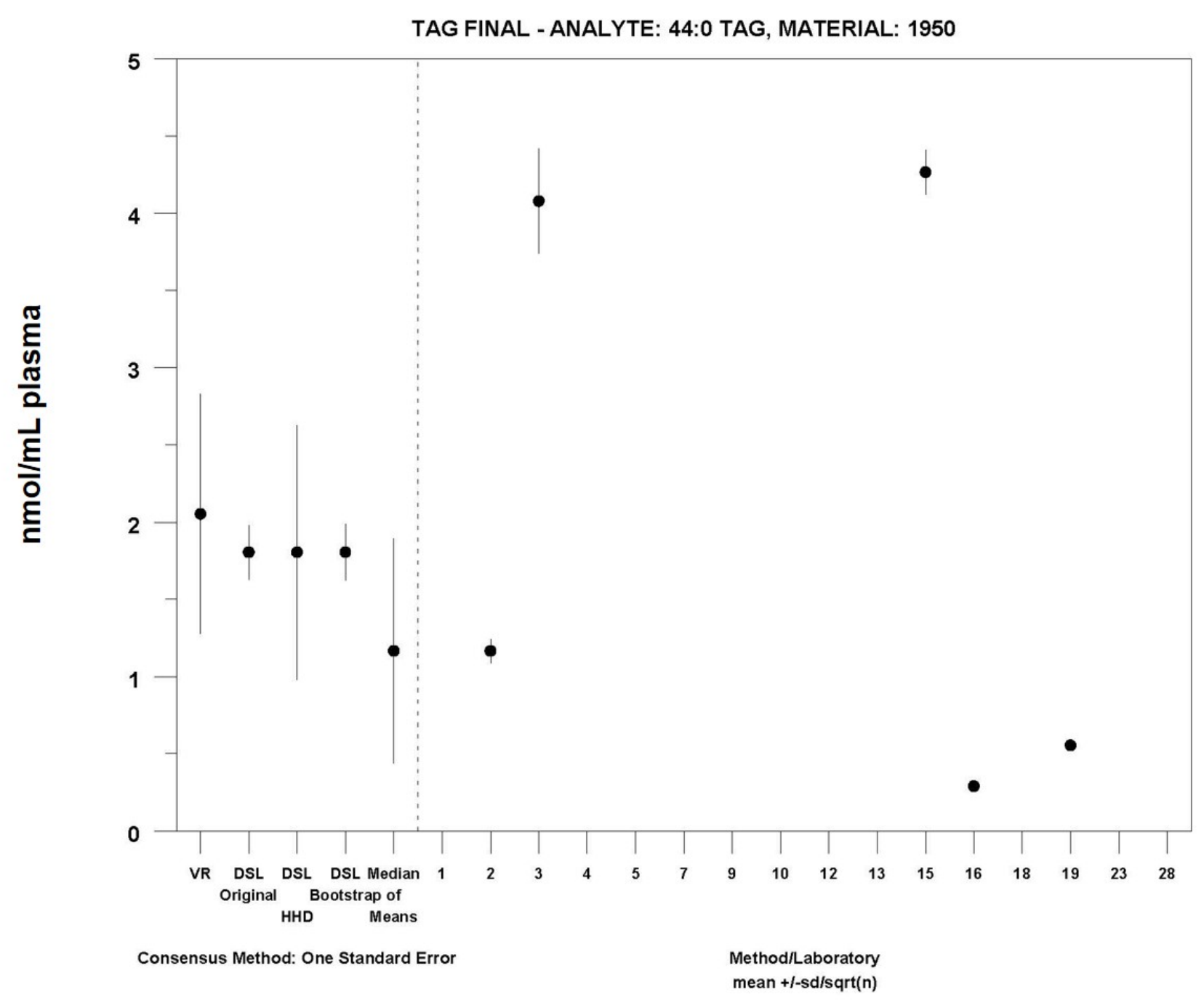

MEDM Location: $1.2 \pm 0.73 \mathrm{nmol} / \mathrm{mL}$ 


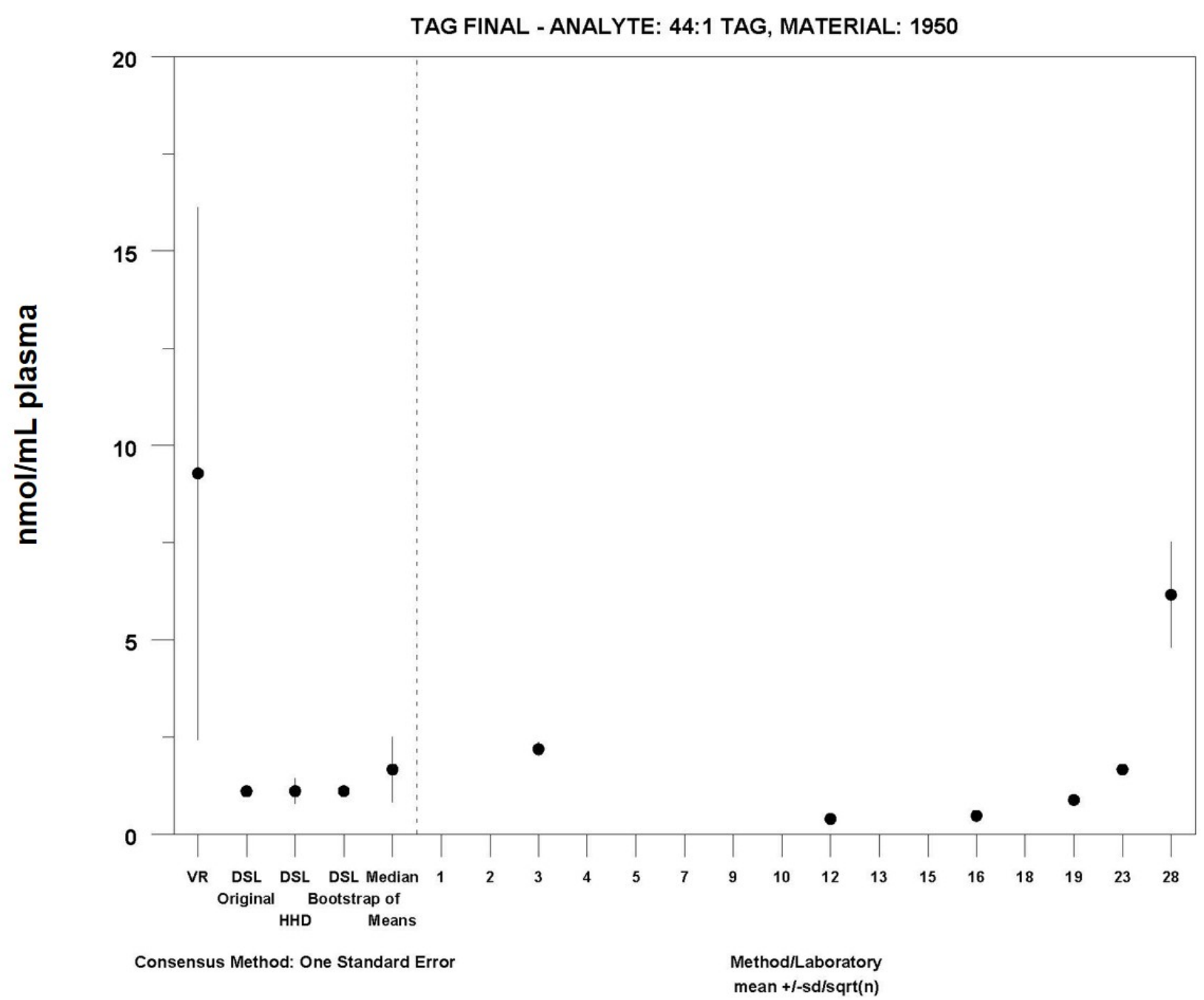

MEDM Location: $1.7 \pm 0.84 \mathrm{nmol} / \mathrm{mL}$

Labs Omitted from Plot (But Not Analysis): 15

15: $55 \pm 6$ 


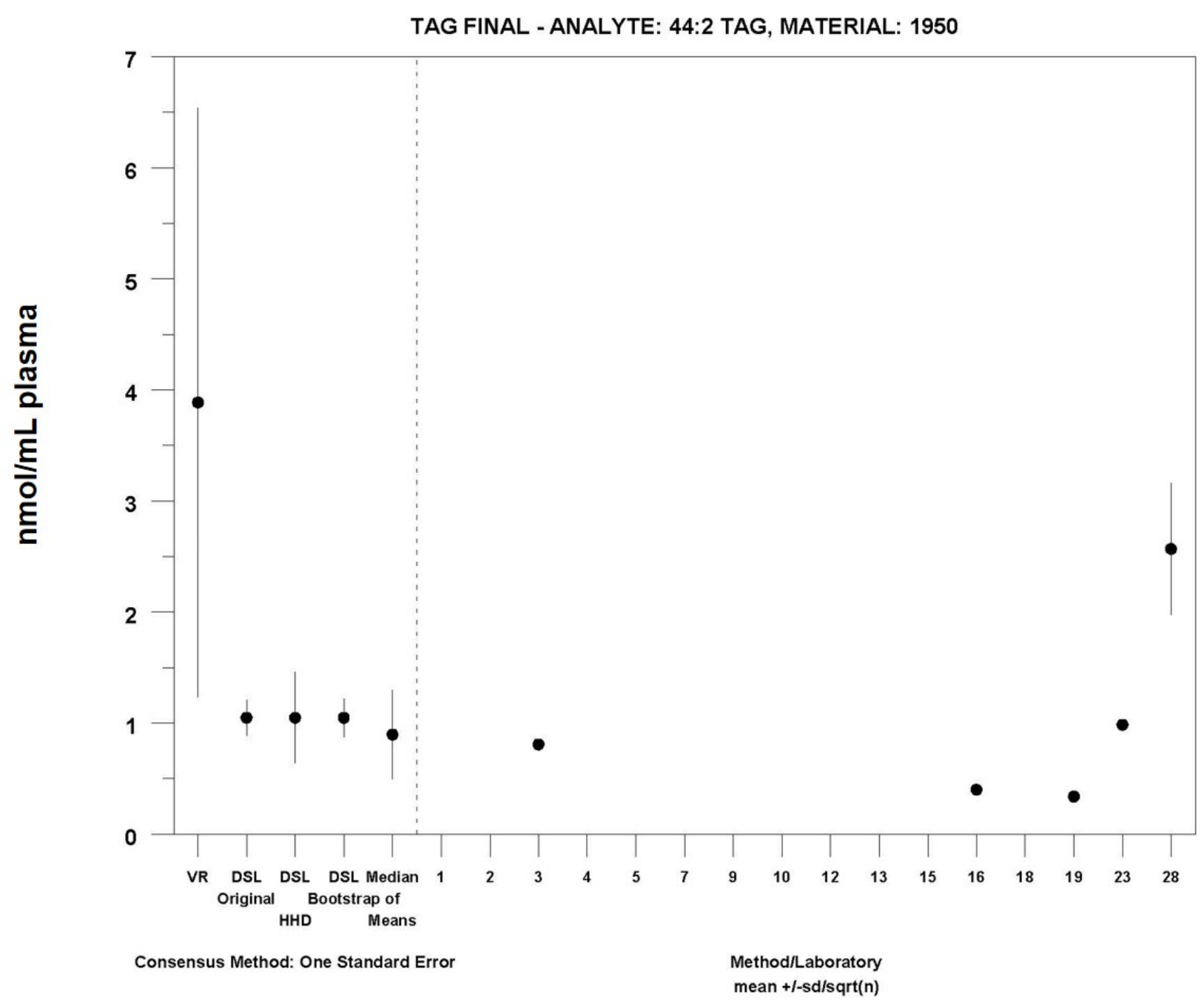

MEDM Location: $0.90 \pm 0.40 \mathrm{nmol} / \mathrm{mL}$

Labs Omitted from Plot (But Not Analysis): 15

15: $19 \pm 2$ 


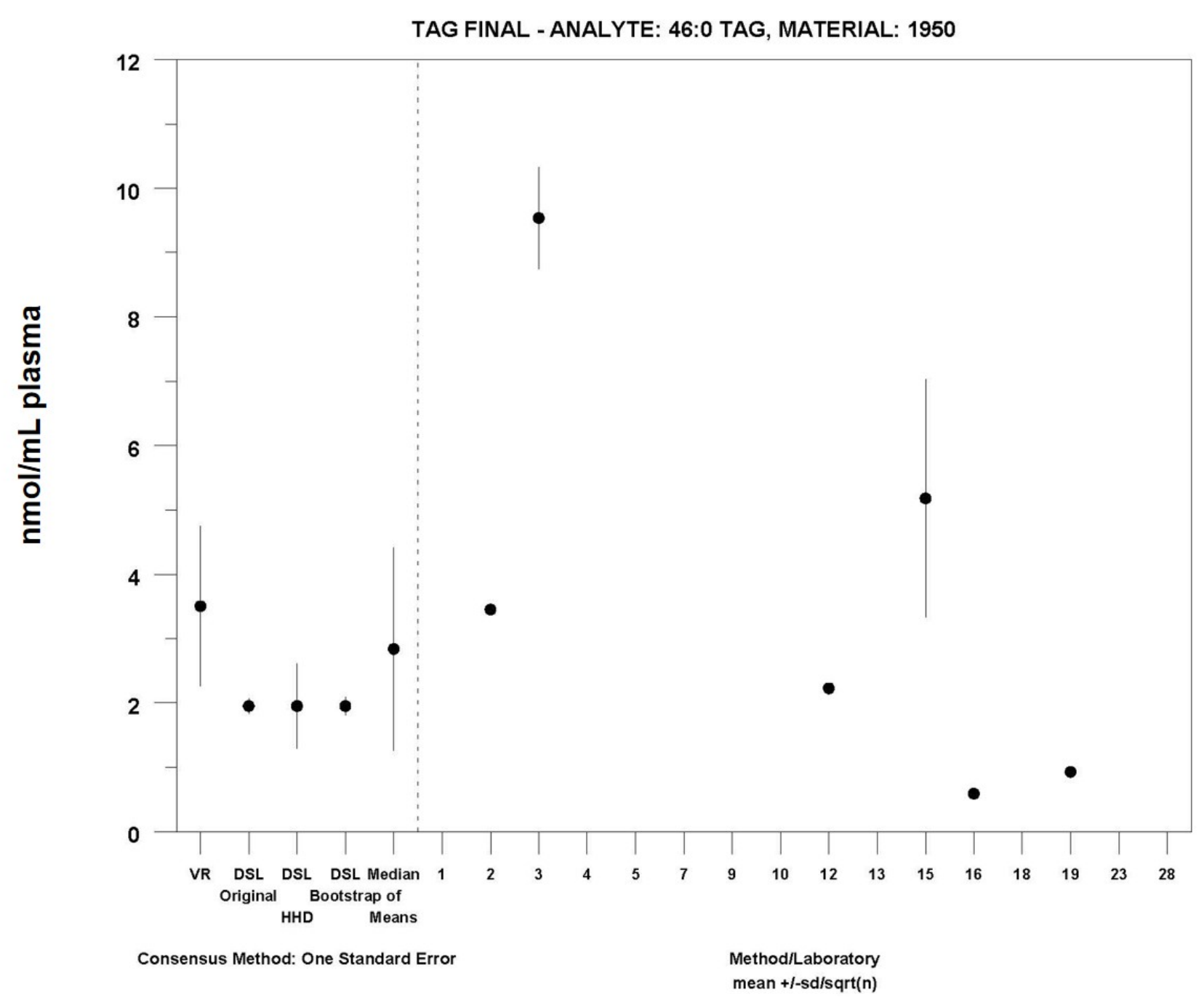

MEDM Location: $2.8 \pm 1.6 \mathrm{nmol} / \mathrm{mL}$ 


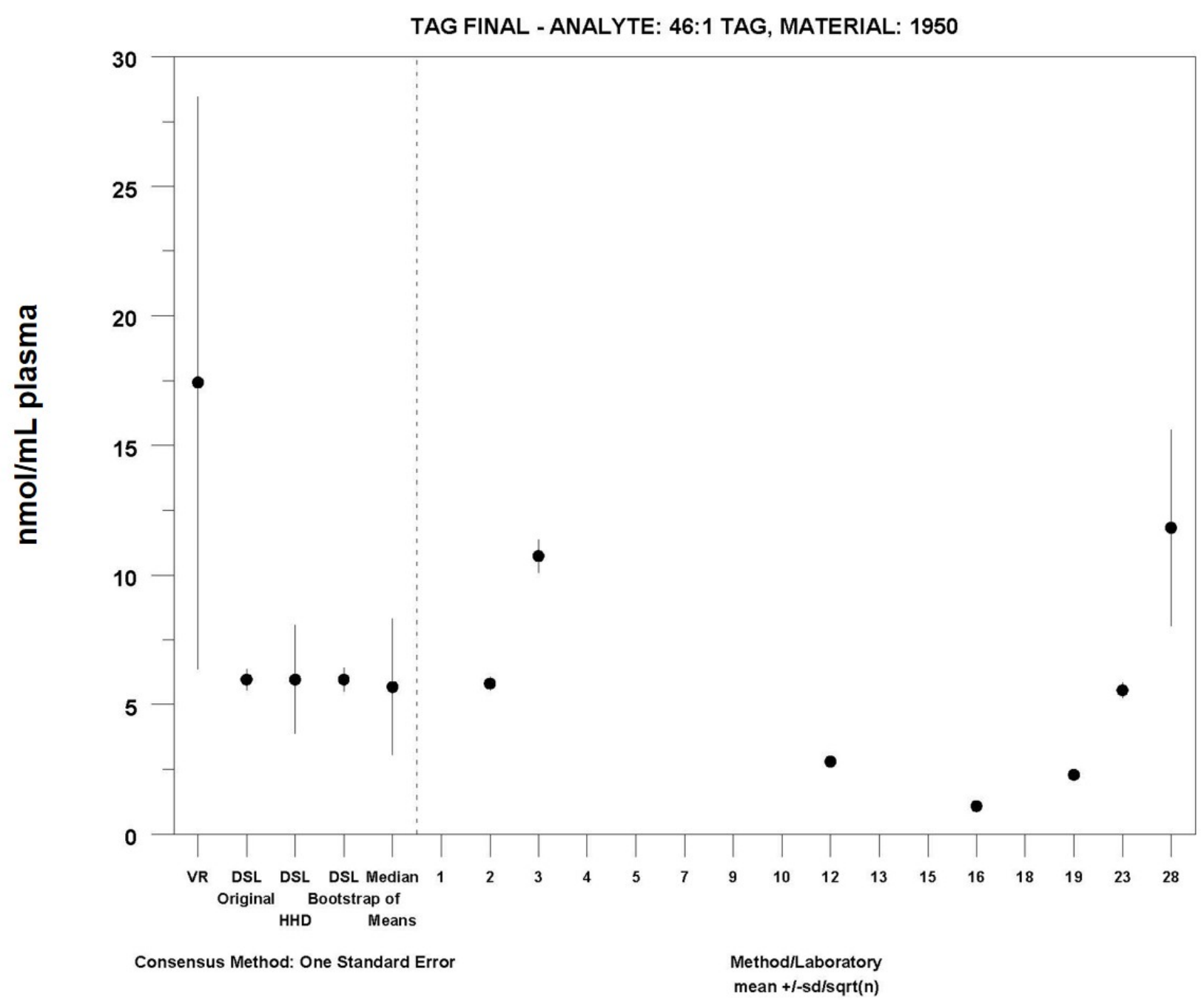

MEDM Location: $5.7 \pm 2.6 \mathrm{nmol} / \mathrm{mL}$

Labs Omitted from Plot (But Not Analysis): 15

15: $100 \pm 5$ 


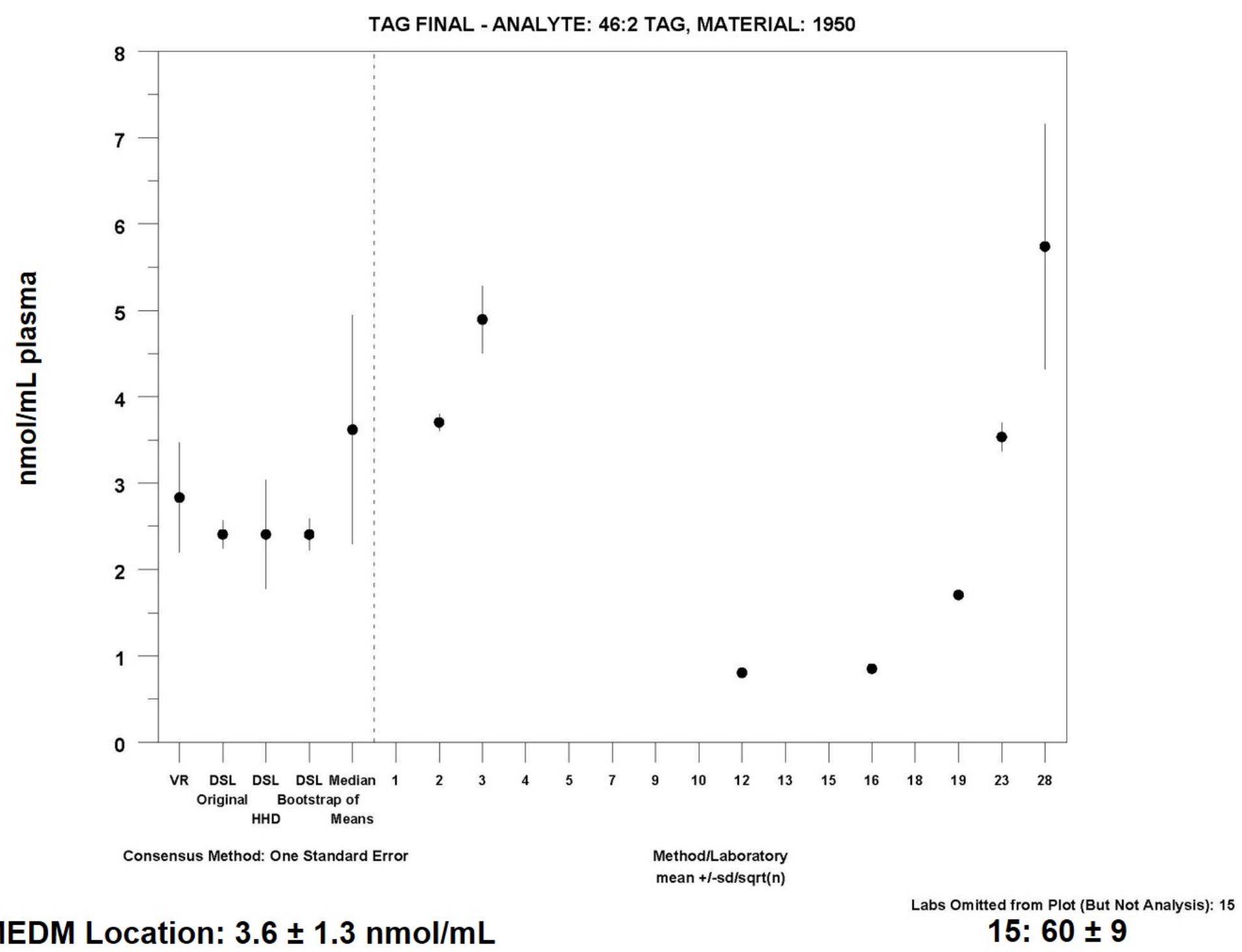




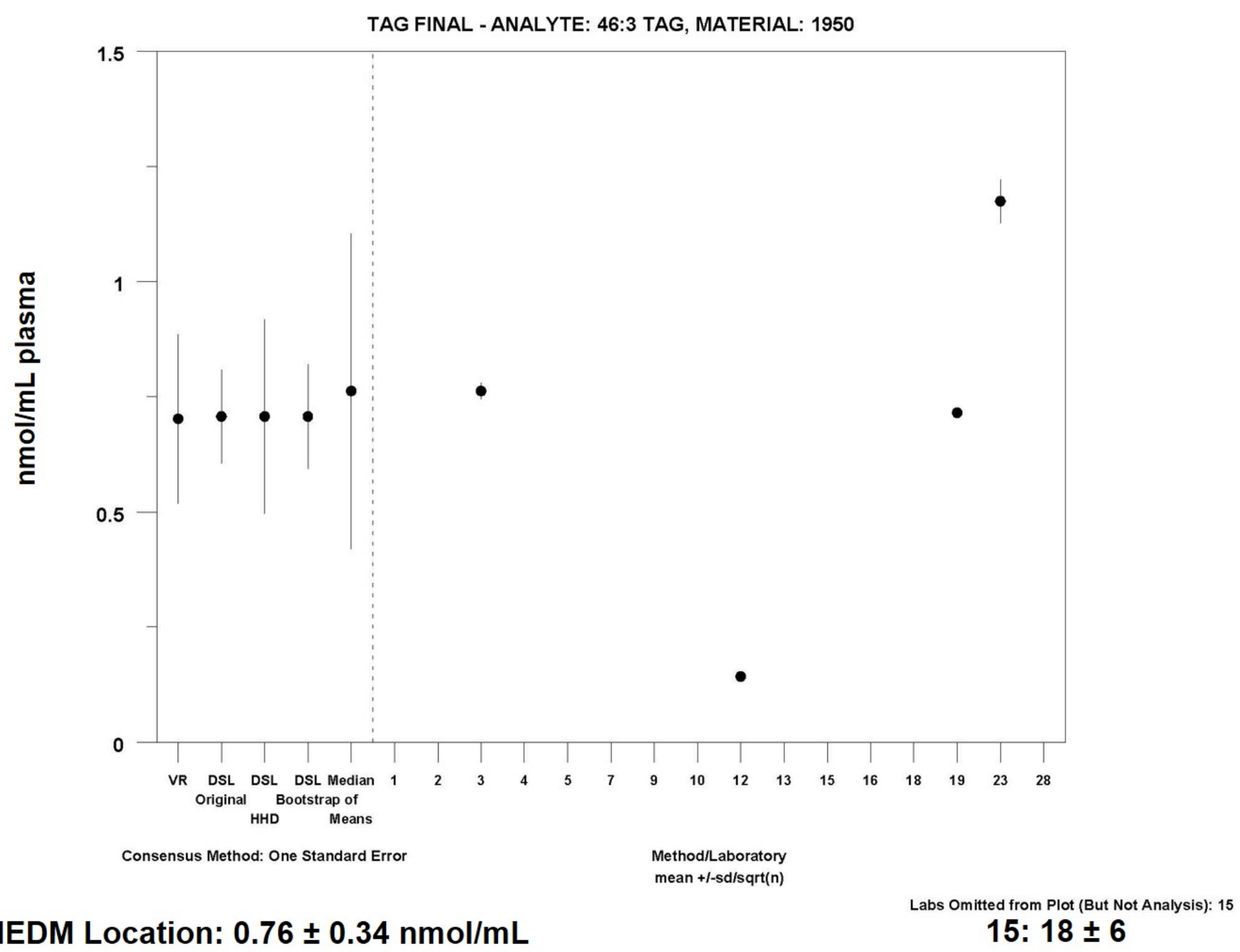




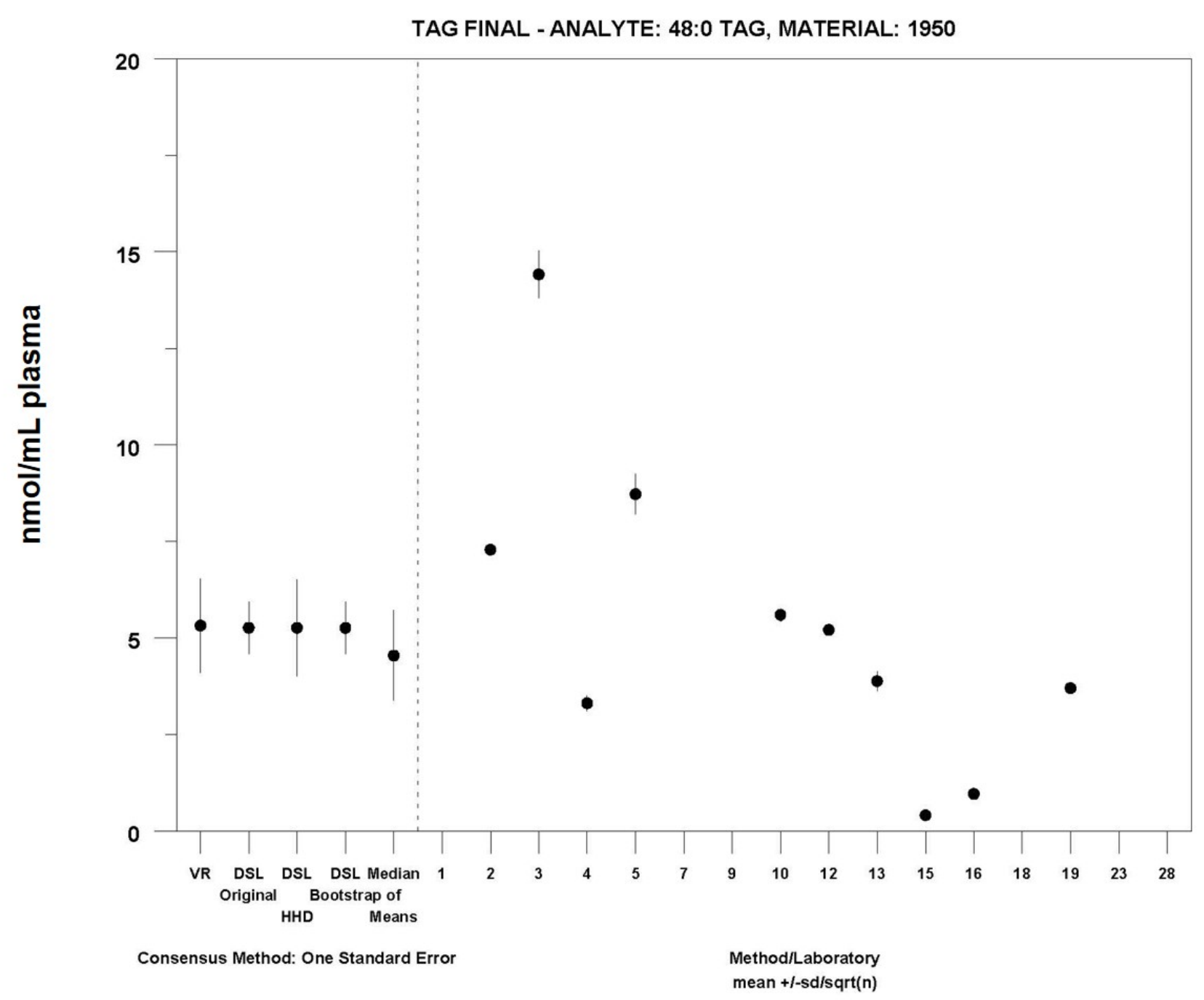

MEDM Location: $4.5 \pm 1.2 \mathrm{nmol} / \mathrm{mL}$ 


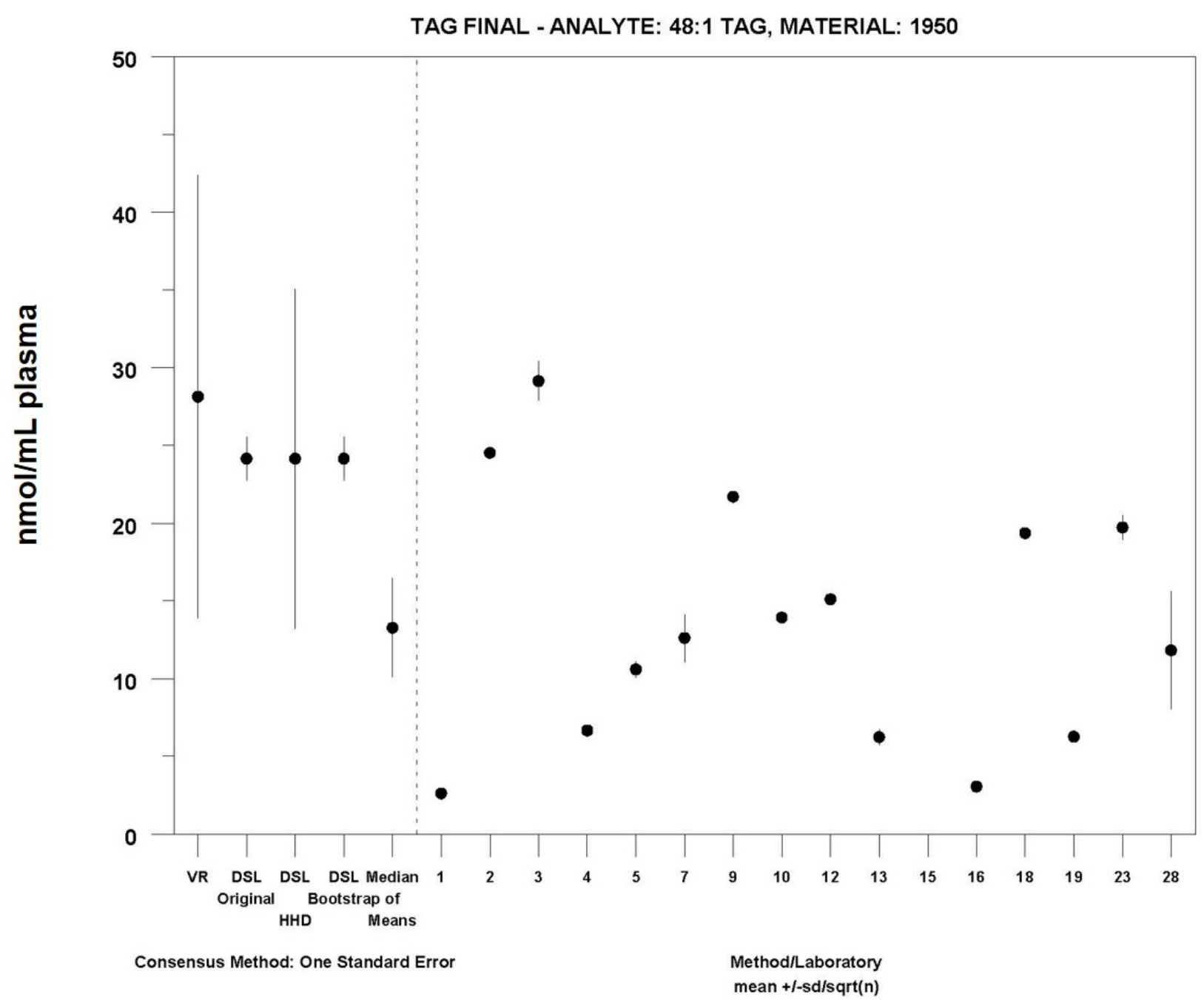

MEDM Location: $13 \pm 3.2 \mathrm{nmol} / \mathrm{mL}$

Labs Omitted from Plot (But Not Analysis): 15

15: $248 \pm 6$ 


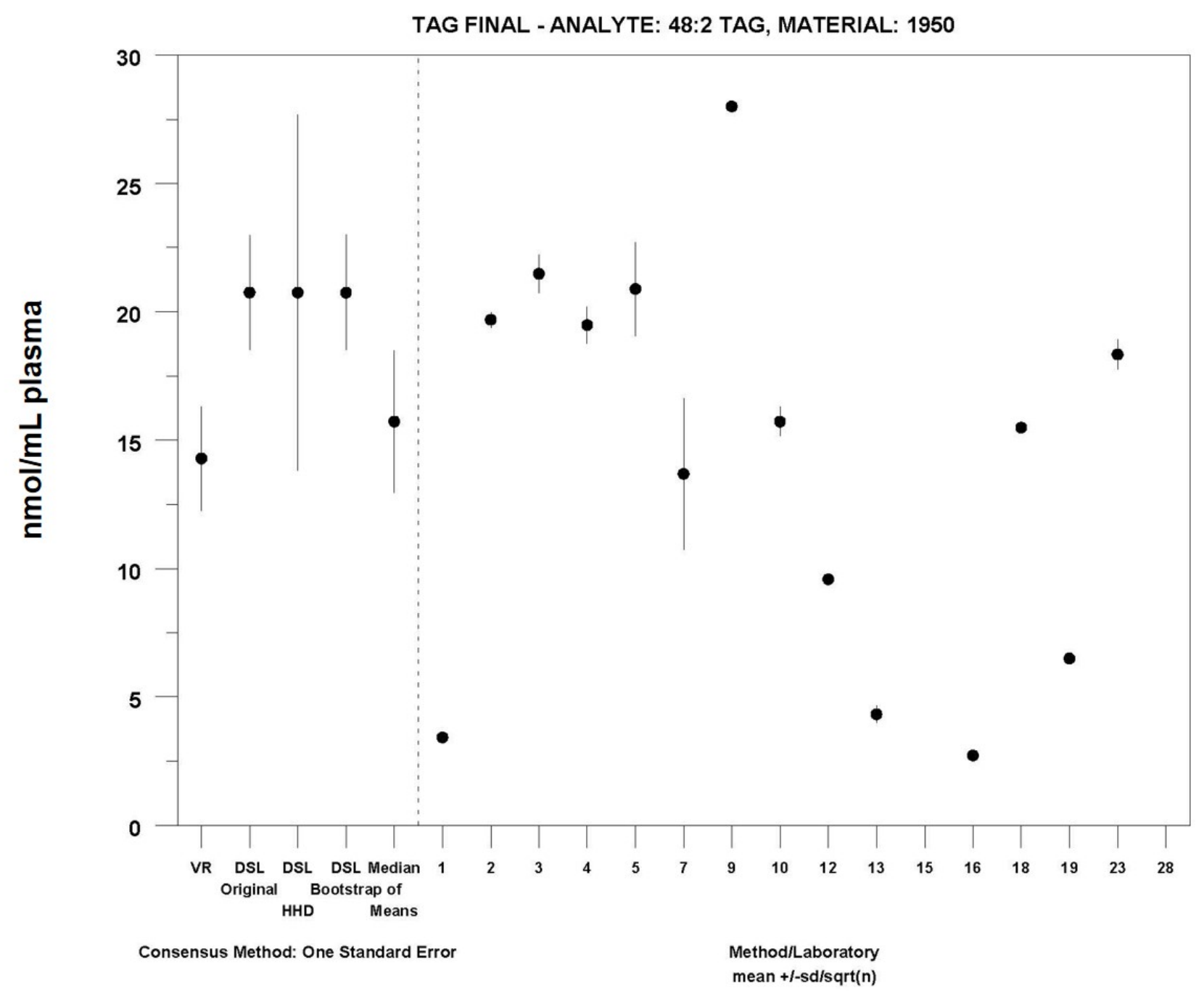

MEDM Location: $16 \pm 2.8 \mathrm{nmol} / \mathrm{mL}$

Labs Omitted from Plot (But Not Analysis): 15 15: $170 \pm 12$ 


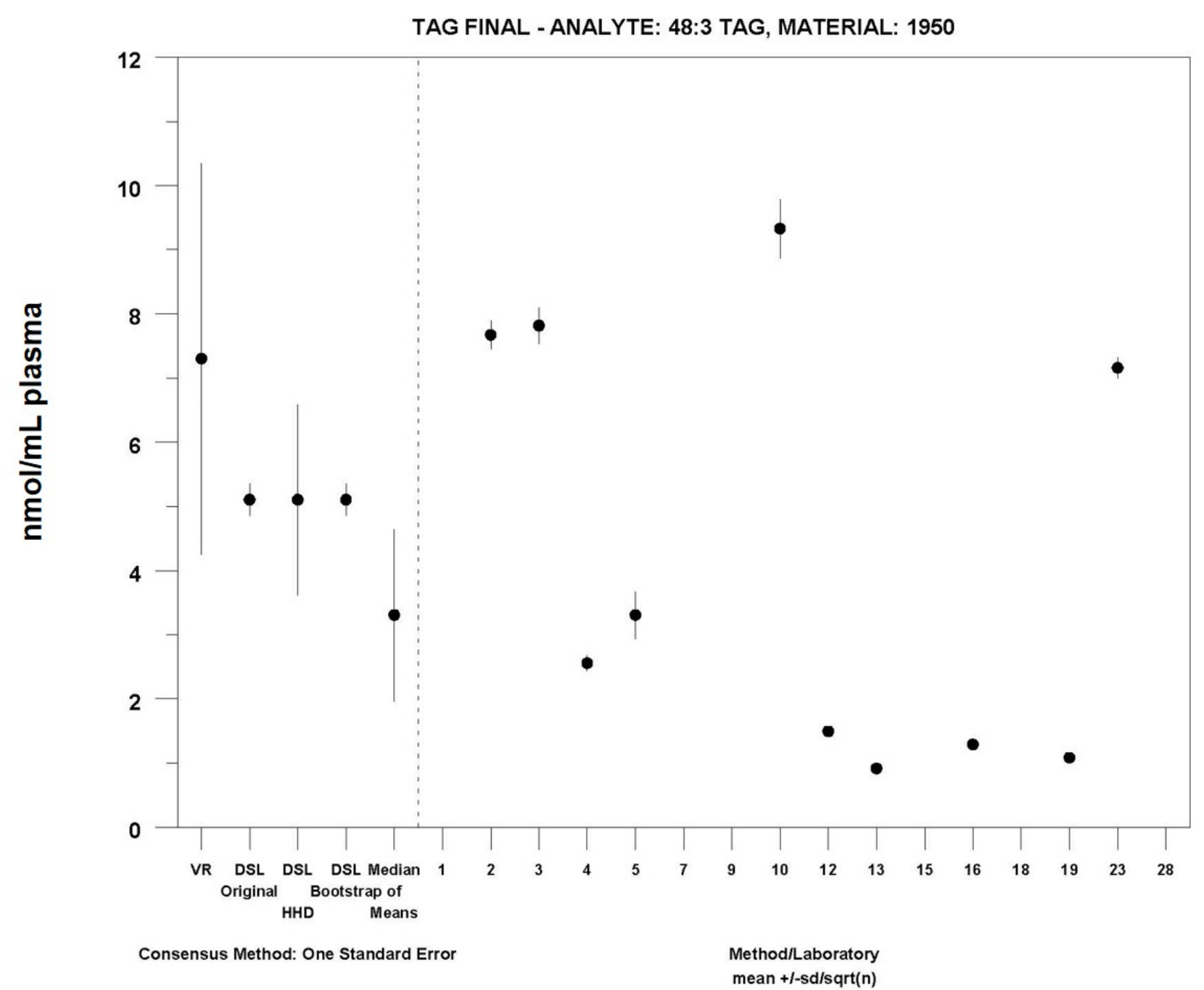

MEDM Location: $3.3 \pm 1.3 \mathrm{nmol} / \mathrm{mL}$

Labs Omitted from Plot (But Not Analysis): 15 15: $38 \pm 2$ 


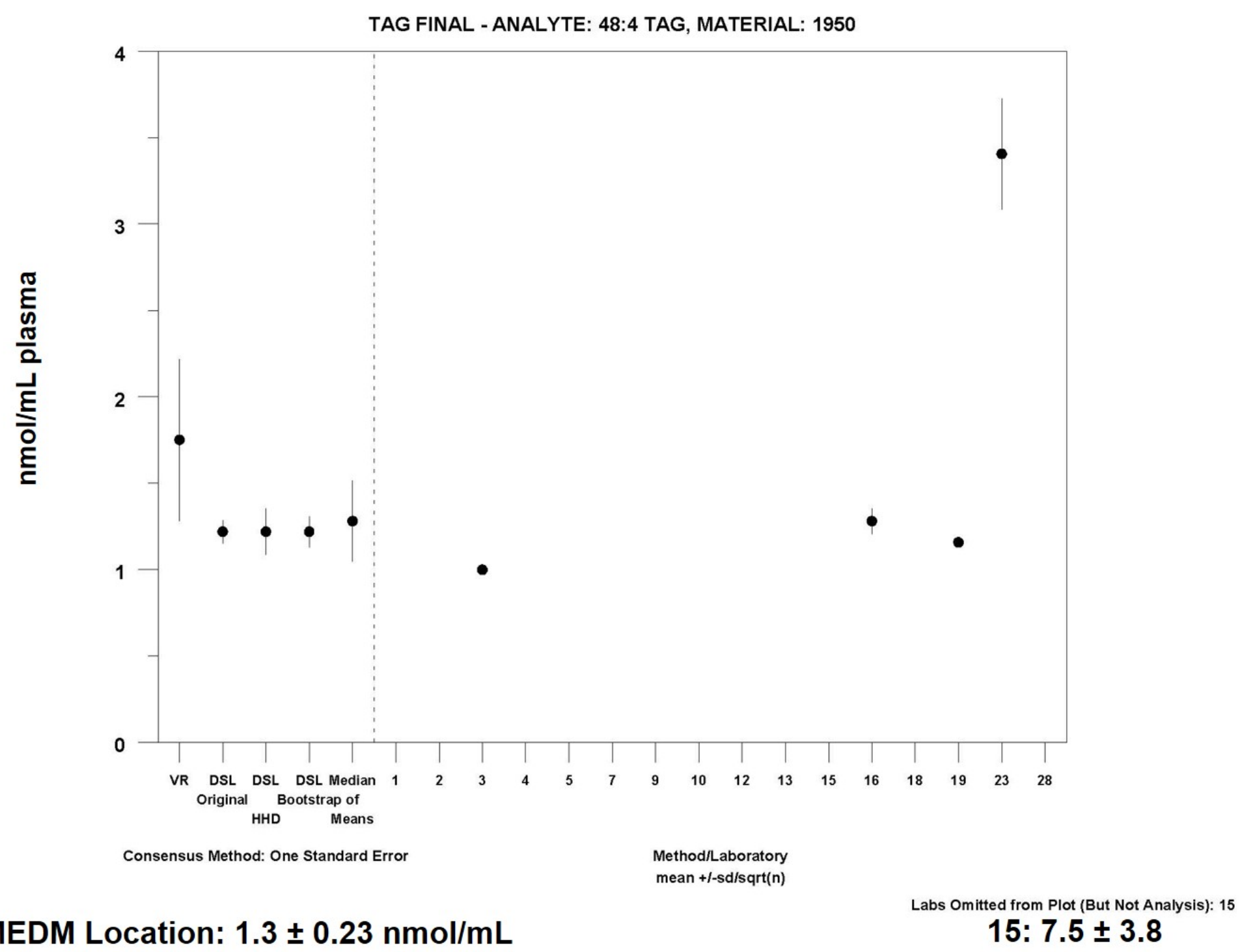




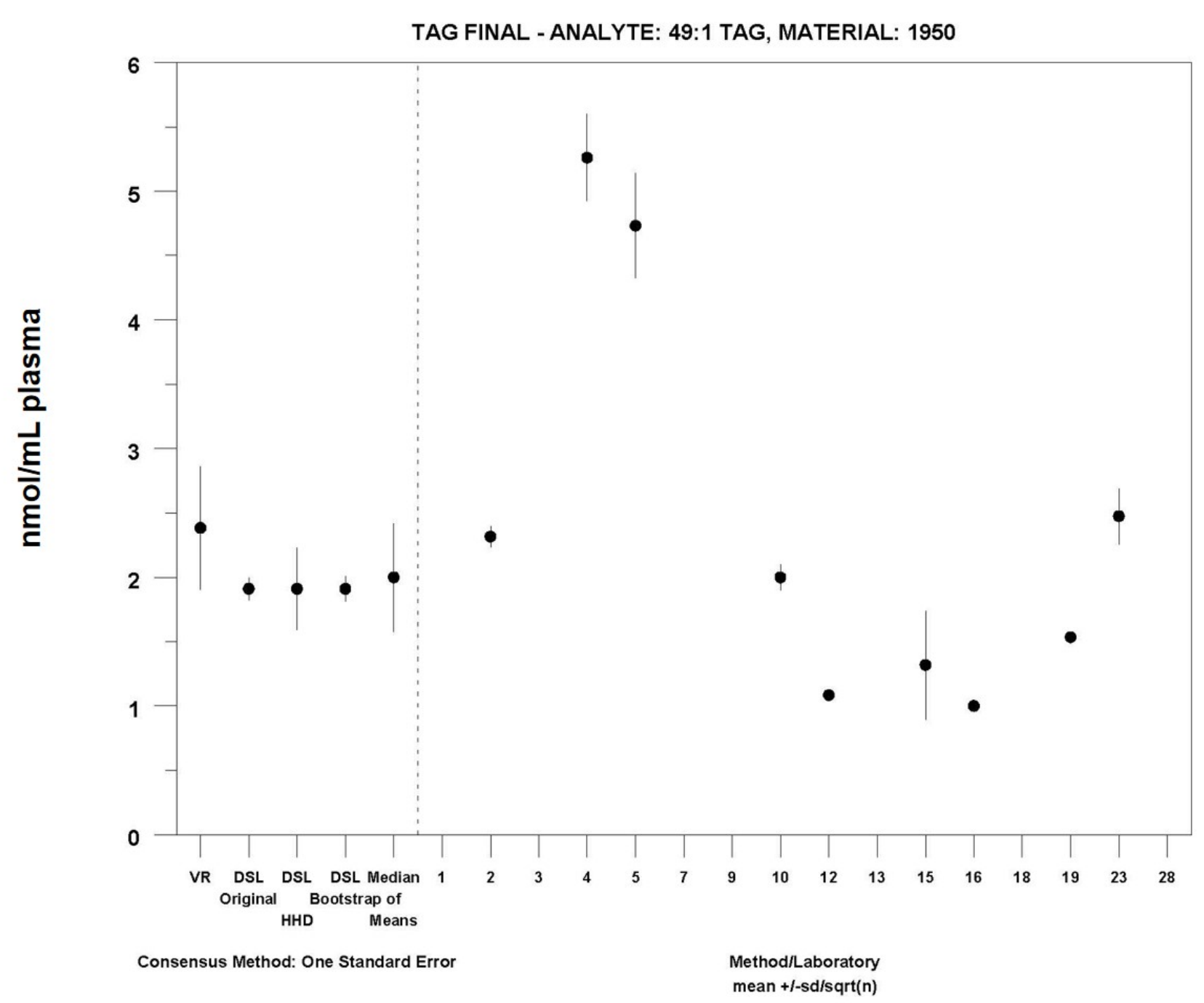

MEDM Location: $2.0 \pm 0.42 \mathrm{nmol} / \mathrm{mL}$ 


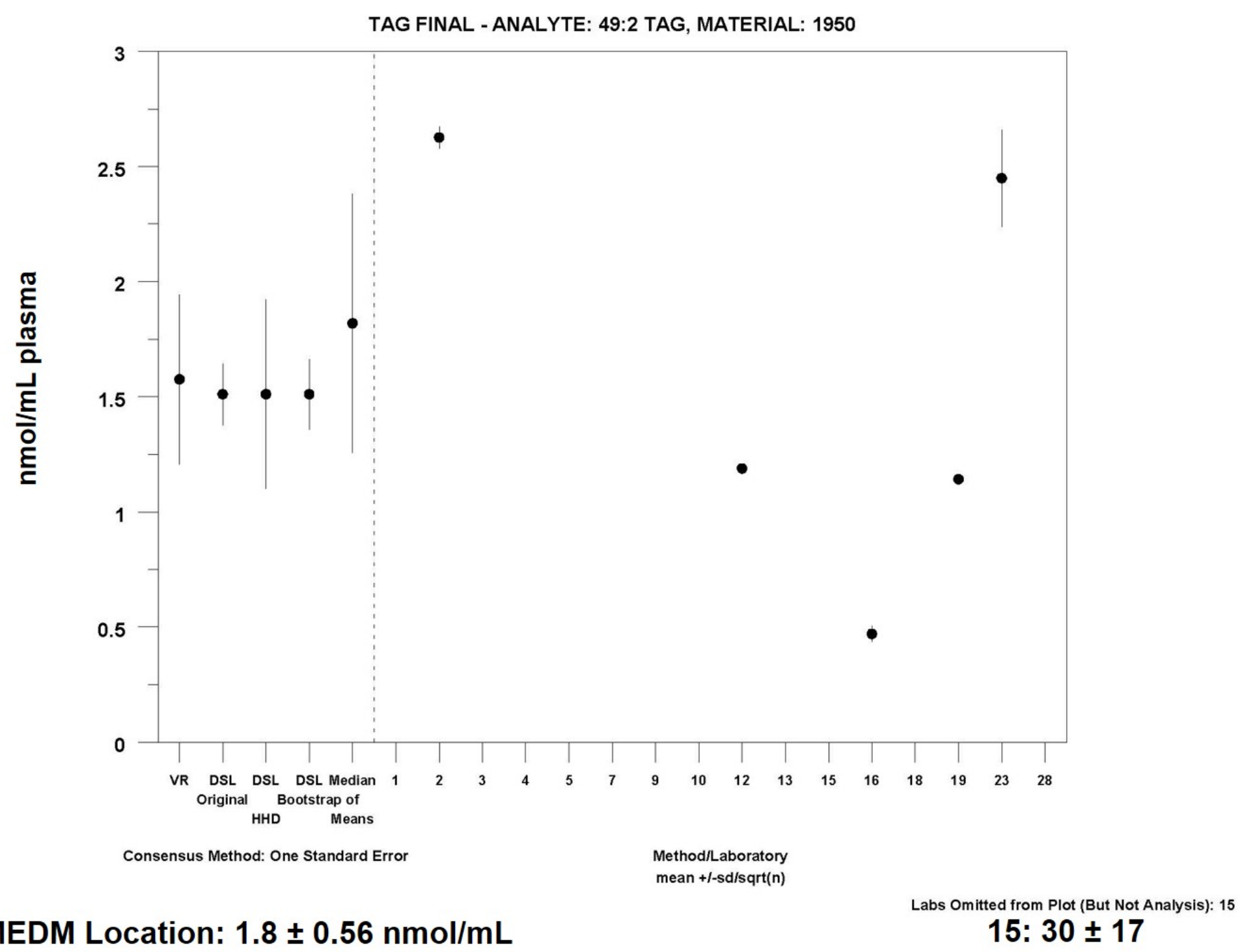




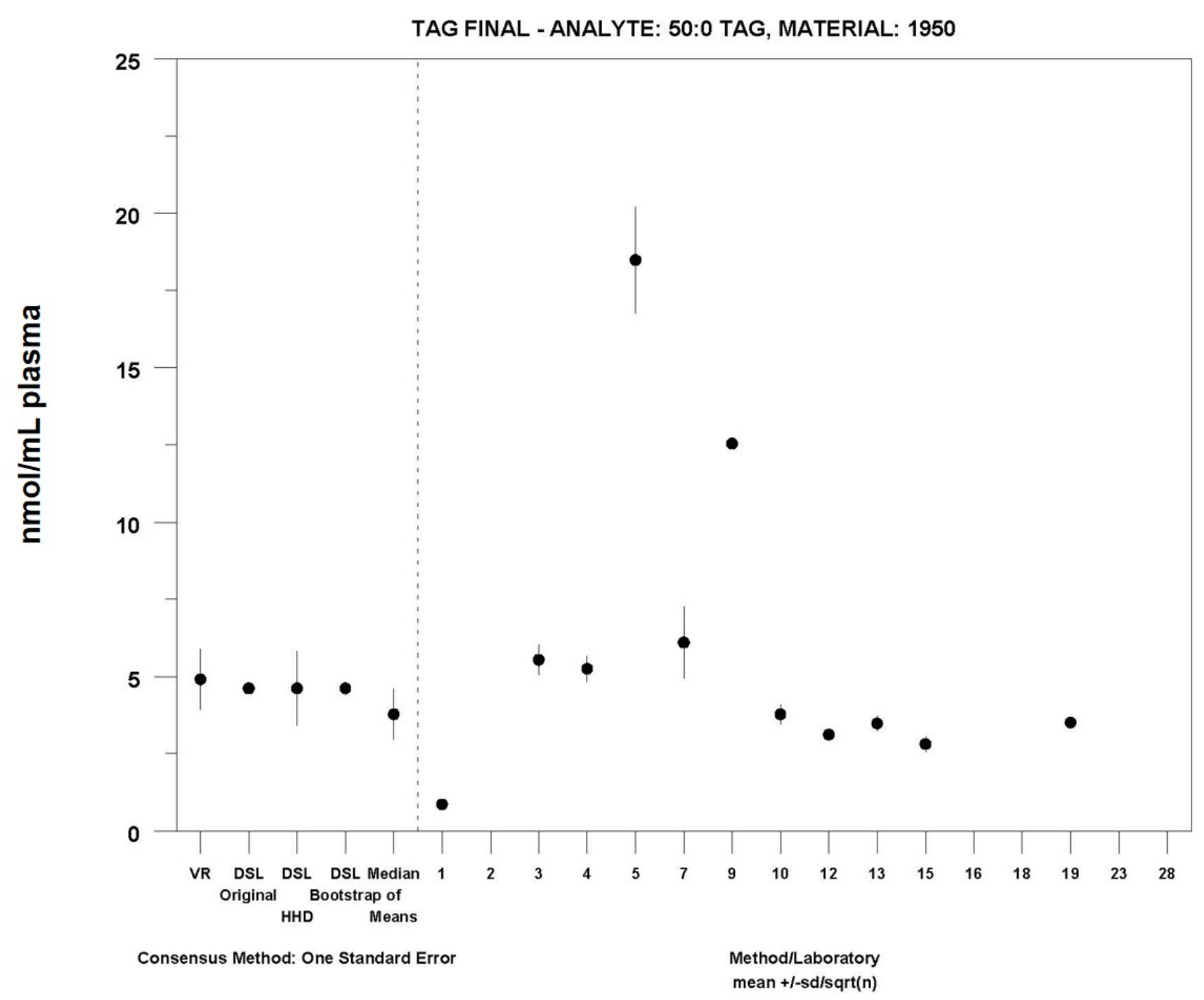

MEDM Location: $3.8 \pm 0.83 \mathrm{nmol} / \mathrm{mL}$ 


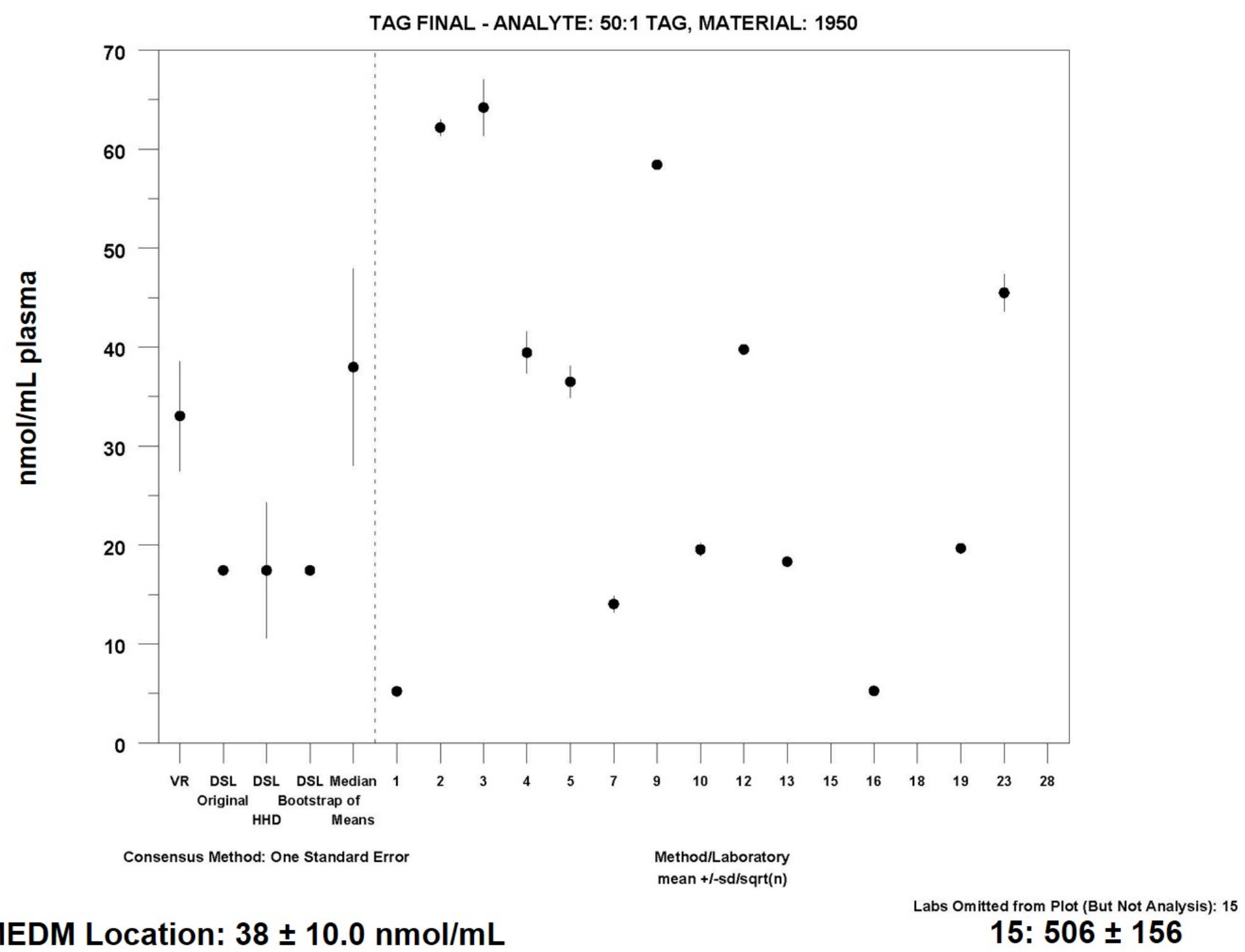




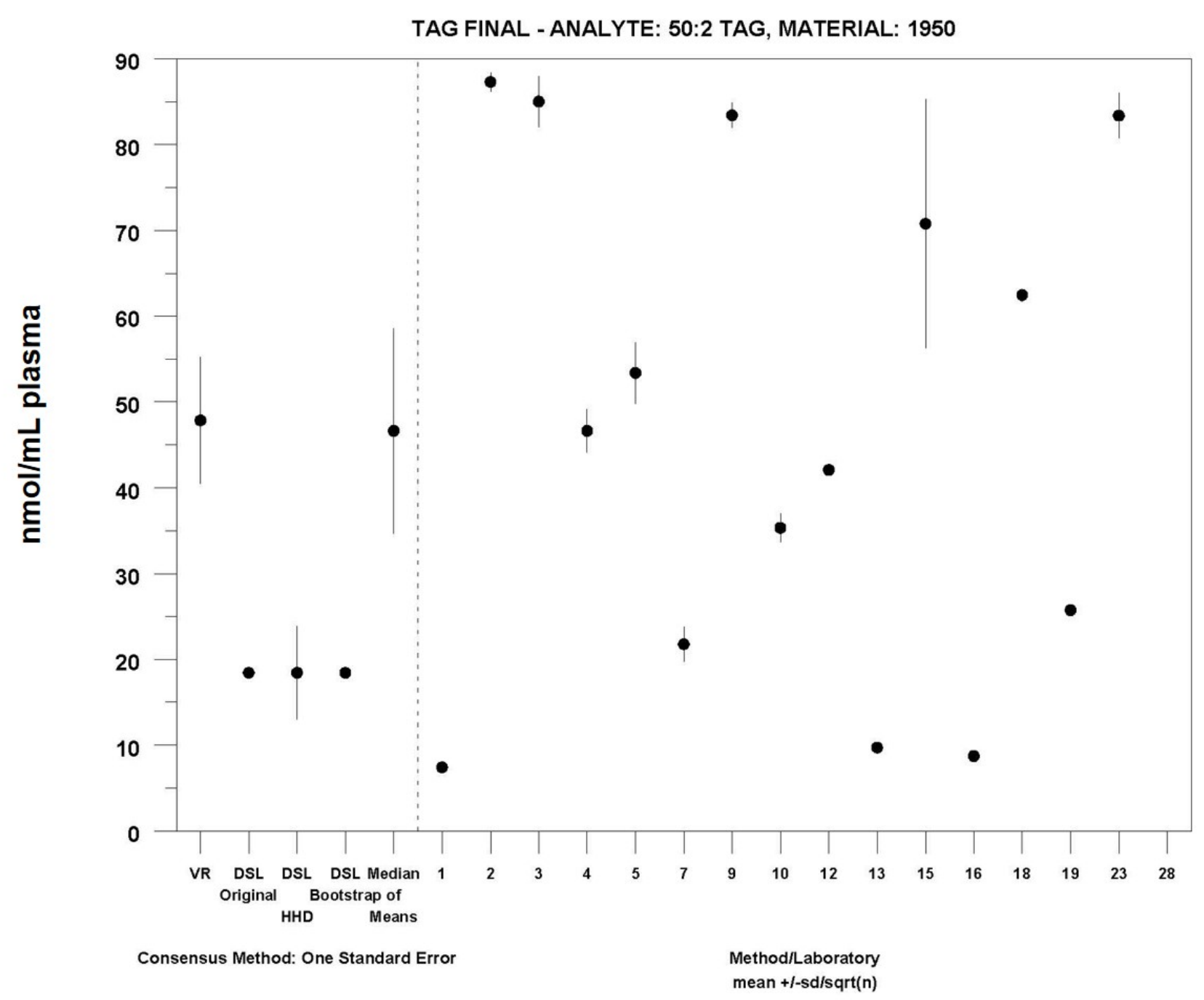

MEDM Location: $47 \pm 12 \mathrm{nmol} / \mathrm{mL}$ 


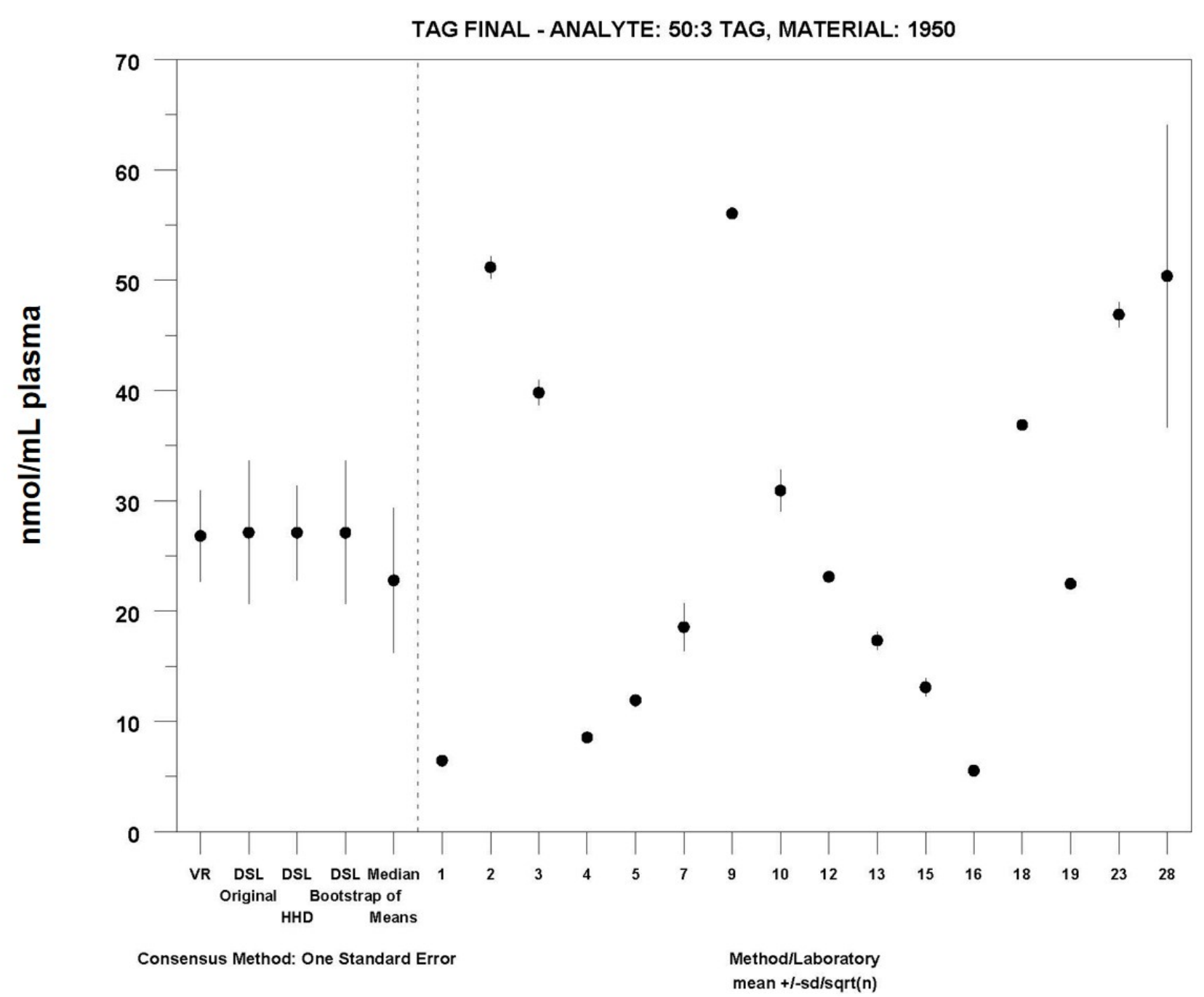

MEDM Location: $23 \pm 6.6 \mathrm{nmol} / \mathrm{mL}$ 


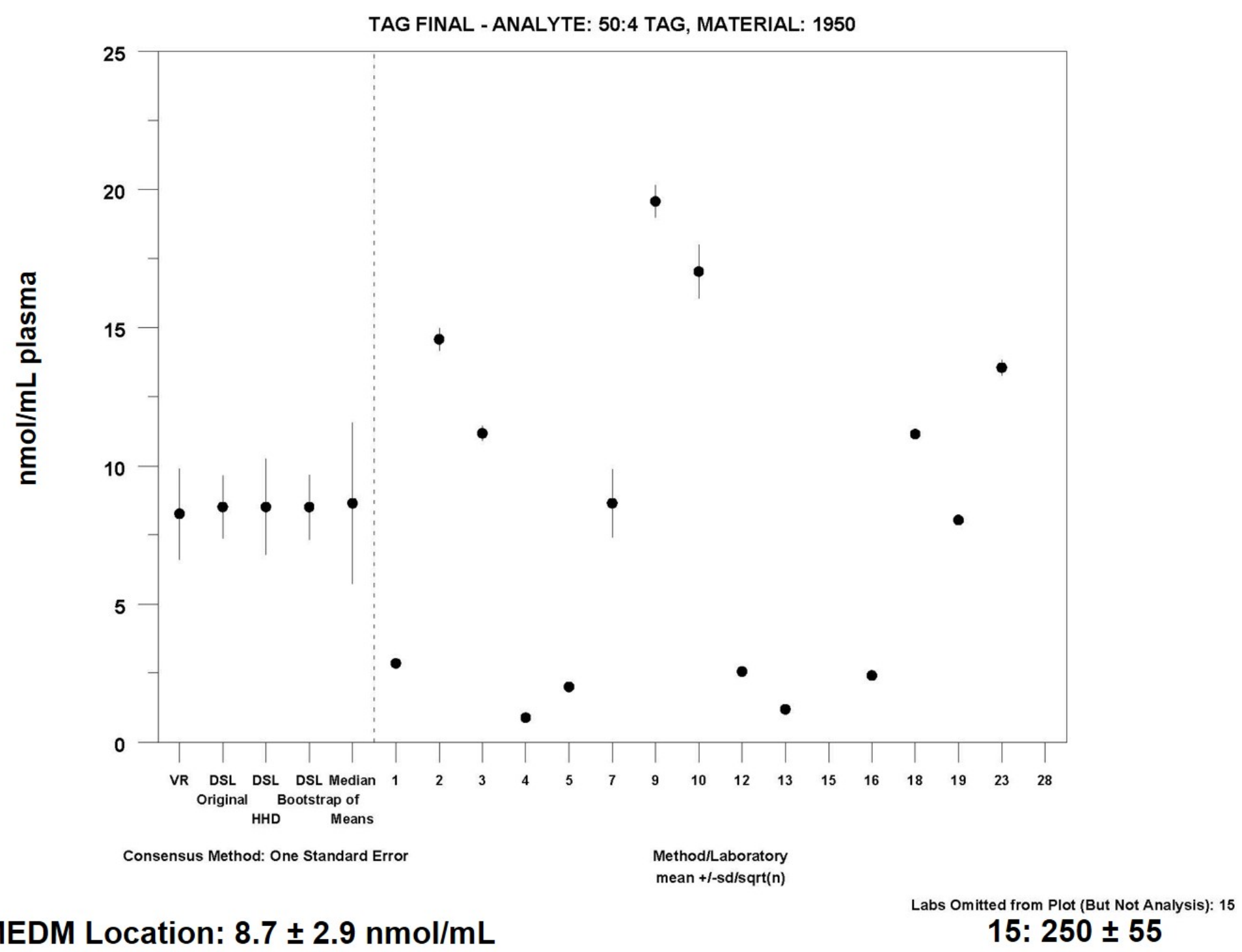




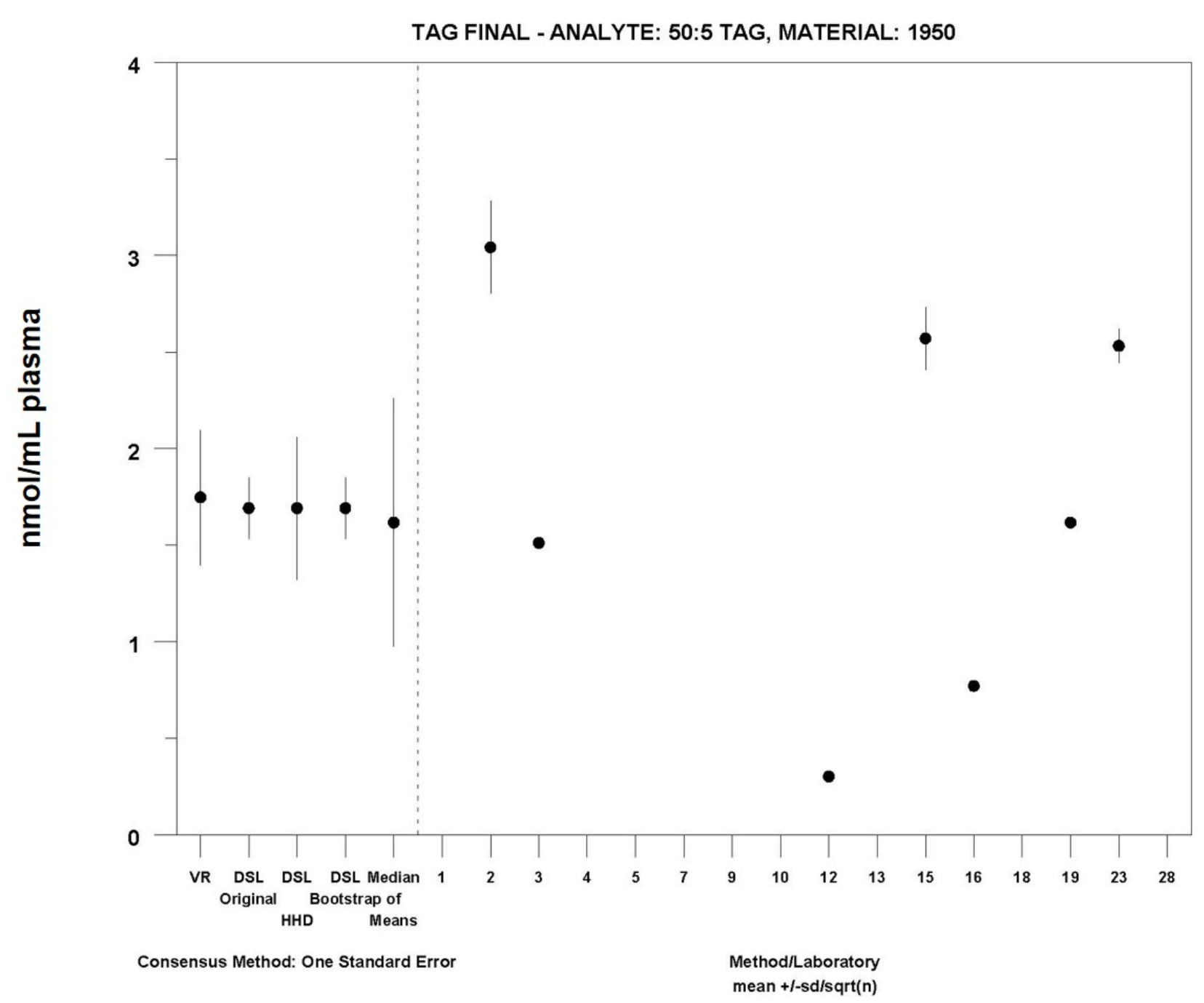

MEDM Location: $1.6 \pm 0.64 \mathrm{nmol} / \mathrm{mL}$ 


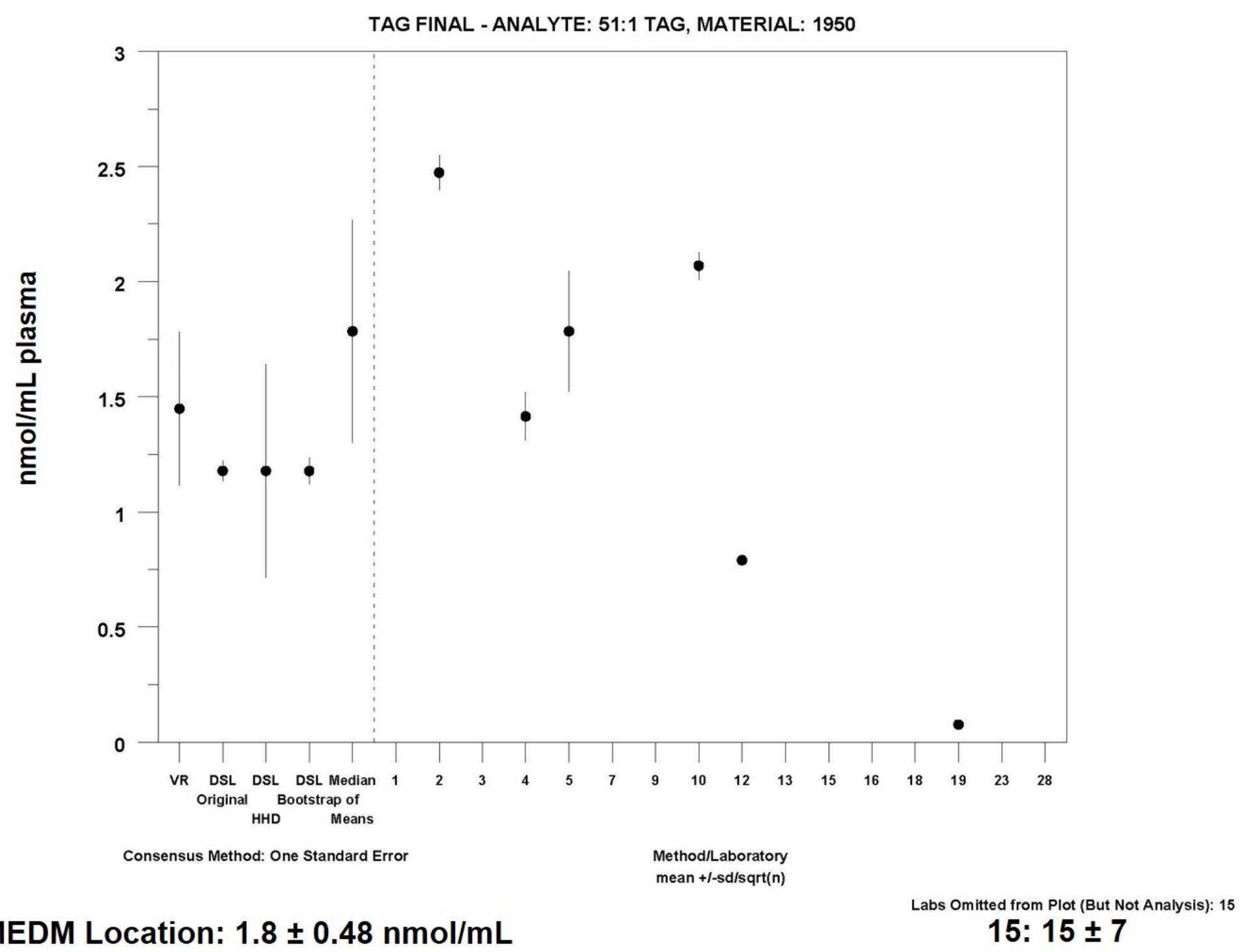




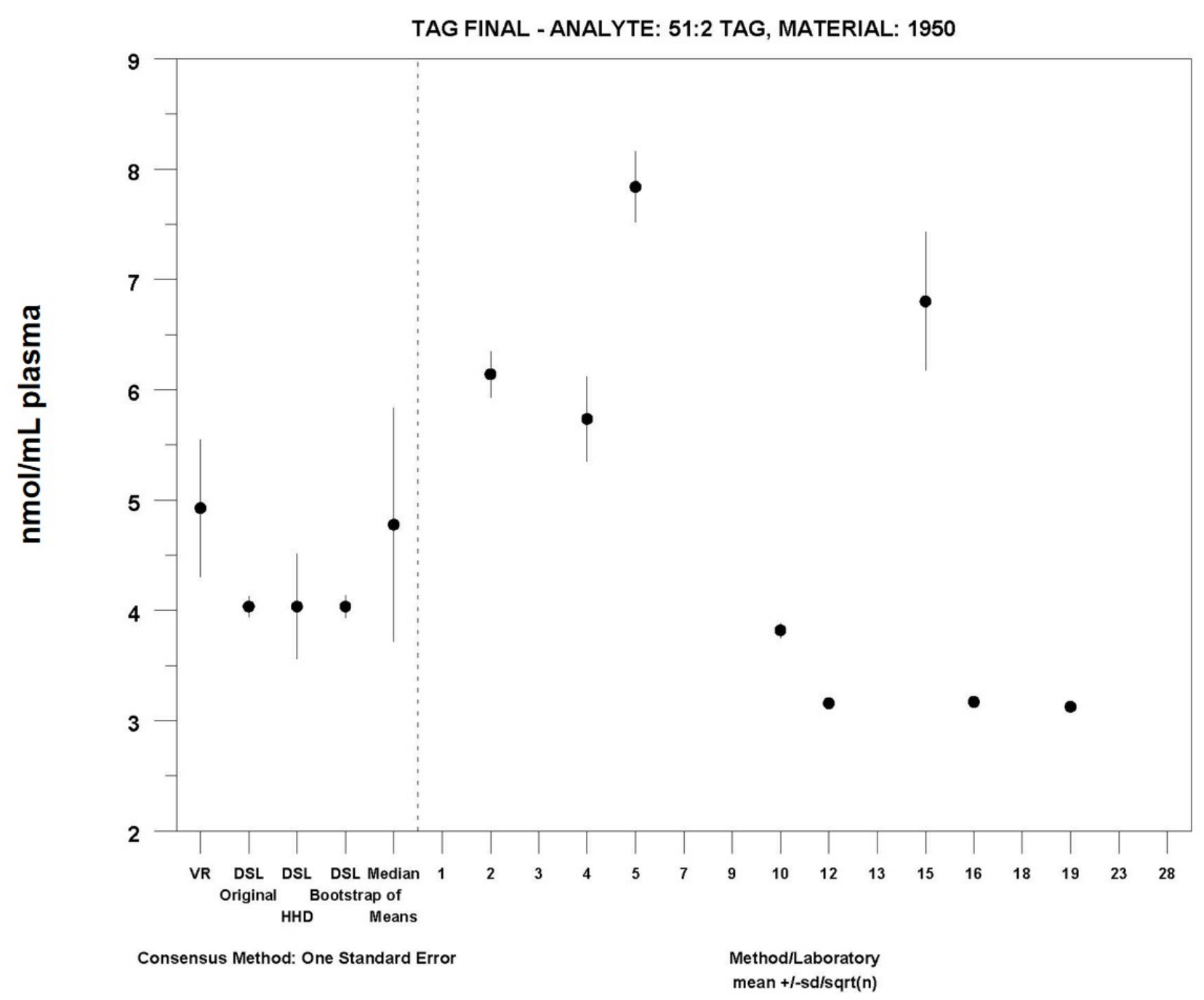

MEDM Location: $4.8 \pm 1.1 \mathrm{nmol} / \mathrm{mL}$ 


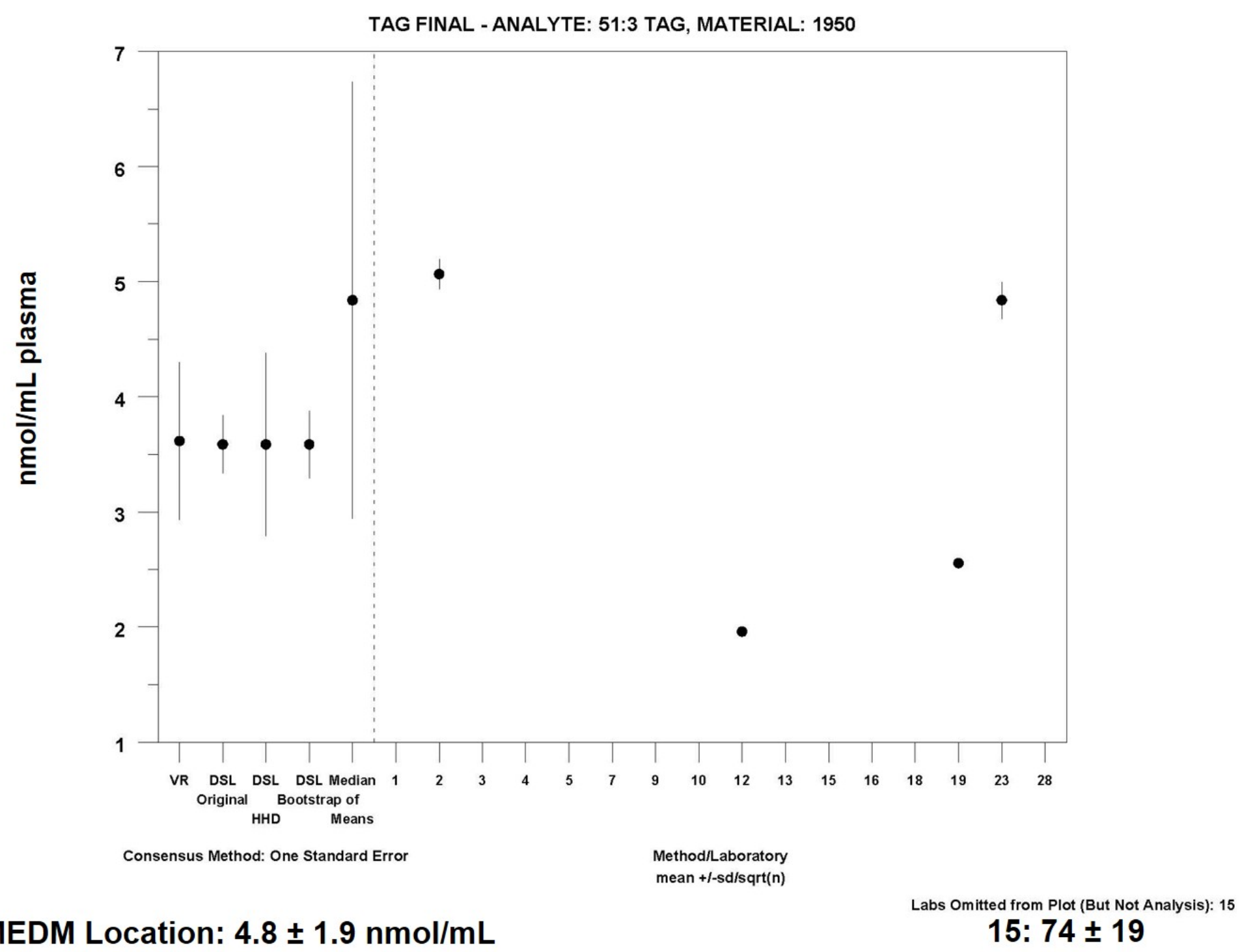




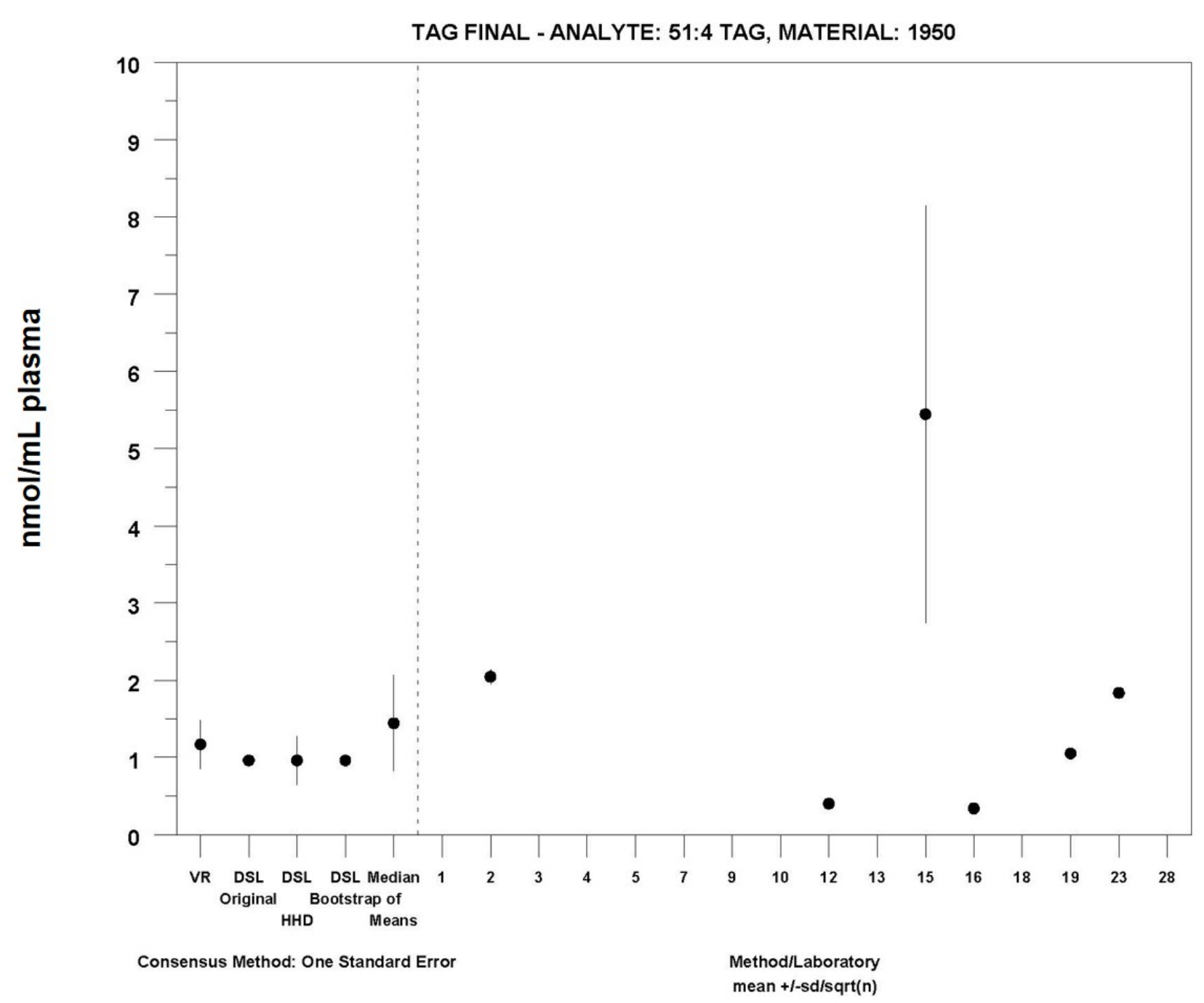

MEDM Location: $1.4 \pm 0.62 \mathrm{nmol} / \mathrm{mL}$ 


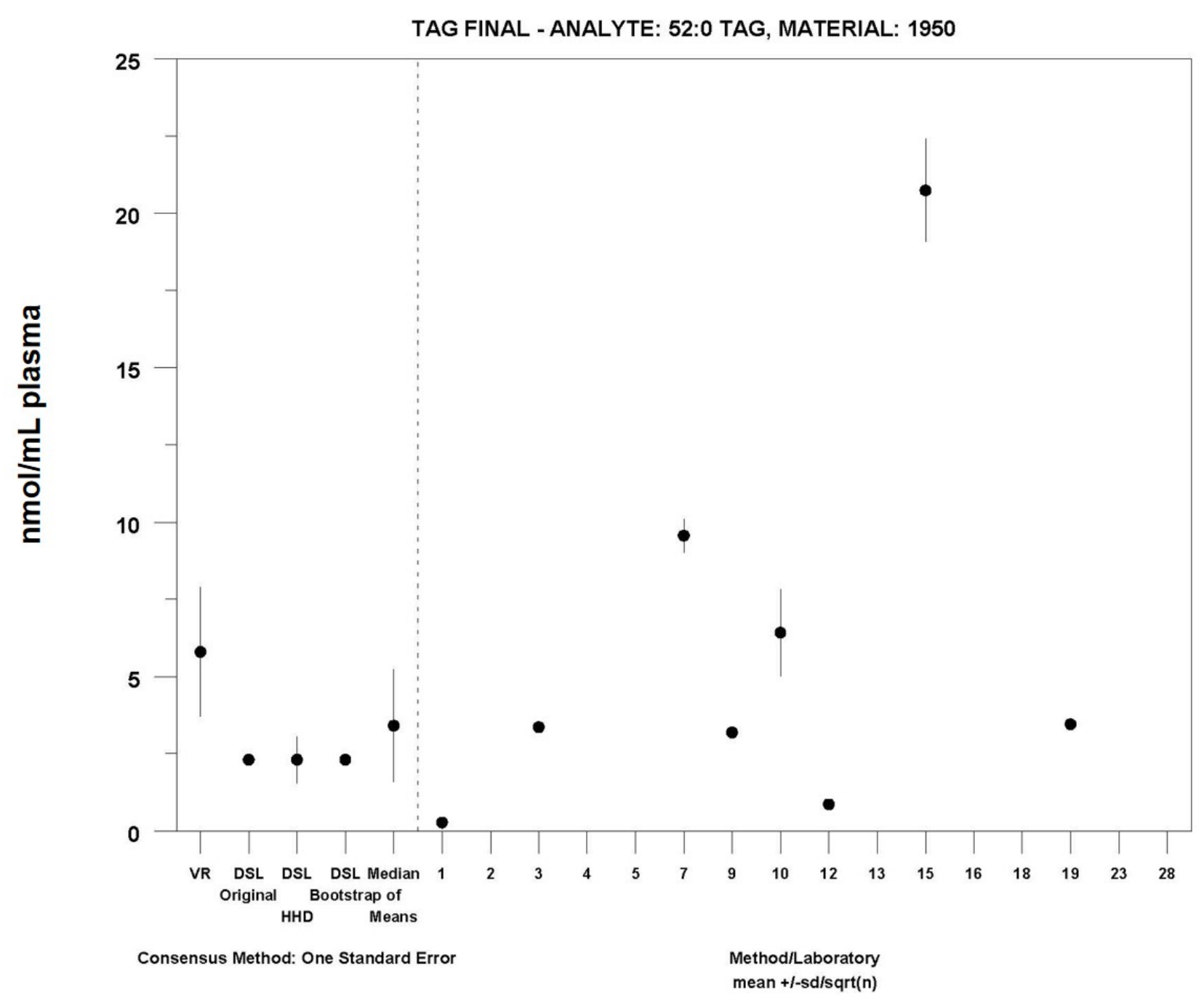

MEDM Location: $3.4 \pm 1.8 \mathrm{nmol} / \mathrm{mL}$ 


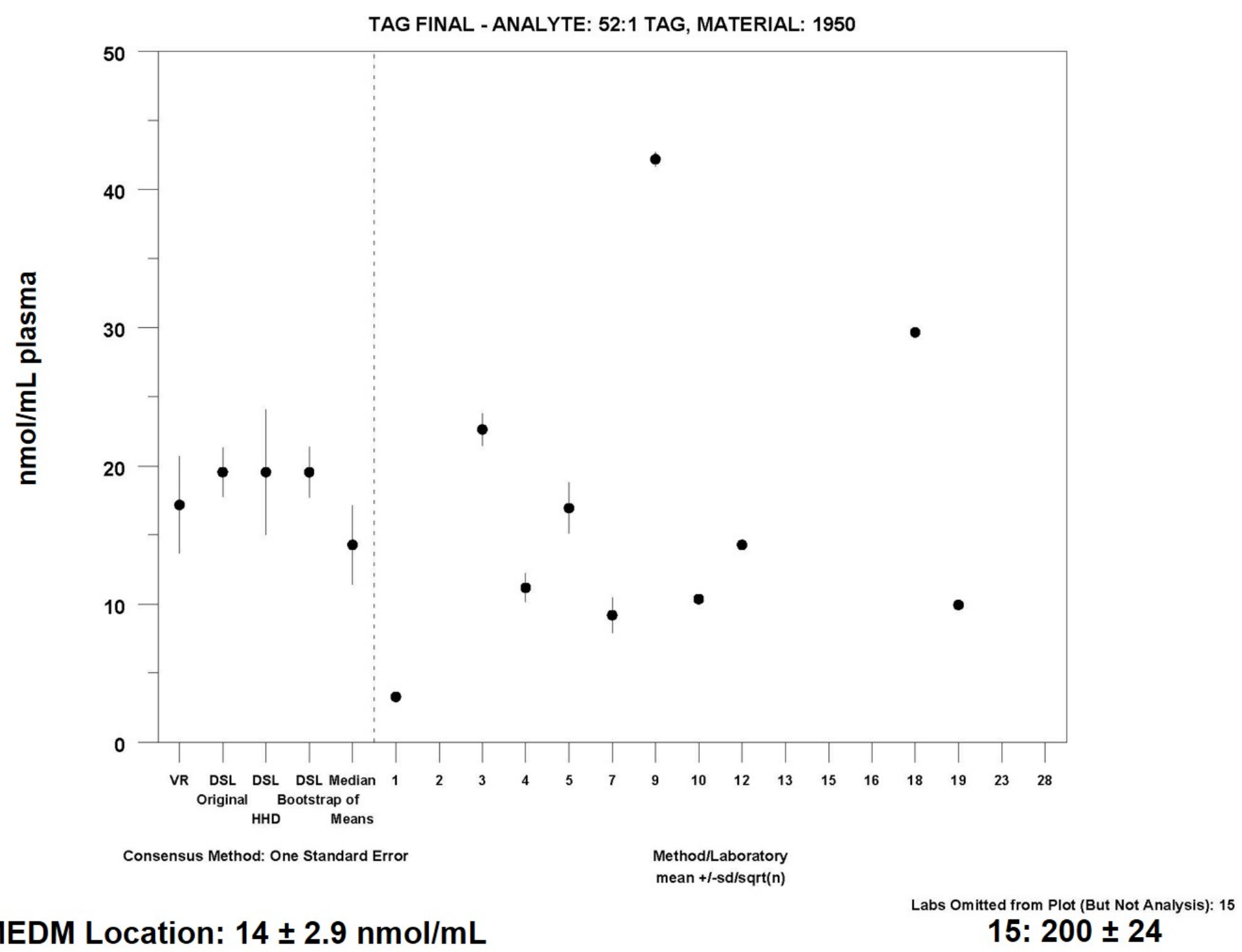




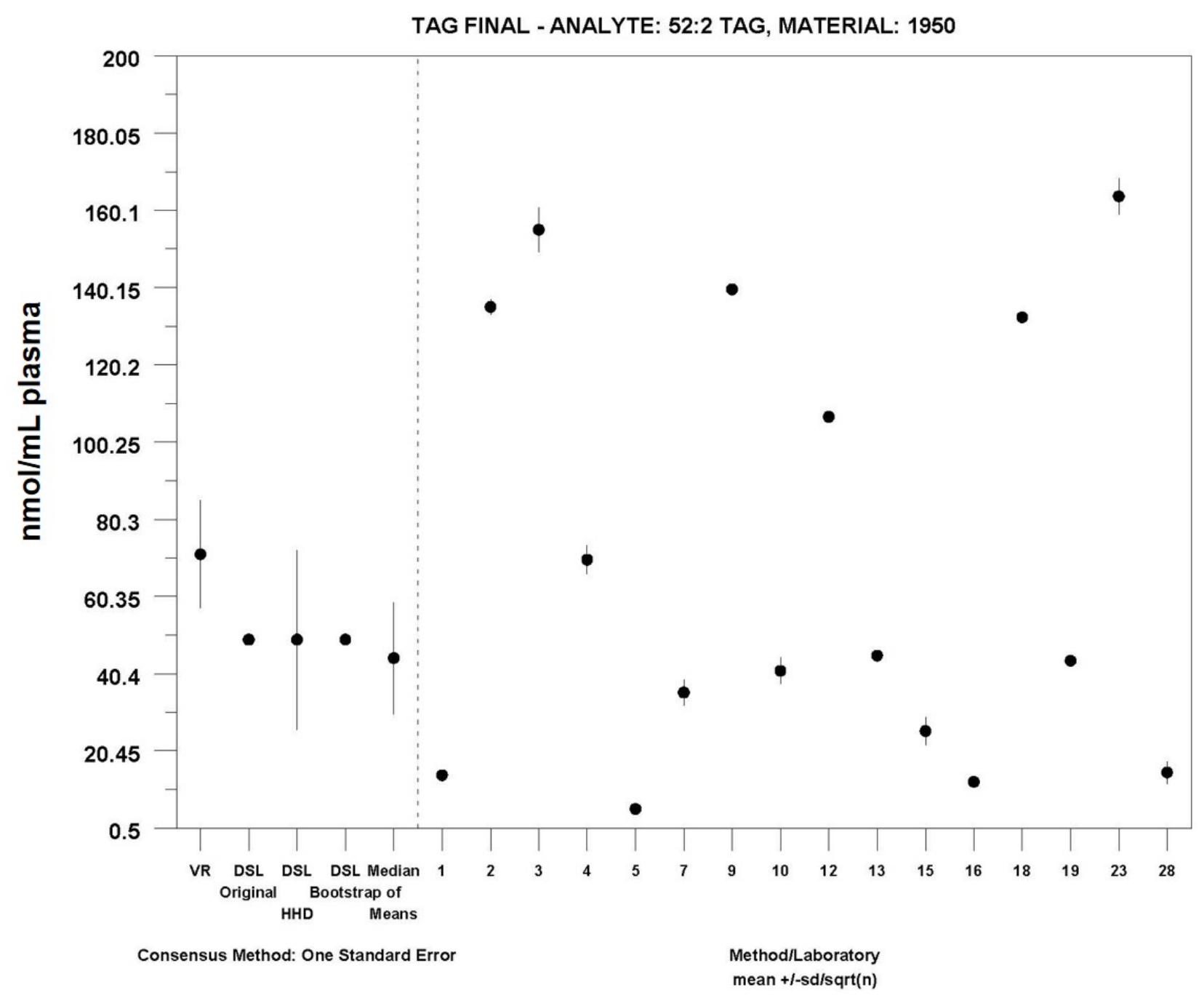

MEDM Location: $44 \pm 14 \mathrm{nmol} / \mathrm{mL}$ 


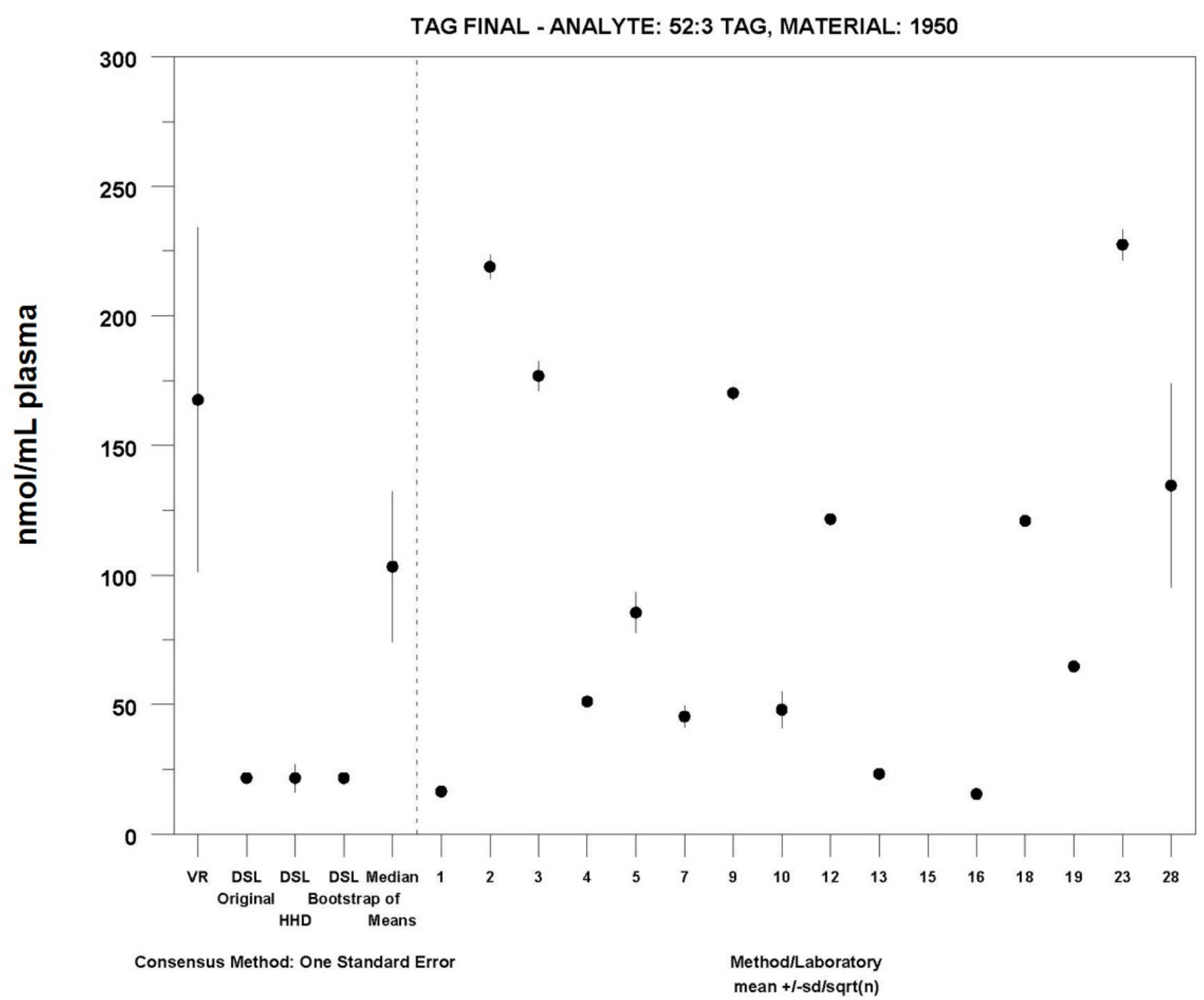

MEDM Location: $100 \pm 29 \mathrm{nmol} / \mathrm{mL}$

Labs Omitted from Plot (But Not Analysis): 15

15: $1166 \pm 35$ 


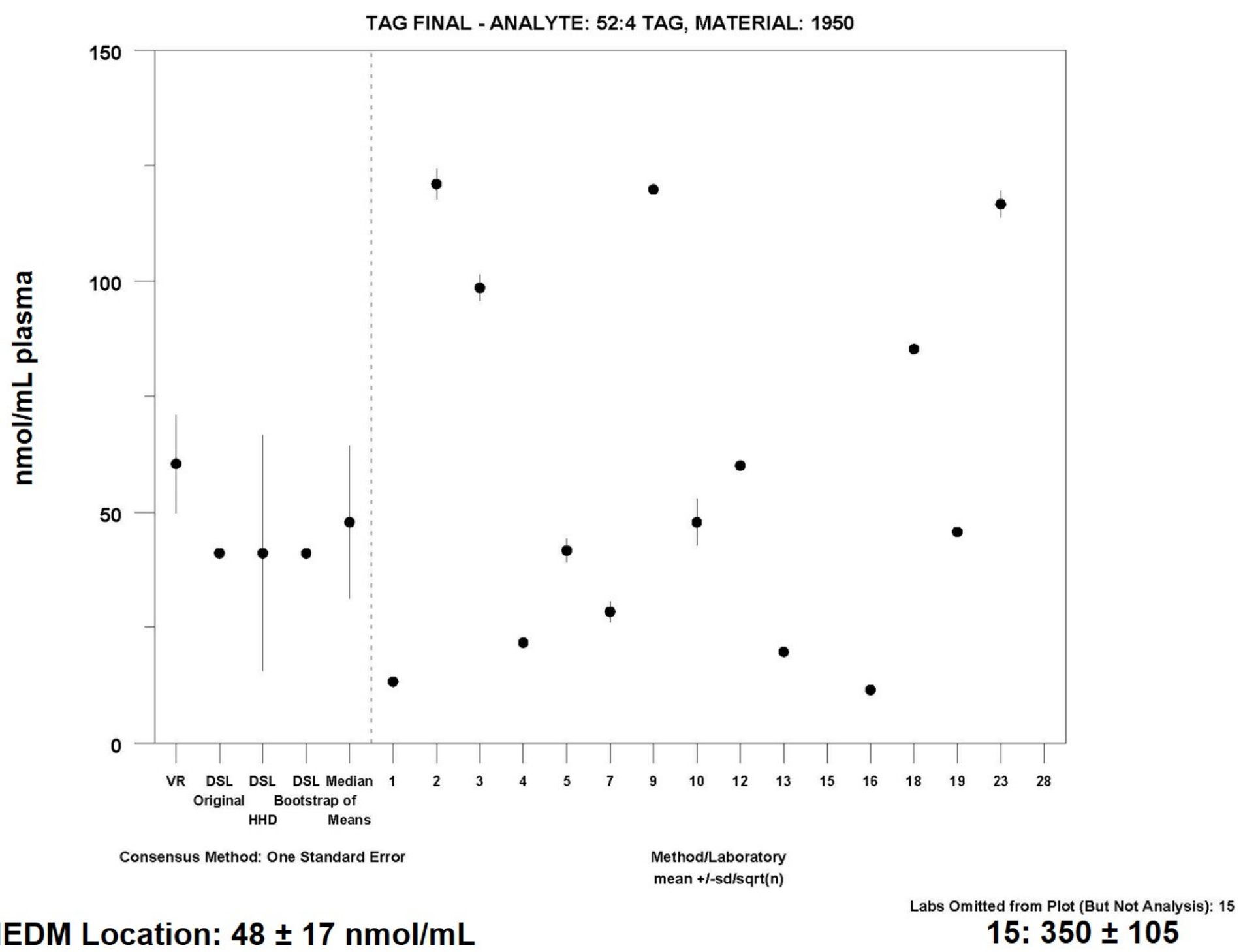




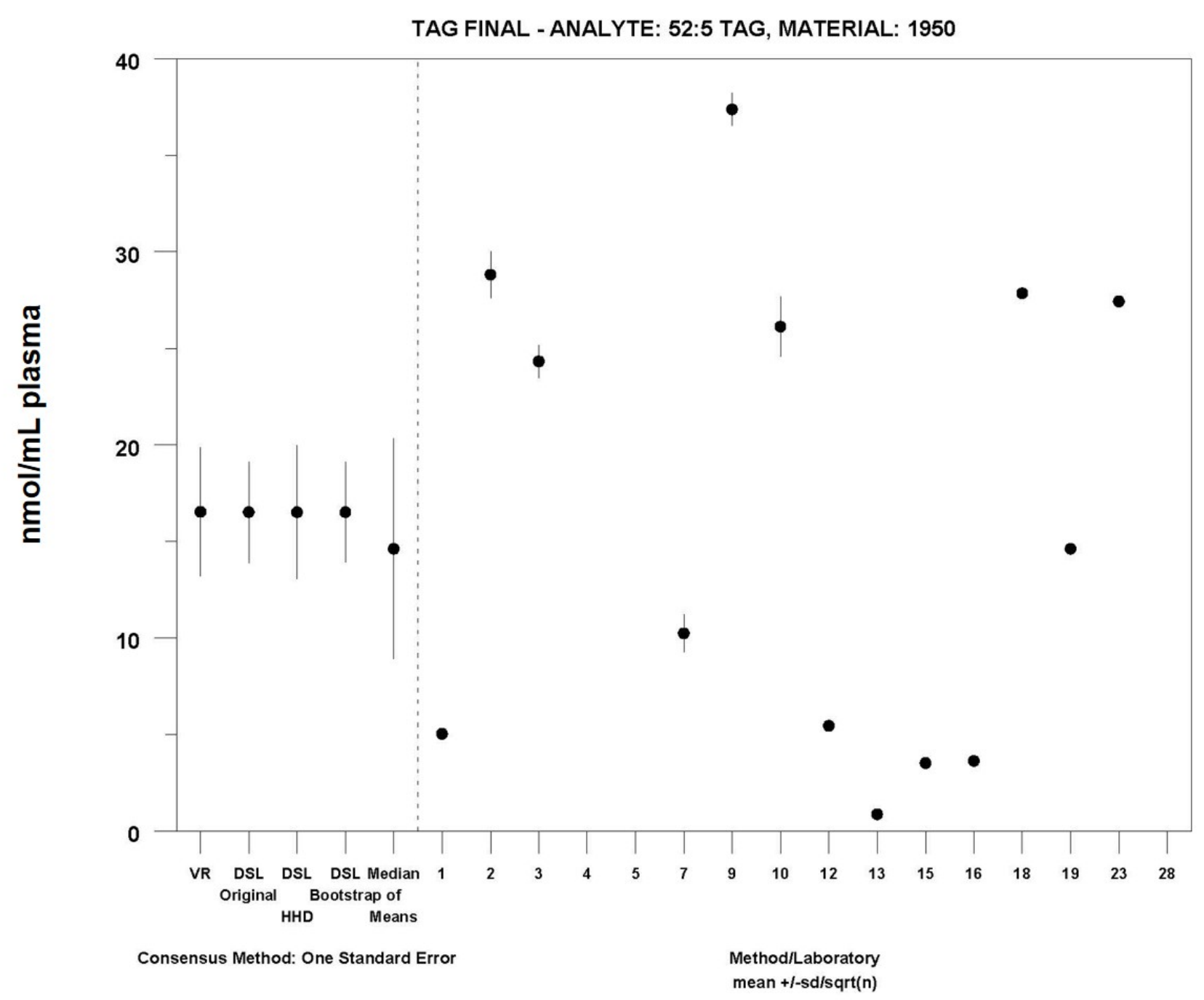

MEDM Location: $15 \pm 5.7 \mathrm{nmol} / \mathrm{mL}$ 


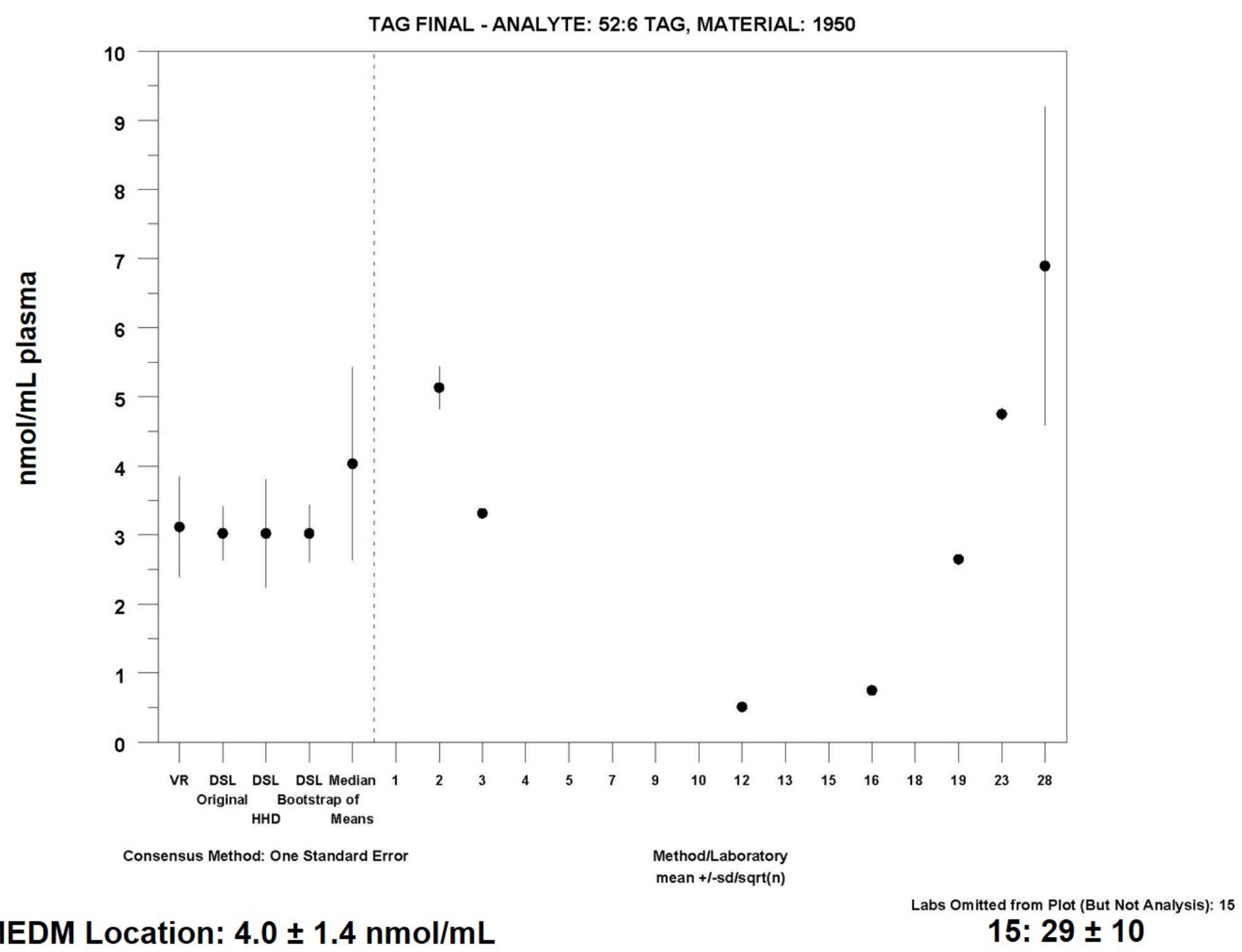




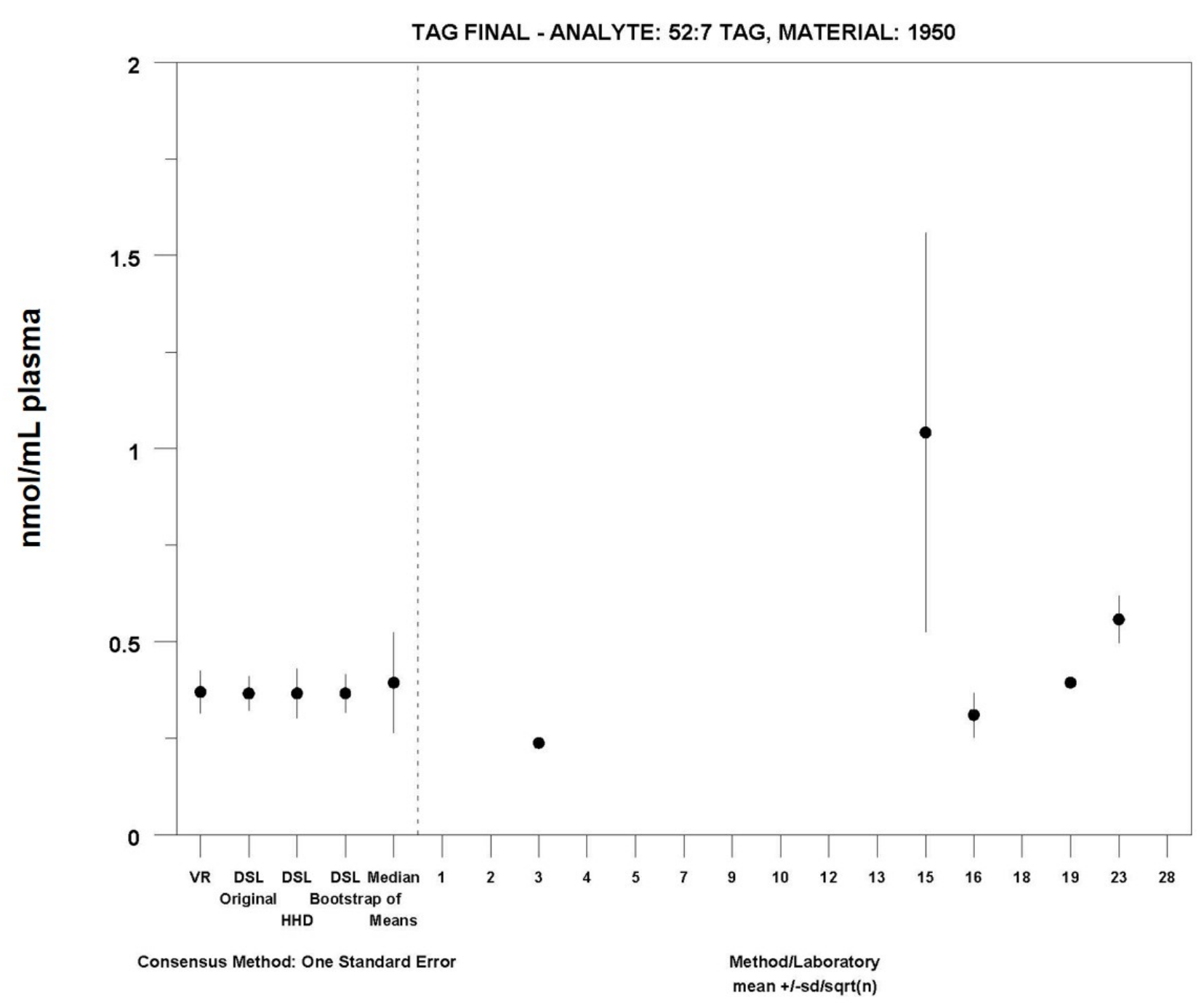

MEDM Location: $0.39 \pm 0.13 \mathrm{nmol} / \mathrm{mL}$ 


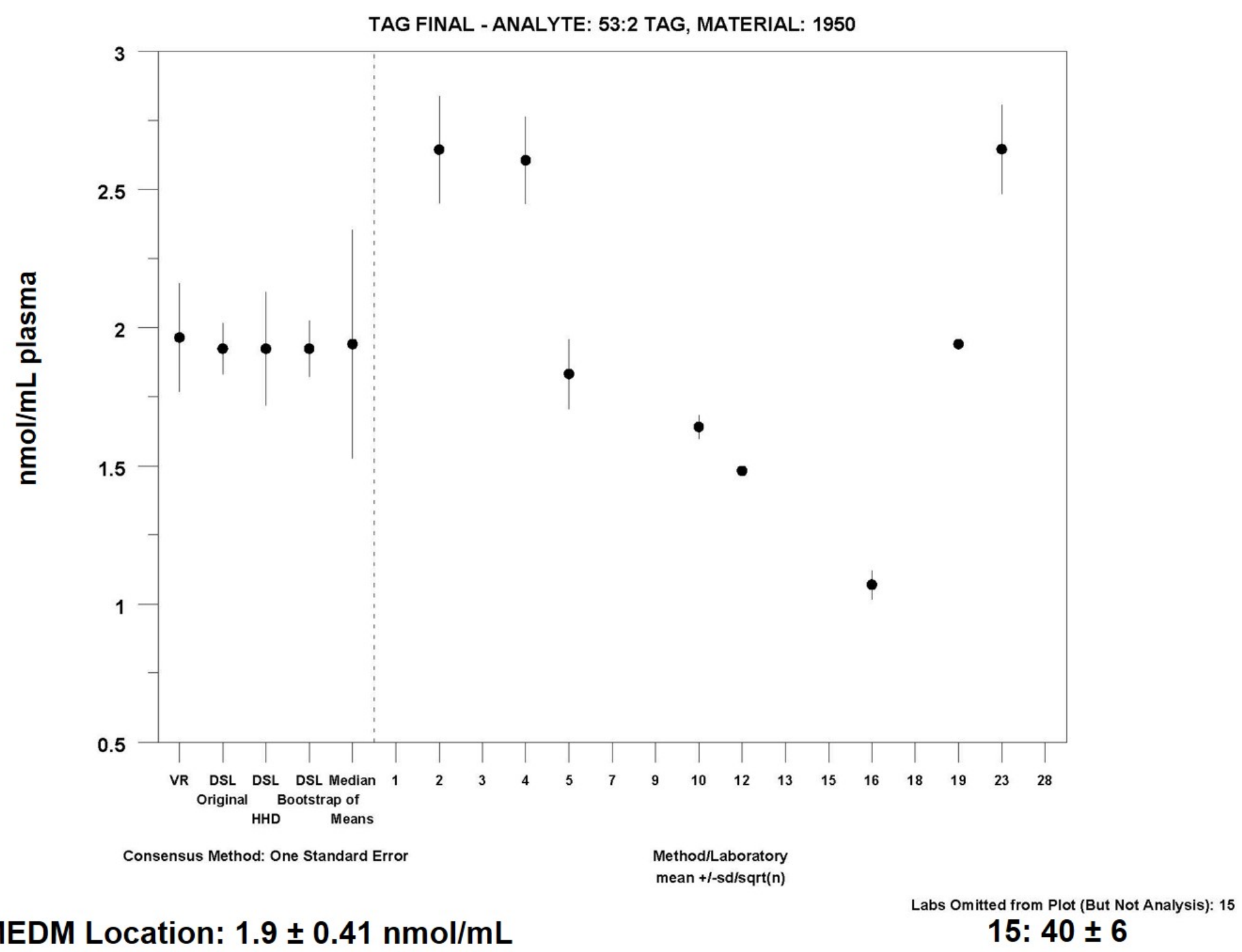




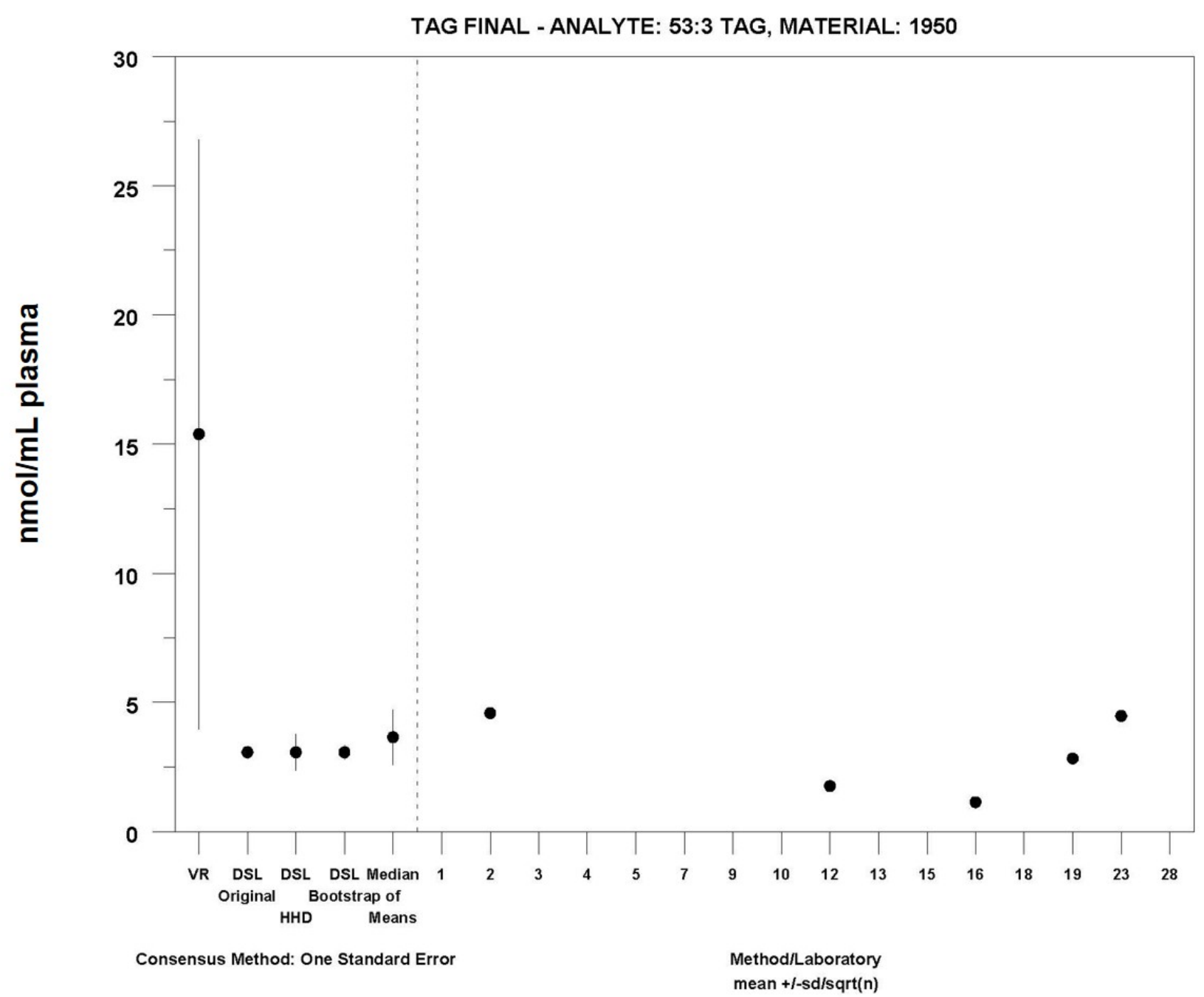

MEDM Location: $3.7 \pm 1.1 \mathrm{nmol} / \mathrm{mL}$

Labs Omitted from Plot (But Not Analysis): 15

15: $80 \pm 9$ 


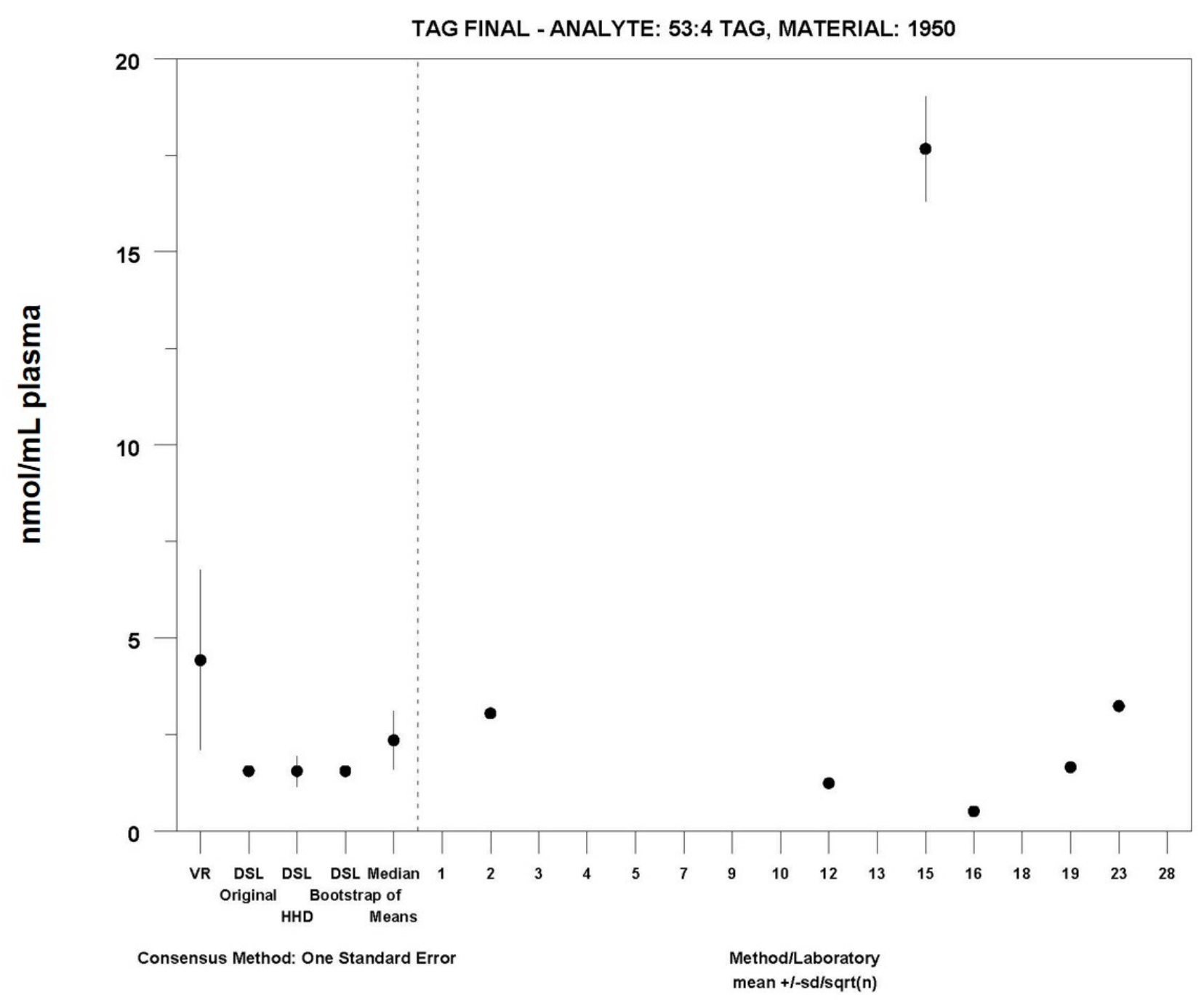

MEDM Location: $2.4 \pm 0.76 \mathrm{nmol} / \mathrm{mL}$ 


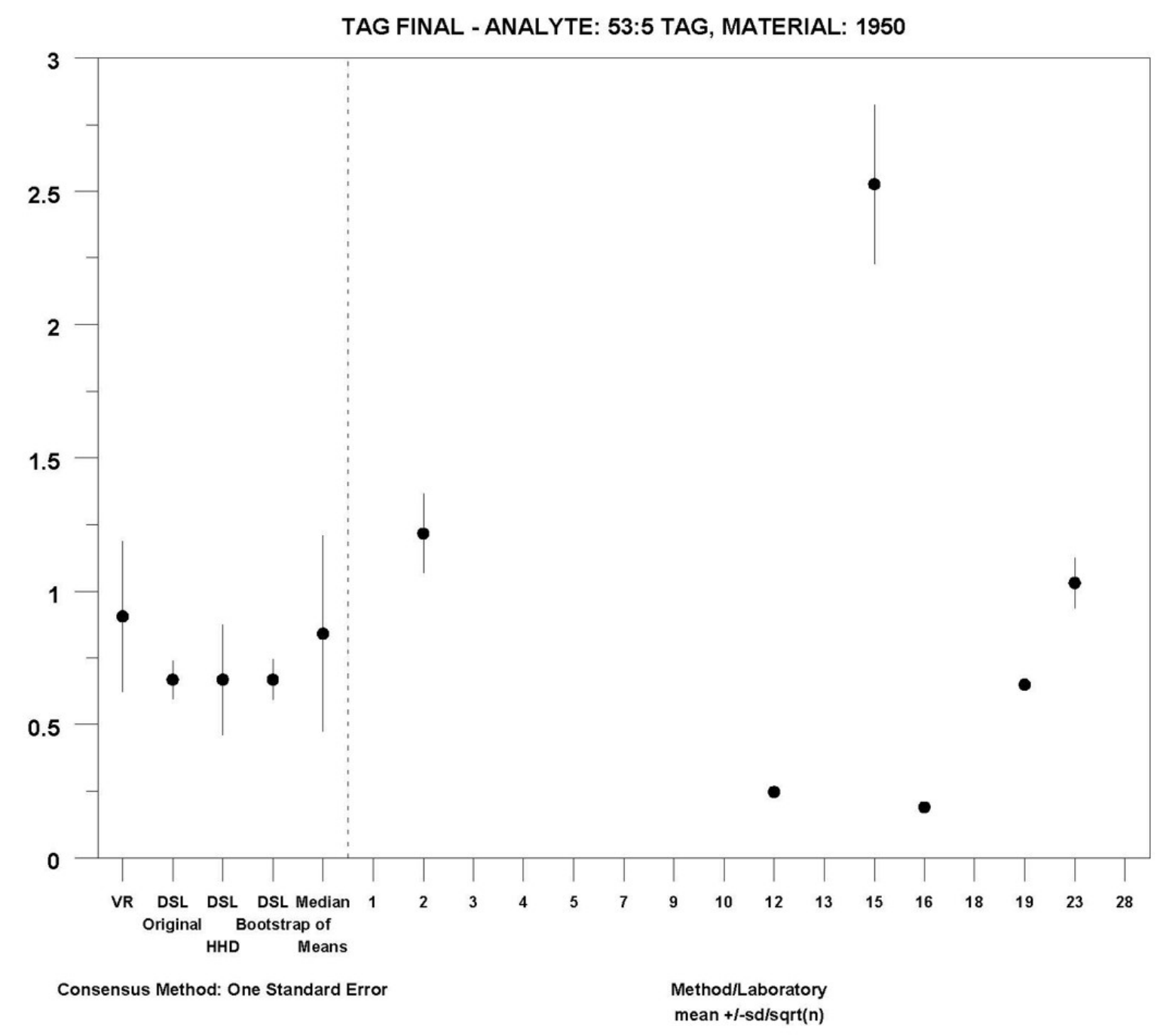

MEDM Location: $0.84 \pm 0.37 \mathrm{nmol} / \mathrm{mL}$ 


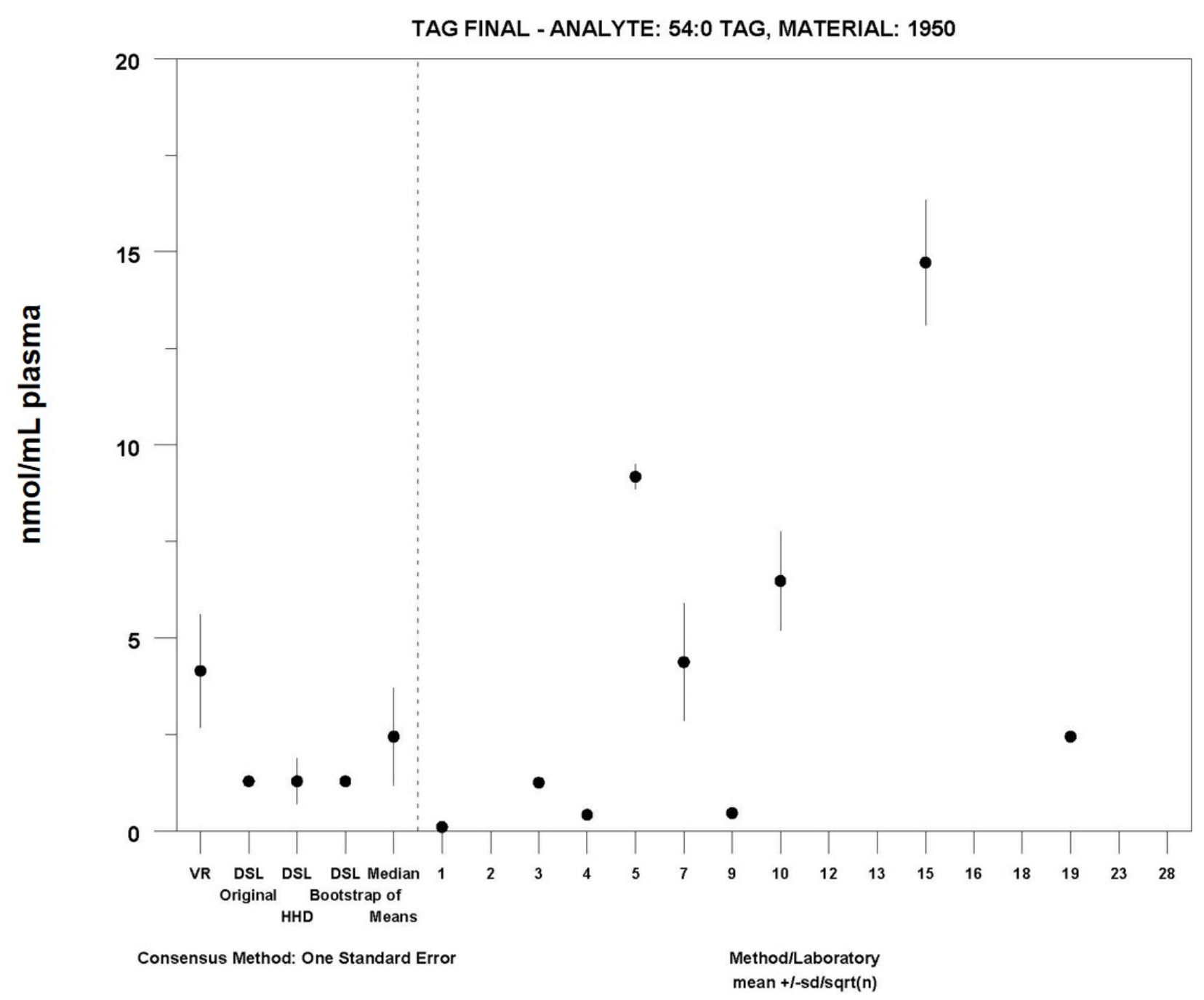

MEDM Location: $2.4 \pm 1.3 \mathrm{nmol} / \mathrm{mL}$ 


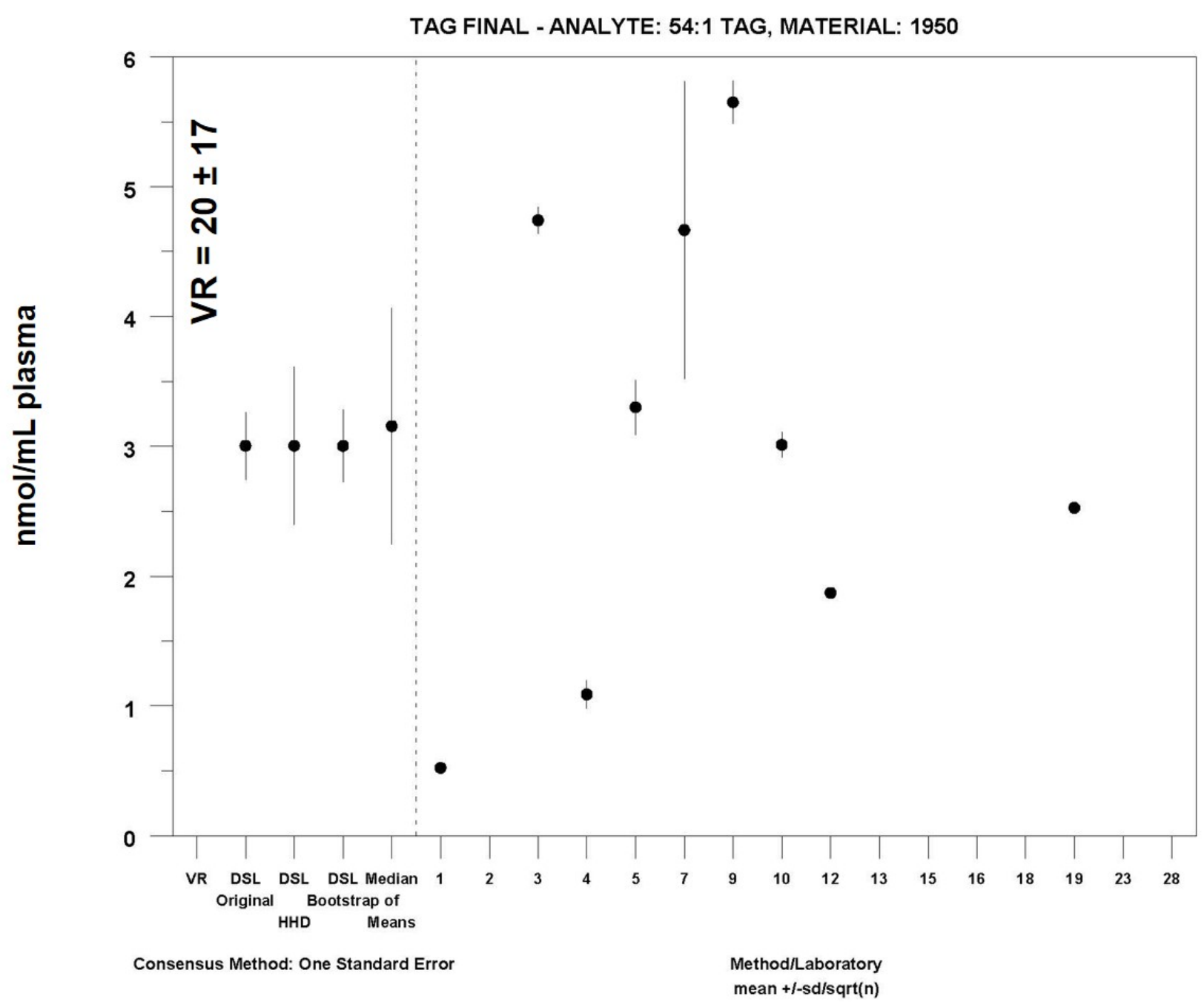

MEDM Location: $3.2 \pm 0.91 \mathrm{nmol} / \mathrm{mL}$

Labs Omitted from Plot (But Not Analysis): 15

15: $184 \pm 18$ 


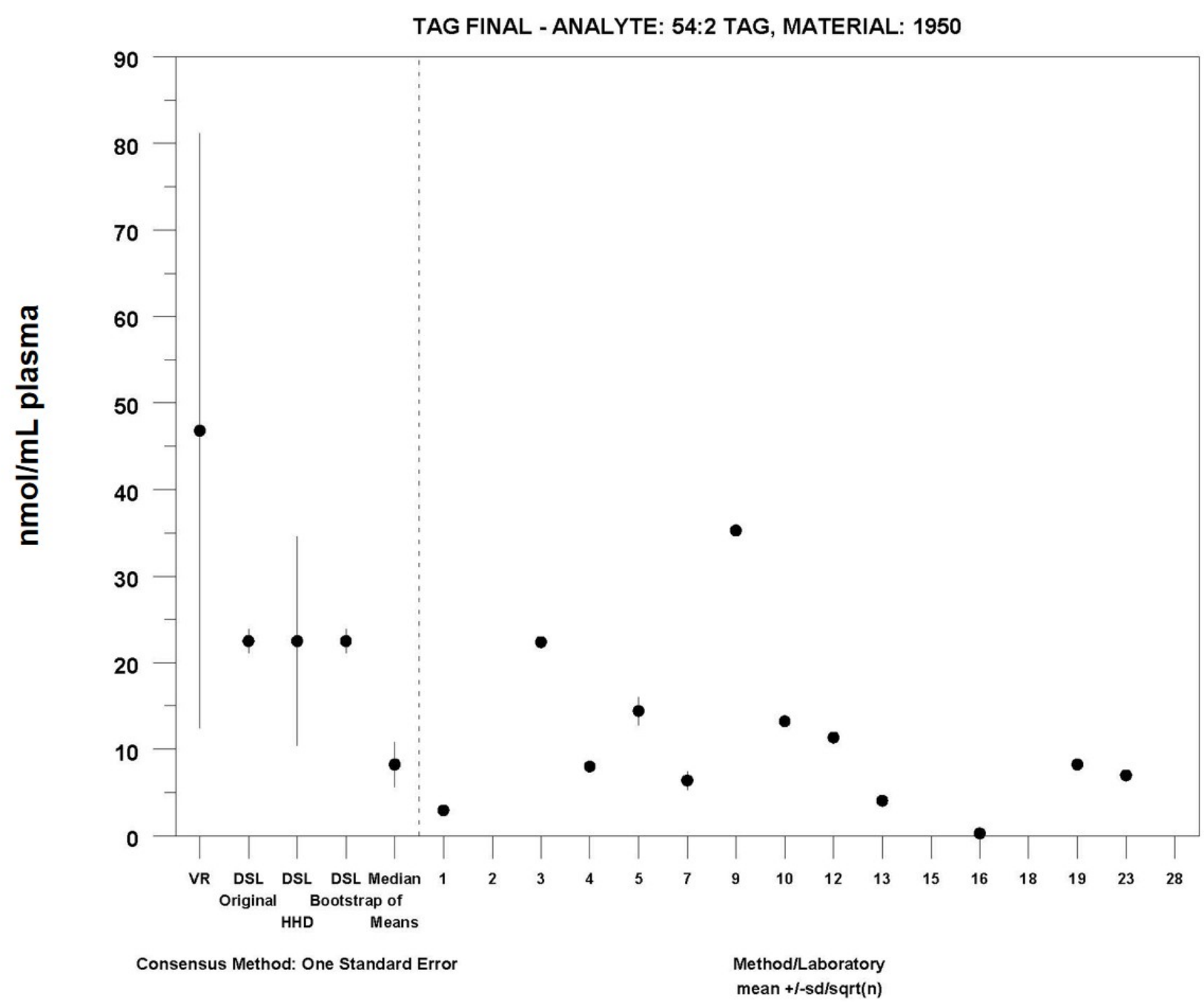

MEDM Location: $8.2 \pm 2.6 \mathrm{nmol} / \mathrm{mL}$

Labs Omitted from Plot (But Not Analysis): 15

15: $477 \pm 13$ 


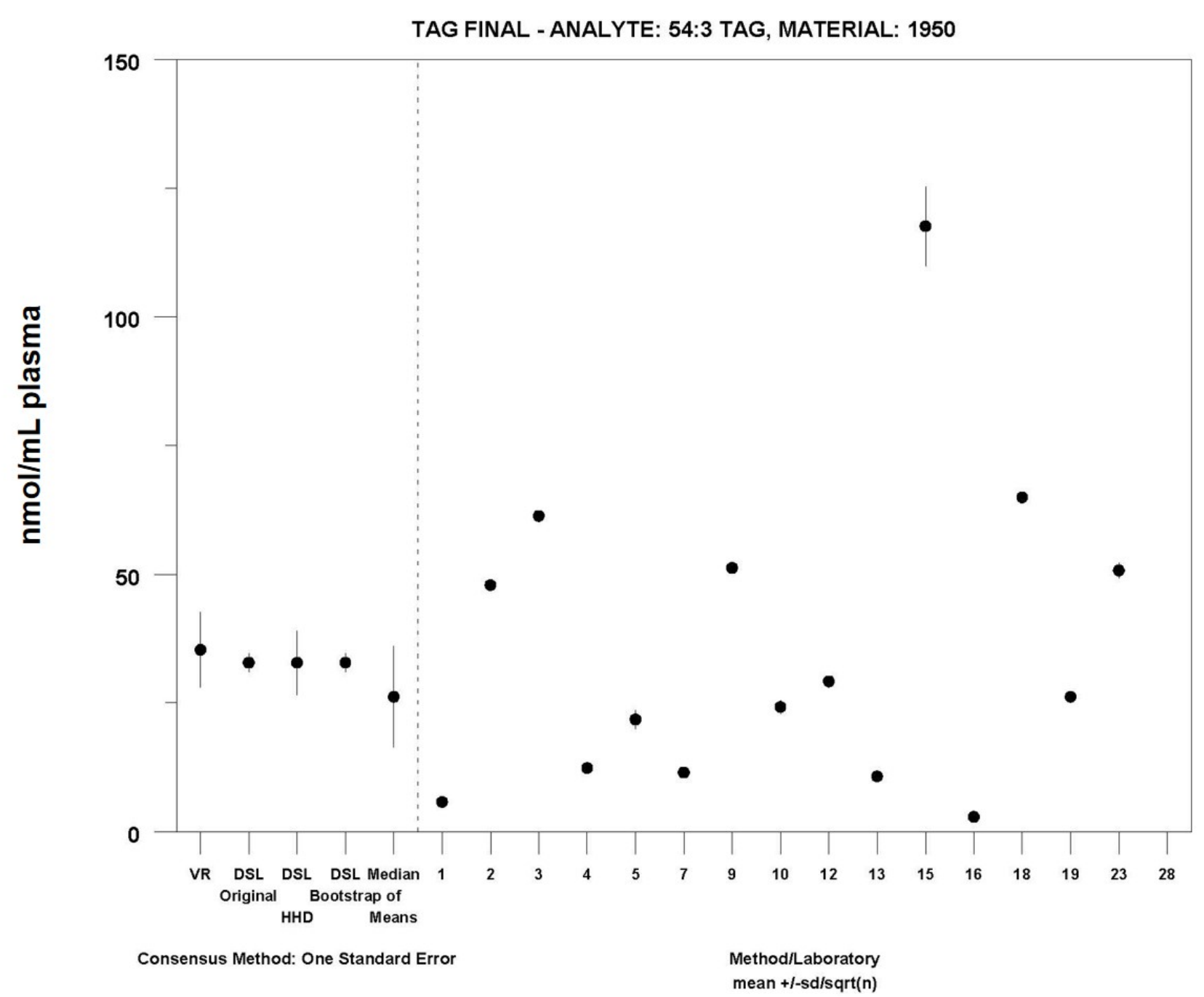

MEDM Location: $26 \pm 9.8 \mathrm{nmol} / \mathrm{mL}$ 


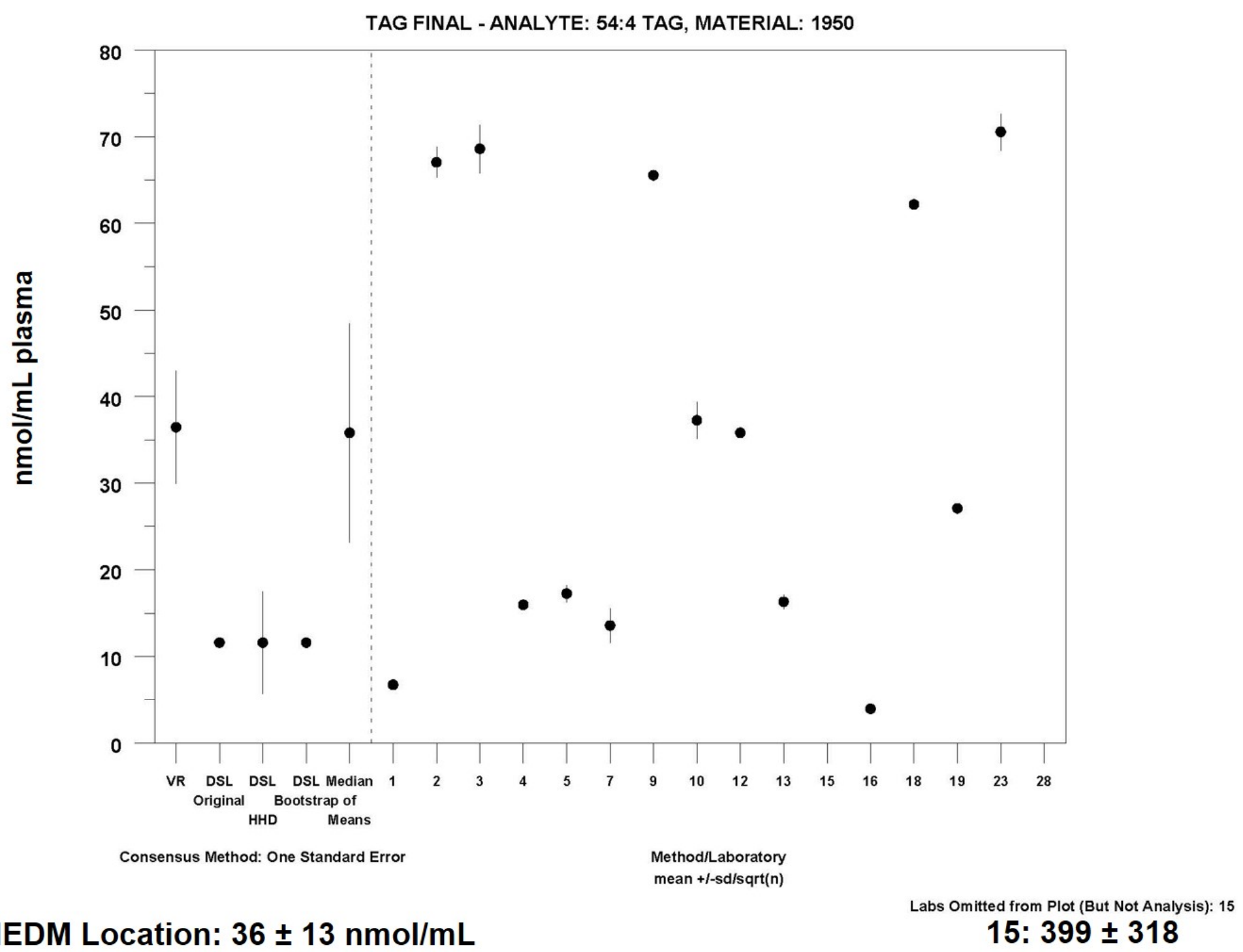




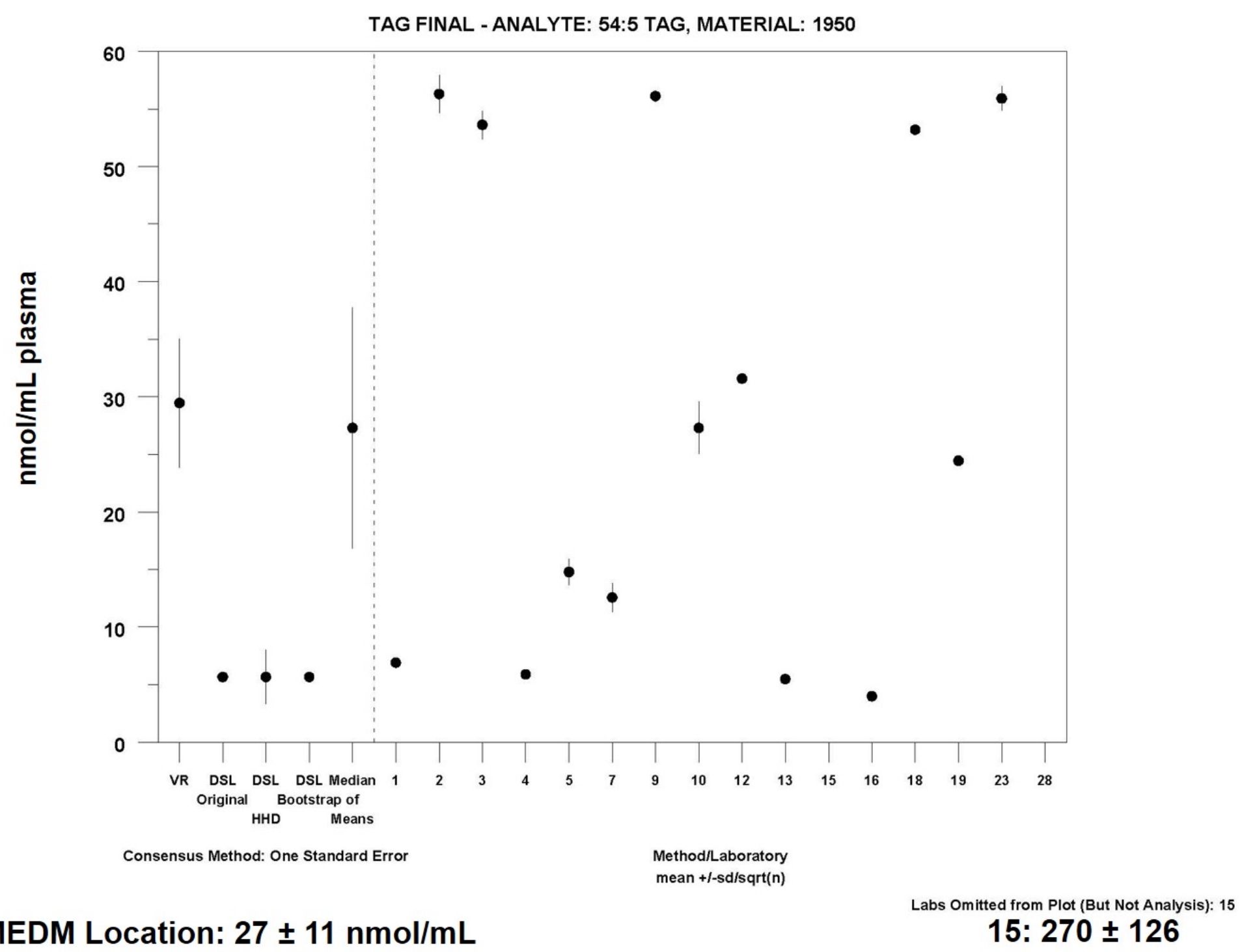




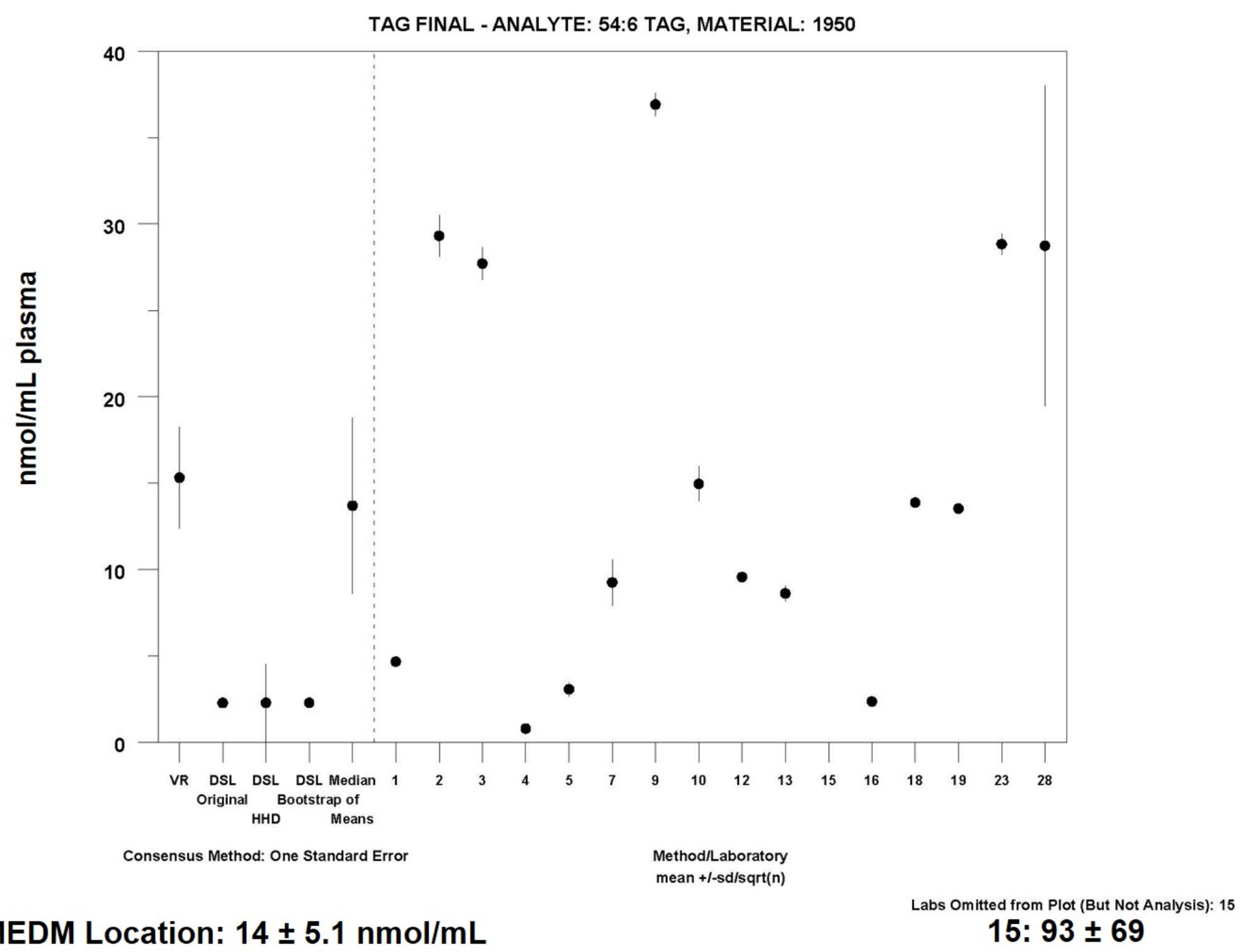




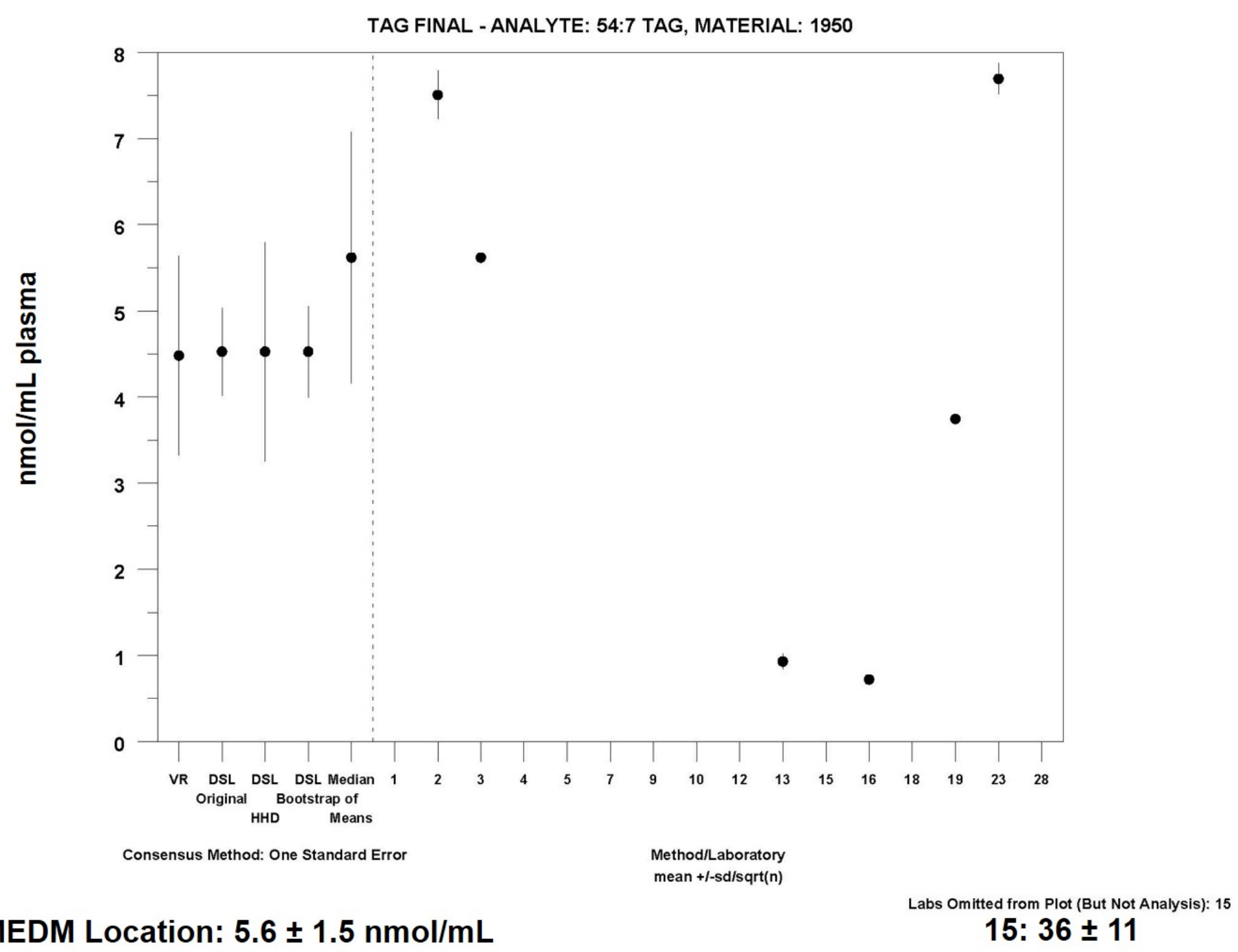




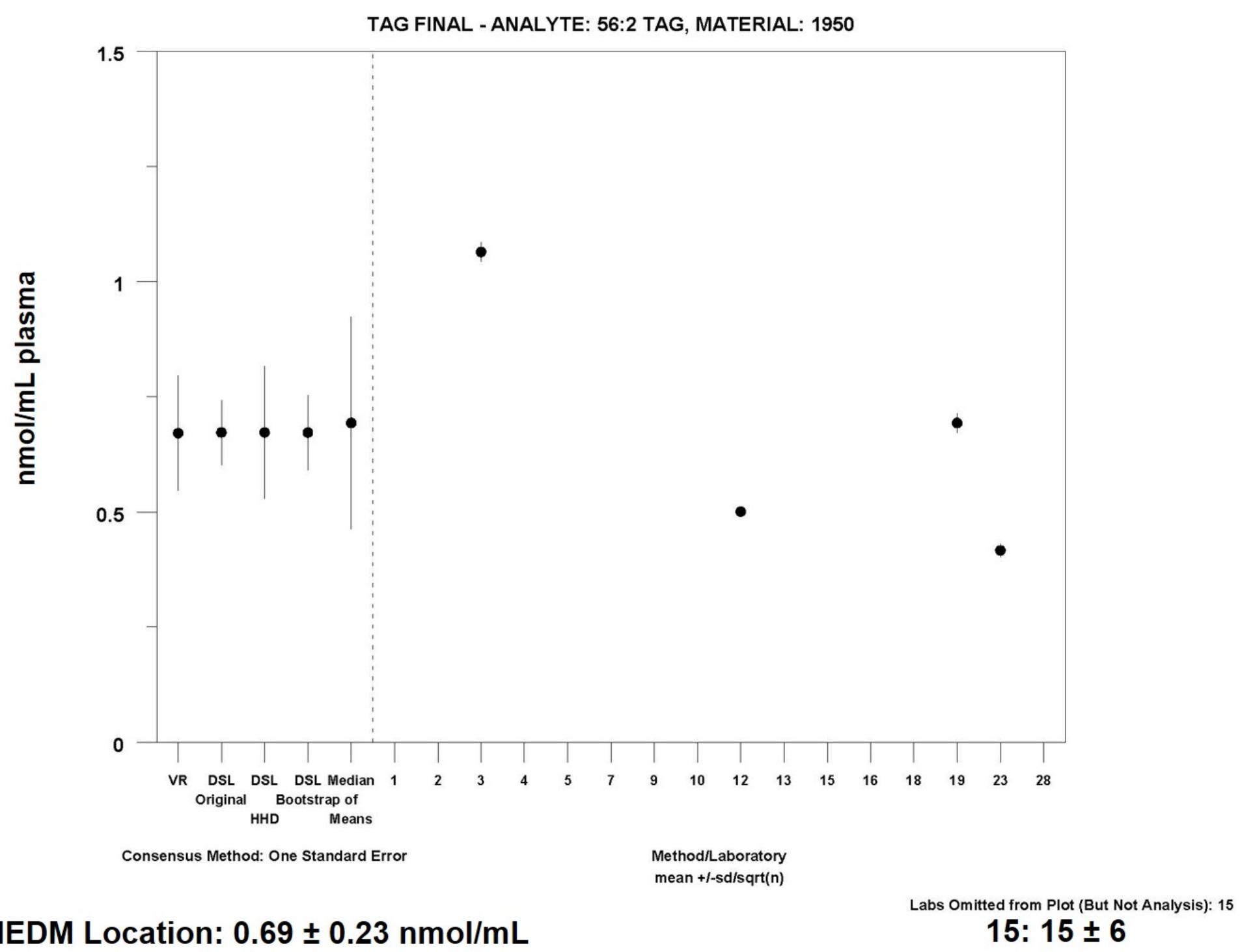




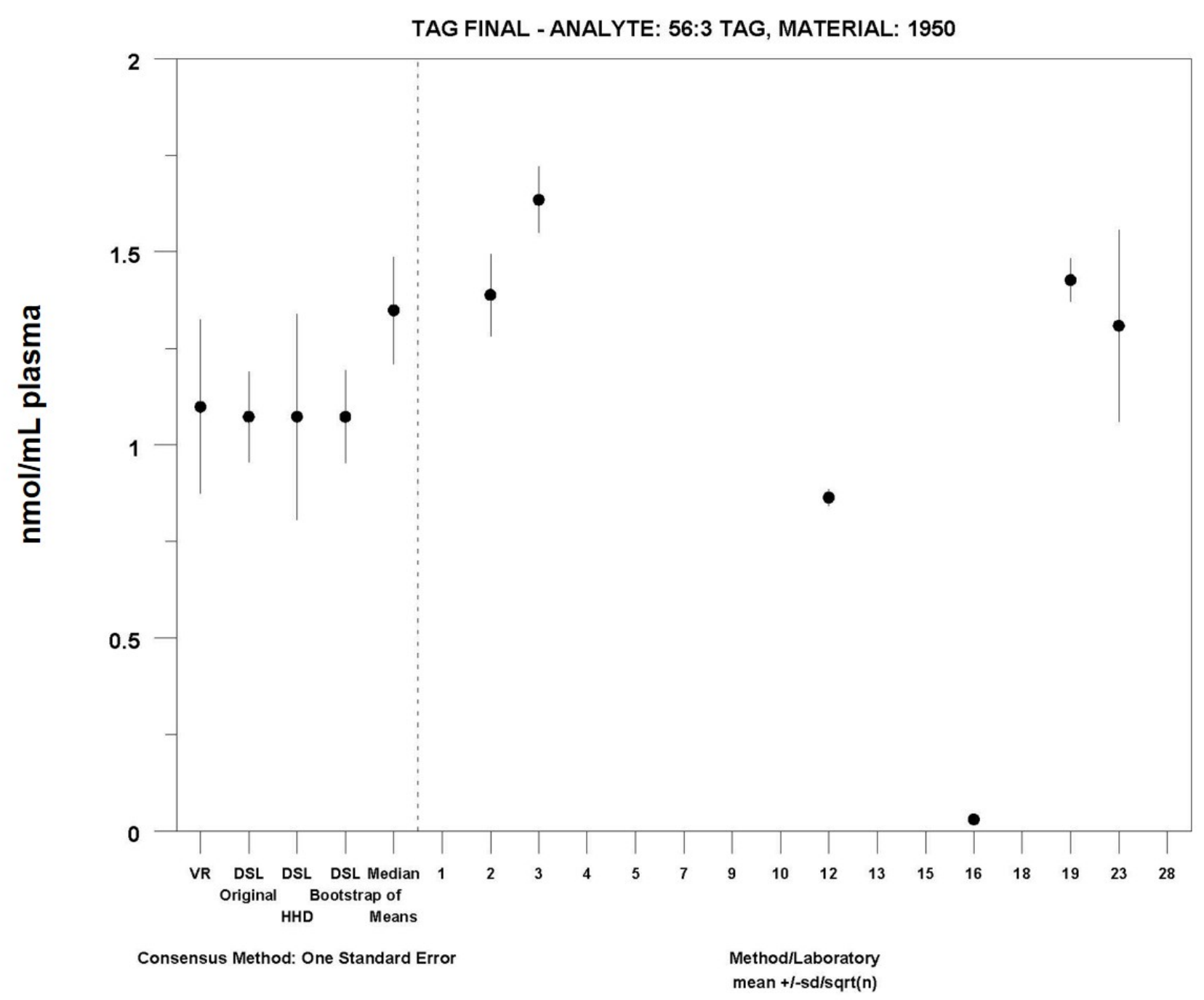

MEDM Location: $1.4 \pm 0.14 \mathrm{nmol} / \mathrm{mL}$ 


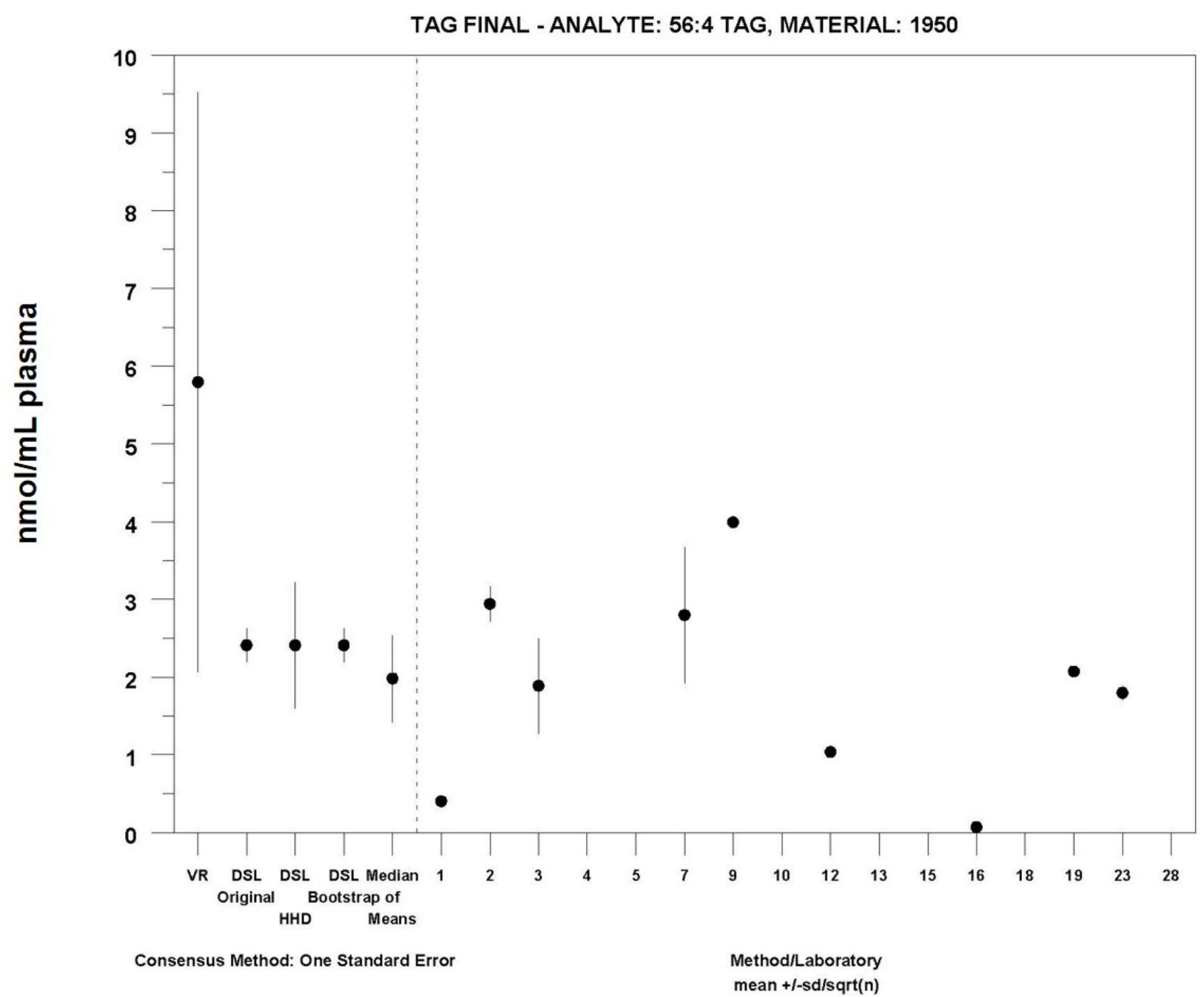

MEDM Location: $2.0 \pm 0.56 \mathrm{nmol} / \mathrm{mL}$

Labs Omitted from Plot (But Not Analysis): 15

15: $42 \pm 3$ 


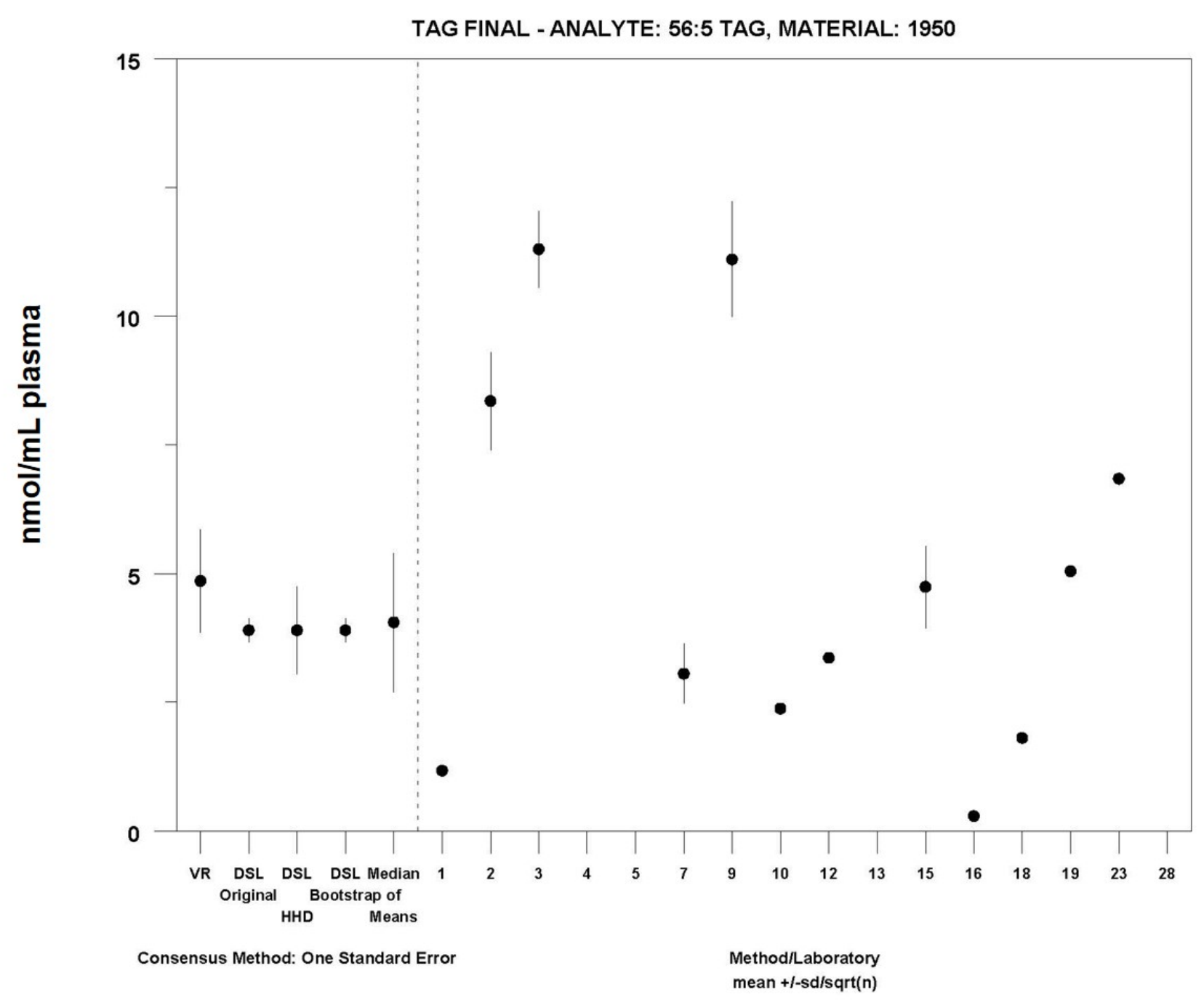

MEDM Location: $4.1 \pm 1.4 \mathrm{nmol} / \mathrm{mL}$ 


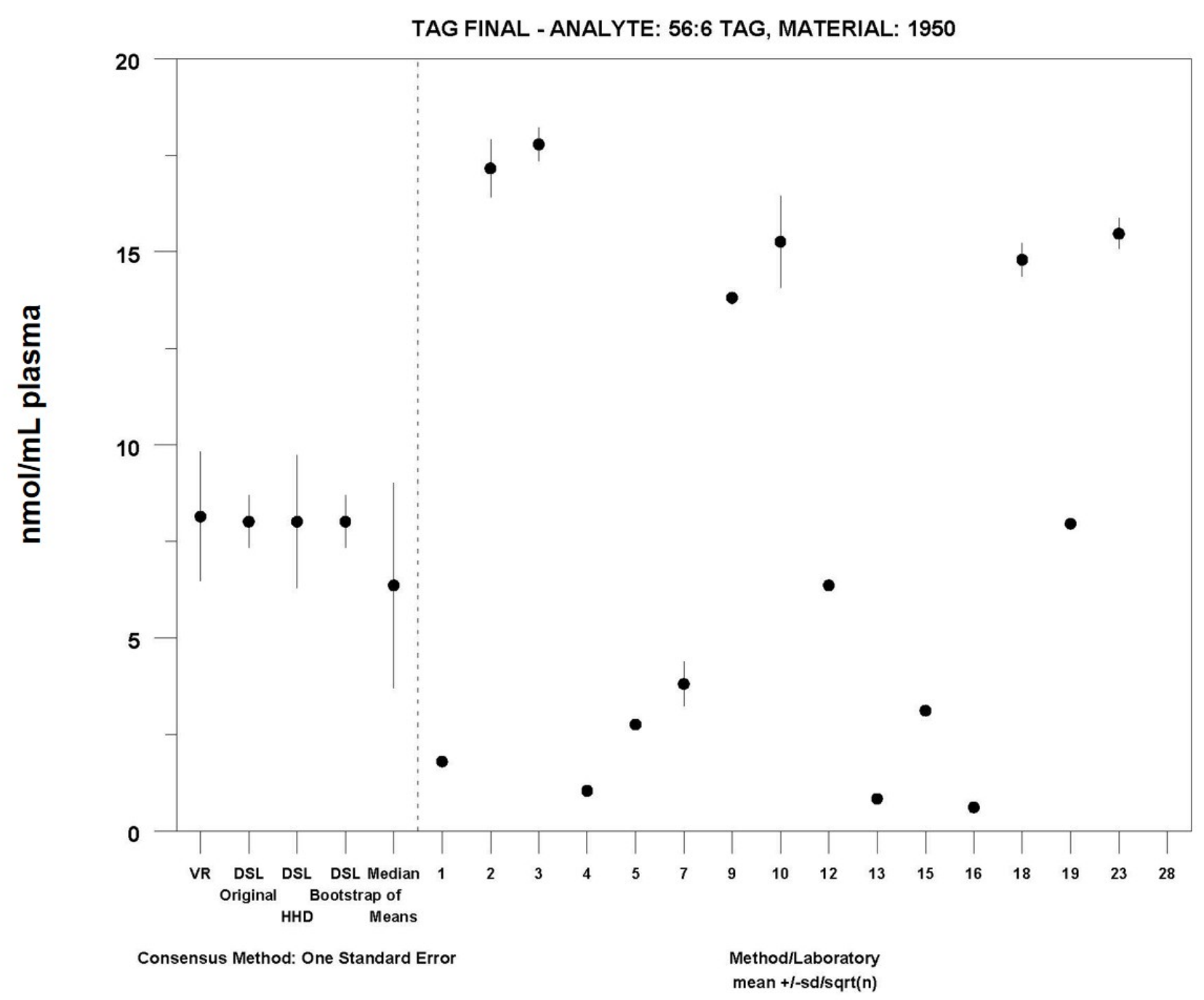

MEDM Location: $6.4 \pm 2.7 \mathrm{nmol} / \mathrm{mL}$ 


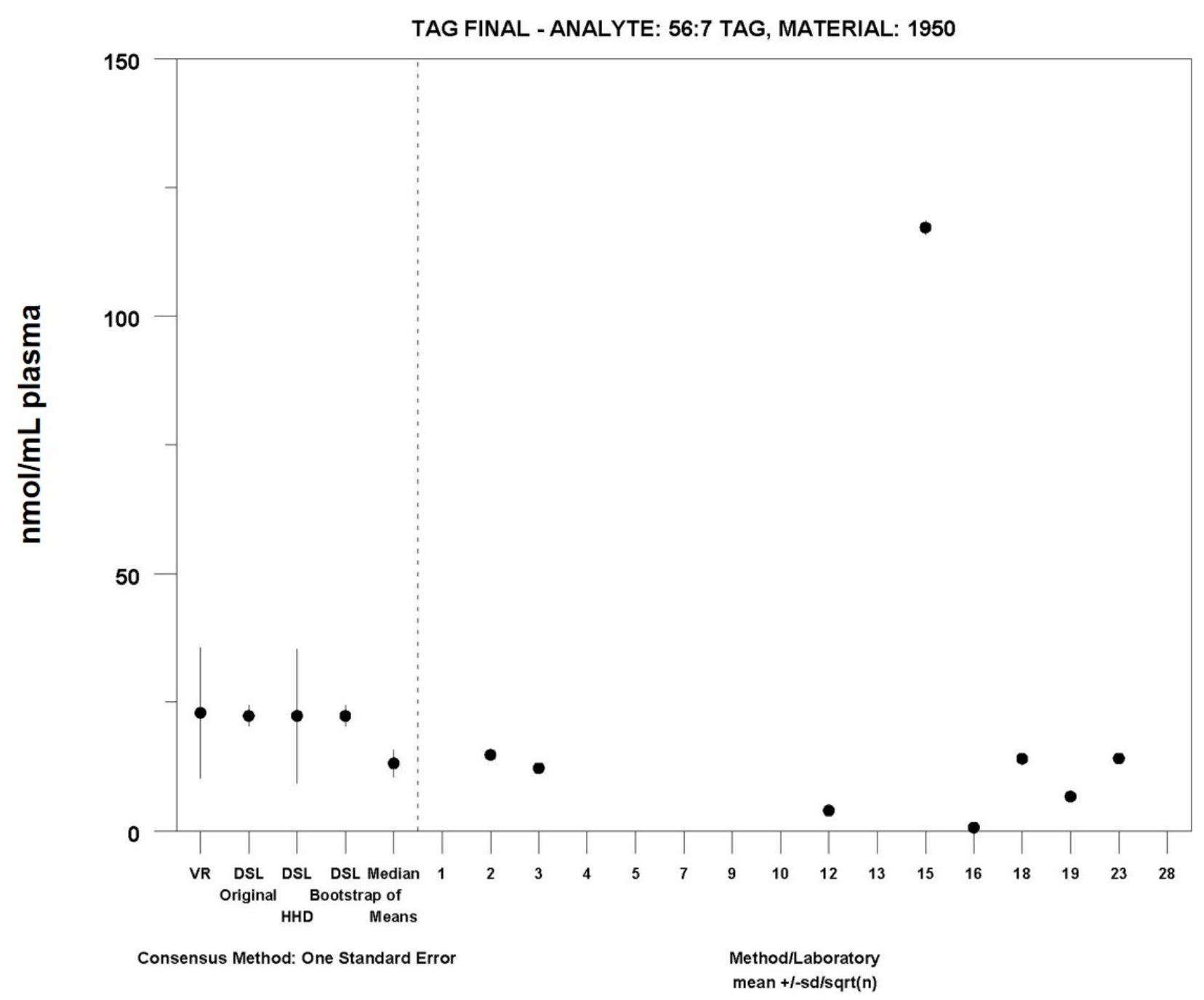

MEDM Location: $13 \pm 2.7 \mathrm{nmol} / \mathrm{mL}$ 


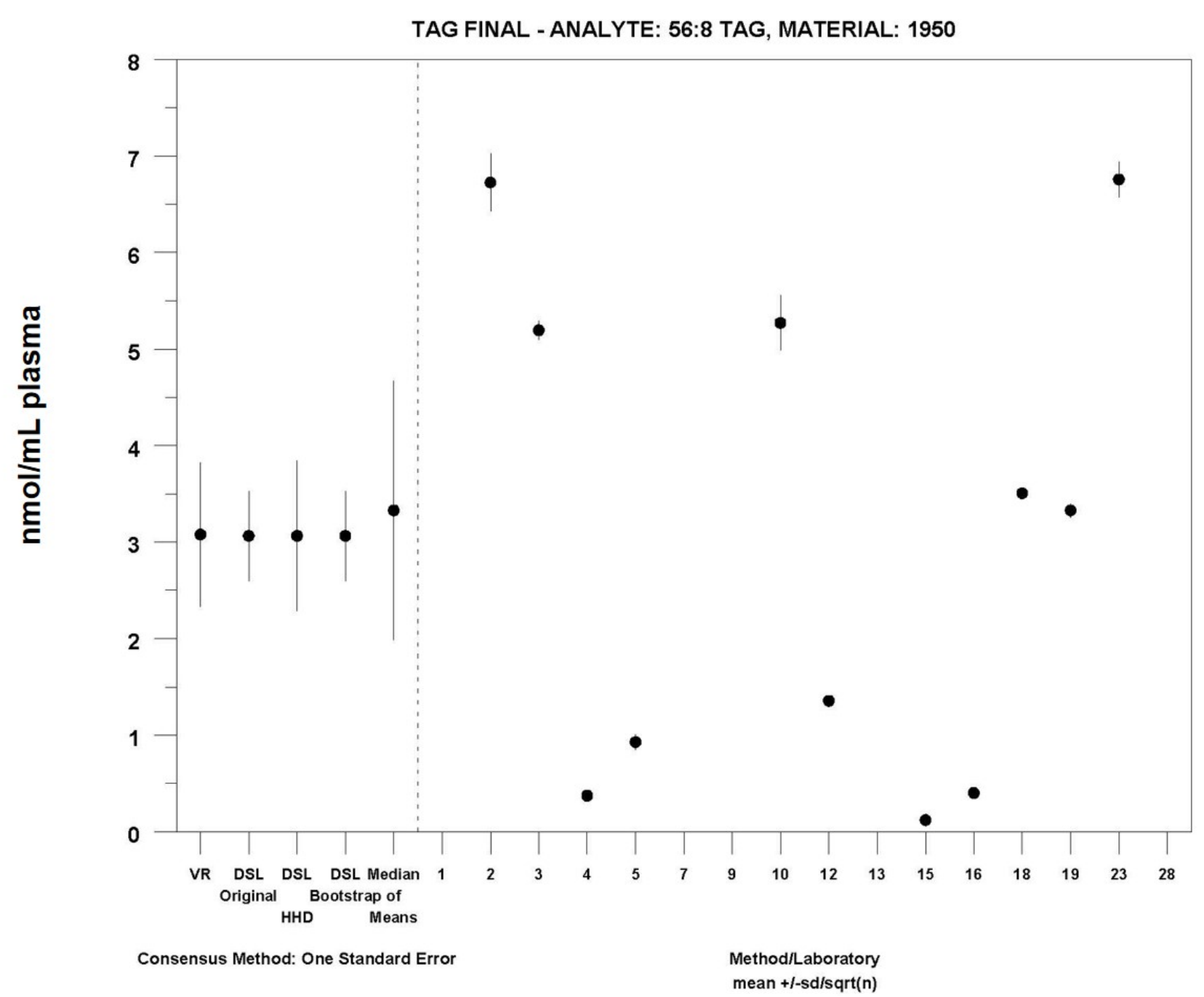

MEDM Location: $3.3 \pm 1.3 \mathrm{nmol} / \mathrm{mL}$ 


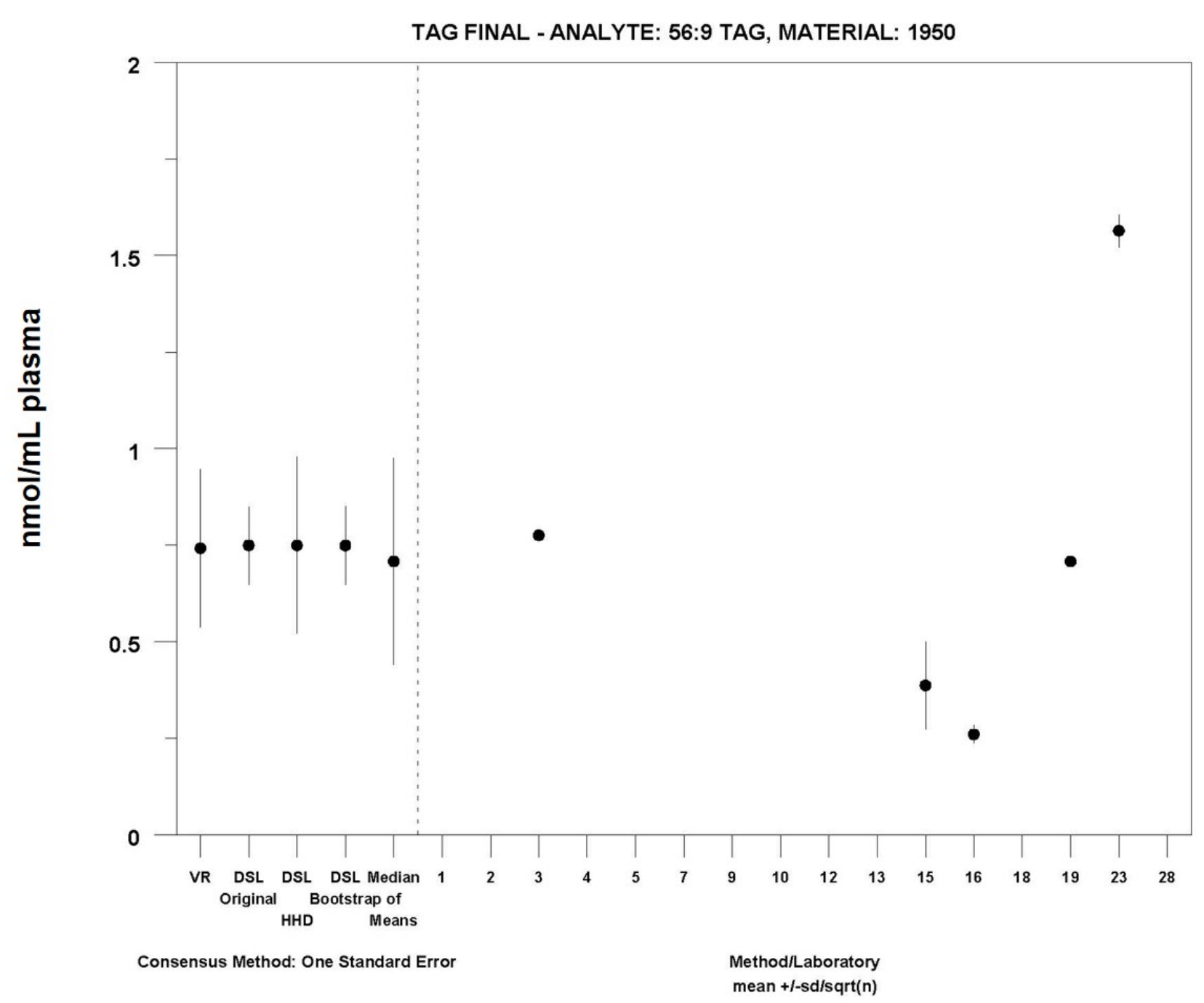

MEDM Location: $0.71 \pm 0.27 \mathrm{nmol} / \mathrm{mL}$ 


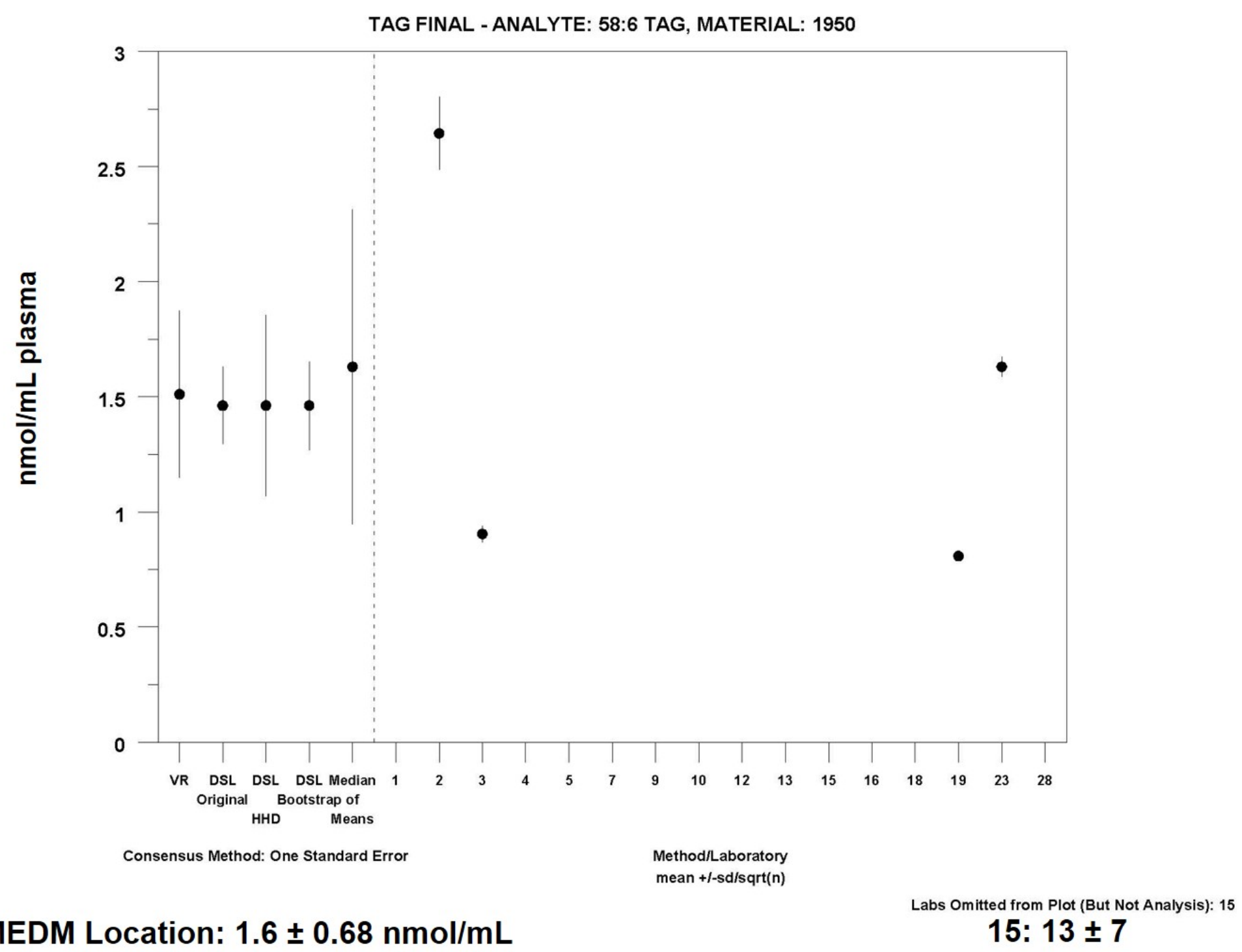




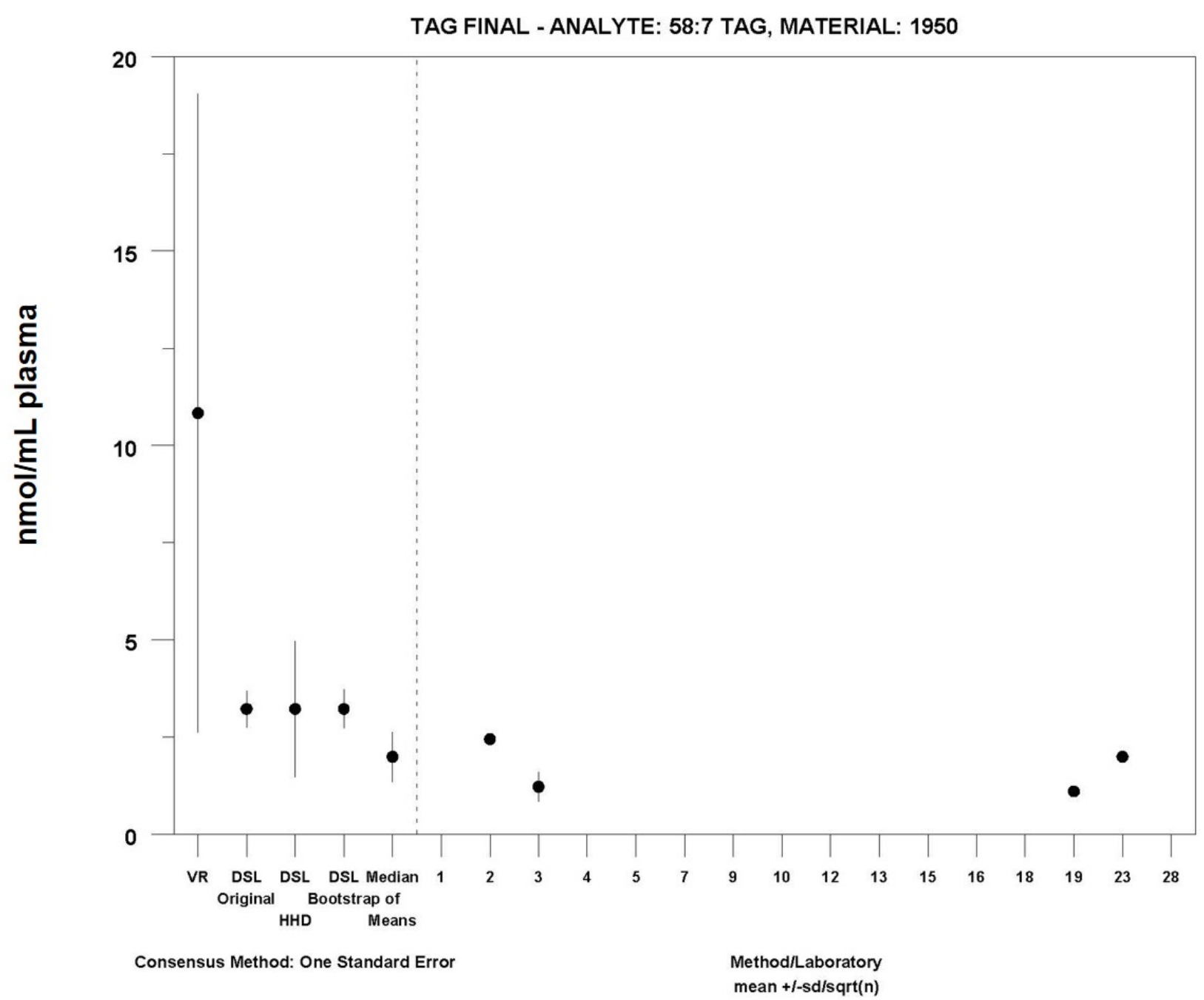

MEDM Location: $2.0 \pm 0.64 \mathrm{nmol} / \mathrm{mL}$

Labs Omitted from Plot (But Not Analysis): 15

15: $48 \pm 4$ 


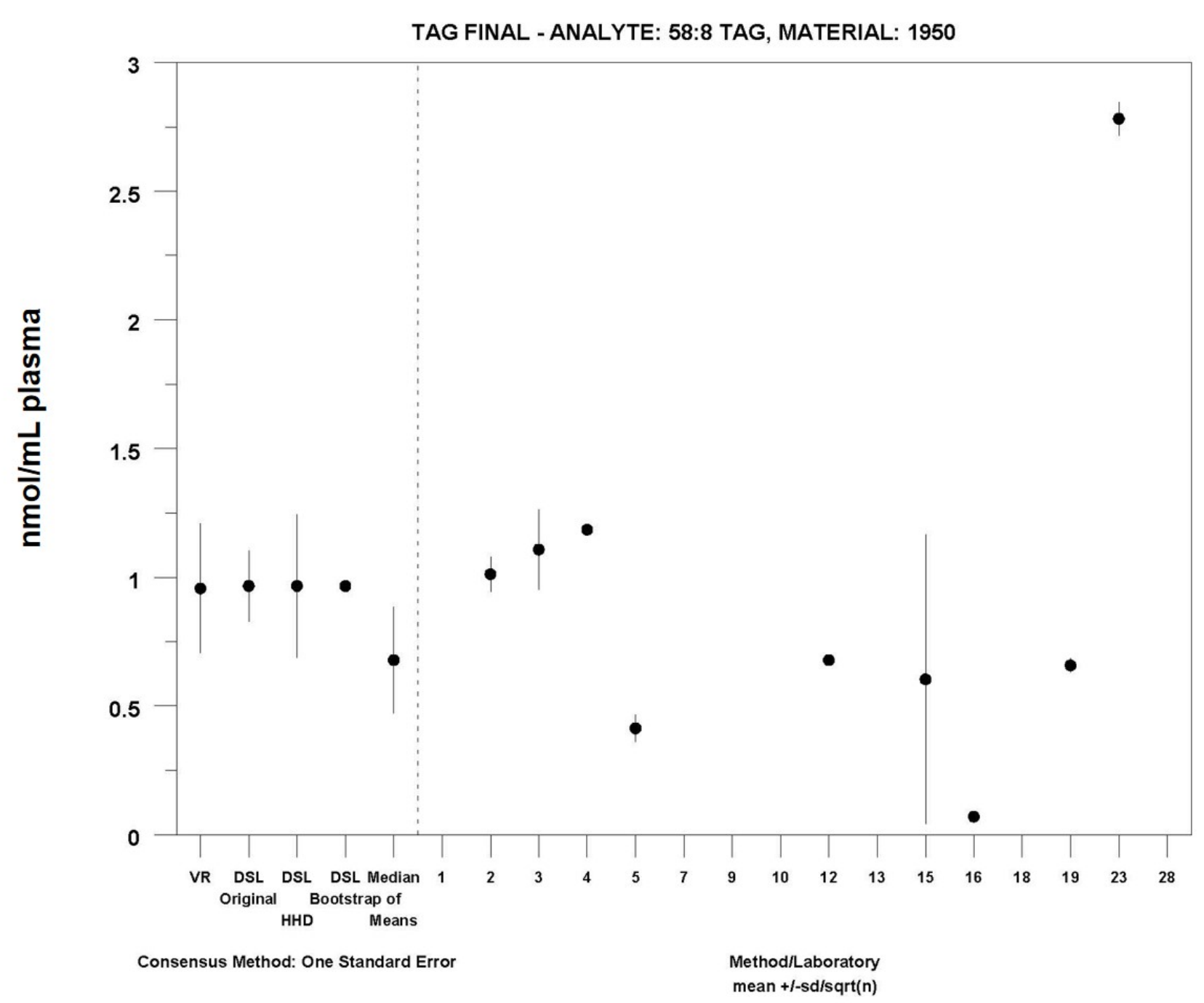

MEDM Location: $0.68 \pm 0.21 \mathrm{nmol} / \mathrm{mL}$ 


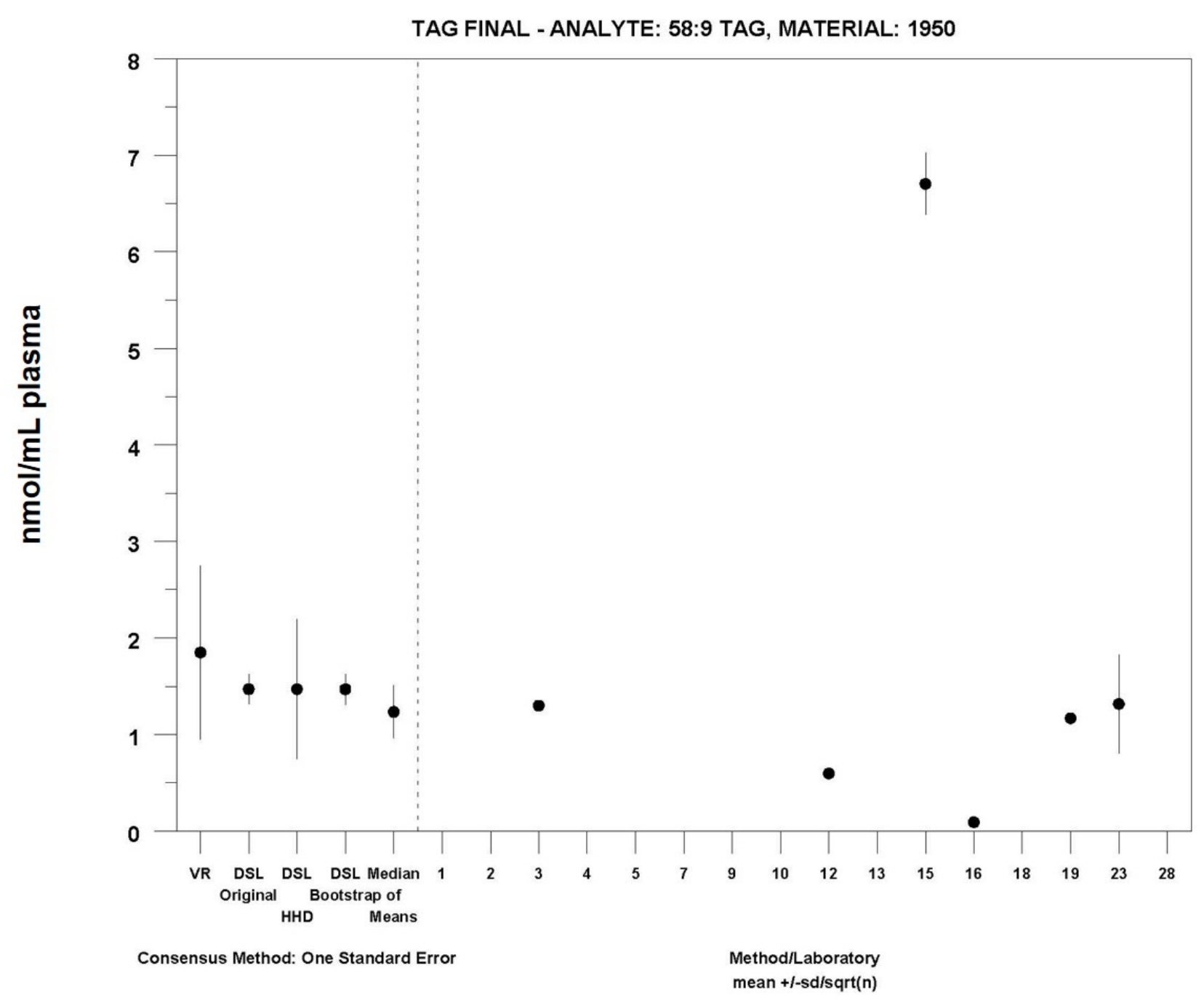

MEDM Location: $1.2 \pm 0.27 \mathrm{nmol} / \mathrm{mL}$ 
Table S14. Top-50 ranked MEDM locations by concentration (nmol/mL) in SRM 1950

\begin{tabular}{|c|c|c|c|}
\hline Lipid & \# of Labs & $\begin{array}{c}\text { MEDM Location } \\
(\mathrm{nmol} / \mathrm{mL})\end{array}$ & COD (\%) \\
\hline TAG 54:6 & 16 & 13.7 & 37 \\
\hline PC 40:6 & 17 & 13.9 & 19 \\
\hline TAG 52:1 & 11 & 14.3 & 20 \\
\hline FFA 18:0 & 5 & 14.5 & 62 \\
\hline TAG 52:5 & 13 & 14.6 & 39 \\
\hline CE 18:0 & 7 & 15.2 & 25 \\
\hline SM d34:2 & 17 & 15.7 & 14 \\
\hline TAG 48:2 & 15 & 15.7 & 18 \\
\hline CE 14:0 & 7 & 16.0 & 37 \\
\hline $\mathrm{SM} d 42: 3$ & 12 & 17.4 & 27 \\
\hline LPC 18:1 & 19 & 18.4 & 13 \\
\hline SM d42:1 & 21 & 19.8 & 28 \\
\hline SM d40:1 & 17 & 20.0 & 25 \\
\hline SM d36:1 & 22 & 20.2 & 18 \\
\hline LPC 18:2 & 19 & 22.1 & 13 \\
\hline TAG 50:3 & 16 & 22.8 & 29 \\
\hline TAG 54:3 & 15 & 26.2 & 37 \\
\hline PC 38:3 & 14 & 26.2 & 20 \\
\hline PC 36:1 & 17 & 26.3 & 17 \\
\hline LPC 18:0 & 20 & 26.9 & 12 \\
\hline TAG 54:5 & 15 & 27.3 & 38 \\
\hline CE 20:3 & 13 & 35.0 & 35 \\
\hline TAG 54:4 & 15 & 35.8 & 35 \\
\hline CE 22:6 & 11 & 37.0 & 26 \\
\hline CE 20:5 & 12 & 37.6 & 23 \\
\hline TAG 50:1 & 14 & 38.0 & 26 \\
\hline PC 38:6 & 18 & 40.5 & 11 \\
\hline PC 38:5 & 18 & 42.2 & 19 \\
\hline FFA $16: 0$ & 5 & 42.5 & 31 \\
\hline SM d42:2 & 18 & 43.8 & 25 \\
\hline FFA $18: 2$ & 6 & 44.3 & 49 \\
\hline TAG 52:2 & 16 & 44.4 & 33 \\
\hline TAG 50:2 & 15 & 46.6 & 26 \\
\hline TAG 52:4 & 15 & 47.8 & 35 \\
\hline LPC 16:0 & 20 & 72.9 & 15 \\
\hline CE 18:3 & 13 & 84.1 & 28 \\
\hline
\end{tabular}




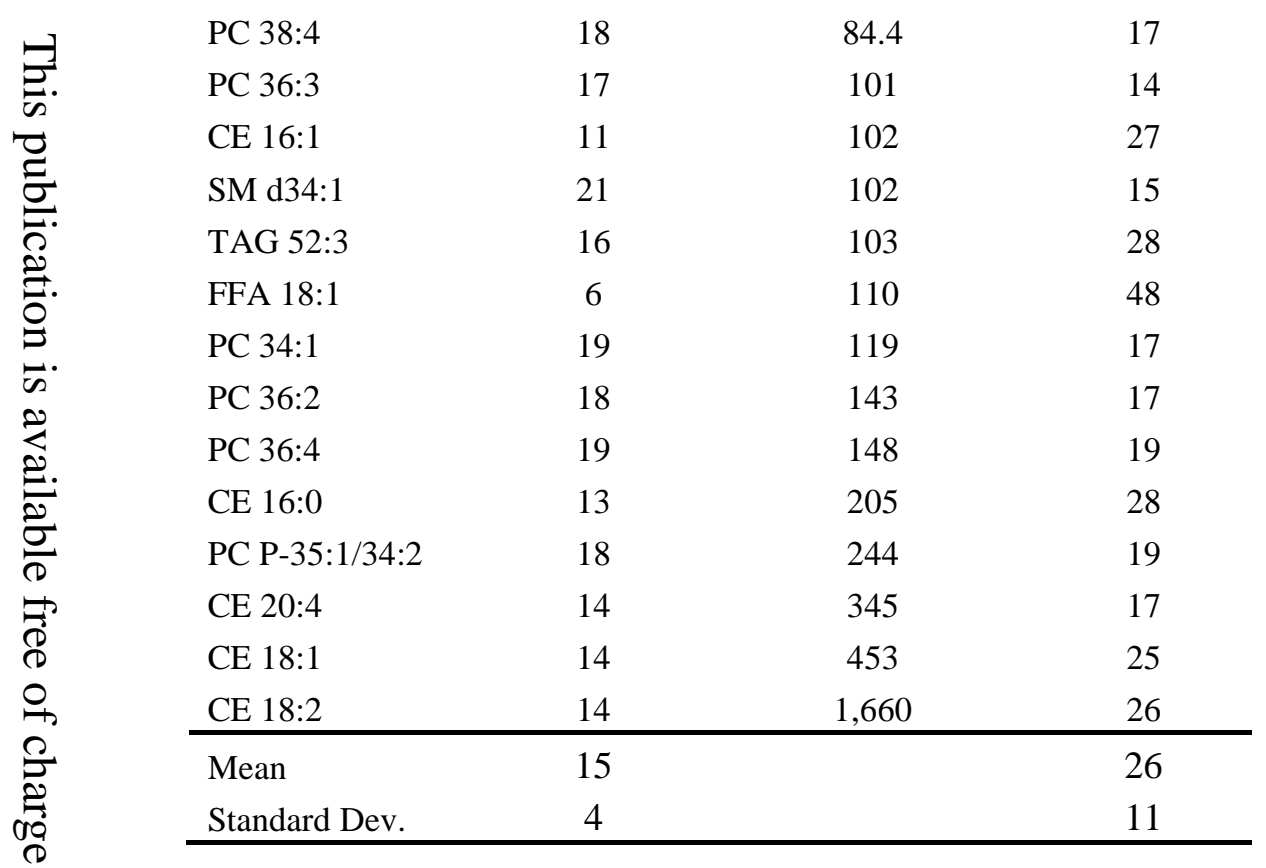


Table S15. Bottom-50 ranked MEDM locations by concentration (nmol/mL) in SRM 1950

\begin{tabular}{|c|c|c|c|}
\hline Lipid & \# of Labs & $\begin{array}{c}\text { MEDM Location } \\
(\mathrm{nmol} / \mathrm{mL})\end{array}$ & COD (\%) \\
\hline 15-HETE & 5 & 0.00239 & 27 \\
\hline TLCA & 5 & 0.00269 & 26 \\
\hline 12-HETE & 5 & 0.00680 & 23 \\
\hline 5-HETE & 5 & 0.0102 & 13 \\
\hline LPC 22:1 & 5 & 0.0129 & 36 \\
\hline LCA & 8 & 0.0141 & 26 \\
\hline TAG 42:2 & 6 & 0.157 & 41 \\
\hline GLCA & 6 & 0.0245 & 7 \\
\hline LPC 22:0 & 5 & 0.0253 & 7 \\
\hline CER d36:2 & 7 & 0.0256 & 56 \\
\hline TCA & 9 & 0.0260 & 22 \\
\hline PC 34:5 & 5 & 0.0343 & 13 \\
\hline LPE 22:1 & 5 & 0.0363 & 82 \\
\hline TDCA & 8 & 0.0402 & 16 \\
\hline CER d44:2 & 7 & 0.0442 & 49 \\
\hline CER d34:0 & 5 & 0.0450 & 70 \\
\hline LPC 24:0 & 5 & 0.0460 & 33 \\
\hline PC O-30:1/P-30:0 & 7 & 0.0473 & 20 \\
\hline CER d32:1 & 8 & 0.0513 & 42 \\
\hline CER d44:1 & 7 & 0.0631 & 49 \\
\hline PC O-40:2/P-40:1 & 5 & 0.0688 & 30 \\
\hline PC O-30:0/29:0 & 7 & 0.0720 & 36 \\
\hline PC 42:6 & 5 & 0.0790 & 52 \\
\hline DAG 40:5 & 5 & 0.0838 & 63 \\
\hline TCDCA & 9 & 0.0839 & 6 \\
\hline LPC 20:0 & 7 & 0.100 & 34 \\
\hline UDCA & 8 & 0.106 & 22 \\
\hline CER d38:1 & 16 & 0.109 & 20 \\
\hline PC O-34:4/P-34:3 & 6 & 0.119 & 66 \\
\hline CER d36:1 & 14 & 0.122 & 17 \\
\hline $\mathrm{CA}$ & 9 & 0.122 & 28 \\
\hline LPC 22:4 & 8 & 0.123 & 33 \\
\hline HexCer d36:1 & 5 & 0.127 & 34 \\
\hline CER d40:2 & 6 & 0.145 & 14 \\
\hline GUDCA & 6 & 0.146 & 16 \\
\hline LPC O-18:0 & 6 & 0.158 & 36 \\
\hline LPC 20:1 & 13 & 0.194 & 12 \\
\hline SM d31:1 & 5 & 0.194 & 25 \\
\hline SM d37:2 & 5 & 0.207 & 50 \\
\hline CER d42:3 & 5 & 0.228 & 62 \\
\hline
\end{tabular}




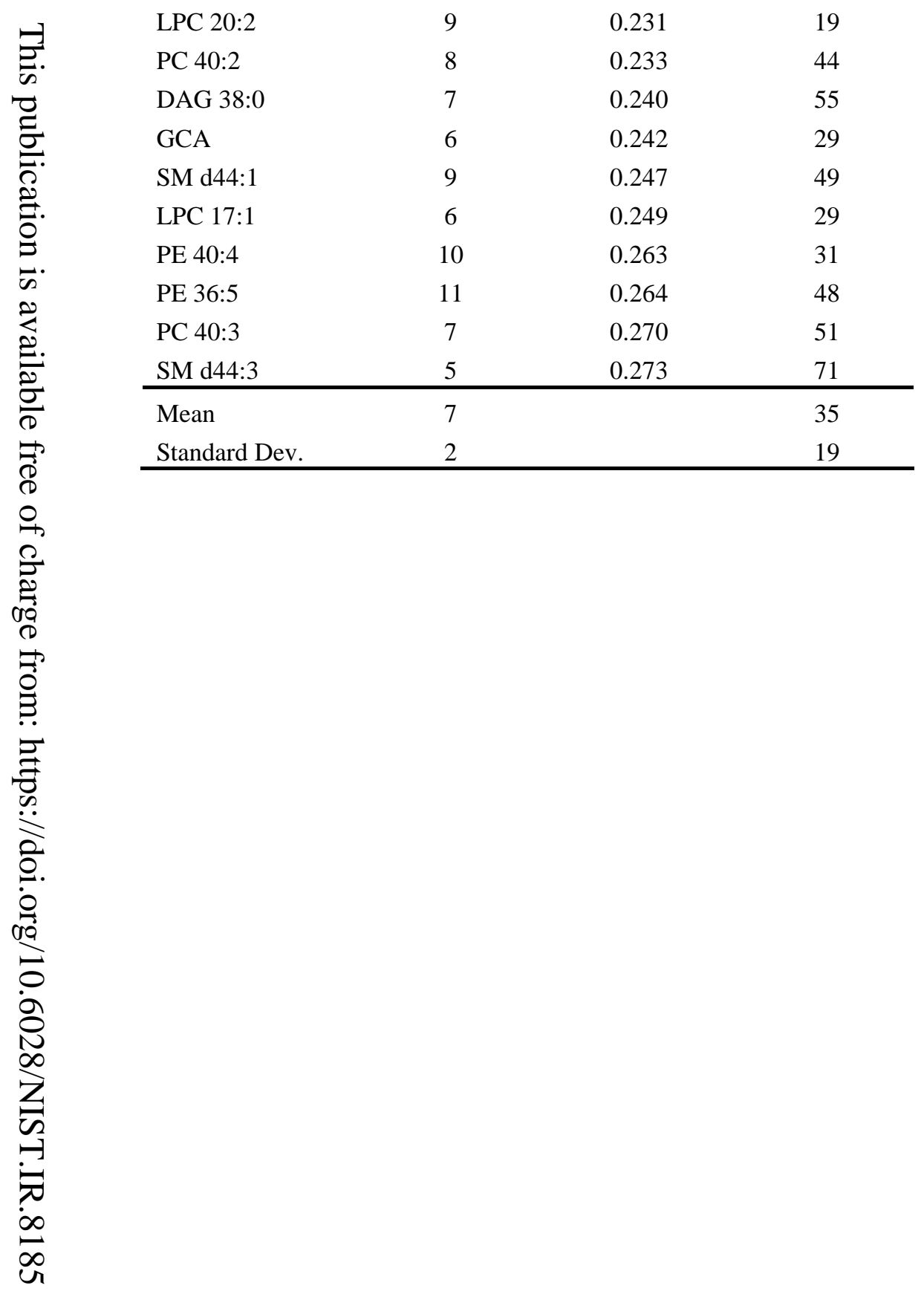


Zeta-score plots are provided for lipids organized by lipid (measured by at least five participating laboratories). Each dot represents a single laboratory measurement for that lipid. The $\zeta$-scores were calculated using the MEDM location as the target. The distance for each point from the mean indicates how many $x$ times the number of combined standard uncertainties the submitted value is above or below the MEDM location. The plots were truncated at \pm 10 to maintain visual resolution. Absolute value mean $\zeta$-scores were calculated two ways: (1) setting truncated values to 10 (first $\zeta$ score average listed) and (2) using the original outlier $\zeta$-score in the calculation. 
Summary of Zeta-Scores by Compound for Bile Acids: Material 1950
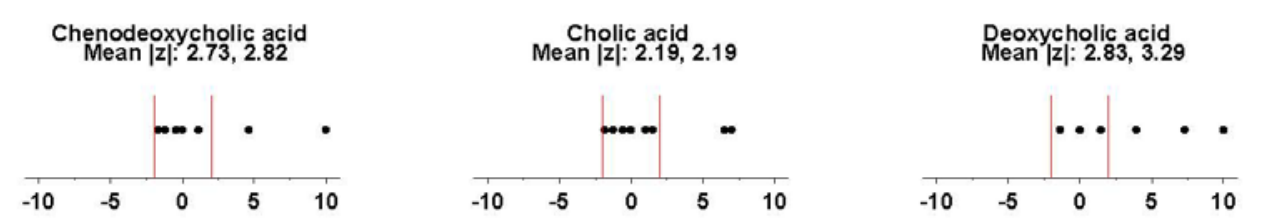

Glycochenodeoxycholicacid
Mean $|z|: 1.44,1.44$
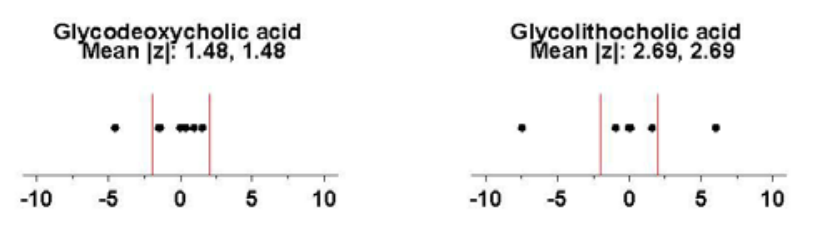

Glycoursodeoxycholic acid
Mean $|z|: 1.06,1.06$
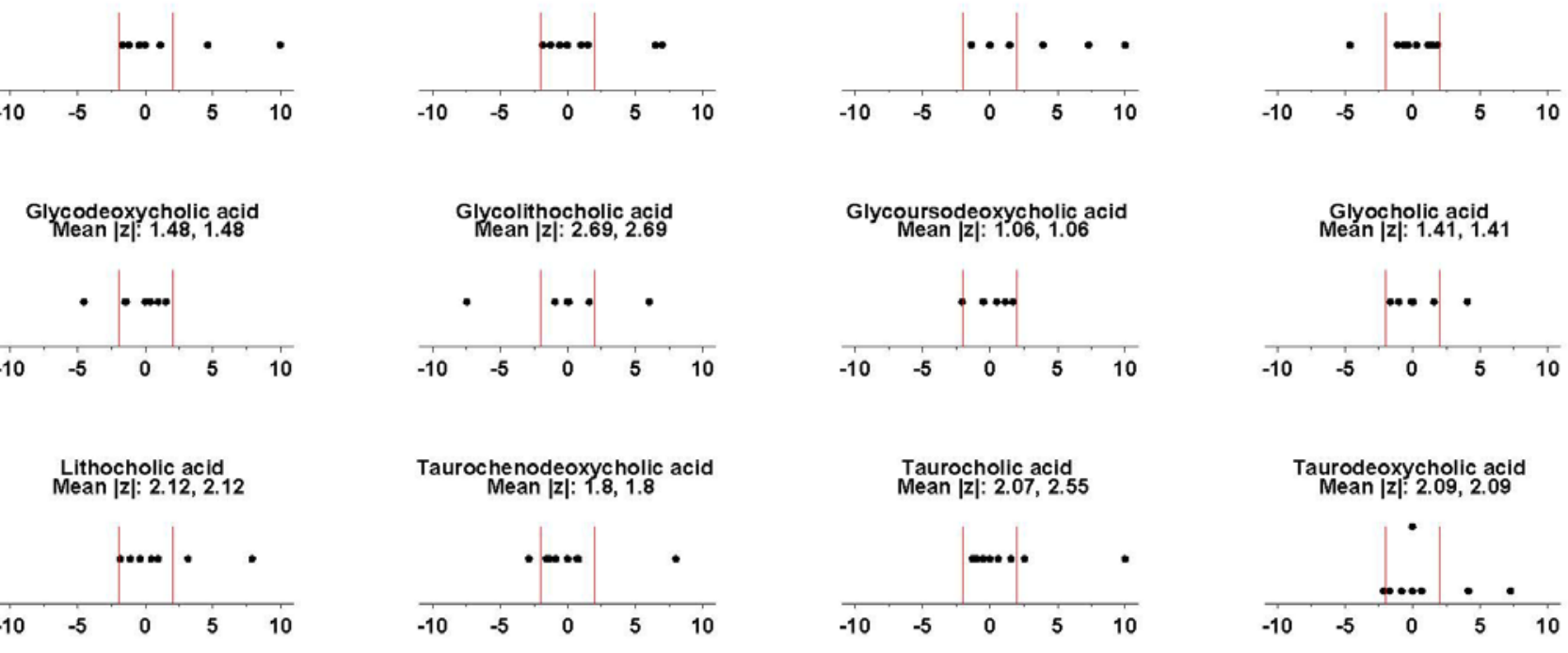

Glyocholic acid
Mean $|z|: 1.41,1.41$

Taurolithocholic acid
Mean $|z|: 1.03,1.03$

Ursodeoxycholic acid
Mean $|z|: 2.99,3.6$
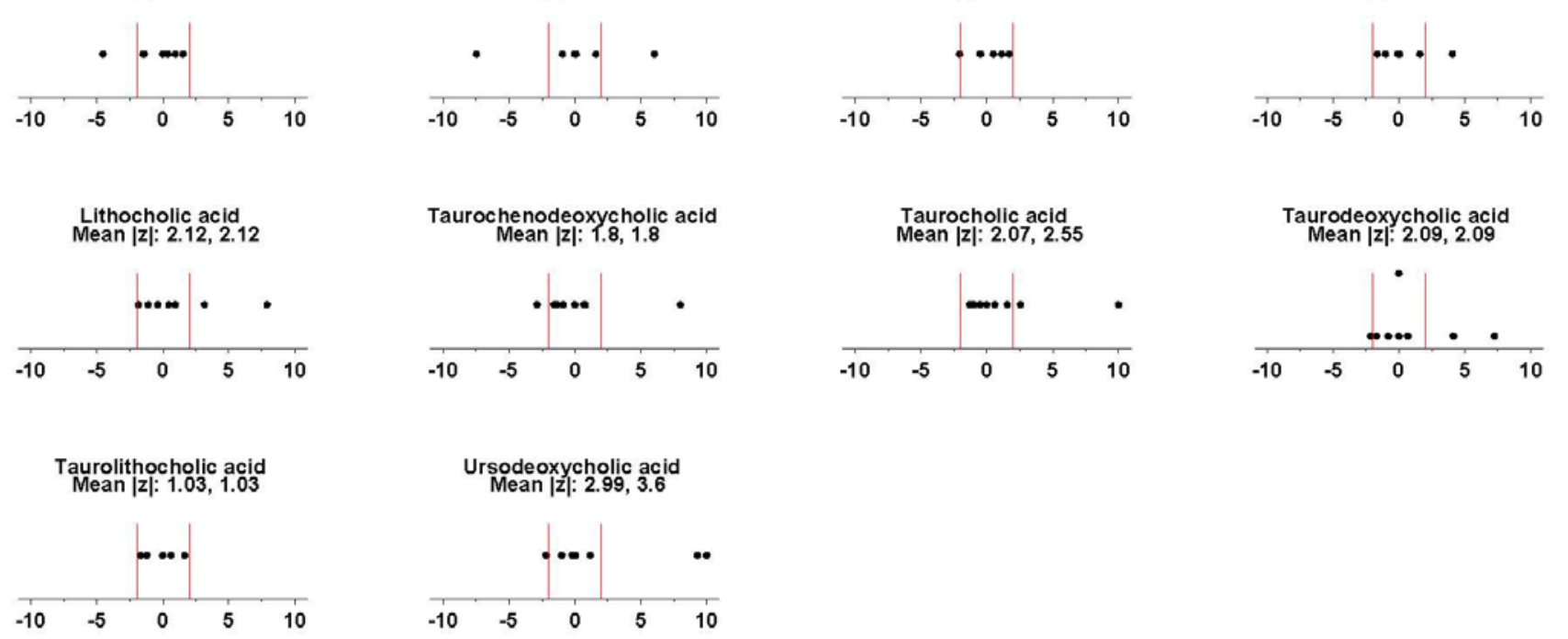

10

*Z-Scores truncated at $+l-10$ 
Summary of Zeta-Scores by Compound for CE Final: Material 1950
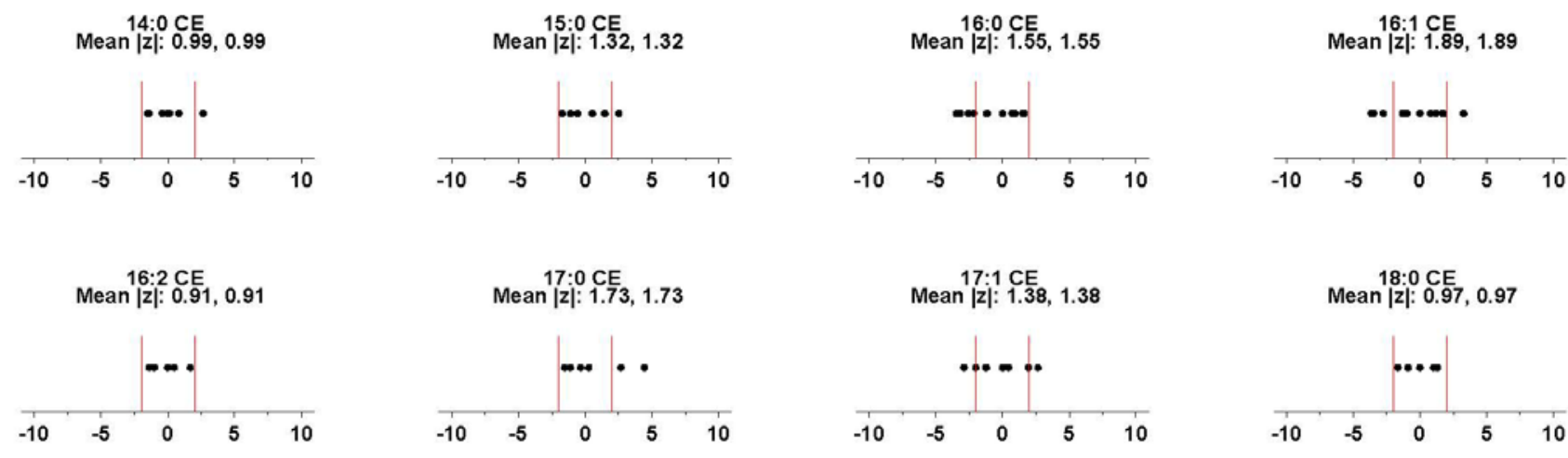

18:0 $\mathrm{CE}$
Mean $|\mathrm{z}|: 0.97,0.97$
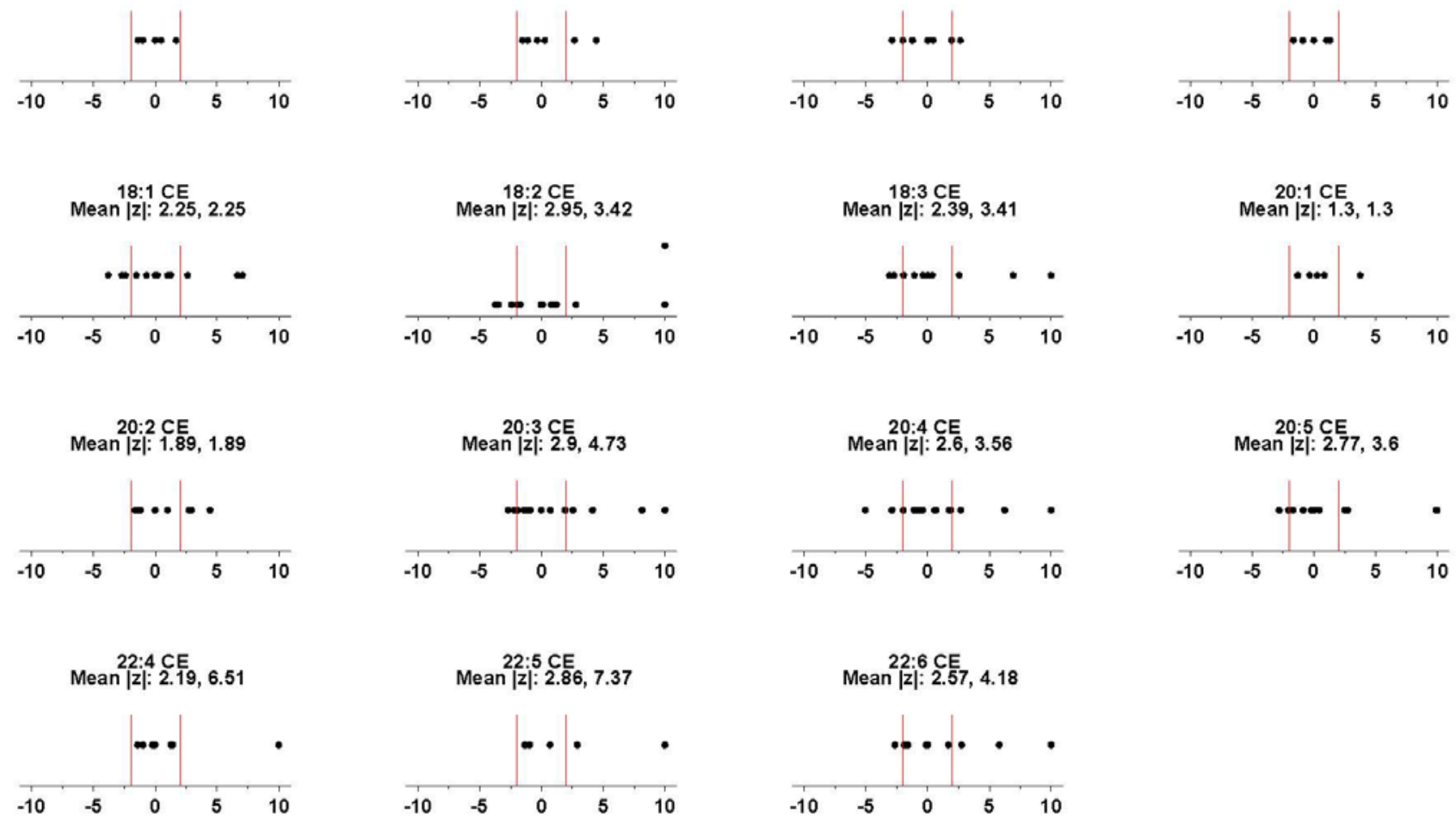

*Z-Scores truncated at $+1-10$ 
Summary of Zeta-Scores by Compound for CER Final: Material 1950
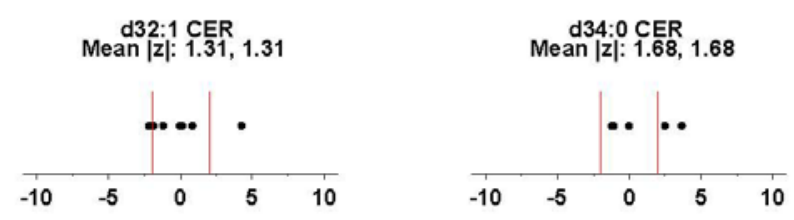

$d 38: 1$
Mean $|z|: 3.12,9.61$

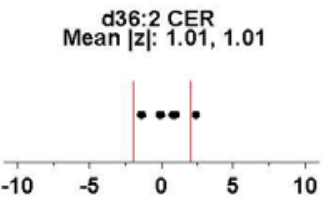

$\stackrel{d 41: 1}{\operatorname{CER}} \operatorname{Mean}|z|: 1.49,1.49$

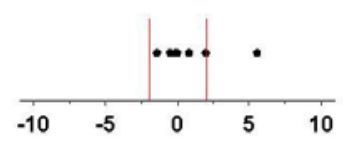

Mean $|z|: 2.58$ : $2.58,3.95$

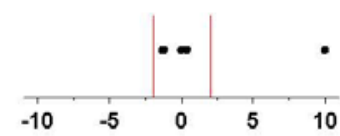

18:0 Hexosyl-Cer $($ d 18:1)
Mean $|z|: 1.23,1.23$

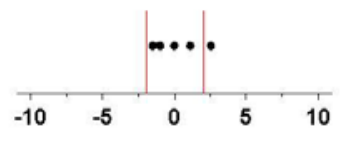

$\mathrm{d} 42: 0$ CER
Mean $|z|: 2.61,2.61$

$\mathrm{d} 44: 1$ CER
Mean $|z|: 0.98,0.98$

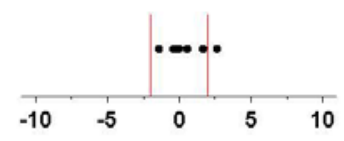

22:0 Hexosyl-Cer $(d 18: 1)$
Mean $|z|: 1.13,1.13$

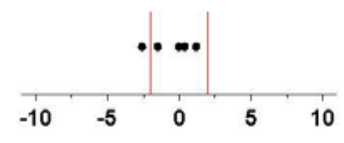

$\mathrm{d} 34: 1 \mathrm{CER}$
Mean $|z|: 2.81,3.41$

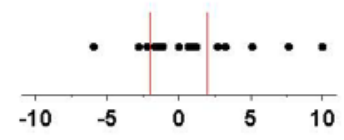

$\mathrm{d} 40: 1$
Mean $|z|: 2.16,2.39$
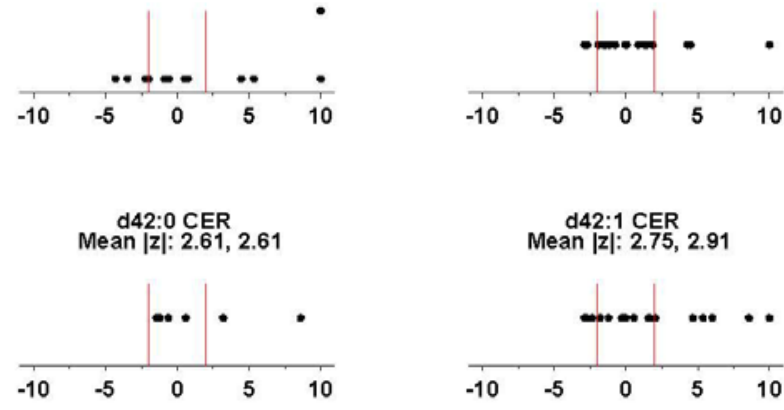

$$
\begin{gathered}
\mathrm{d} 42: 1 \text { CER } \\
\text { Mean }|z|: 2.75,2.91
\end{gathered}
$$

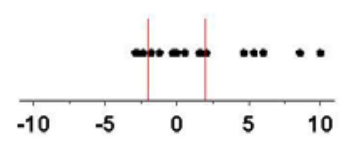

$$
\begin{aligned}
& \mathrm{d} 44: 2 \mathrm{CER} \\
& \text { Mean }|z|: 1.63,1.63
\end{aligned}
$$

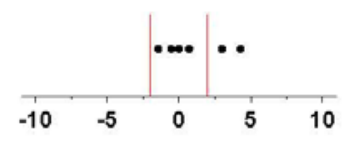

24:0 Hexosyl-Cer (d18:1)
Mean $|z|: 1.52,1.52$

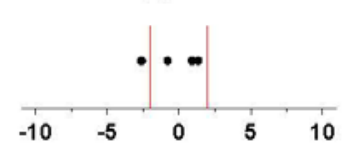

$\operatorname{d36:1} \underset{\operatorname{CER}}{\operatorname{Coan}|z|: 2.14,2.32}$

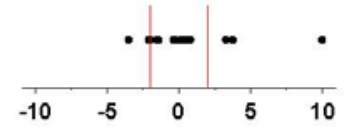

$\mathrm{d} 40: 2 \mathrm{CER}$
Mean $|z|: 2.75,4.38$

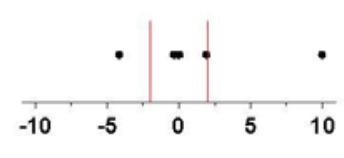

$\mathrm{d} 42: 2$ CER
Mean $|z|: 2.97,3.48$

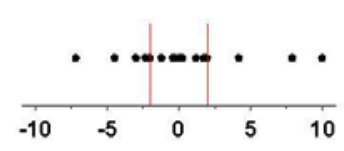

16:0 Hexosyl-Cer(d18:1))
Mean |z|: 1.06, 1.06

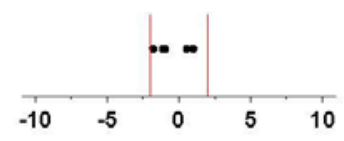

24:1 Hexosyl-Cer(d18:1)
Mean |z| $1.25,1.25$

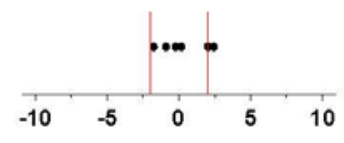

*Z-Scores truncated at $+/-10$ 


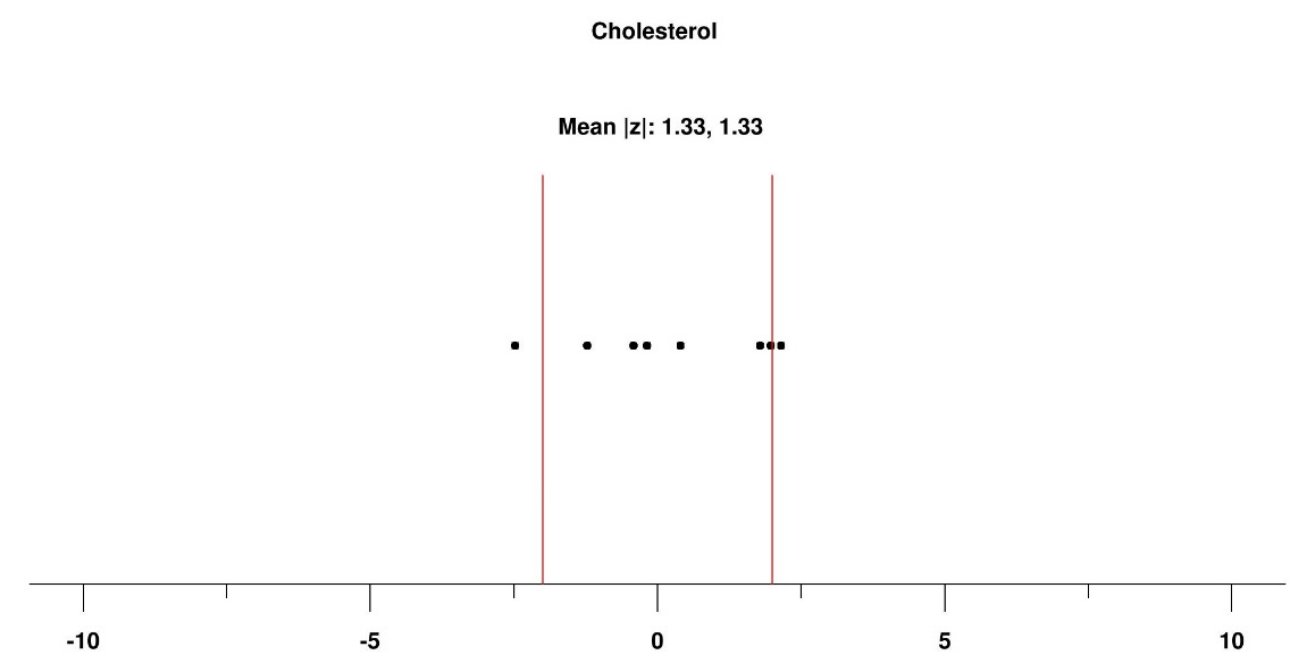


Summary of Zeta-Scores by Compound for DAG Final: Material 1950
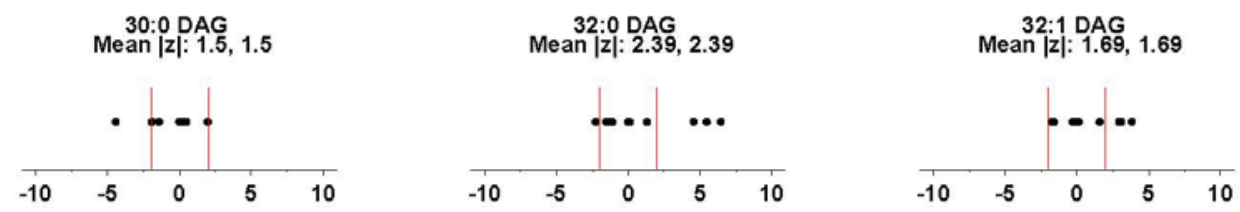

$32: 2 \mathrm{DAG}$
Mean $|z|: 1.77,1.77$
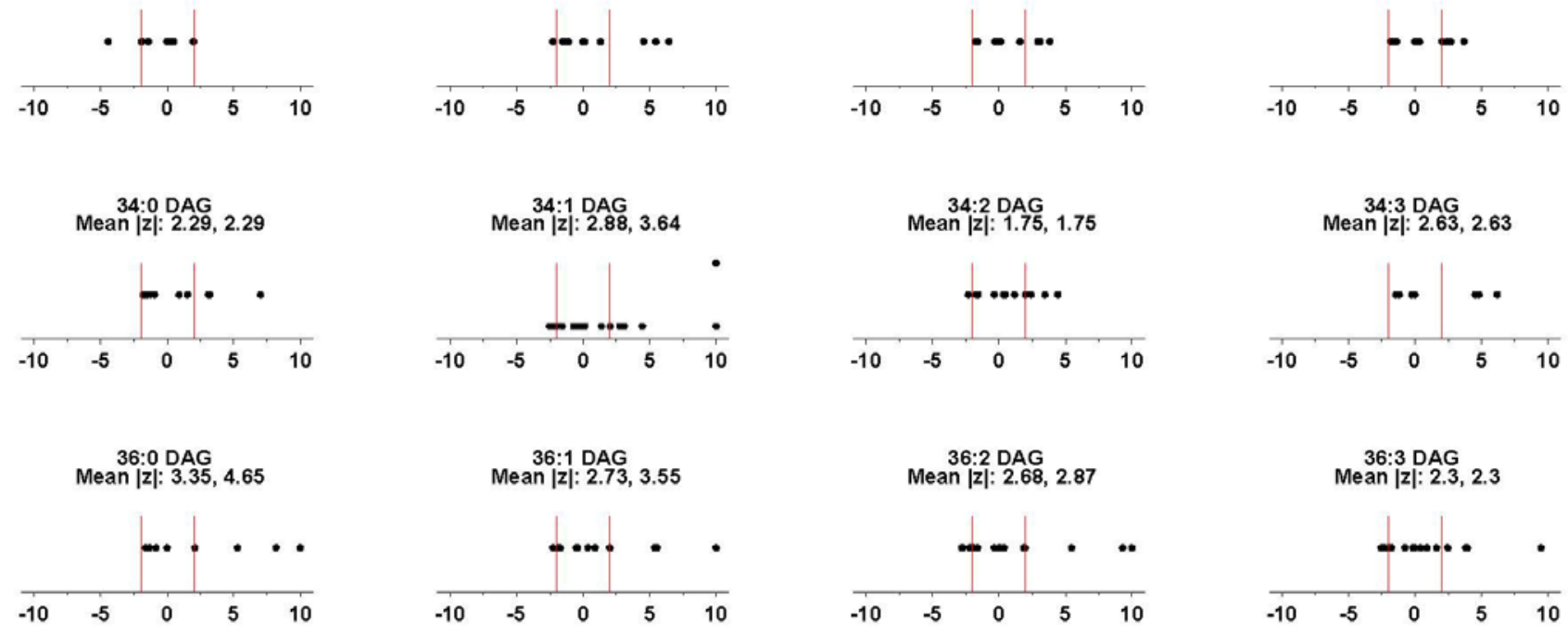

$34: 3 \mathrm{DAG}$
$\operatorname{Mean}|z|: 2.63,2.63$
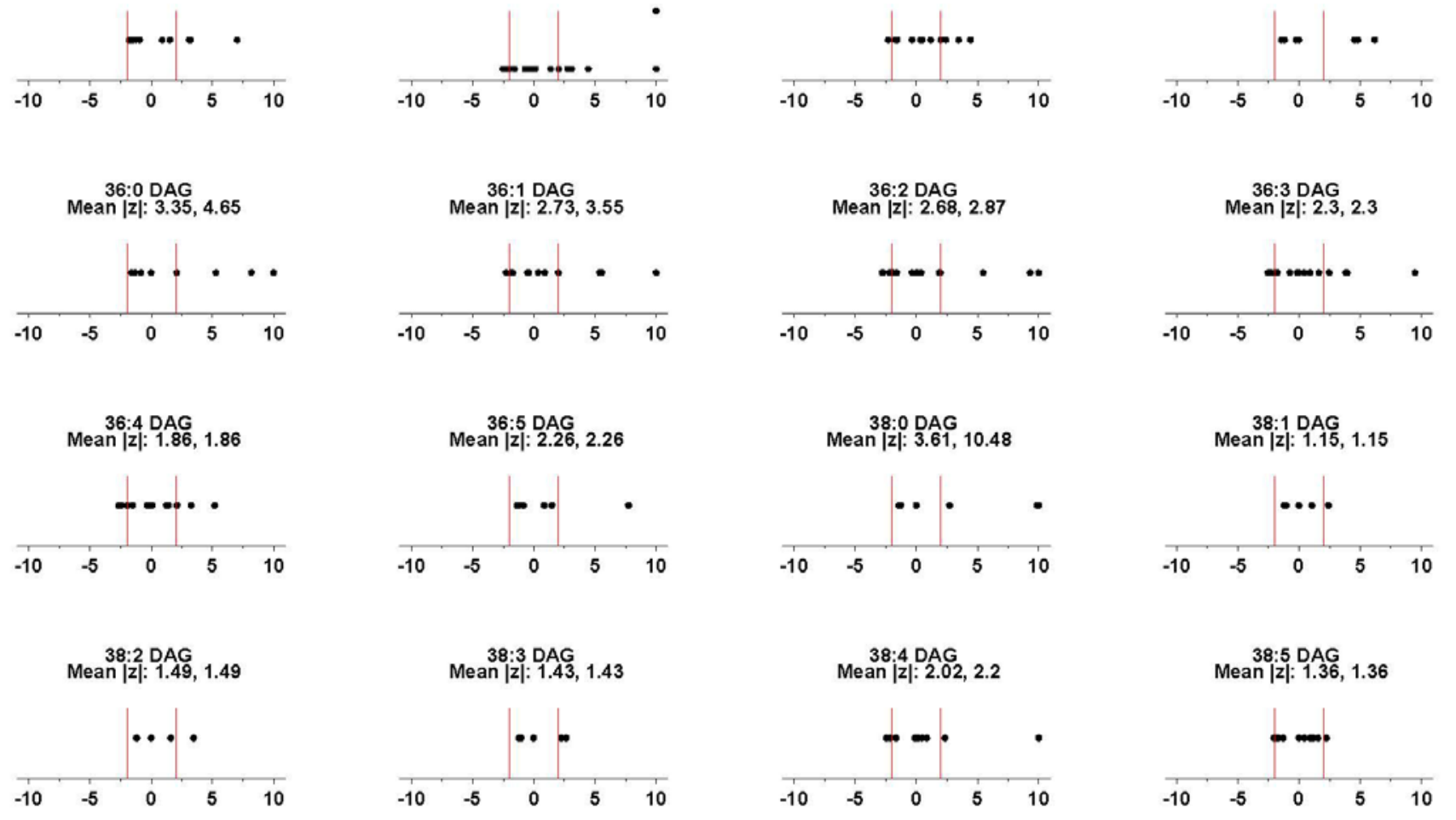

*Z-Scores truncated at $+/-10$ 
Summary of Zeta-Scores by Compound for DAG Final: Material 1950
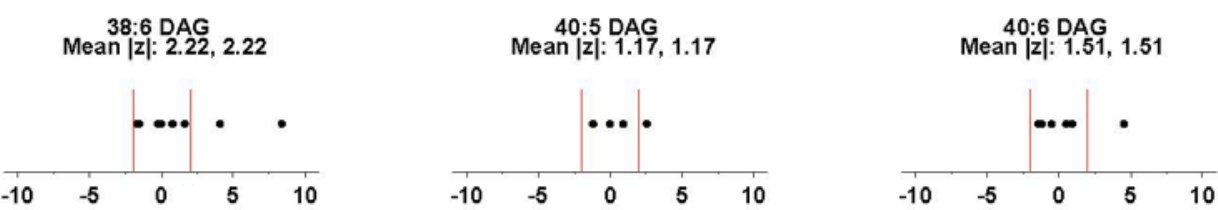

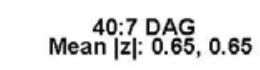

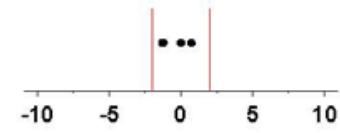

*Z-Scores truncated at +/-10 
Summary of Zeta-Scores by Compound for Eico Final: Material 1950
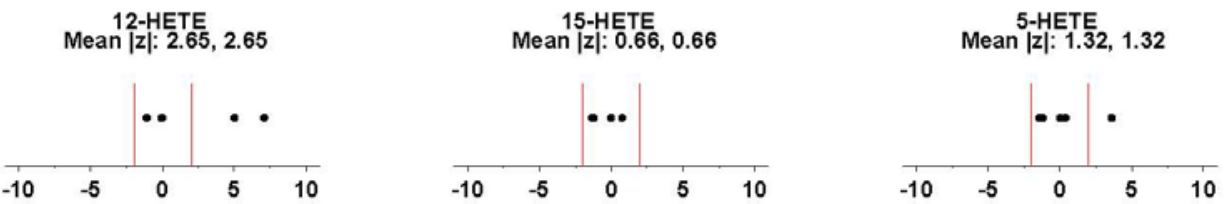

*Z-Scores truncated at $+l-10$ 
Summary of Zeta-Scores by Compound for Fatty Acids: Material 1950
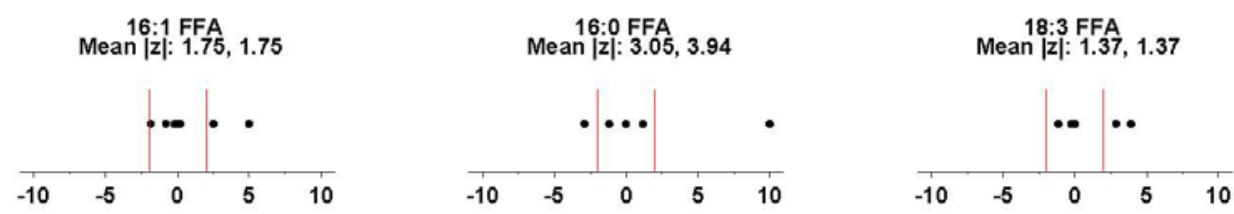

$18: 2$ FFA
Mean $|z|: 1.99$,
1.99
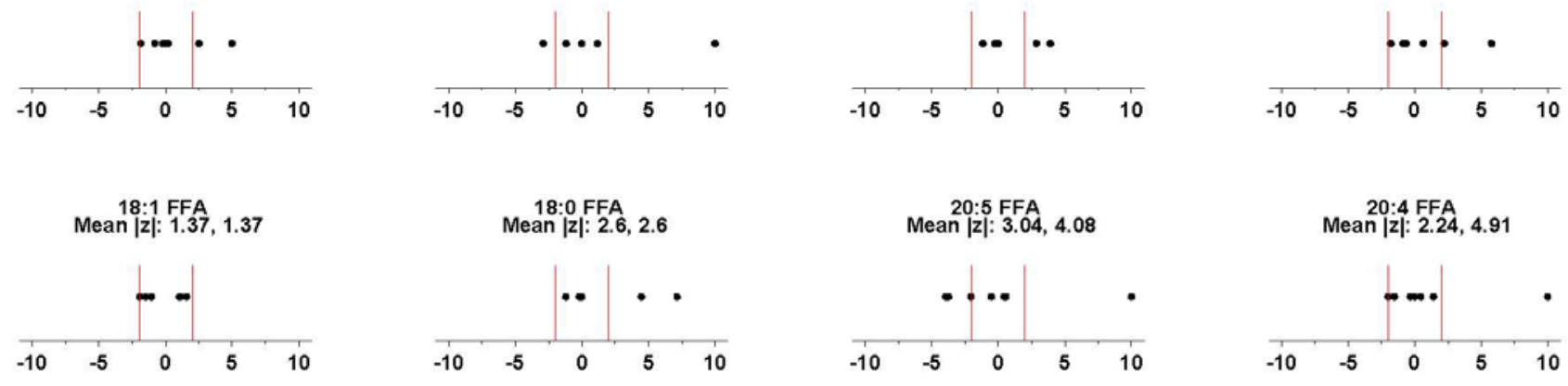

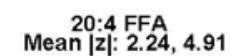

22:6 FFA

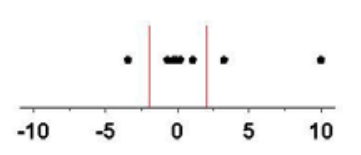

*Z-Scores truncated at $+/-10$ 
Summary of Zeta-Scores by Compound for LPC Final: Material 1950
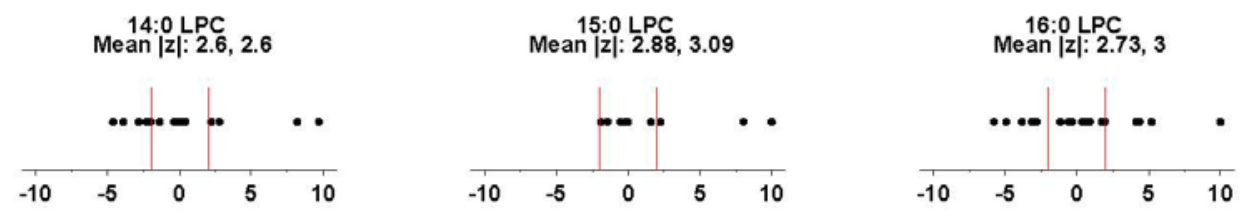

$16: 1$
$\operatorname{Mean}|z|: 2.97,3.63$

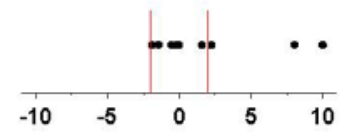

Mean $|z 7|: \mid \frac{L P C}{2.51,2.51}$
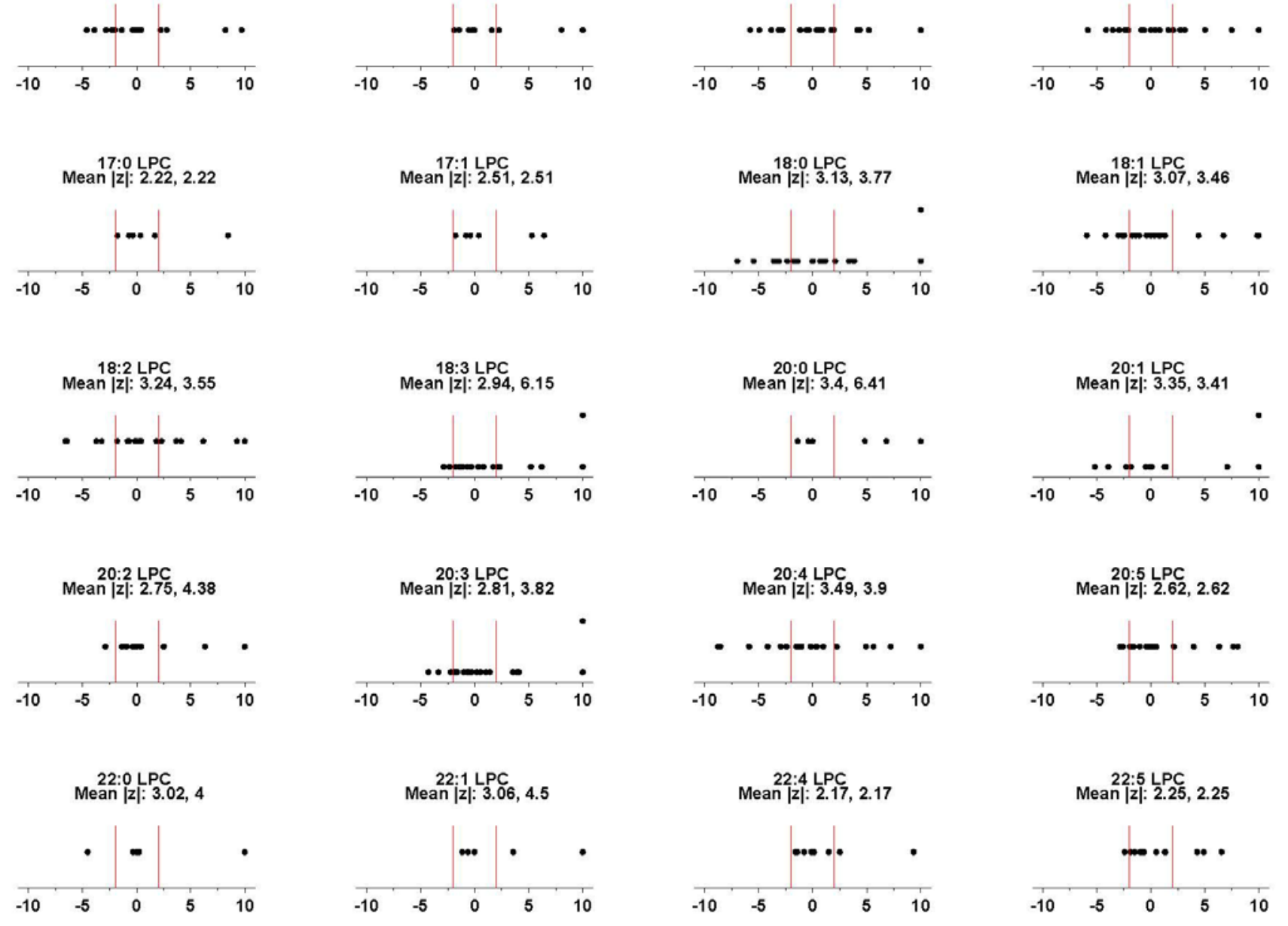

"Z-Scores truncated at $+l-10$ 


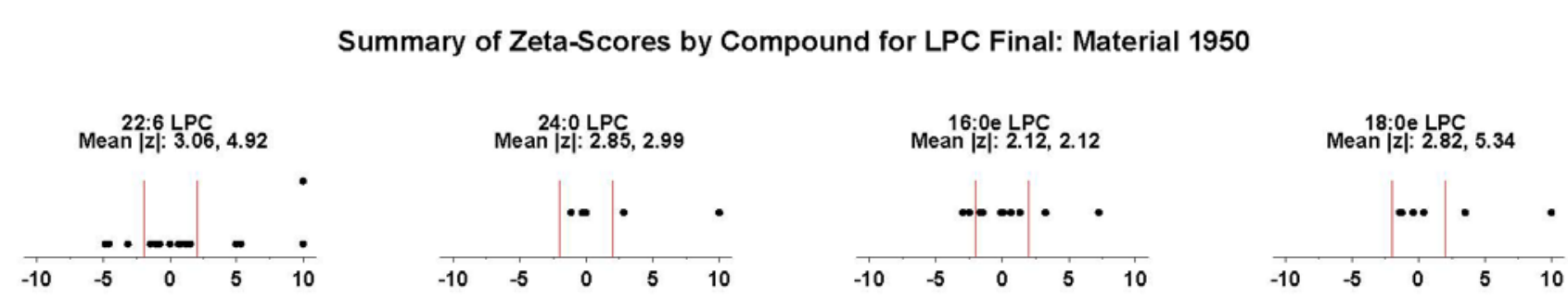

16:0p $L P C$
Mean |z|: $2.34,2.34$

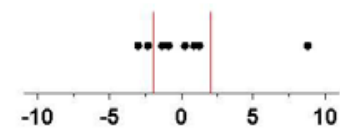

*Z-Scores truncated at $+/-10$ 


\section{Summary of Zeta-Scores by Compound for LPE Final: Material 1950}
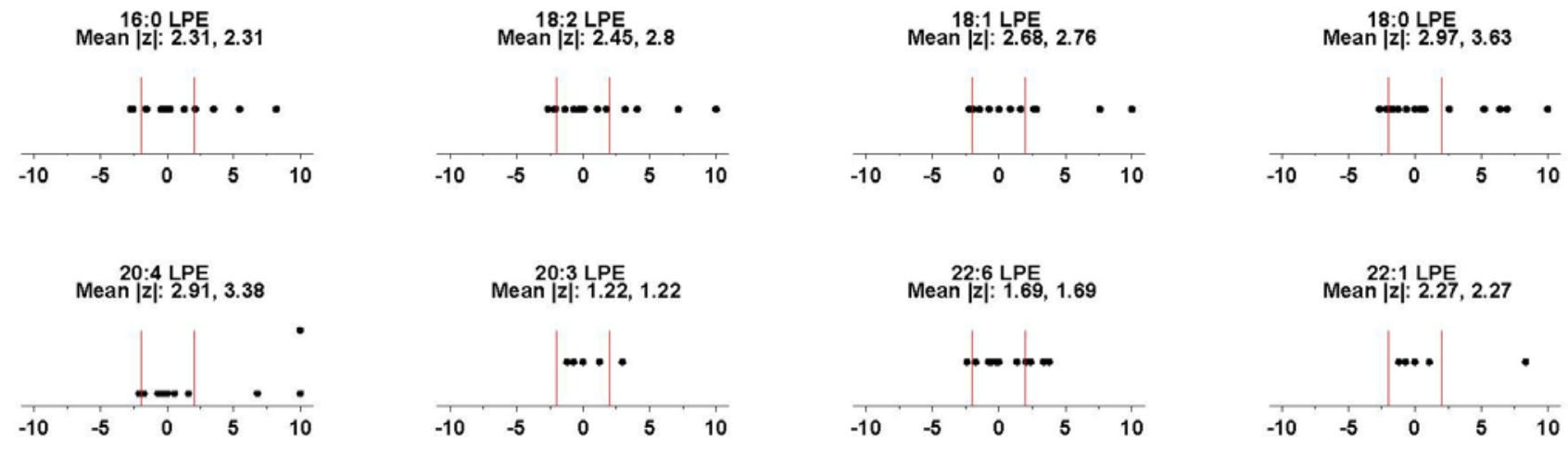

*Z-Scores truncated at $+/-10$ 
Summary of Zeta-Scores by Compound for Other Final: Material 1950
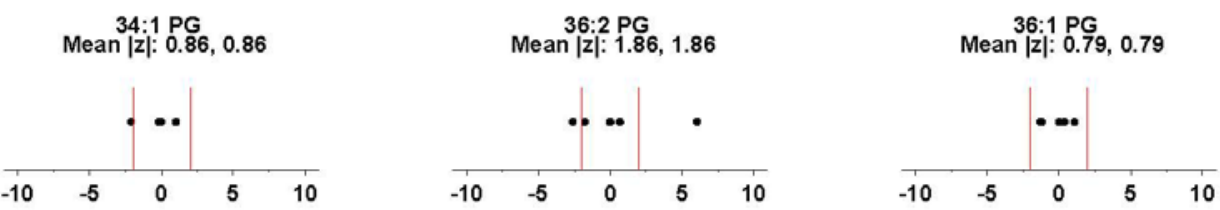

*Z-Scores truncated at $+/-10$ 
Summary of Zeta-Scores by Compound for PC Final: Material 1950
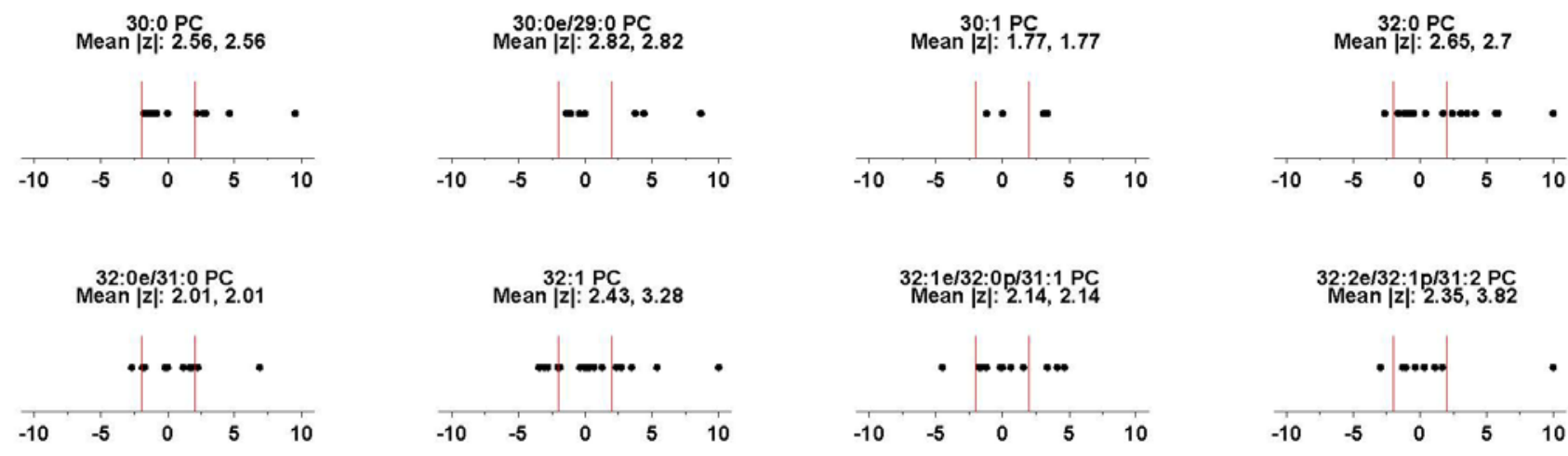

\section{2:1e/32:00/31:1 PC
Mean $|z|: 2.14,2.14$}

32:2e/32: $1 \mathrm{p} / 31: 2 \mathrm{PC}$
Mean $|z|: 2.35,3.82$
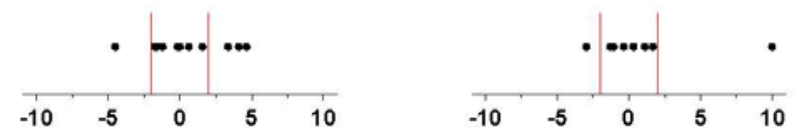

$$
\begin{gathered}
32: 3 \text { PC } \\
\text { Mean }|z|: 2.39,2.39
\end{gathered}
$$

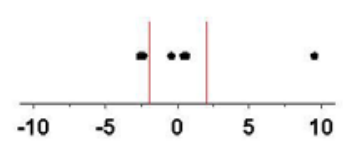

$$
\operatorname{Mean} \mid \begin{aligned}
& 33: 1 / 32: 2 \\
& \mid z C \\
& 2.62,2.62
\end{aligned}
$$

$$
\operatorname{Meann}|z 4| 0 \mathrm{PC}
$$

34:0e/33:0 PC
Mean $|z|: 1.8,1.8$
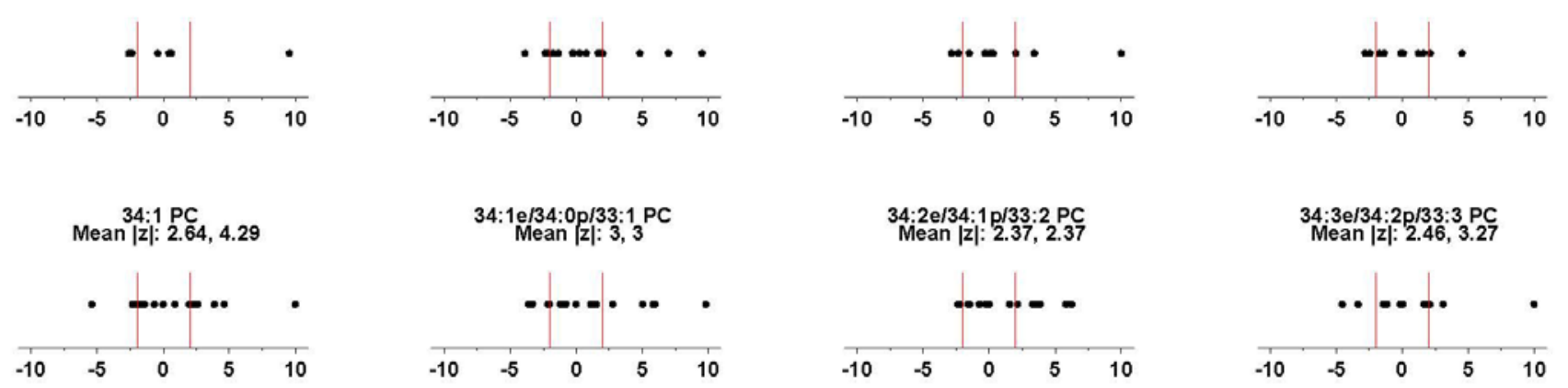

\section{$34: 2 e / 34: 1 / 3 / 3: 2 \mathrm{PC}$
Mean $|z|: 2.37,2.37$}

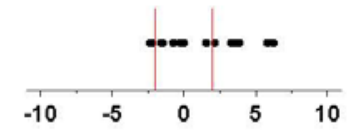

\section{$34: 3 \mathrm{e} / 34: 2 \mathrm{p} / 33: 3 \mathrm{PC}$
Mean $|z|: 2.46,3.27$}
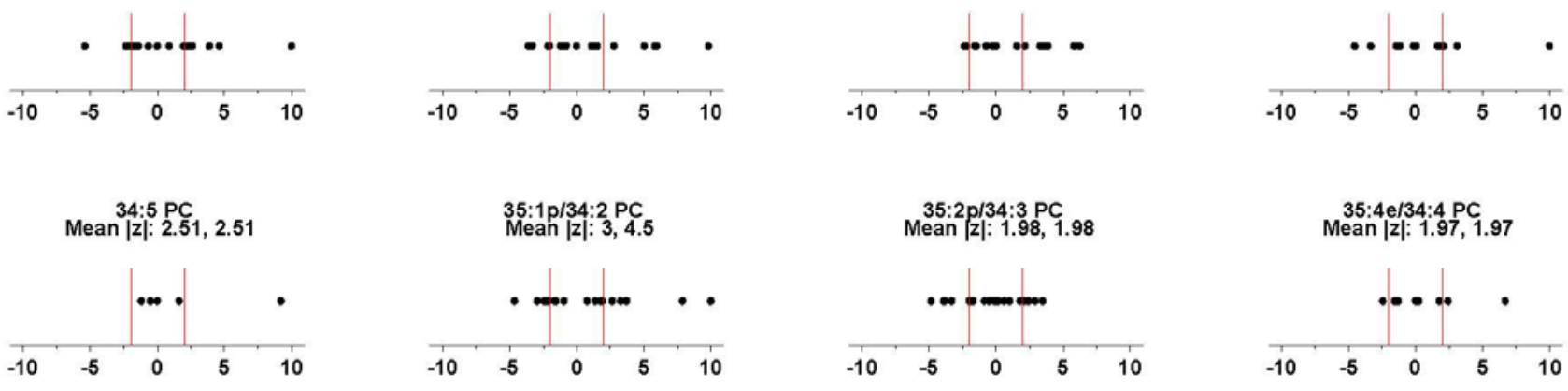

"Z-Scores truncated at $+1-10$ 
Summary of Zeta-Scores by Compound for PC Final: Material 1950
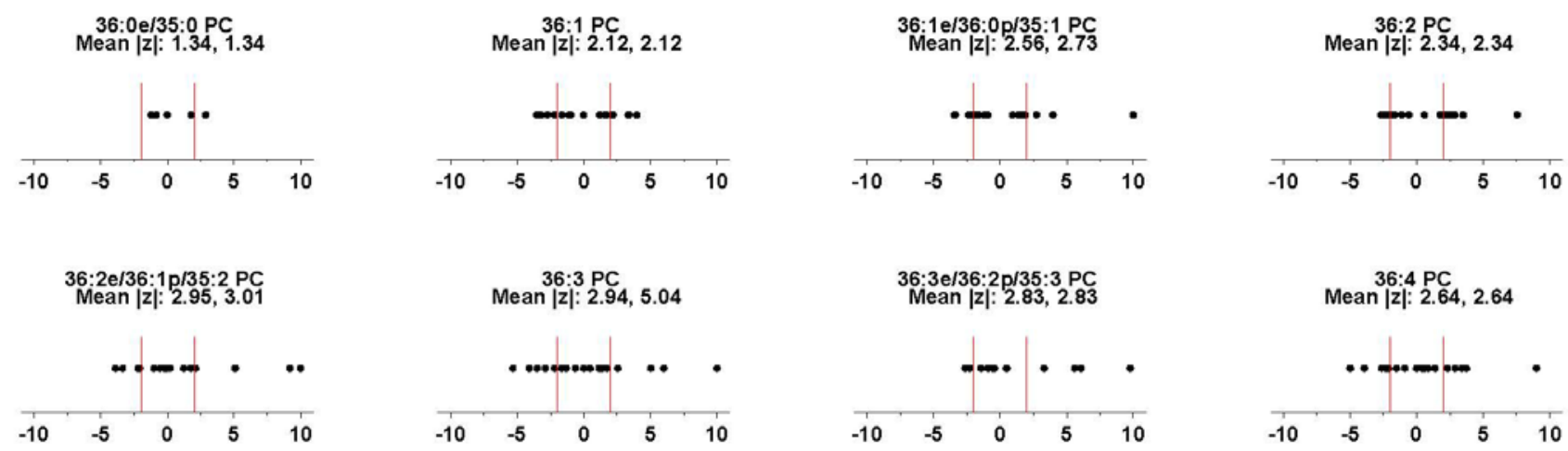

$36: 3 e / 36: 2 p / 35: 3 P C$
Mean $|z|: 2.83,2.83$

Mean $|z|: 2.64,2.64$
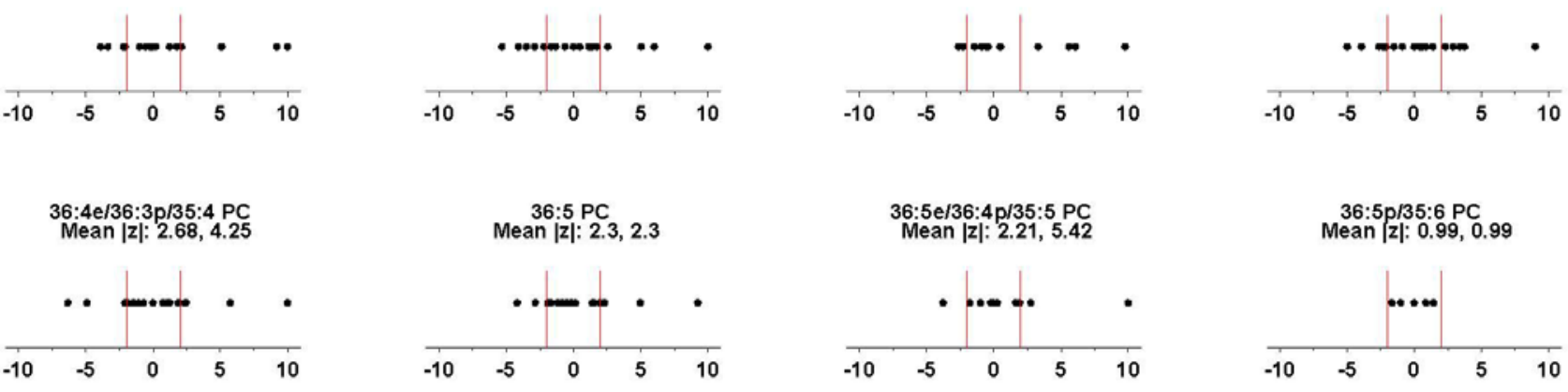

$36: 5 p / 35: 6$ PC
Mean $|z|: 0.99,0.99$

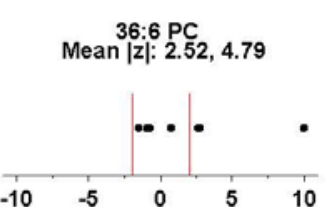

$$
\text { Mean }|z|: 0 \text { : } 1.04,1.04
$$

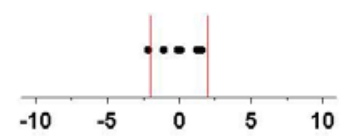

Mean $38: 1$ PC $0.96,0.96$
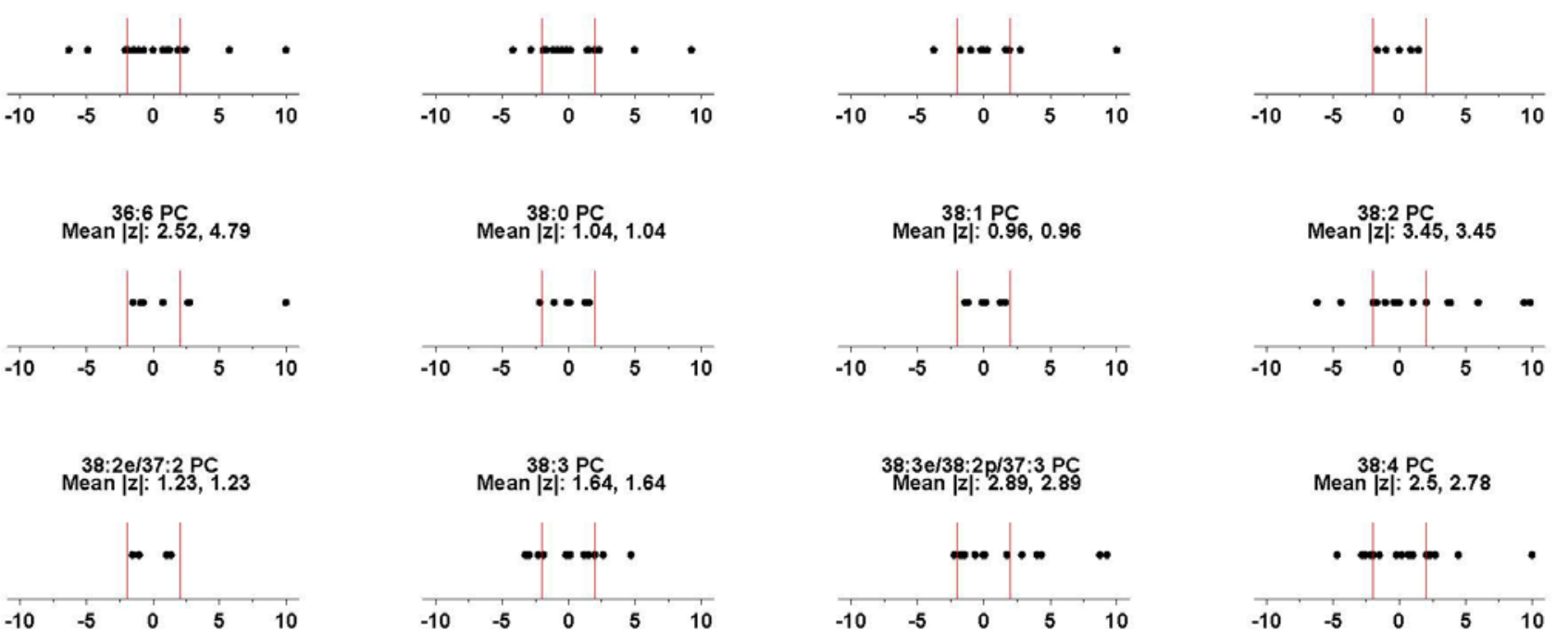

" $Z$-Scores truncated at $+1-10$ 
Summary of Zeta-Scores by Compound for PC Final: Material 1950
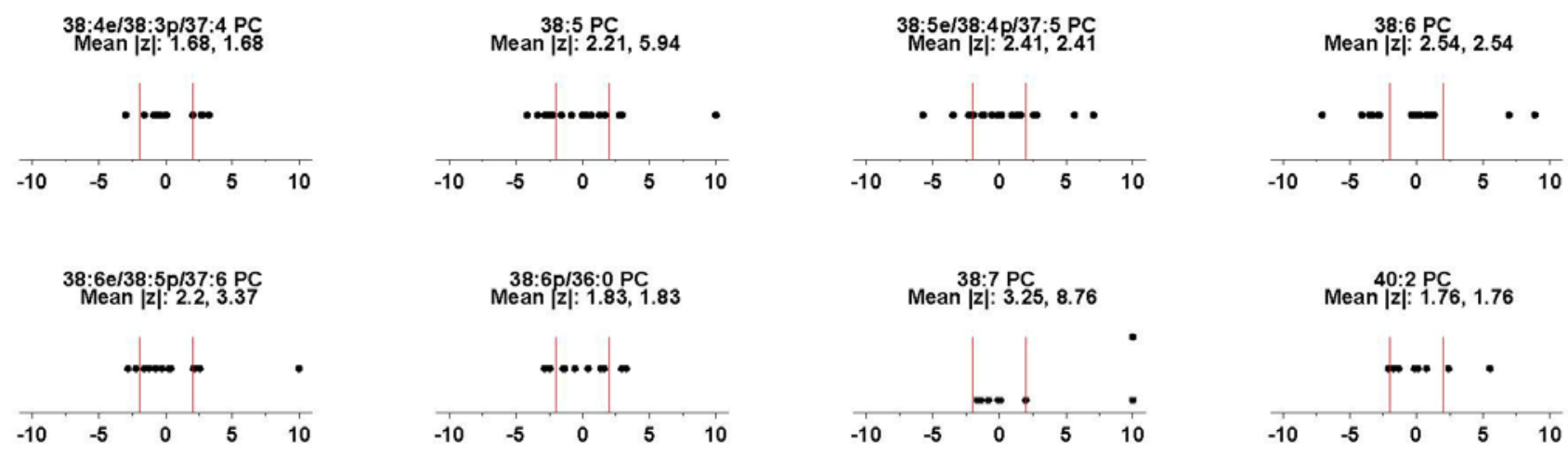

$$
\operatorname{Mean}|z|: 1.76,1.76
$$
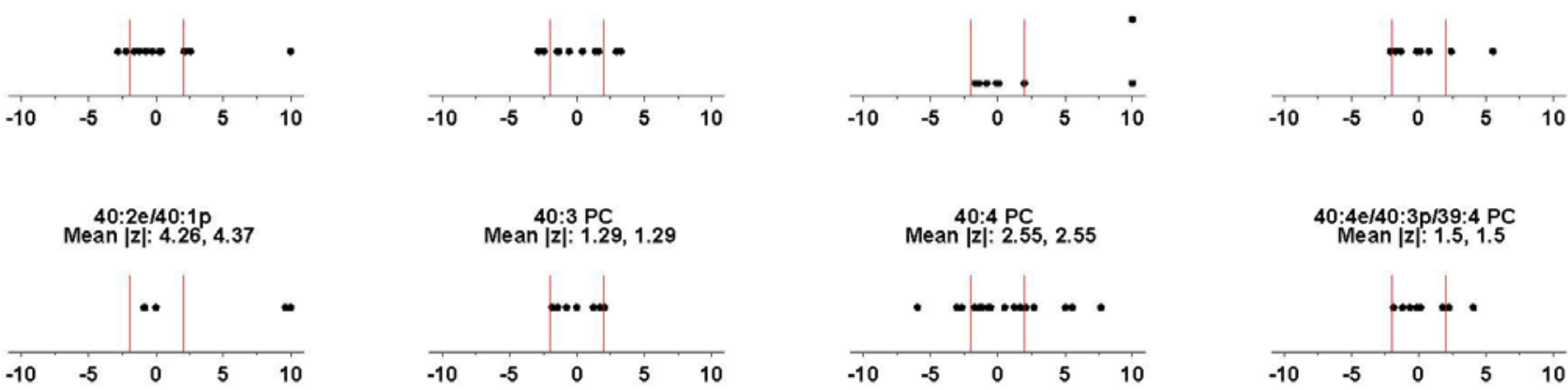

\section{$40: 4 e / 40: 3 p / 39: 4 \mathrm{PC}$
Mean $|z|: 1.5,1.5$}
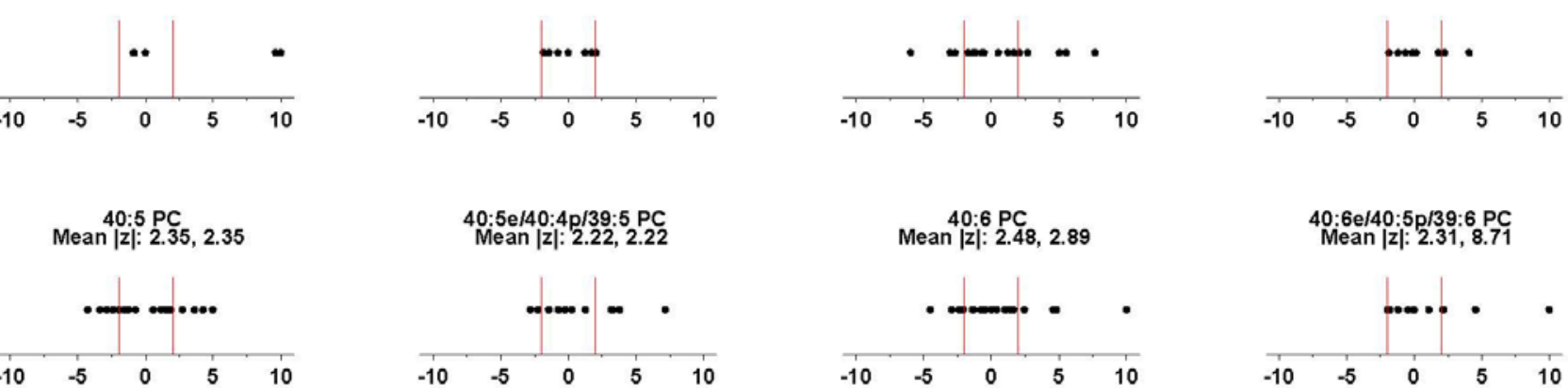

$40: 6 \mathrm{PC}$
$\operatorname{Mean}|z|: 2.48,2.89$

\section{$40: 6 e / 40: 5 \mathrm{p} / 39: 6 \mathrm{PC}$
Mean $|z|: 2.31,8.71$}
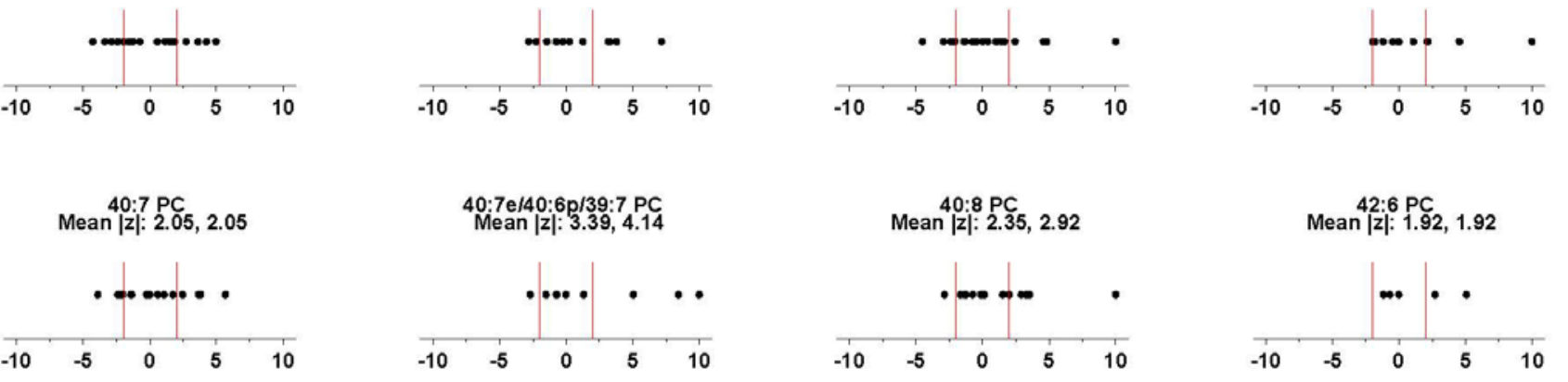

*Z-Scores truncated at $+1-10$ 
Summary of Zeta-Scores by Compound for PC Final: Material 1950
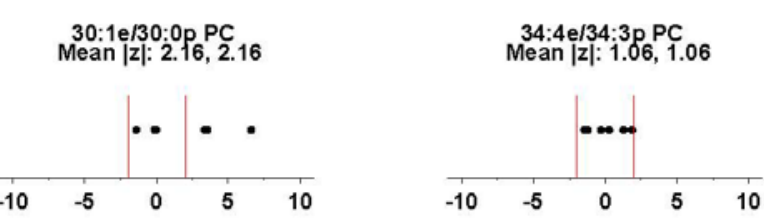

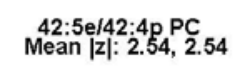

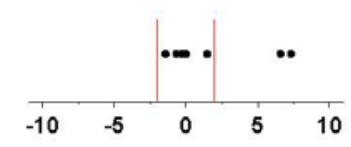

"Z-Scores truncated at +1-10 
Summary of Zeta-Scores by Compound for PE Final: Material 1950
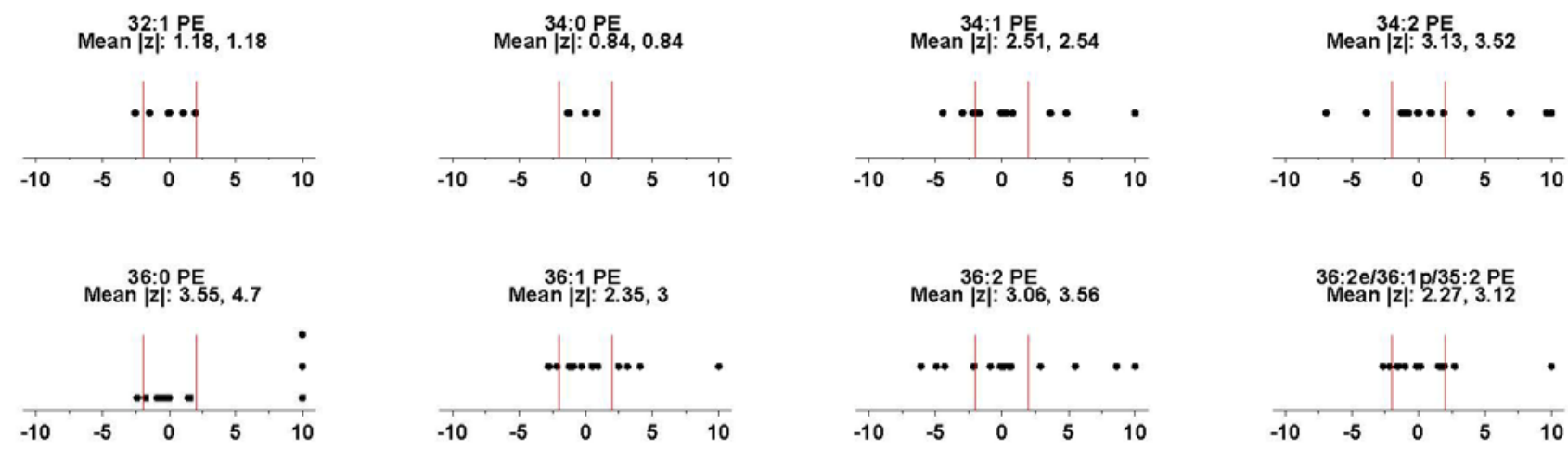

36:2e/36:1 $\mathrm{p} / 35: 2 \mathrm{PE}$
Mean $|\mathrm{z}|: 2.27,3.12$
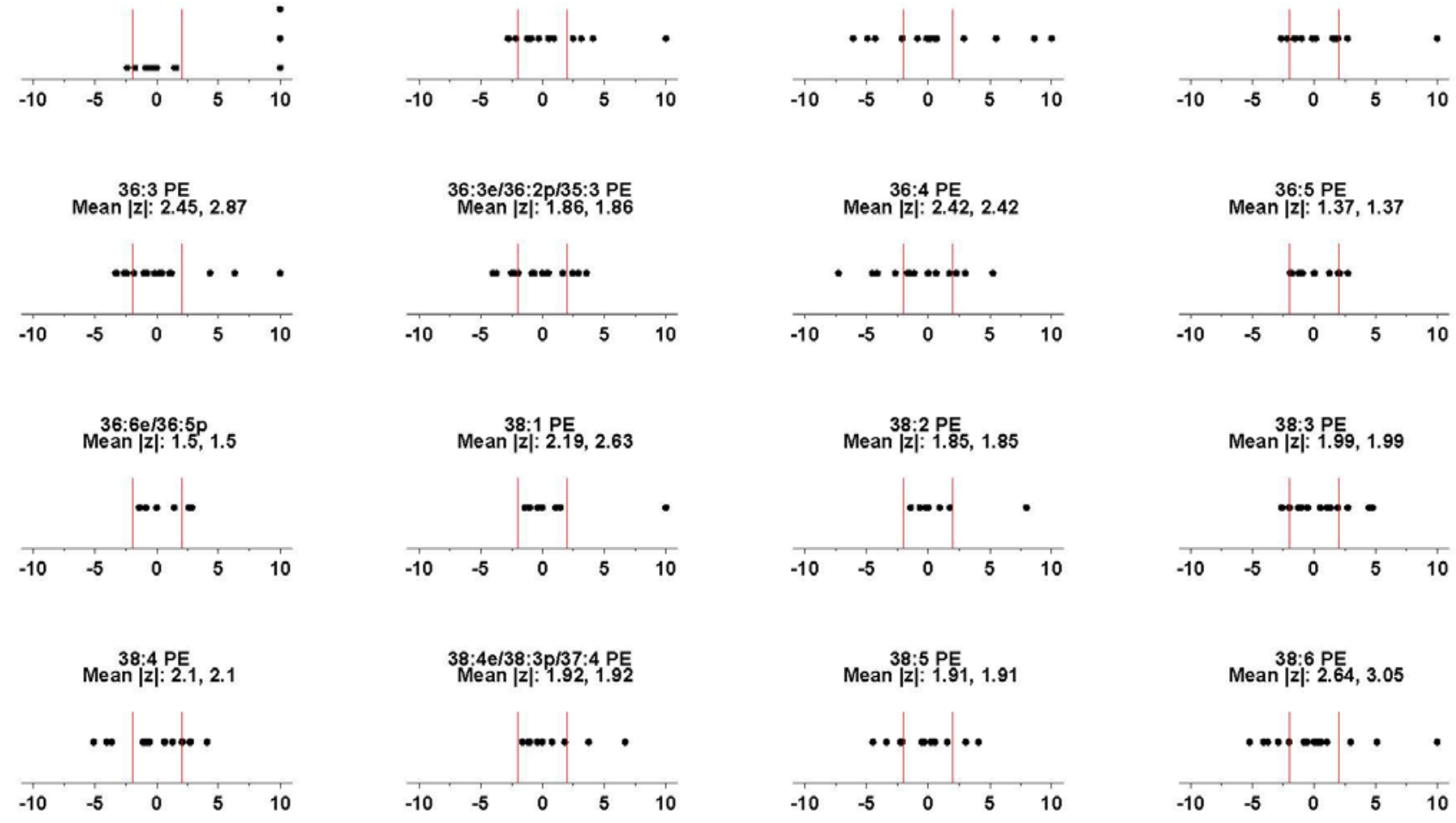

*Z-Scores truncated at +/-10 
Summary of Zeta-Scores by Compound for PE Final: Material 1950
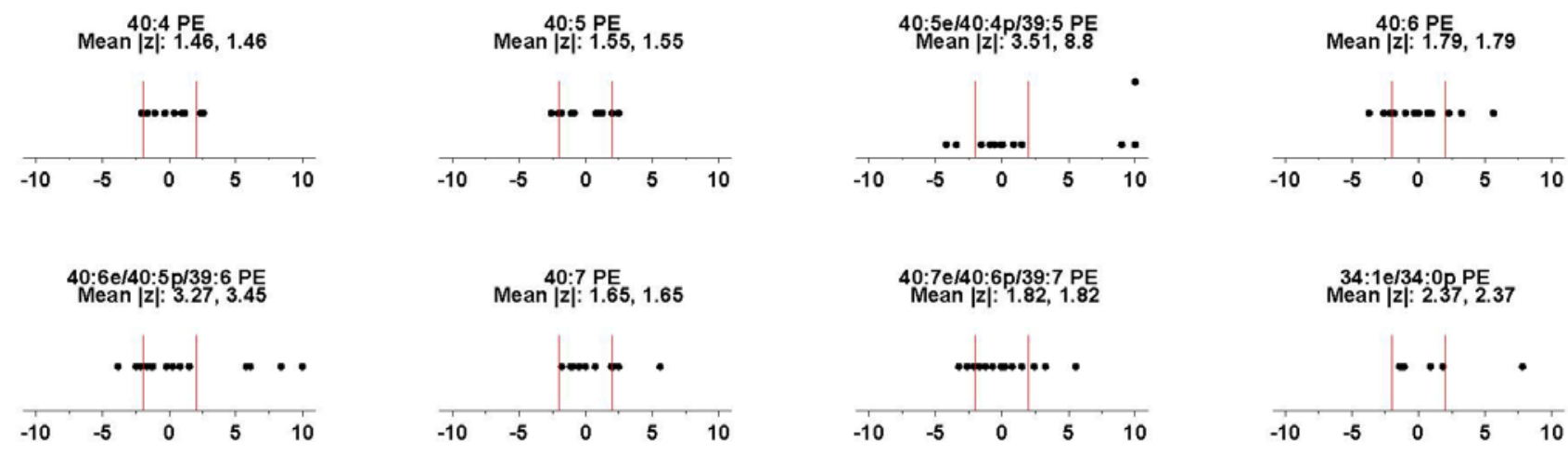

\section{0:7e/40:6p/39:7 PE
Mean $|\mathrm{z}|: 1.82,1.82$}

$34: 1 \mathrm{e} / 34: 0 \mathrm{PE}$
Mean $|z|: 2.37,2.37$
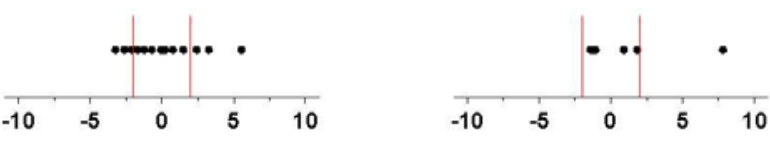

$$
\begin{aligned}
& 34: 2 e / 34: 19 P E \\
& \text { Mean }|2| \mid 1.67,1.67
\end{aligned}
$$

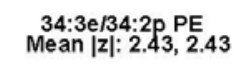
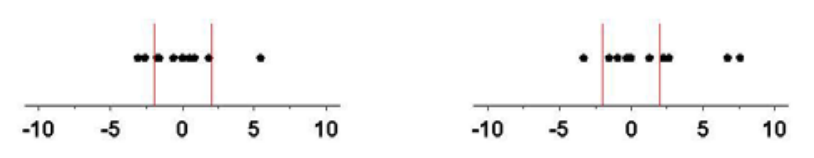

$$
\begin{aligned}
& \text { 36:4e/36: } 3 \mathrm{PE} \\
& \text { Mean }|z|: 3.73,3.34
\end{aligned}
$$

36:5e/36:40 PE
Mean $|2|: 2.4,3.82$
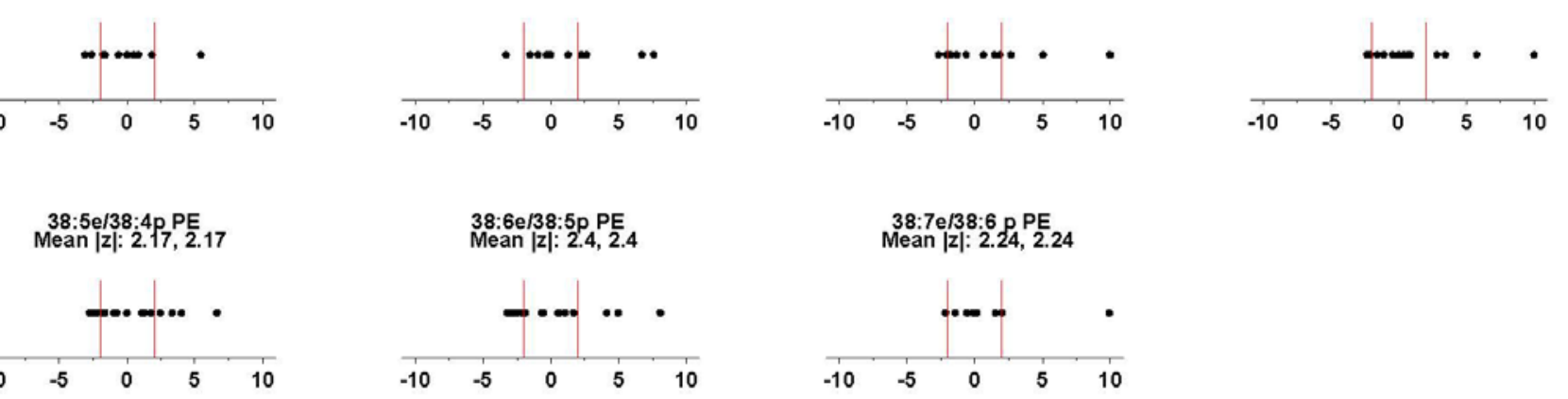

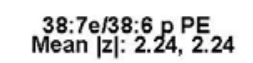

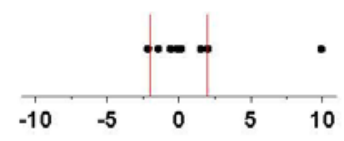

"Z-Scores truncated at $+/-10$ 
Summary of Zeta-Scores by Compound for PI Final: Material 1950
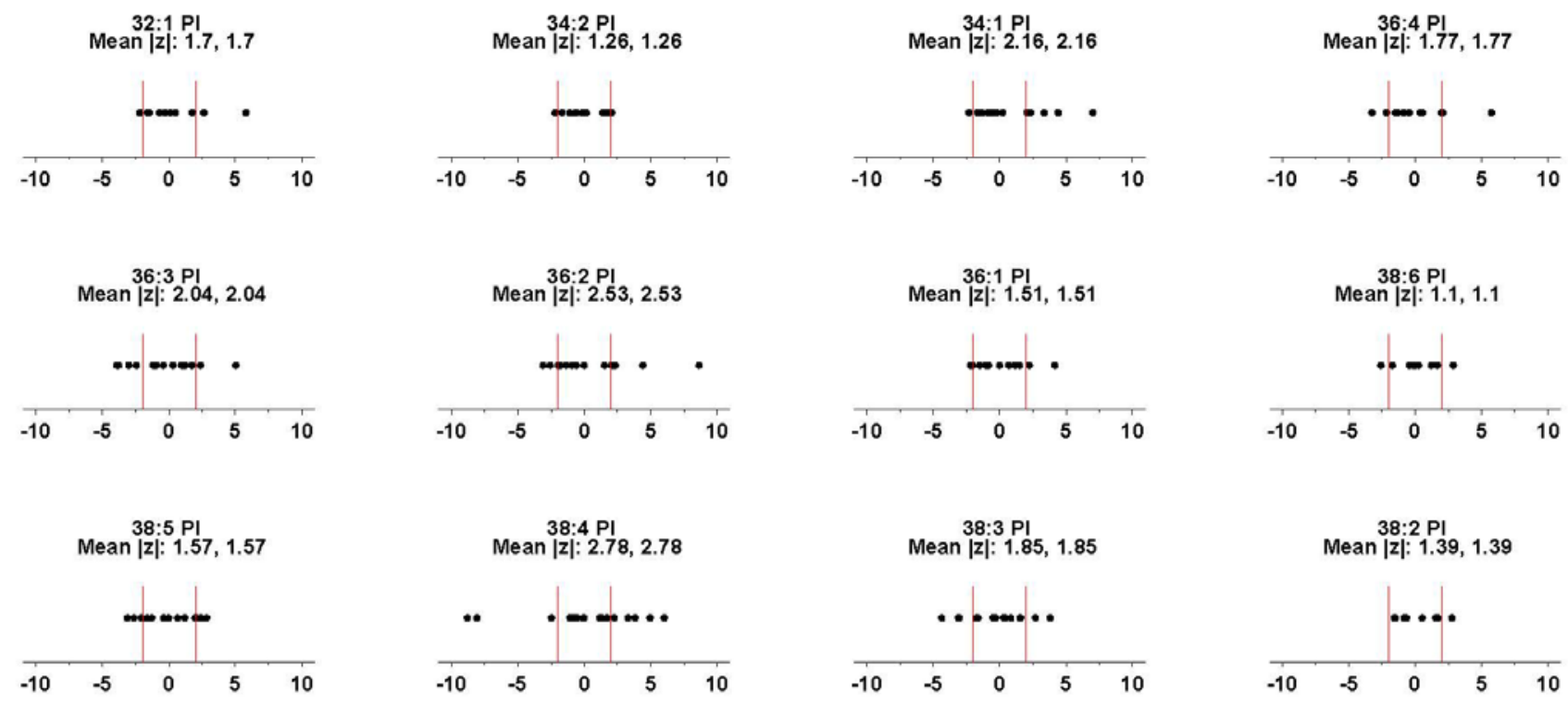

$$
\begin{gathered}
38: 2 \text { PI } \\
\text { Mean }|z|: 1.39,1.39
\end{gathered}
$$
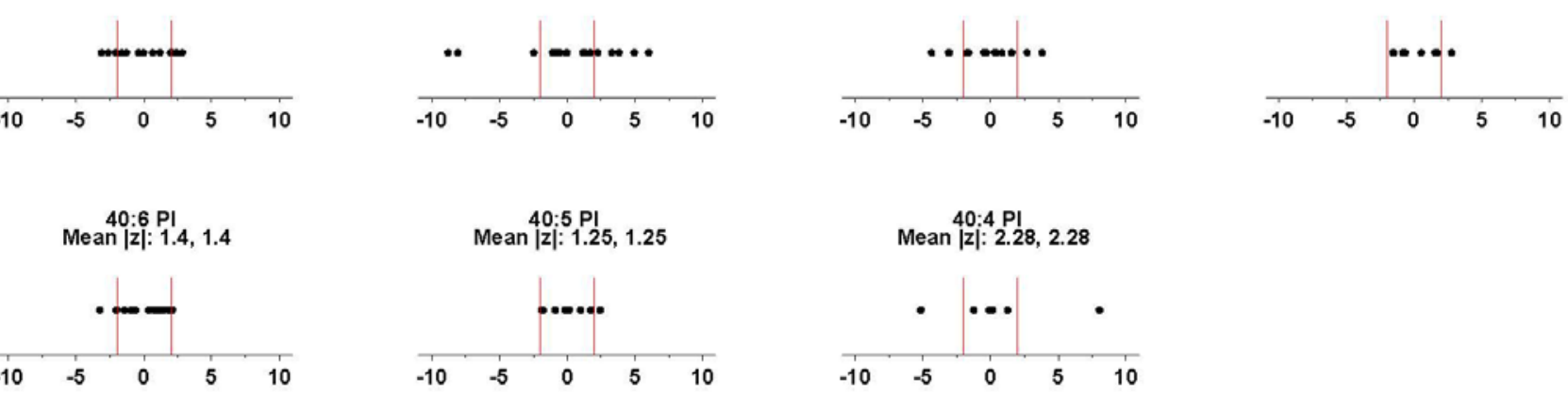

"Z-Scores truncated at $+/-10$ 
Summary of Zeta-Scores by Compound for PS Final: Material 1950

38:4 PS

Mean |z|: 1.19, 1.19

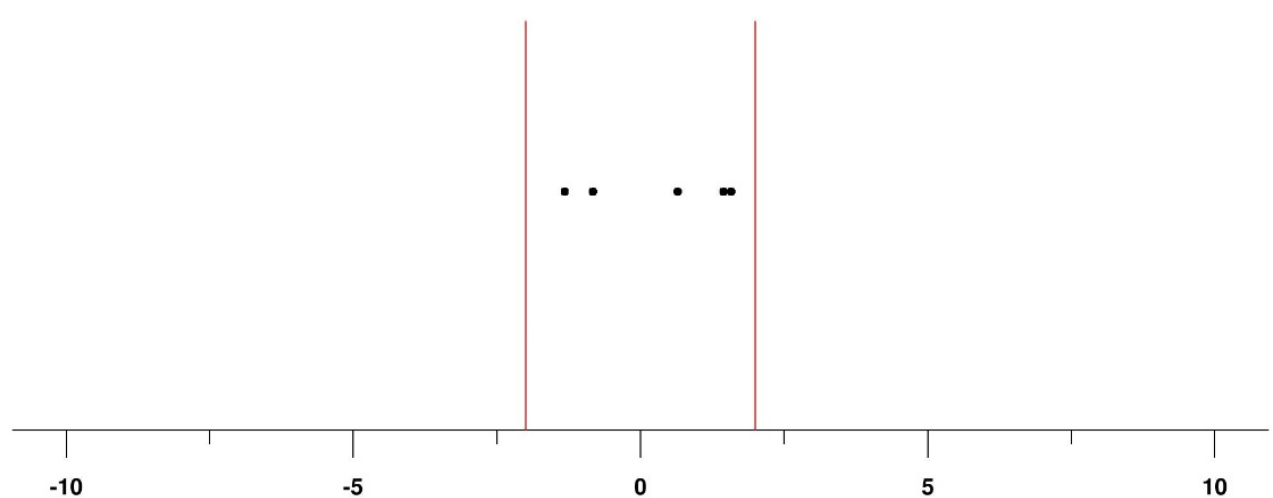

*Z-Scores truncated at $+/-10$ 
Summary of Zeta-Scores by Compound for SM Final: Material 1950
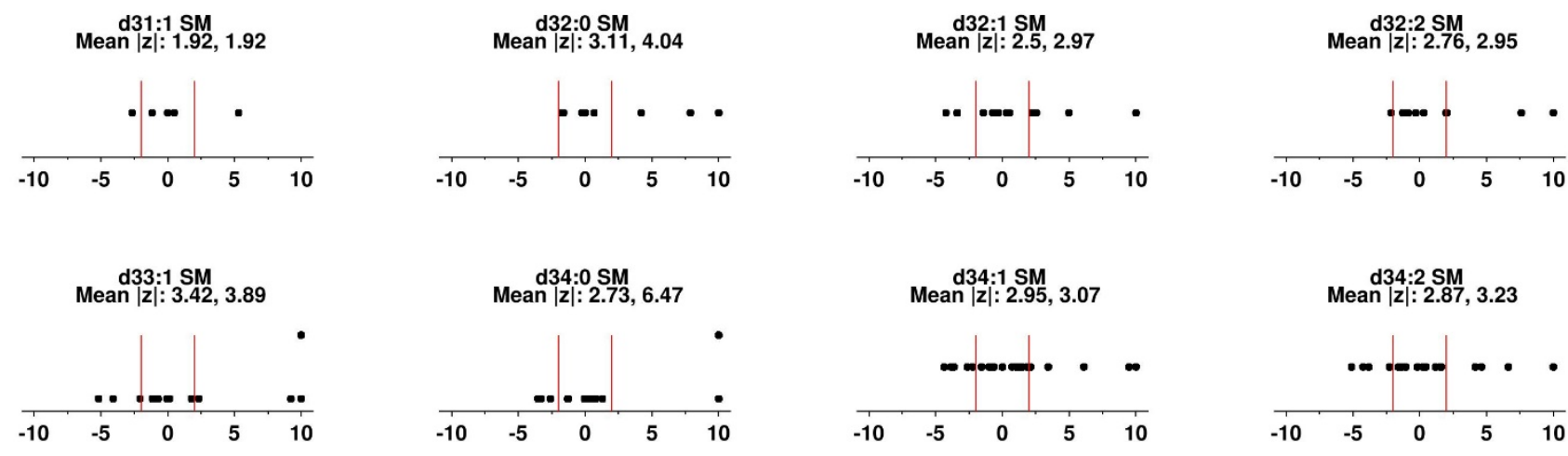

$d 34: 2 \mathrm{SM}$
$\operatorname{Mean}|z|: 2.87,3.23$
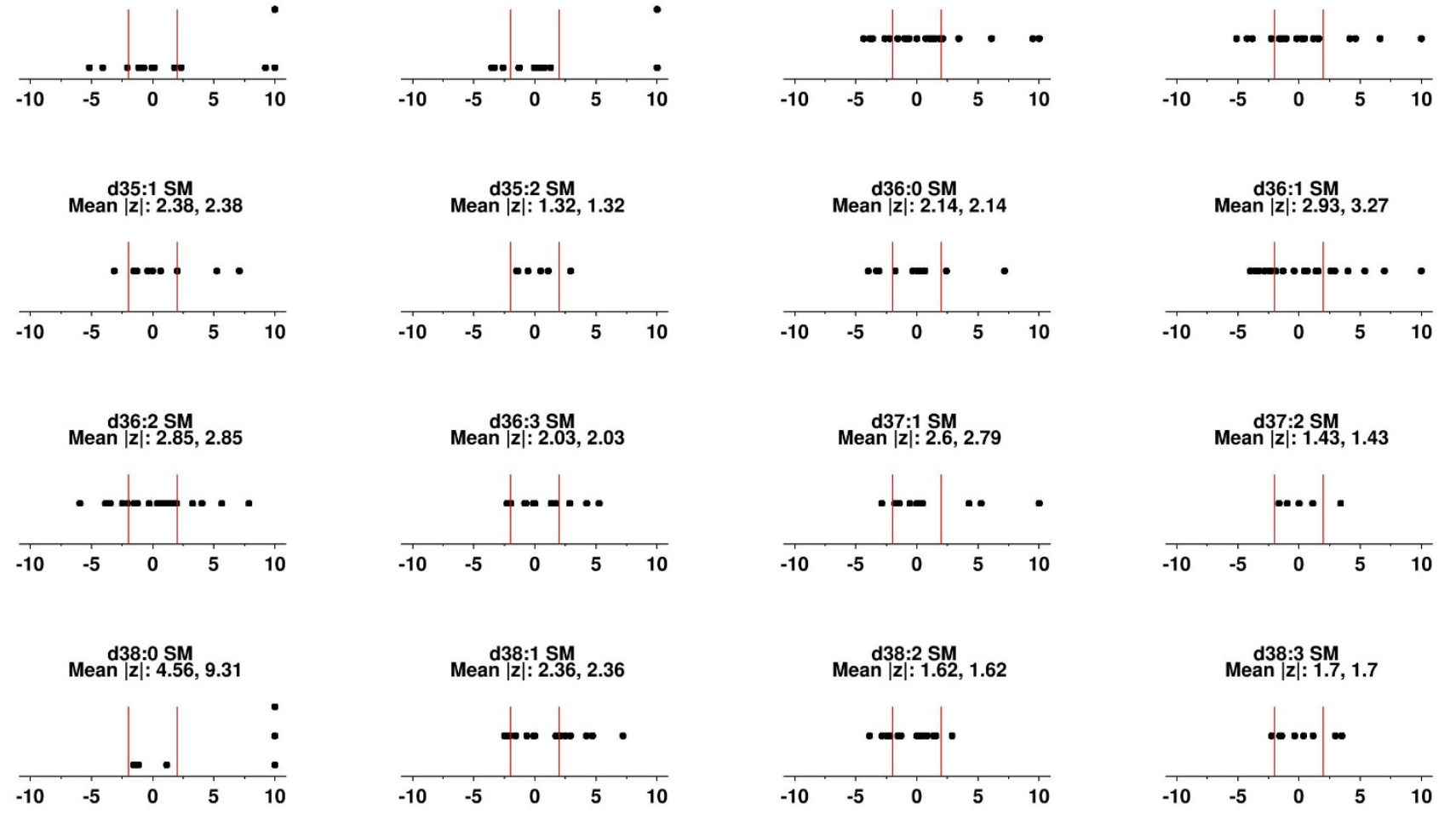

${ }^{\star}$ Z-Scores truncated at $+/-10$ 
Summary of Zeta-Scores by Compound for SM Final: Material 1950
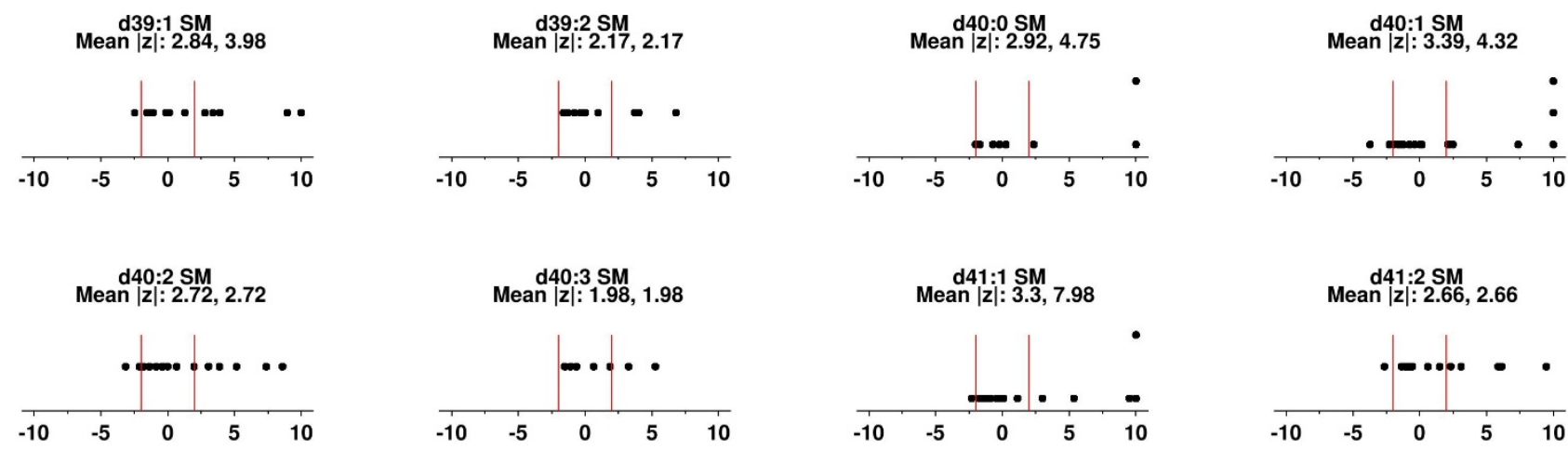

$\operatorname{Mean}|z|: 2 \mathrm{SM}$ :2.66, 2.66
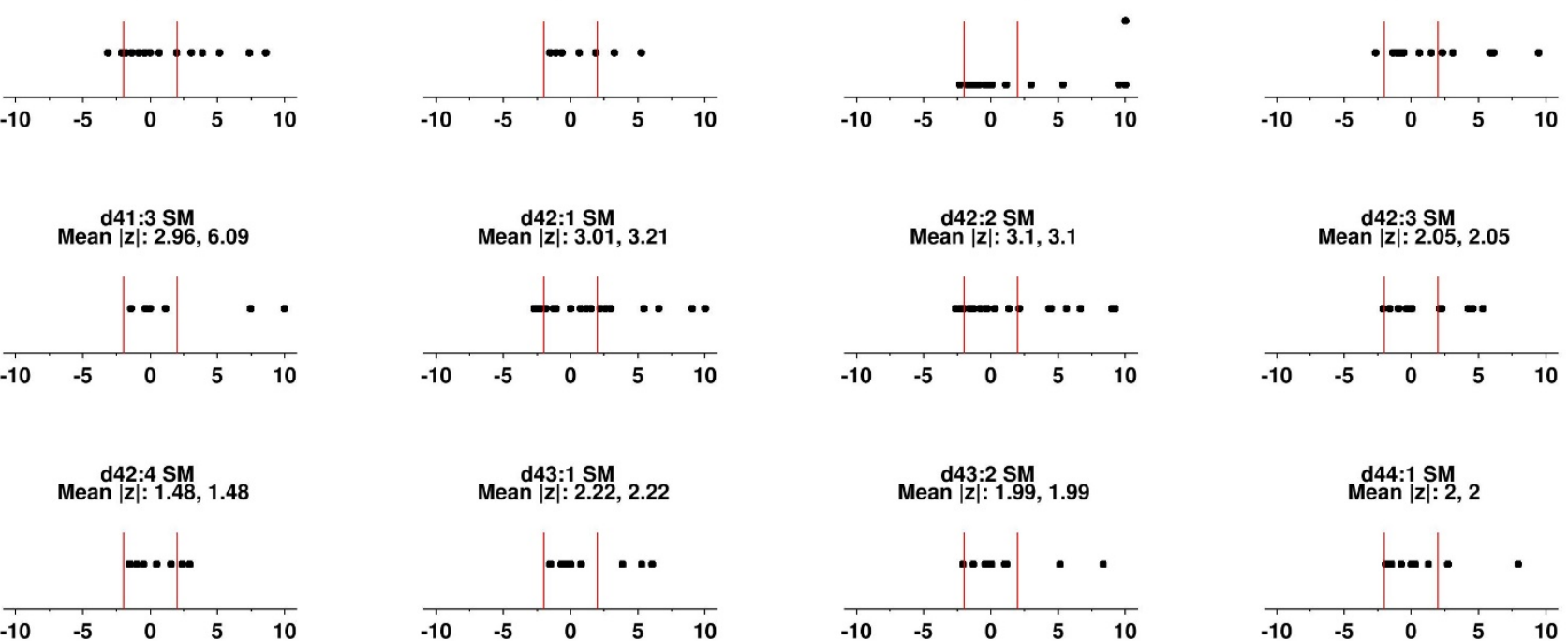

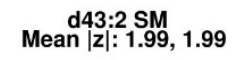

$$
\begin{gathered}
d 42: 3 \text { SM } \\
\text { Mean }|z|: 2.05,2.05
\end{gathered}
$$
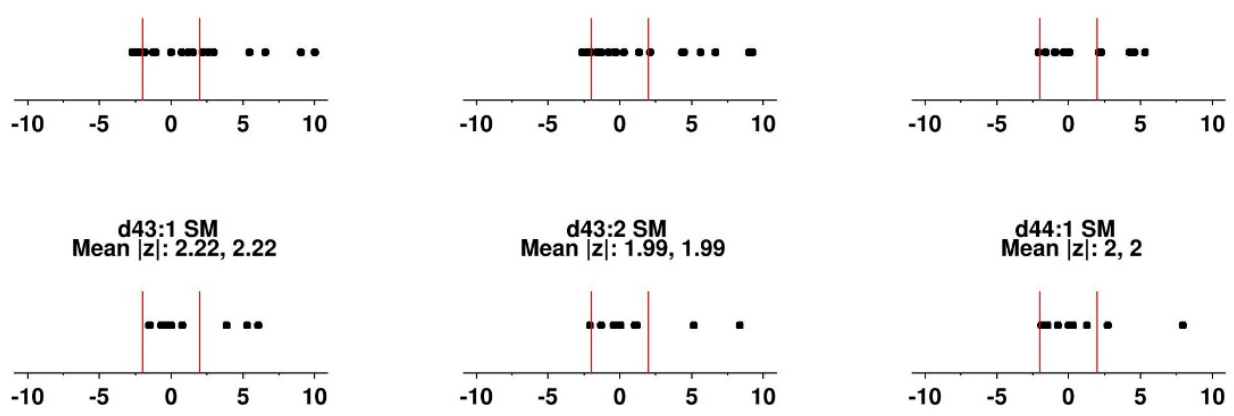

$\stackrel{\text { d44:1 SM }}{\text { Mean }|z|: 2,2}$

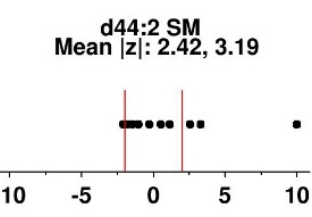

Mean $|z|: 2.95,4.76$

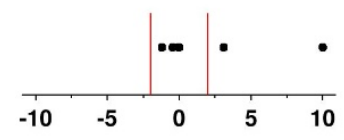

*Z-Scores truncated at $+/-10$ 


\section{Summary of Zeta-Scores by Compound for Tag Final: Material 1950}
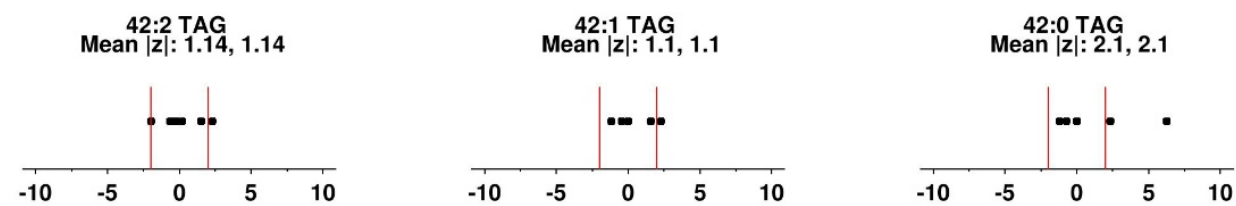

$44: 2 T A G$
$M e a n|z|: 2.19,2.19$
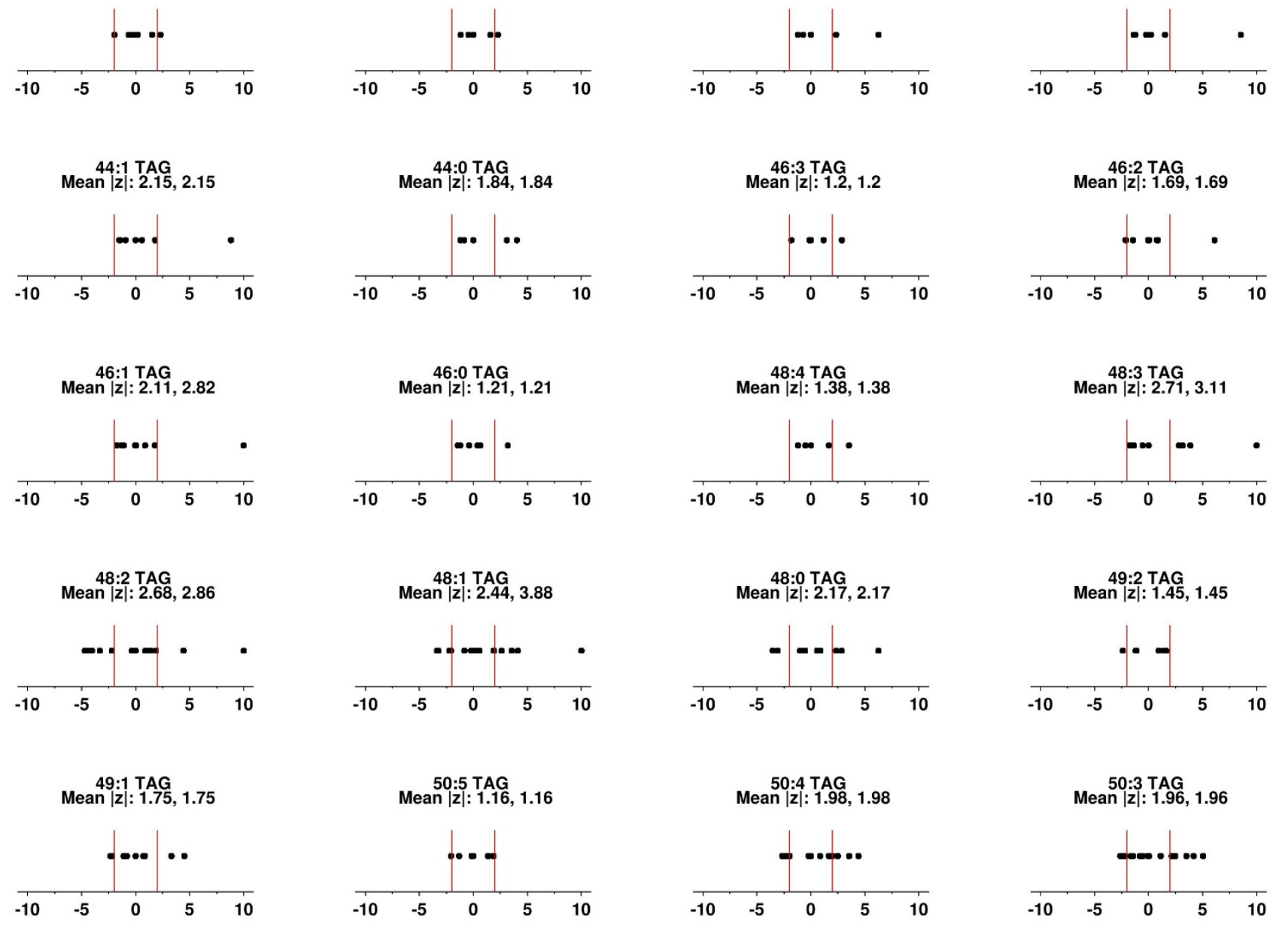

*Z-Scores truncated at $+/-10$ 


\section{Summary of Zeta-Scores by Compound for Tag Final: Material 1950}
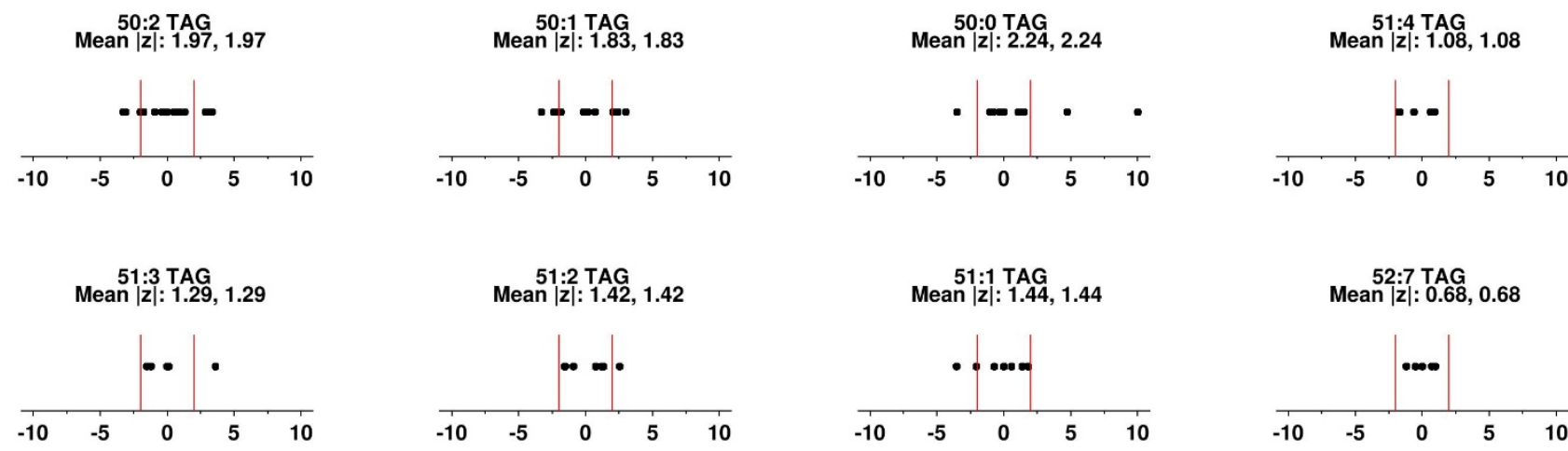

$52: 7$ TAG
Mean $|z|: 0.68,0.68$
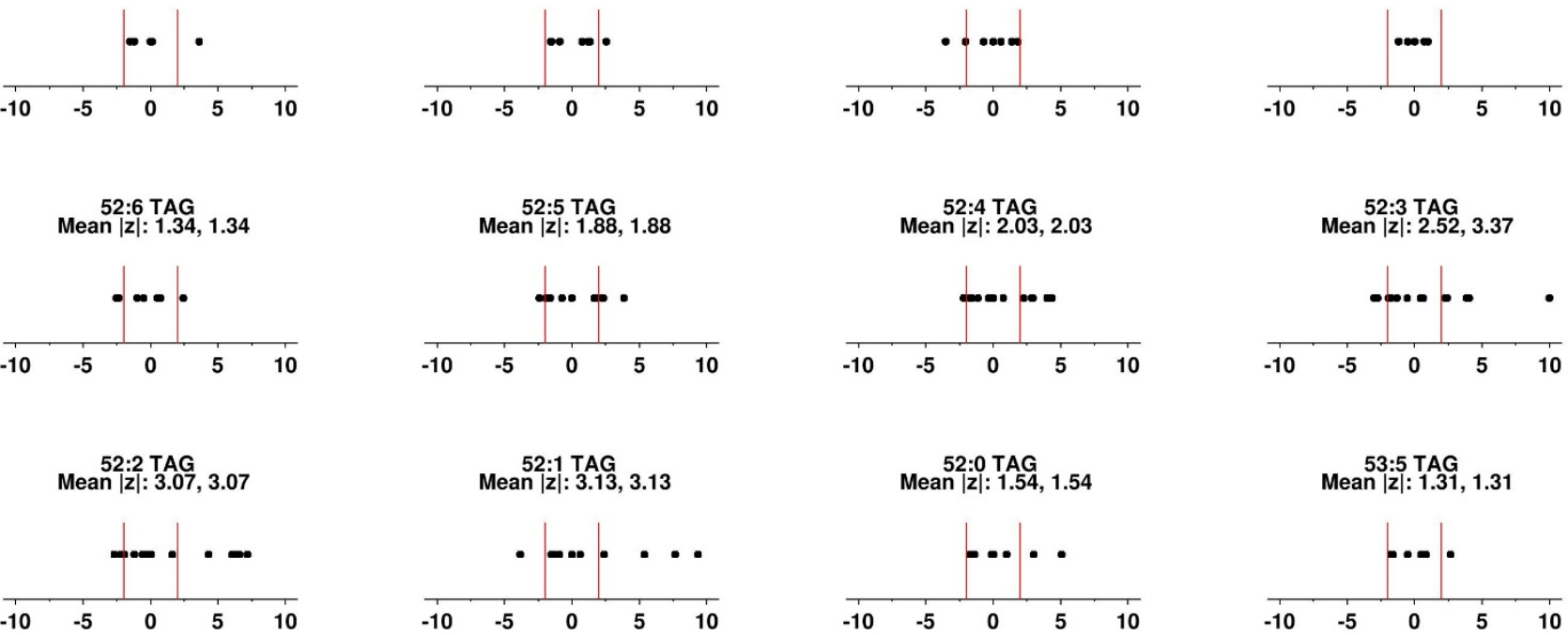

$$
\begin{gathered}
\text { 52:0 TAG } \\
\text { Mean |z|: } 1.54,1.54
\end{gathered}
$$

$$
\begin{gathered}
52: 3 \text { TAG } \\
\text { Mean }|z|: 2.52,3.37
\end{gathered}
$$
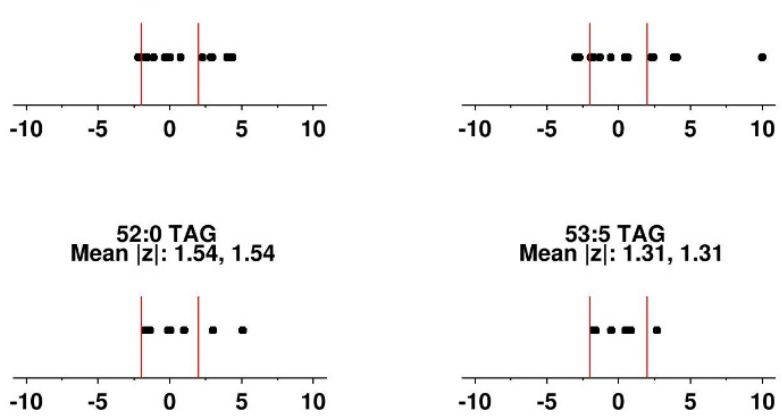

$$
\begin{gathered}
\text { 53:5 TAG } \\
\text { Mean }|z|: 1.31,1.31
\end{gathered}
$$
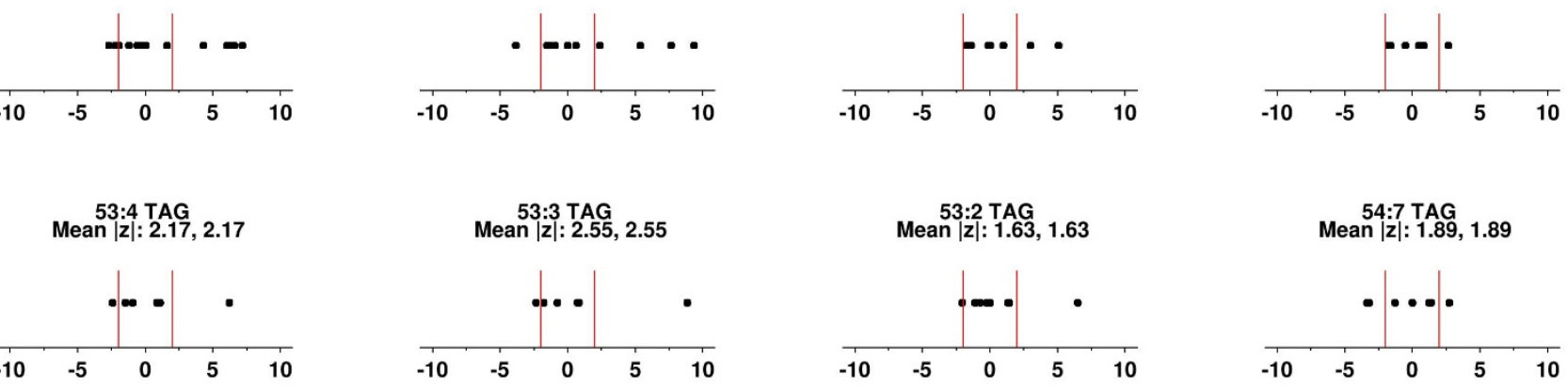

*Z-Scores truncated at $+/-10$ 


\section{Summary of Zeta-Scores by Compound for Tag Final: Material 1950}
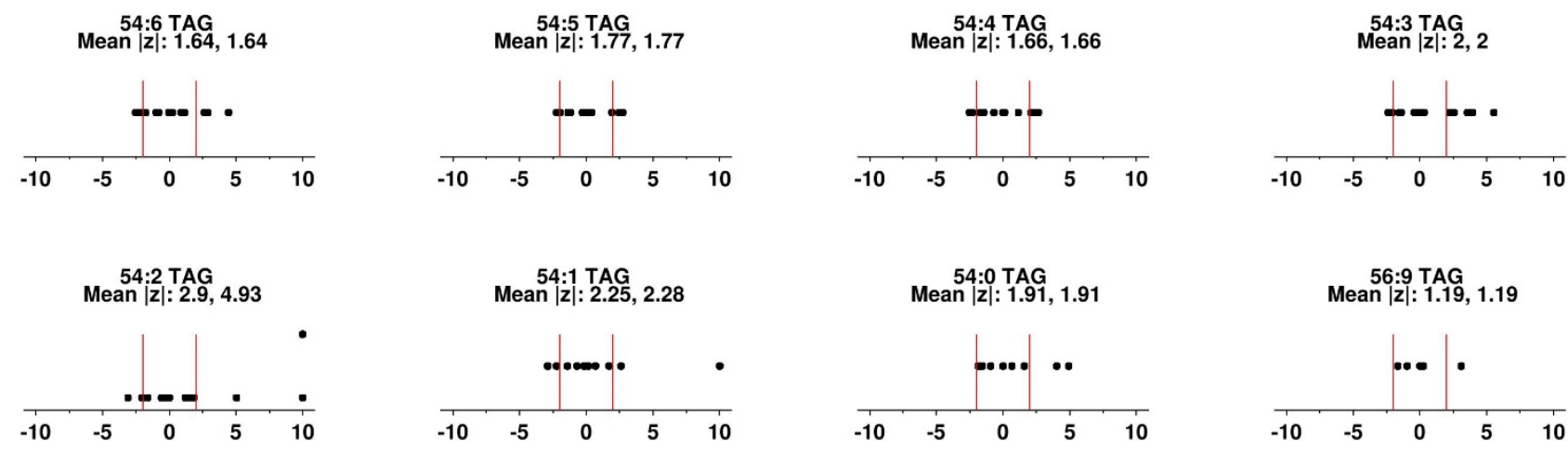

56:9 TAG
Mean $|z|: 1.19,1.19$
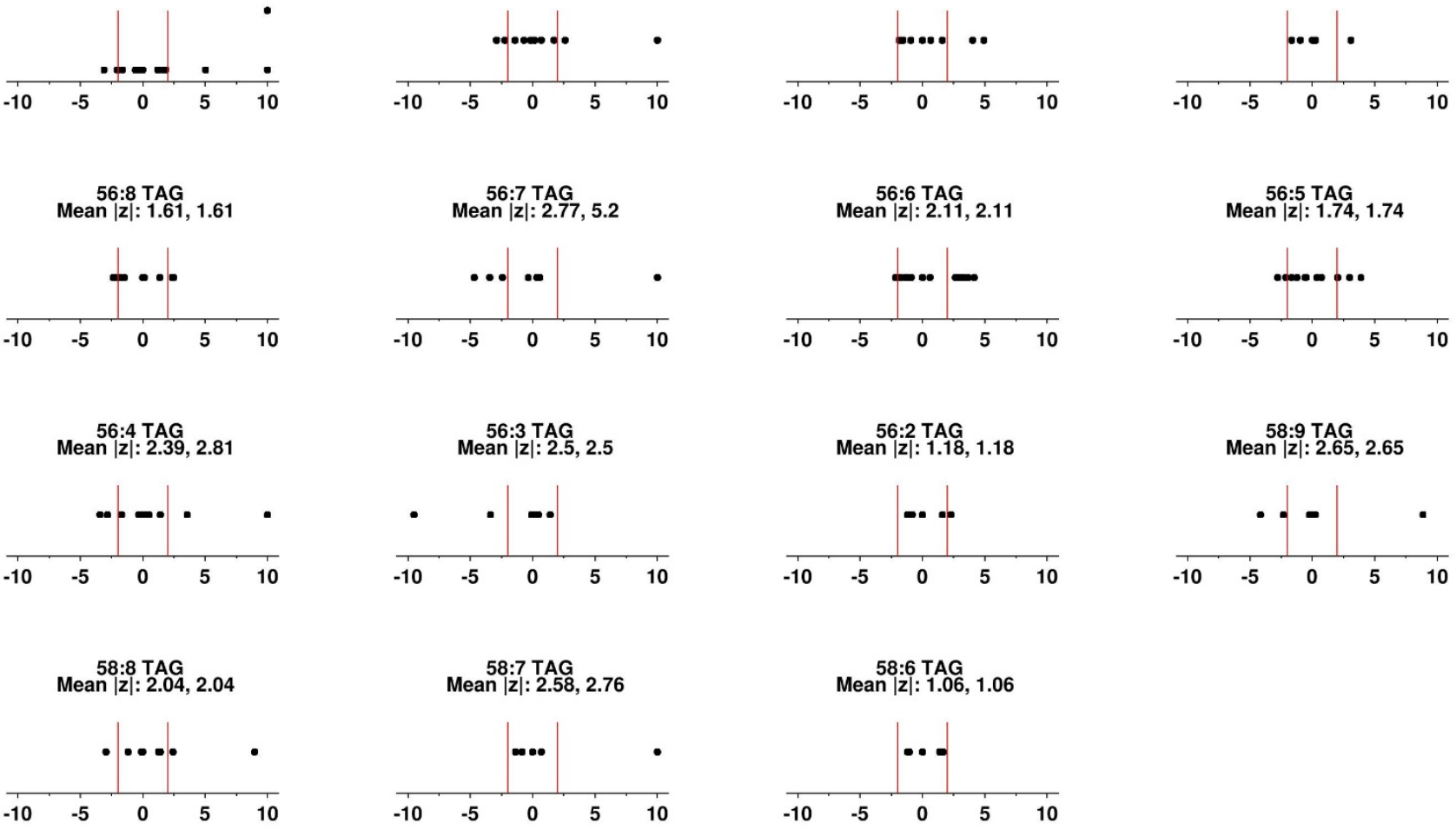

$$
\begin{aligned}
& \text { 58:6 TAG } \\
& \operatorname{Mean}|z|: 1.06,1.06
\end{aligned}
$$

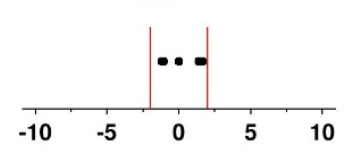

*Z-Scores truncated at +/-10 
Appendix F: Zeta-Scores Organized by Laboratory

Zeta-score plots provided for all consensus lipids ( $\mathrm{n} \geq 5$ participating laboratories reporting), organized by participating laboratory and presented on a lipid class basis. Each dot represents a single laboratory measurement for a reported lipid using the MEDM location as the target. The distance for each point from the mean indicates that the measurement is $x$ times the number of combined standard uncertainties the submitted values is above or below the MEDM location. The plots were truncated at \pm 10 to maintain visual resolution. Absolute value mean $\zeta$-scores were calculated two ways: (1) setting truncated values to 10 (first $\zeta$-score average listed) and (2) using the original outlier $\zeta$-score in the calculation. The purpose of this organization was to identify the laboratories that consistently measured outside the consensus location with high frequency and magnitude. 

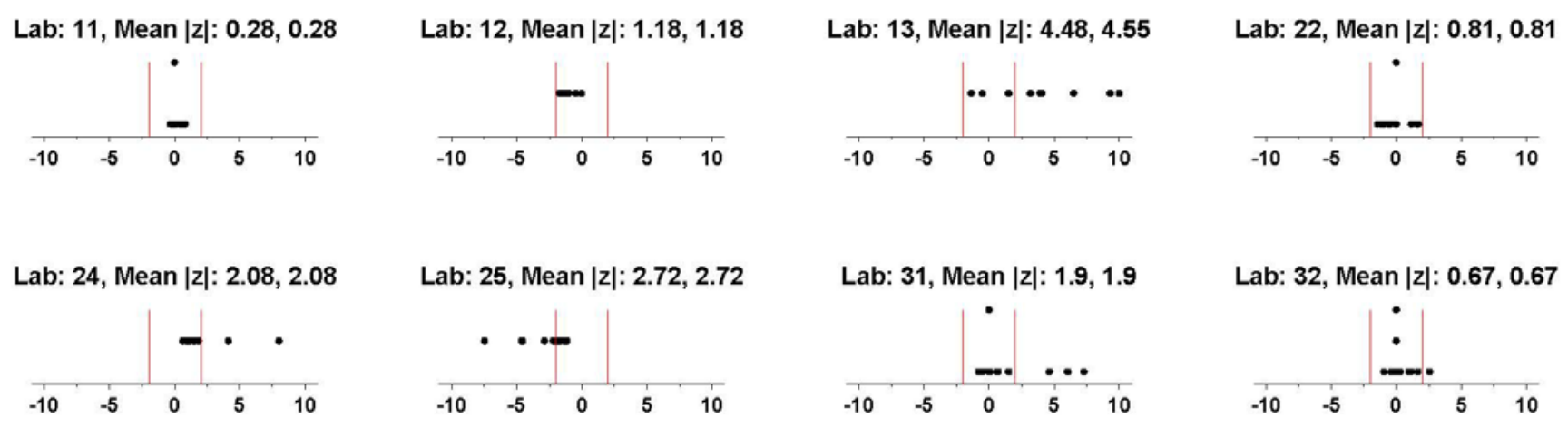

Lab: 34 , Mean |z|: 7.58, 9.48

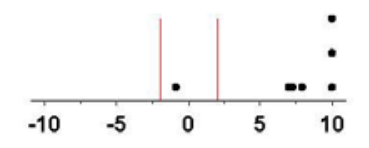




\section{Summary of Zeta-Scores by Laboratory for CE Final}

Lab: 1, Mean $|z|:$ 2.56, 2.56

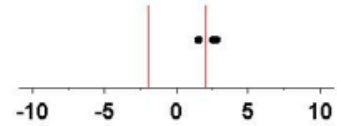

Lab: 7 , Mean $|z|: 4.14,4.17$

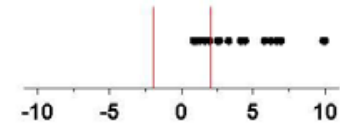

Lab: 13, Mean |z|: 1.5, 1.5

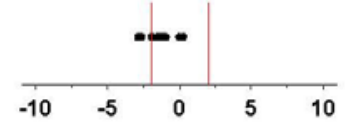

Lab: 19, Mean |z|: 6.67, 16.79

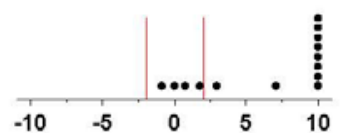

Lab: 2, Mean $|z|: 0.97,0.97$

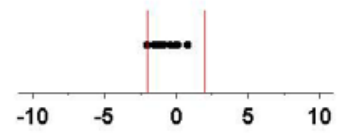

Lab: 9 , Mean $|z|: 3.49,3.49$

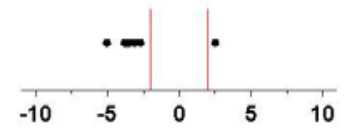

Lab: 15, Mean $|z|: 2.79,2.79$

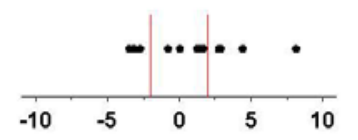

Lab: 23, Mean $|z|:$ 0.76, 0.76

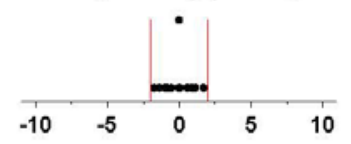

Lab: 4, Mean $|z|: 1.07,1.07$

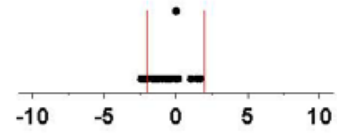

Lab: 10, Mean $|z|:$ 1.53, 1.53

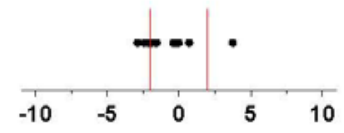

Lab: 16, Mean $|z|: 0.82,0.82$

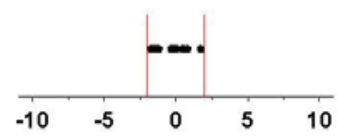

Lab: 5 , Mean $|z|: 0.63,0.63$

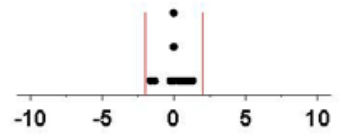

Lab: 12 , Mean $|z|: 1.83,1.83$

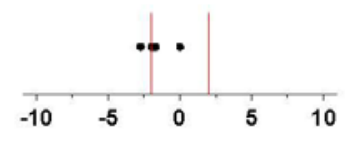

Lab: 18, Mean $|z|:$ 1.55, 1.55

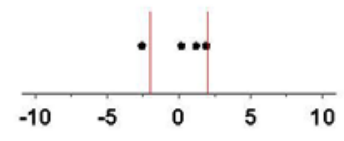

"Z-Scores truncated at +/-10 


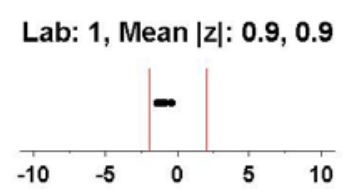

Lab: 5, Mean $|z|: 0.75,0.75$

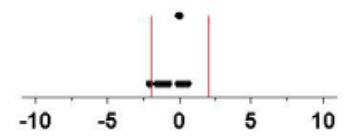

Lab: 11, Mean $|z|:$ 1.78, 1.78

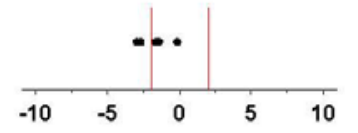

Lab: 15 , Mean |z|: 5.08, 7.02

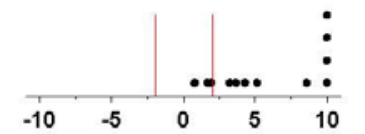

Lab: 23, Mean |z|: 0.59, 0.59

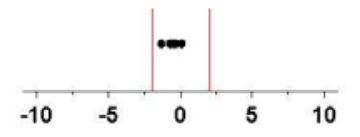

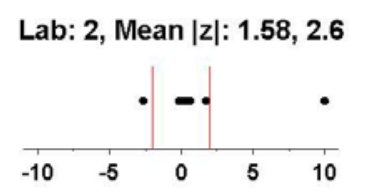

Lab: 7 , Mean $|z|: 0.88,0.88$

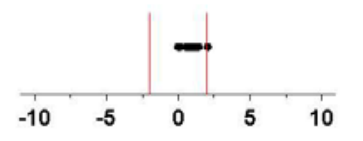

Lab: 12, Mean |z|: 0.91, 0.91

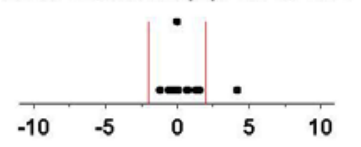

Lab: 18, Mean |z|: 6.88, 20.74

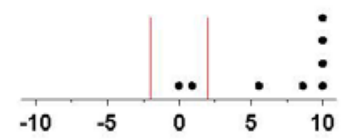

Lab: 24, Mean |z|: 2.16, 2.16

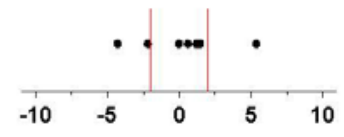

Lab: 3, Mean $|z|: 0.95,0.95$

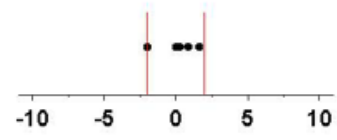

Lab: 9, Mean $|z|: \mathbf{3 . 7 4 ,} 3.74$

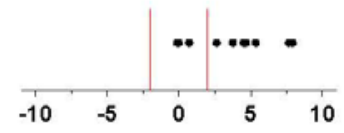

Lab: 13, Mean |z|: 2.25, 2.25

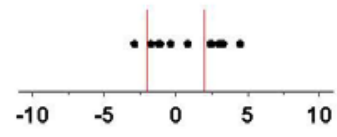

Lab: 19, Mean $|z|: 2.23,2.23$

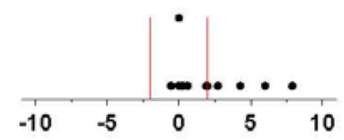

Lab: 27 , Mean $|z|:$ 4.08, 4.08

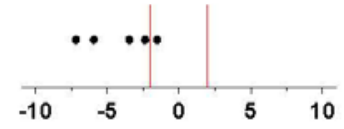

Lab: 4, Mean $|z|:$ 1.38, 1.38

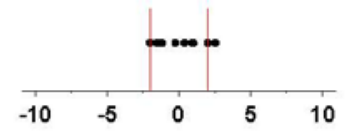

Lab: 10, Mean |z|: 2.09, 2.09

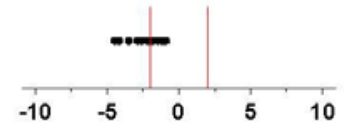

Lab: 14, Mean |z|: 1.29, 1.29

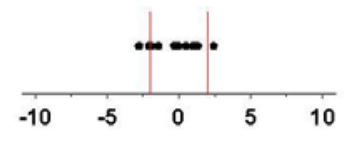

Lab: 20, Mean |z|: 1.2, 1.2

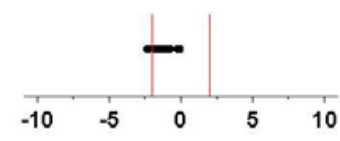

*Z-Scores truncated at $+/-10$ 
Summary of Zeta-Scores by Laboratory for Cholesterol Final

Lab: 2, Mean |z|: 2.15, 2.15

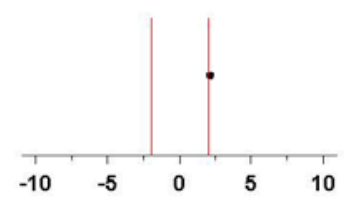

Lab: 7, Mean |z|: 0.18, 0.18

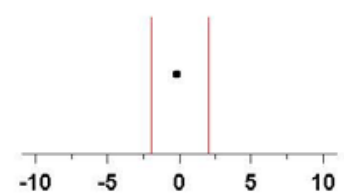

Lab: 3 , Mean $|z|: 0.42,0.42$

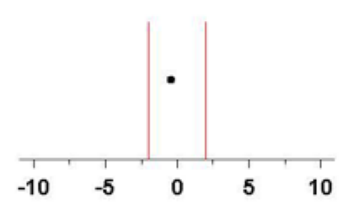

Lab: 9, Mean |z|: 2.48, 2.48

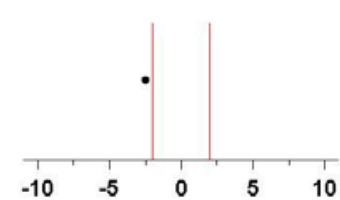

Lab: 4 , Mean $|z|: 0.4,0.4$

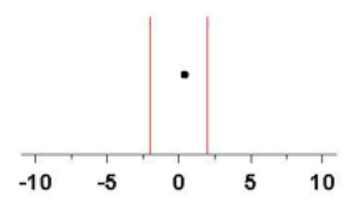

Lab: 12, Mean |z|: 1.22, 1.22

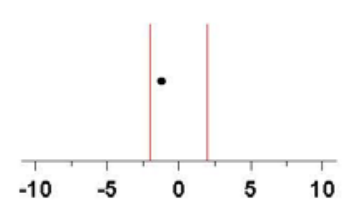

Lab: 5, Mean $|z|: 1.97,1.97$

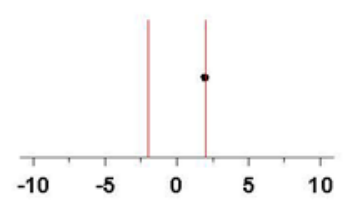

Lab: 23, Mean |z|: 1.79, 1.79

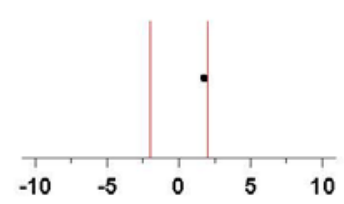

*Zeta-Scores truncated at $+/-10$ 
Lab: 1, Mean $|z|: 2.27,2.27$

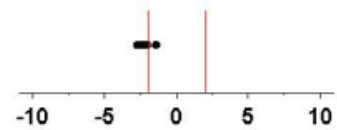

Lab: 5 , Mean $|z|: 1.73,1.73$

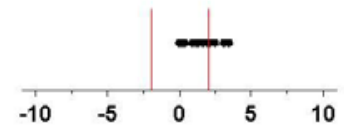

Lab: 12, Mean |z|: 1.56, 1.56

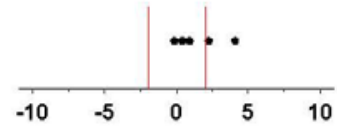

Lab: 16, Mean |z|: 1.17, 1.17

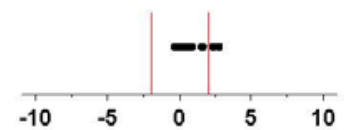

Lab: 28 , Mean $|z|:$ 6.35, 8.46

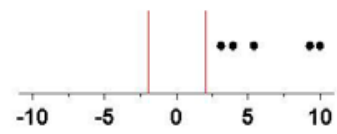

Lab: 2, Mean |z|: 0.55, 0.55

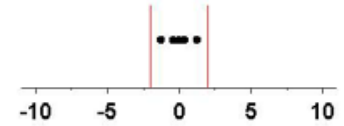

Lab: 7, Mean $|z|:$ 2.21, 2.21

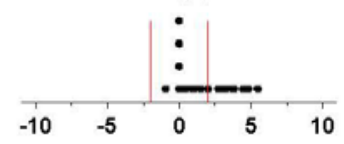

Lab: 13, Mean $|z|:$ 1.96, 1.96

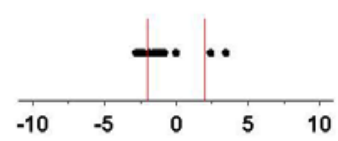

Lab: 18, Mean $|z|: 0,0$

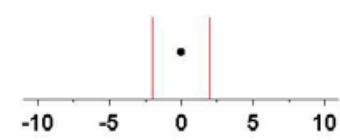

Lab: 3, Mean $|z|: 0.94,0.94$

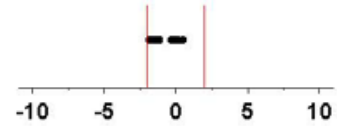

Lab: 9, Mean $|z|: 1.58,1.58$

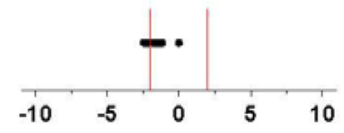

Lab: 14, Mean $|z|:$ 1.61, 1.61

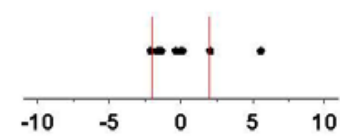

Lab: 19, Mean |z|: 2.59, 3.12

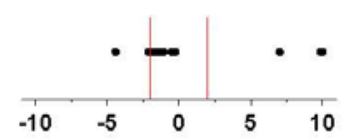

Lab: 4 , Mean $|z|: 0.82,0.82$

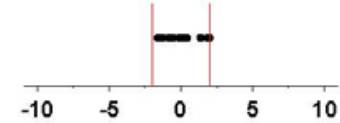

Lab: 10, Mean $|z|: 2.9,5.73$

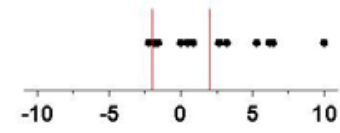

Lab: 15 , Mean $|z|: 4.65,5.34$

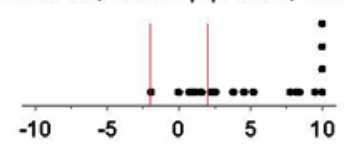

Lab: 23 , Mean |z|: 0.81, 0.81

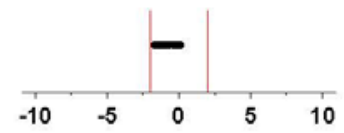

*Z-Scores truncated at $+/-10$ 
Summary of Zeta-Scores by Laboratory for Eico Final

Lab: 8 , Mean $|z|: 0.4,0.4$

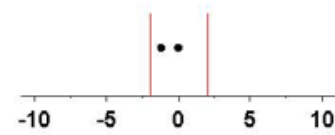

Lab: 32 , Mean $|z|: 0.27,0.27$

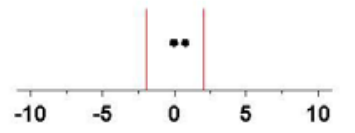

Lab: 20, Mean |z|: 2.25, 2.25

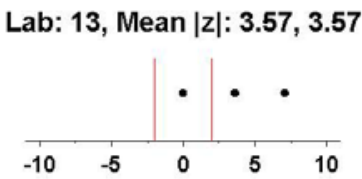

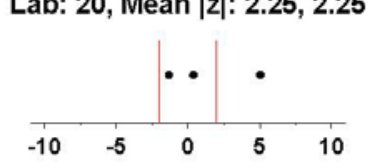

Lab: $\mathbf{3 0}$, Mean $|z|: \mathbf{2 . 2 5}, \mathbf{2 . 2 8}$

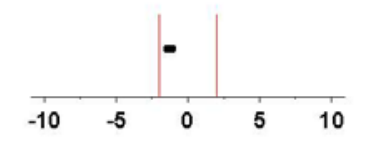


Summary of Zeta-Scores by Laboratory for Fatty Acids
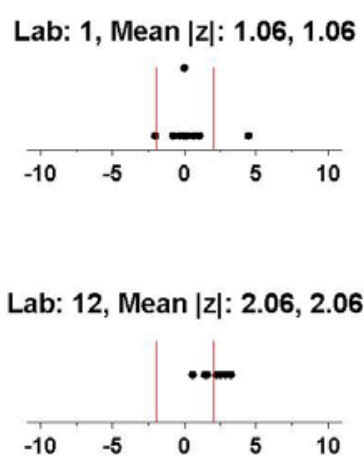

Lab: 8 , Mean |z|: 1.03, 1.03

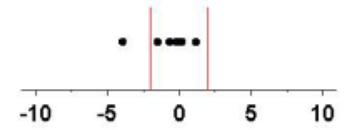

Lab: 16, Mean |z|: 0.74, 0.74

Lab: 9, Mean |z|: 2.12, 2.12

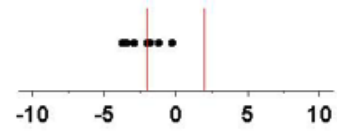

Lab: 20, Mean $|z|:$ 0.61, 0.61

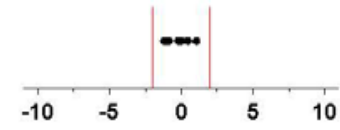

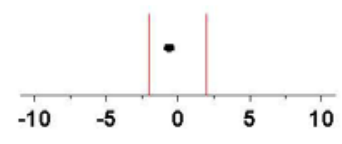

Lab: 11, Mean $|z|:$ 7, 11.86

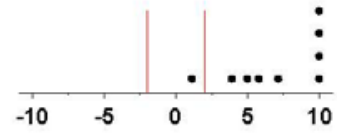

Lab: 32, Mean |z|: 0.25, 0.25

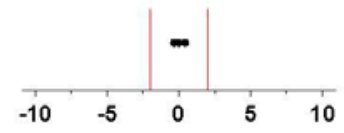

*Z-Scores truncated at $+/-10$ 

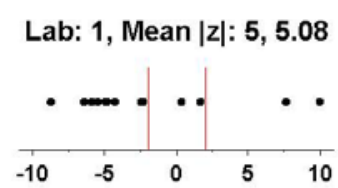

Lab: 5, Mean |z|: 1.04, 1.04

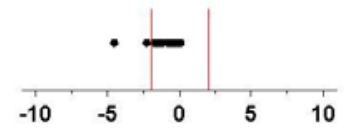

Lab: 10, Mean |z|: 2.19, 2.19

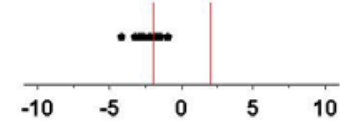

Lab: 15, Mean |z|: 6.17, 7.54

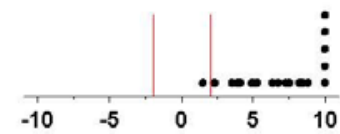

Lab: 19, Mean |z|: 0.65, 0.65

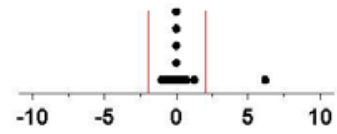

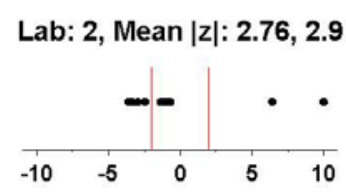

Lab: 6, Mean $|z|: 0.94,0.94$

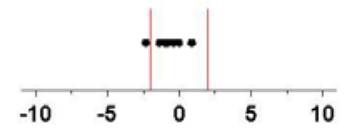

Lab: 11, Mean |z|: 1.94, 1.94

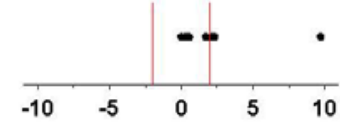

Lab: 16, Mean $|z|: 1.84,1.84$

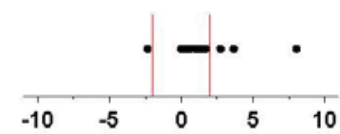

Lab: 23, Mean $|z|:$ 2.75, 2.75

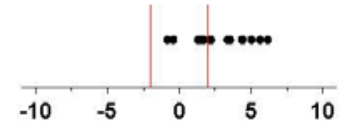

Lab: 3, Mean $|z|:$ 1.44, 1.44

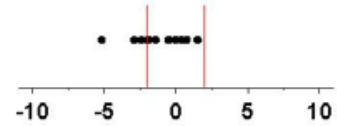

Lab: 7, Mean |z|: 6.24, 7.08

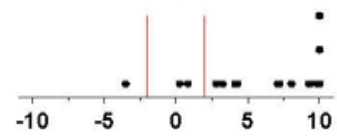

Lab: 12, Mean $|z|: 1.53,1.53$

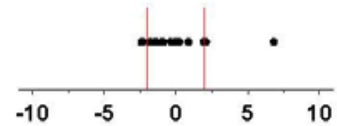

Lab: 17, Mean |z|: 4.79, 6.94

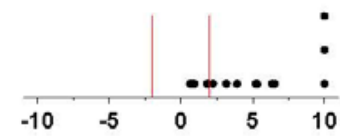

Lab: 26, Mean |z|: 1.35, 1.35

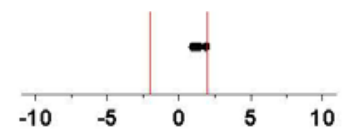

Lab: 4, Mean $|z|: 0.55,0.55$

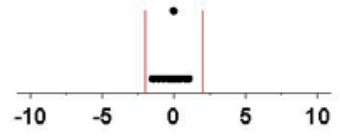

Lab: 9, Mean |z|: 2.87, 2.87

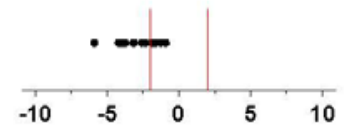

Lab: 13, Mean |z|: 1.9, 1.9

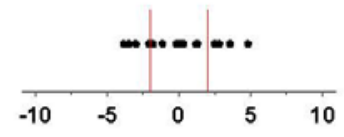

Lab: 18, Mean |z|: 10, 26.18

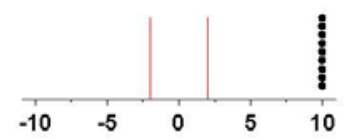

Lab: 28, Mean |z|: 4.96, 4.96

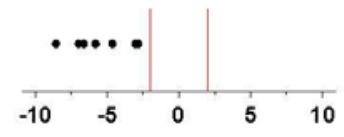

*Z-Scores truncated at $+/-10$ 
Lab: 1, Mean |z|: 2.35, 2.35

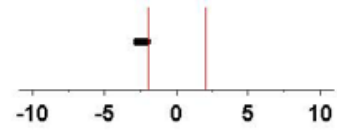

Lab: 5, Mean $|z|: 0.64,0.64$

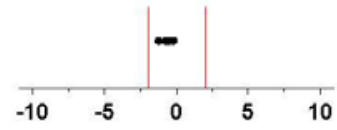

Lab: 12, Mean $|z|: 0.23,0.23$

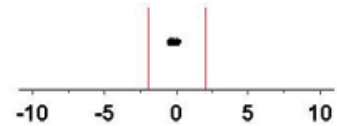

Lab: 17, Mean |z|: 0.9, 0.9

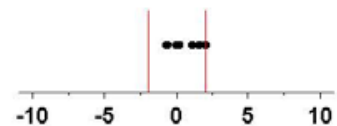

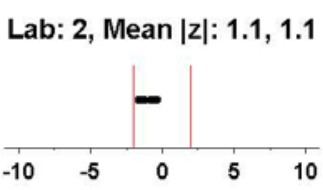

Lab: 7 , Mean $|z|: 1.97,1.97$

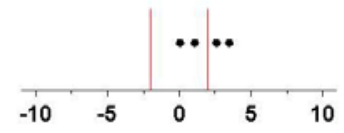

Lab: 13 , Mean $|z|: 4.71,4.89$

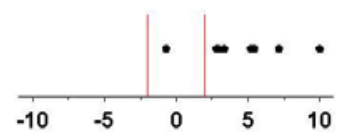

Lab: 18, Mean $|z|:$ 6.92, 6.92

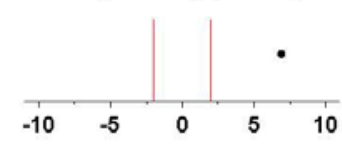

Lab: 3, Mean $|z|:$ 1.76, 1.76

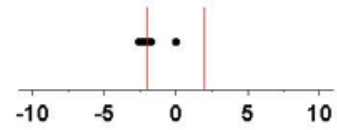

Lab: 9, Mean |z|: 2.94, 2.94

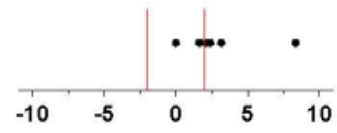

Lab: 15, Mean $|z|: \mathbf{5 . 4 4 ,} 5.44$

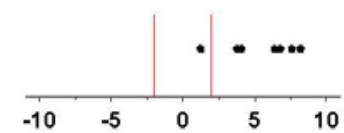

Lab: 19, Mean |z|: 0.19, 0.19

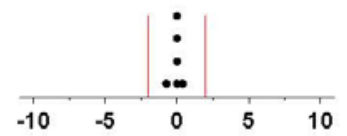

Lab: 4, Mean |z|: 1.1, 1.1

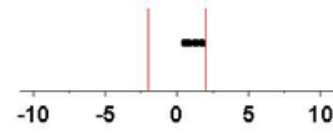

Lab: 11, Mean |z|: 2.06, 2.06

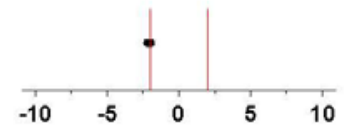

Lab: 16, Mean |z|: 1.71, 1.71

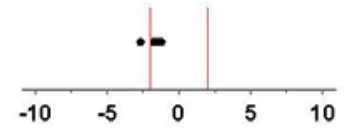

Lab: 25, Mean |z|: 8, 12.23

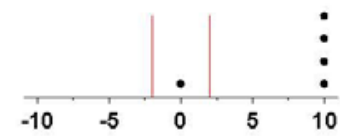

*Z-Scores truncated at $+/-10$ 
Lab: 4, Mean |z|: 1.48, 1.48

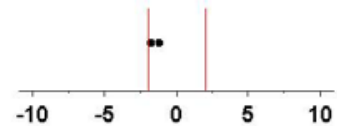

Lab: 9 , Mean |z|: $0.35,0.35$

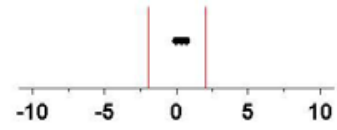

Lab: 5, Mean |z|: 2, 2

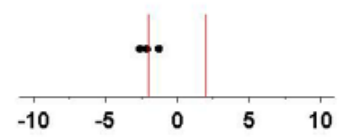

Lab: 15, Mean |z|: 2.71, 2.71

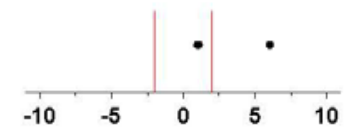

Lab: 6, Mean |z|: 0.54, 0.54

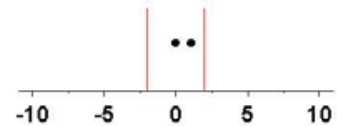

Lab: 7, Mean |z|: 0.06, 0.06

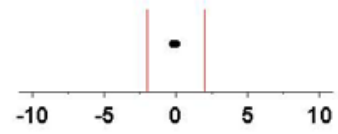

*Z-Scores truncated at $+/-10$ 
Lab: 1, Mean |z|: 2.4, 2.4

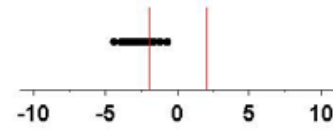

Lab: 5, Mean |z|: 1.33, 1.33

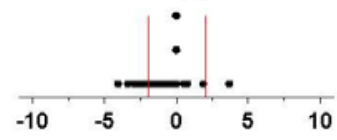

Lab: 10, Mean |z|: 2.39, 2.39

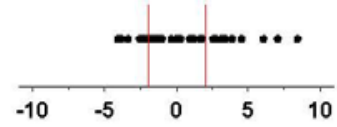

Lab: 16, Mean |z|: 2.89, 2.89

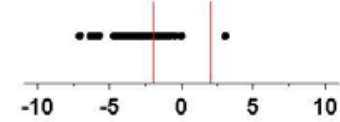

Lab: 23 , Mean $|z|: 0.81,0.81$

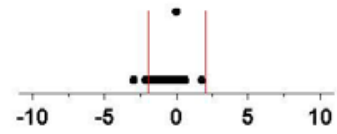

Lab: 2, Mean $|z|:$ 1.78, 1.82

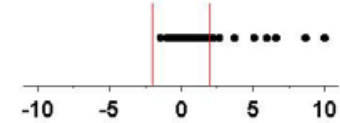

Lab: 6 , Mean $|z|:$ 4.1, 4.24

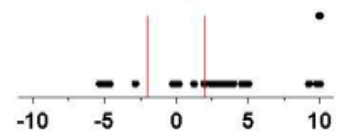

Lab: 11, Mean $|z|: 1.99,1.99$

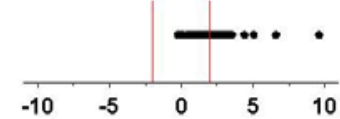

Lab: 17, Mean |z|: 1.94, 1.94

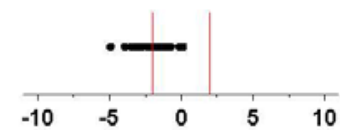

Lab: 26, Mean $|z|:$ 1.22, 1.22

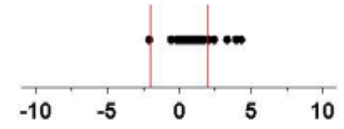

Lab: 3, Mean |z|: 2.64, 2.64

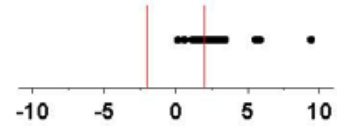

Lab: 7, Mean $|z|: 3.63,3.63$

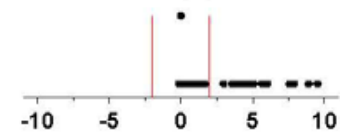

Lab: 12, Mean |z|: 2.21, 2.21

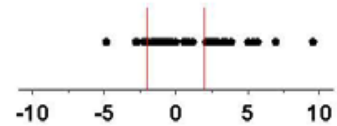

Lab: 18, Mean |z|: 1.72, 2.52

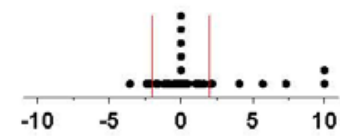

Lab: 28, Mean |z|: 5.19, 5.19

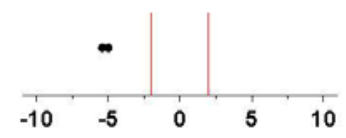

Lab: 4, Mean |z|: 2.76, 2.76

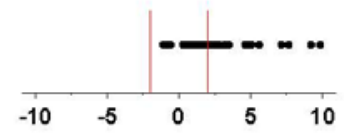

Lab: 9, Mean |z|: 1.74, 1.74

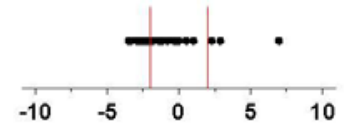

Lab: 15, Mean $|z|:$ 5.75, 12.31

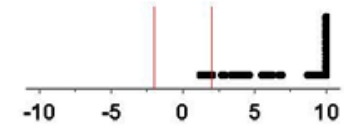

Lab: 19, Mean $|z|:$ 1.02, 1.02

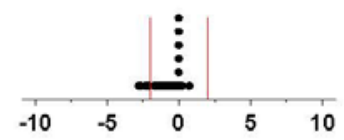

$\begin{array}{lllll}-10 & -5 & 0 & 5 & 10\end{array}$

*Z-Scores truncated at $+/-10$ 
Lab: 1, Mean |z|: 1.46, 1.46

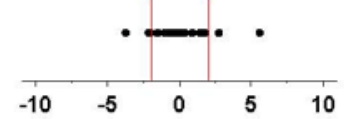

Lab: 5, Mean |z|: 0.89, 0.89

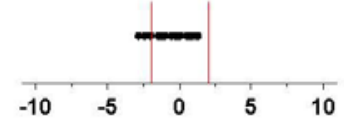

Lab: 10 , Mean $|z|: 1.18,1.18$

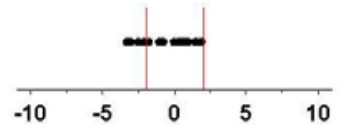

Lab: 16, Mean |z|: 1.89, 1.89

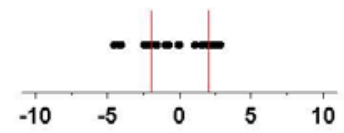

Lab: 25, Mean |z|: 2.42, 2.42

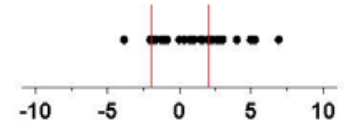

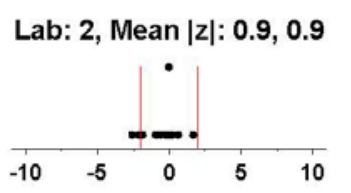

Lab: 6, Mean $|z|: 1.16,1.16$

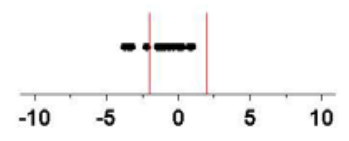

Lab: 11, Mean $|z|:$ 1.58, 1.58

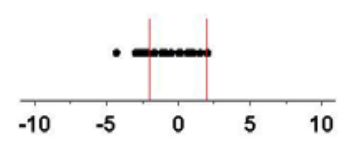

Lab: 17, Mean |z|: 2.55, 2.55

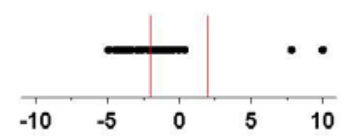

Lab: 3, Mean |z|: 1.78, 1.78

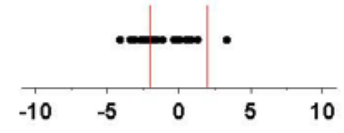

Lab: 7, Mean |z|: 2.52, 3.01

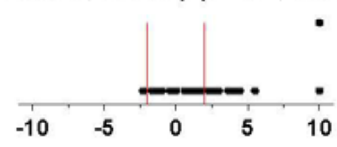

Lab: 12, Mean |z|: 1.93, 1.93

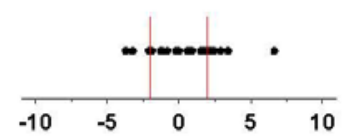

Lab: 18, Mean |z|: 1.29, 1.29

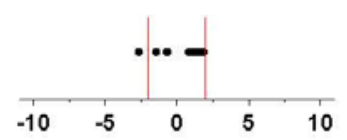

Lab: 4, Mean |z|: 1.44, 1.44

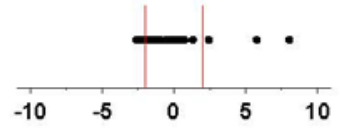

Lab: 9, Mean |z|: 5.54, 6.97

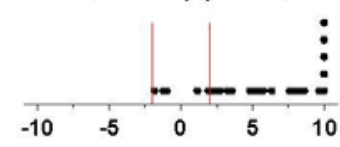

Lab: 15, Mean |z|: 4.75, 6.2

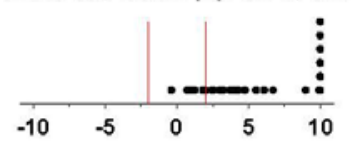

Lab: 19, Mean |z|: 3.59, 5.61

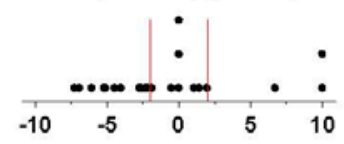

"Z-Scores truncated at $+/-10$ 


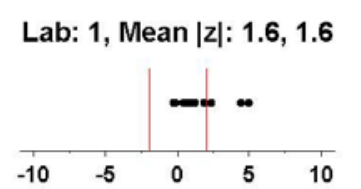

Lab: 5, Mean $|z|:$ 1.2, 1.2

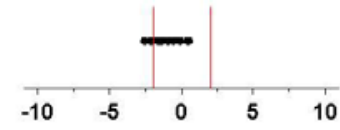

Lab: 10, Mean |z|: 0.94, 0.94

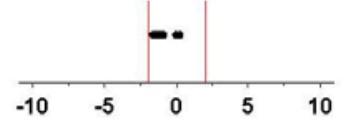

Lab: 17, Mean |z|: 2.49, 2.49

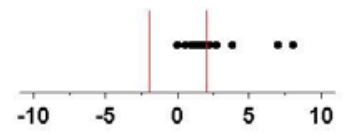

Lab: 28 , Mean |z|: 4.47, 4.47

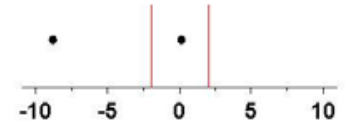

Lab: 2, Mean $|z|:$ 2.08, 2.08

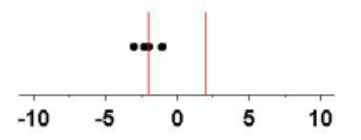

Lab: 6, Mean $|z|: 1.87,1.87$

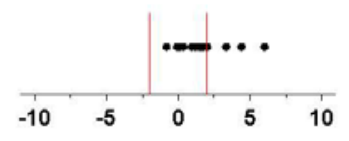

Lab: 12, Mean $|z|: 1.53,1.53$

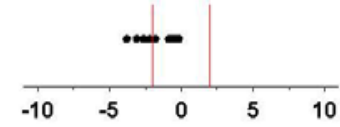

Lab: 18, Mean $|z|:$ 8.06, 8.06

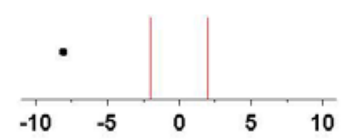

Lab: 3, Mean |z|: 1.58, 1.58

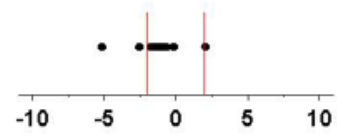

Lab: 7, Mean |z|: 2.08, 2.08

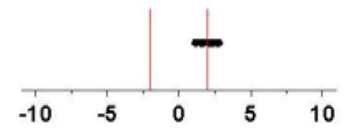

Lab: 15, Mean |z|: $3.07,3.07$

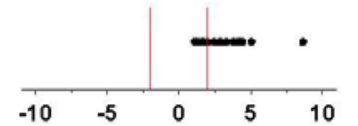

Lab: 19, Mean $|z|: 1.07,1.07$

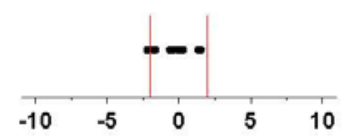

Lab: 4, Mean $|z|:$ 1.01, 1.01

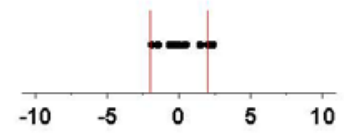

Lab: 9, Mean $|z|:$ 1.73, 1.73

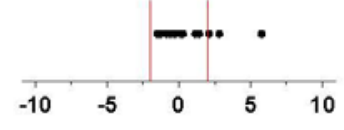

Lab: 16, Mean |z|: 2.62, 2.62

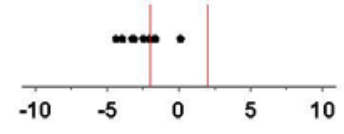

Lab: 23, Mean |z|: 0.93, 0.93

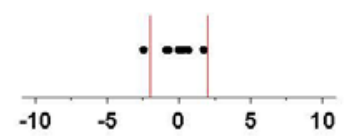

"Z-Scores truncated at +1-10 


\section{Summary of Zeta-Scores by Laboratory for PS Final}

Lab: 4, Mean $|z|:$ 1.45, 1.45

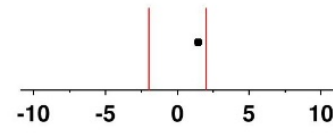

Lab: 9, Mean $|z|: 0.65,0.65$

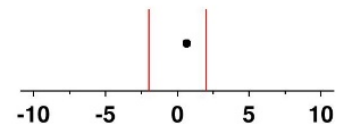

Lab: 5, Mean $|z|:$ 1.32, 1.32

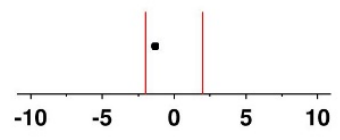

Lab: 15, Mean $|z|:$ 1.58, 1.58

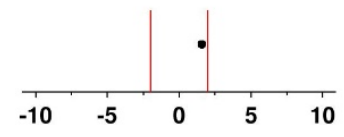

Lab: 6, Mean $|z|:$ 1.32, 1.32

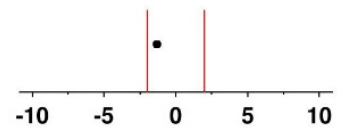

Lab: 7 , Mean $|z|: 0.83,0.83$

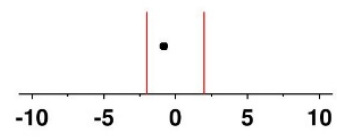

*Z-Scores truncated at $+/-10$ 
Lab: 1, Mean $|z|: 2.73,2.73$

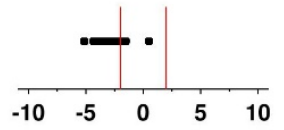

Lab: 6, Mean $|z|: 3.6,3.6$

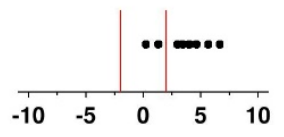

Lab: 2, Mean $|z|:$ 2.2, 2.2

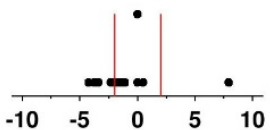

Lab: 7, Mean $|z|: 5.19,5.37$

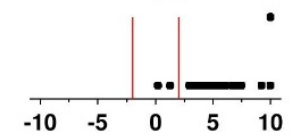

Lab: 3, Mean $|z|: 1.28,1.28$

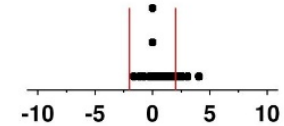

Lab: 9 , Mean $|z|: 1.04,1.04$

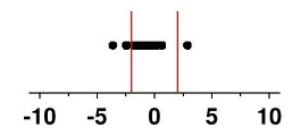

Lab: 4 , Mean $|z|: 1.33,1.33$

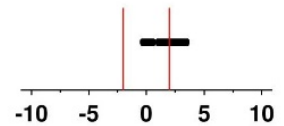

Lab: 10, Mean $|z|: 0.82,0.82$

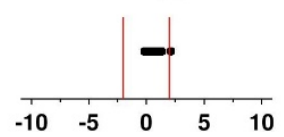

Lab: 5, Mean $|z|: 0.7,0.7$

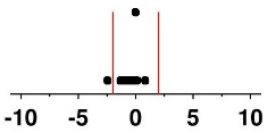

Lab: 11, Mean $|z|: 0.74,0.74$

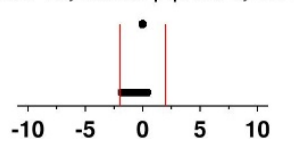

Lab: 12, Mean $|z|: 6.34,7.2$ Lab: 13, Mean $|z|: 6.39,14.29$ Lab: 14 , Mean $|z|: 1.97,1.97$ Lab: 15 , Mean $|z|: 6.22,11.13$ Lab: 16, Mean $|z|: 1.12,1.12$
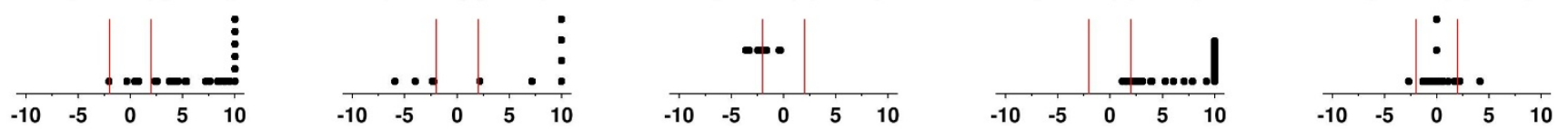

Lab: 17, Mean $|z|: 1.25,1.25$

Lab: 18 , Mean $|z|: 3.35,3.35$

Lab: 19, Mean $|z|: 1.83,1.83$

Lab: 20 , Mean $|z|: 0.9,0.9$

Lab: 23, Mean $|z|: 1.31,1.31$
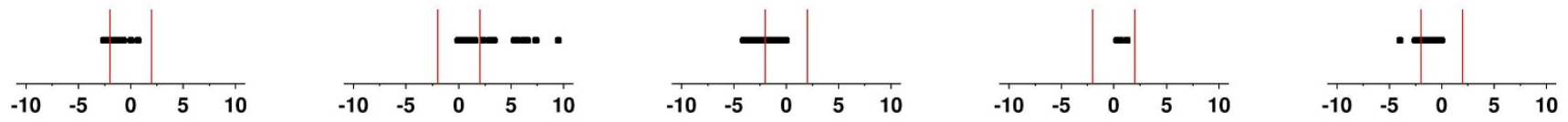

Lab: 26 , Mean $|z|: 2.08,2.08$

Lab: 27 , Mean $|z|: 2.9,2.9$
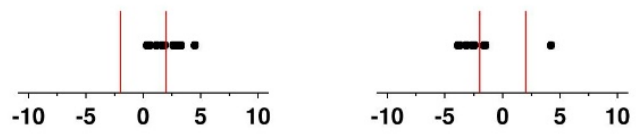

${ }^{\star}$ Z-Scores truncated at $+/-10$ 


\section{Summary of Zeta-Scores by Laboratory for TAG Final}

Lab: 1, Mean $|z|:$ 2.53, 2.53

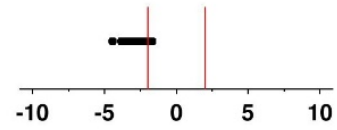

Lab: 5, Mean $|z|: 1.5,1.5$

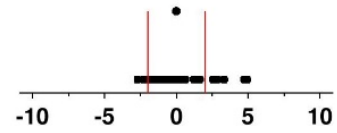

Lab: 12, Mean $|z|:$ 1.32, 1.32

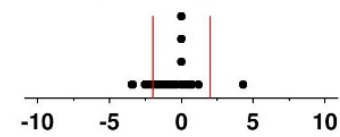

Lab: 18, Mean |z|: 2.04, 2.04

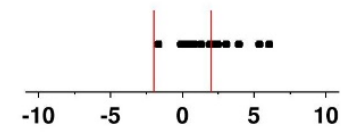

Lab: 2, Mean $|z|:$ 1.82, 1.82

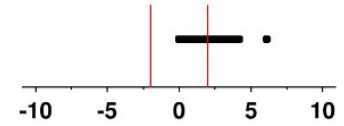

Lab: 7, Mean $|z|:$ 1.07, 1.07

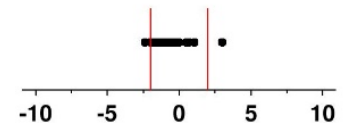

Lab: 13, Mean $|z|: 1.86,1.86$

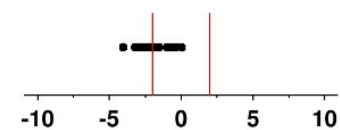

Lab: 19, Mean $|z|: 0.78,0.78$

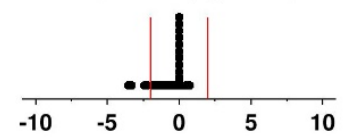

Lab: 3, Mean $|z|:$ 1.88, 1.88

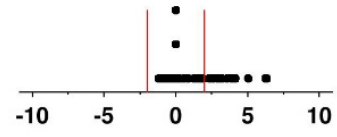

Lab: 9, Mean |z|: 4.04, 4.04

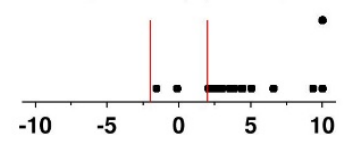

Lab: 15, Mean $|z|:$ 4.29, 6.02

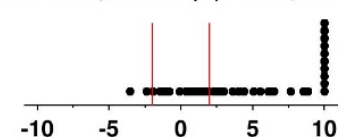

Lab: 23, Mean $|z|:$ 1.71, 1.71

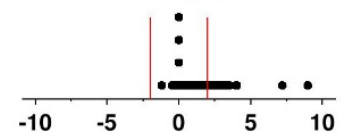

Lab: 4, Mean $|z|: 1.57,1.57$

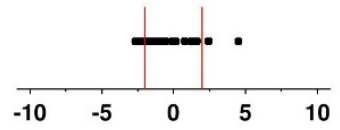

Lab: 10, Mean $|z|: 1,1$

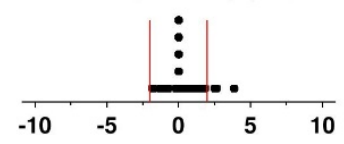

Lab: 16, Mean $|z|: 2.42,2.42$

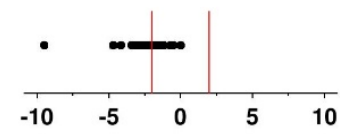

Lab: 28, Mean $|z|:$ 1.17, 1.17

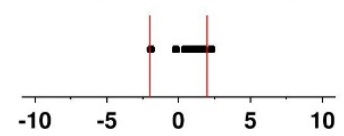

'Z-Scores truncated at $+/-10$ 


\section{Appendix G: Interlaboratory Exercise Participants}

For this exercise, lipid data was submitted from the following laboratories with contributing investigators listed (see below). The order of the listing does NOT correspond to the laboratory number identification codes used in this report, which were randomly assigned upon receipt of data.

The Roskamp Institute

2040 Whitfield Avenue

Sarasota, FL, 34243 USA

Laila Abdullah

Steno Diabetes Center

Copenhagen

DK-2820 Gentofte, Denmark

Linda Ahonen

Department of Pharmaceutical Sciences

986025 Nebraska Medical Center

Omaha, NE 68198-6025 USA

Yazen Alnouti, Rhishikesh Thakare

Department of Chemistry and Biochemistry and Department of Pharmacology

School of Medicine

University of California, San Diego

9500 Gilman Drive

La Jolla, CA 92093-0601 USA

Aaron Armando, Edward A. Dennis, Jiang Jiang

Department of Medicine and Department of Pharmacology

School of Medicine, University of California, San Diego

9500 Gilman Drive, La Jolla, CA 92093-0601 USA

Oswald Quehenberger

Division of Signal Transduction

Beth Israel Deaconess Medical Center

Boston, MA USA

John M. Asara, Min Yuan, Susanne B. Breitkopf

Division of Metabolomics

Research Center for Transomics Medicine 


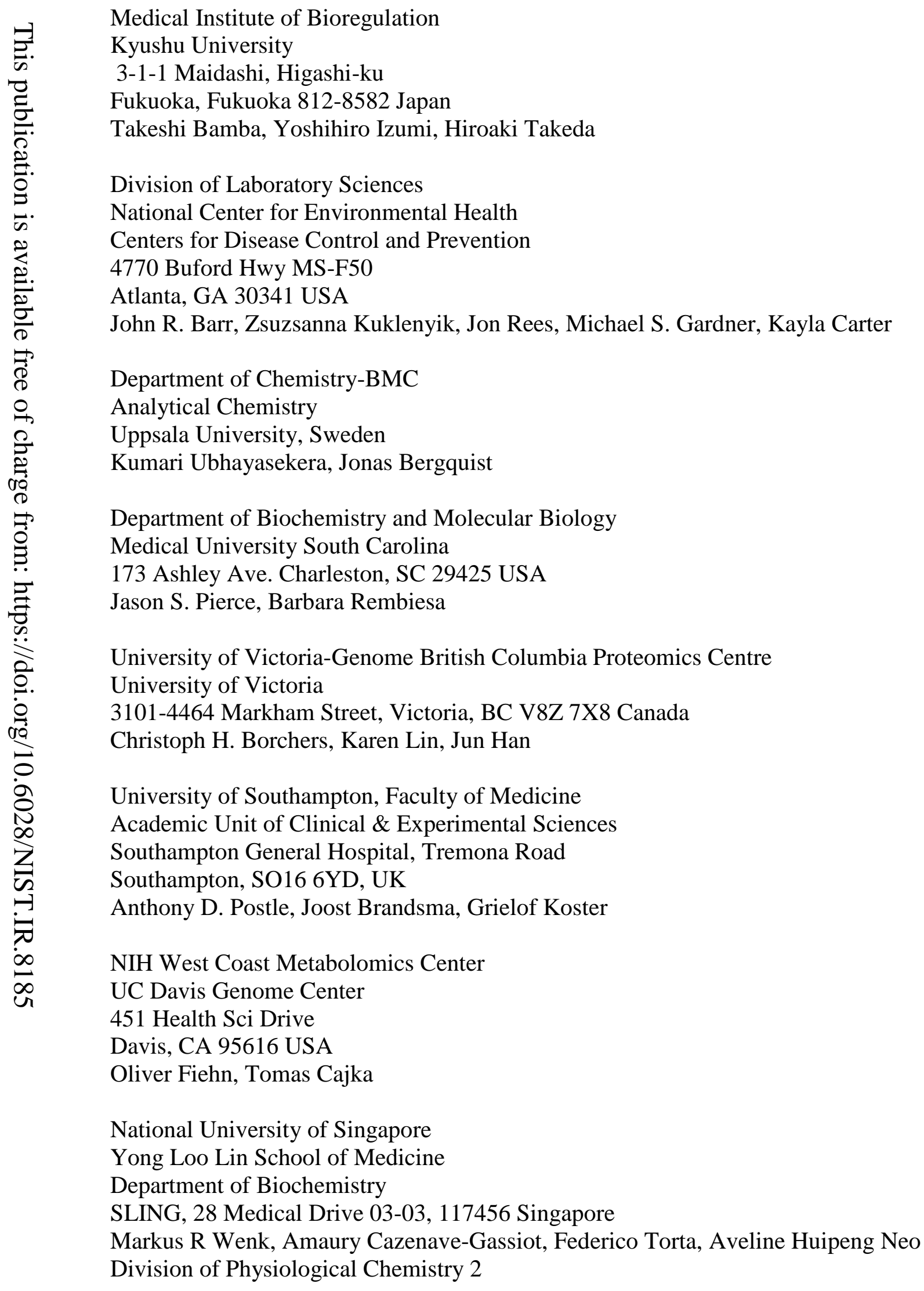




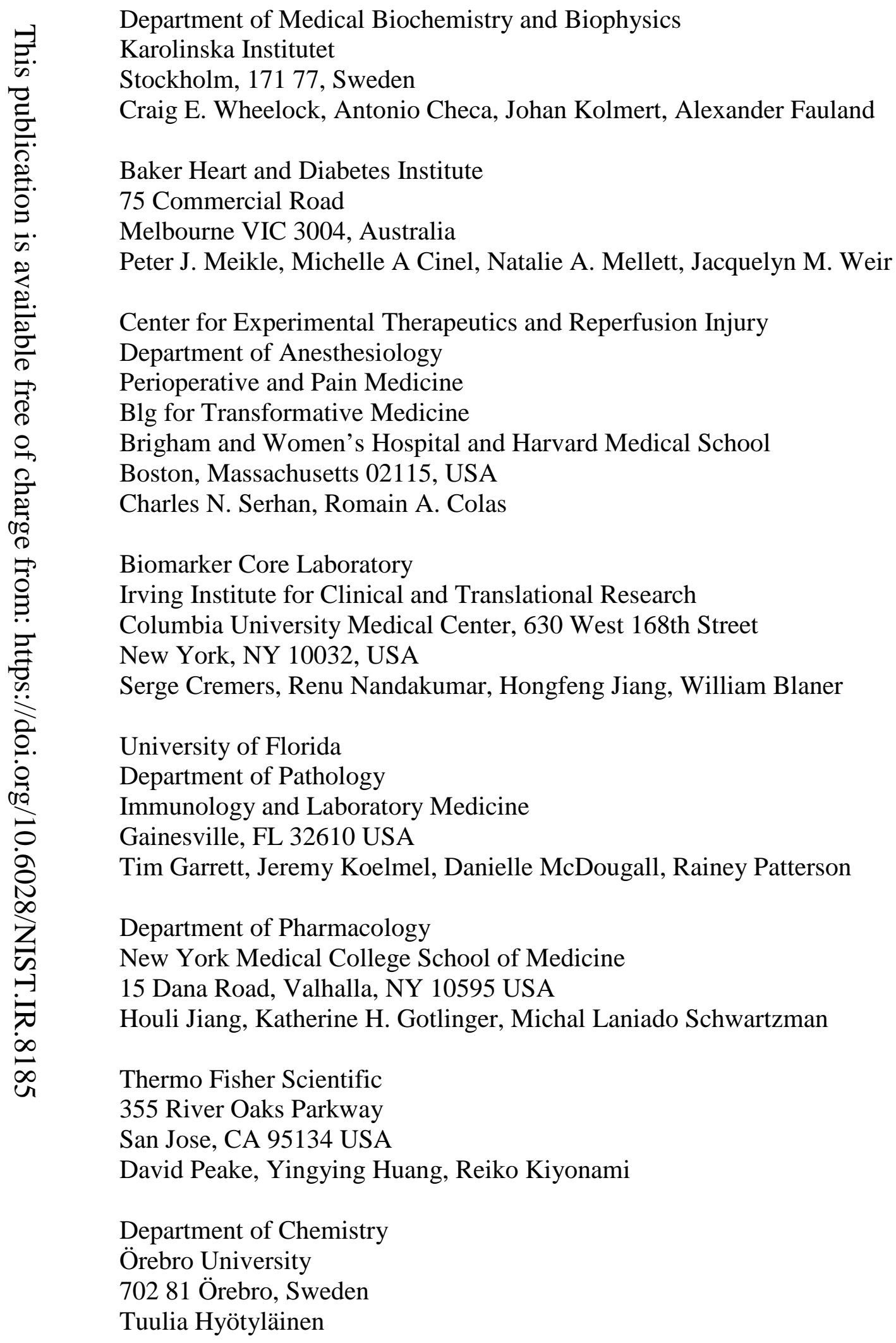




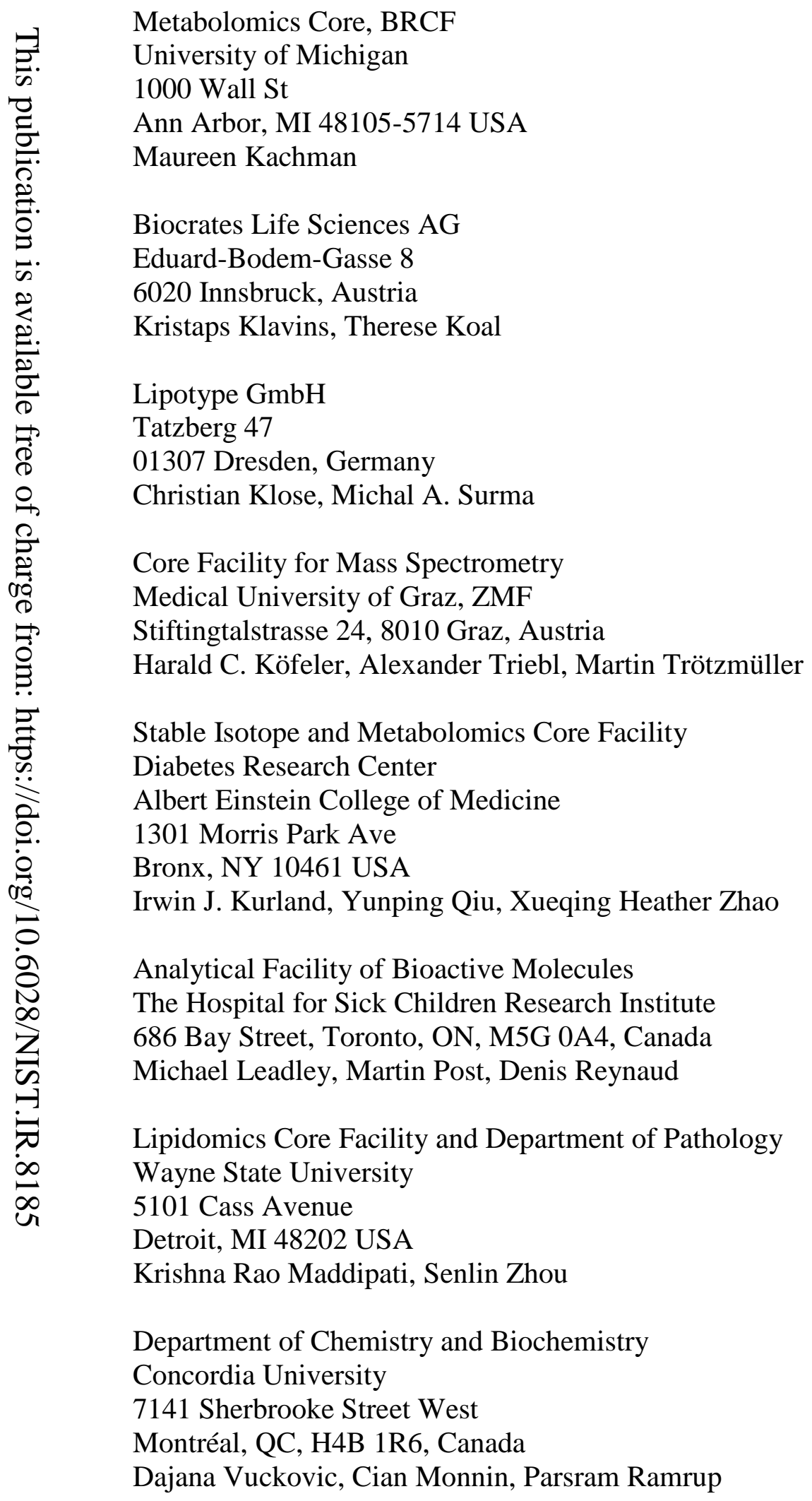




$\begin{array}{ll} & \text { Proteomics and Metabolomics Shared Resource } \\ & \text { Duke University School of Medicine, B02 Levine Science Research Center } \\ & \text { 450 Research Drive } \\ \text { Durham, NC 27710 USA } & \text { M. Arthur Moseley, Lisa St. John-Williams, J. Will Thompson } \\ & \text { Turku Centre for Biotechnology } \\ \text { University of Turku and Åbo Akademi University }\end{array}$

